ร1"

\title{
is
}





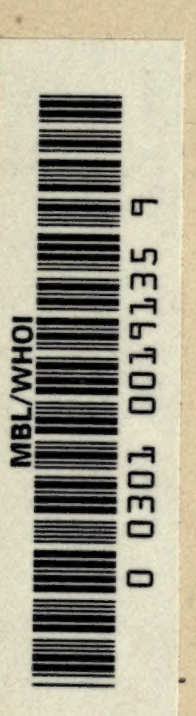





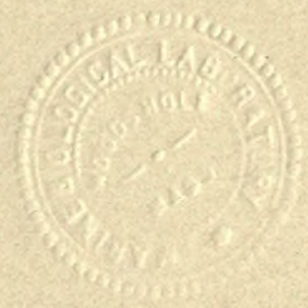





\section{ANATOMY \\ OF THE \\ BRAIN AND SPINAL CORD \\ SANTEE}





\section{ANATOMY}

OF THE

\section{BRAIN AND SPINAL CORD}

WITH SPECIAL REFERENCE TO

\section{MECHANISM AND FUNCTION}

FOR STUDENTS AND PRACTITIONERS

BY

HARRIS E. SANTEE, M. D., Ph. D.

PROFBSSOR L $P$ ANATOMY IN THE COLLEGE OF PHYSICIANS AND SURGEONS, MEDICAL DEPARTMBNT, UNIVERSITY OF ILLINOIS; PROFESSOR OF ANATOMY IN JENNBR MBDICAL COLLBgE, CHICAGO; MEMBER OF ASSOCIATION OF AMERICAN ANATOMISTS.

FOURTH EDITION, REVISED AND ENLARGED

WITH 128 ILLUSTRATIONS, 33 OF WHICH ARE PRINTED IN COLORS

PHILADELPHIA

P. BLAKISTON'S SON \& CO.

1012 WALNUT STREET

1907 
Copyright, rgo7, By P. Blakiston's Son \& Co.

$$
21
$$




\section{PREFACE}

THE author hopes that the present edition of this work may receive from his colleagues the same generous consideration accorded to the former editions. His endeavor has been to set forth the present status of the anatomy of the human brain and spinal cord: To do this the facts have been gleaned from many sources; and, so far as was possible, from the original sources. Being designed for a text-book, the subject-matter is presented in the order found convenient to the dissector. The description proceeds from the gross structures to the constituent neurones in each successive region. Wherever the embryology will assist the student to comprehend the adult forms, the development is briefly given in the text; but a special chapter is also devoted to embryology, which presents a concise and connected statement of the development of the entire brain and spinal cord.

The special objects held in view throughout the book are the location of functional centers and the tracing of their afferent, associative and efferent connections. Particular emphasis is laid upon the origin, course, termination and function of conduction paths as they are met in the regular study, and the more important and better known of these paths are summed up in a final chapter on the tracing of impulses. Function is everywhere correlated with structure; and so far as present knowledge permits, the function of each group of neurones is given in connection with its anatomical description.

The BNA Nomenclature is followed almost without exception, the English equivalents of the Latin terms being very largely employed.

Keeping pace with the lectures, every student is expected to dissect the human brain in the laboratory, exposing, studying and sketching every macroscopic structure as. it occurs in the work; 
and, then with the microscope, examine the minute structure and picture the histology of the same parts. For these purposes the class should be taken in small sections, divided into groups of two to four students, and each group should be provided with a well hardened human brain. It is desirable that each student should receive a well stained microscopic section of every important part. This is, however, often impossible; and the instructor may get along with considerable satisfaction, by having the students exchange, if he has but a few sets of slides.

The author wishes to acknowledge his indebtedness to recent literature and to standard works on anatomy. McMurrich's "Development of The Human Body," Barker's "BNA" and Barker's "Nervous System," the work of Dr. Alfred W. Campbell, "Histological Studies on the Localization of Cerebral Function," the "Text-book of Anatomy" and "Memoirs" of D. J. Cunningham, F. R. S., and the fourth edition of Morris's "Human Anatomy" have been especially useful.

I desire to express my appreciation of the kindly assistance of my colleague, Prof. Wm. T. Eckley, M. D.; and to say that the artistic merit of the new illustrations in this book belongs to my friend and pupil, Mr. Zan D. Klopper, of Chicago, who sketched the original drawings from my own specimens. My thanks are also due to the publishers of this little monograph, for many favors and courtesies shown me, particularly for allowing me to select illustrations from Gordinier, McMurrich and Morris, works published by them.

Harris E. SANTEE. 


\section{TABLE OF CONTENTS.}

\section{CHAPTER I. \\ THE MENINGES OF THE BRAIN.}

Dura Mater of the Brain:....................... I

Structure and relations........................ I

Processes ................................. I

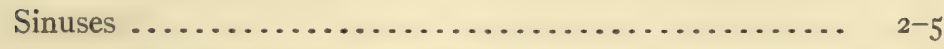

Arachnoid granulations (Pacchioni) ............... 5

Arteries ................................ $6-7$

Nerves ................................ 7

Contrasted with dura of spinal cord............... 7

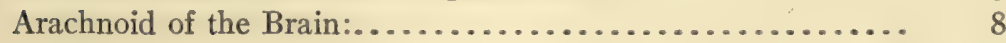

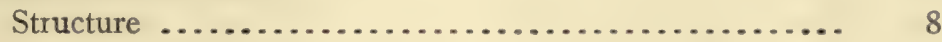

Relations, subarachnoid spaces................ 8 8-9

Vessels and nerves......................... 9

Contrasted with arachnoid of the cord.............. 9

Pia Mater of the Brain:......................... 9

Structure and relations..................... 9-10

Chorioid tela of third and fourth ventricles .......... ro

Arteries and veins......................... 10

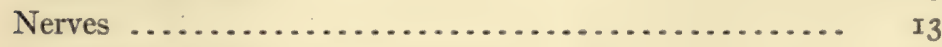

Contrasted with pia of spinal cord................. $x_{3}$

Blood Supply of the Brain:..................... I4-30

Carotid and vertebral arteries................... I4

A. Cerebral Circulation, Arteries:.................. I4-2I

Arterial circle (Willisi) and branches............... I4-I5

Ar. Cortical system of arteries................. I5-18

Anterior cerebral artery...................... $\quad$ x6

Middle cerebral artery...................... $16-17$

Posterior cerebral artery...................... ${ }^{17}$

Chorioidal arteries, posterior and anterior.......... I7-18

A2. Ganglionic system of arteries ............... I8-2r 
Antero-median, ganglionic arteries............... I8

Antero-lateral ganglionic arteries................ r8

Postero-median ganglionic arteries.............. I8-2I

Postero-lateral ganglionic arteries................ 2I

The Veins of the Cerebrum:.................. $2 \mathrm{I}-24$

Internal veins of the cerebrum.................. $2 \mathrm{I}$

Great vein of the cerebrum (Galeni) .............. $2 \mathrm{I}-22$

External veins of the cerebrum.................. 22-23

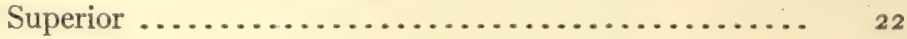

Medial................................22-23

Inferior .............................. ${ }^{23}$

Lymphatics of cerebrum..................... $23-24$

B. Circulation of the Rhombencephalon:............ 24-30

B. The medulla oblongata..................... 24

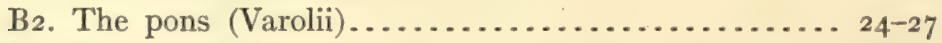

B3. The cerebellum.................................. 270

Superior cerebellar artery ................... 27-28

Anterior inferior cerebellar artery.............. 29

Posterior inferior cerebellar artery............... 29

Internal cerebellar veins.................... 29

External cerebellar veins.................... 29-30

Superior ............................ 29

Inferior .............................. 29-30

Lymphatics of cerebellum................... 30

Table I. Embryologic Divisions of the Brain........... 30-33

Components of cerebrum.................... 33

Components of rhombencephalon............... 33

CHAPTER II.

\section{GENERAL CONSIDERATION OF THE BRAIN.}

Embryonic Brain Vesicles......................... 34

Their cavities-the ventricles .................. 34

Superior view................................. 34-35

Posterior view ................................. $35-36$

Inferior view................................ $36-4 \mathrm{I}$

Anterior area............................ 36-37

Middle area ................................. $37-38$

Posterior area............................ $3^{8-4 \mathrm{I}}$ 
Roots of the Twelve Cerebral Nerves:.............. 42-49

Nuclei, genetic and terminal.................... 42

Olfactory nerves........................... $\quad 42$

Optic nerve............................... 42

Oculo-motor nerve....................... 42-45

Trochlear nerve............................. 45

Trigeminal nerve.......................... 45

Abducent nerve........................... 45

Facial nerve.......................... $45^{-46}$

Intermediate nerve..................... $45-46$

Acustic nerve............................. 46

Glossopharyngeal nerve........................ ${ }_{46} 6$

Vagus nerve............................. $46-49$

Accessory nerve............................ 49

Hypoglossal nerve.......................... 49

CHAPTER III.

THE CEREBRUM.

Subdivisions:

End-brain.......................... 50

Inter-brain .............................. 50

Mid-brain............................... 50

Section I. The Fore-brain or Prosencephalon. ..... 50-I44

Exterior surface of fore-brain ............... $5^{\mathrm{I}-98}$

Definition of fissure and sulcus................ $5^{2}$

Subdivisions and borders.................... $5^{2}$

Convex, medial and basal.................. $5^{2}$

Fissures and sulci of convex surface........... $5^{2-73}$

Longitudinal fissure of cerebrum............. $5^{2-55}$

Transverse fissure of cerebrum............... 55

Lateral fissure of cerebrum (Sylvii)........... $55-56$

Sulcus centralis (Rolandi)................... ${ }_{56}^{6}$

Occipito-parietal sulcus................... ${ }_{56}^{6-59}$

Lobes and gyri of convex surface............. 59 59.73

Frontal lobe, its gyri and sulci............... $59-63$

Parietal lobe, its gyri and sulci................ . 63-67

Occipital lobe, its gyri and sulci............. 67-69 
Temporal lobe, its gyri and sulci.................

Superior surface.........................

External surface....................... 70-71

Island (Reili), its sulci and gyri.............. $7 \mathrm{I}-73$

The base of the fore-brain................... $73-85$

Frontal lobe, inferior surface.............. $73-74$

Island (Reili), inferior surface............... . 74-77

Rhinencephalon .......................... $\quad 77-79$

Olfactory lobe....................... $\quad 77-79$

Olfactory bulb ....................... $77-78$

Olfactory tract and striæ ................ $\quad 78$

Olfactory triangle....................... $\quad 79$

Parolfactory area (Brocæ)................ $\quad 79$

Anterior perforated substance............... $\quad 79$

Tentorial area of basal surface.............. $79-82$

Chorioidal fissure....................... 80

Hippocampal fissure...................... 80

Ectorhinal sulcus...................... $80-8 \mathrm{I}$

Fissura collateralis..................... $8 \mathrm{I}$

Inferior temporal sulcus................... $8 \mathrm{I}$

Gyrus fusiformis.................... $8 \mathrm{I}$

Gyrus lingualis......................... $8 \mathrm{I}$

Limbic lobe, inferior part................ $8 \mathrm{x}-82$

Gyrus hippocampi and uncus.............. $8 \mathrm{I}-82$

Dentate fascia........................ $\quad 8_{2}$

Hypothalamus....................... . $8_{2}-85$

Pars optica hypothalami................. $\quad 82-84$

Lamina cinerea terminalis................ $8_{3}$

Optic chiasma, nerves and tracts.......... $83-84$

Tuber cinereum and infundibulum........... 84

Hypophysis (pituitary body).............. $\quad 84$

Pars mammillaris hypothalami.............. 82 and 84

Corpora mammillaria................... $\quad 84$

Fissures and sulci of medial and tentorial surface ..... $84-98$

Sulcus cinguli (calloso-marginal).............. 86

Subparietal sulcus .......................... $\quad 86$

Callosal sulcus......................... $86-89$

Occipito-parietal sulcus.................... $\quad 89$

Calcarine fissure...................... $89-90$

Hippocampal fissure.................... 90 
Chorioidal fissure....................... 90

Collateral fissure........................ 9. 90.93

Ectorhinal sulcus......................... 90 90-93

Inferior temporal sulcus .................... 93

Gyri of medial and tentorial surface........... 9 93-98

Gyrus fornicatus....................... 93-97

Gyrus cinguli......................... 93-94

Gyrus hippocampi..................... 94-97

Uncus......................... $94-97$

Lobus pyraformis................... 97

Limbic lobe, rhinencephalon................ 97

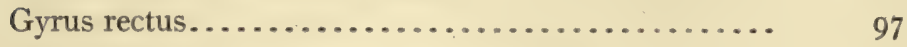

Gyrus frontalis superior (g. marginalis)......... 97

Lobulus paracentralis..................... 97

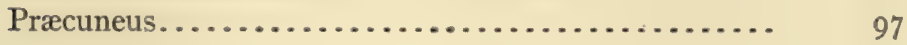

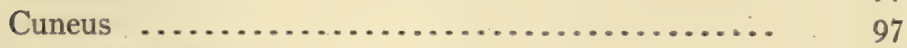

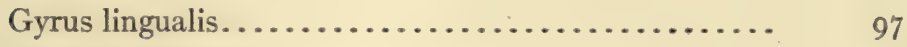

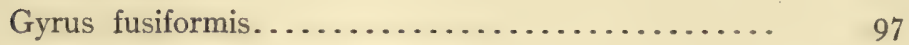

Summary of lobes of the cerebrum............. 98

Neopallium.......................... . . 98

Rhinencephalon (archipallium).............. 98

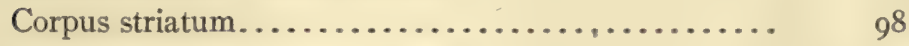

Interior surface of the fore-brain............. 98-I44

Internal capsule. . . . . . . . . . . . . . . . . . . 99-104

Inferior lamina..................... 9. 99-100

Motor fibers..................... I00

Sensory fibers..................... 100

Superior lamina...................... 100-104

Genu, frontal and occipital parts........... 103

Motor fibers......................... I0 3

Common sensory fibers................. I03-104

Special sense fibers.................... 104

Corpus callosum:...................... 104-108

Upper surface......................... 107

Gyri supracallosus and subcallosus........... 107

Inferior surface...................... I07

Borders, posterior and anterior............... 107-108

Splenium, rostrum, genu, truncus........... I08

Boundaries of general cavity of fore-brain ......... I08-109

Body of fornix..................... Io9 
Crus fornicis........................ IIo

Columnæ fornicis........................ n

Septum pellucidum........................ III

Fifth ventricle....................... III-II

Lateral ventricle and its boundaries........... II2-I27

Central part (body)..................... II .

Corpus striatum.................... II5-II9

Lentiform nucleus................... II6

Nucleus caudatus................... I16-II9

Stria terminalis................. II

Thalamus......................... II

Chorioid plexus of lateral ventricle.......... I20

Anterior horn of the lateral ventricle ............ I20-123

Posterior horn......................... I ${ }_{12}$

Inferior horn...................... I23-127

Trigonum collaterale................... $\quad$ I24

Hippocampus, its digitations ............. I24

Chorioid epithelium..................... I24

Third Ventricle and Inter-Brain............. I27-I44

Posterior commissure.................... ${ }_{132}$

Roof epithelium....................... 132

Pineal body. . . . . . . . . . . . . . . . . . . . ${ }^{132-135}$

Chorioid tela of third ventricle............. I35

Anterior commissure................... 136-139

Lamina terminalis ...................... I39

Thalamus.......................... I39-143

Extremities-anterior and posterior........... I I0

Surfaces-medial, superior, lateral and inferior.. I40-I43

Tegmental hypothalamic region............. I43

Nucleus hypothalamicus (Luysi)............. $\quad{ }_{143}$

Lateral geniculate body................... I ${ }_{43}$

Medial geniculate body................. I43-I 44

Section II. The Mid Brain (Mesencephalon):. . . . . I44-I64

Surfaces-superior, inferior, anterior, posterior ....... I44-146

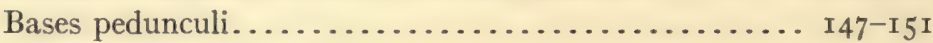

Intermediate bundle..................... I4

Temporo-pontal tract....................... ${ }_{148}$

Pyramidal tract ........................ I48

Fronto-pontal tract....................... ${ }_{15}$ 
PAGE

Substantia nigra....................... ${ }^{I_{5}} \mathrm{I}$

Tegmenta............................ $\mathbf{1 5}^{2} \mathbf{5}^{2} \mathbf{1 6}_{3}$

Cerebral aqueduct (Sylvii)................... ${ }^{1} 5^{2}$

Nuclei of oculo-motor and trochlear nerves......... ${ }^{152-155}$

Mesencephalic nucleus of trigeminal nerve........ $\quad 155$

Formatio-reticularis..................... ${ }_{155}-163$

Tegmental decussations..................... ${ }^{155}$

Tracts of fibers in the tegmentum........... $155-163$

Medial (or posterior) longitudinal bundle........ $155^{-15} 8$

Anterior longitudinal bundle ................ I58-159

Fillet or lemniscus.................. 159-16r

Medial fillet-superior fillet............... I59-I60

Lateral fillet ...................... I60-I6I

Spino-thalamic tract..................... I6I-I62

Brachium conjunctivum................ $\quad{ }_{162}$

Rubro-spinal tract........................ ${ }_{162}$

Olivary fasciculus..................... $\quad \mathrm{I}_{62}$

Descending root of trigeminal nerve............ $\quad{ }_{16}$

Quadrigeminal lamina ..................... I6 $6_{3}-164$

Colliculus superior...................... $\quad{ }_{16} 6_{3}$

Colliculus inferior...................... I6 $33^{-164}$

Brachium superius......................... I6

Brachium inferius ..................... I6

Section III. Structure of the Cerebrum:......... r64-24I

The neurone or nerve cell................. I67-I73

Cell-body, perikaryon, or neurone center......... $\quad$ I67

Dendrites ............................. ${ }_{16} 67$

Axone................................ ${ }_{168}$

Myelin sheath .............................. ${ }_{16} 68$

Types of neurones (three).................. $\quad$ I68

Orders of neurones (Ist, 2 d, etc.)............. I68-I 7 I

Functions of neurones................... I7

Degeneration of neurones.................. I 1 I-I 72

Development of neurones................. I $172-173$

Sustentacular tissue...................... $\quad{ }^{173}$

Epiblastic ............................ $173-174$

Neuroglia and ependyma................ I73-174

Mesoblastic connective tissue................. 174

Cortical gray matter...................... I74-204 
Cortical or cerebral localization $\ldots \ldots \ldots \ldots \ldots \ldots \ldots \times \ldots \ldots$

Motor area, emissive.................. $\quad$ I77

Psychic motor area....................... ${ }_{17} 8$

Common sensory area.................. ${ }_{17} 8$

Psychic sensory area..................... $\quad{ }_{17} 8$

Acustic center........................ I8I

Optic center......................... I8I

Olfactory and gustatory centers............. $18 \mathrm{I}$

Naming center........................ I $8 \mathrm{r}$

Centers of intonation, equilibration and orientation $18 \mathrm{I}$

Anterior association center, abstract conceptions.... $\quad$ I82

Posterior association center, concrete conceptions.... I82-183

Middle association center................. ${ }_{18} 8_{3}$

Cell and fiber lamination $. . \ldots \ldots \ldots \ldots \ldots \ldots \ldots . . . \ldots \ldots 3^{-204}$

Plexiform layer....................... I $84-187$

Layer of small pyramids ............... 184 and 187

Layer of medium-sized pyramids.......... I84 and 187

External layer of large pyramids......... 184 and $\mathrm{I} 87-\mathrm{I} 88$

Layer of stellate cells................. I84 and 188

Internal layer of large pyramids........ I84 and I88-I9I

Layer of fusiform cells................. 184 and $19 \mathrm{r}$

Radiations of Meynert....................... I92

Association fibers of Meynert................. 192

Atypical cortex: ........................ $192-204$

Visual cortex.......................... I92-I95

Olfactory cortex...................... 195

Olfactory bulb...................... I95-196

Uncus hippocampi.................... 196-199

Nucleus amygdalæ................... $\quad$ I99

Subiculum.......................... 199

Fascia dentata......................... 200

Trigonum olfactorium, etc.............. 200-203

Gyrus cinguli........................ $\quad 203$

Claustrum.......................... $\quad 204$

Ganglionar gray matter..................... 204-22 I

Corpus striatum........................ 204-208

Centrifugal fibers....................... 207-208

Centripetal fibers......................... 208

Thalamus........................... 208-217

Thalamic nuclei...................... 208-2I I 
White matter of the thalamus.

Occipito-thalamic radiation (optic) .............. 216-2I7

Temporo-thalamic radiation (acustic)

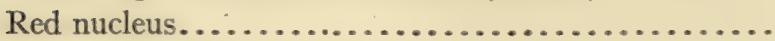

Nucleus hypothalamicus......................

Metathalamus.

Lateral geniculate body......................

Medial geniculate body ................... 218-219

Superior colliculi of corpora quadrigemina........ 219-220

Anterior longitudinal bundle ................ $2 I^{2} 9^{-220}$

Inferior colliculi of corpora quadrigemina......... 220

Nucleus lateralis superior................... 220

Substantia nigra......................... 22I

Central or ventricular gray matter.............. 22 I-224

Hypothalamus......................... 22I-222

Pars optica........................... 221

- Pars mammillaris........................ 22I-222

Massa intermedia (middle commissure)........... 222

Stratum griseum centrale of mid-brain.......... 222-224

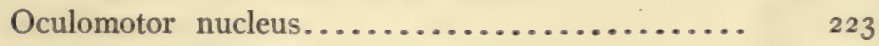

Trochlear nucleus........................ 223

Trigeminal nucleus of mid-brain.............. 224

Projection fibers of the cerebrum............. 224-234

Corticifugal, or motor fibers .................225-23I

Intermediate tract........................ 225

Fronto-pontal tract....................... 225

Tempora-pontal tract.................... 225-226

Pyramidal tract...................... 226-23I

Head and neck fibers.................. 227

Upper extremity fibers.................. $\quad 228$

Trunk fibers......................... $\quad 228$

Lower extremity fibers.................... 228-23I

Destruction of by clot, etc................. 231

Sensory or corticipetal fibers................. 23I 234

Medial fillet, spino-thalamic tract and brachium confunctivum ........................ 23 I

Olfactory projection fibers................. 232

Cortical fillet (common sensory) ............. 232-233

Taste fibers (not located)................. 233 
PAG

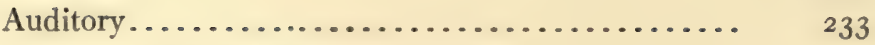

Lateral fillet and brachium inferius.......... 233

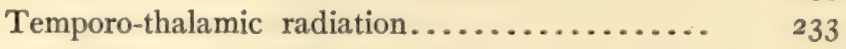

Occipito-thalamic radiation (optic)............ 234

Commissural fibers of cerebrum .................. 234-236

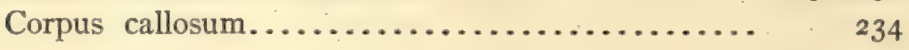

Anterior commissure.................... 234-235

Commissura hippocampi.................... ${ }_{236}$

Association fibers of cerebrum ................ 236-24I

Short association fibers.................. 236-238

Long association fibers.................. 238-24I

Cingulum of gyrus fornicatus................... 238

Fornix ............................ 238-240

Uncinate fasciculus.................... 240

Superior longitudinal fasciculus.............. 240

Inferior longitudinal fasciculus. . . . . . . . . . . 240 24I

Fasciculus occipito-frontalis................ 24I

Perpendicular fasciculus................... 24 I

CHAPTER IV.

\section{THE RHOMBENCEPHALON.}

Section I. The Cerebellum:.................. 242-266

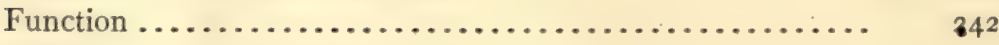

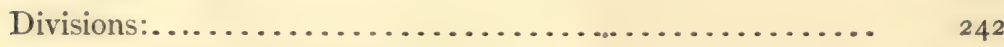

Cerebellar hemispheres................... 242-243

Vermis cerebelli or worm................... 243

Cerebellar notches, anterior and posterior......... 244

Medullary Body:.......................... 244-247

Inferior medullary velum ................... 244

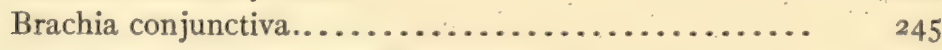

Superior medullary velum (Vieussensi)........... 246

Corpora restiformia..................... 246-247

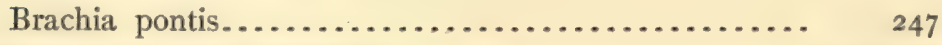

Horizontal sulcus of cerebellum ............... 247-248

Superior Surface of Cerebellum:................ 248-25I

Sulci of upper surface..................... 248-249

Precentral sulcus........................... 248

Postcentral sulcus....................... 249 
Predeclivil sulcus............................... $\quad 249$

Postdeclivil sulcus....................... 249

Lobes of superior surface..................... 250-25I

Lobus lingulæ.......................... 250

Lobus centralis......................... $\quad 250$

Lobus culminis monticuli.................. $\quad 250$

Lobus declivis monticuli.................... $\quad 25 \mathrm{I}$

Lobus folii vermis.................... $25 \mathrm{I}$

Inferior Surface of Cerebellum:.................. $25^{1-256}$

Sulci of lower surface..................... 252-253

Postnodular sulcus........................ $\quad 25^{2}$

Prepyramidal sulcus ..................... $25^{2-253}$

Postpyramidal sulcus....................... $\quad 253$

Midgracile and postgracile..................... $\quad 253$

Lobes of lower surface. . . . . . . . . . . . . . . . $253^{-256}$

Lobus noduli ........................... 253-254

Lobus uvulæ............................ 254

Lobus pyramidis ........................... 254-255

Lobus tuberis ......................... ${ }^{255^{-2}} 5^{6}$

Cortical Gray Matter of the Cerebellum............. 256-260

Superficial layer............................. $256-258$

Stratum cinereum ....................... $\quad 256$

Stratum gangliosum ..................... 257.

Cells of Purkinje and stellate cells............. 257

Fibers of superficial layer................... $257^{-258}$

Deep layer (stratum granulosum) . . . . . . . . . 25 25-260

Cells of granular layer...................... 258-259

Fibers of granular layer.................... $\quad 260$

Function of stellate, granule and Purkinje cells...... 260

Neuroglia of cerebellum..................... $\quad 260$

Ganglionar Gray Matter of Cerebellum ............. 260-262

Function, relay stations ..................... $\quad 260$

Nucleus dentatus....................... 260-26I

Nn. emboliformis, globosus and fastigii........... 26 I

White Substance-Corpus Medullare: . . . . . . . . . . 26. 262-266

Projection fibers........................ ${ }_{26} 63^{-26} 6_{5}$

Brachium conjunctivum................... 263

Superior medullary velum .................. ${ }_{26}^{26}-264$

Brachium pontis ........................ ${ }_{26} 264$

Corpus restiforme ....................... $26_{4}^{-26}$ 
Commissural fibers

Association fibers......................... $265^{-266}$

Section II. The Pons (Varolir) ................. 266-284

Surfaces:............................ 266-270

Superior and inferior..................... $\quad 266$

Anterior (Tuber annulare).................. 266-269

Posterior-ventricular, and attached part........ 269-270

Transverse fibers of the pons................. 27 I-272

Superficial of pars basilaris pontis............. $27 \mathrm{I}$

Deep transverse of pars basilaris pontis......... $27 \mathrm{I}$

Transverse of pars dorsalis pontis.............27I-272

Trapezoid body........................ 272

Longitudinal fibers of the pons............... $272-278$

Ventral .............................. 272

Dorsal .................................. $272-278$

Medial fillet and superior fillet ............. 273-274

Lateral fillet............................ ${ }_{272}$

Spino-thalamic tract................... $\quad 272$

Ascending anterior cerebello-spinal tract ........ $272-276$

Gowers's tract.......................... $\quad 276$

Medial longitudinal bundle................ $\quad 276$

Anterior longitudinal bundle ............... $\quad 276$

Rubro-spinal tract..................... 276-277

Olivary bundle....................... $\quad{ }_{277}$

Descending root of trigeminal nerve........... $\quad 277$

Spinal tract n. trigemini .................. $\quad 277$

Gray Matter of the pons.................... 277-284

Nucleus pontis ......................... ${ }^{277}$

Stratum nucleare....................... ${ }_{27} 78-284$

Superior olivary nucleus................... 278-279

N. præolivaris and n. semilunaris............. $\quad 279$

Nucleus of trapezoid body ................... $\quad 279$

Nuclei of recticular formation.............. 279-280

Ponto-spinal tracts (Collieri) ................ 280

Nuclei of trigeminal nerve.................. $\quad 28 \mathrm{I}$

Genetic (motor) ............................. $28 \mathrm{I}$

Terminal (sensory) ....................... $28 \mathrm{I}$

Nucleus of abducent nerve................. 28I-282

Nucleus of facial nerve.................... $\quad 282$ 
Salivary nucleus.

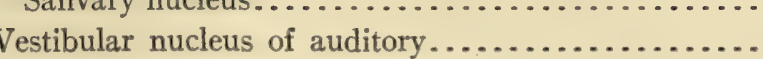

Lesions in pons

Section III. Medulla Oblongata (Myelencephalon).. 284-3i3

Origin

Ventricle

Surfaces

Anterior lateral sulcus.

Posterior lateral sulcus...................... $\quad{ }_{286}$

Anterior surface....................... 286-287

Lateral surface......................... $287-288$

Olive ............................... $\quad{ }_{28} 87$

Lateral column........................ $282_{7}-288$

Posterior surface ...................... 288-290

Restiform body........................ ${ }_{2} 88$

Roof epithelium of fourth ventricle........... $\quad 289$

Floor of fourth ventricle.................. 290

White matter of medulla ................. 290-302

Substantia reticularis ..................... 291

Raphe ............................... 291

Transverse fibers....................... 29I 292

Pyramidal decussation .................. 29I

Arcuate fibers, external and internal ......... 29I-292

Fillet decussation.................... . 292

Cerebello-olivary fibers.................. $\quad 292$

Dorso-ventral fibers.................... 292-293

Anterior external arcuate................. ' 292

Roots of eighth to twelfth cerebral nerves....... 293

Longitudinal fibers of anterior area:............ 293-298

Pyramid, anterior and lateral tracts............ : 294

Medial fillet (interolivary stage) .............. 295

Medial longitudinal bundle (posterior)......... 296

Anterior longitudinal bundle................. $\quad 297$

Longitudinal fibers of lateral area ............. 298-300

Fasciculus lateralis proprius............... $\quad 298$

Descending anterior cerebello-spinal tract........ 298

Ascending anterior cerebello-spinal tract ........ 299

Rubro-spinal tract........................ 300

Longitudinal fibers of posterior area ........... 300-302 
PAGE

Funiculus gracilis...................... 300

Funiculus cuneatus...................... 300

Spinal tract of trigeminal nerve.............. 30I

(Posterior) Cerebello-spinal tract .............. 30I

Restiform body ...................... 301

Vestibular and cochlear nuclei............ 302

Tractus solitarius...................... 302

Gray matter of the medulla .................. 302-313

Nucleus of external arcuate fibers............ 302

Nuclei in floor of ventricle (s. nucleare).......... 303-310

Hypoglossal nucleus ....................... 303

Nucleus lateralis inferior................. 303-304

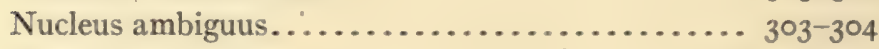

Nuclei alæ cincerex .................. 304 and 306

Nucleus tractus solitarii................... 306

Nucleus tractus spinalis $n$. trigemini........... 306

Vestibular nuclei ..................... 307-308

Cochlear nuclei ..................... 309-310

Special nuclei in medulla .................. $33^{10}-3^{1} 3$

Nucleus funiculi gracilis. . . . . . . . . . . . . 310-31 I

Nucleus funiculi cuneati................. 310-3II

Nucleus olivaris inferior................ $3^{12}$

Section IV. The fourth Ventricle........... 313-325

Boundaries ............................ 31 $3-3$ r4

Floor of fourth ventricle...................... 314-3I8

Colliculus facialis....................... $3{ }^{1} 7$

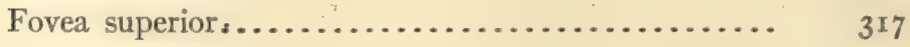

Locus cæruleus ............................... 317

Hypoglossal triangle....................... $33^{17}$

Ala cinerea (trigonum vagi)................. 317

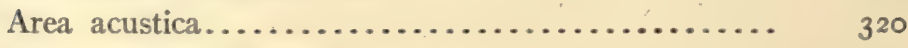

Origin of cerebral nerves..................... 320-325

Table II. Sensory Nerves and Roots.... . . . . . . . 320-32I

Table III. Motor Nerves and Roots ............ 321-322

Terminal nuclei........................... 322-324

Common sensory....................... 322

Cortical and reflex connections............. 322

Special sense.......................... 322-324

Cortical and reflex connections............ 324 
Genetic nuclei. 324-325

Cortical and reflex connections $324-325$

\section{CHAPTER V. \\ MEMBRANES OF THE SPINAL CORD.}

Dura mater................................. 326

Arachnoid .......................................... 326-329

Pia mater ............................... 329-330

Blood Supply of the Spinal Cord................ 330-332

Spinal arteries, anterior and posterior ............. 330-332

Fissural or centrifugal..................... 33 I

Centripetal ............................ $33^{1-332}$

Venæ spinales internæ..................... 332

Root and fissural veins................... 332

Venæ spinales externæ.................... 332

Internal vertebral plexus..................... 332

Lymphatics............................ $\quad 33^{2}$

CHAPTER VI.

THE SPINAL CORD.

Extent

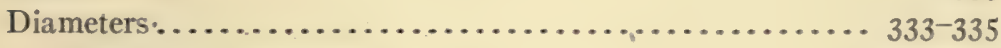

Cervical enlargement..................... 333-334

Lumbar enlargement. ........................ 334-335

Sixth ventricle (canalis centralis spinalis) ............ 337

Fissures of the cord:........................ $337-33^{8}$

Anterior median fissure .................. 337

Posterior median fissure................... $\quad 337$

Posterior lateral sulcus...................... $337-33^{8}$

Anterior root-line (s. lateralis anterior)............ 338

Posterior intermediate sulcus................... $\quad 338$

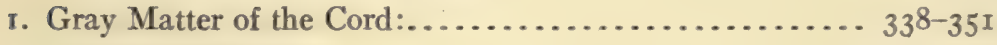

H-shaped column ........................ 339

Substantia gelatinosa ................... 339

Substantia spongiosa...................... $\quad 339$

Gray crescent.............................. 339-349

Anterior columna......................... 339-344

Cells of anterior columna .................. $340-344$ 
Golgi cells.................................. 340

Deiters cells ........................ . 340

Medial column...................... 340

Lateral column .................... 340, 343-344

Cortical connection..................... 343

Reflex mechanism ................... 343-344

Lesions of anterior columna................. 344

Center of crescent and columna lateralis......... 344-347

Intermedio-lateral column of cell-bodies (efferent

sympathetic neurones) ................ 344-347

Posterior columna....................... 347-349

Neurones of head of posterior columna....... 347-348

Nucleus dorsalis (Clarki) terminal nucleus of sym-

pathetic) .......................... 348-349

Gray commissure of spinal cord ............ 350

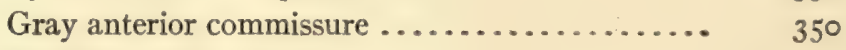

Posterior commissure .................. 350

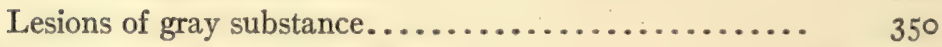

2. White Matter of the Spinal Cord: .............. 35 $5^{\mathrm{I}-362}$

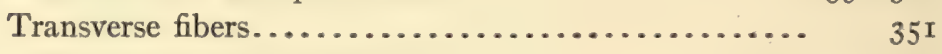

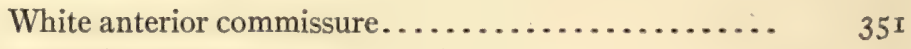

Dorso-ventral fibers...................... $35=352$

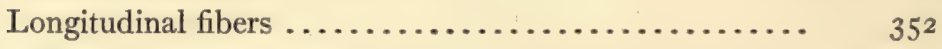

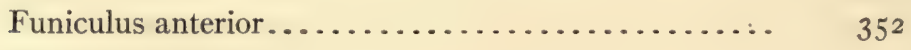

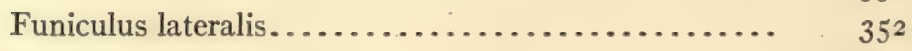

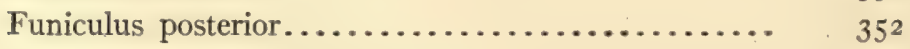

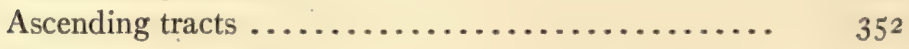

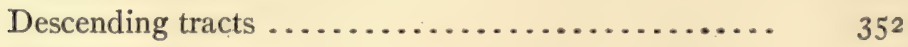

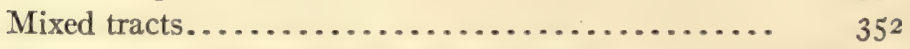

Embryological method of locating tracts........ 352-353

Pathological and experimental method ........... 353

Tracts of the Spinal Cord . . . . . . . . . . . . . . . . 354-362

Antero-lateral fasciculus proprius............... 354

Medial longitudinal bundle................ 354

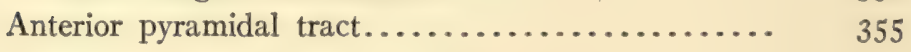

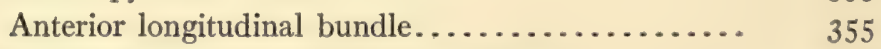

Descending anterior cerebello-spinal tract......... 356

Ascending anterior cerebello-spinal tract......... 356-357

Spino-thalamic tract ..................... 356-357

Triangular tract of Helwig................ 357 
(Posterior) Cerebello-spinal tract ................ 357-358

Spino-vestibular tract ................... $357-35^{8}$

Lateral pyramidal tract.................... 358

Rubro-spinal tract.......................... $\quad 35^{8}$

Lesions of anterior and lateral funiculi.......... $\quad 359$

Marginal tract (Lissaueri) ................... . 359

Entry zone..................................... 359-360

Fasciculus gracilis (Golli).................... 360-36I

Fasciculus cuneatus (Burdachi) ............... 36 I

Descending tracts from posterior roots............ $36 \mathrm{I}$

Descending postero-medial tract (comma, oval, septomarginal and median triangular tract)....... $36 \mathrm{I}$

Descending postero-lateral tract.............. $\quad 362$

Posterior fasciculus proprius................ $\quad 362$

Cornu-commissural tract................... $3^{62}$

Lesions of posterior columns................. ${ }_{362}$

Roots of the spinal nerves..................... $362-365$

Anterior root............................ $\quad 3_{36}$

Apparent origin.......................... $3^{6} 6_{3}$

Real origin (genetic nuclei)............... $\quad 363$

Voluntary motor fibers.................. $3_{36}$

Sympathetic efferent fibers............... $\quad 363$

Lesions............................... 360-364

Posterior root .............................. $364-365$

Spinal ganglion (origin)................... $\quad 3^{64}$

Apparent central termination................. 364

Real central termination, terminal nuclei....... 364

Gray matter of the cord.................. 364-365

Nuclei of medulla oblongata.............. $\quad 365$

Physiological groups of posterior root-fibers...... 365

Lesions of posterior roots................. $\quad 365$

CHAPTER VII.

\section{TRACING OF IMPULSES.}

I. Efferent, or Motor Paths:...................... 366-374

Cerebro-spinal or pyramidal paths............... 366-369

Through spinal nerves...................... $\quad 366$

Through cerebral nerves................... $\quad 369$

Cerebro-pontal paths..................... 369-370

Fronto-pontal........................ 370 
PAGE

Temporo-pontal.......................... $\quad 370$

Intermediate............................ 370

Spinal and cerebral...................... 370

Paths through red nucleus.................. 370-373

Rubro-spinal path, direct.................. 370-373

Indirect via brachium conjunctivum............. $\quad 373$

Ponto-spinal paths (Collieri)................... 373

Medial ponto-spinal tract.................. 373

Lateral ponto-spinal tract................... 373

Short fiber paths in formatio-reticularis.......... 373-374

II. Afferent, or Sensory Paths, General Sensations:...... $377-383$

Tactile, muscular, pain, and temperature sense...... 377

(I). Muscular and tactile impulses from muscles, skin,

etc............................... $377-381$

Through fasciculi gracilis et cuneati........... $377-378$

Direct route......................... 378

Indirect route.......................... 378

Through cerebral nerves and medial fillet....... 378-38I

(II). Muscular and tactile impulses from viscera..... $38 \mathrm{x}$

Through posterior cerebello-spinal tract......... $38 \mathrm{I}$ (III). Paths for pain, temperature and tactile impulses .. $38 \mathrm{I}-382$

Through spino-thalamic tract................. $381-382$

Through ascending anterior cerebello-spinal tract... $381-382$

Through cerebral nerves and spino-thalamic tract... $\quad 382$

Short fiber paths............................ $3^{82-383}$

Afferent Paths-Special Sensations:................. 383-390

Olfactory path ............................. $383_{3}-384$

Optic path................................. $388_{4}-385$

Auditory path ................................. $385^{8-389}$

Cochlear (hearing proper)................. 386

Vestibular (equilibrium) ..................... 386-389

Reflex connections..................... $\quad 389$

Gustatory path............................. $\quad 389$

Lesions of special sense paths................ 389-390

III. Reflex Paths: ............................ 390-394

Reflex arcs............................. 390

(I) Spinal reflexes......................... 390-392

Coordinating reflexes..................... 390

Defecation reflexes .......................... $399^{\mathrm{I}-392}$

(2) Cerebral reflexes......................... 392-393 


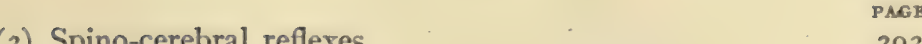

(4) Cerebro-spinal reflexes...................... 393-394

Respiratory reflexes........................ 393

Equilibrium reflexes...................... 393

Pupillary reflexes...................... $\quad 394$

\section{CHAPTER VIII.}

\section{EMBRYOLOGY OF THE BRAIN AND SPINAL CORD.}

Medullary plate and ridges

Neural Tube:........................... 395-400

Brain vesicles..................................... 495 and 402

Ependymal cells.......................... 397

Indifferent cells......................... 397-398

Neuroblasts, neurones ....................... $\quad 398$

Spongioblasts, neuroglia.................... 398-399

Zones, roof-plate and floor-plate .............. 399-400

Neural Crest:........................... 400-40I

Cephalic portion........................... 400

Cerebral nerve ganglia................... 400

Unipolar and bipolar neurones............. 400-40I

Spinal portion of crest................... $40 \mathrm{I}$

The Bratn:................................ 40I-426

Flexures................................ 402

Table IV. Brain Vesicles and Derivatives.......... 403

Table V. Secondary Brain Vesicles .............. 403-404

Telencephalon (end-brain) .................... 404-4I5

Table VI. Derivatives of .................... 404-405

Optic vesicle, cup and retina............... 405

Hemisphere of cerebrum................. 405-415

Rhinencephalon.................... 406-409

Primary fissures...................... 409-410

Secondary sulci and fissure............... 4I0-4II

Transverse fissure of cerebrum............ $4_{4} \mathrm{II}$

Cerebral cortex and medulla ............. 4II-4I2

Fornix ............................ 412

Stria terminalis....................... $4_{412}^{12}$

Internal capsule..................... 412-413

Anterior commissure................. 4I 3 -4I 4

Corpus callosum.................... $4^{114}$ 
PAGE

Septum pellucidum ................... $\quad 415$

Pars optica hypothalami................ 415

Diencephalon (inter-brain) .................. 4I5-4I7

Table VII. Derivatives of...................... 415

Roof-plate............................ 4 . $4 x$

Dorsal lamina......................... $4 \mathrm{I} 7$

Ventral lamina and floor-plate ............. 4 4I7

Mesencephalon (mid-brain).................. 417-418

Table VIII. Derivatives of.................. 4 I $7-4 I 8$

Metencephalon (hind-brain).................. 4I8-424

Table IX. Derivatives of .................. 419

Cerebellum....................... 418-42I

Vermis and hemispheria................ 419-420

Sulci............................ 420-42I

Cortex and ganglia................... $42 I$

Corpus restiforme and brachia............ $42 \mathrm{I}$

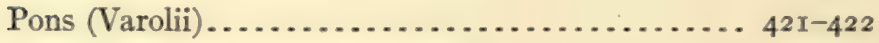

Myelencephalon (medulla oblongata) ............ 422-426

Internal surface....................... 423

External surface........................ 423-424

Table X. Derivatives of Myelencephalon.......... 424

Ependymal layer...................... 424-425

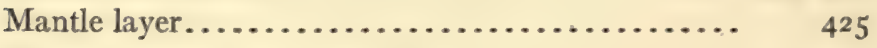

Neuroglia layer, marginal velum ........... 425-426

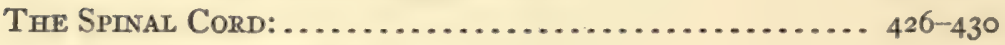

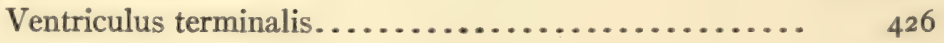

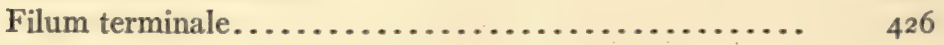

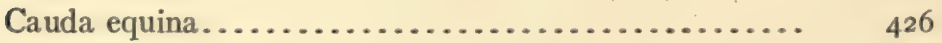

Meninges.............................. 426

Zones, ventral and dorsal................... 426-427

Histologic layers....................... 427-428

Marginal velum, neuroglia layer............... 428

Mantle layer........................... . 428-429

Ependymal layer......................... 429

Longitudinal tracts...................... 429-430

Order of medullation................... 429-430

Fissures of cord. ........................ 430

Posterior median fissure.................... 430

Posterior lateral sulcus .................... 430

Anterior median fissure.................... 430 


\section{LIST OF ILLUSTRATIONS.}

PIG.

I. Sagittal section of skull, showing falx cerebri, falx cerebelli, a part of the tentorium cerebelli and the sinuses of the dura mater. (After Morris's Anatomy)............

2. Upper surface of tentorium cerebelli, tentorial notch and certain sinuses of the dura. (After Morris's Anatomy)..

3. Sinuses of the dura mater in the base of cranium, etc. (After

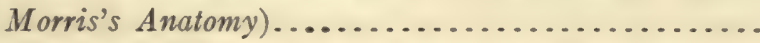

4. Coronal section of meninges showing falx cerebri, superior sagittal sinus and the arachnoid granulations. (Gor-

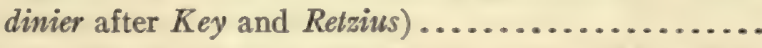

5. Middle meningeal artery inside the cranium. (After Morris's Anatomy)..........................

6. Diagram of pia mater and arachnoid, showing subarachnoid spaces. (After Morris's Anatomy)...............

7. Horizontal section of the cerebrum. Fornix turned back to show the chorioid tela of third ventricle. (Original). II

8. Roof and lateral walls of fourth yentricle and its chorioid plexus. (After Morris's Anatomy).............. I3

9. Arterial circle of Willis and its branches. The base of the brain. (After Morris's Anatomy)............... I5

Io. Arterial circle (Willisi) and base of the cerebrum. (After Gordinier from Quain)................... I9

II. Middle cerebral artery, and its branches. (After Gordinier from Quain)........................ 22

I2 Anterior and posterior cerebral arteries. (After Spalteholz).. 25

I3. Arteries of the medulla oblongata. (Gordinier after Duret)... 27

14. Median section of embryonic brain of third month. (After McMurrich from $H$ is)................... 28

I5. Divisions of the brain, diagrammatic. (After Morris's Anatomy)................................. $3 \mathrm{I}$

I6. Neural tube and brain vesicles. (After Morris's Anatomy)..

I7. Diagrammatic horizontal section of vertebrate brain, showing vesicles and ventricles. (After Morris from Huxley) $3^{6}$ xxvii 
I8. Diagrammatic median section of vertebrate brain showing vesicles, ventricles and olfactory diverticulum. (After Morris from Huxley)................... 37

I9. Antero-superior surface of the brain. (Original)....... 39

20. The posterior aspect of the brain. (Original).......... 43

21. The base of brain. (Original).................. 47

22. Latero-superior aspect of the brain, showing great fissures,

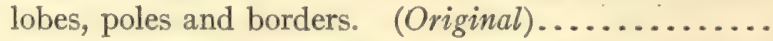

23. The convex surface of the cerebrum, showing the fissures and

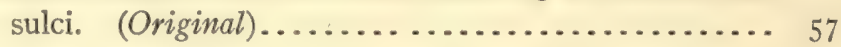

24. Gyri of the convex surface of the cerebrum. (Original).... 6 6 I

25. Lateral aspect of the brain. Part of frontal and parietal lobes are cut away to show the island (Reili) and the superior surface of the temporal lobe, arachnoid granulations,

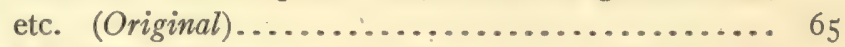

26. Base of the fore-brain and cut surface of mid-brain. Right temporal pole is cut away. (Original)........... 75

27. The median section of the brain. (Original) .......... 87

28. Medial surface of left cerebral hemisphere, showing lobes and sulci. (Original) ....................... 9

29. Gyri on medial surface of hemisphere. (Original)....... 95

30. Transverse section of the brain, directed from the pons obliquely upward and forward, showing internal capsule, corpus callosum, ganglia and ventricles of the forebrain. (Original) ....................... Ior

3I. Horizontal section of right cerebral hemisphere cutting corpus callosum, internal capsule, corpus striatum, thalamus, and the island. (Original).................... 105

32. Sagittal section of basal part of right cerebral hemisphere showing inferior lamina of internal capsule, hippocampus, inferior horn of lateral ventricle. (Original).... II3

33. Diagram of internal capsule in colors. (Original)......... I I 5

34. Dorsal surface of corpus callosum, cerebral hemisphere cut away to expose it. (Original).................. II7

35. Horizontal section of cerebrum, cutting splenium and genu of corpus callosum, showing lateral ventricles, septum pelIucidum, fornix and transverse temporal gyri. (Original) ............................... I 2 I

36. Horizontal section of cerebrum just below splenium of corpus callosum, showing commissura hippocampi, fornix, sep- 
PIG.

tum pellucidum, the island and lateral ventricles. (Or-

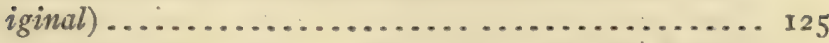

37. Horizontal section of cerebrum. Fornix turned back, showing chorioid tael of third ventricle, and internal cerebral veins. (Original)...................... 129

38. Transverse section of left cerebral hemisphere cutting the splenium and showing the posterior horn and the floor of the inferior horn of the lateral ventricle. (Original) I3I

39. The inferior and posterior horns of the lateral ventricle, shown by removal of their lateral walls. (Original)....... I33

40. Horizontal section of cerebrum through genu and below splenium of corpus callosum, fornix and chorioid tela turned back, to show inter-brain and third ventricle. (Or-

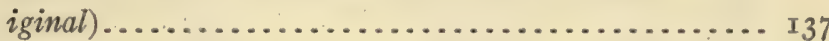

4I. Lateral and dorsal view of the ventricles. Diagrammatic. (Original) ............................. I4

42. Transverse section of brain, cutting corpora mammillaria. (After Told. Morris's Anatomy)............... I45

43. The region of the mid-brain showing pulvinar of the thalamus, the geniculate bodies, the corpora quadrigemina and brachia, the pineal body, the optic tract and the fourth nerve. (Original) .......................... I47

44. The dorsal or posterior aspect of the inter-brain, the mid-brain, the pons and the medulla. (Original) ............ I49

45. Anterior aspect of the mid-brain, pons, and medulla. (Orig-

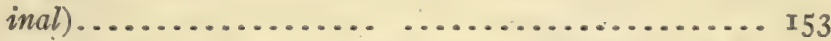

46. Transverse section through the corpora mammillaria and the. superior colliculi of the corpora quadrigemina. (Orig-

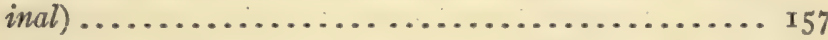

47. Section of the mid-brain through superior colliculi and the apparent origin of the oculo-motor nerve. (Original) .. I59

48. Section of the mid-brain cutting the inferior colliculi of the cor-

pora quadrigemina. (Original)................ I6I

49. Varieties of neurones in the human nervous system. (After Morris's Anatomy) ......................... I65

50. Motor neurone. (After Barker's Nervous System)........ I69

51. An efferent neurone and an afferent neurone. (After Brubaker's Physiology)...................... I7I

52. Diagram showing development of neurones in the spinal cord.

(McMurrich after Schäffer)................ I72 
rig.

53. Neuroglia cells and ependyma cells of the spinal cord. (After Lenhossek, Gordinier's Nervous System) .......... I73

54. Cortical areas on convex surface of cerebral hemisphere. (Original) ............................. I75

55. Cortical areas on the medial and tentorial surface of the cerebral hemisphere. (Original)................. I79

56. Cortical areas after C. K. Mills. Convex surface of cerebral hemisphere. (Brubaker's Physiology)........... I8z

57. Cortical areas after C. K. Mills. Medial and tentorial surface of cerebral hemisphere. (Brubaker's Physiology).... I 83

58. Cell and fiber lamination in the posterior half of the anterior central gyrus. The motor area. (After A.W. Campbell)

59. Cell and fiber lamination in the anterior half of the posterior central gyrus. The common sensory area. (After A. W. Campbell $) \ldots \ldots \ldots \ldots \ldots \ldots \ldots \ldots \ldots \ldots \ldots \ldots$

6o. Cell and fiber lamination in the calcarine region. Receptive visual area. (After A.W. Campbell) ............ I93

6r. Cell and fiber lamination in the uncus hippocampi (lobus pyraformis). The area of smell. (After $A . W_{.}$Campbell) I97

62. Transverse section of the hippocampal region. (After Edinger) 201

63. Chief elements of the olfactory bulb. (Gordinier after Van

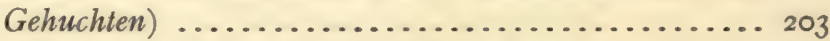

64. Horizontal section of the cerebrum through genu and below splenium of corpus callosum, fornix and chorioid tela turned back to show inter-brain and third ventricle. (Original).

65. Dissection of brain to show geniculate bodies, optic tract, nucleus amygdalæ, etc. (After Morris's Anatomy) ... 207

66. Transverse section of the brain in the line of the pyramidal tracts, showing basal ganglia, internal capsules, corpus callosum, lateral and third ventricles, etc. Viewed from front. (Morris's Anatomy after Toldt)........ 209

67. The optic path. (Original)....................... 213

68. Section of mid-brain through superior colliculi and the apparent origin of the oculomotor nerve. (Original).... 2I5

69. Section of the mid-brain cutting the inferior colliculi of the corpora quadrigemina. (Original)................ 216

70. Horizontal and sagittal section through internal capsule, much

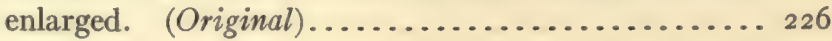


PIG.

7I. Diagram of internal capsule in colors. (Original).

72. A diagram showing motor and sensory paths, motor red, sensory blue. (After Gordinier's Central Nervous System).............................

73. Transverse section of cerebrum, cutting corpus callosum, anterior commissure and optic chiasma. Viewed from front. Commissural fibers. (Morris's Anatomy after Toldt $)$.............................

74. Diagram of association fibers in the cerebral hemisphere.

(Gordinier and Quain after Meynert)........... 237

75. Fasciculus occipito-frontalis. Stria terminalis and fasiculus uncinatus. (Gordinier after Dejerine) ............. 239

76. Dorsal view of inter-brain, mid-brain and cerebellum. Superior surface of cerebellum. (Original).......... 243

77. Anterior aspect of cerebellum. (Original)............. 245 78. Dissection of rhombencephalon to show brachium conjunctivum, brachium pontis and corpus restiforme. (Gordinier, Sappey after Hirschfeld and Leveille)....... 246

79. Median section of cerebellum, pons and medulla. (Original) 247 80. Inferior surface of cerebellum. (Original)............. $25^{2}$ 8r. Sagittal section of cerebellum, cutting nucleus dentatus. (Original) .................................... 255

82. Section of cerebellar gyrus made parallel with its free border.

Diagrammatic. (Cunningham after Kölliker)...... $25^{8}$

83. Section across a cerebellar gyrus at a right angle to the free border. Diagrammatic. (Gordinier after Van Gehuchten)............................... 259

84. Horizontal section of cerebellum cutting nuclei and brachia conjunctiva. (Morris's Anatomy after Toldt).......26r

85. Anterior aspect of mid-brain, pons and medulla. (After Morris's Anatomy)........................267

86. Dorsal surface of pons and medulla. (Morris's Anatomy modified from Spalteholz) ................... 270

87. Superior transverse section of the pons. (Original)....... 275

88. Inferior transverse section of the pons together with the cerebellum. (Original) ...................... 277 89. Diagram of the auditory paths in the pons. (After Morris's Anatomy) .............................. 280

90. Section of embyronic medulla, embryo measuring $9.1 \mathrm{~mm}$. in length. (Gordinier and Minot after His) ......... 285 
91. Roof and lateral walls of fourth ventricle, and its chorioid plexuses. (After Morris's Anatomy) ............ 289

92. Section of medulla oblongata near the pons. (Original) ... 295

93. Section of the medulla oblongata at the middle of olive. (Original).............................. 297

94. Section of the medulla oblongata at the fillet decussation. (Or-

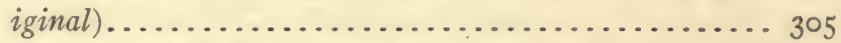

95. Section of the medulla oblongata at the pyramidal decussation.

(Original) ..................................

96. Nuclei of the cerebral nerves in the medulla, pons, mid-brain, inter-brain, and olfactory bulb. Motor (or genetic) nuclei red, terminal (or sensory) nuclei blue. (After Morris's Anatomy) ......................... 3 I 5

97. Meninges of the spinal cord. A. Transverse section. (After Key and Retzius.) B. Anterior view. (After Ellis.)

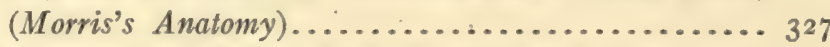

98. Diagrammatic section of the spinal meninges and spinal cord.

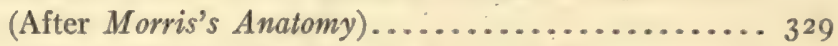

99. The arteries and veins in the spinal cord. Diagrammatic.

(After Morris's Anatomy)....................... 33I

I00. Posterior view of the spinal cord, the dura mater and the arachnoid being laid open and turned aside. (Brubaker

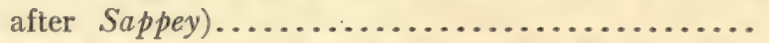

IOI. Sections of the spinal cord: A. The cervical. B. The thoracic. C. The lumbar, and D. The lower sacral. (Or-

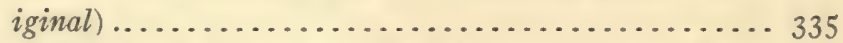

102. Tracts of fibers and columns of cells, in the cervical and thoracic regions of the cord. Diagrammatic. (In part after Bruce and Cunningham)..................... $34 \mathrm{I}$ 103. Tracts of fibers and columns of cells in the lumbar and sacral regions of the cord. Diagrammatic. (In part after Bruce and Cunningham)..................... 345 104. The roots of the spinal nerves. Diagrammatic. (Original). 349 105. Direct motor paths from cerebral cortex, to cerebral and spinal nerve. Diagrammatic. (Original)........... 367 Io6. Indirect motor paths to the spinal nerves. Diagrammatic.

(Original) .....................................

107. Common sensory paths, muscular and tactile, by way of the posterior column and (posterior) cerebello-spinal tract. Diagrammatic. (Original) 
FIG.

I08. Common sensory paths, pain, temperature and touch, by way of ascending anterior cerebello-spinal and spino-thalamic tracts. Diagrammatic. (Original) ......... 379 Io9. Chief elements of the olfactory bulb. (Gordinier after Van

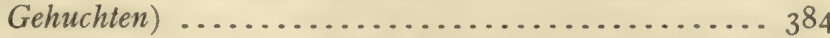

I Io. The chief retinal elements. (After Brubaker's Physiology).. 385 III. The optic path. (Original) .......................... 387 I12. A simple spinal reflex arc. (Brubaker after Morat and Dayon)................................... 391

II3. A more complicated spinal reflex arc, involving the fasciculi proprii. (Brubaker after Kölliker) ............. 392 II4. Medullary groove, neural tube, etc. (Gordinier after E. A.

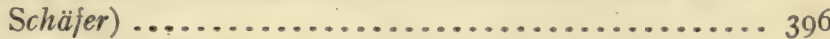

II5. Two histologic layers in the embryonic spinal cord, embryo $4.25 \mathrm{~mm}$. long. (McMurrich after His)......... 397 I 6. Diagram showing development of neurones in the spinal cord.

(McMurrich after Schäfer) ................. 398

II7. Ventral and dorsal zones of the spinal cord. (Gordinier and Quain after Kölliker).................... 399 II8. Median section of embryonic brain of the third month. (McMurrich after His) ...................... 406

II9. Transverse section through the fore-brain of a four and one half weeks' embryo. (Gordinier and Quain after His) 407 I20. Diagrammatic sagittal section of vertebrate brain. (Morris's Anatomy after Huxley) .................... 409

I2I. Medial sagittal section through the brain of an embryo of three months showing the primitive fissures on the medial surface of the cerebral hemisphere. (McMurrich after Mihalkovicz) .................. 4II

I22. The fossa cerebri lateralis, in embryonic brain of fourth month. (After McMurrich's Development of the Human Body) ............................... 413

I23. Permanent fissures and sulci on the convex surface of the cerebrum as seen in a seven months' embryo. (McMurrich after Cunningham) ............... 4I4

I24. Dorsal view of an embroynic brain, the roof of the lateral ventricles having been cut away. Embryo of $12.6 \mathrm{~mm}$. (McMurrich after $H$ is) ...................... 416 I25. Transverse section of medulla from an embryo of $9.1 \mathrm{~mm}$.

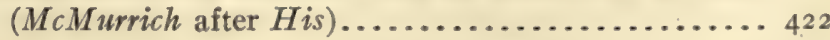


PIG:

I26. Transverse section of the medulla from an embryo of eight weeks. (McMurrich after His)............... 423

I27. Transverse section of the spinal cord, from an embryo of (A) four and one-half weeks, and (B) of three months. (McMurrich after $H i$ is) .......................... 427

I28. Mode of origin of anterior and posterior roots of spinal nerves. Diagrammatic. (Brubaker and Edinger after His)

\section{ERRATA.}

Page 30. Paragraph two, last line, last word should be brainvesicles.

Page 57. Fig. 23. "Intraparietal sulcus," and wherever found should be written Interparietal sulcus.

Page 108. Paragraph four, fourth line, second word is sulcus, instead of "fissure."

Page 184. Paragraph one, last line and first word is Alfred, instead of "Arthur."

Page 264. Fourth line, the phrase "anterior ascending cerebellospinal tract," and wherever it occurs, should read Ascending anterior cerebello-spinal tract.

Page 265. Paragraph two, third line, the phrase "anterior descending cerebello-spinal tract," should stand Descending anterior cerebello-spinal tract: likewise, wherever the quoted phrase is found.

Page 277. Third line, last word is (Horsley).

Page 286. Paragraph three, last sentence, the phrase "fasciculus cerebello-spinalis," and wherever else it occurs should stand thusfasciculus cerebello-spinalis (posterior).

Page 288. Paragraph three, fifth line, the substantive "cerebellospinal fasciculus" should have "posterior" prefixed so as to read posterior cerebello-spinal fasciculus. This is a necessary variation from the BNA and applies wherever the phrase is found.

Page 29r. Last line, there should be added a fifth item, viz., (e) the medullary striæ. 
Page 319. Fifth line, common sensory should be added under intermediate nerve.

Page 35r. Paragraph three, third item, (3) should be omitted, as no posterior root-fibers decussate. Intrinsic fibers only cross through the gray commissure (Mott and Russell).

Page 358. To the last sentence should be added: and according to Sir Victor Horsley has to do with locomotion(Brain, 1906).

Page 4ro. Fourth line, "callosal fissure" should be callosal sulcus. 



\section{BRAIN AND SPINAL CORD.}

\section{CHAPTER I. \\ THE MENINGES OF THE BRAIN. \\ (Meninges Encephali.)}

Three membranes invest the brain and spinal cord. They are, from without inward, the dura mater, the arachnoid, and the pia mater. Each membrane forms a protecting sheath for the cerebral or spinal nerves piercing it.

\section{THE DURA MATER OF THE BRAIN. \\ (Dura Mater Encephali.)}

Structure and Relations. - It is a very dense and inelastic membrane composed of white fibrous and yellow elastic tissue lined with flat endothelial cells, which constitute its internal surface. In children it is closely adherent to the cranial bones of which it forms the real periosteum; but it is attached chiefly at the foramina and along the sutures in adults. The dura of the brain is made up of two layers which are separable up to the eighth or tenth year. The external layer constitutes the endosteum of the cranial bones. It is their nutrient membrane. Through the cranial foramina and sutures it is continuous with the external periosteum. In the adult the internal layer of the dura separates from the outer layer only over the apex of the petrous bone, to form Meckel's space for the semilunar ganglion (Gasseri); at the foramina, to form sheaths for the nerves; and, along the sinuses, to form their internal boundary and to produce the great incomplete partitions, called processes, which project centrally into the great fissures of the brain.

Processes. (Processus durce matris). -From the inner surface of the dura the great processes are given off. The falx cerebri 
and falx cerebelli hang vertically in the longitudinal fissure of the cerebrum and the posterior notch of the cerebellum; and, into the transverse fissure of the cerebrum, extends horizontally the tentorium cerebelli. The falx cerebri (Figs. I, and 4 ) is attached in front to the crista galli and behind to the internal occipital protuberance and superior surface of the tentorium; the falx cerebelli (Fig. I) continues from the inferior surface of the tentorium, along the occipital crest, to the posterior border of the foramen magnum. The bony attachment of the tentorium cerebelli (Fig. 2) is to the internal protuberance and the lateral arms of the crucial ridge forward to the petrous bone; and, then, it is along the superior border of the petrous bone to the clinoid processes of the sphenoid. Between its clinoid attachments there is a deep bay, the incisura tentorii, which transmits the midbrain. The diaphragma sellæ is a small centrally perforated sheet of dura which covers the hypophyseal fossa.

Sinuses. (Sinus durce matris).-Large venous passages lined with endothelial cells, and called sinuses, are situated between the layers of the dura (Figs. I, 2, 3 and 4). In the convex and in the free border of the falx cerebri are, respectively, the superior sagittal sinus (s. sagittalis superior) and the inferior sagittal sinus (s. sagittalis inferior). The superior (Fig.. I) extends from the foramen cæcum back to the confluens sinuum (torcular Herophili,) located at the internal occipital protuberance. Having run through the posterior two-thirds of the concave border of the falx cerebri, the inferior sagittal sinus joins the great cerebral vein at the margin of the tentorium and forms the straight sinus (s. rectus). The latter runs through the middle of the tentorium to the confluens (Fig. 2). The occipital sinus (s. occipitalis) traverses the falx cerebelli from the foramen magnum upward to the same point. In the confluens sinuum the transverse sinuses (s. transversi) rise (Fig. 2). Grooving the horizontal arms of the crucial ridge, each runs outward in the tentorium to the base of the petrous bone, where it receives the superior petrosal sinus; it then turns downward through the sigmoid fossa, communicates with the occipital sinus and unites with the inferior petrosal sinus in the jugular foramen. Situated on either side 
of the sella Turcica is a continuation of the ophthalmic vein, the large cavernous sinus (s. cavernosus) (Fig. 3), which receives at the sphenoidal fissure the spheno-parietal sinus (s. alce parve), the course of which is along the posterior border of the lesser wing of the sphenoid bone. At the posterior clinoid process the

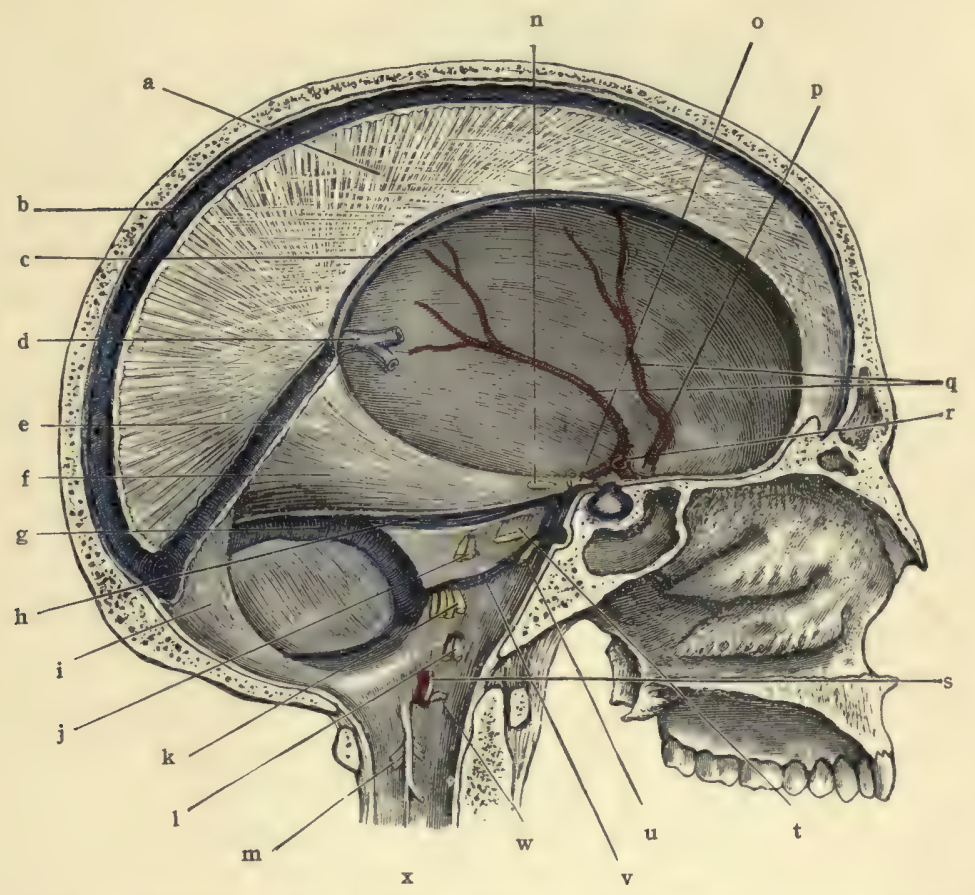

Fig. I.-Sagittal section of skull, showing falx cerebri, falx cerebelli, part of tentorium cerebelli and sinuses. (After Morris's Anatomy.)

a. Falx cerebri. b. Superior sagittal sinus. c. Inferior sagittal sinus, d. Great cerebral vein. e. Straight sinus. f. Tentorium cerebelli. g. Transverse sinus. h. Superior petrosal sinus. i. Falx cerebelli. j. Seventh and eighth nerves, k. Ninth, tenth, and eleventh nerves. 1. Twelf th nerve. m. Second cervical nerve. n. Fourth nerve. 0. Third nerve. p. Second nerve. q. Middle meningeal artery. r. Internal carotid artery. s. Vertebral artery: t. Fif th nerve. u. Sixth nerve. v. Inferior petrosal sinus. w. First cervical nerve. $\mathbf{x}$. Ligamentum denticulatum.

cavernous sinus divides into the superior petrosal sinus (s. petrosus superior) and the inferior petrosal sinus (s. petrosus inferior). The sinus intercavernosus anterior and sinus intercavernosus posterior extend across the hypophyseal fossa, and join the two cavernous sinuses together, and these four communicating sinuses 
constitute the circular sinus (s. circularis) (Fig. 3). From the bifurcation of the cavernous sinus at the apex of the petrous bone, the petrosal sinuses run outward along the corresponding superior and inferior borders of that bone. $\mathbf{a}$ The!superior petrosal sinus

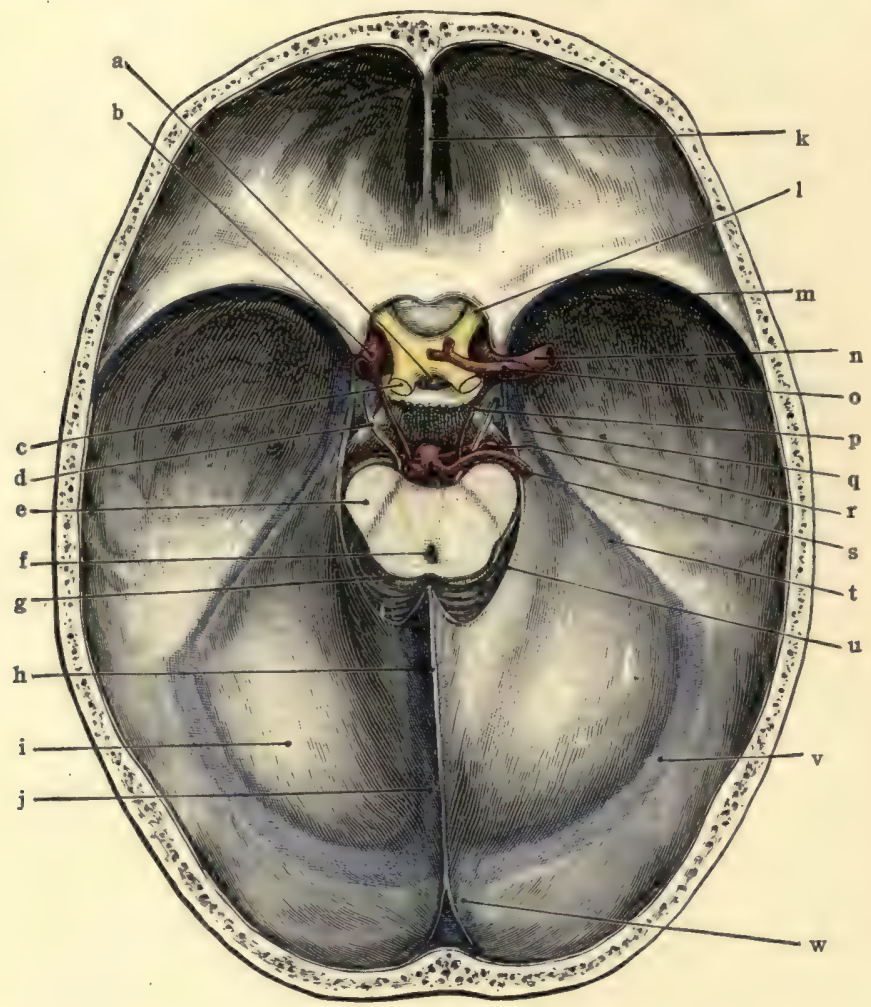

Fig. 2.-Horizontal section of skull, showing tentorium cerebelli, tentorial notch and sinuses. (After Morris's Anatomy.)

a. Infundibulum. b. Internal carotid artery. c. Optic tract. d. Third nerve, e. Basis pedunculi. f. Cerebral aqueduct (Sylvii). E. Quadrigeminal body. h. Falx cerebri. i. Tentorium cerebelli. j. Straight sinus. k. Crista galli. 1. Optic nerve. m. Sphenoparietal sinus. n. Middle cerebral artery. o. Anterior cerebral artery. p. Posterior communicating artery. q. Cavernous sinus. r. Superior cerebellar artery. s. Posterior cerebral artery. t. Superior petrosal sinus. u. Free border of tentorium bounding tentorial notch. v. Transverse sinus, w. Superior sagittal sinus.

(Figs. I and 2) empties into the transverse sinus at the base of the petrous bone; the inferior petrosal simus, in its course to the jugular foramen, is joined to its fellow, across the basilar process of the occipital bone, by the basilar plexus ( $p$. basilaris) and, in 
the jugular foramen, unites with the transverse sinus in forming the internal jugular vein.

Arachnoid Granulations (Fig. 25).-Along and within the

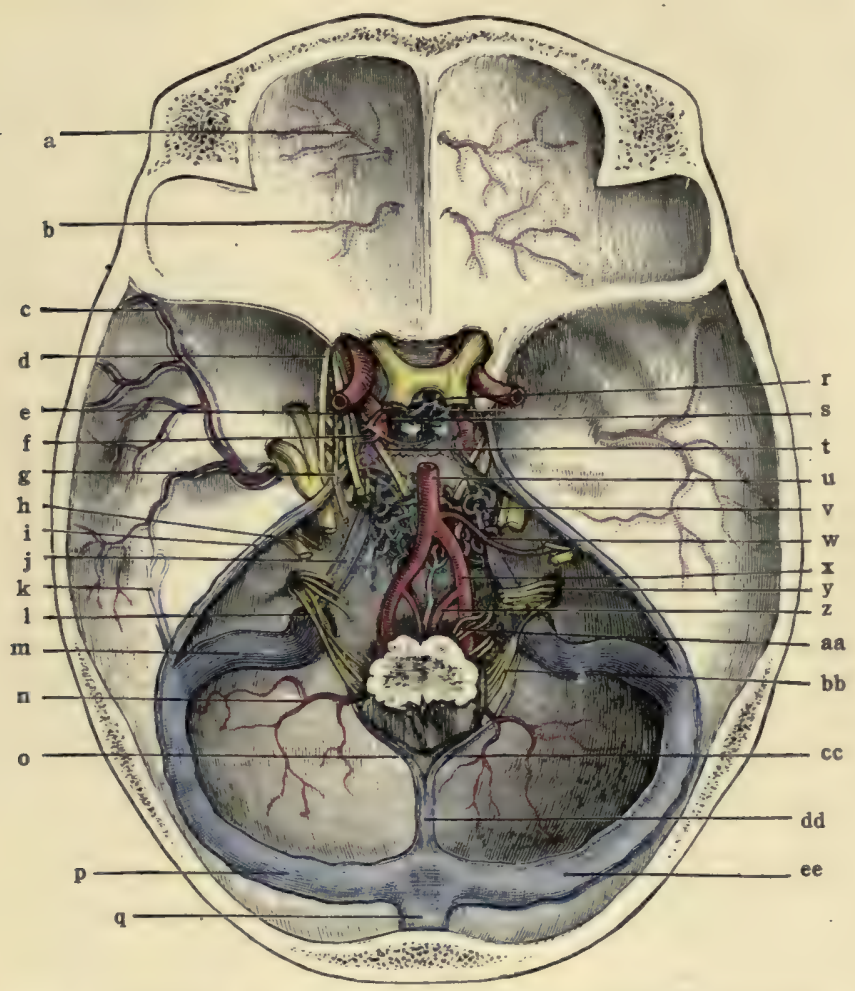

Fig. 3. - Sinuses in the base of the cranium, also meningeal arteries. (After Morris's Anatomy.)

a. Meningeal branch of anterior ethmoidal artery. b. Meningeal branch of posterior ethmoidal artery. c. Middle meningeal artery. d. Ophthalmic division of fifth nerve. e. Third nerve. f. Cavernous sinus, g. Fourth nerve, h. Auditory and facial nerves, i. Superior petrosal sinus. j. Inferior petrosal sinus. k. Petro-squamosal sinus, 1. Accessory nerve. $\mathrm{m}$. Sigmoid part of transverse sinus. n. Posterior meningeal branch of vertebral artery. o. Left marginal sinus. p. Left transverse sinus, q. Superior sagittal sinus. r. Circular sinus. s. Carotid artery, t. Sixth nerve. u. Basilar artery. v. Basilar plexus of veins. w. Auditory artery. x. Vertebral artery. y. Glossopharyngeal and vagus nerves. z. Anterior spinal artery. aa. Hypoglossal nerve. bb. Accessory nerve. cc. Right marginal sinus. dd. Occipital sinus, ee. Right transverse sinus.

superior sagittal, the straight, the transverse, the petrosal and the cavernous sinuses are the gramulationes arachnoideales (Pacchionian bodies). These granulations are enlarged villi of the 
arachnoid (Fig. 4) and seem to afford an outlet for the subarachnoid fluid into the sinuses. They are said to make their appearance about the tenth year and to be more numerous and larger in the male sex. Some of them by absorption produce depressions in the cranial bones called foveole granulares.

The arteries which supply the dura are (I) The anterior meningeal from the anterior ethmoidal branch of the ophthalmic. (2) The middle meningeals, viz., the great and the small middle meningeal from the internal maxillary, the meningeal branch

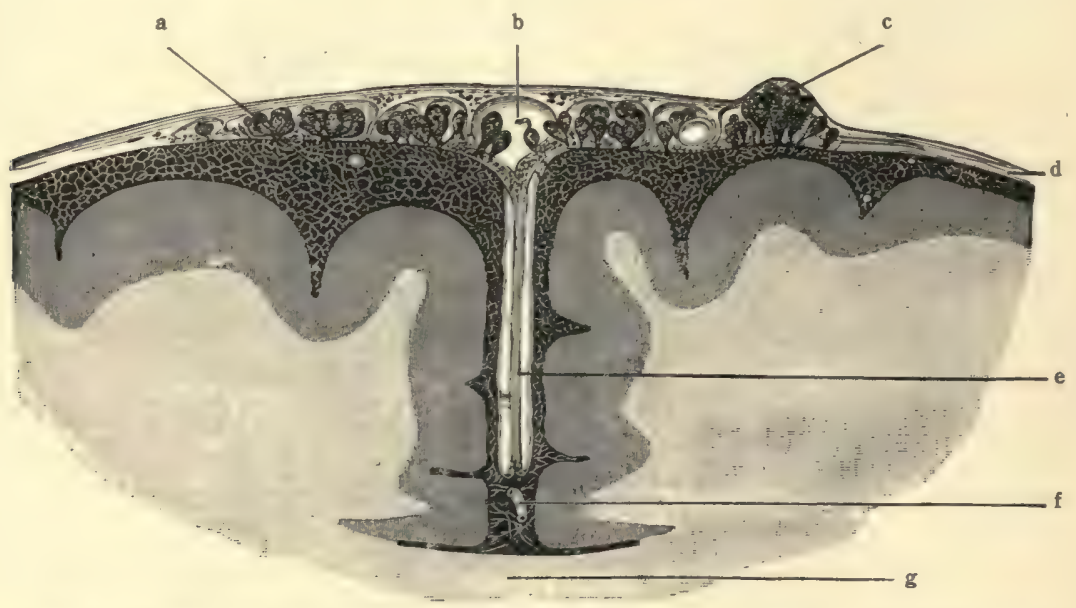

Fig. 4.-Coronal section showing falx cerebri, superior sagittal sinus and arachnoid granulations. (Gordinier after Key and Retzius.)

a. Subarachnoid space. b. Superior sagittal sinus. c. Arachnoid granulations (Pacchioni). d. Dura mater. e. Falx cerebri. f. Anterior cerebral artery. g. Corpus callosum.

of the lacrimal and of the internal carotid, and the meningeal branch of the ascending pharyngeal which enters the middle fossa of the cranium through the lacerate foramen. (3) The posterior meningeal arteries, which rise from the ascending pharyngeal, the occipital and the vertebral and are distributed to the dura over the posterior cranial fossa.

The great middle meningeal artery (arteria meningea media) is much the largest and is most important (Fig. 5). It overlies the motor and somæsthetic areas of the brain. Like the other meningeal arteries it is usually accompanied by two veins. Ascend- 
ing from the foramen spinosum it divides near the upper border of the squamosa into two large branches, the anterior and posterior. The posterior runs horizontally backward just below the squamoparietal suture and then ascends over the posterior half of the parietal bone. The anterior branch runs upward a half-inch behind the coronal suture. It may be located, according to Quain, at one inch, at one inch and a half, and at two inches

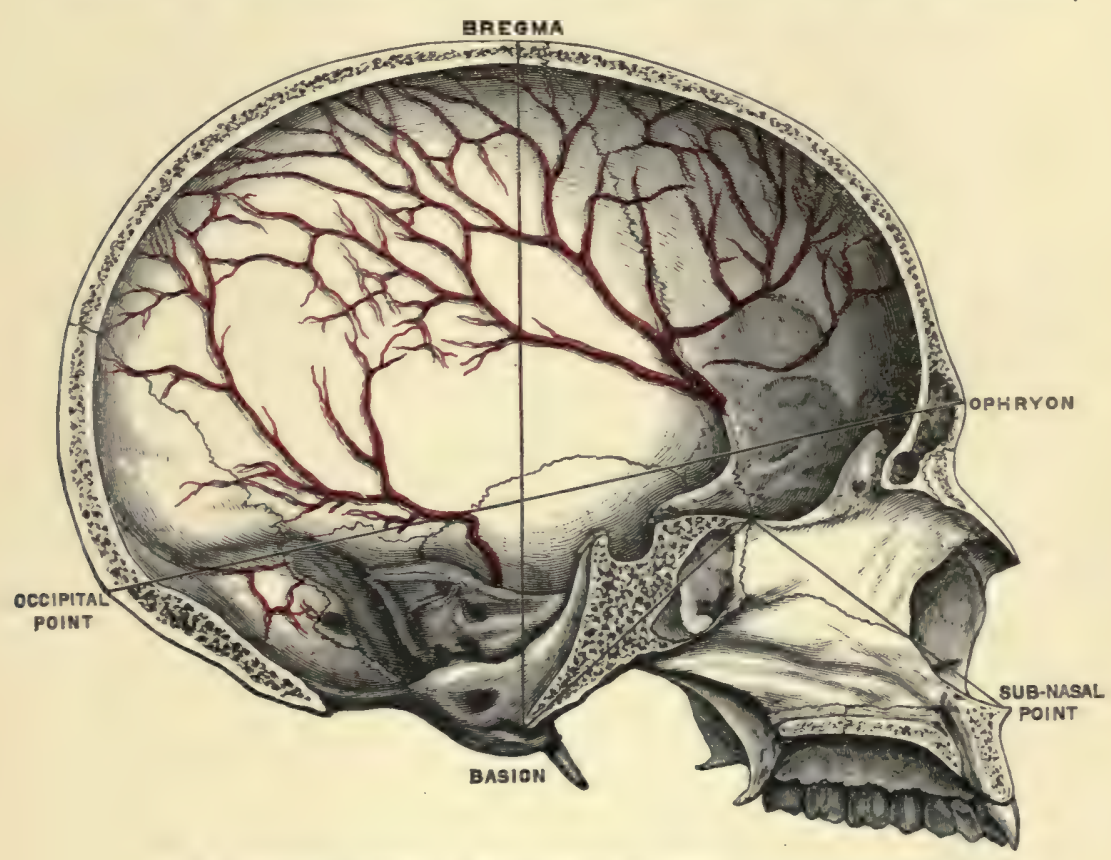

Fig. 5.-Median section of skull showing middle meningeal artery.

(After Morris's Anatomy.)

behind the zygomatic process of the frontal bone and above the zygomatic process of the temporal bone.

The following nerves give branches to the dura:--Trochlear, ophthalmic, semilunar ganglion, vagus and hypoglossal of the cercbral group; and the sympathetic. The motor fibers supply the meningeal arteries.

Six Points of Difference in the Dura of the Cord.-Absence of processes, of sinuses, of arachnoid granulations, and of periosteal 
function. It is covered on both surfaces by endothelium and is separated from the vertebræ by areolar tissue, fat and the plexus of internal vertebral veins.

\section{THE ARACHNOID OF THE BRAIN.}

\section{(Arachnoidea Encephali.)}

In structure it is a delicate, fibrous, web-like membrane covered externally with endothelium. Internally it is joined to the pia mater by innumerable fibrous trabeculæ, the subarachnoid tissue (Fig. 4). The trabeculæ are ensheathed and all subarachnoid spaces lined with a single layer of endothelial cells, hence both surfaces are formed of endothelium. Conical elevations of fibrous tissue with their investing endothelium constitute the villi seen on the outer surface.*

Relations. - The arachnoid follows the inner surface of the dura and is prolonged, as a sheath, upon the nerves which pierce it. It does not dip into the sulci of the cerebrum (Fig. 6); but only into the lateral fissure and the longitudinal fissure, and does not reach to the bottom of the latter. From the pia it is separated by the subarachnoid spaces (cava subarachnoidealia). The anterior subarachnoid space (Fig. 6) includes the cisterna pontis, cisterna interpeduncularis, cisterna chiasmatis and cisterna fossæ lateralis cerebri. It is located in front of the medulla, pons and mid-brain and between the temporal lobes of the cerebrum. The posterior subarachnoid space (Fig. 6) is located behind the medulla and cerebellum. It embraces the cisterna cerebellomedullaris, a space between the medulla and cerebellum, and the cisterna venæ cerebri magnæ, situated along the straight sinus and the great cerebral vein. These two, the anterior and posterior, are the largest subarachnoid spaces and they contain much of the subarachnoid fluid. But in the cerebral sulci and fissures there are streams of this fluid which constitute the subarachnoid rivulets. The anterior subarachnoid space has slit-like com-

* Some authors consider this membrane, as just described above, merely as a visceral layer of the arachnoid, and regard the endothelial lining of the dura mater as its parietal layer. According to such, therefore, the subdural space becomes the arachnoid space. 
munications with the inferior horn of the lateral ventricle; the posterior space communicates with the fourth ventricle (Fig. 8) through the median aperture (apertura mediana ventriculi quarti, Magendi) and the lateral apertures (aperturce laterales ventriculi quarti, Key and Retzii).

The vessels seen for a short distance in the arachnoid belong to the pia mater. Its nerves are doubtful. Perhaps branches of the mandibular, of the facial and of the accessory supply it.

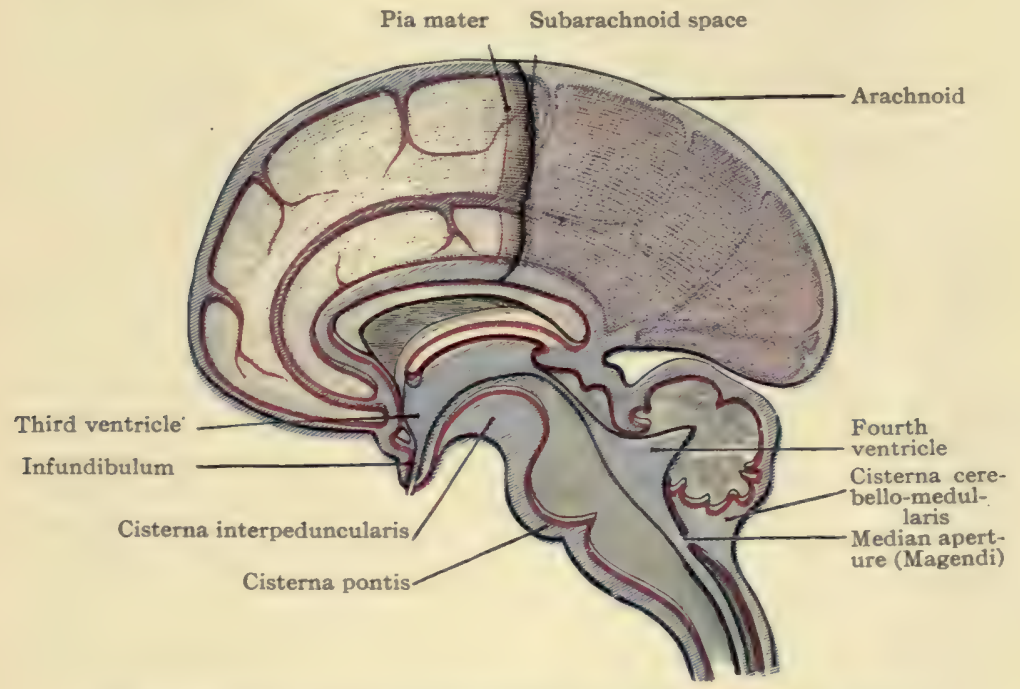

Fig. 6. -Diagram of pia and arachnoid, showing subarachnoid spaces.

(After Morris's Anatomy.)

In the arachnoid of the cord fewer trabeculæ join it to the pia; and these, in great part, are collected to form a fenestrated septum in the posterior median line (Fig. 97, A). The external spinal veins are covered by the spinal arachnoid, they lie between it and the pia.

THE PIA MATER OF THE BRAIN.

(Pia Mater Encephali.)

Structure and Relations.--It is a vascular membrane composed of a close network of veins and arteries held together by 
fibro-elastic areolar tissue (Fig. 9). The endothelium covering its outer surface is continuous with that ensheathing the subarachnoid trabeculæ. The pia closely follows the brain surface (Fig. 6). Internally, it sends supporting trabeculæ into the brain, which transmit blood-vessels; and externally it forms an investing sheath for each cerebral nerve.

Folds. - Two important processes are formed by the pia mater: (I) The chorioid tela of the third ventricle (tela chorioidea ventriculi tertii) is pushed forward into the anterior part of the transverse fissure of the cerebrum between the fornix and the interbrain (Fig. 6). Hence the old name, velum interpositum. It is triangular in shape, with apex directed forward (Fig. 7). Each lateral border is tucked into the chorioidal fissure of the cerebral hemisphere and enters into the floor of the lateral ventricle, while the median part of the fold is in the roof of the third ventricle. Between the two layers of this chorioid tela is some areolar tissue through which run backward the two internal cerebral veins and unite near the base of the tela to form the great cerebral vein. The chorioid plexuses of the lateral and the third ventricles occupy, respectively, the lateral borders and the median area of this chorioid tela. (2) A second fold of pia mater is tucked into the transverse fissure of the cerebellum, dorsal to the medulla oblongata and ventral to the posterior median part of the cerebellum (Fig. 6). It is called the chorioid tela of the fourth ventricle (tela chorioidea ventriculi quarti) because its inferior layer enters into the roof and contains the chorioid plexus of that ventricle. This lower layer invests the posterior surface of the medulla and the roof-epithelium of the fourth ventricle (Fig. 8). It is pierced by three foramina which are situated as follows: One over each lateral angle of the fourth ventricle, the lateral apertures (Key and Retzii), and one over its inferior angle. The latter is the largest and is called the median aperture (Magendi). Those three foramina establish communication between the posterior subarachnoid space and the fourth ventricle.

The arteries of the pia mater supply the brain (Figs. 9, IO, II and I2). They are the anterior, middle and posterior cerebrals; the anterior and posterior chorioidals; and the anterior and pos- 


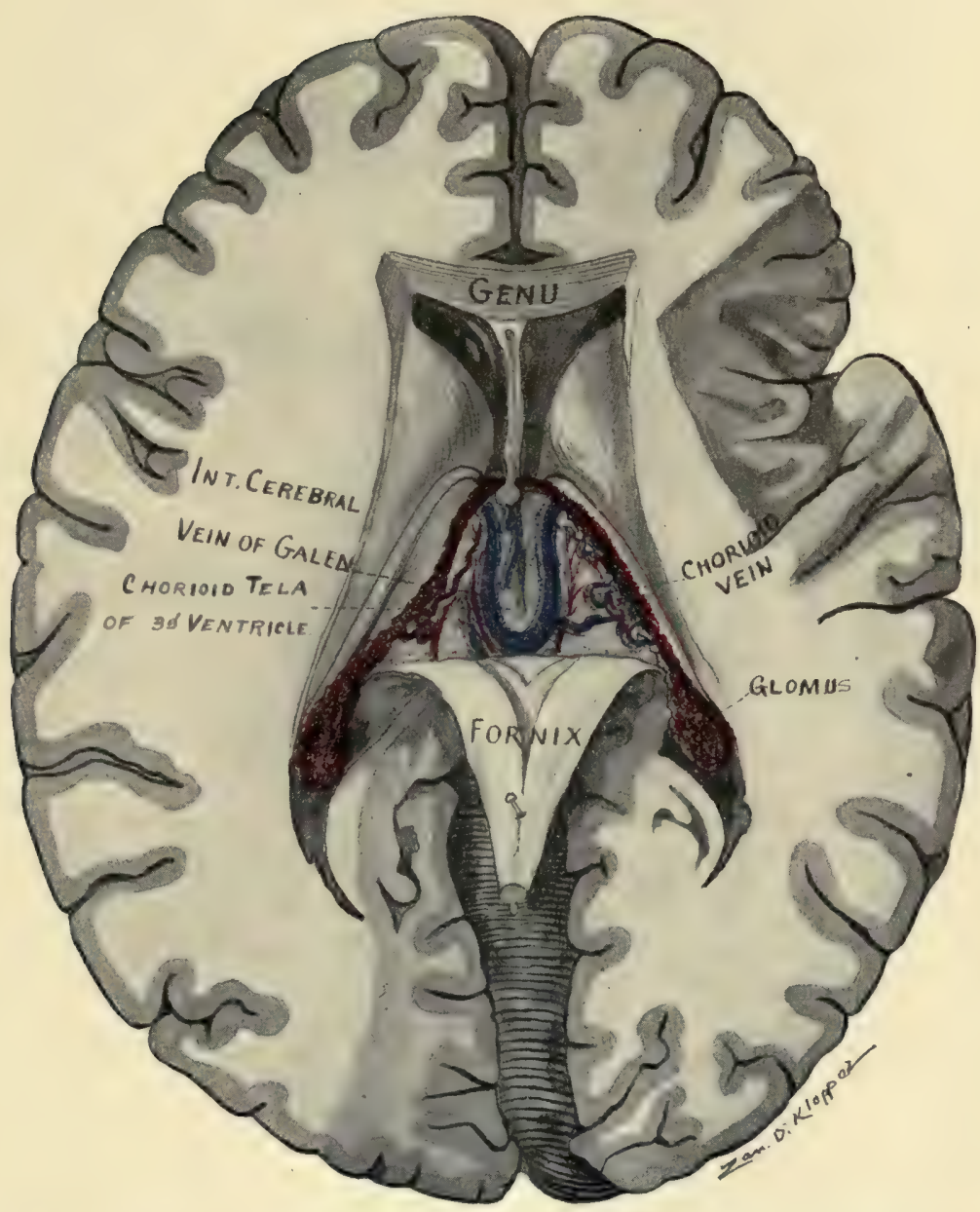

Fig. 7.-Horizontal section of cerebrum. Fornix turned back, showing chorioid tela of third ventricle, and internal cerebral veins. (Original.) 

terior inferior cerebellar and the superior cerebellar with many branches.

The veins are more numerous than the arteries in the pia: the internal and great cerebral veins, the veins of the chorioid plexuses of the lateral, third and fourth ventricles and the basilar vein; the cerebral veins, superior, medial and inferior; and the superior and inferior cerebellar veins. All of them empty into the sinuses (see page 2).

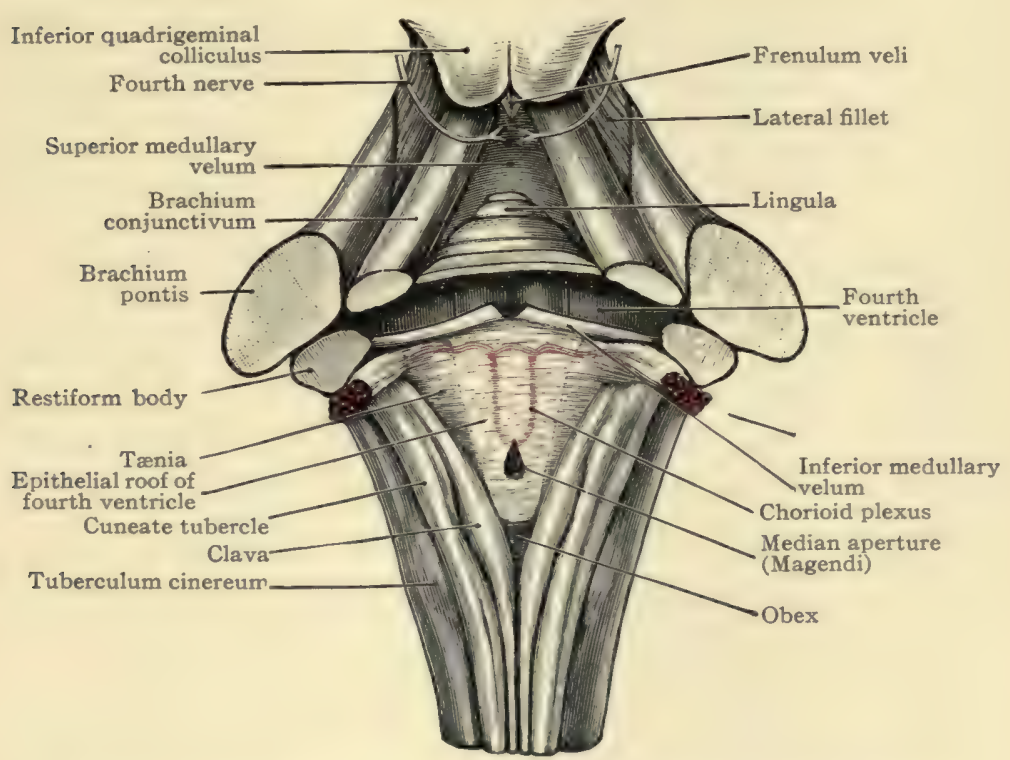

Fig. 8.-Roof and lateral walls of fourth ventricle, and its chorioid plexuses.

(After Morris's Anatomy.)

Seven cerebral nerves - $3 \mathrm{~d}$, 5th, 6th, 7 th, 9 th, Ioth and IIthand the sympathetic supply the pia mater and its blood-vessels.

The pia mater of the spinal cord has two layers, the outer of which is the more vascular and contains the spinal arteries and the tributaries of the external spinal veins. It forms three processes, namely, the anterior septum, which occupies the anterior median fissure, and the ligamentum denticulatum of each side. 


\section{THE BLOOD SUPPLY OF THE BRAIN.}

The brain is furnished with blood by the internal carotid and vertebral arteries (Fig. 9). The internal carotid artery ( $a$. carotis interna) gives origin to the anterior and the middle cerebral, the anterior chorioidal and the posterior communicating; the vertebral artery (a. vertebralis) gives off the anterior and posterior spinal and the posterior inferior cerebellar and then unites with its fellow at the inferior border of the pons and forms the basilar artery. The basilar artery (a. basilaris) runs upward the length of the pons and terminates in the two posterior cerebral arteries and, furthermore, gives off the following collateral branches, viz., the anterior inferior cerebellar, the pontal, the internal auditory and the superior cerebellar. Certain of these arteries form a wonderful circular anastomosis at the base of the brain, called the arterial circle and the distribution of that circle is to the cerebrum (Figs. 9 and Io); while the rhombencephalon (pons, cerebellum and medulla) is supplied by the remainder of the arteries above enumerated. It is therefore convenient to describe the circulation of the brain under two heads: (A) The circulation of the cerebrum, and (B) The circulation of the rhombencephalon.

\section{A. THE CEREBRAL CIRCULATION, ARTERIES.}

The Arterial Circle. (Circulus arteriosis, Willisi).-The arteries which supply the cerebrum freely communicate in the arterial circle, which is really a heptagon extending from a point in the longitudinal fissure anterior to the optic chiasma, back to the pons (Fig. 9). It is about an inch and a half long, and from a half to one inch in transverse diameter. In front are the anterior cerebral arteries converging forward from the internal carotids and, through the anterior communicating artery (a. communicans anterior), uniting just as they enter the longitudinal fissure of the cerebrum. These vessels form three sides of the heptagon and the front of the circle. On either side, the posterior communicating artery (a.communicans posterior) which connects the internal carotid with the posterior cerebral artery, forms the lateral bound- 
ary of the circle. The posterior cerebral arteries bound the circle behind, and so complete it (Fig. Io). The large distal branches of the arteries which are connected with the arterial circle are distributed chiefly to the cortex and medulla of the hemispheres; while the small proximal branches supply the

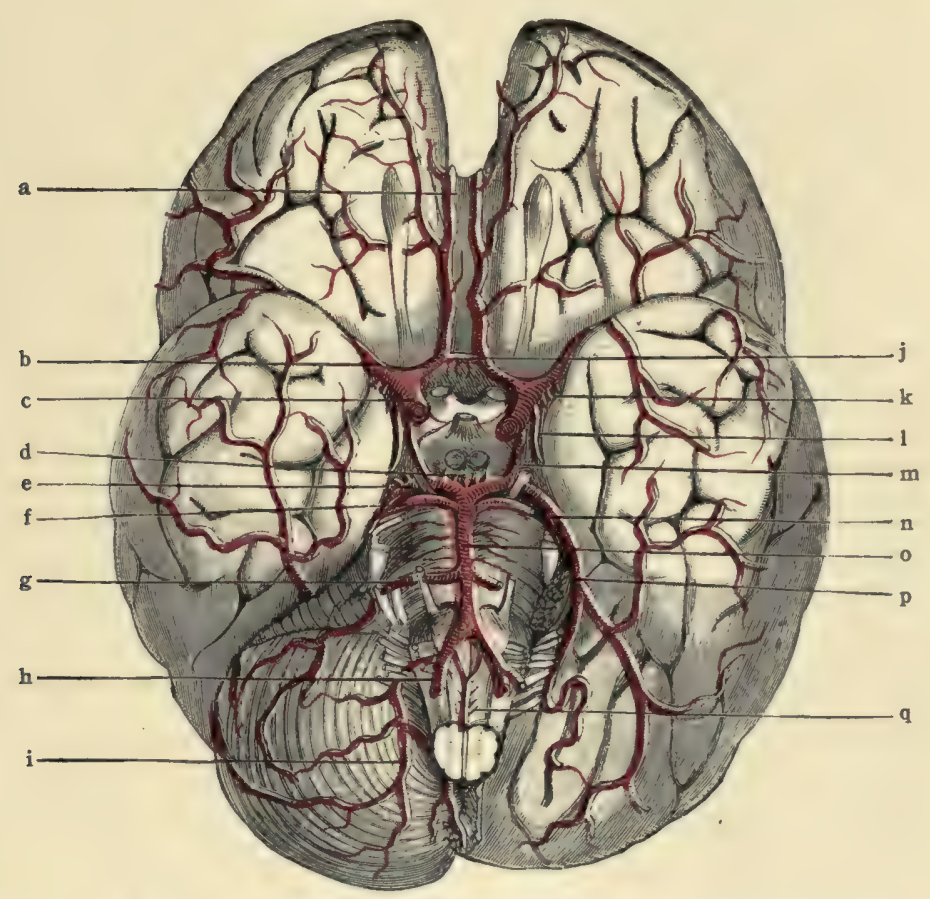

Fig. 9.-Arterial circle and its branches on the base of the brain.

(After Morris's Anatomy.)

a. Anterior cerebral artery, b. Middle cerebral artery. c. Internal carotid artery. d. Postero-median ganglionic. e. Posterior cerebral artery. f. Superior cerebellar artery. g. Anterior inferior cerebellar artery. h. Vertebral artery. i. Posterior inferior cerebellar artery. j. Anterior communicating artery. k. Antero-lateral ganglionic. 1. Anterior chorioid. m. Posterior communicating artery. n. Posterior chorioid. o. Basilar artery. p. Hemisphere of cerebellum cut away. q. Anterior spinal artery.

ganglia and the inter-brain. The former belong to the cortical system (AI), the latter to the ganglionic system (A2).

A1. The Cortical System of Arteries.-The cortical arterial system comprises the distal portions of the anterior, middle and posterior cerebral and the chorioidal arteries. The branches of 
these great vessels pierce the hemispheres perpendicularly to the surface. They are distributed, the short, to the cortex, and the long, to the medulla of the hemispheres. To a limited extent they anastomose with one another, but they do not communicate with the ganglionic system.

The anterior cerebral artery (a. cerebri anterior, Figs. 9 and I2), a branch of the internal carotid, runs forward and toward the median line above the optic nerve and enters the longitudinal fissure; it is here joined to its mate by a very short artery, the anterior communicating. Winding around the genu of the corpus callosum, it runs back on the medial surface of the hemisphere to the occipito-parietal sulcus. It gives origin to the antero-median ganglionic arteries, and to four groups of cortical branches: (I) The internal orbital artery (Figs. 9 and Io) which supplies the medial orbital gyrus, the gyrus rectus, the optic chiasma and the olfactory bulb, tract, medial and intermediate striæ, triangle, and parolfactory area. (2) The anterior internal frontal (Fig. I2) which enters the anterior parts of the gyrus cinguli and superior frontal gyrus on the medial surface and of the superior and middle frontal gyri on the convex surface. (3) The middle internal frontal branches, which are distributed to the middle part of the gyrus cinguli, to the paracentral lobule and to the upper portions of the superior frontal and the anterior and posterior central gyri. The posterior internal frontal branches, which run back to the occipito-parietal sulcus. They supply nearly the whole corpus callosum, the posterior half of the gyrus cinguli, a part of the paracentral lobule, the præcuneus, and the superior parietal lobule.

The middle cerebral artery (a. cerebri media, Figs. Io and II) crosses the anterior perforated spot and runs in the lateral fissure of the cerebrum to the posterior sulcus circularis (Reili) where it breaks up into several parieto-temporal branches. It gives origin to the antero-lateral ganglionic arteries, and to four cortical branches: (I) The lateral orbital branches are distributed to the anterior and posterior orbital and the inferior frontal gyri. (2) The ascending frontal, two branches, which follow the precentral and central sulci, supply the anterior central gyrus and 
the posterior fourth of the middle frontal gyrus. (3) The ascending parietal, whose course is along the interparietal sulcus, furnishes blood to the posterior central gyrus and the adjacent parts of the superior and inferior parietal lobules. (4) The parieto-temporal arteries, which comprise two polar branches to the temporal lobe and a large posterior branch. The latter runs in the posterior ramus of the lateral cerebral fissure to its upturned posterior end and there bifurcates into a parietal and a temporal branch, which just pass the anterior limit of the occipital lobe. The entire distribution of the parieto-temporal arteries is to the temporal pole and to the superior, middle and part of the inferior temporal gyri; to the major parts of the supramarginal, angular and post-parietal gyri, and to a very small portion of the superior and lateral occipital gyri.

The posterior cerebral artery (a. cerebri posterior), a terminal branch of the basilar, lies in the posterior boundary of the arterial circle and is joined to the internal carotid by the posterior communicating artery (Figs. Io and I2). It winds backward between the midbrain and gyrus hippocampi to the tentorial surface of the cerebral hemisphere where, just beyond the splenium of the corpus callosum, it terminates in the calcarine and occipitoparietal branches. From the posterior cerebral arteries originate the postero-median and the postero-lateral ganglionic, and two or more posterior chorioidal arteries and three cortical branches: (I) The temporal branches, often an anterior, middle and posterior temporal, which supply the hippocampal and the fusiform gyri and a part of the lingual and of the inferior temporal gyrus.

(2) The calcarine artery, which runs along the fissure of the same name and supplies the cuneate and lingual gyri; also the pole and the lateral and superior gyri of the occipital lobe. The occipito-parietal artery, a single branch, which runs along the sulcus occipito-parietalis over the supero-medial border to the convex surface of the cerebral hemisphere and is distributed to the cuneus, the præcuneus and the superior occipital gyrus.

The posterior chorioidal arteries (arteria chorioidece posteriores, Figs. 7, 9 and Io) two or more in number are branches of 
the posterior cerebral which run forward in the transverse and chorioidal fissures of the cerebrum to the chorioid plexuses of the lateral and third ventricles (Fig. 7).

The anterior chorioidal arteries (a. chorioidea anterior) rises from the internal carotid artery just proximal to its anterior and middle cerebral branches, and runs backward and outward along the optic tract to the anterior inferior end of the chorioidal fissure, which it enters (Fig. IO). It terminates in the chorioid plexus of the inferior horn of the lateral ventricle, and gives collateral branches to the optic tract, the gyrus hippocampi, the fascia dentata, the hippocampus, the crus of the fornix and the posterior part of the internal capsule.

A2. The Ganglionic System of Arteries.-Small arteries from the arterial circle and from the cerebral arteries near the circle constitute this system (Fig. IO). The arteries pass to their distribution without communicating with one another or with the cortical arteries. They are the end-arteries of Cohnheim. Between the cortical and ganglionic systems, there is an area poorly supplied with blood. That is the area of cerebral softening in old age. The ganglionic system of arteries is made up of six groups of small vessels: The antero-median, the right and left antero-lateral, the postero-median and the right and left posterolateral.

The antero-median ganglionic arteries rise from the anterior cerebrals in front of the optic chiasma (Fig. Io). They supply the chiasma, the lamina terminalis, the rostrum of the corpus callosum, the septum pellucidum and the head of the caudate nucleus.

The antero-lateral ganglionic arteries take their origin, on either side, from the middle cerebral artery, a little outside the arterial circle (Fig. Io). They pierce the anterior perforated substance and are distributed to the striated body, internal capsule and thalamus. The largest one of this group is the lenticulostriate artery. It supplies the greater part of the corpus striatum. On account of its frequent rupture, it is called the artery of cerebral hemorrhage (Charcot).

Postero-median Ganglionic Arteries.-These are branches 


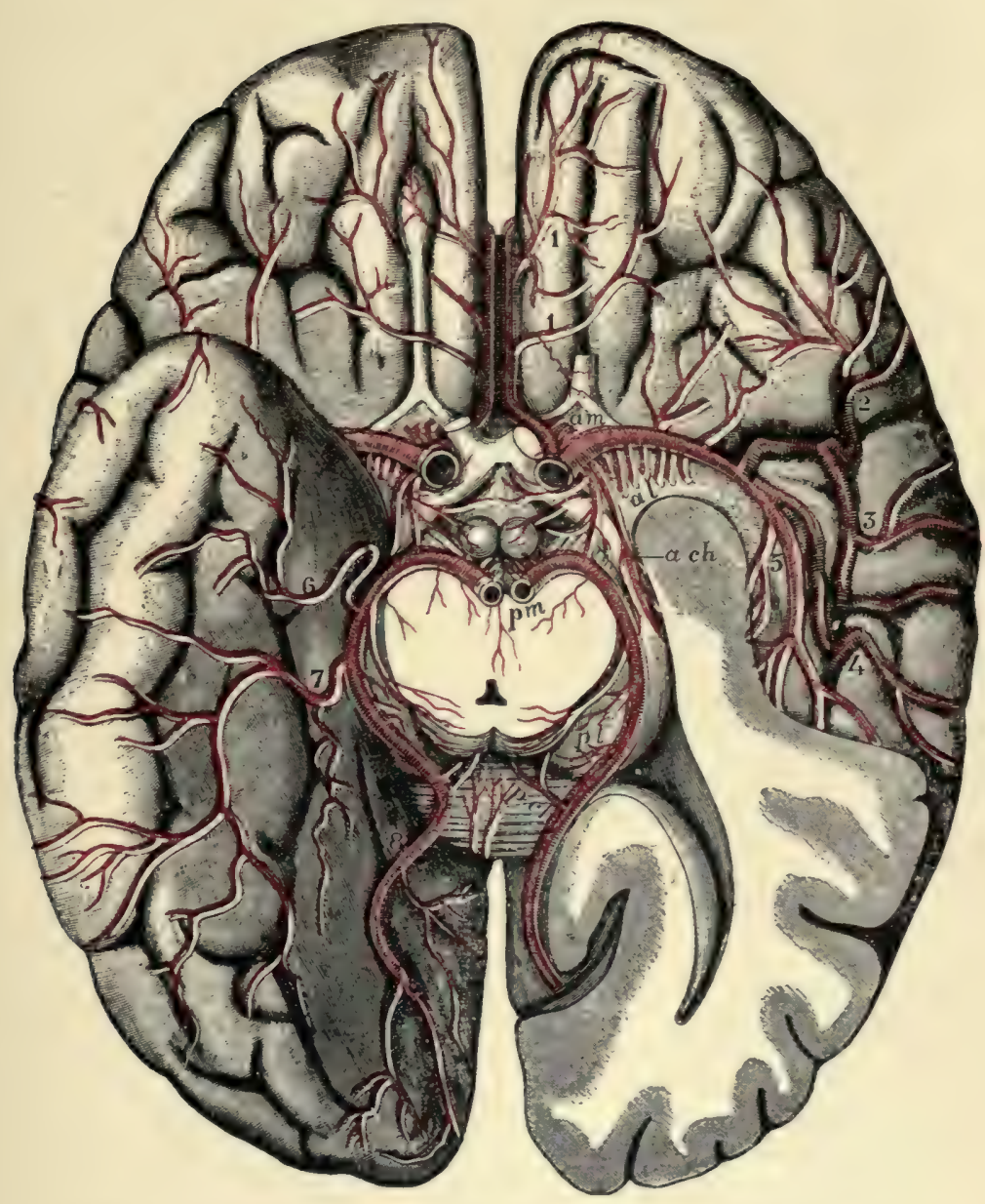

Fig. I0.-Arterial circle and its branches on the base of the cerebrum.

(Gordinier after Duret.)

On the left side of the brain the temporal lobe is cut away so as to open the inferior and posterior horns of the lateral ventricle. The mid-brain is divided close above the pons and the posterior cerebral arteries are cut at their origin from the basilar.

Ganglionic arteries: am. Antero-median group arising from the anterior cerebral. al. Antero-lateral group, from the middle cerebral. $p m, p l$ (on the optic thalamus). Posteromedian and postero-lateral groups, from the posterior cerebral.

Chorioidal arteries: $a \mathrm{ch}$. Anterior, from the internal carotid. $p c h$ (on the splenium). Posterior, from the posterior cerebral.

Cortical arteries: 1, I. Inferior internal frontal, from the anterior cerebral. 2. Inferior external frontal. 3. Ascending frontal. 4. Ascending parietal, and 5, temporo-parietal from the middle cerebral. 6. Anterior temporal, 7 , posterior temporal, and $\mathbf{8}$, occipital, from the posterior cerebral. 

of the posterior cerebral and posterior communicating arteries (Figs. 9 and 10). They supply the interpeduncular structures, the peduncles and, after piercing the posterior perforated substance, the walls of the third ventricle and the medial parts of the thalami.

Postero-lateral Ganglionic Arteries.-They rise, on either side, from the posterior cerebral artery after it has wound around the base of the peduncle (Fig. 10). They are distributed to the posterior part of the thalamus; the geniculate, quadrigeminal and pineal bodies; the quadrigeminal brachia and the pedunculus cerebri. The superior cerebellar arteries send several branches to the dorsum of the mid-brain, and complete the arterial supply of the cerebrum.

\section{VEINS OF THE CEREBRUM.}

The Internal Veins of the Cerebrum. - The veins of the cerebrum (vence cerebri) are classed as internal and external. The trunks of the internal veins are located largely in the chorioid tela of the third ventricle, near the apex of which the internal cerebral vein is formed: while at the base of this chorioid tela the internal cerebral vein unites with its mate in forming the great cerebral vein.

The internal cerebral vein (v. cerebri interna) is formed by the union of the chorioidal, the terminal and the vein of the septum pellucidum. It runs backward between the layers of the chorioid tela of the third ventricle (Fig. 7), receiving several small collaterals from the tela, from the pineal and quadrigeminal bodies and the corpus callosum; and, finally, it receives the basilar vein from the inferior surface of the cerebral hemisphere. Under the splenium of the corpus callosum it joins the internal cerebral vein of the opposite side and forms the great cerebral vein.

The great cerebral vein ( $v$ cerebri magna, Galeni) is a short, thick, median trunk, a half-inch long (Fig. I). At the posterior border of the tentorial notch it is joined by the inferior sagittal sinus and then continued as the sinus rectus. This short vein receives collateral tributaries from the gyrus cinguli, from the 
medial and tentorial surfaces of the occipital lobe and from the superior surface of the cerebellum (Cunningham).

Small nameless internal veins issue from all parts of the exterior surface of the cerebrum and form the external veins.

The External Veins of the Cerebrum.--The external cerebral veins (vence cerebri externa) are numerous and of large size. They ramify in the pia mater and in the subarachnoid space. They empty into the dural sinuses, as a rule, against the current in the sinuses, and they form two principal groups: The superior

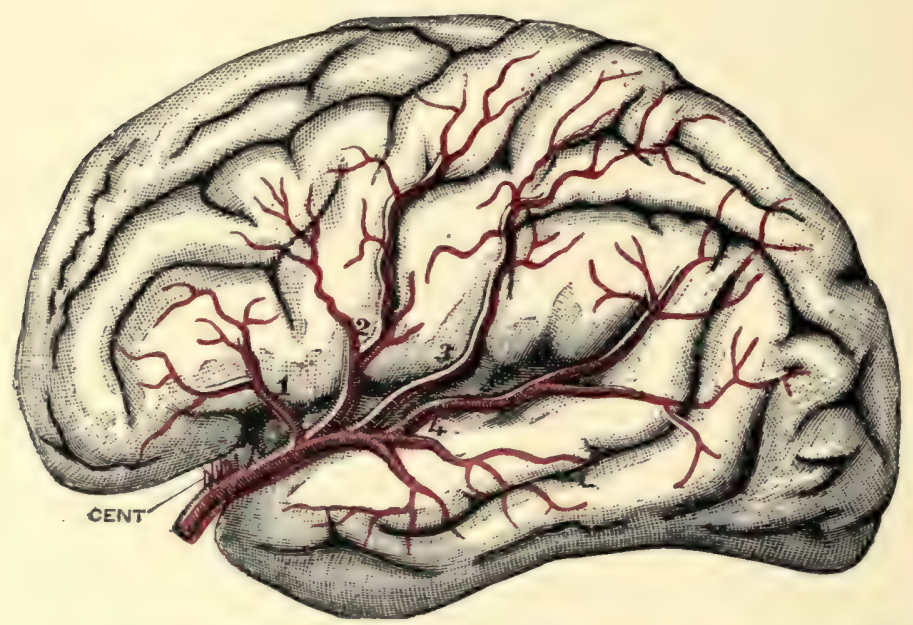

Fig. I I.-Middle cerebral artery and branches. (Gordinier after Quain and Charcot.)

CENT. Antero-lateral group of ganglionic arteries. 1. Inferior external frontal artery. 2. Ascending frontal artery. 3. Ascending parietal artery. 4. Parieto-temporal artery.

cerebral and the inferior eerebral, and a very small group, on the medial cerebral surface, called the medial cerebral veins.

The superior cerebral veins (vence cerebri superiores), twelve or more in number, carry away the blood from the superior surface of the hemisphere. They run obliquely upward and forward into the superior sagittal sinus. Just before emptying into the sinus they receive most of the medial veins.

The Medial Cerebral Veins. (Vencerebri mediales).-They drain the marginal part of the medial surface of the hemisphere. 
The veins of this group which do not empty into the superior cerebral veins unite and form the inferior sagittal sinus, and the anterior cerebral vein which drains much of the medial surface.

The inferior cerebral veins (vence cerebri inferiores) drain the base of the cerebrum and the lower border of its convex surface. On the tentorial surface of the hemisphere, from three to five of these veins empty into the transverse and superior petrosal sinuses. Those from the temporal and frontal lobes empty into the spheno-parietal sinus and cavernous sinus, excepting the small anterior cerebral vein and the deep middle cerebral vein, which unite with the inferior striate veins in forming the basilar vein. The anterior cerebral vein accompanies the artery of the same name. It drains the gyrus cinguli and corpus callosum, chiefly; and, in the fossa lateralis cerebri, unites with vessels that descend from the corpus striatum, the inferior striate veins, and with the deep middle cerebral vein. The deep vena cerebri media drains the insula and the opercula, in part, and deep in the fissure runs medianward to the fossa lateralis cerebri and helps to form the basilar. The basilar vein ( $v$. basilaris), is formed at the anterior perforated spot by the deep middle cerebral, the inferior striate and the anterior cerebral veins. Running backward it receives additional blood from the interpeduncular structures, the hippocampal gyrus and the inferior horn of the lateral ventricle, and from the mid-brain, as it winds around it to empty into the corresponding internal cerebral vein near its termination. In the fissura lateralis cerebri (Sylvii) runs also a superficial vein, called the superficial middle cerebral (v. cerebri media) which receives tributaries from the surfaces adjacent to the posterior ramus and the stem of that fissure and empties into the cavernous sinus; but it may have two other outlets, viz., the transverse sinus and the superior sagittal sinus. The connection occasionally established between the superficial middle cerebral vein and the transverse sinus is called the posterior anastomotic vein; while the great anastomotic vein (of Trolard) is produced when it joins one of the superior cerebral veins. The great anastomotic vein connects the superior sagittal with the cavernous sinus.

There are no lymphatic vessels in either the brain or spinal 
cord; perivascular lymph spaces carry the fluid from the interior to the subarachnoid spaces.

\section{B. THE CIRCULATION OF THE RHOMBENCEPHALON.}

B1. The medulla oblongata is supplied with blood by the following branches of the vertebral artery: The posterior and the anterior spinal, the posterior inferior cerebellar and several short bulbar arteries (Fig. 9). The posterior inferior cerebellar (a. cerebelli inferior posterior) winds from before backward around the medulla, runs between the vagus and accessory nerves, enters the vallecula cerebelli and gives branches to the medulla and to the chorioid tela of the fourth ventricle. The anterior spinal artery (a. spinalis anterior) formed by the y-like union of a branch from each vertebral artery, descends along the anterior median fissure; and the posterior spinal artery (a. spinalis posterior) of either side, rising from the vertebral near the lower end of the medulla, descends in front of the posterior lateral sulcus. Both distribute branches along their course. The branches for the most part enter the median raphe or follow the roots of the bulbar nerves, suggesting the centrifugal and centripetal arteries of the spinal cord (Fig. I3). The veins pursue much the same course as the arteries. The anterior median vein joins the ventral veins of the pons and is drained into the cerebellar veins or directly into the superior petrosal sinus. The posterior median vein bifurcates $y$-like at the middle of the medulla and the two branches wind around the medulla to its anterior surface and empty into the inferior petrosal sinus or the basilar plexus. Issuing from the medulla with the roots of the ninth to the twelfth cerebral nerves are three or four small veins, the radicular veins, which run into the occipital and inferior petrosal sinuses (Cunningham). Both arteries and veins possess perivascular lymph spaces, but there are in the medulla no lymphatic vessels.

B2. The pons Varolii is supplied by the pontal, the superior cerebellar and the posterior cerebral branches of the basilar artery (Fig. 9). The short and transverse branches of the basilar artery, the pontal arteries ( $a$ a. pontales), furnish the greater portion of blood to the basilar area of the pons, while the superior cere- 

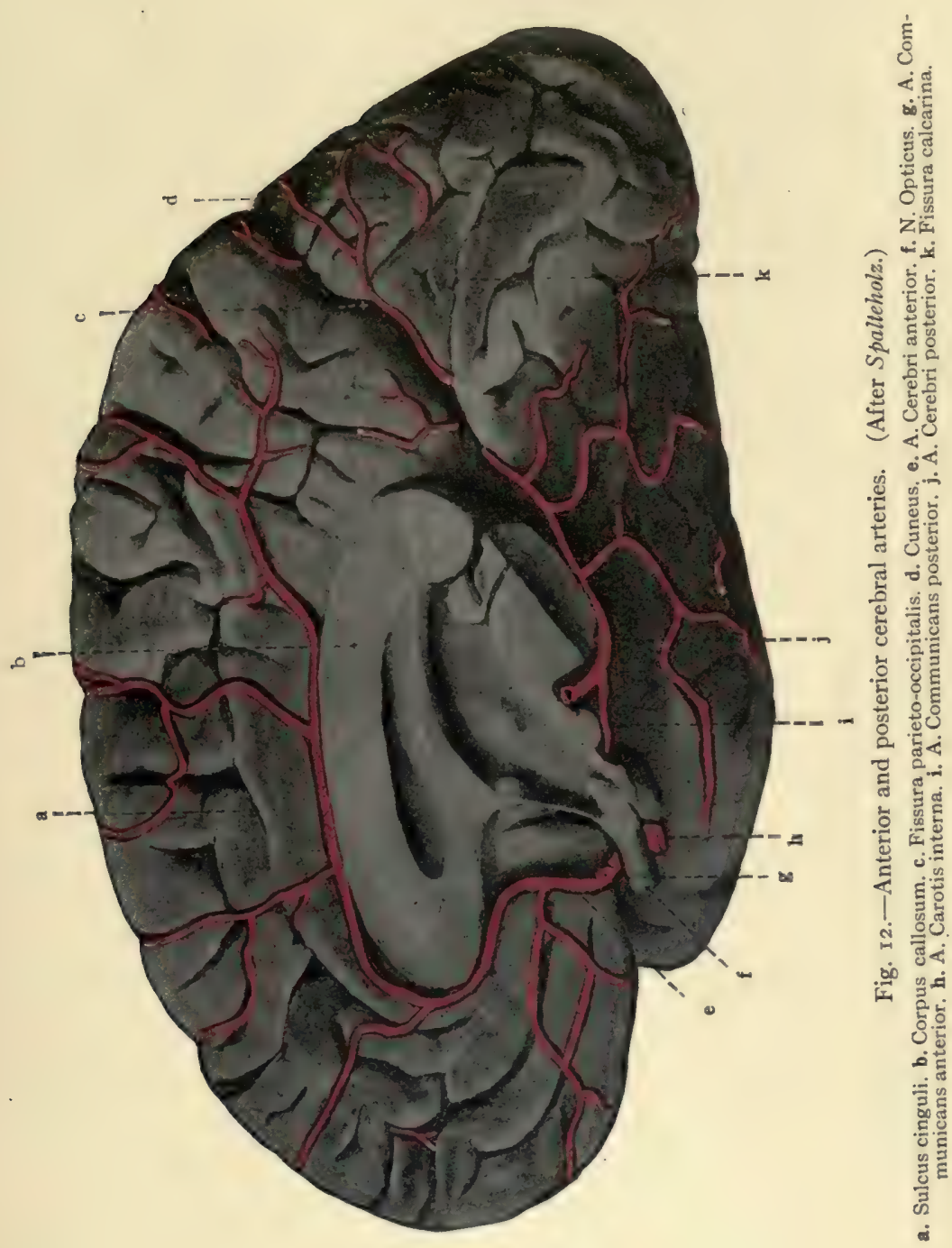

bellar artery supplies the superior medullary velum and the brachia conjunctiva cerebelli. The branches enter the median raphe, also the substance of the pons elsewhere, especially along the nerve roots, and run at right angles to the surface into it. The deep veins of the pons run forward and form a plexus on its surface which, according to Cunningham, is drained by a superior efferent into the basilar vein and by an inferior efferent into the cerebellar veins or the superior petrosal sinus. There are no

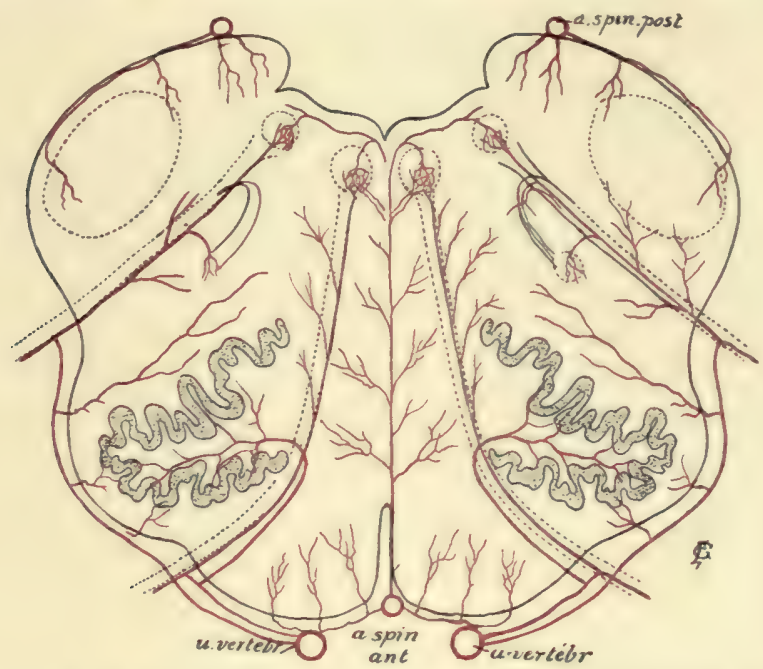

Fig. 13.-Arteries of the medulla oblongata. (Modified from Gordinier after Duret.) a.spin.post. Posterior spinal artery. a.vertebr. Vertebral artery. a.spin.ant. Anterior spinal artery.

lymphatic vessels in the pons; but, as elsewhere in the central nervous system, there are lymph spaces about the blood-vessels.

B3. The blood supply of the cerebellum is furnished by three pairs of arteries (Fig. 9). The superior cerebellar, from the basilar, supplies all the superior surface except a narrow zone at the posterior border; the anterior inferior cerebellar, also from the basilar, and the posterior inferior cerebellar, from the vertebral, supply the inferior surface and the posterior part of the superior surface.

The Superior Cerebellar Artery. (A. cerebelli superior).Rising from the basilar just behind the posterior cerebral, from 
which it is separated by the oculomotor nerve, it winds dorsally around the mid-brain to the sulcus lateralis, where it bifurcates into a medial and a lateral branch (Fig. 9). The medial branch continues along the trochlear nerve in the groove between the cerebellum and the mid-brain almost to the median line; and then, bending backward, runs along the superior worm of the cerebellum to its posterior extremity. It distributes branches to the geniculate bodies, corpora quadrigemina, tela chorioidea

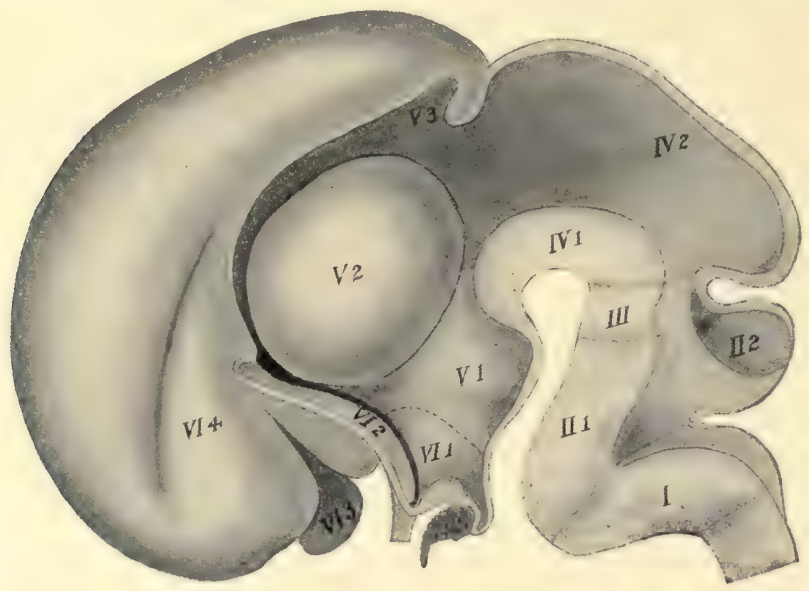

Fig. 14. - Median section of embryonic brain of the third month.

(McMurrich after $H$ is.)

I. Myelencephalon. II. Metencephalon: I, Pons, 2, Cerebellum. III. Isthmus rhombencephali. IV. Mesencephalon: I, Pedunculi, 2, Corpora quadrigemina. V. Diencephalon: I, Pars mammillaris hypothalami, 2 , Thalamus, 3 , Epithalamus. VI. Telencephalon: 1 , Pars optica hypothalami, 2, Corpus striatum, 3, Rhinencephalon, 4, Neopallium.

ventriculi tertii and posterior surface of the pons, besides the vermis superior cerebelli and the medial part of the superior surface of the hemisphere. The lateral branch of the superior cerebellar artery passes from its point of origin near the sulcus lateralis of the mid-brain onto the superior surface of the cerebellum. It runs backward a half-inch from the border of that surface, giving off collaterals along its course. The lateral branch, together with the medial, supplies the superior cerebellar surface almost as far back as the horizontal sulcus of the cerebellum, along which the superior cerebellar artery anastomoses with both the inferior cerebellar arteries. 
The anterior inferior cerebellar artery (a. cerebelli anterior inferior, Fig. 9) is given off by the basilar near the junction of its inferior and middle thirds. (Sometimes it is replaced by two or three small vessels.) It runs lateralward, behind the flocculus, keeping close to the anterior border of the hemisphere. In its course it passes anterior to the abducent nerve and posterior to the facial and auditory nerves. It supplies the anterior part of the under surface and border of the cerebellar hemisphere.

The posterior inferior cerebellar artery $(a$. cerebelli inferior posterior, Fig. 9) is the largest branch of the vertebral and is given off just before the vertebral arteries unite and form the basilar. Passing first between the root-bundles of the hypoglossal nerve and then between those of the accessory and vagus nerves, the posterior inferior cerebellar artery bends at a right angle backward and runs between the medulla and the cerebellar hemisphere, where it divides into a medial and a lateral branch. The medial branch follows the sulcus valleculæ and gives branches to the medial part of the hemisphere and the vermis inferior. It anastomoses with its fellow of the opposite side. The lateral branch, runs lateralward from the posterior cerebellar notch over the inferior surface of the hemisphere; its terminal branches wind around the postero-lateral border and communicate with the superior cerebellar artery on the upper surface of the hemisphere. The undivided trunk of the posterior inferior cerebellar artery gives small branches to the medulla oblongata and supplies the chorioid tela of the fourth ventricle.

The internal cerebellar veins bring the blood from the interior of the organ and pour it into the superior and inferior external veins.

The superior external cerebellar veins (vence cerebelli superiores) converge forward into a medial vein, which empties into the great cerebral vein, and several lateral veins, which end in the transverse or the superior petrosal sinus.

The inferior external cerebellar veins (vence cerebelli inferiores) also form one small medial vein, which runs backward and upward either into the straight or transverse sinus, and a number of lateral veins. The lateral inferior cerebellar veins terminate in the inferior petrosal and in the occipital sinus. 
Lymphatics. - There are no lymphatic vessels in the cerebellum, but the perivascular lymph spaces carry out the lymph and pour it chiefly into the subarachnoid space.

\section{TABLE I.}

\section{EMBRYOLOGIC DIVISIONS OF THE BRAIN.}

In accordance with its development the brain or encephalon is naturally divided into three embryologic divisions which comprise the derivatives of the anterior, the middle and the posterior brain-vessels (Fig. I4).

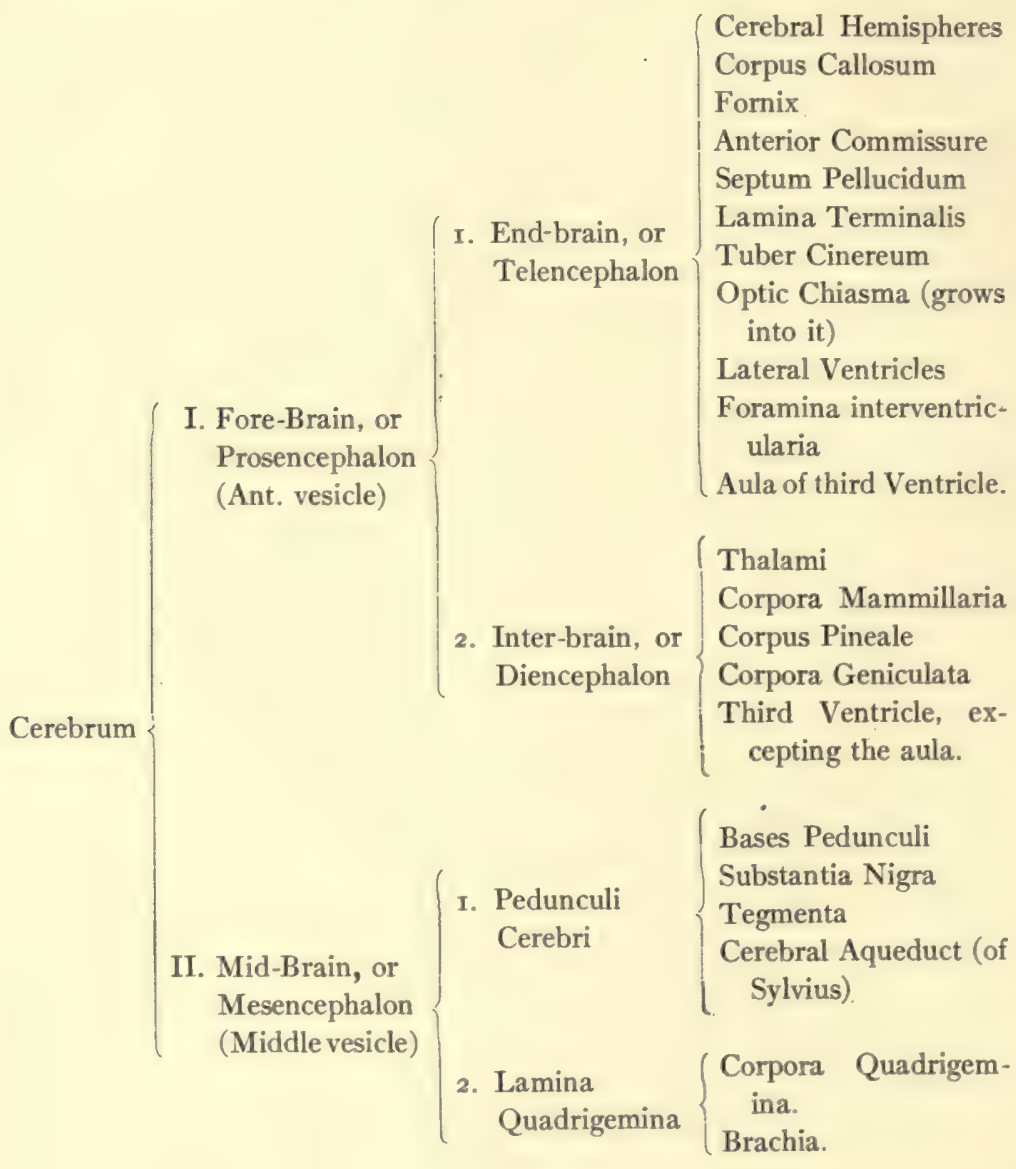




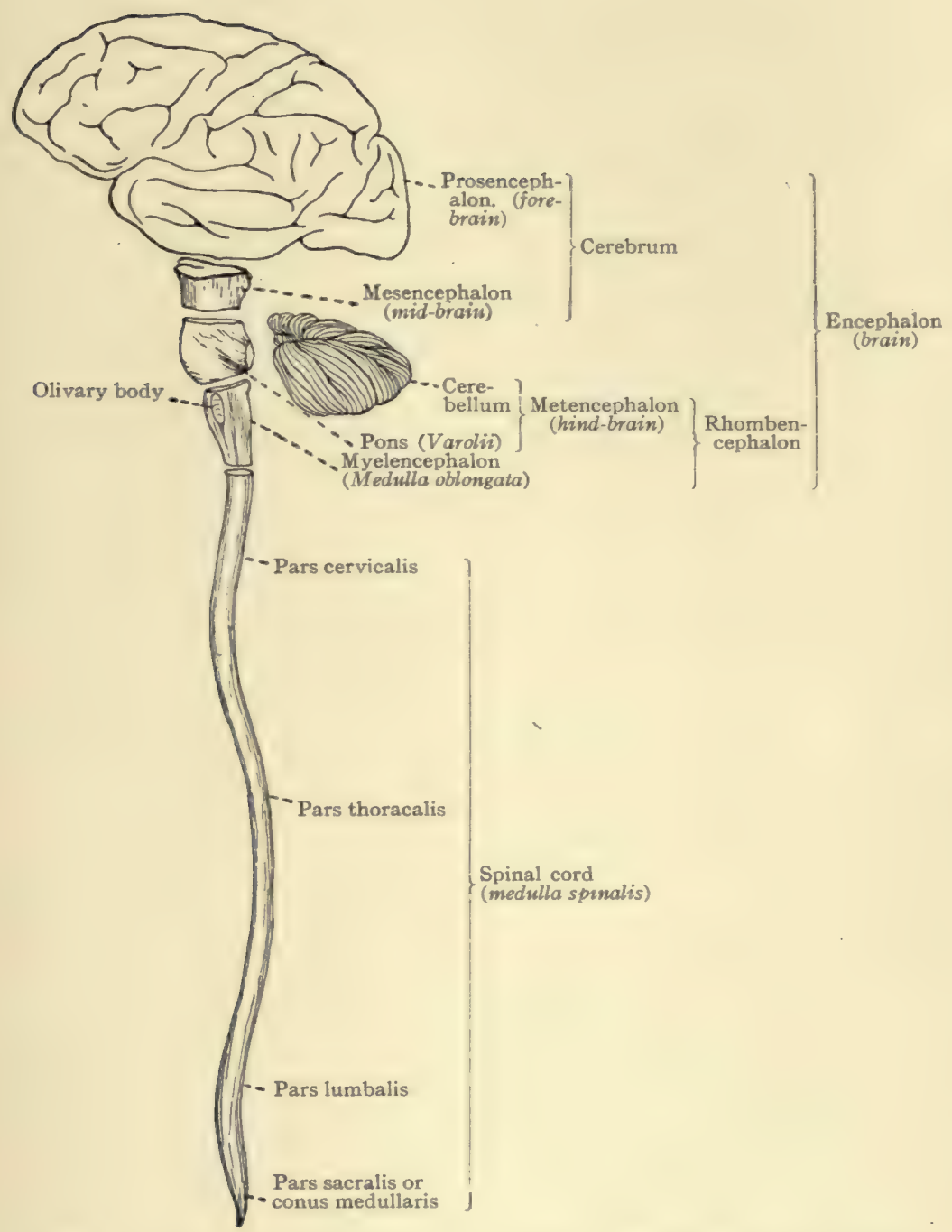

Fig. 15.-Divisions of the brain. Diagrammatic. (After Morris's Anatomy.) 

III. Rhombencephalon (Post. vesicle)
Isthmus Rhombencephali

Cerebellum, Pons

Upper half of Fourth Ventricle

2. Myelencephalon (After-brain)
Medulla Oblongata

Lower half of Fourth Ventricle

The cerebrum embraces the fore-brain and the mid-brain, as shown by the table. So we may make a more comprehensive division of the brain into only two grand divisions: The great brain or cerebrum and the rhombencephalon (Fig. I5). We may now simplify the above table as follows:

I. Cerebrum, embracing-

End-brain, or Cerebral Hemispheres, etc.

Inter-brain

Mid-brain.

II. Rhombencephalon, comprising-

Isthmus

Cerebellum

Pons

Medulla Oblongata. 
CHAPTER II.

\section{GENERAL CONSIDERATIONS OF THE BRAIN OR ENCEPHALON.}

Before taking up the special study of the cerebrum the student should notice certain prominent features of the entire brain. To do this the arachnoid and pia mater must be removed, and great care and patience should be exercised to preserve the integrity of the brain substance and to guard against evulsion of the roots of the cerebral nerves.

The human brain forms the greatly expanded superior extremity of the cerebro-spinal axis. It is derived from three sack-like dilatations of the epiblastic neural tube, called the anterior, the middle and the posterior brain-vesicles (Fig. I6).

Cavities. - The cavities of these brain-vesicles constitute the adult ventricles, which form a continuous median series extending from the canal of the spinal cord up to the level of the cerebral hemispheres; at that level the central cavity bifurcates into a branch for each hemisphere of the cerebrum (Figs. I 7 and 18). Thus is formed the lateral ventricle in the cerebral hemisphere and, below the cerebral hemispheres, the median series of cavities comprises the third ventricle in the inter-brain, the cerebral aqueduct in the mid-brain, and the fourth ventricle in the hindbrain and after-brain. The walls of these simple embryonic cavities undergo wonderful development and specialization; ultimately they produce all the multiform and complicated structures of the adult human brain.

Superior View. - The superior surface of the brain is markedly convex (Figs. I9 and 22). It is elliptical in outline, the major axis being contained in the median line; the greatest transverse axis is situated a little behind the middle and runs between the points which, when the brain is in the skull, underlie the tubera parietalia. This surface is closely adapted to the interior of the 
calvaria. Only the great convoluted hemispheres of the cerebrum are visible from the superior view-point. The two hemispheres are separated by a deep, median cleft, called the longitudinal fissure of the cerebrum (fissura longitudinalis cerebri) from which the falx cerebri has been removed.

Posterior View. - When the brain is viewed from behind, three great structures and two transverse fissures are visible (Fig. 20):

$\mathbf{A}$
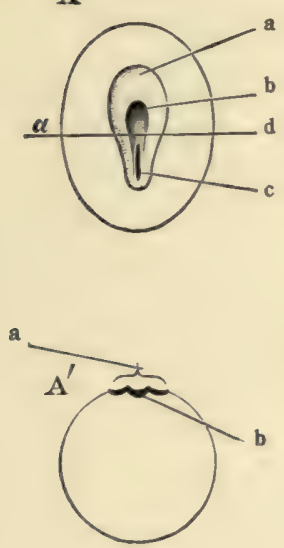

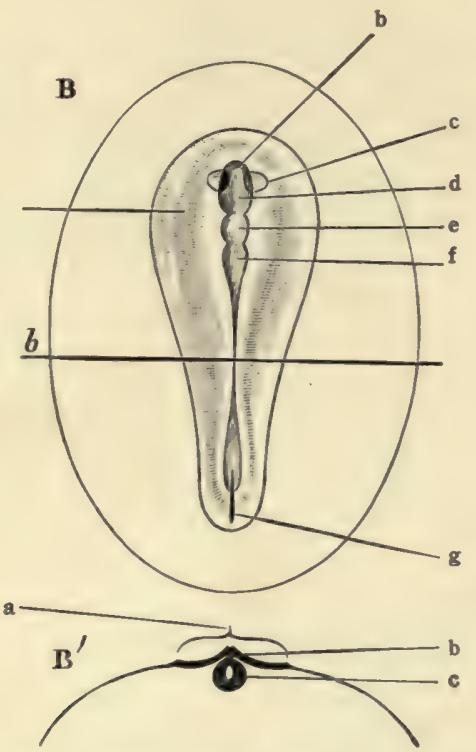

Fig. I6.-Diagrams of surface views and sections of germinal areas showing the development of the primitive streak, neural groove, neural tube and brain vesicles. (After Morris's Anatomy.)

A. Earlier stage, a. Germinal area, b. Neural groove. c. Primitive streak. B. Later stage. a. Germinal area. b. Fore-brain (rudiment of cerebral hemispheres). c. Optic vesicle. d. First cerebral vesicle. e. Second cerebral vesicle. f. Third cerebral vesicle. g. Primitive streak. $\mathbf{A}^{\prime}$. Section through area $\mathbf{A}$ along the line $a$, a. Germinal area. b. Neural groove, $\mathbf{B}^{\prime}$. Section through area $\mathbf{B}$ along line $b$. a. Germinal area. b. Neural crest. c. Neural tube.

First, the occipital end of the cerebral hemispheres with their irregular gyri and sulci; second, the transversely laminated cerebellum, lying below the cerebrum and separated from it by the transverse fissure of the cerebrum (fissura transversa cerebri); and third, the inferior extremity of a relatively small median structure, the medulla oblongata. The cerebellum is especially 
characterized by its parallel crescentic sulci, which give it a stratified appearance. It shows a partial subdivision into lateral hemispheres produced by a posterior median depression, called the posterior cerebellar notch, and by a longitudinal groove on its inferior surface, called the vallecula cerebelli. The vallecula is fitted over the posterior surface of the medulla. Thecerebellum is, therefore, separated from the medulla oblongata by a sharply

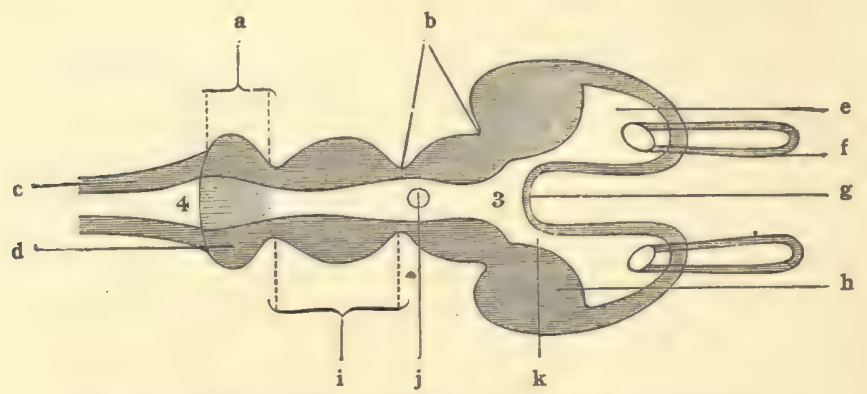

Fig. 17.-Diagrammatic horizontal section of vertebrate brain.

(Morris's Anatomy after $H$ uxley.)

a. Metencephalon. b. Thalamus. c. Medulla oblongata. d. Cerebellum. e. Lateral ventricle. f. Olfactory diverticulum. g. Lamina terminalis. h. Corpus striatum. i. Mid-brain. j. Pineal body. $\mathbf{k}$. Interventricular foramen.

curved, rainbow-shaped fissure. That fissure is the transverse fissure of the cerebellum (fissura transversa cerebelli) which, as already pointed out, is bridged over by the arachnoid and contains the cisterna cerebello-medullaris.

Inferior View.-The base of the brain presents three areas, situated in three successive levels, which correspond in location and extent to the great fossæ in the base of the cranium (Figs. 21 and 27). The anterior area, situated in the anterior cranial fossa, occupies the highest level; the middle area is intermediate in position; it occupies the middle fossa and together with the anterior area comprises all of the base of the cerebrum which is visible in the complete brain; and, the posterior area, which is but the base of the rhombencephalon, is situated at the lowest level in the posterior fossa of the cranium.

The anterior area of the base of the brain is divided into lateral halves by the longitudinal fissure of the cerebrum, and 
separated from the middle area by the fossa and fissura lateralis cerebri. The frontal lobe of the cerebral hemisphere, on either side of the longitudinal fissure, makes up nearly all this area. The inferior surface of the frontal lobe is concave and is adapted to the convex orbital plate of the frontal bone; its medial border is most prominent and presents, near the longitudinal fissure, an elongated gray mass, the olfactory bulb (if it has not been torn

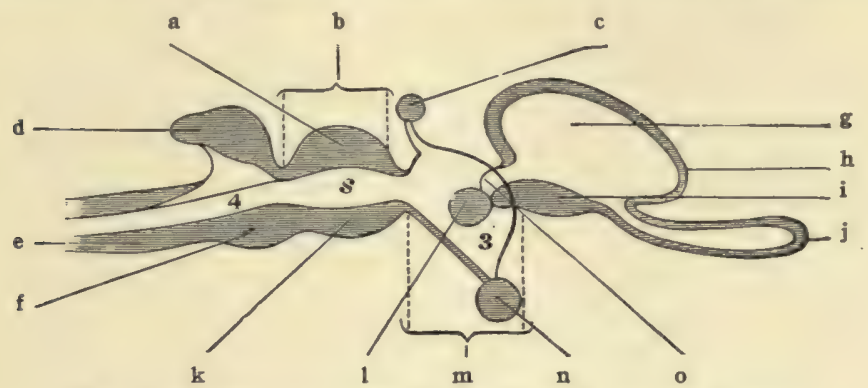

Fig. I8.-Diagrammatic sagittal section of vertebrate brain. (Morris's Anatomy after Huxley.)

a. Corpora quadrigemina. b. Mid-brain. c. Pineal body. d. Cerebellum (hind-brain). e. Medulla oblongata (after-brain). f. Pons Varolii (hind-brain). g. Lateral ventricle. h. Cerebral hemisphere. i. Corpus striatum. j. Olfactory diverticulum. k. Pedunculi cerebri. 1. Thalamus, m. Inter-brain. n. Hypophysis. o. Interventicular foramen. 4. Fourth ventricle. s. Aqueduct of cerebrum. 3. Third ventricle.

off) and a white strand, the olfactory tract. Running backward from the bulb, parallel with the longitudinal fissure of the cerebrum to the fossa lateralis cerebri, the olfactory tract is seen to bifurcate into two distinct striæ, a medial and a lateral.

The middle area of the inferior surface of the brain is prominent laterally where it is formed by the temporal lobes of the cerebrum. It is depressed in its median portion and thus adapted to the hypophyseal region of the cranial floor. This median hypophyseal region extends from the end of the longitudinal fissure, in front, backward to a great white, transversely striated eminence, called the pons; it contains several important structures, viz., the bases pedunculi; posterior perforated substance; the mammillary bodies; tuber cinereum and stem of the infundibulum; optic chiasma, tracts and nerves; lamina cinerea terminalis; and the anterior perforated substance.

Issuing from the under surface of the cerebral hemisphere and 
running downward toward the median line, there may be seen a white striated band, a half-inch broad, called the basis pedunculi, which, on approximating its fellow in the median plane disappears into the pons. Anteriorly, the X-like optic chiasma (chiasma opticum) is easily identified near the longitudinal fissure; its anterior limbs are the optic nerves and its posterior, the optic tracts (Fig. 2I). The optic tract, when traced backward and outward, under the overhanging temporal lobe, is observed to cross the basis pedunculi at its point of emergence from the cerebral hemisphere. Thus the optic tract and the basis pedunculi form the lateral boundary of a diamond-shaped space extending from the optic chiasma, in front, backward to the pons. This is commonly called the interpeduncular space. You observe in it three structures: (I) A gray eminence just behind the optic chiasma called the tuber cinereum; (2) a pair of white, nipple-like bodies, an eighth of an inch in diameter, known as the white or mammillary bodies (corpora mammillaria), and (3) a triangular, perforated mass of dark gray substance, called the posterior perforated substance (substantia perforata posterior). In the normal condition, the infundibulum projects downward and forward from the center of the tuber cinereum and connects it with the hypophysis cerebri; but it is usually broken in removing the brain and the hypophysis left behind in the hypophyseal fossa.

If the optic chiasma be drawn slightly downward and backward, a transverse and nearly vertical sheet of gray matter will be seen extending upward from it, between the cerebral hemispheres, toward the corpus callosum. That is the lamina cinerea terminalis. It bounds posteriorly the frontal part of the longitudinal fissure of the cerebrum. Lateral to the optic chiasma and anterior to the optic tract, the gray substance is perforated by many vessels; it is called the anterior perforated substance (substantia perforata anterior) to distinguish it from a similar posterior region located between the bases pedunculi.

Posterior Area.--The posterior area of the base of the brain is formed by the pons, the cerebellum, and the medulla oblongata, which constitute the rhombencephalon (Fig. 2I). The pons and medulla are median structures. They are separated by a 


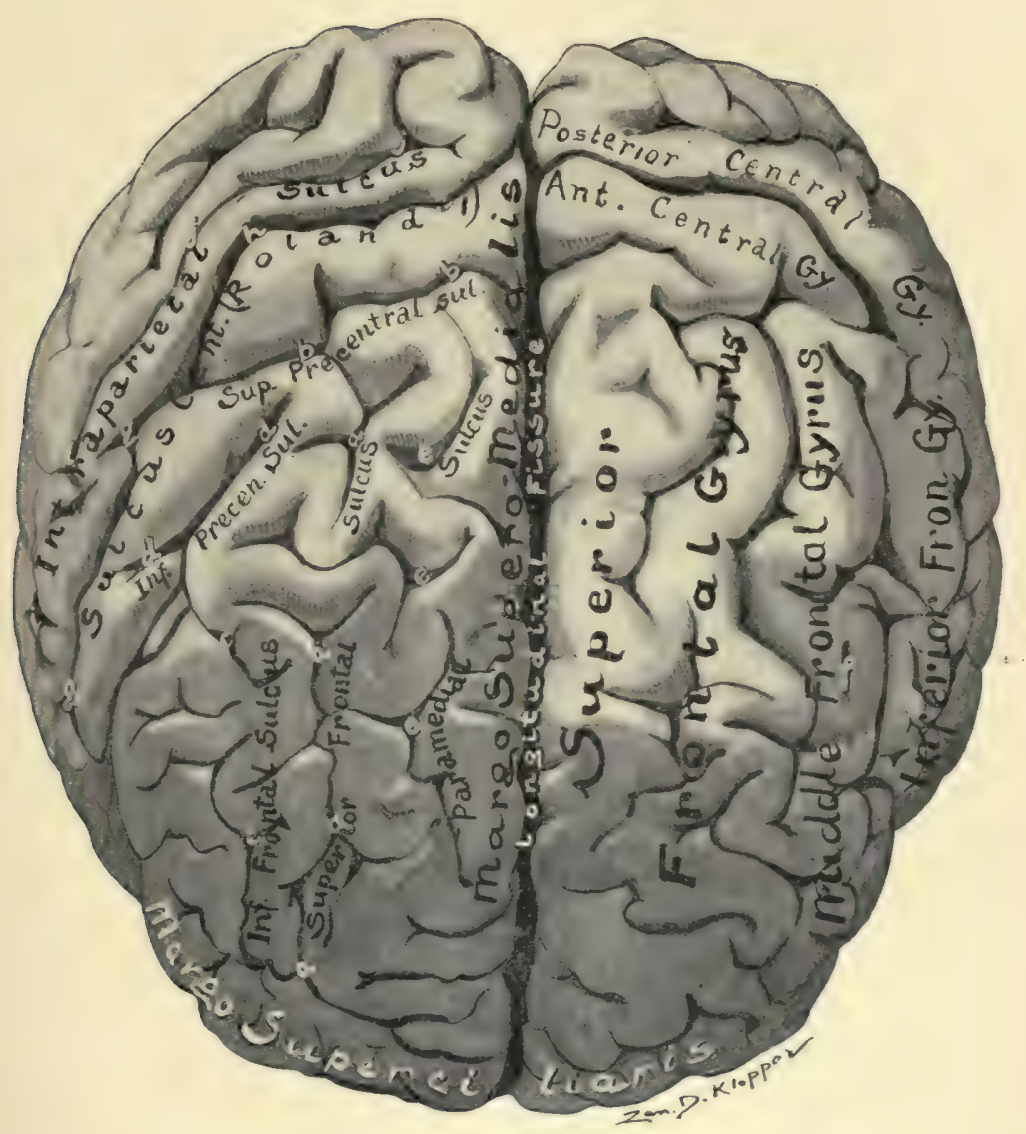

Fig. 19.-Fronto-superior surface of cerebrum. (Original.) 

well marked transverse groove containing the roots of the sixth, the seventh, the intermediate and the eighth cerebral nerves. The transverse strands of the pons traced lateralward are observed to form a large round bundle, called the brachium pontis, which extends into the hemisphere of the cerebellum on either side. Between those pontine strands, at the lateral border of the pons, there should be noticed the roots of the great trigeminal nerve. A sagittal line through this nerve at its attachment to the pons may be regarded as the boundary between the pons and the cerebellar hemisphere. The hemispheres of the cerebellum form the lateral part of the posterior area; their stratified appearance is already familiar. Inferior to the pons is the medulla oblongata. The medulla is about an inch long and three-quarters of an inch broad near the pons, but measures less than one-half inch in width at the lower end. It is partially divided into lateral halves by the anterior median fissure, which is deep, above, but is almost obliterated in the lower half of the medulla by the crossing of the lateral pyramidal tracts, the decussatio pyramidum. On either side of the anterior median fissure, the student should notice, in this order, the pyramid, the olive, and the restiform body. The pyramid (pyramis) bounds the anterior median fissure. It is an eighth of an inch in width, is most prominent near the pons and tapers off inferiorly because about 80 per cent. of its fibers cross over to the opposite side and sink backward in the medulla. It is bounded laterally by a slight longitudinal furrow, the anterior lateral sulcus (sulcus lateralis anterior) which contains the roots of the twelfth cerebral nerve, and separates the pyramid from the olive and from a flat surface, called the lateral funiculus of the medulla. The olive (oliva) occupies the upper half of the lateral surface of the medulla; the lateral funiculus, the lower half. The olive is equal in breadth to the pyramid. It is quite prominent, is white in color and is elliptical in outline. The posterior lateral sulcus (sulcus lateralis posterior) separates it from the restiform body. The roots of the ninth, tenth and eleventh cerebral nerves, which are contained in that groove and the restiform body which lies beyond it, can be seen only by pressing aside the hemisphere of the cerebellum. 
The Roots of the Twelve Cerebral Nerves (Fig. 2I).-The cerebral nerves (nervi cerebrales) are numbered from before backward according to the order of their points of attachment to the brain surface. Those points of attachment are, for the motor roots, points of exit from the brain (apparent origins); and are points of entrance into the brain (apparent central terminations), for all the sensory roots. The genetic nucleus (mucleus originis), which is the real origin of each motor root, and the terminal nucleus (nucleus terminalis), which contains the real central termination of every sensory root, are imbedded within the brain substance and do not at present concern us.

1. The olfactory nerves (nervi olfactorii) are the first. .They are the nerves of smell. They are composed of twenty or thirty scattered bundles of non-medullated fibers which rise from the olfactory cells in the nasal mucous membrane and, passing through the cribriform plate of the ethmoid bone, enter the under surface of the olfactory bulb. The surface of the bulb is, therefore, their apparent central termination. The fibers proceed some distance into the gray substance of the olfactory bulb, which constitutes the terminal mucleus of the first nerves, and there branch richly and end in relation with the mitral and bush-cells (real central termination).

2. Optic Nerve. (Nervus opticus).-The second nerve, the nerve of sight, is really a brain tract rather than a nerve, and its fibers are imbedded in neuroglia. It rises in the ganglionar layer of the retina. Passing through the chorioid and sclera of the eyeball and the optic foramen of the sphenoid bone, it enters into the optic chiasma where the "nerve" is said to end; but the fibers of the nerve continue without interruption through the optic tracts and their lateral roots to the inter-brain and the midbrain, whose surfaces they pierce (apparent central termination); they end (real central termination) in the lateral geniculate body, in the pulvinar of the thalamus, and in the superior colliculus of the quadrigeminal bodies.

3. The oculomotor nerve ( $n$. oculomotorius) is the great motor nerve to the eye (Fig. 2I). It issues from the mid-brain at the medial border of the basis pedunculi, which is its apparent origin, 


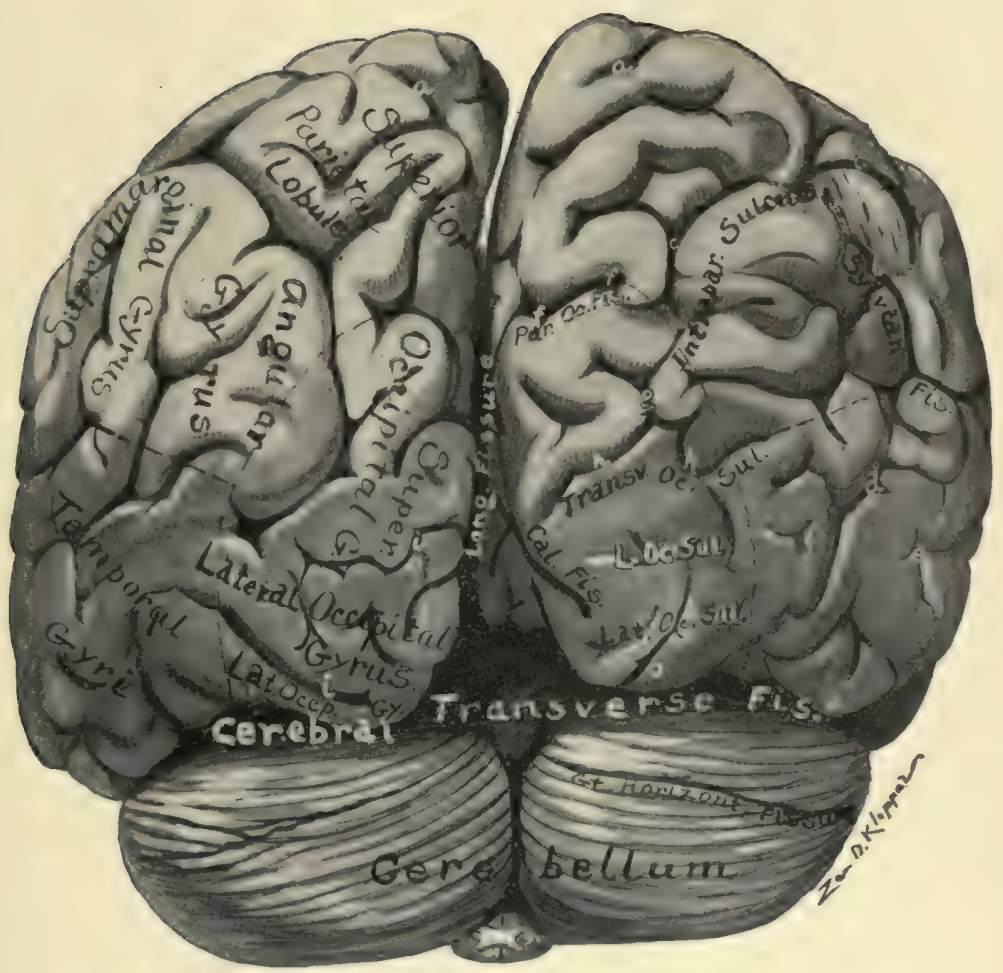

Fig. 20.-Posterior view of the brain. (Original.) 

but its real origin is in a mass of gray substance, the genetic nucleus ( $n$. originis), situated within the depths of the mid-brain.

4. Trochlear Nerve. (N.trochlearis).-The fourth is a motor nerve to the eye and is the smallest of the cerebral nerves. It may be seen winding forward over the basis pedunculi (Fig. 2I). Its apparent origin is from the dorsal surface of the brain stem at the junction of the mid-brain with the hind-brain (the isthmus, Fig. 44); this apparent origin cannot be seen in the complete brain. The genetic nucleus of the fourth nerve is located below that of the third in the mid-brain.

5. Trigeminal Nerve. ( $N$. trigeminus).-The trigeminal nerve is a mixed nerve, motor and sensory (Fig. 2I). It is attached to the ventral surface of the pons a little above the middle of its lateral border. The small anterior motor root emerges from this point (apparent origin); but this is the apparent central termination of the large sensory root, which rises in the semilunar ganglion (Gasseri) and enters the pons close to the emergence of the motor root.

6. The abducent nerve ( $n$. abducens) is a motor nerve to the eye. It issues from the pons at its inferior border, or from the transverse groove between the pons and the medulla, just above the pyramid of the medulla and nearly in line with the anterior lateral sulcus (Fig. 2I).

In the transverse groove between the pons and the medulla, lateralward from the root of the sixth nerve, are the roots of the seventh, intermediate and eighth. The seventh is smaller in diameter than the eighth and medial to it in position; the intermediate is between these two (Figs. 2I and 45).

7. The facial nerve ( $n$. facialis) is the motor nerve to the muscles of expression (Figs. 2 I and 45). Rising from a nucleus in the pons, its emergence from the transverse groove between the medulla and pons constitutes its apparent origin. The intermediate nerve ( $n$. intermedius) is so closely associated with the facial nerve that many regard it as the sensory root of that nerve; but the intermediate nerve is in reality a mixed nerve with efferent fibers of vasodilator, secretory and trophic functions and afferent fibers whose function is taste. The efferent fibers rise from the 
salivary nucleus (the dorsal part of the facial nucleus) in the pons. They issue from the transverse ponto-medullary groove between the facial and auditory nerves (apparent origin) at the point where the afferent fibers enter the brain. The sensory part of the intermediate nerve, which is the nerve of taste to the anterior part of the tongue, takes its origin in the ganglion geniculi situated within the canalis facialis (Follopii); its apparent central termination is in the ponto-medullary groove.

8. The acustic nerve ( $n$. acusticus) is a sensory nerve, having the double function of hearing and equilibrium (Figs. 2I, 44 and 45 ). It rises from the spiral and vestibular ganglia situated in the petrous bone, and its apparent central termination is in the bottom of the transverse groove separating the pons from the medulla. The roots of both the seventh and eighth nerves are near the upper end of the posterior lateral sulcus of the medulla oblongata.

9. Glossopharngeal Nerve. ( $N$. glossopharyngeus).-This is a complex mixed nerve, containing efferent fibers (motor, vasodilator, secretory and trophic) and afferent fibers, which are both common sensory and gustatory. It is joined to the medulla in the bottom of the superior end of the posterior lateral sulcus (Figs. 2I and 45). This point of attachment is the apparent origin of its efferent and the apparent central termination of its afferent fibers. The latter fibers rise in the superior and petrosal glossopharyngeal ganglia situated in the jugular foramen. The genetic nucleus of the efferent fibers is located inside the medulla.

Behind the ninth nerve in the same groove are the roots of the tenth and eleventh nerves. The roots of the ninth and tenth are situated between the olive and the restiform body; but, if the nerve trunks have been cut, it is impossible to determine which of the ten or a dozen root bundles belong to each of them.

10. The Vagus Nerve. (N.vagus).-The efferent fibers of the vagus, like the glossopharyngeal, take their apparent origin from the posterior lateral sulcus, and in the same sulcus the afferent fibers enter the medulla, apparent central termination (Figs. 2I and 45 ). It is a very complex nerve. Its efferent fibers comprise motor, inhibito-motor, vasodilator, secretory, trophic and inhibito- 


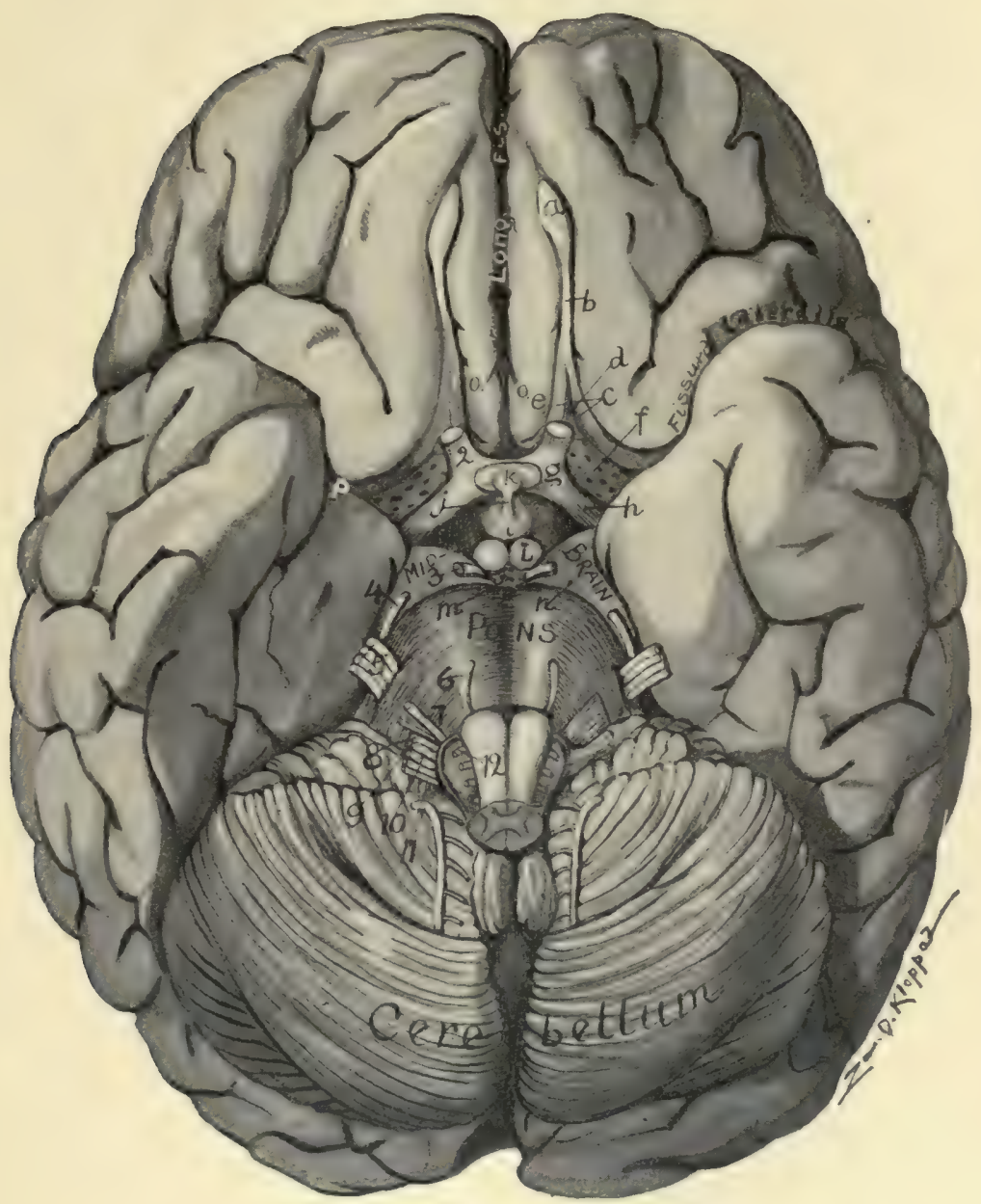

Fig. 21.-Base of brain. (Original.)

a. Olfactory bulb, b. Olfactory tract. c. Medial and lateral olfactory striæ. d. Trigonum olfactorium. e. Area parolf actoria (Brocæ). f. Anterior perforated substance. g. Optic chiasma. h. Optic tract. i. Tuber cinereum. j. Infundibulum. k. Hypophysis. 1. Corpus mammillare. $m$. Posterior perforated substance. n. Basis pedunculi. o. Sulcus parclfactorius anterior. 2 to $\mathbf{2}$, the cerebral nerves. 

secretory fibers (Pawlow). The afferent or sensory fibers of the vagus rise in the jugular and nodular ganglia of the nerve ( $g$. jugulare and g. nodosum) within and just below the jugular foramen. Within the medulla are the genetic nuclei of the efferent fibers.

11. The accessory nerve ( $n$. accessorius) is composed of a cerebral and a spinal root both of which are efferent in function (Fig. 45). The cerebral root (radix cerebralis) rises within the medulla and issues from the posterior lateral sulcus below the level of the olive and immediately inferior to the roots of the vagus (apparent origin). This is distributed entirely by way of the vagus. The spinal root (radix spinalis), having taken its $a p$ parent origin from the lateral surface of the spinal cord and passed through the foramen magnum, joins the cerebral (accessory) root near the jugular foramen.

12. Hypoglossal Nerve. (N. hypoglossus). - The twelfth is the great motor nerve to the tongue (Figs. 2I and 45). A half dozen or more radicals make it up; they rise in the medulla and issue in linear series from the anterior lateral sulcus of the medulla between the pyramid and the olive (apparent origin). The root bundles which emerge from the same sulcus below the level of the olive belong to the anterior root of the first cervical nerve. The student should now turn back to Table I. Study it carefully and identify all the primary and secondary divisions of the brain (Figs. 14, I5, I6, I7, 18, and 28). 


\section{CHAPTER III.}

\section{THE CEREBRUM.}

The cerebrum with its great hemispheres is that part of the brain which especially characterizes man. In man only do the hemispheres reach such predominant development. Though they are mere outgrowths of the anterior brain-vesicle in the beginning, they completely overshadow all other parts of the brain by the seventh month of embryonic life, extending farther forward, backward and lateralward than any other part. Within the cerebral hemispheres lies the physical basis of all mental function; they constitute the central mechanism of thought and consciousness.

Reference to the table given above shows that the cerebrum is made up of three parts: (I) The end-brain, which includes the cerebral hemispheres and their connecting links; (2) the inter-brain, comprising the thalami and their associated nuclei, which with the former constituted the fore-brain; and (3) the mid-brain (Figs. I7, I8, and 27). The cerebrum is an ovoid mass, flattened inferiorly, which fills the vault of the cranium and rests, below, upon the floor of the cranial cavity in the anterior and middle fossæ and upon the tentorium cerebelli over the posterior fossa (Fig. 2). Viewed from above, it is sufficiently round to suggest a sphere; and, being divided in the median line by the longitudinal fissure, the lateral halves are called hemispheres. The most anterior point is the frontal pole, and the most posterior is the occipital pole (Fig. 22). In the floor of the longitudinal fissure of the cerebrum the corpus callosum can be seen joining the hemispheres together; and beneath it, concealed from view, are the formix and anterior commissure. 'Those are the connecting links, proper, of the hemispheres (Figs. 34, 36 and 37). Inferior to them is found the inter-brain. The latter form an additional union of the hemispheres, as may be seen by viewing 
the base of the brain. Just caudal to the inter-brain is the midbrain which occupies the tentorial notch of the dura mater; and, situated in the median line, is so overhung by the cerebral hemispheres as to reveal only its anterior surface. It resembles the inter-brain in this respect. Inferiorly the mid-brain joins the rhombencephalon. Their plane of union cuts the isthmus (Fig. 44).

In studying the gross structures of the cerebrum it is most convenient to divide it into its embryologic divisions, viz., the fore-brain and the mid-brain.

\section{SECTION I. THE FORE-BRAIN OR PROSENCEPHALON.}

Fore-brain $\begin{cases}\text { r. End-brain }\left\{\begin{array}{l}\text { Cerebral Hemispheres and their connecting } \\ \text { links- } \\ \text { Corpus Callosum } \\ \text { Commissura Anterior } \\ \text { Commissura Hippocampi (Fornix). }\end{array}\right. \\ \text { 2. Inter-brain }\left\{\begin{array}{l}\text { Thalami } \\ \text { Mammillary Bodies (of hypothalamus) } \\ \text { Geniculate Bodies (metathalamus) } \\ \text { Pineal Body (of epithalamus). }\end{array}\right.\end{cases}$

In order to fix important landmarks and to learn the location and relations of the gross structures of the fore-brain it is necessary to study in detail the topography of the exterior surface and the interior surface. It is that with which the present section deals. For the minute anatomy of the cerebral structures, see Section III of the Cerebrum.

\section{EXTERIOR SURFACE OF FORE-BRAIN.}

The exterior surface of the fore-brain is composed of a thin sheet of gray matter varying in thickness from one-sixth to onequarter of an inch. That gray matter forms a bark-like covering for the underlying white substance and is, therefore, called the cortex (Figs. 34 and 35). It is thrown into irregular elongated 
folds named convolutions, or gyri, by deep linear depressions, which greatly increase the relative amount of cortical substance. The linear depressions are called fissures, or sulci; and, in consequence of them, the gray substance is increased in bulk to fifty-eight and one-half per cent. of the entire cerebrum (DeRegibus).

The name fissure is properly applied, first to those deep furrows which represent clefts between embryonic vesicles, viz., the median, verticle cleft between the cerebral hemispheres, and the two arched clefts, one between the cerebellum and the cerebral hemispheres and the other between the cerebellum and the posterior surface of the medulla oblongata (Figs. I9 and 20); and, second, the deep linear depressions in the cerebral hemisphere which indent the entire ventricular wall and produce eminences on the interior surface are properly called fissures. All other furrows in the cerebral surface are called sulci.

The exterior surface of the fore-brain is divided by distinct borders into three regions, namely, the convex surface, the medial surface, and the basal surface (Figs. 22, 26 and 27). The basal surface comprises the orbital and tentorial areas, separated by the stem of the fissura lateralis cerebri (Sylvii). The convex surface is separated from the medial surface by the superomedial border (margo supero-medialis), from the tentorial area of the basal surface by the infero-lateral border (margo infero-lateralis, or $m$. occipitalis lateralis), and from the orbital area of the basal surface by the superciliary border (margo superciliaris). The medial orbital border (margo orbitalis medialis) separates the orbital area of the basal surface from the medial surface, and the medial occipital border (margo occipitalis medialis) divides the medial surface from the tentorial area of the basal surface (Figs. 19, 22 and 26).

\section{FISSURES AND SULCI OF CONVEX SURFACE.}

The convex surface of the cerebral hemisphere (facies convexa cerebri) is related to two very extensive fissures, viz., the longitudinal and the transverse. The longitudinal fissure of the cerebrum (fissura longitudinalis cerebri) is the vertical median 


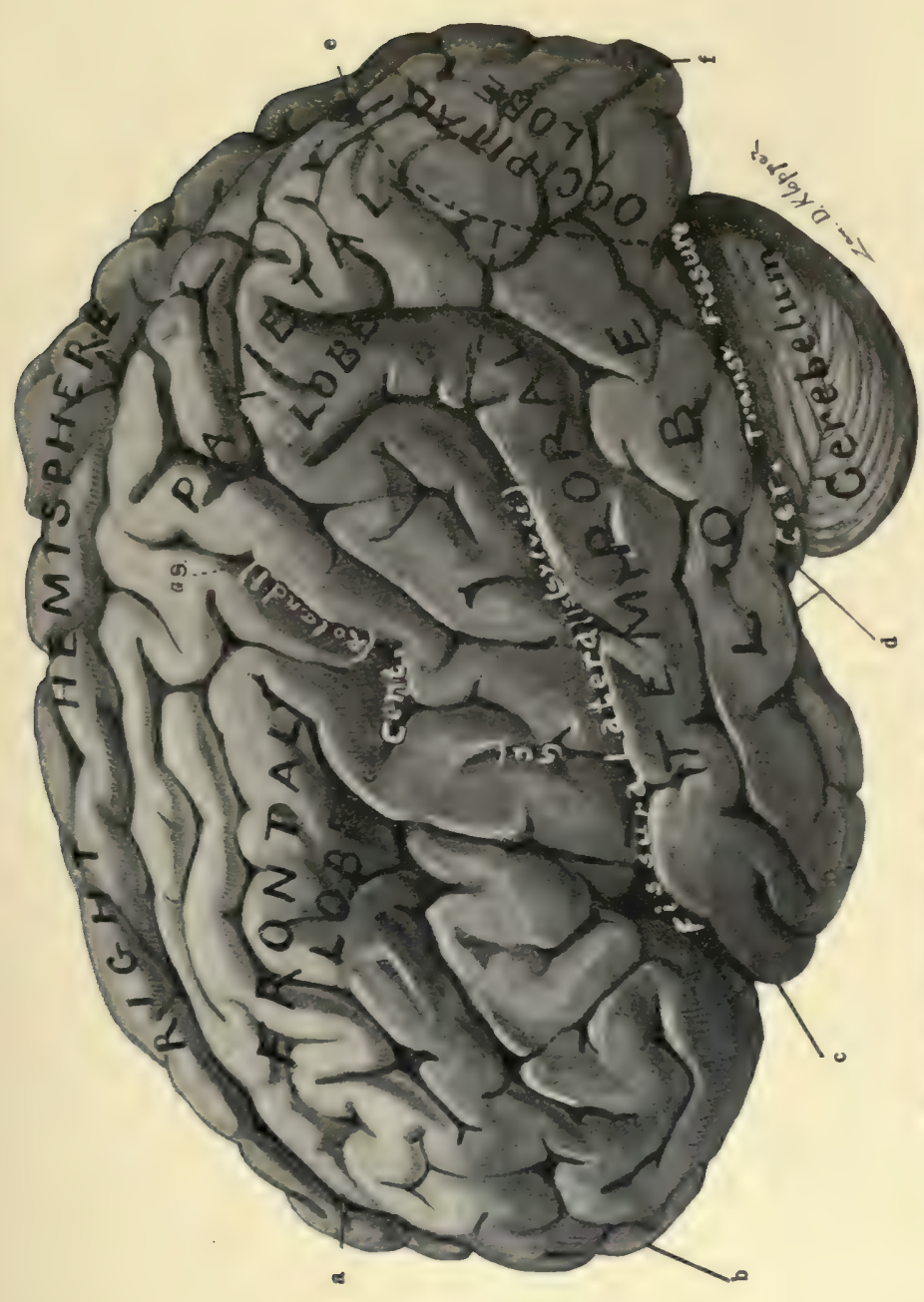

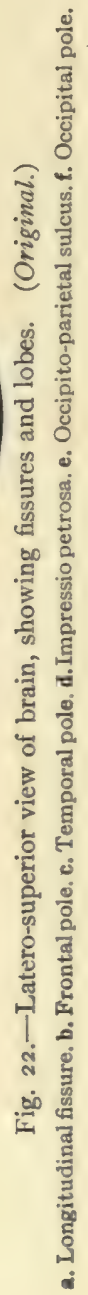



cleft between the hemispheres of the cerebrum (Figs. I9 and 22). It contains the falx cerebri (Fig. I). Its floor is formed by the corpus callosum. The cerebrum is separated from the cerebellum by the transverse fissure of the cerebrum (fissura transversa cerebri, Figs. 20, 27, I and 6). This fissure continues forward above the mid-brain, and terminates in the cerebrum between the inter-brain and the fornix, where it is continuous, by its lateral extremities, with the chorioidal fissures of the hemispheres. The tentorium occupies the posterior part. The anterior part of the transverse fissure contains the chorioid tela of the third ventricle.

There are three great furrows in the convex surface of each cerebral hemisphere which form interlobar boundaries and constitute very important landmarks: The fissura cerebri lateralis, the sulcus centralis, and the sulcus occipito-parietalis (Figs. 22 and 23 ).

The lateral fissure (fissura cerebri lateralis, [Sylvii]) begins in the fossa of the same name at the base of the brain (Fig. 2I). It runs outward between the frontal and the temporal lobe, along the lesser wing of the sphenoid bone; and, turning upward, on the convex surface, it divides three-fourth inch behind the Sylvian point into an anterior horizontal, and anterior ascending and a posterior ramus (Fig. 23). Into the frontal lobe project the small anterior rami. They are separated by the foot (posterior end) of the inferior frontal gyrus, called the pars triangularis. Below the anterior horizontal ramus is a knuckle of the same frontal gyrus which forms the pars orbitalis; and, between the ascending and posterior rami, is located the pars opercularis, constituting the connecting gyrus between the anterior and posterior central gyri. The inferior frontal gyrus forms the frontal part of the operculum (pars frontalis operculi). The operculum (operculum, a cover) covers the island. The posterior limb of the lateral cerebral fissure separates the temporal lobe from the parietal. Near the crotch and within the fissure is situated the island. A line drawn from the Sylvian point, one and one-quarter inches behind the zygomatic process of the frontal bone and one and a half inches above that of the temporal, backward to the subparietal 
point, three-quarters of an inch below the tuber parietale, lies directly over the posterior ramus of this fissure.

The Sulcus Centralis (Rolandi, Figs. 22, 23, 24 and 27).Beginning just above the posterior limb of the lateral cerebral fissure, is the central sulcus, which extends upward and backward to the longitudinal fissure of the cerebrum. Its upper extremity is about half an inch (or 5.7 per cent.) behind the middle of a line drawn from the nasal eminence to the external occipital protuberance. With this sagittal meridian the sulcus centralis forms an anterior angle of 69 to 74 degrees. ' The average Rolandic angle is $7 \mathrm{I}^{\circ} 7^{\prime}$ (Cunningham). The sulcus centralis is three and three-eighths inches long and forms the boundary between the frontal and the parietal lobe. It is developed in two parts a superior third and an inferior two-thirds, which join at an angle open backward, called the genu superius; both parts may present an anterior concavity. Often a concealed gyrus separates the two parts of the sulcus at the genu superius (Fig. 22). This superior genu is in line with the superior frontal sulcus and marks the probable location of the trunk center and the boundary between the arm and leg areas in the anterior central gyrus. There is a less constant angle, the genu inferius, in the lower part of the central sulcus; it is in line with the inferior frontal sulcus and marks the lower limit of the arm area and the upper limit of the face area.

The Occipito-parietal Sulcus. (Sulcus occipito-parietalis).If the line on the skull locating the posterior limb of the lateral cerebral fissure be extended back to the sagittal meridian its posterior end marks the location of the occipito-parietal sulcus. The sulcus is located one-sixth of an inch above the lambda in the adult, and is from one and a half to two inches above the occipital pole. The greater part of the occipito-parietal sulcus is situated on the medial surface of the cerebral hemisphere, hence, it is divided into an internal part and an external part which are continuous through the supero-medial border (Figs. 20, 22 and 28). To the extent of its depth, which is about one inch, the external occipito-parietal sulcus separates the occipital from the parietal lobe on the convex surface of the hemisphere. Cunningham 

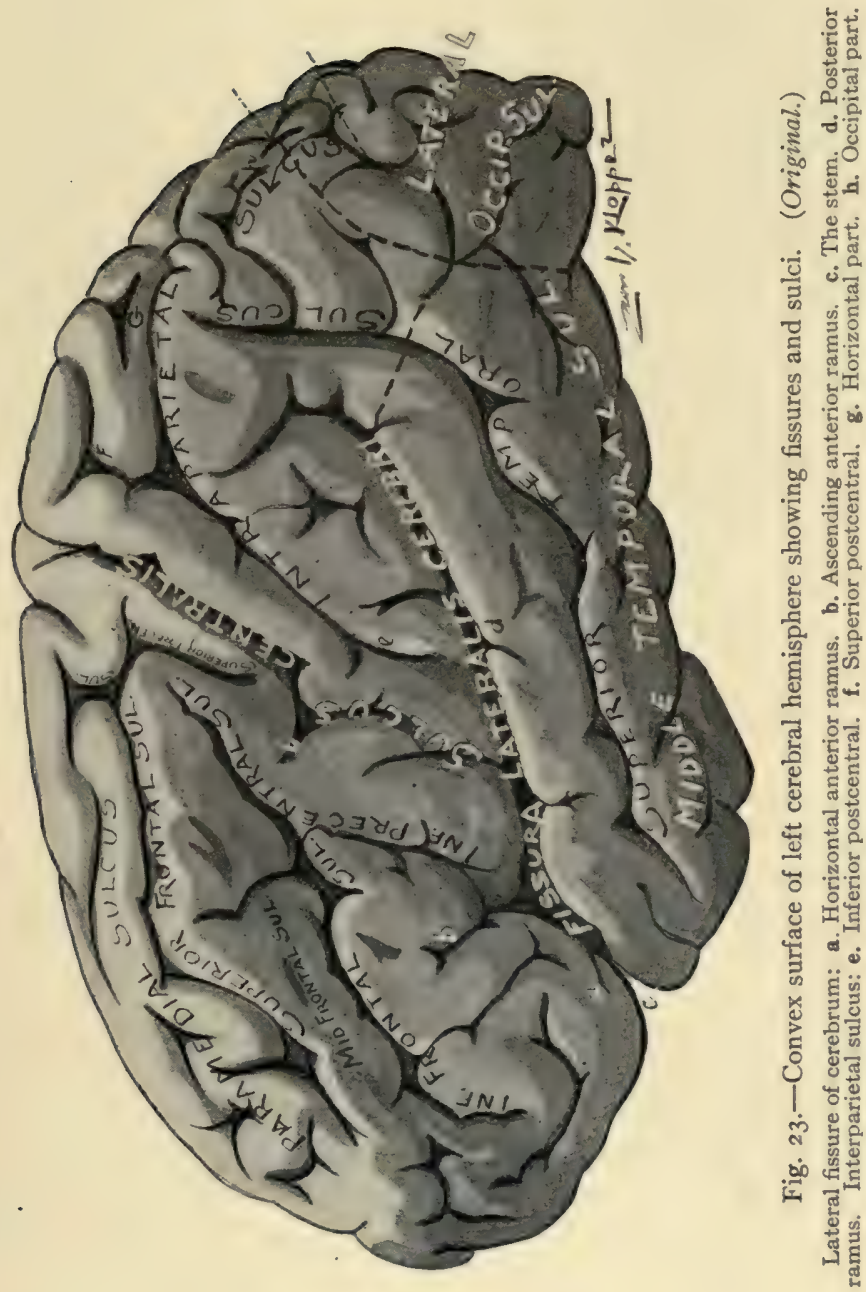

considers the occipito-parietal sulcus a true fissure because in the embryo it produces a ventricular eminence, though it disappears during development.*

\section{LOBES AND GYRI OF THE CONVEX SURFACE.}

(1) The frontal lobe (lobus frontalis) comprises the anterior polar region of the hemisphere and forms a part of all three surfaces (Figs. 22, 28 and 26). On the convex surface, it extends as far back as the central sulcus and the lateral cerebral fissure; on the basal surface, it is bounded behind by the stem of the lateral cerebral fissure and the anterior perforated spot; and it is limited posteriorly by the sulcus cinguli on the medial surface of the cerebral hemisphere.

On the convex surface, the frontal lobe has the following sulci and gyri (Figs. 23 and 24):

Sulci $\left\{\begin{array}{l}\text { Superior precentral (s. præcentralis superior) } \\ \text { Inferior precentral (s. præcentralis inferior) } \\ \text { Superior frontal (s. frontalis superior) } \\ \text { Inferior frontal (s. frontalis inferior) } \\ \text { Middle frontal (s. frontalis medius) } \\ \text { Paramedial (s. paramedialis). }\end{array}\right.$

Gyri $\left\{\begin{array}{l}\text { Anterior central (g. centralis anterior) } \\ \text { Superior frontal (g. frontalis superior) } \\ \text { Middle frontal (g. frontalis medius) } \\ \text { Inferior frontal (g. frontalis inferior). }\end{array}\right.$

The precentral sulci (Fig. 23) are parallel with the central sulcus and are located about a half inch in front of it, the lower end of the inferior precentral being insinuated between the central sulcus and the ascending ramus of the lateral fissure of the cerebrum. They form the anterior boundary of the anterior central gyrus. The superior frontal sulcus and the inferior frontal sulcus are respectively continuous with the corresponding precentral sulcus from which they trend downward and forward parallel

* The name of this sulcus is written "occipito-parietal" rather than "parieto-occipital;" this is a simpler word to pronounce as it avoids having "oocc" in the middle of it. 
with the supero-medial border of the hemisphere. They separate from each other three gyri of nearly equal width, viz., the superior, middle and inferior frontal gyri (Fig. 24).

The superior frontal gyrus is incompletely divided in the human brain by an interrupted sulcus, called the sulcus paramedialis (Fig. 23) which is located near the supero-medial border of the hemisphere and is said by Cunningham to be better developed in the higher types of the human race and to be rare in the higher apes.

A series of shallow furrows, described by Eberstaller as the middle frontal sulcus (s. frontalis medius, Fig. 23) partially subdivides the middle frontal gyrus into an upper and a lower part. The middle frontal sulcus, not found below the anthropoid apes (Cunningham), is best marked anteriorly and, at the superciliary border of the hemisphere, bifurcates and forms a horizontal furrow, the fronto-marginal sulcus. The posterior end, the foot, of the middle frontal gyrus, like that of the superior and inferior frontal, lies in the psychic-motor zone of the brain. It contains the writing center (Gordinier) in the left hemisphere of right-handed people.

The inferior frontal gyrus is highly developed in the human brain, especially in the left hemisphere of right-handed people. It is deeply cleft along its lower border by the anterior ascending and anterior horizontal rami of the lateral fissure of the cerebrum and is thus divided into a pars orbitalis, situated below the anterior horizontal ramus, a pars triangularis, inclosed between the anterior horizontal and ascending rami, and a pars basilaris, located between the anterior ascending ramus of the lateral fissure and the inferior precentral sulcus. The pars basilaris constitutes the foot of the inferior frontal gyrus and is continuous with the gyrus centralis anterior; on the left side it contains the speech center. The anterior portions of the superior middle and inferior frontal gyri comprise a psychic center, center of attention, volition, inhibition, etc., "of abstract concept" (Mills).

The anterior central gyrus ( $g$. centralis anterior) lies between the precentral sulci and the central sulcus. It is joined to the posterior central gyrus by the paracentral lobule, above the 

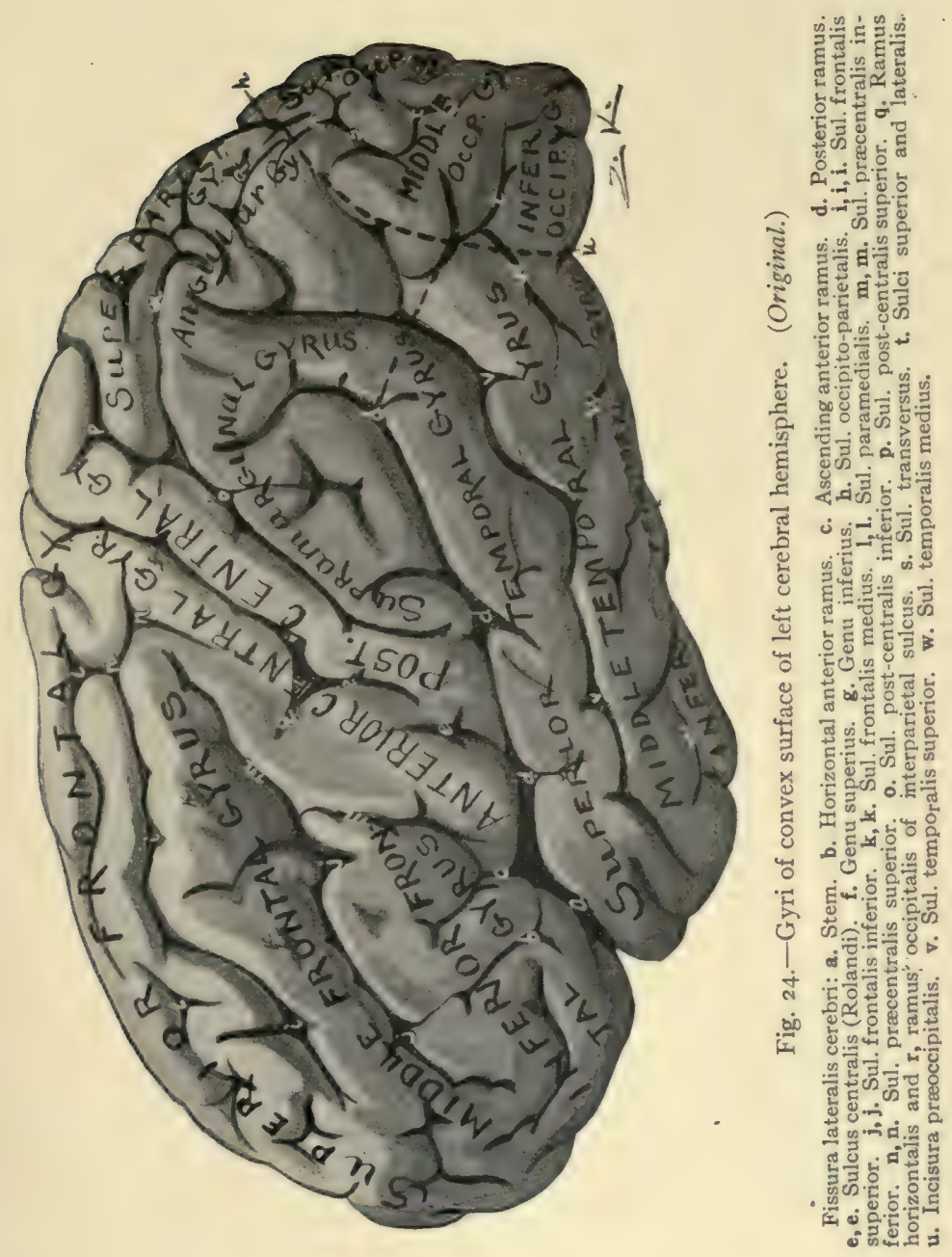

central sulcus, and by the fronto-parietal part of the operculum, below it. The anterior central gyrus, together with the paracentral lobule constitutes the emissive motor zone of the human cerebrum.

(2) The parietal lobe (lobus parietalis) is situated behind the central sulcus and above the posterior limb of the lateral fissure of the cerebrum (Figs. 22 and 28). From the curve near the posterior end of the latter to the occipito-parietal sulcus the lobe is separated from the temporal, below, and the occipital, behind, by an imaginary line. This imaginary line runs backward parallel with the infero-lateral border of the hemisphere to the boundary of the occipital lobe; and then, obliquely upward toward the supero-medial border in a line drawn from the preoccipital notch to the occipito-parietal sulcus. Extending over the supero-medial border, the lobe on the medial surface is inclosed between the occipito-parietal sulcus behind and the marginal part of the sulcus cinguli in front, and is bounded antero-inferiorly by the subparietal sulcus.

On the convex surface of the hemisphere the parietal lobe possesses the following sulci and gyri (Figs. 23 and 24).

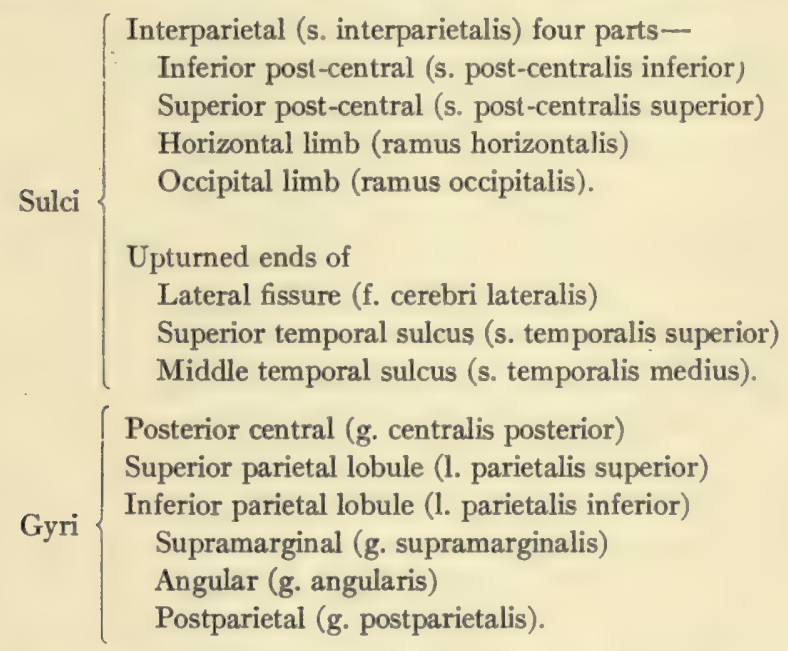

The interparietal sulcus (Figs. 20 and 23) is the only one belonging to the parietal lobe. The inferior and superior post- 
central sulci, constituting its anterior parts, are parallel with the central sulcus and are located a half or three-quarters of an inch behind it, separated from the central sulcus by the gyrus centralis posterior. The post-central sulci are often not continuous. The inferior is about twice the length of the superior, in this resembling the central sulcus, and usually it is joined at its upper end to the horizontal limb of the interparietal sulcus. The horizontal part of the sulcus lies about an inch below the supero-medial border of the hemisphere with which it is parallel; it separates the superior parietal lobule from the inferior parietal lobule and is continued as ramus occipitalis into the occipital lobe where it bifurcates.

The posterior central gyrus reaches from the posterior limb of the lateral fissure upward and backward, between the central and post-central sulci, to the longitudinal fissure of the cerebrum (Fig. 24). It is joined to the anterior-central gyrus around the ends of the central sulcus by superficial aninectant gyri (gyri transitivi) and sometimes is connected with it by a buried gyrus (g. profundus transitivus) which, deeply, separates the superior from the inferior part of the central sulcus. The annectant gyrus which closes the central sulcus superiorly and links together the central gyri is the paracentral lobule (lobulus paracentralis); the fronto-parietal part of the operculum joins them below the central sulcus. The posterior central gyrus and paracentral lobule constitute the receptive area of common sensation, the somesthetic area, so far as it extends on the convex surface.

The superior parietal lobule (Figs. 20 and 24) forms the supero-medial border of the hemisphere from the superior postcentral to the occipito-parietal sulcus. It is separated from the inferior parietal lobule by the horizontal part of the interparietal sulcus; posteriorly, it is joined to the occipital lobe by a curved annectant gyrus, called the arcus occipito-parietalis, which closes the superior end of the occipito-parietal sulcus; and, over the supero-medial border, it is continuous with the præcuneus of the medial surface. In the præcuneus and the superior parietal lobule Mills locates the stereognostic center (Figs. 56 and 57).

The Inferior Parietal Lobule.-The inferior parietal lobule is incompletely divided into two or three gyri. Named from 


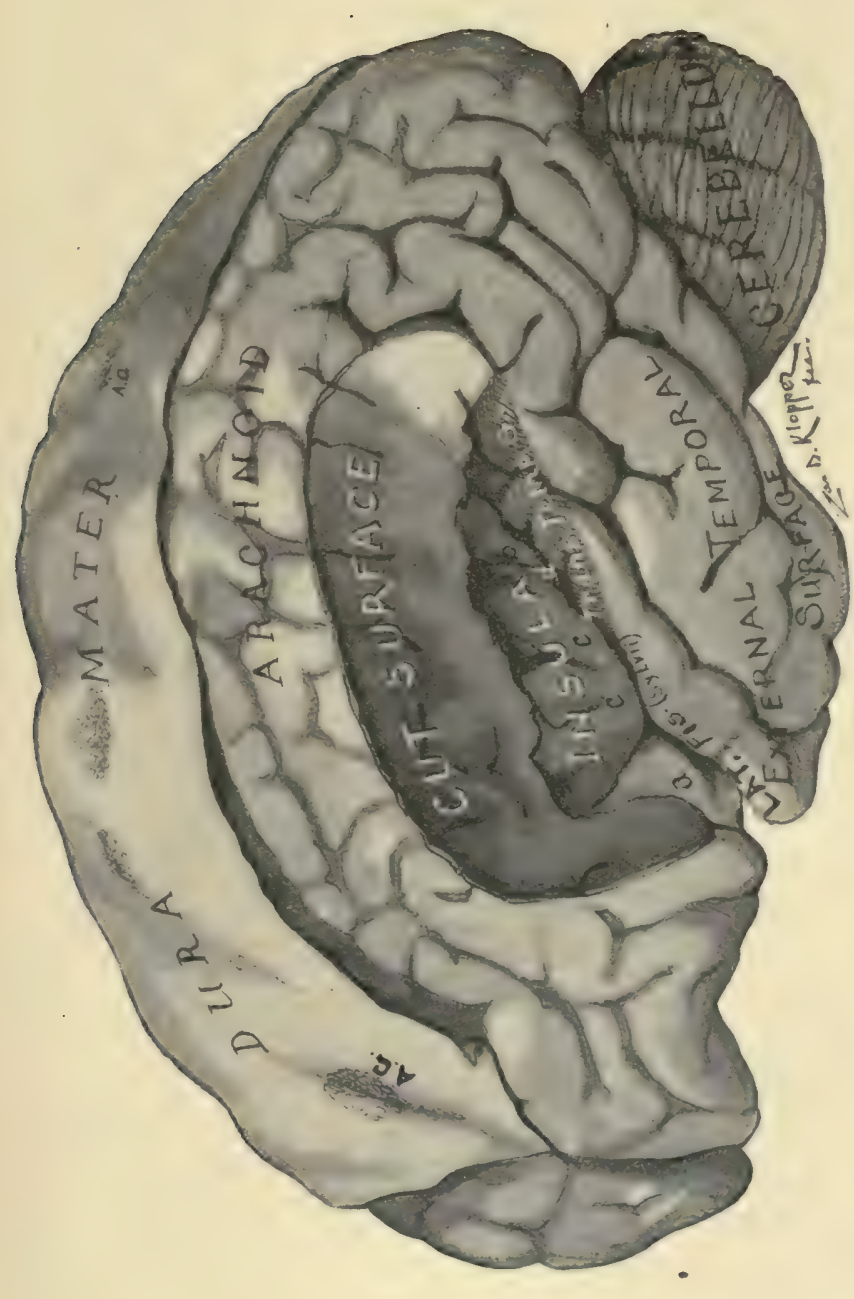

를 हैं

है

巳ี

3.

क

$\rightarrow-$

तो

旅

可

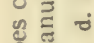

娄

증 흔

苋芯芯

라

ฮี

듕든 든

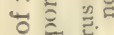

है छ

$⿻ 10$

. ⿷匚े

ปู่

क

पँ ऊ

흥. 홀

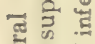

농

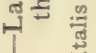

is

iा है 

before backward they are as follows: The supramarginal, the angular and the post-parietal (Figs. 20 and 24).

The supramarginal gyrus arches over and closes the upturned end of the posterior ramus of the lateral fissure of the cerebrum (Fig. 24). The anterior segment of the arch is continuous with the posterior central gyrus and is comprised in the psychic-sensory area; the posterior segment of the arch fuses with the angular gyrus, behind, and the superior temporal gyrus, below, and belongs to the psychic-sensory area, also, the motor memory center (Fig. 56).

The angular gyrus forms an arch over the end of the superior temporal sulcus (Figs. 20 and 24). The angular arch is in direct continuity with the superior and middle temporal gyri and the posterior segment of it is continuous with the post-parietal gyrus when that gyrus is present.

The post-parietal gyrus is present only when the middle temporal sulcus bends upward and terminates in the parietal lobe; in which case this gyrus curves over and closes that sulcus (not figured). It connects the posterior ends of the inferior and middle temporal gyri and also blends with the superior occipital gyrus. The angular, post-parietal and superior occipital gyri on the left side, according to Mills and others, constitute the center for visual memories. The studies of A. W. Campbell render it probable that the visual cortex does not extend into the parietal lobe of man at all. The receptive visual center for macular vision (vision in the macula lutea of the retina) is likewise located in the angular or post-parietal gyrus by Mills, but it is probably situated on the medial surface of the occipital lobe; unlike the memory center, this is present in both hemispheres.

(3) Occipital Lobe. (Lobus occipitalis, Figs. 20, 22, 24, 26 and 28). - The occipital lobe forms the posterior pole of the hemisphere. With the parietal and temporal lobes it is directly continuous, being marked off from them by an imaginary line drawn from the preoccipital notch to the occipito-parietal sulcus. This sulcus, on the convex surface, bounds it to the extent of about an inch; rarely, the external perpendicular sulcus bounds it in front. On the medial and basal surfaces of the hemisphere the 
occipital lobe extends from the occipito-parietal sulcus to the preoccipital notch, and is separated from the temporal lobe on the basal surface by an imaginary line drawn from this notch toward the posterior end of the corpus callosum, to the isthmus of the limbic lobe. The occipital lobe has the form of a triangular pyramid whose borders are the supero-medial, the infero-lateral and the medial occipital borders of the cerebral hemisphere. Those borders meet at its apex, the occipital pole.

The occipital lobe is somewhat rudimentary in man, though present only in apes and men (Cunningham). It makes its appearance at the fourth month in utero, and is distinctly outlined by fissures on all three surfaces at the sixth month, when it resembles the same lobe in the adult ape's brain (Cunningham Memoirs). After the sixth month the fissural boundaries largely disappear from the human brain on the convex and basal surfaces; hence, the artificial boundaries in the adult. Its rudimentary character probably accounts for the great variability in the sulci and gyri of the occipital lobe. On the convex surface they are usually as follows:

$$
\begin{aligned}
& \text { Sulci }\left\{\begin{array}{l}
\text { Ramus occipitalis of interparietal sulcus. } \\
\text { Transverse occipital (s. occipitalis transversus) } \\
\text { Lateral occipital (ss. occipitales laterales). }
\end{array}\right. \\
& \text { Gyri }\left\{\begin{array}{l}
\text { Superior occipital (gg. occipitales superiores) } \\
\text { Lateral occipital (gg. occipitales laterales). }
\end{array}\right.
\end{aligned}
$$

The lateral occipital sulcus is the only one properly belonging to the convex surface of the occipital lobe (sometimes there are two of them). It divides that surface almost equally into a superior and lateral gyrus, both of which may be double. The sulcus begins near the supero-medial border. It follows a meridian which is nearly parallel with the infero-lateral border of the lobe and runs forward, often presenting one interruption, to the occipito-temporal boundary line where is bifurcates and forms a short perpendicular sulcus, which represents the affenspalte of the ape (Elliot Smith). When the lateral occipital sulcus is double there are two lateral gyri (Figs. 20, 23 and 24).

The occipital limb of the interparietal sulcus descends in 
the occipital lobe a variable distance (Fig. 20). It is not always continuous with the horizontal limb. Running about an inch from the supero-medial border of the hemisphere, it passes the occipito-parietal sulcus, from which it is separated by the arcus occipilo-parietalis, and bifurcates in the superior occipital gyrus into two more or less oblique branches, constituting the transverse occipital sulcus. The medial end of the transverse sulcus may or may not cut the supero-medial border of the lobe.

The lateral occipital gyrus (Figs. 20 and 24) lies below the lateral occipital sulcus and extends from the occipital pole forward along the infero-lateral border of the hemisphere to the preoccipital notch. Sometimes it is divided into two lateral gyri by an inferior lateral sulcus. It is continuous with the inferior temporal gyrus, except rarely, when the two are separated by the external perpendicular sulcus.

The superior occipital gyrus forms the upper half of the convex surface of the lobe (Figs. 20 and 24). It is incompletely separated from the lateral occipital gyrus by the lateral occipital sulcus and is often divided into two gyri. It is continuous with the post-parietal gyrus around the lateral end of the transverse occipital sulcus; and, around the medial end of that sulcus, it is joined to the superior parietal lobule by the arcus occipitoparietalis. The latter is a sharply curved annectant gyrus which bounds the occipito-parietal sulcus. When the middle temporal sulcus does not turn upward at its posterior end and terminate in the parietal lobe, the superior occipital gyrus is continuous in front with the middle temporal and angular gyri. As already mentioned, the superior occipital gyrus, according to Mills, belongs to the center for visual memories.

(4) The temporal lobe (lobus temporalis, Figs. 20, 22, 23, 24, 25 and 26) is that part of the cerebral hemisphere behind the main stem and below the posterior limb of the lateral cerebral fissure. It rests in the middle fossa of the skull; forms the temporal pole of the hemisphere; and is continuous posteriorly with the occipital and parietal lobes, from which it is marked off only by the imaginary lines already described. On the basal surface of the hemisphere, the temporal lobe along its medial 
border is separated from the limbic lobe by the collateral fissure and by a short furrow, which is shallow in the adult human brain, called the sulcus ecto-rhinalis. The temporal lobe is attached to the hemisphere posteriorly and medially; but it presents three free surfaces - a superior, an external and an inferior-which meet at the anterior point, called the temporal pole.

The superior surface of the temporal lobe forms the inferior wall of the lateral cerebral fissure (Fig. 25). It looks somewhat medianward toward the island and constitutes the temporal part of the operculum. It is separated from the island by the inferior circular sulcus (s. circularis insula). Anteriorly, the superior surface of the temporal lobe is smooth; posteriorly, it possesses one or two shallow transverse temporal sulci which feebly outline two or three gyri, called the transverse temporal gyri of Heschl (gyri temporales transversi). These transverse gyri belong to the receptive auditory center (Figs. 3I, 35, 36 and 54).

The external surface of the temporal lobe presents the following sulci and gyri (Figs. 23 and 24):

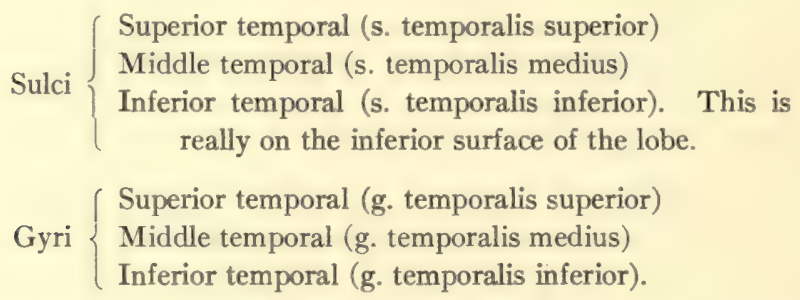

The superior temporal and middle temporal sulci (Fig. 23) divide the external surface into three nearly equal gyri; they run parallel with each other, with the infero-lateral border and with the lateral cerebral fissure. The superior temporal sulcus, like the lateral fissure, bends upward at its posterior extremity; it terminates in the concavity of the angular gyrus. The middle temporal sulcus is usually an interrupted one. It may present an upward curve at its posterior end which is bounded and closed by the post-parietal gyrus; or it may continue in its original direction toward the occipital lobe.

The inferior temporal sulcus, situated in the tentorial area of 
the basal surface, runs interruptedly close to the infero-lateral border of the hemisphere and parallel with it (Fig. 26). It separates the inferior temporal gyrus from the fusiform gyrus.

The superior temporal, the middle temporal and the inferior temporal gyri are of nearly equal width (Figs. 24 and 26). They fuse with one another and with the fusiform gyrus at the temporal pole. The superior temporal gyrus is continuous with the supramarginal and angular gyri, posteriorly: in its third and fourth fifths and in the transverse temporal gyri is the receptive auditory center (Barker).

The middle temporal gyrus fuses at its posterior end with the angular gyrus and either with the post-parietal or the superior occipital. Along the superior temporal sulcus, in the middle two-fourths of the superior and middle temporal gyri is the center for auditory memories, the psychic auditory center. This center is in the left hemisphere of right handed people (Figs. 54 and 56).

The inferior temporal gyrus forms the infero-lateral border of the hemisphere (Figs. 24 and 26). It is continuous with the lateral occipital gyrus and sometimes, also, with the superior occipital and post-parietal gyri. If this gyrus and the lower half of the middle temporal gyrus be divided into four equal parts, each fourth, according to Mills, belongs to a definite center. From behind forward they are the center of orientation; the center of equilibration (?); the naming center; and, in the anterior fourth and the pole of the temporal lobe, the center of intonation (Fig. 56).

(5) The island (insula, Reili) is also called the central lobe (Figs. 25, 26, 3I and 36). It is situated in the medial wall of the lateral fissure of the cerebrum, between the frontal, parietal and temporal lobes, whose growth, after the fifth month in utero, gradually covers it over. At the end of the first year of extrauterine life it is entirely concealed by temporal, parietal, and frontal parts of the operculum. The island is thus separated from the general surface of the cerebral hemisphere by a distance of half or three-quarters of an inch. It is triangular in shape. Its apex is directed downward and forward toward the fossa lateralis cerebri, and is called the pole (polus insula). If the lips of the lateral fissure be widely separated, the sulcus circularis insulæ 
may be seen separating the island from the frontal, parietal and temporal lobes (Figs. 25, 26 and 36). The circular sulcus is lacking only at the antero-inferior part, at the pole, where the cortex of the island is continuous with that of the posterior orbital gyrus and with the anterior perforated substance, and is on the same level as the orbital area of the basal surface. The imaginary line separating the anterior perforated substance from the island is called the threshold of the island (limen insulce).

In the island there is one named sulcus and four to six gyri which have a radial or fan-like grouping (Fig. 25):

$$
\begin{aligned}
& \text { Sulcus }\{\text { Central of the island (s. centralis insulae). } \\
& \text { Gyri }\left\{\begin{array}{l}
\text { Short (gyri breves), three or four of them. } \\
\text { Long (gyrus longus-furcalis). }
\end{array}\right.
\end{aligned}
$$

The sulcus centralis insulce begins at the apex, or pole, of the island and runs obliquely upward and backward dividing the lobe into two lobules (Fig. 25). It is in the same transverse plane as the central sulcus (of Rolando). In front of it, is the precentral lobule composed of the short gyri and continuous with the frontal lobe; the post-central lobule lies behind it and is in continuity with the parietal, temporal and limbic lobes.

The gyri breves insulæ, three or four in number, are separated by shallow. furrows which diverge upward and backward from the smooth apex of the precentral lobule (Fig. 25). They are joined to the orbital operculum by a short annectant gyrus (gyrus transversus insulce of Eberstaller) which extends from the apex around the lower end of the anterior circular sulcus. Their connection under the circular sulcus with the foot of the inferior frontal gyrus suggests a participation in the speech center, and they are figured by Mills in that center. However, the paraphasia which results from lesions in the short gyri may be due to the involvement of an association tract of fibers running underneath them.

Gyrus Longus (Furcalis) (Fig. 25).- It lies behind the central sulcus of the island and trends obliquely backward and upward. Posteriorly, it bifurcates for a short distance forming two short branches which are continuous under the circular sulcus with the 
parietal lobe; it fuses with the temporal lobe and the gyrus hippocampi of the limbic lobe, inferiorly.

The olfactory lobe and the limbic lobe, comprising the rhinencephalon and a part of the neopallium, also belong to the cerebral hemisphere; but no part of either can be seen on the convex surface (Figs. 28, $2 \mathrm{I}$ and I8).

\section{THE BASE OF THE FORE-BRAIN.}

The basal or inferior surface of the fore-brain comprises the inferior surface, first, of the end-brain, including the pars optica hypothalami and the cerebral hemispheres; and, second, of the inter-brain, which embraces the pars mammillaris hypothalami. It is completely exposed only when a section is made through the mid-brain and the rhombencephalon removed (Figs. 21 and 26). This should now be done with a thin, moistened brain-knife. Make the section from before backward and upward, at a right angle to the axis of the mid-brain. Now notice, first, the section of the mid-brain and, just anterior to that, the median structures of the fore-brain, occupying the center of the field; and, second, the surrounding inferior surface of the cerebral hemispheres. The latter form the very large peripheral zone.

The base of the cerebral hemisphere extends from the frontal to the occipital pole. In front it is composed of the orbital area bounded by the medial orbital and superciliary borders; and, posteriorly, is made up of the tentorial area, which is bounded, laterally, by the infero-lateral border, and, medially, by the chorioidal fissure and the medial occipital margin of the hemisphere (Fig. 26). The orbital area embraces the inferior surface of the frontal lobe and of the island, and the whole olfactory lobe; while the inferior surface of the temporal and occipital lobes, and the gyrus hippocampi and fascia dentata of the limbic lobe are included in the tentorial area.

Frontal Lobe, Inferior Surface (Fig. 26).--The inferior surface of the frontal lobe, resting on the orbital plate of the frontal bone, is often called the orbital lobe. It is separated from its fellow by the longitudinal fissure of the cerebrum, and is bounded behind 
by the lateral fossa and lateral fissure of the cerebrum, overlapped by the temporal lobe. More accurately, the posterior boundary is the anterior perforated substance and the anterior part of the circular sulcus. The orbital lobe is concave transversely and is divided by the triradiate or $H$-shaped sulcus orbitalis, made up of the medial orbital, the transverse orbital and the lateral orbital sulci; and by the olfactory sulcus, which is close to the longitudinal fissure and parallel with it. Five gyri are thus formed:

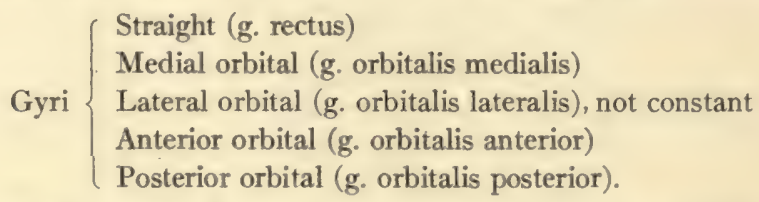

The gyrus rectus (Fig. 26) forms the medial border of this surface. It is separated from the medial orbital gyrus by the sulcus olfactorius in which lie the olfactory bulb and tract. Over on the medial surface it forms a part of the marginal gyrus and it joins the superior frontal at the frontal pole. Posteriorly, the gyrus rectus is separated from the parolfactory area (of Broca) by a slight furrow, the anterior parolfactory sulcus.

The medial orbital gyrus lies between the sulcus of the same name and the sulcus olfactorius (Fig. 26). It extends from the frontal pole to the anterior perforated substance and the island. The anterior and posterior orbital gyri lie within the $\mathrm{H}$-shaped orbital sulcus separated from each other by the transverse orbital sulcus. The former is continuous with the frontal gyri at the superciliary border; the latter is only partially separated, behind, from the island by the anterior circular sulcus; the posterior orbital gyrus is likewise continuous with the posterior end of the lateral orbital gyrus and with the orbital portion of the inferior frontal. The lateral orbital gyrus, which is a distinct gyrus only when the lateral orbital sulcus is long, is situated external to the H-shaped sulcus. It is continuous with both middle and inferior frontal gyri at the superciliary border of the hemisphere.

The Island (of Reil), Inferior Surface (Fig. 26). - If the anterior part of the temporal lobe be removed, the under surface of the island (insula) is brought into view. The circular sulcus 


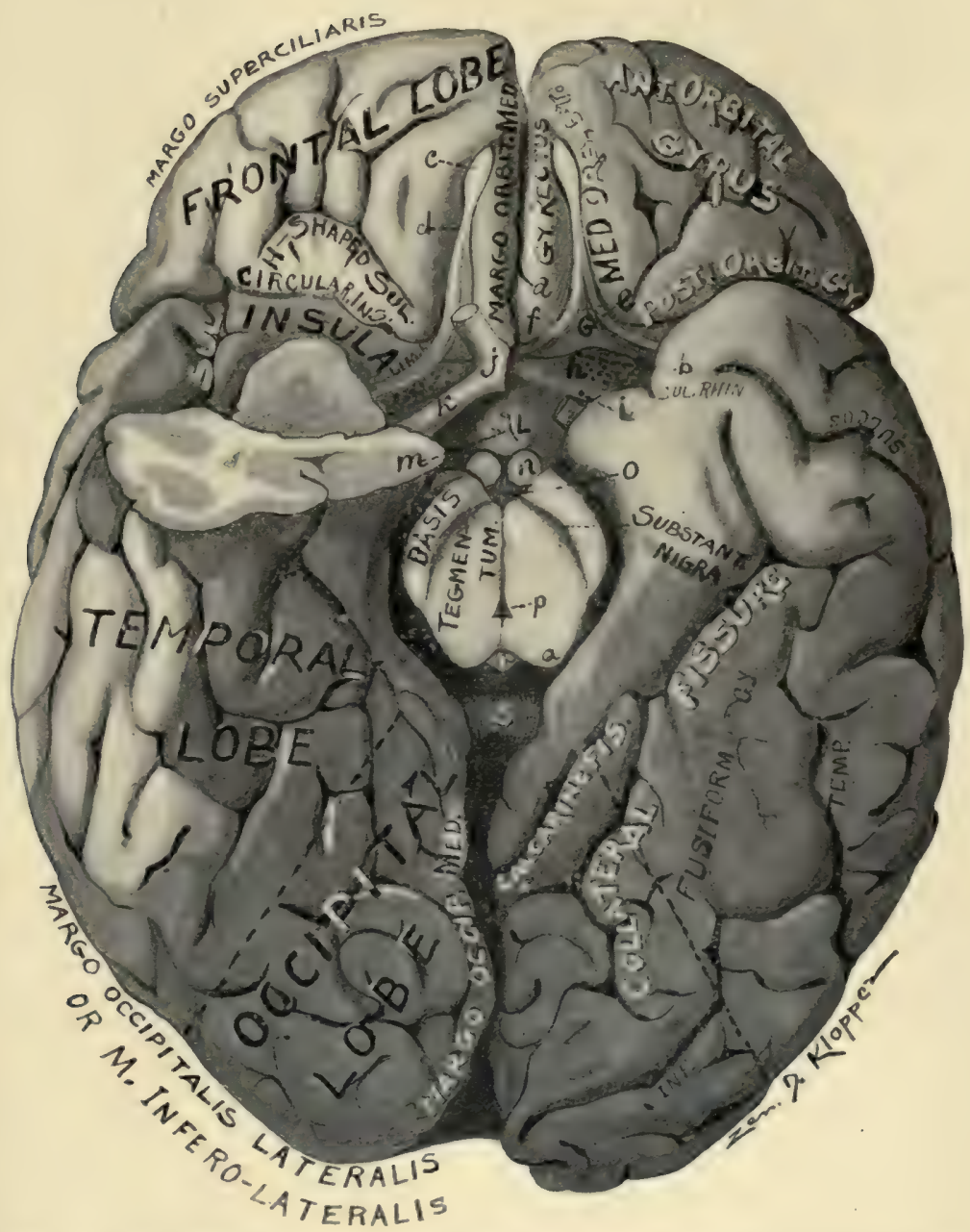

Fig. 26.--Base of fore-brain and cut surface of mid-brain. Right temporal pole cut away, to show inferior surface of the island: (Original.)

a. Sulcus parolfactorius anterior. b. Sulcus parolfactorius posterior. c. Olfactory bulb. d. Olfactory tract. e. Olfactory striæ. f. Area parolfactoria. g. Trigonum olfactorium. h. Substantia perforata anterior. i. Gyrus subcallosus (peduncle of corpus callosum). j. Optic chiasma. k. Optic tract. 1. Tuber cinereum. m. Infundibulum. n. Corpus mammillare. o. Substantia perforata posterior. p. Aqueductus cerebri. q. Quadrigeminal colliculus. r. Corpus pineale. S. Splenium. 

bounds it on two sides and separates it from the posterior orbital gyrus, in front; and from the temporal lobe, behind. Laterally it is separated from the frontal and the parietal parts of the operculum by an antero-posterior cleft continuous with the lateral cerebral fissure.

The insula is continuous with the anterior perforated substance, and the area of transition from one to the other is called the threshold, or limen insula (Fig. 26).

Rhinencephalon.-The smelling brain belongs to the basal surface. It is rudimentary in man. Many connected parts make it up. It is divided into two parts by the sulcus parolfactorius posterior. These are designated as the pars anterior rhinencephali and the pars posterior rhinencephali. The pars anterior of the rhinencephalon embraces, first, the oljactory lobe, which is made up of the olfactory bulb, tract, triangle and the medial and intermediate strix; and second, the area parolfactoria. In the pars posterior rhinencephali are included the anterior perforated substance, the gyrus subcallosus, the lateral olfactory stria, and the limen insulæ.

Olfactory Lobe. (Lobus olfactorius).-There is one lobe that is studied only on the basal surface of the fore-brain. That is the olfactory lobe (Fig. 26). Belonging to the pars anterior rhinencephali, it comprises many connected parts; and the reason for calling them the olfactory lobe is found in the lower animals and in the human embryo, where it exists as a prominent hollow process of the cerebral hemisphere (Figs. I7 and 18).

$$
\text { Olfactory Lobe }\left\{\begin{array}{l}
\text { Bulbus olfactorius } \\
\text { Tractus olfactorius } \\
\text { Trigonum olfactorium } \\
\text { Stria medialis } \\
\text { Stria intermedia. }
\end{array}\right.
$$

The olfactory bulb (bulbus olfactorius) is an ovoid mass of brain matter about half an inch long, one-sixth of an inch wide and a quarter of an inch in vertical diameter (Fig. 26). It is lodged in the olfactory sulcus of the frontal lobe and rests upon the cribriform plate of the ethmoid bone through which it receives the twenty or thirty olfactory nerves. The center of the bulb 
is formed by a gray core derived from the ependymal lining of the embryonic ventricle. The gray core is surrounded by a white sheath of medullated fibers running longitudinally; posterior to the bulb these fibers form the olfactory tract. Five layers of gray substance thicker on the ventral side, surround the white sheath and constitute the surface of the bulb. The gray substance forms the terminal nucleus of the olfactory nerves and gives origin to the fibers of the olfactory tract.

Olfactory Tract (Tractus olfactorius). - The tract is triangular in section, slightly more than an inch long and one line in width (Fig. 26). It is partially concealed in the olfactory sulcus, and is a continuation backward of the medullated mitral axones which ensheath the gray core of the olfactory bulb. At its posterior end the olfactory tract divides into three striæ-lateral, intermediate and medial, two of which are easily seen. These strice olfactorice are continuous with the three angles of the tract. The lateral and medial striæ diverge and inclose the olfactory triangle between them. The lateral stria (stria lateralis) courses outward and backward and terminates in the uncus at the anterior extremity of the hippocampal gyrus. According to Retzius, the lateral olfactory stria terminates in the rudimentary gyri, circumambiens and semilunaris, which form the anterior end of the hippocampal gyrus. The lateral stria bounds on the outer side the anterior perforated space. The medial stria (stria medialis) bends sharply inward, toward the median line, and runs between the triangle and parolfactory area (of Broca). Its fibers turn into Broca's area and the gyrus cinguli, chiefly, but some of them enter the triangle, the gyrus subcallosus, and the hippocampus through septum pellucidum and fornix. Thus the medial and lateral striæ unite the opposite ends of the gyrus fornicatus. From the dorsal angle of the olfactory tract, a bundle of fibers proceeds into the triangle and frontal lobe, constituting the intermediate stria (stria intermedia). The intermediate olfactory stria is often not visible on the surface, as it turns at once upward into the frontal lobe. Upon reaching the level of the anterior commissure, it passes through that commissure to the opposite hemisphere, where it divides into two bundles; one of which (the commissural 
bundle) turns down into the intermediate stria of the opposite olfactory tract, and the other (the decussating bundle) runs backward and outward toward the uncinate region of the limbic lobe.

The Olfactory Triangle and the Parolfactory Area (of Broca).The triangular portion of the cortex between the medial and lateral olfactory striæ, called the triangle (trigonum olfactorium) is continuous medially with the area parolfactoria. The medial stria marks the boundary between them (Figs. 26 and 27). Both are bounded behind by the sulcus parolfactorius posterior (transverse part), and the oblique part of the same fissure separates the parolfactory area from the gyrus subcallosus (peduncle of the corpus callosum). The area parolfactoria (Broca) is limited in front by a slight curved depression, the sulcus parolfactorius anterior. On the medial surface, this area joins the gyrus cinguli.

The anterior perforated substance (substantia perforata anterior) of the pars posterior rhinencephali requires further mention (Fig. 26). It is separated from the triangle by a very faint groove, the posterior sulcus parolfactorius. Medially, it is in direct continuity with the tuber cinereum. The optic tract bounds it, postero-medially. Laterally, it forms the limen insula in the floor of the fossa cerebri lateralis, where it is overlapped by the temporal lobe. Superiorly, it is continuous with the base of the corpus striatum. Coursing along the inner and outer border of the anterior perforated substance are, respectively, the gyrus subcallosus and lateral olfactory stria, which converge and meet in the hippocampal gyrus. The perforations of this area are for the antero-lateral ganglionic arteries.

Tentorial Area of the Basal Surface (Figs. 26, 24 and 28).From the temporal pole backward, the basal surface of the cerebral hemisphere presents three nearly parallel gyri, viz., the inferior temporal gyrus, which forms the infero-lateral border; the fusiform gyrus, the middle one, and the gyrus hippocampi which lies next the mid-brain. The last belongs to the gyrus fornicatus of the limbic lobe; it is continuous, posteriorly, with the lingual gyrus, which forms a part of the medial occipital border of the cerebral hemisphere. The fusiform and inferior temporal gyri belong to the inferior surface of the temporal and 
occipital lobes. These two lobes are directly continuous with each other on their inferior surfaces, and are only separated arbitrarily by an imaginary line drawn from the preoccipital notch to the anterior end of the calcarine fissure. They are only partially separated from the gyrus hippocampi; the ectorhinal sulcus (s. rhinalis) and the anterior part of the collateral fissure lie between the temporal lobe and the hippocampal gyrus of the limbic lobe; while the inferior surface of the occipital lobe is separated from the gyrus cinguli, of the limbic lobe, by the anterior calcarine fissure. The fissures and sulci of the tentorial area are the following:

Chorioidal fissure (f. chorioidea)

Hippocampal fissure (f. hippocampi)

Ectorhinal sulcus (s. ectorhinalis)

Collateral fissure (f. collateralis)

Inferior temporal sulcus (s. temporalis inferior).

The chorioidal fissure ( $f$. chorioidea) forms a part of the medial boundary of the tentorial area (Figs. 26 and 28). At the surface it appears to be identical with the hippocampal fissure; but, upon looking deeper, the two are found to be separated by the fascia dentata and the crus of the fornix. This fissure is separated from the inferior horn of the lateral ventricle, only by a layer of epithelium, derived from the roof plate of the telencephalon. It contains the chorioid plexus of the inferior horn.

Hippocampal Fissure. (F. hippocampi, Fig. 28).-Along the medial and concave border of the hippocampal gyrus is the crescentic fissure known as the hippocampal fissure. The fissure in front is closed by the uncus. It extends backward to the splenium of the corpus callosum where, in the adult, it is continuous with the furrow behind and above the corpus callosum, called the callosal sulcus. The hippocampal is a true fissure as it indents the whole ventricular wall; the long ventricular eminence produced by it is the hippocampus seen in the inferior horn of the lateral ventricle (see medial surface of the cerebral hemisphere).

Ectorhinal Sulcus. (Incisura temporalis, Figs. 26 and 29).Midway between the temporal pole and the hook-point of the hippocampal gyrus is a slight notch, called the ectorhinal sulcus, 
which represents an important lateral boundary of the rhinencephalon in animals with highly developed sense of smell. It indicates in man the boundary between the hippocampal and fusiform gyri. A half inch behind the ectorhinal sulcus is the anterior end of the collateral fissure.

Fissura Collateralis (Figs. 26 and 28).-The collateral fissure extends in a somewhat curved course from near the temporal pole almost to the occipital pole. Its anterior two-thirds separates the hippocampal from the fusiform gyrus; its posterior one-third completes the medial and upper boundary of the fusiform gyrus and separates it from the gyrus lingualis.

Inferior Temporal Sulcus (Fig. 26).--Only one sulcus belongs wholly within the inferior surface of the temporal and occipital lobes. It extends from a point near the occipital pole forward along the infero-lateral border of the hemisphere almost to the temporal pole, and incompletely separates the inferior temporal gyrus and the lateral occipital gyrus from the gyrus fusiformis. Very frequently the sulcus has two or more interruptions. It may be called the temporo-occipital sulcus.

Gyrus Fusiformis.-One gyrus only is found entirely within the inferior temporo-occipital region (Figs. 26 and 29). That is the fusiform (temporo-occipital gyrus). It extends from near the occipital pole forward and forms the temporal pole. The posterior nine-tenths of its medial boundary is formed by the collateral fissure and the anterior one-tenth by an imaginary line and the ectorhinal sulcus; laterally, it is bounded by the inferior temporal sulcus.

Gyrus Lingualis.-The gyrus lingualis lies above and medial to the posterior one-third of the collateral fissure; inferior and lateral from the calcarine fissure. It is continuous with the gyrus hippocampi of the limbic lobe in front. The gyrus lingualis (Fig. 29) forms nearly all of the medial occipital border of the hemisphere. It contains a part of the receptive visual center (Figs. 55 and 57 ).

Limbic Lobe (Lobus Limbus), Inferior Part.-The gyrus hippocampi of this lobe is visible on the inferior surface of the fore-brain (Fig. 26). Notice how this crescentic gyrus embraces 
in its concavity the section of the mid-brain. It is separated from the fusiform gyrus by the collateral fissure and the ectorhinal sulcus; and bounded medially by the hippocampal fissure. The anterior end of the gyrus is flexed inward and backward over the end of the hippocampal fissure and the whole anterior part constitutes the uncus hippocampi. . The region of the uncus is somewhat irregular and appears to comprise the gyrus circumambiens and the gyrus semilunaris described by Retzius. It represents the greater part of the lobus pyraformis of osmatic mammals and is probably the chief receptive center of smell; it receives the lateral stria of the olfactory tract and fuses with a low oblique ridge, the gyrus subcallosus (or peduncle of the corpus callosum).

If the hippocampal gyrus be drawn downward somewhat, a rudimentary gyrus may be seen between the hippocampal and chorioidal fissures. That is the dentate fascia. It is continuous with the reflected part of the uncus in front, and behind is in continuity with the fasciola cinerea and gyrus supracallosus. Like the hippocampal gyrus it forms a part of the limbic lobe. We shall recur to this lobe on the medial surface of the hemisphere.

Having studied the basal structures of the cerebral hemispheres, it is now in order to examine the median structures in the inferior surface of the fore-brain. They occupy the interpeduncular or hypophyseal region. They constitute the hypothalamus and form part of the floor of the third ventricle.

The hypothalamus is the name applied to the fore-brain structures under the thalamus. Posteriorly it blends with the mid-brain. Its free portion is divided into two parts, viz., the pars optica hypothalami and the pars mammillaris hypothalami. The former belongs to the telencephalon, the latter to the diencephalon. They include the following:

Pars Optica Hypothalami $\left\{\begin{array}{l}\text { Lamina cinerea terminalis } \\ \text { Optic chiasma (chiasma opticum) } \\ \text { Tuber cinereum } \\ \text { Infundibulum and } \\ \text { Hypophysis. }\end{array}\right.$

Pars Mammillaris Hypothalami Corpora mammillaria. 
The lamina cinerea terminalis (Fig. 27) is most superior of the median structures. It is a thin lamina of ash-colored (cinereum) gray matter closing the end of the neural tube. It extends from the anterior superior surface of the optic chiasma upward and backward to the anterior commissure, just in front of which it becomes continuous with the lamina rostralis of the corpus callosum. Laterally, it is continuous with the cortex of the cerebral hemisphere. Behind it, is the third ventricle; in front of it, a part of the longitudinal fissure of the cerebrum.

Optic Chiasma. (Chiasma opticum).-The optic chiasma is a quadrilateral sheet of nerve fibers whose anterior angles receive the optic nerves and whose posterior angles give off the optic tracts (Fig. 2I). With the nerves and tracts attached, it is $\mathrm{x}$ shape. The chiasma is a median structure and is situated beneath the lamina cinerea, in the optic groove of the sphenoid bone. The fibers of the optic nerves and tracts compose it. There are three sets of these fibers, namely, the intercerebral, the direct, and the decussating. A fourth group of fibers, called the interretinal and said to be commissural for the retinæ, has been hitherto described, but their existence is very doubtful. The intercerebral fibers are not found in the optic nerves, but form the inferior commissure (Guddeni) which joins together the medial geniculate bodies (Fig. 43). The direct (or temporal) and decussating (or nasal) fibers run through nerve and tract and join the retina with the brain on the same and the opposite side, respectively In most vertebrates below mammals, and in the mouse and guinea pig, it is said that the optic fibers all decussate in the chiasma. Normally in man and the higher mammals, the temporal half of each retina contributes to the chiasma direct fibers and the nasal half crossed fibers (Fig. 67). The optic nerves (nervi optici) extend from the foramen scleræ of each eyeball back to the front of the chiasma, through the optic foramina; they rise in the ganglionar cells of the retinæ, which are connected with the rods and cones by the bipolar neurones. The optic tracts (tractus optici) connect the chiasma with the brain. Each tract winds outward and backward around the cerebral peduncle, and divides into a medial and a lateral root (Fig. 43). The roots 
wind under the thalamus and disappear at the corresponding geniculate body. The lateral root contains all the retinal fibers, the medial root has nothing to do with vision. The fibers of the lateral root (radix lateralis) may be traced to the lateral geniculate body (80 per cent., Von Monokow), to the pulvinar of the thalamus (nearly all the 20 per cent. remaining), and the rest to the superior quadrigeminal colliculus. The optic radiation of the capsule connects these centers with the medial occipital cortex. The medial root rises and ends in the medial geniculate body and thalamus. Its fibers form the commissura inferior (Guddeni).

Tuber Cinereum. - The posterior border of the optic chiasma is continuous with the tuber cinereum (Figs. 2I and 26). Here the gray matter is thickened and centrally prominent. The bulbous infundibulum projects downward from it to rest in the sella Turcica, where it forms the posterior lobe of the hypophysis. The upper end of the infundibulum is hollow (funnel-like). Its cavity forms the lowest part of the third ventricle. In man the bulb of the infundibulum is solid at maturity, though hollow in the embryo. It is composed largely of fibrous tissue, notwithstanding the fact that it is developed from the floor of the telencephalon. From the base (superior end) of the infundibulum, the tuber cinereum extends in continuity with the anterior perforated substance on each side of it; and, behind, the corpora mammillaria mark the boundary between it and the posterior perforated substance of the mid-brain.

The lamina cinerea and tuber cinereum form the inferior gray commissure of the fore-brain.

The hypophysis (pituitary body, Fig. 2I) is composed of two lobes bound together by connective tissue. A sheet of dura mater (diaphragma sella) holds them in the hypophyseal fossa. The anterior lobe, the larger, is derived from the epithelium of the mouth cavity; and, in structure, resembles the thyroid gland. Its closed vesicles, lined with columnar epithelium (in part ciliated), contains a viscid jelly-like material (pituita), which suggested the old name for the body. The anterior lobe is hollowed out on its posterior surface (kidney-shape) and receives the posterior lobe, the infundibulum, into the concavity. The hypophysis 
has an internal secretion which appears to have an inhibitive action on growth and has been found diseased in acromegalia.

Corpora Mammillaria (Figs. $2 \mathrm{I}$ and 26). - Two white bodies (corpora albicantia), as large as a small pea, are situated one on either side of the median line, between the tuber cinereum and the pigmented gray matter of the posterior perforated substance. Each is formed superficially by a loop in the columna of the fornix and is, therefore, composed of white substance at the surface. There is gray matter in the interior which forms a medial and a lateral nucleus (Fig. 46). In the medial nucleus the fornix fibers terminate and an ascending bundle rises, called the fasciculus thalamo-mammillaris (Vicq d'Azyri). The latter terminates in the anterior nucleus of the thalamus. From the medial nucleus of the corpus mamillare also rises the tegmental part of the fasciculus pedunculo-mammillaris; the basilar portion of the same bundle rises in the lateral nucleus and both parts of this pedunculomammillary bundle run downward into the mid-brain to an unknown termination.

Immediately behind the corpora mammillaria is the posterior perforated substance (Figs. 2I and 26). This is the exposed part of the substantia nigra of the mid-brain, perforated for the passage of the postero-median ganglionic arteries. The pons and bases pedunculi bound it behind. Issuing from the inner side of the basis pedunculi is the large oculomotor nerve; and coursing over its surface from. behind forward, is the smaller trochlear nerve. The bases pedunculi will be described with the mid-brain to which they belong.

\section{FISSURES OF THE MEDIAL AND TENTORIAL SURFACE.}

To expose the medial surface of the cerebral hemispheres; a median sagittal section must be made through the connecting links of the hemispheres and the inter-brain, dividing the forebrain into lateral halves. Separate the lips of the longitudinal fissure of the cerebrum; drop the moistened brain-knife down onto the corpus callosum; and make one quick sweep of the knife toward you. Of the surface now exposed the middle onethird is produced by section. 
It is convenient to study the tentorial area of the basal surface with the medial surface (Fig. 26). In this medial and tentorial surface there are six important sulci and four fissures (Fig. 28).

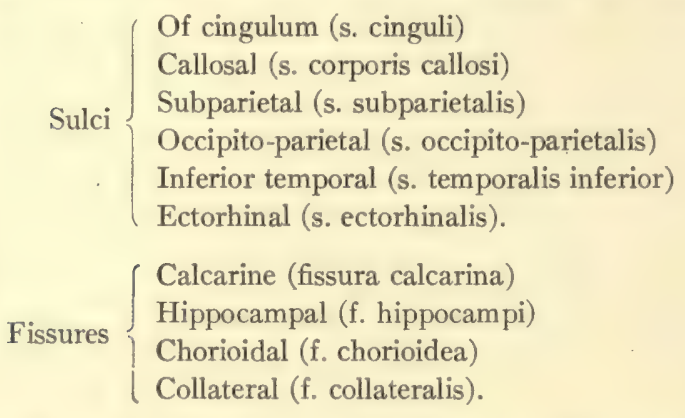

Sulcus Cinguli (Calloso-marginal Sulcus).-Beginning under the middle cut surface and extending in a curve forward, upward, and backward, until it half encircles the corpus callosum; and, then, turning upward to the supero-medial border and ending just behind the central sulcus is the sulcus cinguli (Figs. 27 and 28). It separates the gyrus cinguli and a marginal gyrus, including the straight and superior frontal, from one another by its anterior part; and, by its marginal end, separates the paracentral lobule from the præcuneus. The sulcus cinguli is usually interrupted by one annectant gyrus and often by two. These indicate its development in three separate parts.

At its beginning under the corpus callosum, the sulcus cinguli is almost continuous with a small curved sulcus, which runs nearly vertically downward, called the anterior parolfactory sulcus (Figs. 28 and 29). Behind that little sulcus there is a small curved gyrus, the parolfactory area (of Broca), which is continuous with the gyrus cinguli and bounded behind by another slight sulcus, called the posterior parolfactory sulcus. The latter separates the area parolfactoria from the gyrus subcallosus.

Subparietal Sulcus.-About one inch above and behind the posterior end of the corpus callosum there is an irregular sulcus, called the subparietal, which separates the gyrus cinguli of the limbic lobe from the præcuneus of the parietal lobe (Fig. 28).

The callosal sulcus is the deep furrow between the corpus 

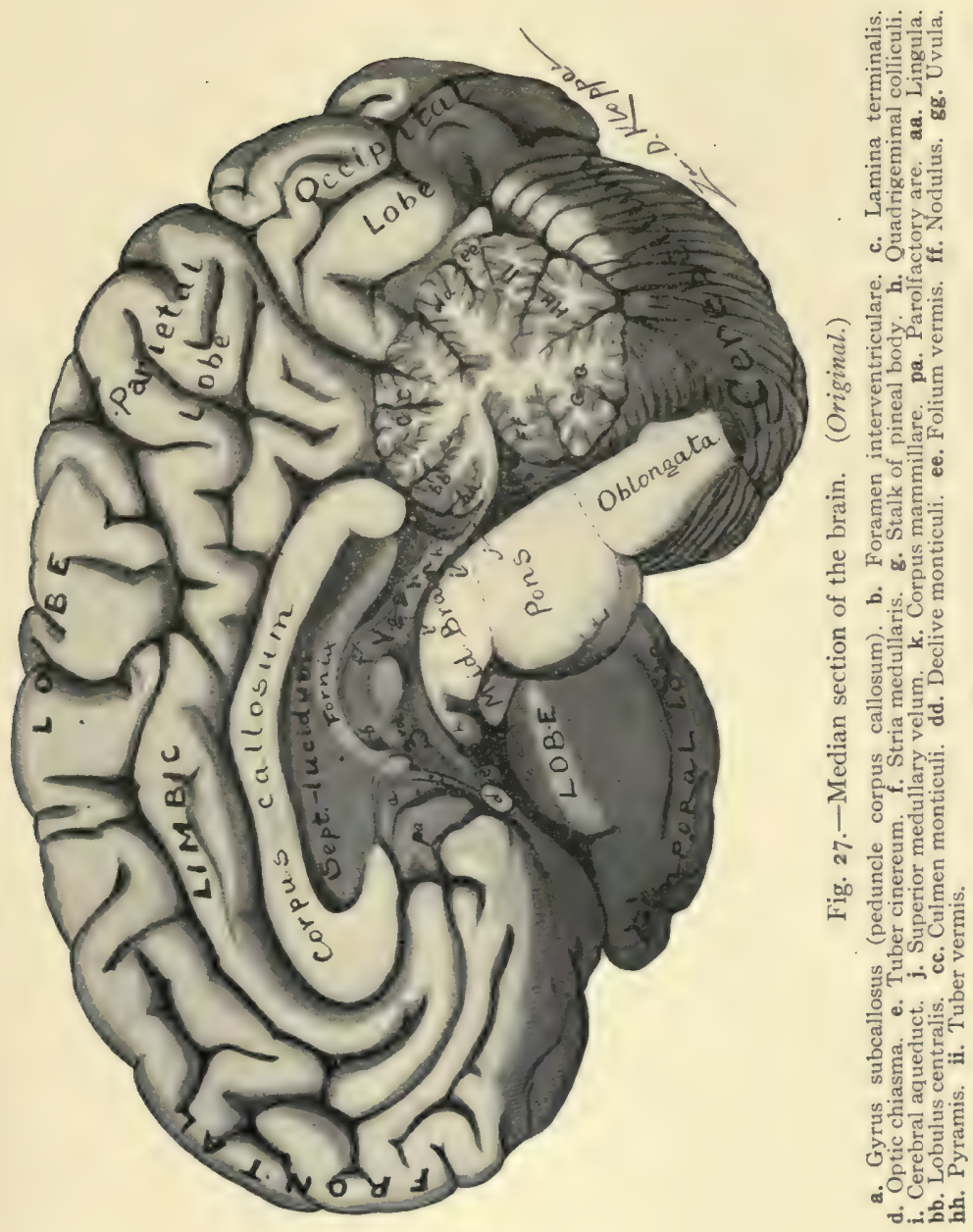

callosum and the gyrus cinguli. It follows the convexity of the corpus callosum and was formerly called the ventricle of it (Fig. 28). The callosal sulcus, behind the corpus callosum, is continuous with the hippocampal fissure.

The occipito-parietal sulcus (Figs. 27, 28 and 20), the internal part, extends downward from the supero-medial border to the middle of the calcarine fissure. The two form a lambdashaped fissure $<$ (Fig. 28); the lambda being tilted toward the frontal pole has one anterior and two posterior rami. The anterior ramus and the lower of the posterior rami constitute the calcarine fissure; the posterior superior ramus, is the occipito-parietal sulcus. This latter sulcus cuts the supero-medial border at the junction of the posterior one-sixth with the anterior five-sixths of that border; it is situated about two inches above the occipital pole, and lies one-sixth of an inch anterior to the point in the skull called the lambda. It separates the parietal lobe from the cuneus of the occipital lobe. The occipito-parietal sulcus is a deep one. In the embryo the primary occipito-parietal fissure produces an eminence in the posterior horn of the lateral ventricle (Cunningham). It is then a true fissure. But that primitive fissure and the ventricular eminence entirely disappear, and the adult sulcus is a secondary and superficial furrow, hence it is properly called a sulcus and not a fissure. At the inferior end of the occipito-parietal sulcus a buried annectant gyrus, the gyrus cunei, separates the occipito-parietal sulcus from the calcarine fissure, with which superficially it is continuous.

The calcarine fissure begins a quarter of an inch below the posterior end of the corpus callosum and runs backward and slightly upward to the lower end of the occipito-parietal sulcus; and, then, curves downward to a point near the occipital pole; where it ends bifid (Figs. 28 and 26). It is thus divided by the sulcus occipito-parietalis into an anterior calcarine and a posterior calcarine fissure. These three furrows are continuous with one another superficially in the human brain; but buried annectant gyri actually separate them from each other: the gyrus cunei separates the occipito-parietal sulcus from the calcarine fissure 
and the anterior calcarine fissure is separated from the posterior calcarine by the gyrus cuneo-lingualis (Cunningham). The anterior calcarine fissure indents the medial wall of the posterior horn of the lateral ventricle, producing the calcar avis.

Hippocampal Fissure (Figs. 26 and 28).-A crescentic fissure, convex downward begins under the splenium of the corpus callosum in continuity with the callosal sulcus, and winds forward beneath the thalamus to within an inch of the temporal pole, where it is closed by the uncus. It is the hippocampal fissure. On the surface of the temporal lobe this fissure appears to be identical with the temporal extension of the chorioidal fissure; but, deeply, it lies posterior to that fissure and is separated from it by the fascia dentata. The hippocampal fissure produces a long ridge in the inferior horn of the lateral ventricle, called the hippocampus.

The chorioidal fissure (Figs, 28 and 39) describes about two-thirds of a circumference along the concavity of the fornix. It extends from the foramen interventriculare backward over the thalamus; and then downward and forward along the hippocampal fissure, but separated from it by the dentate fascia. The chorioidal fissure is a complete one, involving the whole hemisphere wall. A single layer of epithelium separates it from the lateral ventricle. The pia mater, dipping into it, forms the chorioid plexus of that ventricle. The fissure is peculiar in the fact that between the inter-brain and the fornix there is a transverse slit by means of which it is continuous with the same fissure on the opposite side. In this antero-superior part, which is in direct continuity with the transverse fissure of the cerebrum, is the border of the chorioid tela of the third ventricle.

Collateral Fissure.-The collateral is a long fissure (Figs. 26 and 28). It reaches from near the occipital almost to the temporal pole. It is situated below and parallel with the calcarine and hippocampal fissures, being separated from the former by the lingual gyrus and from the latter by the hippocampal gyrus. The gyrus fusiformis lies below and external to this fissure. Anterior to the collateral fissure, there is a small sulcus between the gyrus hippocampi and the temporal pole, called the ectorhinal 


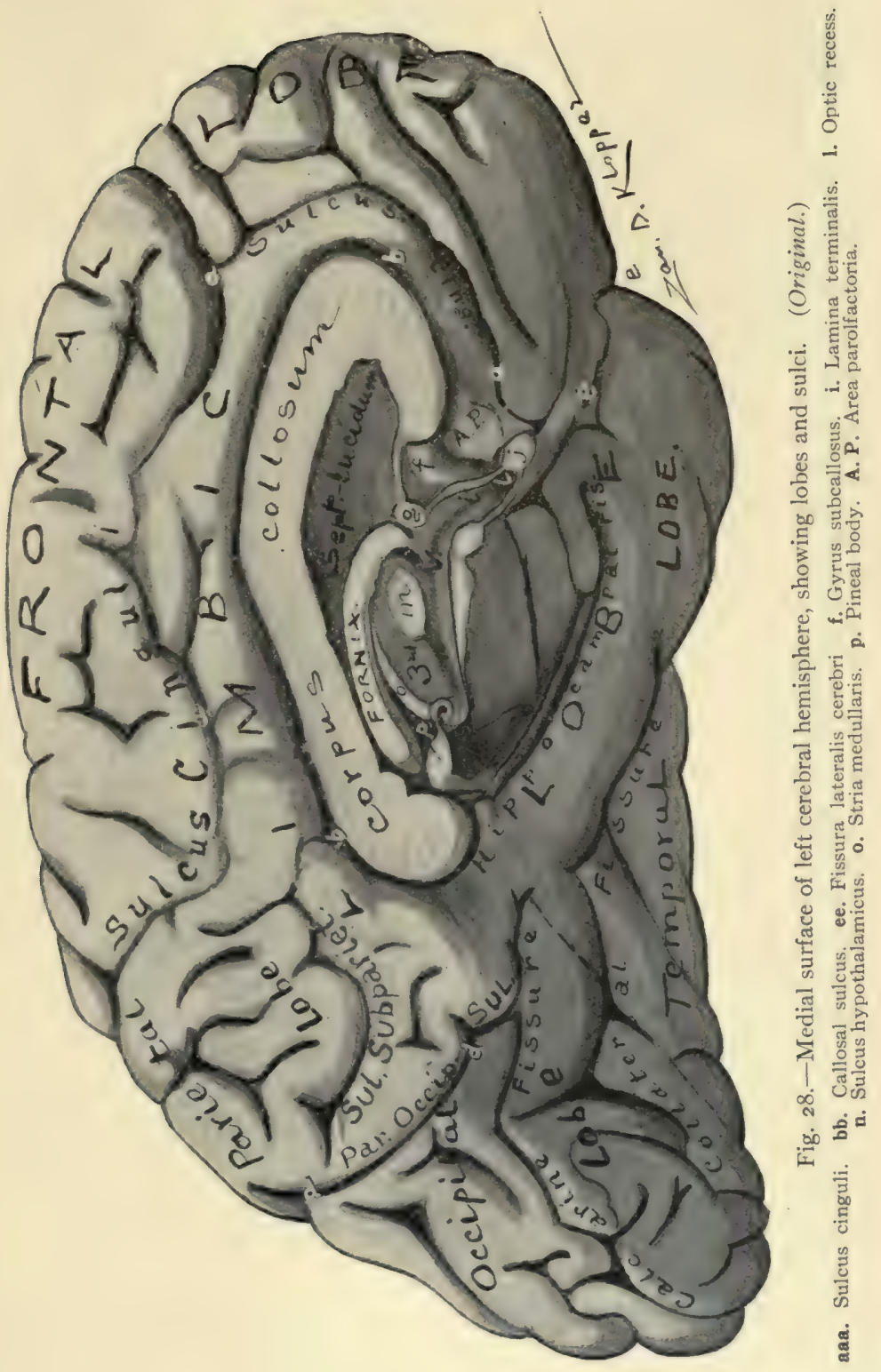



sulcus, which represents a very important fissure ( $f$. rhinalis) in osmatic animals. The collateral fissure is occasionally interrupted by two annectant gyri and divided into a temporal, an occipital and an intermediate part. This signifies a persistence of its embryonic condition. The intermediate portion, sometimes assisted by the anterior part, indents the ventricular wall and produces the eminentia collateralis in the inferior horn of the lateral ventricle.

The inferior temporal sulcus is usually a series of indentations rather than a continuous sulcus (Figs. 26 and 28). It is about equal in extent to the collateral fissure from which it is separated by the fusiform gyrus. It is parallel with the inferolateral border of the cerebral hemisphere. As the inferior temporal gyrus, which forms this border reaches over onto the tentorial area a variable distance, even in the two sides of the same brain, the position of the inferior temporal sulcus it not constant; but it is usually one-quarter or one-half inch medial to the border.

\section{LOBES AND GYRI OF MEDIAL AND TENTORIAL SURFACE.}

The gyri form two concentric rings, interrupted antero-inferiorly at the fossa cerebri lateralis, which encircle the corpus callosum and thalamus (Fig. 28). The two rings are scparated from one another by a broken fissure, the limbic fissure, made up of the sulcus cinguli (except its marginal end), the subparietal sulcus, and the anterior part of the calcarine and of the collateral fissures.

Gyrus Fornicatus. - The gyrus cinguli and the gyrus hippocampi, joined together at the posterior border of the corpus callosum by the isthmus, and together constituting the gyrus fornicatus, form the central ring. The gyrus cinguli begins anteriorly under the corpus callosum in continuity with the area parolfactoria anterior to the fossa cerebri lateralis; and the hippocampal terminates as uncus just behind that fossa. The gyrus fornicatus forms the chief part of the limbic lobe.

The gyrus cinguli is the arched gyrus which is inclosed between the callosal sulcus and the sulcus cinguli, except above the posterior 
end of the corpus callosum; there it is bounded on its convexity by the subparietal sulcus (Fig. 28). Underneath the corpus callosum, the anterior end of the gyrus cinguli is continuous with a small vertical gyrus, called the area parolfactoria (Brocæ), which is embraced between the anterior and posterior parolfactory sulci and is continuous with the area of the same name on the base of the cerebral hemisphere. This part belongs to the cortical area of smell. The posterior end of the callosal gyrus is almost separated from the hippocampal gyrus by the anterior calcarine fissure; the narrow link left between this fissure and the splenium of the callosum is the isthmus gyri fornicati. It is claimed by Schäfer and others, that the superior part of the gyrus cinguli constitutes a portion of the somæsthetic area; but the histological investigations of Dr. A. W. Campbell appear to disprove such a claim. According to Paul Flechsig, the gyrus cinguli contains the center of taste. He locates the center in the posterior part of the gyrus adjacent to the splenium of the corpus callosum; it forms a thin zone bounding the callosal sulcus (Fig. 55).

The gyrus hippocampi (Figs. 26 and 28) extends downward and forward, along the hippocampal fissure, from the isthmus to within a half-inch of the temporal pole. Its anterior extremity is separated from the pole by the ectorhinal sulcus, and is bent upward and backward over the end of the hippocampal fissure, forming a sharply curved hook, the uncus. The hippocampal gyrus is bounded below and laterally by the collateral fissure. Posteriorly, it is continuous with the gyrus lingualis. The reflected part of the uncus hippocampi is continuous with a concealed gyrus, located between the hippocampal and chorioidal fissures, viz., the dentate fascia; a narrow band, which winds over the uncus near its free point and is called the fremulum of Giacomin establishes this junction with the dentate fascia.

The uncus and the area parolfactoria constitute the greater part of the receptive center of smell (Figs. 55 and 57). In the uncus anterior to the end of the hippocampal fissure, Retzius locates the gyrus circumambiens and gyrus semilunaris, which he has identified in the human embryo; and he declares them to contain the end of the lateral olfactory stria and of the gyrus 

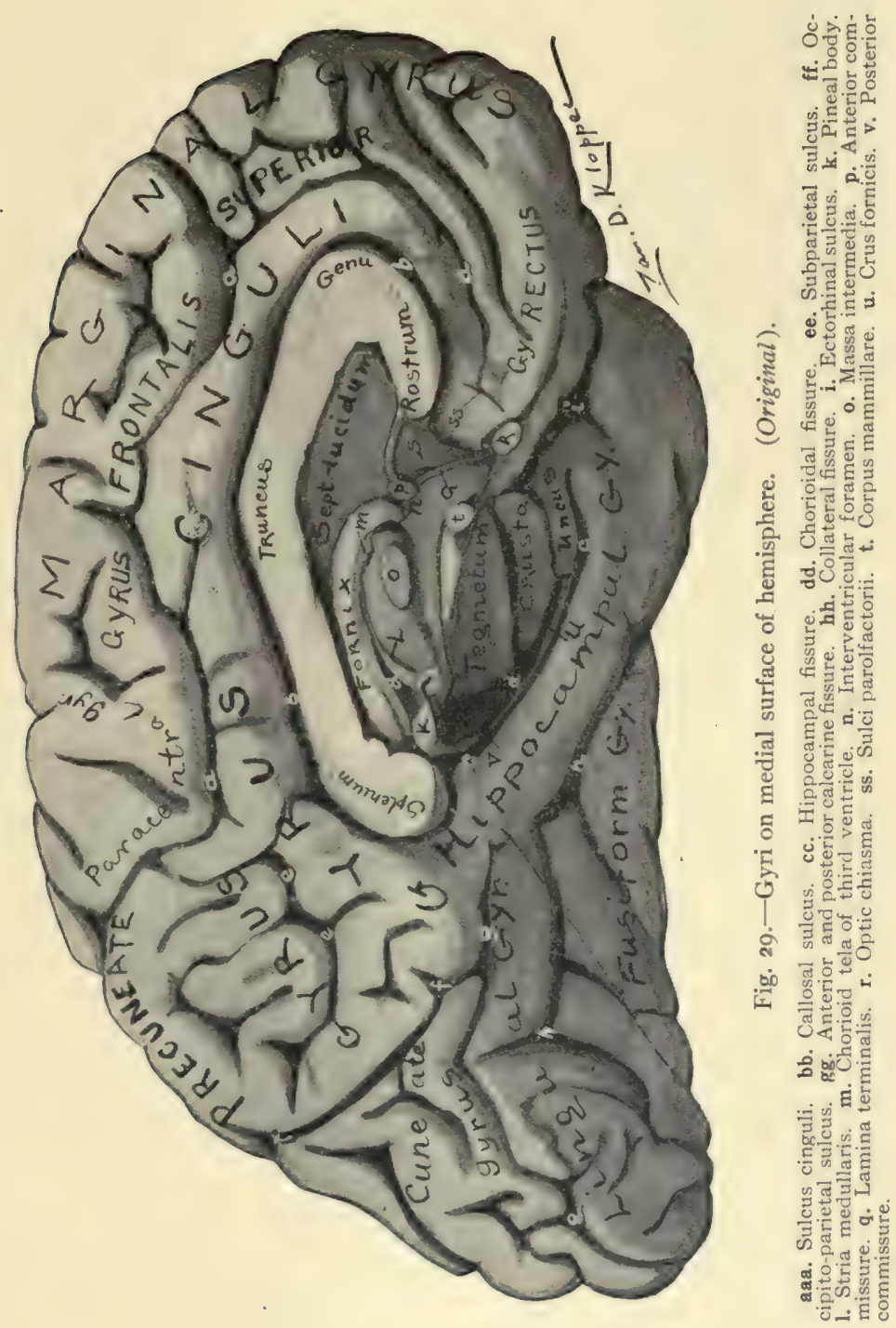

subcallosus, and he thinks they form the most important part of the receptive olfactory center. This region represents the lobus pyraformis of osmatic animals and, according to Elliot Smith (as quoted by Cunningham) it is the only part of the gyrus hippocampi which properly belongs to the rhinencephalon.

Limbic Lobe. - The parts of the limbic lobe may now be enumerated as follows: (I) The gyrus fornicatus ( $g$. cinguli and $g$. hippocampi); (2) the fascia dentata, fasciola cinerea, supracallosal gyrus (longitudinal striæ), and subcallosal gyrus (peduncle of the corpus callosum); (3) one-half of the septum pellucidum; and (4) a lateral half of the fornix. The limbic lobe is rudimentary in the human brain. The structures enumerated above under No. 2 and No. 3 are but faint representatives of the strong dentate gyrus seen in animals having no corpus callosum. The development of the corpus callosum encroaches upon and partially destroys the dentate gyrus. The limbic lobe in part belongs to the rhinencephalon. According to the researches of Elliot Smith the posterior inferior part of the gyrus hippocampi, that part behind the uncus and below the subiculum, and the whole gyrus cinguli belong to the neopallium and not to the rhinencephalon.

The peripheral ring on the medial and tentorial surface of the cerebral hemisphere is composed of five gyri (Figs. 28 and 26). Beginning under the corpus callosum anterior to the area parolfactoria and going forward to the frontal pole, then along the supero-medial border to the occipital pole and, finally, along the infero-lateral border to the temporal pole, these gyri are as follows: The gyrus rectus and gyrus frontalis superior ( $g$. marginalis) ending behind as lobulus paracentralis which closes the superior end of the central sulcus; the præcuneus inclosed between the marginal end of the sulcus cinguli and the sulcus occipito-parietalis; the cuneus, located between the occipito-parietal sulcus and the posterior calcarine fissure; the gyrus lingualis, which forms much of the medial occipital border and lies between the calcarine and collateral fissures; and lastly, the gyrus fusiformis (temporo-occipital gyrus), which lies below the collateral fissure and extends from the temporal pole almost to the occipital pole, 
being separated from the inferior temporal gyrus by an interrupted sulcus near the infero-lateral border of the hemisphere. The inferior temporal gyrus is chiefly on the convex surface.

The paracentral lobule contains the motor center for the opposite foot, just in front of the central sulcus; and immediately behind that sulcus is the superior part of the receptive somesthetic area (Figs. 55 and 57 ). In the præcuneus is a part of the stereognostic center; the remainder is in the superior parietal lobule; this center belongs to the psychic-sensory area. The cuneus and lingual gyrus, along the calcarine fissure of each hemisphere constitute the receptive visual center for the corresponding halves of both retinæ and perhaps for both maculæ luteæ. Probably the anterior part of the fusiform gyrus, that part just below the uncus, contains the center of taste (Mills).

The peripheral ring of gyri seen on this surface belongs to lobes which have their largest exposure on the convex surface of the cerebral hemisphere. Thus seven lobes belong to the exterior surface of each hemisphere.

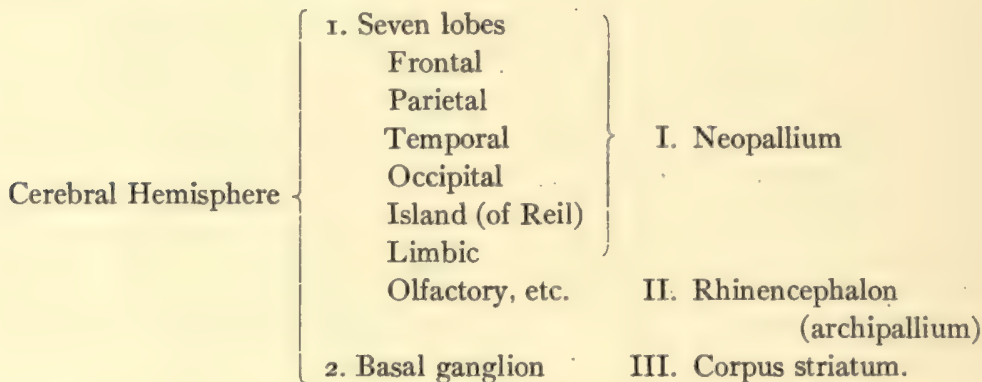

\section{INTERIOR SURFACE OF THE FORE-BRAIN.}

The boundaries of the fore-brain ventricles constitute the interior surface. Considering these cavities together, we notice that they occupy a wedge-like space (Figs. 30 and 42). In shape the wedge is rectangular; and it stands, base upward, against the corpus callosum. Its beveled surfaces look toward the hemispheres. The blade is driven downward as if to split the forebrain into lateral halves, the edge resting on the medial structures at the base of the brain. The space is inclosed laterally between 
the diverging internal capsules, which, within the hemispheres, decussate with the lateral extremities of the corpus callosum. The two structures most necessary to an understanding of the cavities and interior surface of the fore-brain, therefore, are the internal capsule and the corpus callosum.

Internal Capsule. (Capsula Interna).-Looking at the base of the brain, we see two broad bands of nerve fibers, the bases pedunculi, issue from the cerebral hemispheres under cover of the optic tracts and, converging downward and backward, disappear in the pons (Figs. $2 \mathrm{I}$ and 45). Traced in their reverse direction, the fibers of each basis pedunculi enter the hemisphere of the cerebrum and are reinforced by a great number of additional fibers from the thalamus. The fibers then radiate toward the cerebral cortex in the form of a hollow cone or funnel. This funnel-like group of fibers is the internal capsule (Fig. 33). The bell of the funnel opens upward and outward and contains the lentiform nucleus; its solid spout, directed toward the pons and medially, is the basis pedunculi. Antero-inferiorly the fibers in the bell of the funnel diverge to opposite sides of the fissura cerebri lateralis (Sylvii) and produce a break in its continuity, the hiatus Sylvii; otherwise the funnel is complete. As the internal capsule proceeds into the hemisphere, it impales the corpus striatum in such manner as to place the caudate nucleus upon its circumference and to inclose within its walls (to capsulate) the lentiform nucleus. The lentiform nucleus is separated externally from the claustrum by a thin layer of fibers called the external capsule.

The internal capsule is directed obliquely outward and upward and is flattened from above downward. It has, therefore, a superior and an inferior lamina, which, posteriorly, are continuous with each other but, anteriorly, are separated by the hiatus Sylvii.

The inferior lamina (or inferior ramus, as seen in sagittal section) is thick behind but bevels down to a sharp edge anteriorly (Figs. 32, 33, and 70). In front it presents a free border. Its fibers pass outward beneath the lentiform nucleus and, after winding over the inferior horn of the lateral ventricle, terminate in the parietal, the insular and temporal lobes. 
Motor Fibers.-The inferior lamina of the internal capsule contains most of the temporo-pontal tract (tractus cerebrocortico-pontalis temporalis) which extends from the temporal cortex beneath and behind the lentiform nucleus to the nucleus of the pons and nuclei of the motor cerebral nerves. The intermediate tract (tractus intermedius) rises in the lentiform nucleus and runs through the anterior part of the inferior lamina, in its course to the substantia nigra and nucleus pontis in which it terminates.

Sensory Fibers (Figs. 32, 33 and 70).-The following sensory fibers are found in the inferior lamina, viz., the ventral stalk (ansa peduncularis) of the thalamus (common sensory) running from the thalamus chiefly to the somæsthetic cortex; and part of the acustic or temporo-thalamic radiation (special sensory), which extends from the medial geniculate body to the auditory cortex in the superior and transverse temporal gyri. In part at least the ventral stalk rises from nuclei lower down than the thalamus-from the substantia nigra and the nuclei of the gracile and cuneate columns probably. The fibers of the ventral stalk of the thalamus are separated into an upper and a lower lamina by a thin sheet of gray substance. The lower lamina (inferior peduncle of the thalamus) runs for the most part beneath the lentiform nucleus into the external capsule and terminates in the temporal lobe and the island. The upper lamina (ansa lenticularis) enters the lentiform nucleus in two vertical sheets that separate the three zones of that nucleus. Its fibers to a large extent terminate in the lentiform nucleus, whose axones continue the path through the parietal stalk, in the superior lamina of the capsule, to the somæsthetic area of the cortex. In the angle between the inferior horn and the central part of the lateral ventricle, the inferior lamina joins the superior.

The superior lamina (or superior ramus) of the internal capsule contains most of the basis pedunculi (Fig. 30). It is a thick and strong sheet of fibers. Often it is considered as the entire "internal capsule," the inferior lamina being disregarded. The superior lamina extends, fan-like, from the basis pedunculi to the lateral extremity of the corpus callosum, with which it inter- 


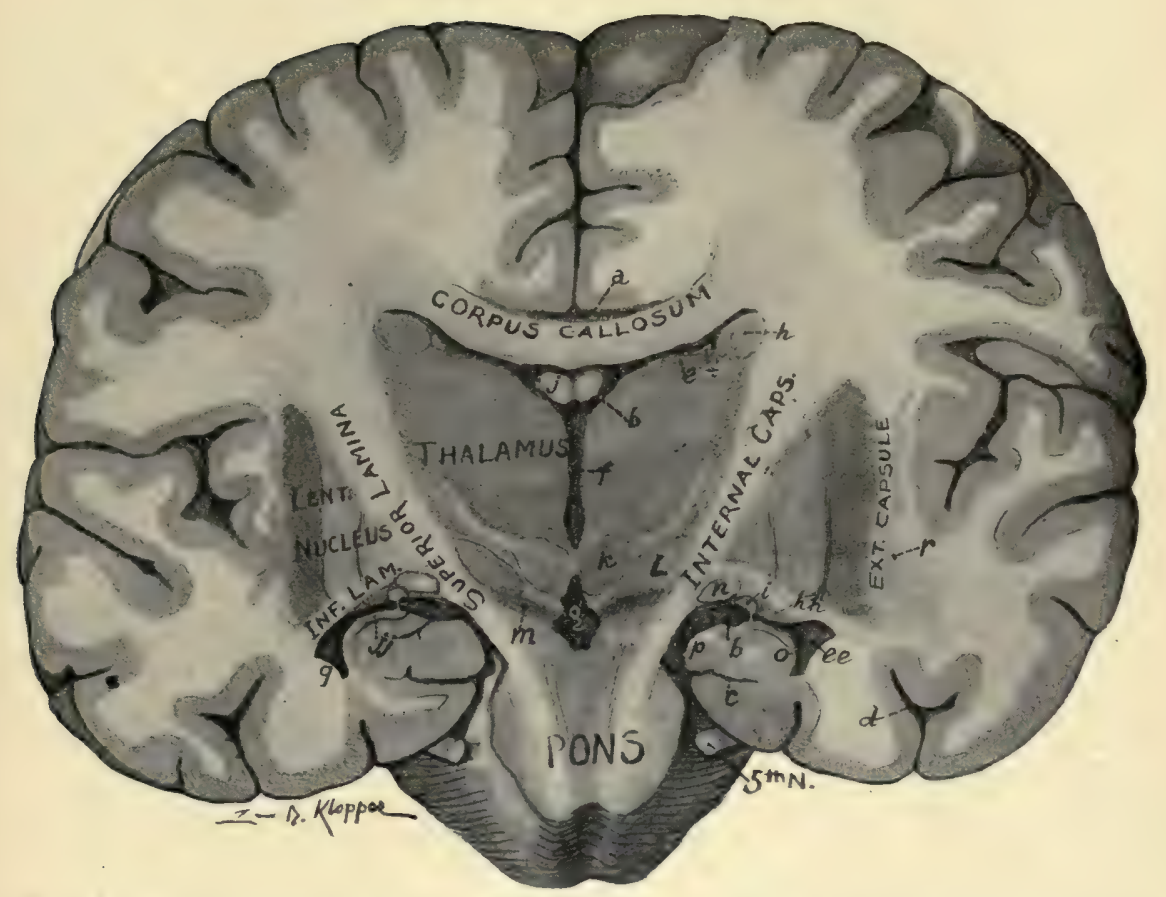

Fig. 30.- Transverse section of the brain, directed from the pons obliquely upward and forward, showing internal capsule, corpus callosum, ganglia and ventricles of the forebrain. (Original.)

a. Callosal sulcus. bb. Chorioidal fissure. c. Hippocampal fissure. d. Collateral fissure. ee. Inferior horn of lateral ventricle. f. Third ventricle. $g$. Fossa interpenduncularis. h. Caudate nucleus. hh. Cauda. i. Stria terminalis. j. Body of fornix. jj. Crus of fornix. k. Red nucleus. I. Hypothalamic nucleus. m. Substantia nigra. o. Hippocampus. p. Dentate fascia. r. Claustrum. 

crosses. It has a free border anteriorly, though imbedded in the corpus striatum (Fig. 32). Upon its medial surface rest the thalamus, below and behind, and the caudate nucleus, above and farther forward. It thus separates the thalamus and caudate nucleus from the lentiform nucleus. A bend seen in horizontal section near the middle of the superior lamina, called the genu, divides it into a frontal part (pars frontalis) and an occipital part (pars occipitalis) which tend slightly outward from the genu and form an angle with each other of about I 20 degrees.

Motor Fibers of Superior Lamina (Figs. 32, 33 and 70).The fibers of the gemu and anterior two-thirds of the occipital part of the internal capsule are very largely motor and constitute the pyramidal tract. They may be traced from the motor area of the cortex (Figs. 54 and $5^{6}$ ) through the internal capsule and the middle three-fifths of the basis pedunculi, and on down into the spinal cord. They end in ramifications about the cell-bodies in the gray matter of the cord and in the motor cerebral nuclei. From these same spinal and cerebral nuclei other fibers rise which constitute the motor part of the spinal and cerebral nerves. The pars frontalis of the internal capsule contains a motor tract which extends from the frontal cortex through the inner one-fifth of the basis pedunculi to the nucleus of the pons and motor nuclei of the cerebral nerves (Flechsig). It is the fronto-pontal tract (tractus cerebro-cortico-pontalis frontalis).

Sensory Fibers of Superior Lamina (Figs. 32, 33 and 70).In both parts of the superior lamina of the capsule there are common sensory fibers which rise chiefly in the thalamus and end in the somæsthetic cortex (Figs. 54 and 56 ). They convey ordinary sensations. In the frontal part is the frontal stalk of the thalamus, which ends chiefly in the caudate and lentiform nuclei, though some of its fibers reach the frontal cortex. The tract is relayed in the lentiform nucleus, whence it is continued to the somæsthetic cortex by way of the parietal stalk, which is entered high up near the corpus callosum. The pars occipitalis of the superior lamina contains the parietal stalk of the thalamus, which conveys ordinary sensations from the thalamus to the somæsthetic cortex, chiefly to the posterior central gyrus. 
In the extreme upper part of the capsule the region of the parietal stalk contains nearly all of the common sensory fibers. The recent investigations of Paul Flechsig show that it is built up of six bundles of fibers, two of which (alpha and gamma) proceed from the globus pallidus and four from the thalamus. According to Flechsig there are corticifugal fibers intermingled with the corticipetal in all of the sensory tracts of the capsule. The common sensory fibers of the internal capsule constitute the cortical fillet.

Special Sense Fibers (Figs. 32, 33 and 70).-At the junction of the superior and inferior laminæ of the internal capsule are the special sense fibers. The optic radiation (radiatio occipitothalamica) and acustic radiation (radiatio temporo-thalamica) occupy the retro-lentiform part of the internal capsule. The former extends through it from the lateral geniculate body and thalamus to the cuneus and lingual gyrus; the latter rises in the medial geniculate body and ends in the superior and transverse temporal gyri. The optic and acustic radiations both contain corticifugal fibers which rise in the visual and auditory cortex, respectively, and run through the capsule to the lateral and medial geniculate bodies and perhaps on to lower nuclei.

Many fibers of the internal capsule give off branches (collaterals) which pass through the corpus callosum to the opposite hemisphere; other fibers may be traced entire through the same course to the cortical cells of the opposite side. A bundle of thalamic fibers has been so traced.

The superior lamina of the internal capsule, proceeding outward and upward into the hemisphere, intermingles with the corpus callosum and enters into the corona radiata. Together with the caudate nucleus, thalamus and stria terminalis (tænia semicircularis), which lie on its medial surface, it forms the entire lateral boundary of the general cavity of the fore-brain. It thus determines the lateral part of the interior surface.

Corpus Callosum (Figs. 29, 30, 34 and 42).-The entire roof of the fore-brain cavity, representing the base of the wedge, is formed by the corpus callosum. A part of the anterior boundary is also formed by it. The corpus callosum is a thick sheet of 


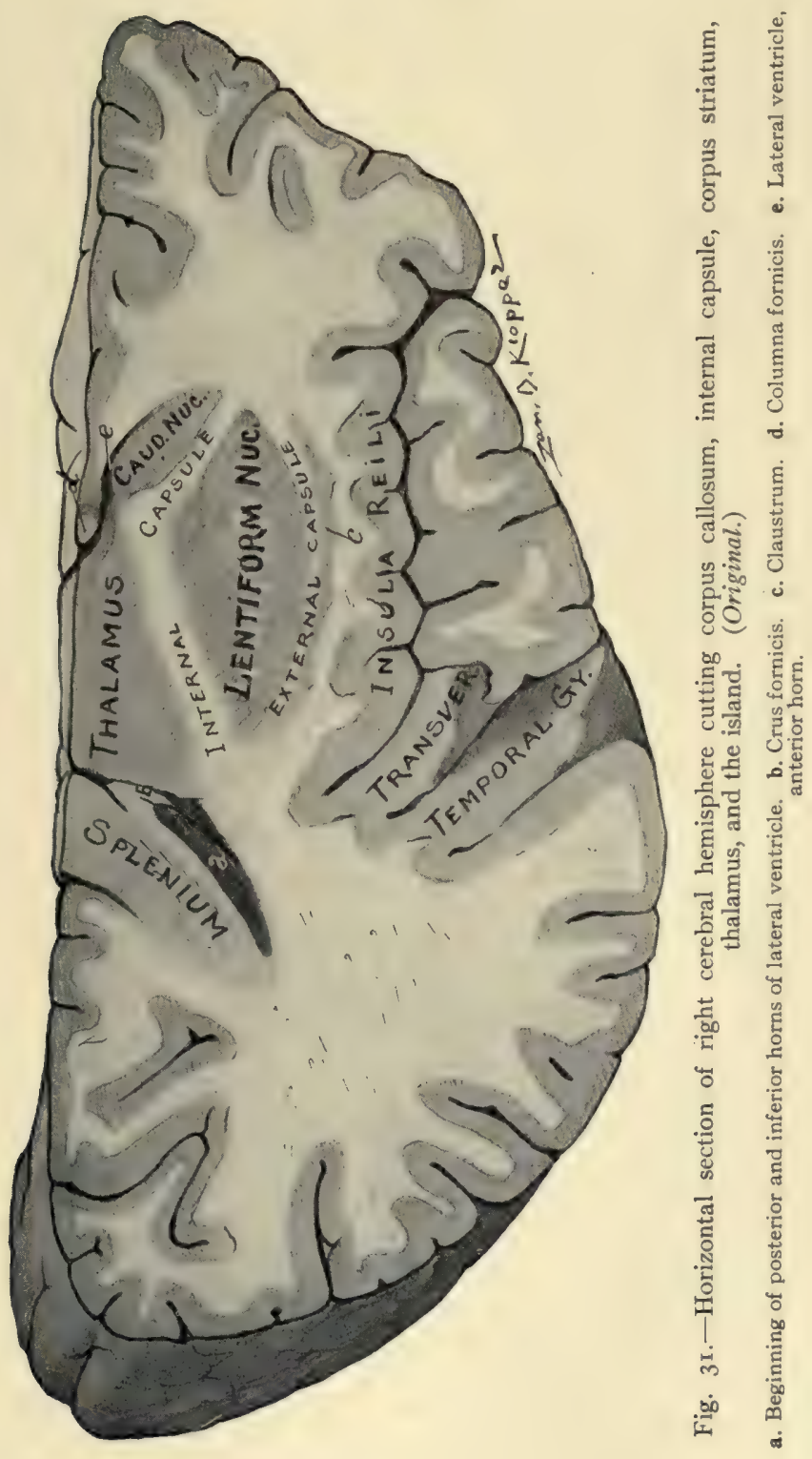



fibers four and a half inches broad, from before backward, which joins the hemispheres together. It constitutes the great commissure, being composed chiefly of those medullated cortical axones which end in arborizations about cortical cells of the opposite hemisphere. It contains some fibers which belong to the internal capsule; and, also, collaterals from capsular and association fibers. The corpus callosum is placed nearer to the anterior than the posterior pole of the hemispheres. Separating the hemispheres above, it is seen in the bottom of the longitudinal fissure. It is about an inch in transverse length.

The upper surface is concave from side to side, and divided in the median line by a longitudinal raphe (Figs. 30 and 34). Transverse striæ are plainly visible. Two longitudinal striæ are also found running on either side of the raphe; one next the raphe, the medial longitudinal stria; and the other near the lateral end of the callosum, the lateral longitudinal stria. The medial and lateral longitudinal striæ are imbedded in a thin sheet of gray substance spread over the corpus callosum; altogether they constitute the gyrus supracallosus. If traced around the posterior border of the callosum, this supracallosal gyrus is found to be continuous with the fasciola cinerea and, through that, with the fascia dentata. The gyrus supracallosus becomes the gyrus subcallosus (peduncle of corpus callosum) after it winds around the anterior border of the corpus callosum. As such it is continued downward between the lamina terminalis and the posterior parolfactory sulcus to the base of the cerebrum, and then across the anterior perforated substance to the uncus. At the anterior and at the posterior border, the corpus callosum is bent downward (scroll-like); hence, it is superiorly convex from before backward.

Its inferior surface (Figs. 29 and 30) is concave antero-posteriorly and near its posterior border is fused with the body of the fornix. Anterior to that fusion, it is joined to the fornix, along the median line, by the septum pellucidum.

The posterior border (Fig. 29) is flexed downward from the horizontal about forty-five degress. Giving passage to the fibers which join the middle and posterior parts of the hemispheres, 
the posterior border is the thickest part of the corpus callosum. It is on that account called the pad, or splenium.

The anterior border is bent downward and then backward, sweeping through I80 degrees of flexion (Fig. 29). It tapers down to a sharp edge, called the rostrum. A very thin sheet-like extension of the rostrum, called the lamina rostralis, proceeds backward from the beak and becomes continuous with the lamina terminalis at the base of the brain. Running downward on either side is a low ridge, continuous with the striæ longitudinales, which constitutes the gyrus subcallosus. Each gyrus subcallosus, after passing across the anterior perforated substance, ends in the uncus of the hippocampal gyrus. The transverse fibers of the rostrum, in the hemisphere, form the floor of the anterior horn - of the lateral ventricle.

Genu and Truncus (Fig. 29).-The down-turned anterior part of the corpus callosum is the genu. It joins the rostrum to the main body, the truncus. The genu forms part of the anterior boundary of the cerebral cavity; the truncus forms the roof. Fibers uniting the frontal lobes of the cerebrum pass through the genu, and in the hemisphere, bound the anterior horn of the lateral ventricle above and in front. Those fibers arching forward and forming the roof of the anterior horn are called the forceps minor. The forceps major, composed of fibers from the splenium which bend backward into the occipital lobe, lies in the roof and inner wall of the posterior horn and produces the eminence called the bulb (Fig. 38).

Each lateral extremity of the corpus callosum is overhung by the gyrus cinguli, which covers the lateral longitudinal stria. Inclosed between the gyrus cinguli and corpus callosum is the callosal fissure (ventricle of the callosum). The lateral extremity of the corpus callosum, within the cerebral hemisphere, intermingles with the superior lamina of the internal capsule and thus stretches entirely across the fore-brain cavities (Figs. 30 and 42 ).

The boundaries of the general cavity of the fore-brain may be given as follows:

Roof (base of wedge) - 
Corpus callosum.

Floor (edge of wedge)-

Tegmenta of mid-brain,

Posterior perforated substance of mid-brain,

Tuber cinereum,

Infundibulum,

Optic chiasma.

Lateral wall (beveled surface)-

Internal capsule (superior lamina),

Caudate nucleus,

Stria terminalis,

Thalamus.

Anterior wall (border of wedge)-

Lamina terminalis,

Anterior commissure,

Genu of corpus callosum.

Posterior wall-

Posterior commissure with cerebral aqueduct beneath it,

Corpora quadrigemina of mid-brain,

Pineal body,

Transverse fissure of cerebrum, containing the chorioid tela of third ventricle,

Splenium of corpus callosum.

The fore-brain cavity thus bounded is subdivided by two partitions (Figs. 29, 35 and 42). The body of the fornix, together with the chorioid tela of the third ventricle and the roof epithelium of the third ventricle, forms a horizontal partition which divides the cavity into an upper and lower chamber. The superior chamber is divided into two lateral chambers, the lateral ventricles, by a double vertical partition, the septum pellucidum. The inferior chamber is the third ventricle

The body of the fornix (corpus fornicis, Figs. 29 and 36) is a triangular sheet of fibers, whose base is attached to the under surface of the splenium of the corpus callosum, and whose bifid apex extends forward to the rostrum and the anterior commissure. Its lateral borders rest on the thalami, the chorioid tela alone intervening (Fig. 42). And the narrow chamber between 
the thalami, the third ventricle, is separated from the broader, superior part of the fore-brain cavity by the body of the fornix together with the chorioid tela and a layer of epithelium. The body of the fornix is produced by the approximation of two bundles of white fibers, one belonging to each hemisphere. These bundles are the crura of the fornix.

The crus fornicis (Figs. 29, $3^{8}$ and 39) may be traced from the uncus and the hippocampus, its chief origin, upward through the inferior horn and into the floor of the body of the lateral ventricle, where it unites with its fellow of the opposite side in forming the body of the fornix. At the apex of the body of the fornix, which is the anterior end, the bundles again separate and become the columnx of the fornix. The crura are united at the back part of the body of the fornix by a few transverse and oblique fibers which form the lyre, or commissura hippocampi (Fig. 36). The commissure is best seen when the corpus callosum and fornix are viewed from below; its fibers connect each crus of the fornix with the hippocampus and uncus of the opposite side.

The columnæ fornicis (Figs. 29 and 40), one on either side pass down in front of the thalami, bounding the foramina interventricularia (Monroi); and then descend to the corpora mammillaria, at the base of the brain. On the way down, each columna (pars libera) passes behind the anterior commissure, beyond which (pars tecta) it pierces the inner part of the thalamus of the same side. The fibers of the columna fornicis for the most part terminate in the medial nucleus of the corpus mammillare, from which other fibers take their origin, forming the fasciculus mammillaris princeps. This bundle divides Y-like; the anterior branch is the fasciculus thalamo-mammillaris (Vicq d'Azyri) and ascends to the anterior nucleus of the thalamus; the posterior bundle is the tegmental part of the fasciculus pedunculo-mammillaris and probably ends in the stratum griseum centrale of the mid-brain.

At the lower border of the interventricular foramen a small bundle of fibers leaves the columna of the fornix and, bending backward, runs as medullary stria along the thalamus to the nucleus of the habenula; some of the fibers decussate through 
the stalk of the pineal body to the opposite nucleus habenulæ and constitute the commissura habenularum. The columna of the fornix is joined by a small fasciculus from the medial root of the olfactory tract, which runs backward to the hippocampus and uncus.

The upper surface of the body of the fornix is convex from before backward (Figs. 29 and 36). It forms the postero-medial part of the floor of the lateral ventricle. Along the median line, it is joined to the corpus callosum by the septum pellucidum.

The septum pellucidum (Figs. 29, 35, 40 and 73), a doublewalled median partition, divides the superior chamber of the fore-brain cavity into lateral halves, the lateral ventricles. The septum pellucidum is crescentic in outline. Its convex border fits into the concave surface of the body, genu and rostrum of the corpus callosum. Its concave border rests upon the fornix. Between the rostrum of the corpus callosum and the anterior commissure, the septum pellucidum is continuous with the gyrus subcallosus with which it is associated in development and function.

The septum pellucidum, like the anterior commissure, corpus callosum and fornix, is developed from the thickened upper border of the lamina terminalis and the adjacent medial wall of the cerebral hemisphere in front of the interventicular foramen. These several structures extend upward and backward with the development and rotation of the hemispheres and, together, roof over the inter-brain. A lymph space, the cavum septi pellucidi, appears in the septum and is commonly called the fifth ventricle. The fore-brain cavity thus embraces four ventricles, viz.:

Two lateral ventricles (the ventricles of the hemispheres),

Fifth ventricle (the ventricle of the septum), and

Third ventricle (ventricle of the inter-brain).

\section{THE FIFTH VENTRICLE.}

(Cavum Septi Pellucidi.)

This is the ventricle of the septum (Figs. 35 and 73). The fifth ventricle is a very narrow, antero-posterior cleft between the walls of the septum pellucidum, with which it coincides in 
extent. It is situated within the concavity of the corpus callosum between the lateral ventricles, above and anterior to the third ventricle. Below and posteriorly it is bounded by the fornix. It is not a part of the embryonic brain cavity, but a mere lymph space. Therefore it does not communicate with any other ventricle, each of the others being a part of the cavity of the neural tube from which both brain and cord are developed. Instead of ependyma, which lines other ventricles, the lining of the fifth is endothelium. A lymph-like fluid fills it.

\section{THE LATERAL VENTRICLE.}

(Ventriculus Lateralis.)

The hemispheres contain the largest of the six ventricles (Figs. $30,35,4 \mathrm{I}, 42$ and 73 ). Situated one on either side of the median line, the ventricles of the hemispheres are very naturally called the lateral ventricles. Each represents a branch of the cavity of the embryonic neural tube (Figs. I 7 and $4 \mathrm{I}$ ). In consequence, the lateral ventricles communicate with all others except the fifth. By the interventricular foramen (of Monro), each directly communicates with the third ventricle; and through that, indirectly, with the fourth and sixth. The foramen interventriculare is situated between the front of the thalamus and the calumna of the fornix (Fig. 29). It extends between the anterior extremity of the third ventricle (the aula) and the junction of the anterior horn with the central part of the lateral ventricle. The lateral ventricles are lined with ependyma, which is a transparent membrane composed of two layers when complete, viz., neuroglia and a covering of columnar ciliated epithelial cells. Over the thalamus (the part seen in the lateral ventricle) and the chorioid plexus, the neurogliar layer is absent.

The lateral ventricle may be studied best in four parts: the central part (or body); the anterior horn; the inferior horn; and the posterior horn.

The central part of the lateral ventricle (Figs. 36, 39 and 40) is the ventricle of the parietal lobe of the cerebrum. The following are its boundaries:

Roof-Corpus callosum. 


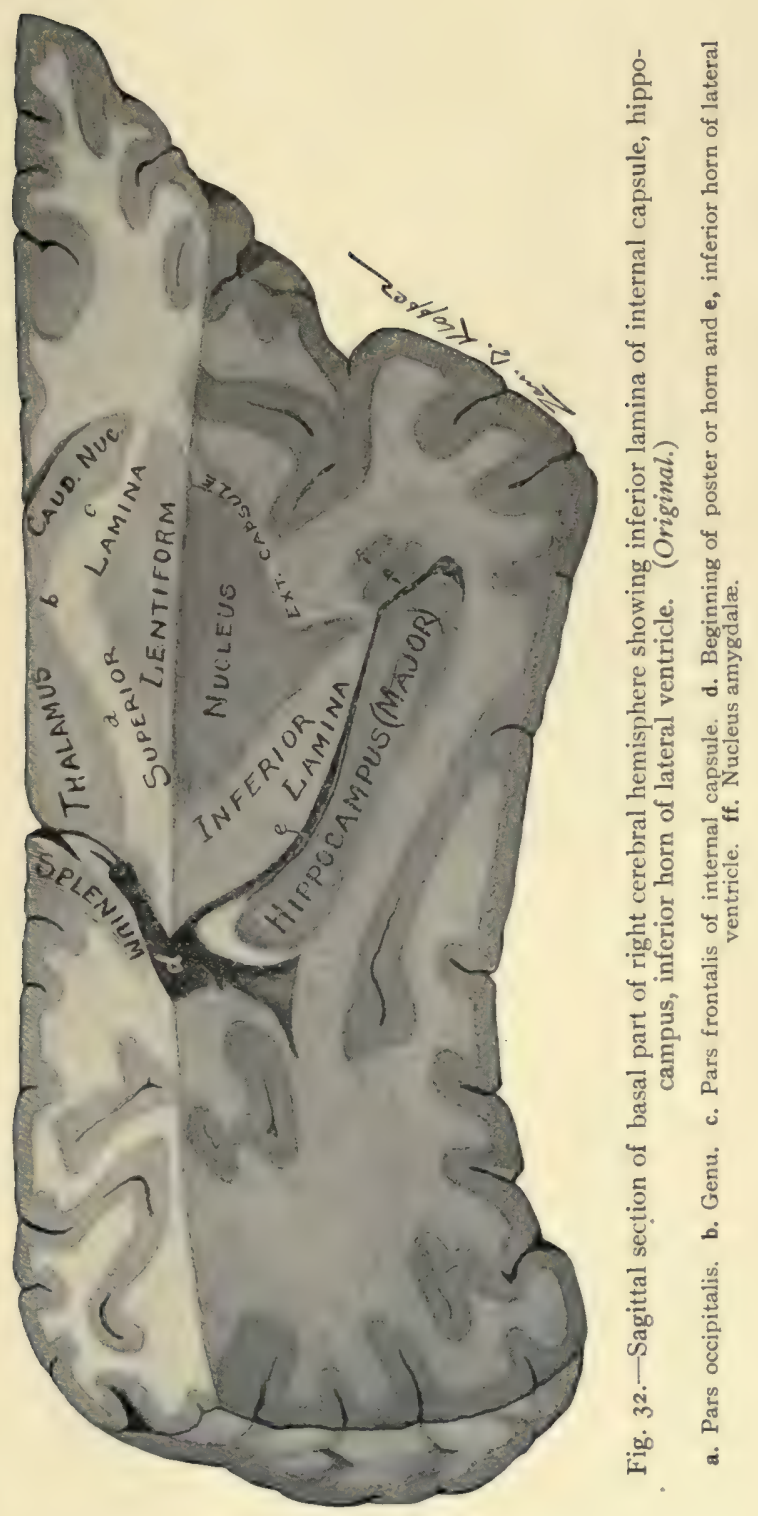



Floor (from before, backward and inward)-

Caudate nucleus of the corpus striatum,

Stria terminalis (tænia semicircularis),

Thalamus (covered by epithelium),

Lamina chorioidea epithelialis and chorioid plexus,

Fornix.

Medial wall-Septum pellucidum.

External wall-Internal capsule.

The corpus callosum forms a complete roof for the central part of the lateral ventricle. The roof inclines upward and outward from the septum pellucidum, the inner wall of the ventricle, to

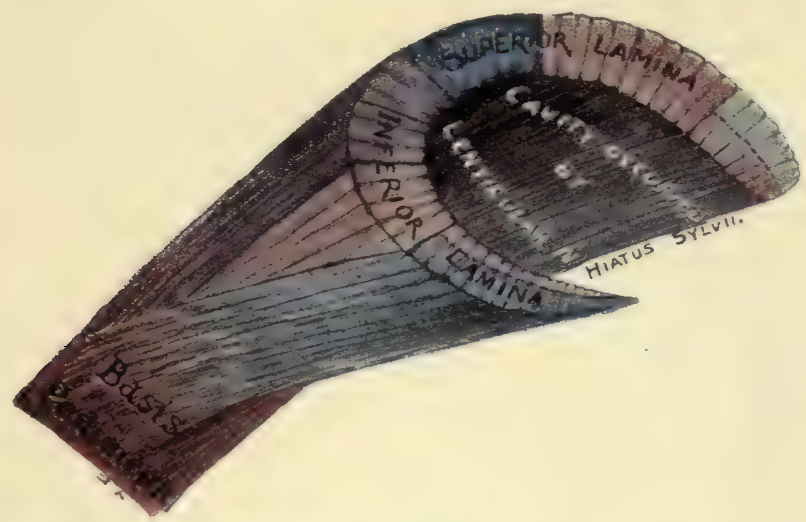

- Fig. 33.-Diagram of internal capsule in colors. (Original.) RED, motor; BLUE, common sensory; PURPLE, special sensory.

the superior lamina of the internal capsule, which forms its outer wall. The floor of the central part of the ventricle is formed by the five parts, as named above, which will now be considered in the order given.

Corpus Striatum (Figs. 30, 3I, 32 and 40).-The striated body is the basal ganglion of the hemisphere. It is an ovoid mass of gray matter imbedded, for the most part, in the cerebral medulla; but it is continuous below with the anterior perforated substance, and extends above to the lateral ventricle. It measures two and a half inches from before backward, an inch and a quarter 
transversely, and, from above downward, one inch and a half. It is placed anterior and external to the thalamus and forms the third of the great divisions of the cerebral hemisphere, viz., the neopallium, the rhinencephalon and the corpus striatum. It is a reddish-gray body, and its streaked appearance is due to the white capsular fibers which pierce it. The striated body is an important relay in the motor conduction path and one of less importance in the sensory path. The internal capsule divides it into two nuclei, namely, the lentiform nucleus (extraventricular part), and the caudate mucleus, which is seen in the boundary of the lateral ventricle. Anterior to the free borders of the superior and inferior capsular laminæ, the two nuclei are united with each other, with the anterior perforated substance and with the lower end of the claustrum.

The lentiform nucleus (nucleus lentiformis) occupies the cone-like cavity of the internal capsule, by whose laminæ it is separated from the ventricle (Fig. 32). It is shorter fore and aft than the caudate nucleus. It resembles a biconvex lens with a somewhat thickened anterior border, when viewed in horizontal section (Fig. 3I). In transverse vertical section through its center, it is triangular in shape. The hypotenuse and base are formed, respectively, by the superior and inferior laminæ of the internal capsule. The external capsule forms the perpendicular and separates the lentiform nucleus from the claustrum. The latter is a thin sheet of isolated gray matter, found just medial to the island (of Reil). In extent and position, fore and aft, the island and lentiform nucleus coincide. The lentiform nucleus is subdivided by two white laminæ, parallel with its external surface, into three zones. (Fig. 30). The outer zone, called the putamen, is deeply pigmented, and, like the caudate nucleus, is of a reddish-gray color; but the two inner zones, having less pigment, are of a pale yellowish tint. They form the globus pallidus.

The nucleus caudatus (the tailed nucleus) is a pear-shaped body of reddish-gray color, situated on the perimeter of the internal capsule (Figs. 30, 32 and 40). It is the intraventricular part of the striated body and forms a strip of the ventricular floor along the outer wall. The head (caput) of the caudate nucleus 


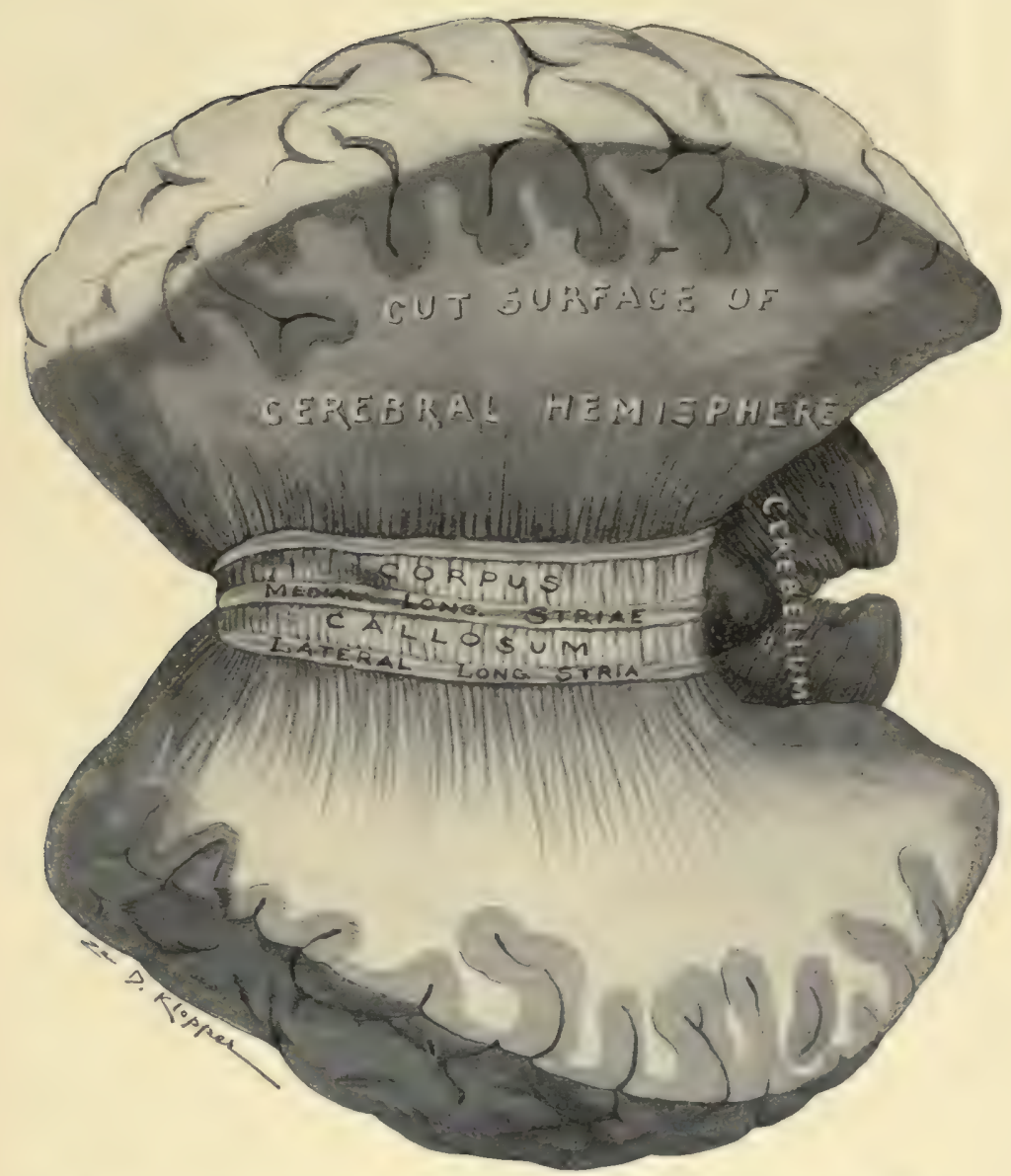

Fig. 34--Dorsal surface of corpus callosum, cerebral hemisphere cut away to expose it. (Original.) 

is directed forward. It is seen in the anterior horn of the lateral ventricle. From the head the nucleus tapers as it proceeds backward through the central part of the ventricle. Its tail (cauda) turns downward in the roof of the inferior horn, and ends in a considerable mass of gray substance called the nucleus amygdalc, which appears to be an ingrowth of cortex from the region of the uncus hippocampi (Fig. 32). The caudate nucleus is covered on its ventricular surface by ependyma. The opposite surface, resting against the fibers of the internal capsule, is irregular and serrated.

The stria terminalis (tænia semicircularis, Figs. 30 and 36 ) lies just medial to the nucleus caudatus. It is a band of white fibers traversing the floor of the central part of the ventricle and the roof of its inferior horn, but covered by the terminal vein and by the ependyma. It may be said to rise from the nucleus amygdalæ. Ascending to the ventricle, it passes forward between the caudate nucleus and the thalamus to the interventricular foramen, where it divides into two bundles. One of them accompanies the columna of the fornix for a little way. The other, passing over the anterior commissure, descends in front of it; and, according to Kölliker, both bundles end in the anterior perforated substance (Cunningham). The thin medial border of the substance covering the vena terminalis, which is attached to the thalamus, is called the lamina affixa; it is present only in the body of the ventricle. The lamina affixa is joined by a single layer of epithelium, the lamina chorioidea epithelialis, to the lateral border of the fornix. This lamina invests the chorioid plexus of the lateral ventricle. If the chorioid epithelium be torn away, the edge which remains on the stria terminalis is called the tania terminalis.

Thalamus (Figs. 30, 31, 36 and 40).-A fusiform part of this ganglion of the inter-brain is visible in the floor of the lateral ventricle, between the stria terminalis and the chorioid plexus. It extends throughout the central part of the ventricle from the interventricular foramen to the inferior horn. A transparent layer of epithelium, the lamina chorioidea epithelialis, extending from the fornix to the stria terminalis and representing the hemi- 
sphere wall, covers it. The thalamus will be described with the third ventricle and inter-brain.

The chorioid plexus (plexus chorioideus, Figs. 35, 36 and 37) of the lateral ventricle is the vascular border of the chorioid tela of the third ventricle. It projects, laterally, from beneath the fornix and its crus through the chorioidal fissure into the floor of the central part of the ventricle and the inner wall of the inferior horn. The epithelium, above mentioned, invests it; and it borders the fornix like a ruffle. It is called chorioid plexus (chorion, a membrane) because it is membrane-like. At the junction of the central part and inferior horn of the lateral ventricle the chorioid plexus presents a large skein-like mass called the glomus chorioideum (Fig. 36). The anterior chorioidal artery from the internal carotid and the postero-lateral chorioidal, a branch of the posterior cerebral, supply the plexus. The former pierces the temporal lobe and enters the apex of the inferior horn of the ventricle; the latter passes in through the transverse and chorioidal fissures of the cerebrum, following the chorioid tela. The chorioidal vein carries the blood away. At the foramen interventriculare, it is joined by the terminal vein of the striated body and the veins of the septum pellucidum and forms the internal cerebral vein. The internal cerebral vein courses backward in the chorioid tela and unites with its fellow of the opposite side, proximal to which union it receives the basilar vein; and then the great cerebral vein (of Galen), uniting with the inferior sagittal sinus, forms the straight sinus.

The floor of the central part of the lateral ventricle is completed by the superior surface of the fornix.

The horns of the lateral ventricle are three in number; the anterior, inferior and posterior (Figs. 39, 40 and 4I).

The anterior horn (cornu anterius, Figs. 35, 36 and 73) projects from the central part of the ventricle forward and outward around the head of the caudate nucleus. It is the ventricle of the frontal lobe and is deep and narrow. Its boundaries are as follows:

Roof-Corpus callosum (forceps minor).

Floor-Rostrum.

Anterior wall-Genu. 


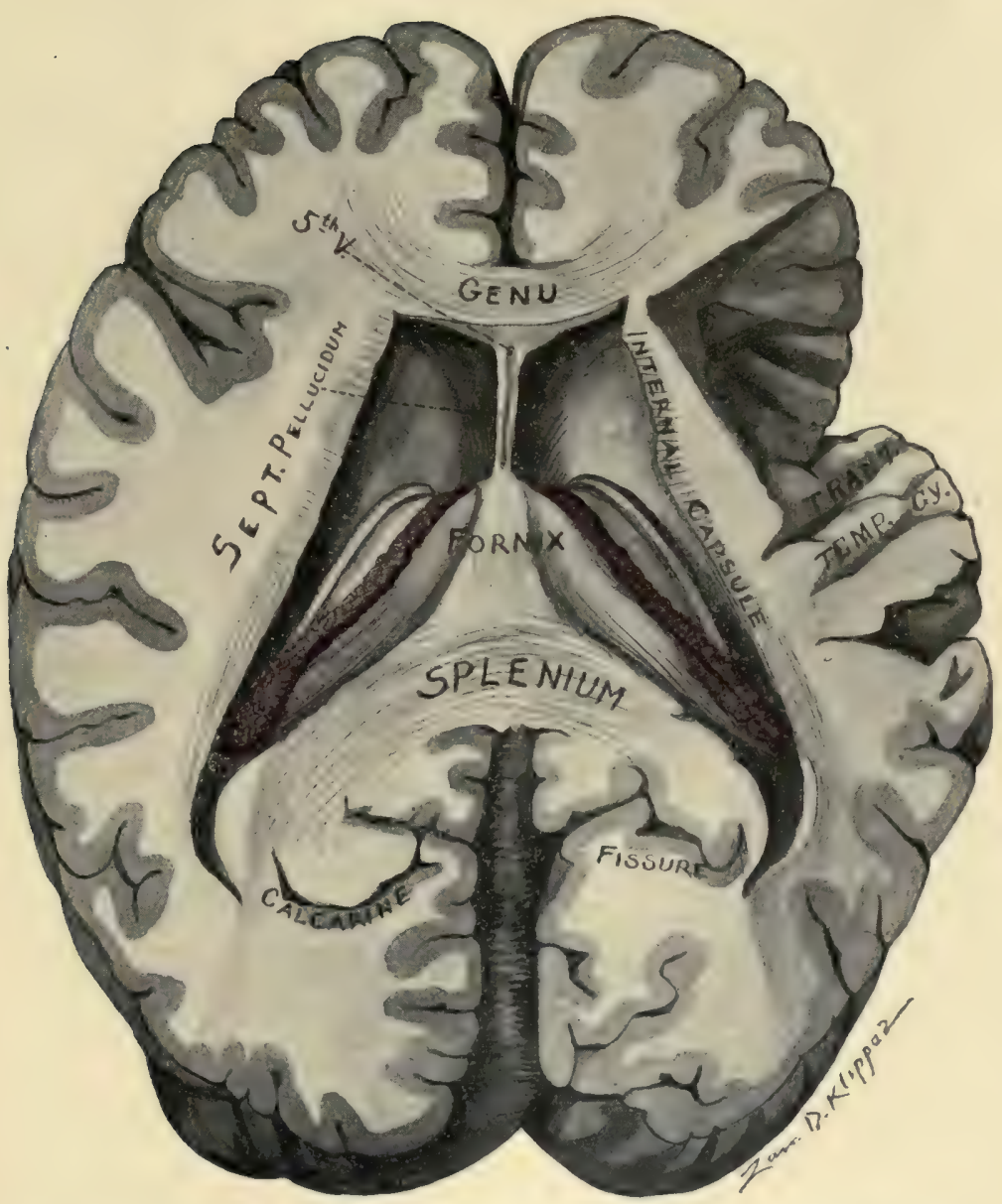

Fig. 35.-Horizontal section of cerebrum, cutting splenium and genu of corpus callosum, showing lateral ventricles, septum pellucidum, fornix and transverse temporal gyri. (Original.) 

Inner wall-Septum pellucidum.

Outer wall-Caudate nucleus.

The posterior horn (cornu posterius, Figs. 35, 36, 38, 39, 40 and $4 \mathrm{I}$ ) is directed backward and downward in a curve concave inward, from the ventricular center into the occipital lobe; and, like the occipital lobe, it first makes its appearance in the fifth month of embryonic life. Its extremity bends medially toward the posterior calcarine fissure, with which the horn is parallel. The anterior calcarine fissure produces the ridge along the inner wall called the calcar avis. The posterior horn is roofed over by fibers from the splenium of the corpus callosum, which turn down outside the horn and also form part of the external boundary. In the external wall and in the roof and floor is also the optic radiation. A well-marked bundle of fibers from the splenium, forceps major, is found passing along the medial border of the roof into the occipital lobe. It produces an eminence above the calcar avis, called the bulb. The anterior extremity of the posterior horn is continuous, inferiorly, with the beginning of the inferior horn. At the junction of the two is a triangular area, the trigonum collaterale.

The inferior horn (cormu inferius, Figs. 32, 38, 39 and 4I) is the ventricle of the temporal lobe. Its course is crescentic, as it follows the perimeter of the internal capsule. It first runs outward and backward from the body of the ventricle, then it turns downward, and finally it proceeds horizontally forward and inward to within an inch of the pole of the temporal lobe. In horizontal section just below the general cavity of the ventricle, the inferior horn is triangular. In that position it has a posterior wall (or, floor in the horizontal part), a medial wall, and a curved antero-lateral wall (or roof in the horizontal portion) which is continuous above with the outer wall and floor of the central part of the ventricle.

The parts found in the walls of the inferior horn may be enumerated as follows:

Roof (or antero-lateral wall)-

Inferior lamina of internal capsule, partially covered by tail of caudate nucleus, stria terminalis and amygdala.

Tapetum Floor (or posterior wall)- 
Eminentia collateralis (trigonum collaterale),

Hippocampus,

Crus of fornix.

Inner wall (medial) -

Epithelium (of hemisphere wall) covering

Pulvinar,

Chorioid plexus,

Chorioidal fissure, and

Dentate fascia.

The structures in the roof of the inferior horn have been sufficiently described. They are easily understood when it is recalled that the roof of the horn is continuous with the outer wall and floor of the central part of the ventricle; the tapetum, the internal capsule (inferior lamina), the cauda, amygdala and the stria terminalis form it.

Beginning at the trigonum collaterale (Figs. 38 and 39) and extending along the outer border of the floor to the end of the inferior horn there is sometimes a low ridge caused by the collateral fissure. It is the eminentia collateralis, and is present only when the anterior part of the collateral fissure, as well as the middle part, is a complete fissure. The short eminence at the entrance to the inferior horn, called the trigonum collaterale, is constant in its presence; it is produced by the middle division of the collateral fissure. In front of this eminence and internal to it is a prominent ridge, the hippocampus, which enlarges downward to a lobulated extremity, called the digitations (digitationes hippocampi, Fig. 39). The ridge is due to the infolding of the floor over the hippocampal fissure on the medial surface of the cerebrum. The ventricular surface of the hippocampus is formed by a lamina of white matter, the alveus, but the deeper part is cortical matter composed almost entirely of pyramidal cell-bodies. The crus of the fornix (fimbria hippocampi) rests in the concavity of the hippocampus, where most of its fibers originate, though a small bundle of them passes beyond it to its origin in the uncus.

The chorioid epithelium (lamina chorioidea epithelialis) (Figs. II8, 62 and 35), representing the hemisphere wall, forms the floor of the chorioidal fissure and the whole medial wall of the 


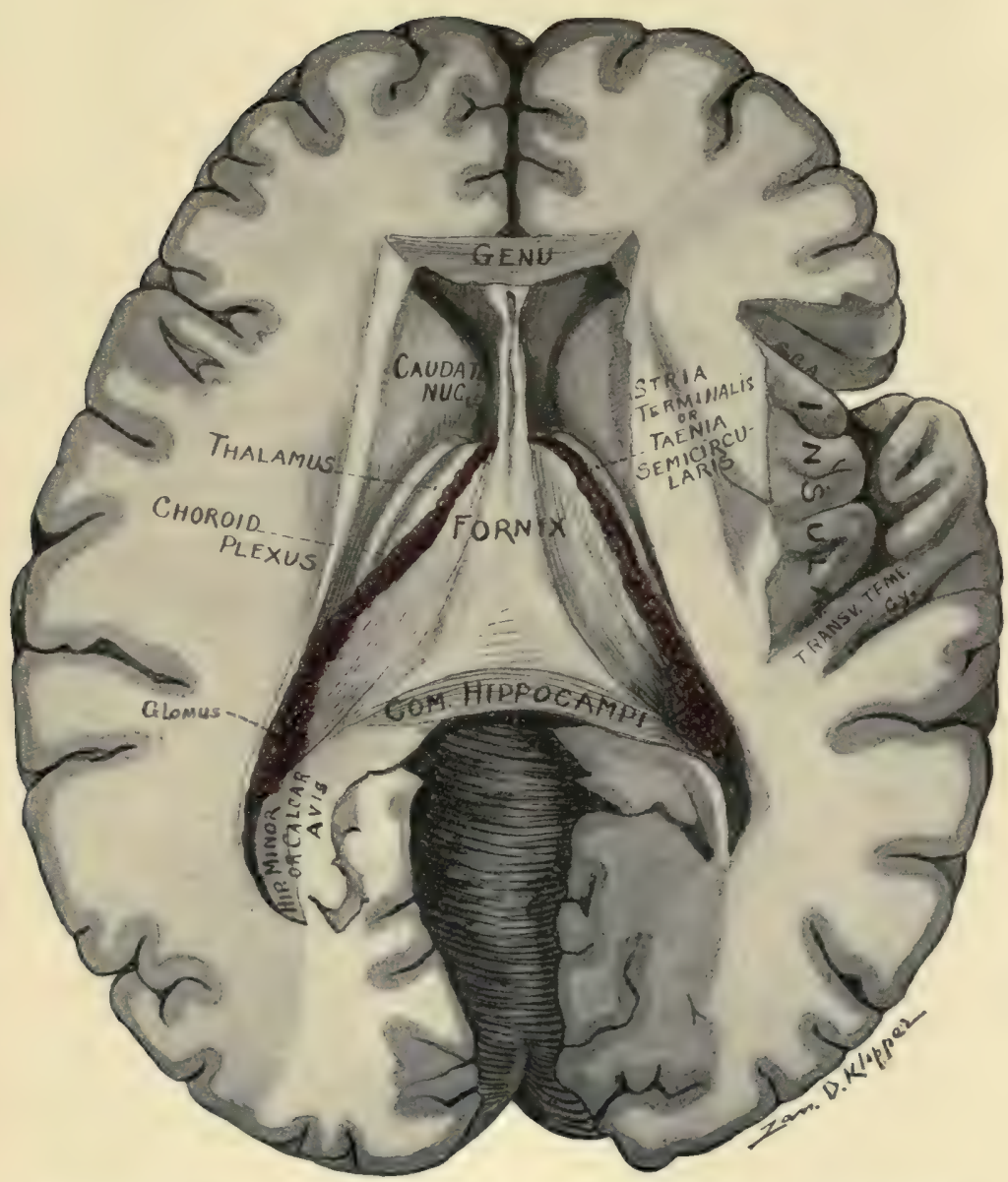

Fig. 36.-Horizontal section of cerebrum just below splenium of corpus callosum, showing commissura hippocampi, fornix, septum pellucidum, the island and lateral ventricles. (Original.)
S. C. A. Sul. circularis anterior.
S. C.P. Sul. circularis posterior. 

inferior horn. It covers the cushion-like projection (the pulvinar) of the thalamus, which forms a small part of both roof and inner wall. Behind, it is attached to the crus of the fornix, from which it extends forward to the stria terminalis. The epithelium covers the chorioidal fissure except at the lower part, where there is a small cleft which forms a communication between the horn and the anterior subarachnoid space. Through the chorioidal fissure a fold of pia mater projects toward the ventricle, and pushing the epithelium before it into the horn, forms the chorioid plexus (Figs. 35 and 36). Bounding this part of the chorioidal fissure below and behind, there is a serrated free border of cortex called the dentate fascia. The dentate fascia (Figs. 38, 42 and 62) folds medially in front of the hippocampal fissure and with the hippocampus, the gyrus hippocampi and the eminentia collateralis forms an $S$-shaped fold of the cortex. The S-shape is perfect in the left hemisphere, when the anterior segment of a coronal section is viewed from behind. The top of the letter is the dentate fascia; the superior curve is the hippocampus, produced by the hippocampal fissure; the lower convexity is the gyrus hippocampi; the lower concavity, open toward the ventricle, is the groove between the hippocampus and the eminentia collateralis.

\section{THE THIRD VENTRICLE AND INTER-BRAIN.}

(Ventriculus Tertius and Diencephalon.)

The inter-brain (diencephalon) is median in position (Figs. $27,28,29,30$ and 40). It is situated beneath the fornix and the layer of epithelium extending from the border of the fornix to the stria terminalis. The chorioid tela of the third ventricle only intervenes between them. Laterally, it is bounded by the superior laminæ of the internal capsules. The ventricle of the inter-brain is the third in number. The third ventricle, therefore, is located in the median plane; and is at a lower level than the ventricles of the hemispheres. Through the interventricular foramina, its anterior part (the aula) communicates with each lateral ventricle, and the cerebral aqueduct connects it, behind, with the fourth ventricle (Figs. I7 and $4 \mathrm{r}$ ). The third ventricle is fissure-like. 
It is a narrow, vertical cleft about an inch in length from before backward and a quarter of an inch broad at its widest part It separates the thalami, and extends almost to the inferior surface of the cerebrum. The roof (Figs. 29, 36, 37, 40 and 42) follows the curve of the fornix and arches from the posterior commissure forward to the anterior commissure. There is a little recess above the anterior commissure and between the columnæ of the fornix, bounded in front by the inferior angle of the septum pellucidum, called the recessus triangularis, in which the roof and anterior wall meet. The anterior wall extends from the triangular recess down to the optic recess, at the angle between the lamina cinerea and the optic chiasma. This angle is so named because, on either side of it, there is a lateral extension of the third ventricle between the lamina terminalis and the columna of the fornix, which is located in the root of the embryonic optic vesicle. The floor (Fig. 28) describes two arches, convex toward the ventricle. The first arch, very convex and short, stretches between the optic recess and the infundibulum, in which the floor reaches its lowest point. The distance from the infundibulum to the anterior orifice of the cerebral aqueduct is spanned by the second arch. It is long and flat. Its posterior extremity is but a sixteenth of an inch below the posterior commissure; the anterior orifice of the cerebral aqueduct separates them. The ventricle is thus contracted behind to the size of the cerebral aqueduct, with which it is continuous. The lateral walls (Figs. 28 and 29) are close together throughout. At one point near the middle they come together and are joined by the massa intermedia (middle commissure). Antero-superiorly, the lateral wall is perforated by the interveniricular foramen (of Monro). That foramen constitutes the slight separation between the front of the thalamus and the columna of the fornix. It opens into the lateral ventricle at the junction of the anterior horn with the central part. The ependyma which lines the third ventricle is continuous through the interventricular foramen with the lining of the lateral ventricle. But one layer of the ependyma is present in the roof of the ventricle; that is the epithelial layer. The third ventricle, like all true ventricles, is occupied by cerebro-spinal fluid. 


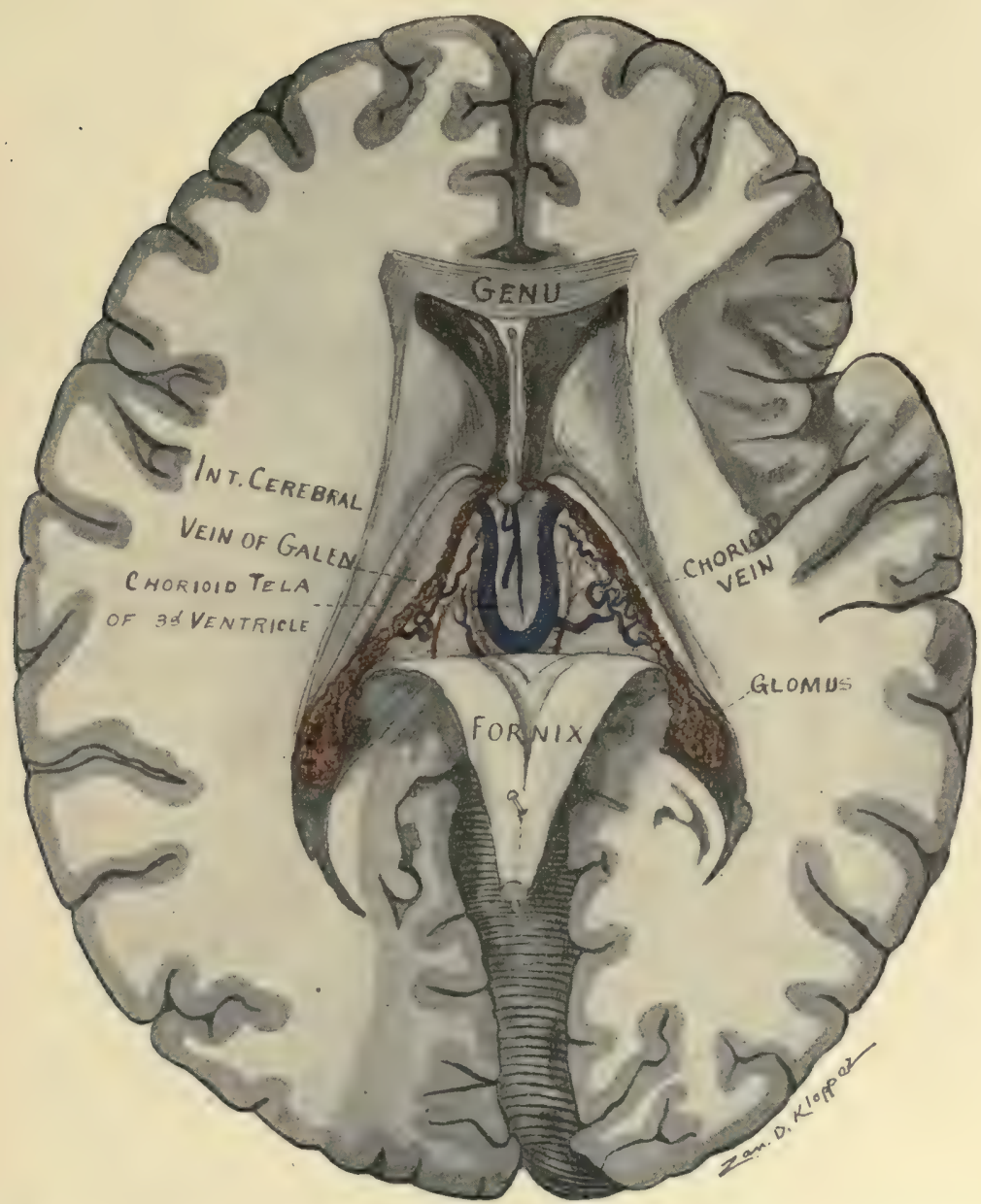

Fig. 37.- Horizontal section of cerebrum. Fornix turned back, showing chorioid tela of third ventricle, and internal cerebral veins. (Original.) 

The following are the boundaries of the third ventricle:

Roof-

Posterior commissure and commissura habenularum,

Roof epithelium and pineal body,

Chorioid tela and plexuses,

Fornix and commissura hippocampi.

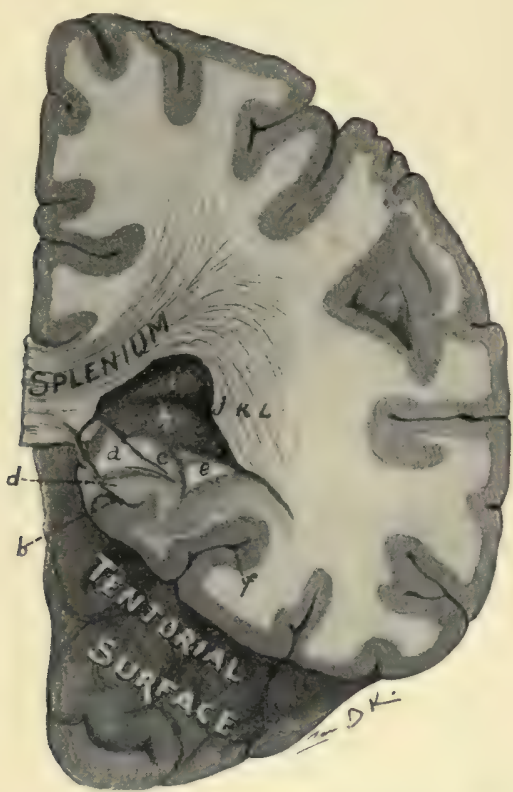

Fig. 38. - Transverse section of left cerebral hemisphere cutting the splenium and showing the posterior horn and the floor of the inferior horn of the lateral ventricle. (Original.)

a. Crus fornicis. b. Fis. hippocampi. c. Hippocampus. d. Fascia dentata. e. Eminentia collateralis. f. Fis. collateralis. h. Calcar avis. i. Bulb caused by forceps major. j. Tapetum. k. Radiatio occipito-thalamica. 1. Fasciculus longitudinalis inferior.

Anterior wall -

Epithelium, covering

Columnæ of fornix, anterior commissure, and

Lamina terminalis.

Floor-

Optic commissure,

Tuber cinereum and infundibulum,

Corpora mammillaria, 
-Posterior perforated substance (of mid-brain),

Tegmenta (of mid-brain).

Posteriorly-

Ventricle is continuous with cerebral aqueduct.

Lateral walls-

Thalamus, and reflected hyporhalamic substance,

Columna of the fornix, and

Foramen interventriculare between them.

Roof.-A band of white fibers passes across the back part of the third ventricle and supports the posterior end of the roof epithelium. That band is the posterior commissure (commissura posterior, Figs. 28 and 40). It crosses immediately in front of the corpora quadrigemina. Beneath it is the anterior orifice of the cerebral aqueduct. The pineal body is above and behind it, and the commissure fuses with the ventral pineal lamina. The posterior commissure stretches from the central gray substance of the mid-brain on one side, over the aqueduct, to the gray substance of the opposite side and also contains decussating fibers of the medial longitudinal bundle (Heald). The commissure is in need of further investigation.

The roof epithelium (Figs. 42 and 37 ) of the third ventricle stretches from the posterior commissure to the anterior commissure, and laterally, is attached to the upper internal border of the thalamus. It is the superficial layer of the ependyma; but it is, here, the only adult representative of the roof of the diencephalon. The roof epithelium presents two longitudinal folds suspended in the ventricle. The lower layer of the chorioid tela of the third ventricle, invests the roof epithelium superiorly; and, dipping down into the longitudinal folds, that inferior layer forms the chorioid plexuses of the third ventricle, which are continuous with those of the lateral ventricles through the interventricular foramina. At the back part in the middle line, there is a pouch-like evagination of the roof of the diencephalon in the embryo, which develops into the pineal body; and there remains a slight pit, called the pineal recess, in the adult condition. A second evagination occurs just above the pineal recess, which forms the epipineal recess.

Pineal Body. (Corpus pineale, Figs. 40, 43 and 76).-It is a 


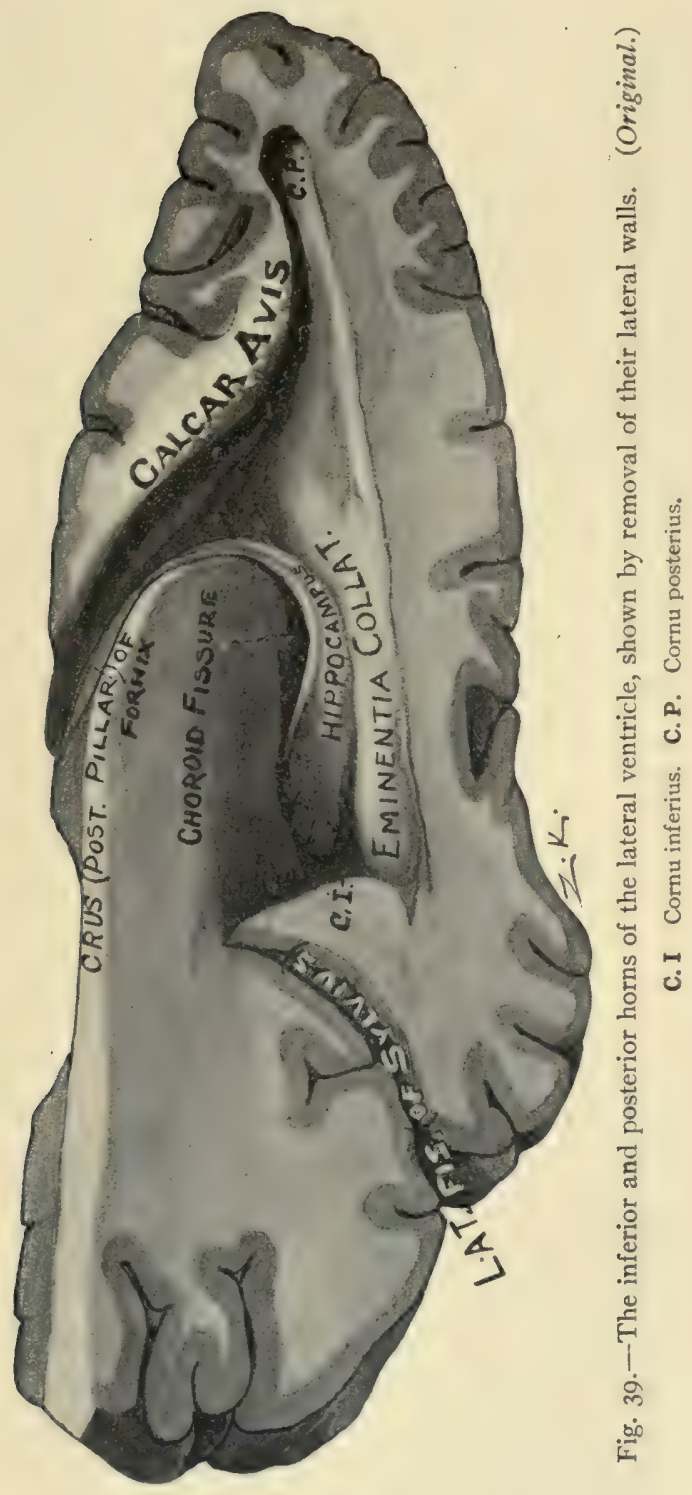



cone-shaped body, a quarter of an inch high and one-sixth of an inch in diameter, joined to the roof of the third ventricle by a flattened stalk or peduncle. It is also called the epiphysis. The pineal body is situated in the floor of the transverse fissure of the cerebrum, directly below the splenium of the corpus callosum and rests between the superior colliculi of the quadrigeminal bodies on the posterior surface of the mid-brain. It is closely invested by pia mater. The pineal stalk splits into a dorsal and a ventral lamina, which are separated by the pineal recess. The ventral lamina fuses with the posterior commissure; but the dorsal stretches forward over the commissure in continuity with the roof epithelium. The border of the dorsal lamina is thickened along the line of attachment to the thalamus and forms the stria medullaris thalami (pineal stria). The thickening is due to the presence of a bundle of fibers derived from the columna of the fornix and the medial stria of the olfactory tract. Between the medullary striæ, at the posterior end, there is a transverse band, called the habenula. The habenula contains the commissura habenularum, through which the fibers of the striæ partially decussate to the nucleus habenulæ in the thalamus.

The interior of the pineal body is made up of closed follicles surrounded by ingrowths of connective tissue. The follicles are filled with epithelial cells mixed with calcareous matter, the brain-sand (acervulus cerebri). Calcareous deposits are found also on the pineal stalk and along the chorioid plexuses. The function of the pineal body is unknown. It is supposed to represent a cyclopian eye. In the Hatteria, a New Zealand lizard, it projects through the parietal foramen and presents an imperfect lens and retina and, in its long stalk, nerve fibers.

The chorioid tela of the third ventricle (velum interpositum, Figs. 37, 40 and 42 ) is the double triangular fold of pia mater spread over the dorsum of the inter-brain. It lies underneath the fornix and the chorioid epithelial lamina which stretches from the body of the fornix lateralward to the stria terminalis. Its apex is just behind the anterior commissure, and its base, directed backward, is continuous, by the upper layer, with the pia mater of the occipital lobes; and, by the inferior layer, it is 
continuous with the pia on the posterior surface of the mid-brain and cerebellum. Each border constitutes the chorioid plexus of the lateral ventricle, and is seen (through the epithelium) in the floor of its central part. The median part of the inferior lamina of the chorioid tela invests the roof epithelium of the third ventricle, and, the lateral portion covers the medial half of the upper surface of each thalamus. This layer forms the two chorioid plexuses of the third ventricle, which depend from its median portion. Between the inferior and superior laminæ is enclosed some connective tissue through which the internal cerebral veins run backward to the base of the tela; there they unite and form the great cerebral vein (Galeni).

Anterior Wall.-The anterior commissure (commissura anterior cerebri, Figs. 29, 40 and 73) is a very distinct round bundle of white fibers about an eighth of an inch in diameter. It is seen in the anterior wall of the third ventricle supporting the roof epithelium. The epithelium there bends down between the columnæ of the fornix and invests the ventricular surface of the commissure. The columnæ of the fornix and the commissure bound the recessus triangularis, in which the roof and anterior wall of the third ventricle meet. The anterior commissure rests upon the upper extremity of the lamina terminalis, between the columnæ fornicis, behind, and the lamina rostralis of the corpus callosum, in front. With the last two structures it is developed in the lamina terminalis. It is the most important connecting link between the hemispheres in vertebrates without a corpus callosum (all below mammals). Bending sharply backward in the cerebral hemisphere the anterior commissure pierces the inferior part of the globus pallidus and then radiates toward the cortex, some of its fibers entering the external capsule. It contains two groups of fibers: (I) The anterior group, which is the commissure of the rhinencephalon, called the pars olfactoria; and (2) the posterior group, the pars occipito-temporalis. The pars olfactoria probably contains two fasciculi: (a) A commissural bundle which bends sharply downward to the olfactory tracts and joins the two olfactory bulbs together; and (b) a decussating bundle which joins each olfactory bulb to the opposite 


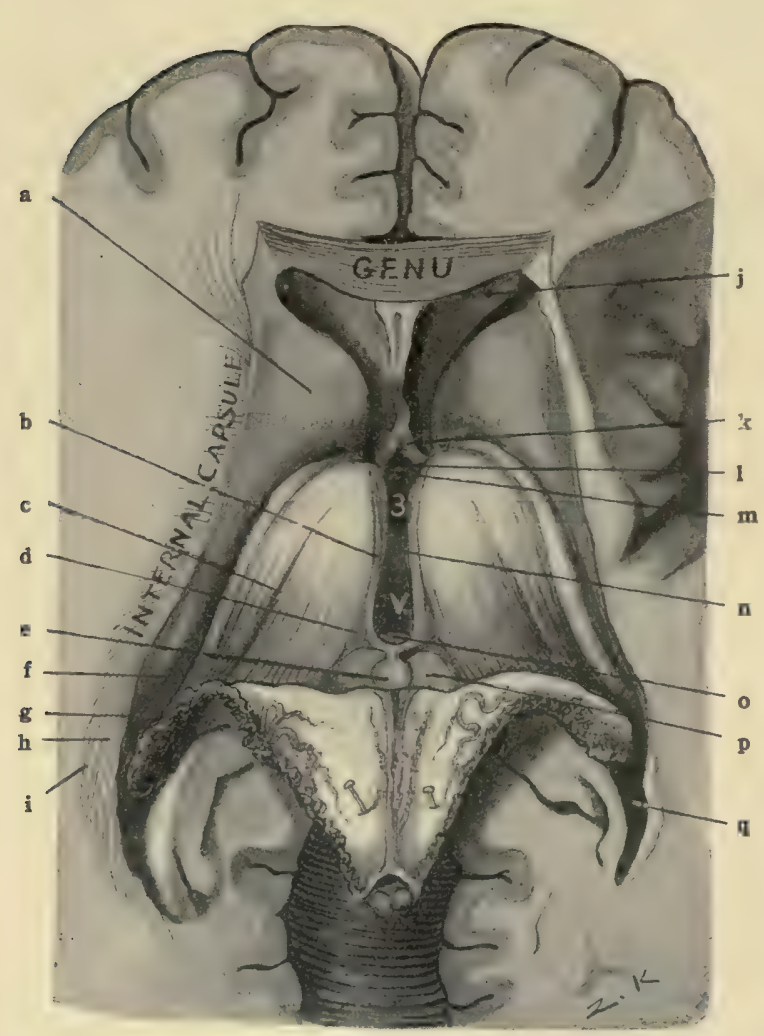

Fig. 40.-Horizontal section of cerebrum through genu and below splenium of corpus callosum. Fornix and chorioid tela turned back, to show inter-brain and third ventricle. (Original.)

a. Head of caudate nucleus. b. Stria medullaris thalami (or pineal stria.) c. Chorioid groove. d. Trigonum habenulae. e. Pineal body. f. Tail of caudate nucleus. g. Tapetum. h. Occipito-thalamic radiation. i. Inferior longitudinal fasciculus. j. Anterior horn of lateral ventricle. k. Columna of fornix. 1. Recessus triangularis. m. Anterior commissure. n. Massa intermedia (or middle commissure). o. Posterior commissure. p. Superior quad. rigeminal colliculus, q. Posterior horn of lateral ventricle. 

uncus and amygdala. The pars occipito-temporalis connects the tentorial areas of the two hemispheres together, regions which are not connected by the corpus callosum. In man it is larger than the pars olfactoria. A thin transverse sheet of gray matter, called the lamina terminalis, extends downward and forward from the anterior commissure to the optic chiasma and completes the anterior wall of the ventricle (Figs. I 7, 28 and 73). Between the chiasma and the lamina terminalis is a sharp angle which terminates on either side in a small pit, called the optic recess.

The floor of the third ventricle is very narrow (Figs. 2 I and 27). It is formed by the interpeduncular structures plus the tegmenta, namely: optic chiasma, tuber cinereum and infundibulum, corpora mammillaria, posterior perforated substance and the tegmenta. The last two are portions of the mid-brain; the others belong to the fore-brain with the surface of which we have already studied them, and all extend laterally beneath the thalami.

The third ventricle has its lateral wall formed chiefly by the thalamus and the columna of the fornix (Figs. 27 and 28). Below a slight longitudinal groove, extending from the optic recess to the cerebral aqueduct and called the sulcus hypothalamicus, the thalamus is covered by upturned hypothalamic gray matter and by the upper part of the central gray substance of the midbrain. The thalamus forms the immediate lateral wall above this hypothalamic groove. The columna of the fornix, diverging from its fellow, proceeds downward and backward to the corpus mammillare through the medial part of the thalamus. In the ventricle, the pars libera of the columna fornicis is covered by the ependymal epithelium. It bounds the interventricular foramen in front.

Thalamus. (Thalamus - a bed, Figs. 40, 42, 43 and 44).-It is the great ganglion of the inter-brain. The thalamus is an important sensory relay station. In it or in the hypothalamic nuclei almost every impulse of general sensation, in its journey to the cerebral cortex, is transferred to a higher neurone. The third ventricle separates the thalami from each other, except at the mid-point where they are joined by the massa intermedia. The thalamus is situated behind and medial to the corpus striatum, 
and projects backward over the mid-brain. Laterally, it rests against the superior lamina of the internal capsule, which separates it from the lentiform nucleus. The thalamus is shaped like an egg, with the small end directed forward. It has an anterior and a posterior extremity and four surfaces: Superior, inferior, medial and lateral.

Extremities. - The anterior extremity of the thalamus is lost in a large group of fibers (frontal stalk) which runs through the frontal part of the internal capsule. The posterior end (Fig. 44) presents a large pillow-like prominence, the pulvinar, and beneath it, are two smaller swellings; the outer one, which forms the lowest point of the thalamus, is the lateral geniculate body; the medial geniculate body is the other. The two geniculate bodies constitute the metathalamus (Fig. 43).

Surfaces.-The medial surface of the thalamus forms the immediate lateral wall of the third ventricle as far down as the sulcus hypothalamicus (Fig. 28). It is joined to the internal surface of the opposite thalamus by the massa intermedia. It is bounded above by the medullary stria. The superior surface is composed of a thin lamina of longitudinal white fibers, derived from the optic tract and radiation, called the stratum zonale. The superior surface of the thalamus is divided by an oblique groove, the chorioidal groove, corresponding in position to the border of the fornix, into two areas-a medial and lateral (Fig. 44). The medial area is covered by the chorioid tela of the third ventricle and the fornix. Internally, it is bounded by the medullary stria of the thalamus. Posteriorly, next the stria, is a triangular depression bounded, behind, by a transverse groove in front of the corpora quadrigemina, and by a slight groove, the sulcus habenulæ, externally. That depressed surface is called the triangle of the habenula (trigonum habenulæ). Beneath the triangle is one of the thalamic nuclei, the nucleus habenula. The lateral area of the superior surface is seen in the floor of the lateral ventricle. It presents an anterior elevation, the anterior tubercle (tuberculum anterior thalami), beneath which is the anterior nucleus of the thalamus. The chorioid sheet of epithelium, extending from the fornix to the stria terminalis, covers this outer 


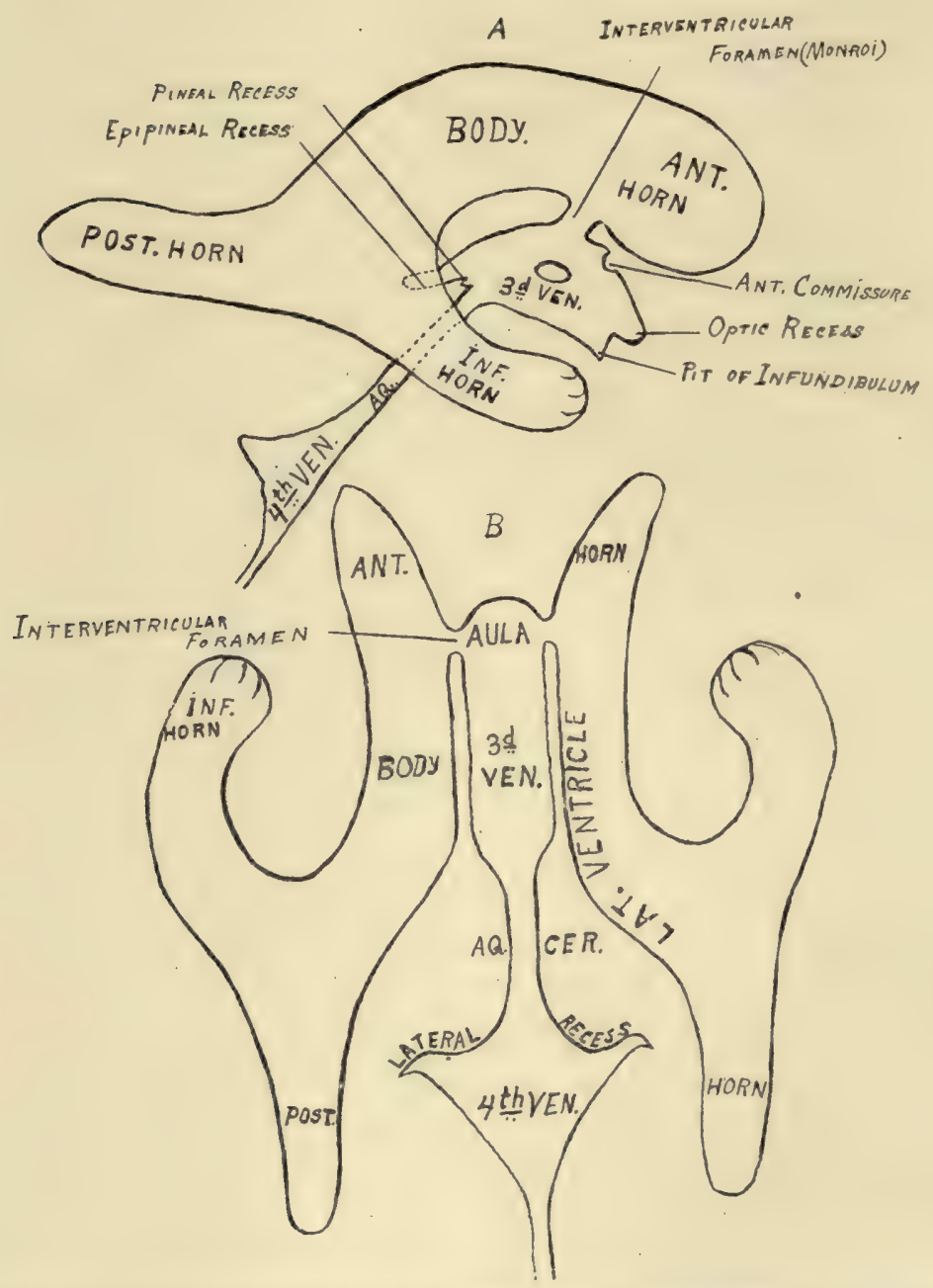

Fig. 4I.-Lateral and dorsal view of the ventricles. Diagrammatic. (Original.)

A. Lateral view of the ventricles. B. Dorsal view of the ventricles. 

area and separates it from the ventricular cavity. A lamina of fibers, the external medullary lamina, forms the lateral surface of the thalamus and rests upon the superior lamina of the internal capsule. Its fibers are continuous with those of the capsule. The inferior surface blends with the superior surface of the tegmentum and substantia nigra, and forms the laminæ and nuclei of the tegmental hypothalamic region. (See below.)

Tegmental Hypothalamic Region (Figs. 30 and 42).-The regio tegmentalis hypothalami is composed of three layers: (I) Stratum dorsale next the thalamus; (2) zona incerta, the middle; and (3) hypothalamic nucleus, the inferior. The nucleus hypothalamicus (Luysi) is ventro-lateral in position and lies between the base of the internal capsule and the zona incerta. Like the substantia nigra just below it, it is composed of pigmented gray matter. The reticular formation of the tegmentum, continuing beneath the thalamus, forms the zona incerta. The stratum dorsale is made up as follows: (a) Fibers from the medial longitudinal bundle (Meynert); (b) the brachium conjunctivum (Forel), in which is the upper end of the red nucleus of the tegmentum; and (c) the medial fillet, which runs lateral and slightly ventral to the red nucleus.

The lateral geniculate body (corpus geniculatum laterale, Fig. 43) forms a slight swelling at the lowest point of the thalamus. It marks the apparent end of the lateral root of the optic tract and is the terminal nucleus of eighty per cent. of its fibers. It is joined to the superior quadrigeminal eminence by the brachium superius. In appearance it is dark colored and laminated; its gray matter, which contains pigmented multipolar cell-bodies, is divided into thick layers by thin laminæ of fibers from the optic tract and radiation. The processes of the multipolar cell-bodies help to form the optic radiation.

The medial geniculate body (corpus geniculatum mediale), also belongs to the inter-brain and, together with the lateral geniculate body, constitutes the metathalamus (Fig. 43). It is placed at the end of the medial root, as the lateral geniculate is at the end of the outer root, of the optic tract. It rises up from the groove between the thalamus and corpora quadrigemina, 
and is joined to the inferior quadrigeminal eminence by the brachium inferius. The brachium superius sweeps around it in front. The medial geniculate body is gray in color and is not laminated. Its cell-bodies are small, and fusiform in shape. They perhaps give origin to the intercerebral fibers (Guddeni) of the optic tract and to a large part of the acustic radiation.

\section{CEREBRUM.}

\section{SECTION II. THE MID-BRAIN.}

(MESENCEPHALON.)

The third division of the cerebrum is the mid-brain (Figs. 44 and 45). It is situated below and behind the inter-brain and forms the connecting link between the fore-brain and the hindbrain. This has suggested the name "isthmus," sometimes applied to it: though isthmus rhombencephali refers only to the constriction below the corpora quadrigemina. The mid-brain is developed from the middle of the brain vesicles, the mesencephalon (Figs. I6, I7 and 18). The cavity of the mesencephalon persists as the cerebral aqueduct, which is reduced to a slender canal by the thickening of its walls, roof and floor, due largely to the ingrowth of fibers from other parts of the brain. The cerebral hemispheres almost conceal the mid-brain from view; they overhang it dorsally, and the temporal lobes, inclosing it between them, bend medially and cover part of its anterior surface. Only the median part of the anterior surface is visible in the complete brain (Fig. 2I). The form of the mid-brain resembles a flattened cylinder. Its axis, a half inch long, is pointed upward and forward, and its long diameter, which varies from an inch to an inch and a half in length, is directed transversely.

\section{SURFACES.}

The mid-brain has four surfaces, viz., the anterior and posterior, which are free, and the superior and inferior, representing the ends of the cylinder, which are attached. The two latter are parallel with each other and are formed by section.

The superior surface, sloping downward and forward, meets 
the anterior surface at an acute angle. Its inclination is that of the posterior end of the floor of the third ventricle. External to the floor of the ventricle, it is attached to the thalami and internal capsules. The blending of it with the thalami forms the structures of the tegmental hypothalamic regions, and the continuations of the extreme lateral portions, the bases pedunculi, enter into the internal capsules of the hemispheres. In the median line

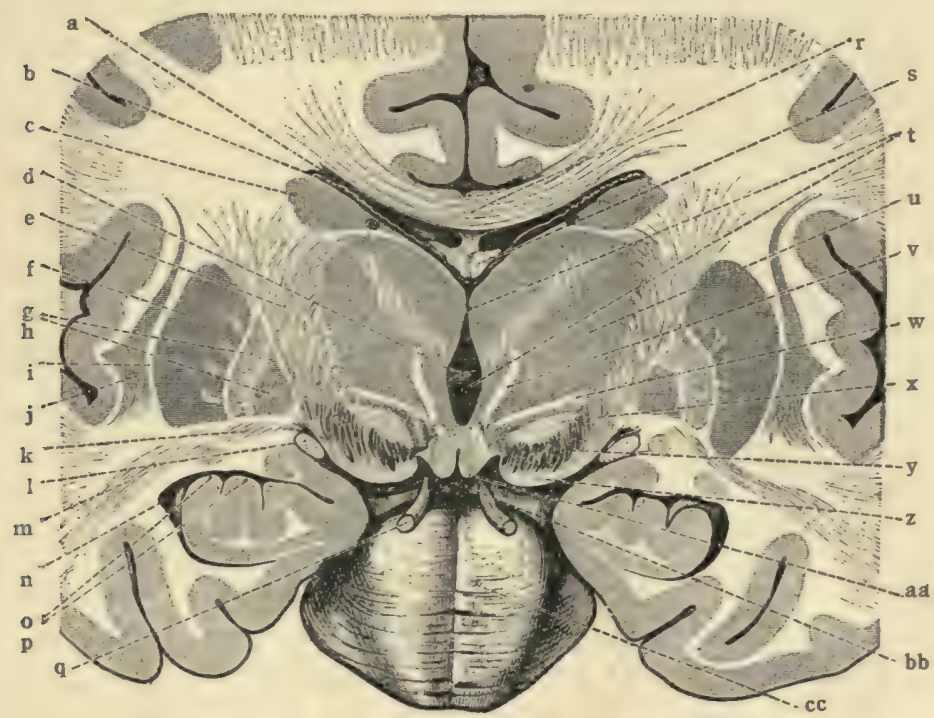

Fig. 42.-Transverse section of brain, cutting corpora mammillaria.

(After Toldt, Morris's Anatomy.)

a. Lateral ventricle (central portion). b. Chorioid plexus of lateral ventricle. c. Caudate nucleus. d. Massa intermedia. e. Internal capsule. Lenticular nucleus: f, Putamen; gh. Zones, globus pallidus. i. External capsule. j. Claustrum. k. Ansa penduncularis. 1. Optic tract. m. Inferior peduncle of thalamus. $\mathbf{n}$. Inferior cornu of lateral ventricle. 0 . Hippocampus. p. Digitations. q. Oculomotor nerve. $\boldsymbol{x}$. Corpus callosum. s. Fornix. t. Third ventricle. u. Thalamus. v. Thalamo-mammillary fasciculus. w. Ansa lenticularis. $\mathbf{x}$. Hy pothalamic nucleus (corpus Luysi). y. Substantia nigra. z. Basis of cerebral peduncle. aa. Corpus mammillare. bb. Interpeduncular fossa. cc. Pons (varolii.)

behind the third ventricle it is attached to the posterior commissure. The superior surface is an inch and a half broad.

The inferior surface joins the upper surface of the pons. It is a little narrower than the superior surface. It is about one inch and a quarter broad and measures an inch dorso-ventrally.

The anterior surface (ventral) of the mid-brain looks forward and downward (Figs. 45, 47 and 48). It is deeply grooved longit- 
udinally by a median sulcus, the fossa interpeduncularis, and is slightly concave from above downward. It is separated on either side from the posterior surface by the sulcus lateralis mesencephali. Though partially concealed by the temporal lobes of the cerebrum, the anterior surface is unattached. It is formed by a prominent band, the basis pedunculi at either side; and by a median structure, the posterior perforated substance which is inclosed between the two bases. The posterior perforated substance forms the floor of the median sulcus. The inner border of the basis pedunculi is free and overhangs the perforated substance slightly. Thus is formed the oculomotor groove (sulcus nervi oculomotorii) between the basis and perforated substance whence the third cerebral nerve takes its apparent origin. The fourth nerve courses forward over the anterior surface, but is not attached to it.

The posterior surface (dorsal) of the mid-brain (Fig. 44), though free, is entirely concealed by the cerebellar and cerebral hemispheres. It forms part of the floor of the transverse fissure of the cerebrum and is covered by pia mater. The lateral sulcus bounds it on each side. From the sulcus lateralis it elevates abruptly toward the median line, where it presents a longitudinal groove. This produces two ridges which are subdivided by a transverse groove into the four eminences, the colliculi of the corpora quadrigemina. On either side, anterior and a little external to the quadrigeminal bodies, is the medial geniculate $b o d y$, joined to the inferior quadrigeminal colliculus by an oblique ridge, called the brachium inferius. The nearly parallel longitudinal ridges below the corpora quadrigemina are formed by the brachia conjunctiva of the cerebellum. The bottom of the groove between them is formed by the superior medullary velum (of Vieussens), whence the trochlear nerve (fourth) is seen issuing.

$$
\text { Mid-brain }\left\{\begin{array}{l}
\text { I. Corpora quadrigemina and Brachia. } \\
\text { II. Pedunculi }\left\{\begin{array}{l}
\text { I. Tegmenta } \\
\text { 2. Substantia nigra } \\
\text { 3. Bases pedunculi. }
\end{array}\right.
\end{array}\right.
$$

The four colliculi of the corpora quadrigemina and the four brachia connecting them with the geniculate bodies constitute 
the quadrigeminal lamina, which forms the greater part of the posterior surface of the mid-brain (Fig. 44). This lamina rests upon the dorsum of the pedunculi cerebri. The pedunculi cerebri are made up of three great divisions, as shown above; named from before backward, they are: (I) The bases pedunculi, comprising the 'anterior part; (2) the substantia nigra, which is the middle part; and (3) the tegmenta, which are united by a median raphe

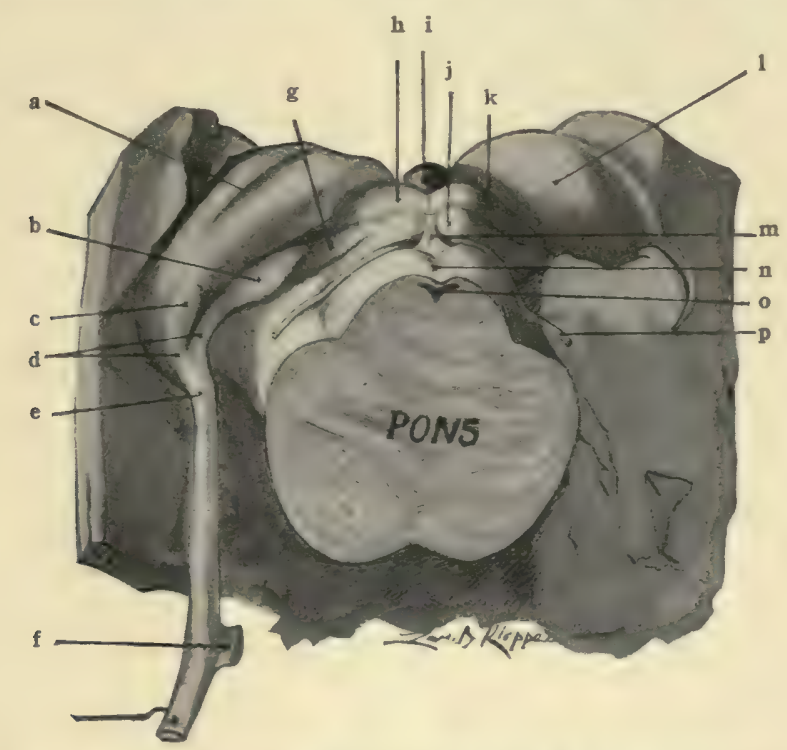

Fig. 43.-The region of the mid-brain showing pulvinar of the thalamus, the geniculate bodies, the corpora quadrigemina and brachia, the pineal body, the optic tract and the fourth nerve. (Original.)

a. Chorioid groove, b. Medial geniculate body, c. Lateral geniculate body. d. Medial and lateral roots of optic tract. e. Optic tract. f. Optic chiasma. g. Brachium inferius. h. Superior colliculus of corpora quad. i. Pineal body. j. Inferior colliculus of corpora quad. k. Brach. superius. 1. Thalamus. m. Frænulum veli. n. Superior medullary velum. o. Fourth ventricle. p. Trochlear nerve.

and lie in the posterior region next the quadrigeminal lamina. In the median plane between the quadrigeminal lamina and the tegmenta runs the cerebral aqueduct.

The bases pedunculi (Figs. 45, 46, 47 and 48) are two rounded bands of medullated fibers, limited by the interpeduncular fossa and lateral sulcus of the mid-brain. Each basis pedunculi is a half-inch broad and is distinctly striated longitudinally. It 
issues from the under surface of the fore-brain at the junction of the hemisphere with the thalamus and, trending toward the median line, descends to the pons. At its superior end it is continuous with the motor tracts of the internal capsule. Four motor tracts make up the basis pedunculi, viz., the intermediate, the temporo-pontal, the pyramidal, and the fronto-pontal.

The deep portion of each basis pedunculi (Fig. 47) is occupied by the intermediate bundle, whose fibers rise in the corpus striatum and terminate in the nucleus pontis (Flechsig). The superficial portion should be studied in three parts.

(I) The outer fifth of each basis contains the temporo-pontal tract (tractus cerebro-cortico-pontalis temporalis). It is composed of efferent fibers which rise in the temporal cortex, in the superior, middle and inferior gyri (Dejerine); and, perhaps, in portions of the occipital lobe (Zacher) and the parietal lobe (Sioli). Proceeding through the inferior lamina and the occipital part of the superior lamina of the internal capsule, and through the lateral part of the basis pedunculi, they terminate chiefly in the nucleus of the pons; a few end in the motor nuclei of cerebral nerves (Spitzka). The fibers are probably interrupted and relayed in the thalamus or lentiform nucleus. They form a segment of the indirect motor path. These fibers are medullated later than the pyramidal tract (Flechsig).

(2) The middle three-fifths of the basis pedunculi (Figs. 47 and 48) is occupied by the pyramidal tract (fasciculus longitudinalis pyramidalis). Its fibers rise in the anterior central gyrus of the cerebral cortex; they run through the genu and anterior two-thirds of the occipital part of the internal capsule, form the middle three-fifths of the basis, a part of the anterior longitudinal fibers of the pons, and the pyramid of the medulla. Below the medulla they are continued in the anterior and lateral pyramidal tracts of the spinal cord. Those fibers of the pyramidal tract which innervate the muscles of speech and of the face run through the genu of the internal capsule and constitute the medial portion of the tract in the mid-brain and the accessory lemniscus (of Bechterew). Immediately behind the face fibers, in the capsula interna, and external to them, in the basis pedunculi, are fibers 


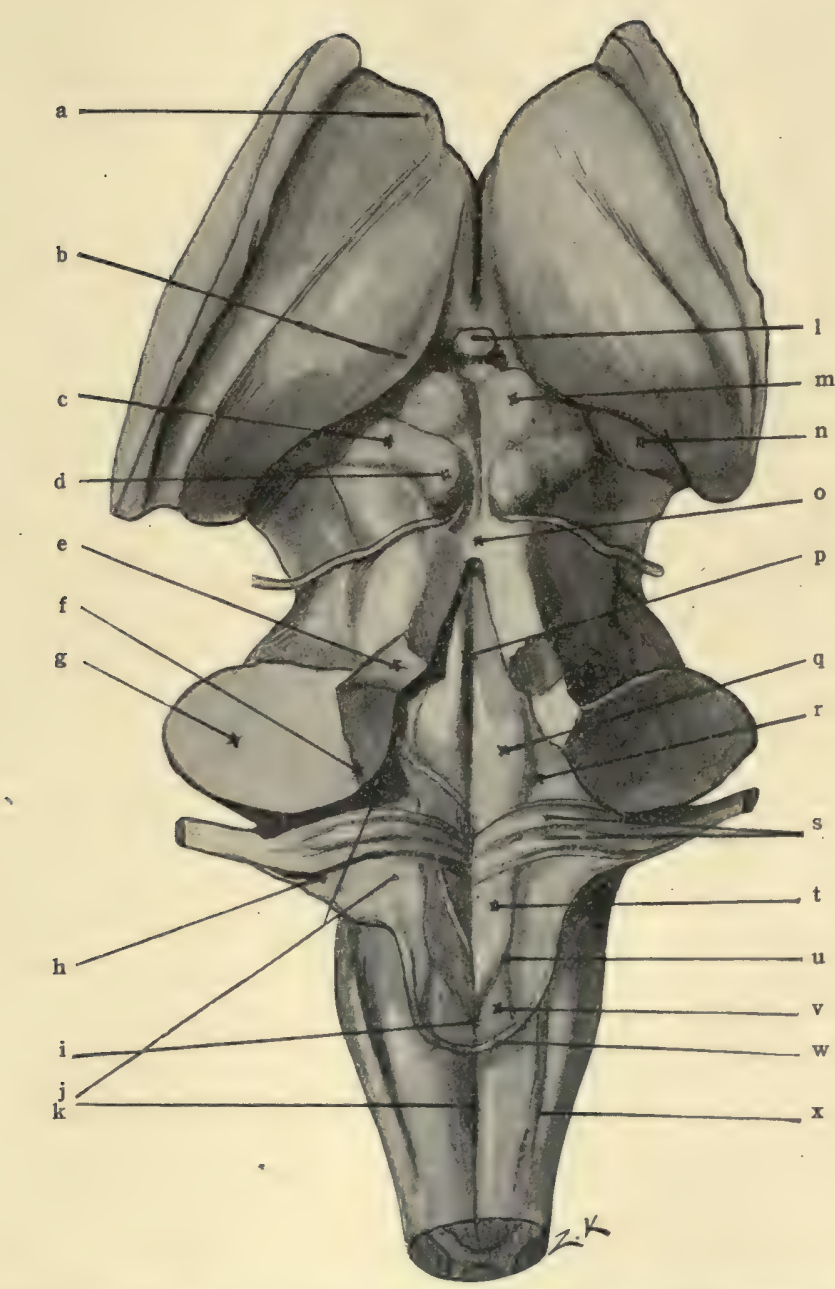

Fig. 44. - The dorsal or posterior aspect of the inter-brain, the mid-brain, the pons and the medulla. (Original.)

a. Anterior tubercle of thalamus. b. Pulvinar of thalamus, c. Brachium inferius. d. Inferior colliculus of corpora quad. e. Brachium conjunctivum. f. Corpus restiforme. g. Brachium pontis. h. Tuberculum acusticum. i. Calamus scriptorius. j. Area acustica. k. Posterior median fissure. 1. Stalk of pineal body. $\mathbf{m}$. Colliculus superior of corpora quad. n. Medial geniculate body. o. Superior medullary velum. p. Median groove. q. Colliculus facialis. $\boldsymbol{r}$. Fovea superior. s. Medullary striæ. t. Trigonum n. hypoglossi. u. Fovea inferior. v. Ala cinerea. w. Tænia ventriculi quarti. x. Posterior lateral sulcus. 

which innervate the muscles of the arm. Still behind these, in the internal capsule, and external to them, in the pyramidal tract of the basis pedunculi, are fibers for the innervation of the trunk and leg muscles.

(3) The inner fifth of the basis pedunculi is composed of the fronto-pontal tract (tractus cerebro-cortico-pontalis frontalis) (Figs. 47 and 48). The origin of the latter is probably in the prefrontal cortex. It is motor. This motor tract is contained in the frontal part of the upper lamina of the internal capsule. According to Hoche, it is interrupted and relayed either in the thalamus or striated body (Barker). Its termination is in the nucleus of the pons and in the motor nuclei of cerebral nerves (Flechsig). It constitutes a stage of an indirect motor path, like the fibers of the outer fifth of the basis pedunculi, and the indirect path is continued to the opposite half of the cerebellum by neurones whose cell-bodies are in the nucleus pontis (Flechsig).

The Substantia Nigra (Figs. 46, 47 and 48). - The central part of the crura cerebri is a sheet of pigmented gray matter. The substantia nigra is visible at the base of the brain between the bases pedunculi, where it is called the posterior perforated substance (substantia perforata posterior), and its margin comes to the surface in each lateral sulcus of the mid-brain. It extends from the pons upward to the corpora mammillaria and nucleus hypothalamicus (Luysi). Dorsal to it are the tegmenta. Transversely, the substantia nigra is convex forward, but it is slightly concave longitudinally. The third nerve pieces it and comes out through the oculomotor groove. It contains small pigmented multipolar cell-bodies, some of which constitute a relay for certain fibers of the medial fillet (Barker). There is a median aggregation of these cells located just in front of the pons, the interpeduncular ganglion (ganglion interpedunculare). According to Forel, this ganglion is connected by a bundle of fibers, the fasciculus retroflexus, with the nucleus habenulæ of the thalamus. The superior portion of the substantia nigra lies ventral to the mucleus hypothalamicus (Luysi) on either side. The nucleus hypothalamicus lies ventro-lateral to the red nucleus, and is separated from it by the zona incerta. 
The Tegmenta (Figs. 46, 47 and 48).-The posterior divisions of the pedunculi cerebri, which cover the other divisions, are in consequence called the tegmenta (tegmentum - a cover). They are united by a median raphe and fit ventrally into the concavity of the substantia nigra. They are bounded by the lateral sulcus of the mid-brain on the free side, where each tegmentum presents a triangle, bounded by the sulcus lateralis, in front; by the brachium inferius, above; and, inferiorly, by the lateral fillet. Dorsally, the tegmenta fuse with the quadrigeminal lamina except along the median line, where the cerebral aqueduct separates them. Each tegmentum at the superior end blends with the thalamus, and helps to form the tegmental hypothalamic structures. Imbedded in that superior portion is the red nucleus (n. ruber) of the tegmentum. (See tegmental hypothalamic region.) Inferiorly, the tegmenta are continued into the reticular formation of the pons.

The Cerebral Aqueduct (Aqueductus Cerebri, Sylvii, Figs. I7, 27 and 47).-The aqueduct is a very slender canal connecting the third and fourth ventricles. So it is the "iter a tertia ad quartum ventriculum." It is situated in the median line under the quadrigeminal lamina and produces a slight beveling of the dorsomedial borders of the tegmenta. It is a half-inch long. In shape it is v-like, above; elliptical, in the middle, with a vertical major axis; and $\mathrm{T}$-form, below, where it joins the fourth ventricle. Its height varies between a sixteenth and an eighth of an inch. Like other ventricles, it is lined with ependyma. A layer of gray matter, thickest on the sides and floor, surrounds the aqueduct of the cerebrum. This is the stratum griseum centrale, which gives rise to some of the fibers of the posterior commissure. The stratum griseum centrale is continuous with the gray matter of the fourth ventricle. In the ventral part of it are the nuclei of the oculomotor (third), the trochlear (fourth) and the trigeminal (fifth) cerebral nerves.

Nuclei of the Oculomotor and Trochlear Nerves (Figs. 47 and 48). - Both nuclei together extend the entire length of the aqueduct, and the oculomotor is prolonged into the wall of the third ventricle, where it receives a bundle of fibers from the opposite 


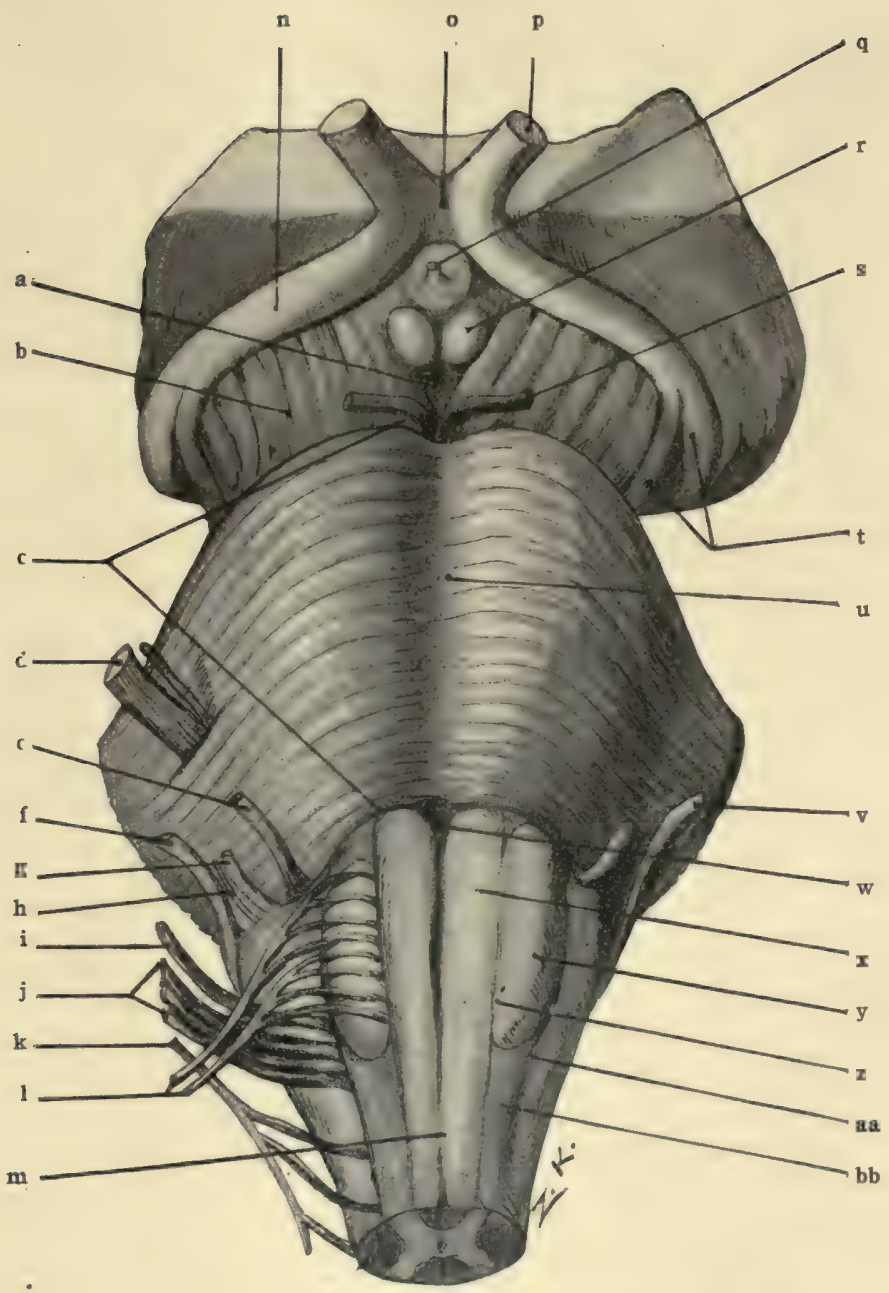

Fig. 45.-Anterior aspect of the mid-brain, pons and medulla. (Original).

a. Interpeduncular fossa. b. Basis pedunculi. c. Pons. d. Trigeminal nerve. e. Abducent nerve. f. Acustic nerve. g. Facial nerve. h. iIntermediate nerve. i. Glossopharyngeal nerve. j. Vagus nerve, k. Accessory nerve. 1. Hypoglossal nerve. m. Anterior median fissure. n. Optic tract. 0. Optic chiasma. p. Optic nerve. q. Stalk of infundibulum. r. Corpus mammillare. s. Oculomotor nerve. t. Medial and lateral roots of optic tract. w. Sulcus basilaris. v. Acustic nerve. w. Foramen cæcum (Vicq d'Azyri). x. Pyramid. y. Olive. z. Anterior lateral sulcus. aa. Posterior lateral sulcus. bb. Funiculus lateralis. 

optic radiation and optic tract. The nucleus of the third nerve (n. oculomotorius), according to Perlia, is composed of seven distinct cell-groups, scattered from the posterior part of the lateral wall of the third ventricle down to the level of the transverse groove between the superior and inferior quadrigeminal colliculi. From these seven cell-groups the axones proceed forward into the nerve of the same side; but there is, in addition, one median group of cell-bodies which sends axones into both nerves. The nuclei are also associated by decussating dendrites. The root fibers run forward through the red nucleus and substantia nigra and issue from the oculomotor groove. The nucleus of the fourth nerve ( $n$. trochlearis) is a single oval mass of cell-bodies situated ventral to the inferior colliculus of the corpora quadrigemina. The root fibers of the fourth nerve, trochlear, proceed dorsally and caudalward from the nucleus. They decussate with the fibers from the opposite nucleus in the superior medullary velum, from which they emerge on either side of the frenulum. They then continue in the opposite nerve around the side and over the anterior surface of the mid-brain. This is the only nerve that decussates en masse between the genetic nucleus and the apparent origin.

The nucleus of the mesencephalic root of the trigeminal nerve is composed of large cell-bodies scattered in the extreme ventrolateral part of the stratum griseum centrale, from the highest level of the mid-brain down to the pons. There is no break between this nucleus and the chief motor nucleus of the fifth nerve formed by the substantia ferruginea under the locus cæruleus. The axones of these large cell-bodies run downward through the nucleus, accumulating gradually until they form a distinct crescentic strand, which joins the chief motor root of the same side.

The opposite pyramidal tracts and, probably, the three homolateral cerebro-pontal tracts (fronto-pontal, temporo-pontal and intermediate tracts) bring these nuclei into relation with the cerebral cortex; and the anterior and the medial longitudinal bundles establish their reflex relation.

Formatio Reticularis (Fig. 47).--Through the greater por- 
tion of the tegmenta there are many oblique fibers interwoven with tracts of longitudinal fibers so as to produce a reticulum or net. Imbedded in the reticular formation ventral to each superior quadrigeminal colliculus is a mass of large cell-bodies constituting the nucleus lateralis superior. This is the first of the reticular nuclei and the only one in the mid-brain. Many of the oblique fibers of the formatio reticularis pass through the median raphe into the opposite tegmentum; they produce the tegmental decussations, which are situated at three successive levels, viz., the superior colliculus, the inferior colliculus and the isthmus rhombencephali.

The tegmental decussations at the level of the superior quadrigeminal colliculus (the fountain decussations) are: (I) The dorsal tegmental decussation (Meynerti) through which the anterior longitudinal bundle crosses. It is situated between the red nuclei but dorsal to them. (2) The middle - the decussation of the fasciculus pedunculo-mammillaris. (3) The ventral tegmental decussation (Foreli) in which the tract from the red nucleus, the rubro-spinal tract, crosses to the opposite side (Fig. 47).

At the level of the inferior colliculus (Fig. 48) is the decussation of the brachium conjunctivum (decussatio brachii conjunctivi). It crosses with its mate to reach the opposite red nucleus.

At the level of the isthmus is located the vestibular commissure, composed of fibers which connect the vestibular nuclei of the auditory nerve. Intermingled with the above bundles of crossing fibers there are many other fibers of the formatio reticularis.

Tracts of Fibers in the Tegmentum (Figs. 46, 47 and 48).In the reticulum of the tegmentum there are eight distinct bundles of longitudinal fibers, viz., the anterior and the medial longitudinal bundles, the fillet, the spino-thalamic tract, the brachium conjunctivum (superior cerebellar peduncle), the rubro-spinal tract, the olivary bundle, and the descending root of the trigeminal nerve.

The medial (or posterior) longitudinal bundle (fasciculus longitudinalis medialis, Fig. 47) is a compact strand of fibers 
running along the median raphe just ventral to the central gray substance. In Weigert-Pal sections it shows clearly as a dark triangle nearly one-sixteenth of an inch on a side. The tract will be found in the same relative position in the pons and medulla oblongata. In addition to several very small strands of fibers which will be explained later, the medial longitudinal bundle is

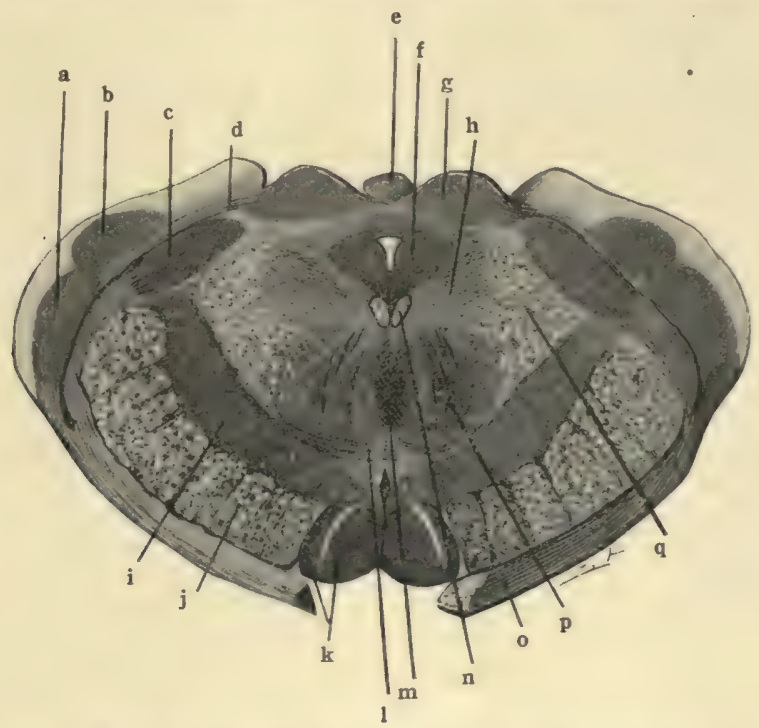

Fig. 46.- Transverse section through the corpora mammillaria and the superior colliculi of the corpora quadrigemina. (Original.)

a. Lateral geniculate body. b. Thalamus. c. Medial geniculate body. d. Brachium superius. e. Pineal body. f. Stratum griseum centrale. g. Superior colliculus of corpora quad. h. Formatio reticularis. i. Substantia nigra j. Basis pedunculi. k. Medial and lateral nuclei of corpus mammillare. 1. Ventral tegmental decussation (Foreli). m. Dorsal tegmental decussation (Meynerti). n. Medial longitudinal bundle. o. Optic tract. p. Red nucleus, q. Medial fillet.

functionally composed of two tracts: (I) An ascending bundle; and (2) a descending bundle.

(I) The ascending part of the medial longitudinal bundle is composed of fibers derived from the gray matter of the spinal cord and from the terminal nuclei of sensory cerebral nerves. It is the continuation of the long ascending fibers of the anterior fasciculus proprius. Possibly a small portion of the tract runs through the posterior commissure to the thalamus and is common sensory in function; but the major part of it decussates in several 
successive strands which end in the opposite motor nuclei of the cerebral nerves. The function of this latter part is reflex.

(2) The descending part of the medial longitudinal bundle is composed chiefly of uncrossed axones from the large cellbodies in the nuclei of the reticular formation. Beginning at the nucleus lateralis superior in the mid-brain, it receives fibers from each reticular nucleus down to the nucleus lateralis inferior of the medulla. It receives the largest accession of fibers in the pons, where the nucleus lateralis medius and the three nuclei centrales are located. On this account James S. Collier suggests that it be called the medial ponto-spinal tract. It has been traced through the anterior fasciculus proprius to the lower part of the spinal cord. Its size is gradually reduced by the ending of a few fibers in the gray substance corresponding to each segment of the cord.

In the mid-brain the medial longitudinal bundle also contains fibers derived-(I) from the oculomotor nucleus, which descend to the pons, and enter into facial nerve through which they supply the muscles of expression above the eye; and (2) from the nucleus of the abducent nerve. Running upward and decussating, the latter strand of fibers terminates in the opposite nucleus of the oculomotor nerve, and thus innervates the internal rectus of that eye. This strand accounts for the conjugate action of the two eyes in both health and disease.

The anterior longitudinal bundle forms a distinct strand situated in the formatio reticularis ventro-lateral to the medial longitudinal bundle (Figs. 47 and 48). It degenerates downward and is a descending tract. It takes its origin in the superior colliculus of the corpora quadrigemina, whence it decussates at once through the dorsal tegmental decussation (Meynerti) and descends through the reticular formation of the pons and medulla; and, then, through the fissural side of the anterior column of the spinal cord until it fades away in the lumbar region. Only by its degeneration has it been located. Its fibers end in the gray matter of the cord on both sides and in the genetic nuclei of cerebral nerves; but, chiefly, in the nuclei of the oculomotor, trochlear and abducent nerves and in the cilio-spinal center of the spinal 
cord. In function the anterior longitudinal bundle is reflex; it is connected with all ocular reflexes which are excited by impulses from the retinæ, such as, accommodation for distance, pupillary contraction and dilatation.

The Fillet or Lemniscus (Figs. 46,47 and 48 ).-Near the upper end of the pons, in the ventral part of the formatio reticularis, the fillet, or lemniscus, forms "a very broad band of fibers

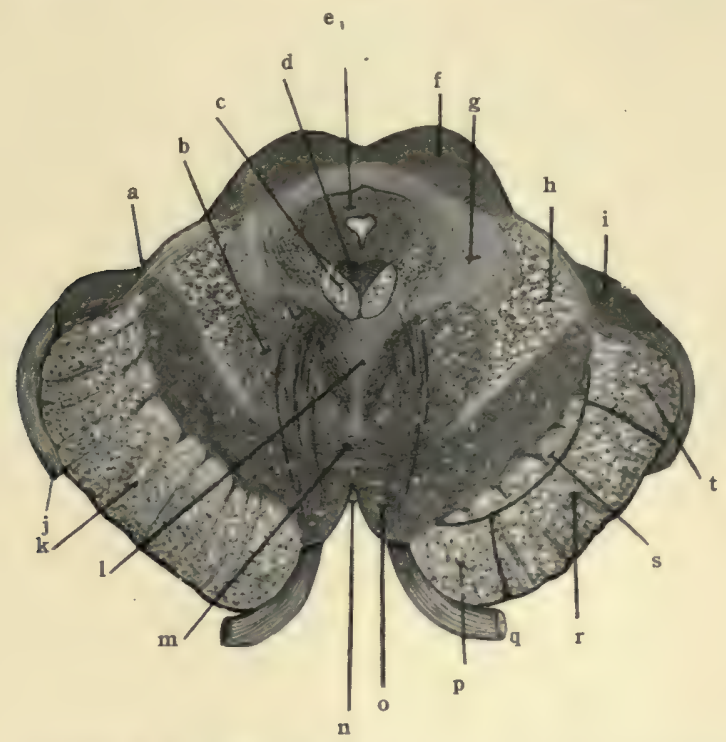

Fig. 47.- Section of the mid-brain through superior colliculi and the apparent origin of the oculomotor nerve. (Original.)

a. Sulcus laternlis of mid-brain. b. Red nucleus. c. Medial longitudinal bundle. d. Oculomotor nucleus. e. Stratum griseum centrale. f. Colliculus superior of corpora quadrigemina. g. Formatio reticularis. h. Medial fillet. i. Medial geniculate body. j. Optic tract. k. Basis pedunculi. I. Dorsal tegmental decussation (Meynerti). m. Ventral tegmental decussation (Foreli). n. Fossa interpeduncularis. o. Substantia nigra. p. Fronto-pontal tract. q. 3d. N. r. Pyramidal tract. 8. Intermediate tract. t. Temporo-pontal tract.

on either side of the median raphe. The fillet is equal in width to half the transverse diameter of the mid-brain. It continues into the ventral portion of the tegmentum, but immediately divides into two fasciculi, viz., the medial fillet, and the lateral fillet. Farther forward a small bundle leaves the lateral part of the medial fillet and runs up to the superior quadrigeminal colliculus. That bundle is called the superior fillet. 
Function.-The fillet forms a segment in the direct sensory tract. It carries spinal and cerebral impulses of the tactile and muscular senses to the corpora quadrigemina and thalamus, and auditory impulses to the inferior quadrigeminal colliculus.

$$
\text { Lemniscus }\left\{\begin{array}{l}
\text { L: Medialis }\{\text { L. Superior. } \\
\text { L. Lateralis }
\end{array}\right.
$$

Medial Fillet. (Lemniscus medialis, Figs. 47 and 48).--The fibers composing the medial fillet rise chiefly in the nucleus funiculi gracilis and nucleus funiculi cuneati of the opposite side of the medulla oblongata. They cross over in the fillet decussation of the medulla; and, excepting a small bundle, terminate in the lateral nucleus of the thalamus. Fibers are added from the terminal nuclei of sensory cerebral nerves which cross the median plane and enter the opposite fillet. Thus connected with all common sensory nerves, and with the vestibular nerve, it enters the mid-brain and divides into two parts. A small bundle of fibers separating from the lateral part and running to the superior quadrigeminal colliculus, forms the superior fillet. It associates ocular movements with sensations from cerebral and spinal nerves. The medial fillet continues to the lateral nucleus of the thalamus, bearing impressions of the tactile and the muscular sense. From the thalamus the impulses are carried by the cortical fillet to the somæsthetic area of the cortex.

The lateral fillet (lemniscus lateralis) forms an oblique ridge on the lateral border of the tegmentum (Fig. 44). It trends upward and inward over the brachium conjunctivum to the inferior quadrigeminal colliculus where some of its fibers terminate. A few fibers continue to the superior colliculus. Its function is auditory conduction. It rises chiefly from the ventral and lateral parts of the cochlear nucleus (principally the opposite one) and ends in the inferior quadrigeminal colliculus. The greater number of its fibers cross through the trapezoid body and medullary striæ, some are uncrossed. It undergoes partial relay in the nucleus of the superior olive and nucleus of the trapezoid body on both sides and the nucleus of the lateral fillet on the same side.

The lateral fillet is only partially relayed in the inferior col- 
liculus of the corpora quadrigemina, being continued directly into the brachium inferius. Auditory conduction therefore proceeds from the inferior colliculus through the brachium inferius to the medial geniculate body and then through the acustic radiation to the temporal cortex. Thus the lateral fillet forms the

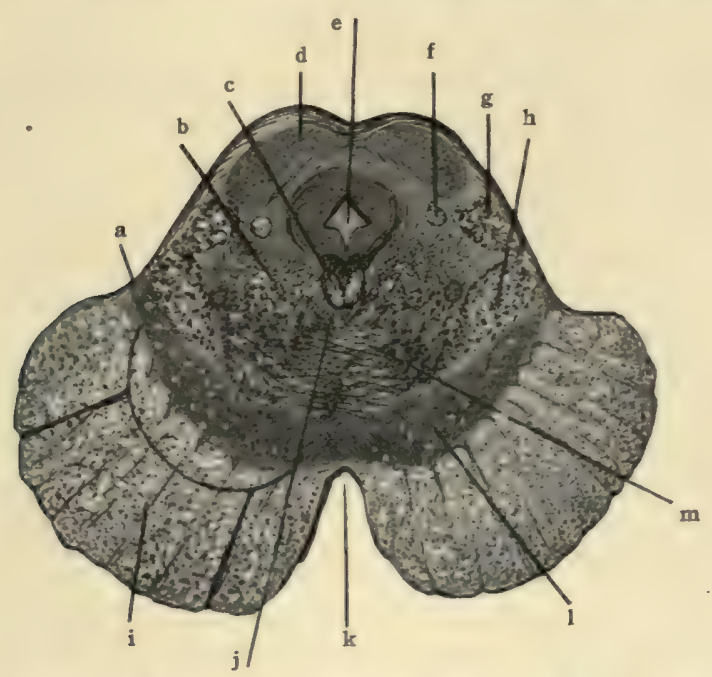

Fig. 48.- Section of the mid-brain cutting the inferior colliculi of the corpora quadrigemina. (Original.)

a. Sulcus lateralis. b. Formatio reticularis. c. Medial longitudinal bundle. d. Nucleus of colliculus inferior. e. Aqueductus cerebri. f. Rubro-spinal tract. g. Lateral fillet. h. Medial filliet. i. Basis pedunculi. j. Location of anterior longitudinal bundle. k. Interpeduncular fossa. 1. Substantia nigra. m. Decussation of brachia conjunctiva.

second stage in the auditory conduction path. The acustic nerve constitutes the first stage, the lateral fillet the second stage, the brachium inferius the third, and the acustic radiation the fourth stage. The last stage ends in the cortex of the superior and the transverse temporal gyri.

The spino-thalamic tract (Figs. 47 and 48 ) is located in the region of the nucleus lateralis superior. It is a loose strand of fibers not isolated from surrounding structures; and, probably, has many relays in its course. The spino-thalamic tract rises in the gray substance of the spinal cord and in the terminal nuclei of the common sensory cerebral nerves. Though it sends some 
fibers to the quadrigeminal bodies, the substantia nigra and the lentiform nucleus, its chief termination is in the lateral nucleus of the thalamus. It is sensory. It conducts tactile, pain and temperature impressions.

Brachium Conjunctivum. (Superior cerebellar peduncle).The brachium conjunctivum forms a ridge on the surface near the median line of the isthmus, which ends above at the inferior quadrigeminal colliculus (Fig. 44). The lateral fillet winds inward over its upper extremity. It is joined to its fellow by a sheet of white matter, the superior medullary velum. The fibers of the brachia conjunctiva bend ventrally beneath the inferior colliculus of the corpora quadrigemina and, for the most part, decussate anterior to the cerebral aqueduct, through the median raphe (Fig. 48). These crossed fibers with the few uncrossed run forward toward the inferior surface of the thalamus, where they inclose the red nucleus, and help to form the stratum dorsale of the hypothalamic region (Forel). Many of the fibers terminate in the red nucleus and from it others rise and proceed forward to the thalamus. Though most of the brachium conjunctivum rises in the cerebellum and forms a segment of an indirect sensory tract, it also contains efferent fibers which rise in the red nucleus.

Rubro-spinal tract (Crossed Descending Tract of the Red Nucleus).-Formed by axones of the red nucleus, it immediately crosses through the ventral tegmental decussation (foreli) and proceeds lateralward to the lateral fillet (Fig. 47). In the lower part of the mid-brain, it is imbedded in the medial part of that fillet. The rubro-spinal tract runs through the medulla and descends in the spinal cord to the lumbar region, where it ends in the center of the gray crescent.

Olivary Fasciculus. - The olivary bundle is a loose strand of fibers traversing the reticular formation lateral to the medial longitudinal bundle, in the upper part of the mid-brain; in the lower region of the mid-brain, it runs closer to the median line and is mingled with the fibers of the brachium conjunctivum as they are about to enter the decussation. The olivary bundle probably rises in the lentiform nucleus and ends in the olivary nucleus of the medulla. 
The descending root of the trigeminal nerve (Fig. 48) rises in the ventro-lateral part of the central gray matter in the mid-brain and is, therefore, called the mesencephalic root. It is a motor root. It occupies a thin crescentic area, just at the lateral border of the stratum griseum centrale, which thickens as it proceeds downward toward the pons. This root extends the entire length of the mid-brain; but it is made up of very few fibers in the upper part and only assumes a distinct shape and outline when the level of the inferior quadrigeminal colliculus is reached. It is continued to the middle of the pons in the same lateral relation to the gray substance; and medial to the brachium conjunctivum cerebelli it joins the main part of the motor root and bends forward toward the anterior surface.

The Quadrigeminal Lamina (Lamina quadrigemina).-The quadrigeminal lamina forms the fourth great division of the midbrain. It rests upon the dorsum of the tegmenta, entering into a large part of the posterior surface of the mesencephalon. A crucial groove shapes its surface into four eminences, called colliculi (colliculi superiores and inferiores) (Fig. 44).

The colliculus superior, of either side, is larger than the inferior colliculus and is circular in outline. It has resting upon its medial half the pineal body. It is joined to the lateral geniculate body by a band of fibers almost entirely concealed by the pulvinar of the thalamus. That band is the brachium superius. The superior colliculus is made up of gray substance for the most part (Figs. 46 and 47). It is composed of a superficial white layer, the stratum zonale, and a thick laminated gray layer, the stratum griseum. Within the stratum griseum many fibers end; a few from the lateral fillet, all of the superior fillet, and nearly all of the brachium superius. The stratum griseum gives origin to the anterior longitudinal bundle and, probably, to a few fibers that run through the brachium superius into the optic nerve. It constitutes an optic-reflex center.

The colliculus inferior of the corpora quadrigemina is elongated transversely (Fig. 44). It is joined to the medial geniculate body by an oblique ridge, called the brachium inferius, and it forms the termination of two ridges that approach it from below, 
due to the lateral fillet, and the brachium conjunctivum of the cerebellum. Its surface is made up of medullated fibers continuous with the lateral fillet and brachium inferius, which forms the stratum zonale; gray substance, called the nucleus of the inferior colliculus, constitutes its deep portion. This nucleus receives a part of the lateral fillet and gives rise to a portion of the brachium inferius; it forms a partial relay in the acustic path.

Brachium Superius (Figs. 43 and 44).-It connects the lateral geniculate body with the superior quadrigeminal colliculus; but, excepting its extreme posterior end, it is buried in the substance of the thalamus. Its course is anterior and internal to the medial geniculate body. The brachium superius is composed chiefly of efferent fibers from the optic radiation, a tract partially relayed in the lateral geniculate body; it probably contains, also, a few optic fibers from the lateral root of the optic tract and a few from the stratum griseum of the superior quadrigeminal colliculus. Optic reflex impulses are conducted by the brachium superius.

Brachium Inferius (Figs. 43 and 44).-The inferior arm, connecting the inferior quadrigeminal colliculus and the medial geniculate body, is visible through its whole length. It forms the superior boundary of the triangle of the lateral fillet; the lateral fillet and the sulcus lateralis of the mid-brain form the other two sides. Two sets of fibers make up the brachium inferius, viz., the lateral fillet fibers and the axones from the nucleus colliculi inferioris. They all terminate in the medial geniculate body. The brachium inferius forms the third segment of the acustic path.

\section{CEREBRUM.}

\section{SECTION III. THE STRUCTURE OF THE CEREBRUM. ITS GRAY AND WHITE MATTER.}

We have noticed in the mid-brain that the basis pedunculi and much of the tegmentum are white matter, while the substantia nigra and quadrigeminal colliculi are composed chiefly of gray substance. Gray matter forms nearly all of the inter-brain. The deep part of the cerebral hemisphere is white substance, imbedded 


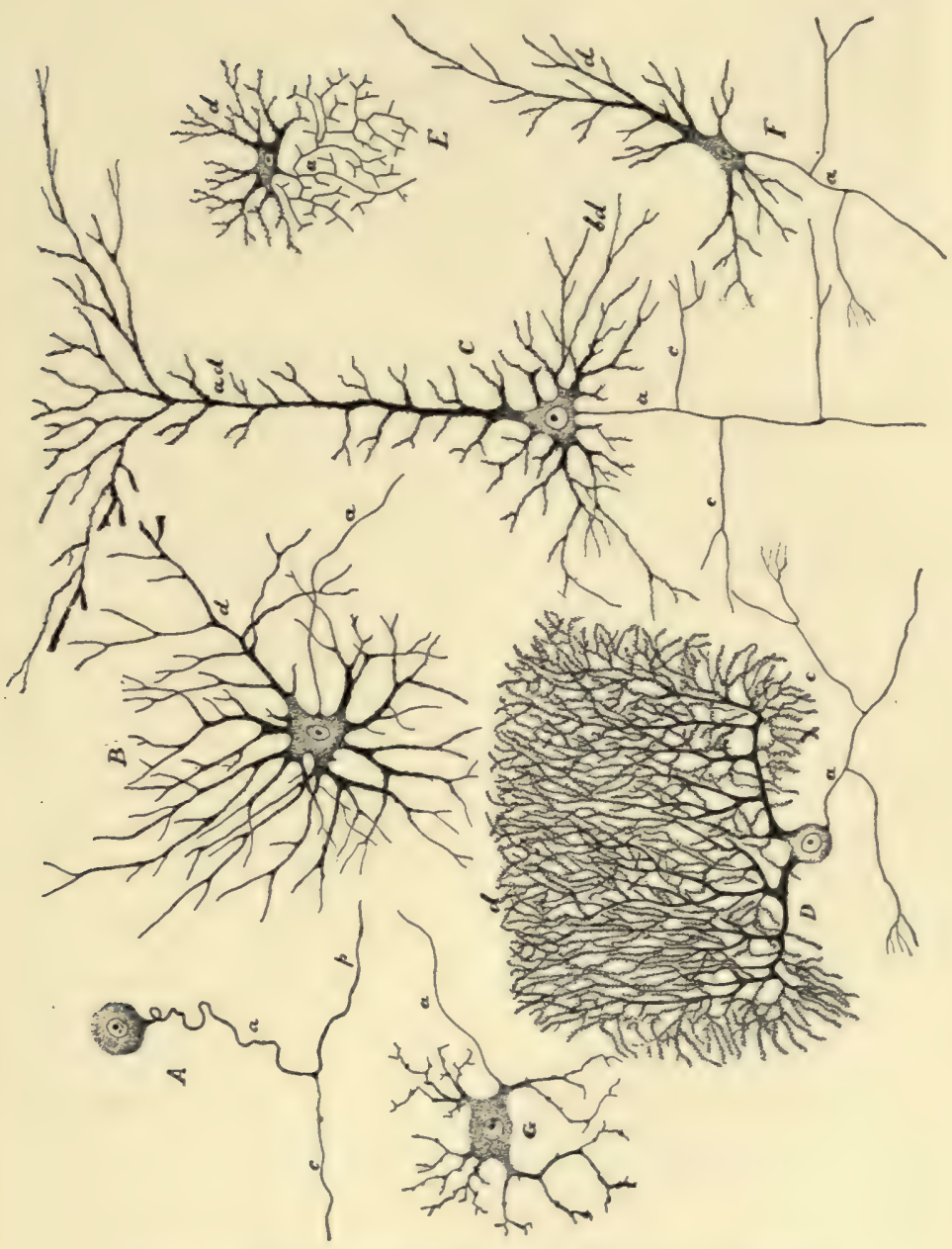

Fig. 49.-Varieties of neurones in the human nervous system.

(After Morris's Anatomy.)

A. From spinal ganglion. B. From ventral horn of spinal cord. C. Pyramidal cell from cerebral cortex. $D$. Purkinje cell from cerebellar cortex. $E$. Golgi cell of type II from spinal cord. $F$. Fusiform cell from cerebral cortex. $G$. Sympathetic, $a$, axone; $d$, dendrites; $c$. collateral branches; ad, apical dendrites; $b d$, basal dendrites; $c c$, central process; $p$, perpheral process, the dendrite. 

in which is the gray corpus striatum. A thin envelope of gray substance, called the cortex, forms the surface of the hemisphere. The entire cerebral substance, both the white and the gray, is composed of neurones and sustentacular tissue, the latter being made up of neuroglia, ependyma and mesoblastic connective tissue. Of necessity the cerebrum possesses a rich blood supply.

The Neurone (Fig. 49).- The essential element in the nervous system is the neurone. The neurone comprises the cell-body, its processes and end-organs. The cell-body, perikaryon, or neurone center, is a granular reticulated mass of protoplasm of variable form. It ranges in man between four microns and one hundred and thirty-five microns in diameter. The size of the cell-body is usually proportionate to the length of the processes. Its shape, which is spherical, fusiform, pyramidal, stellate or polygonal, is dependent upon the number and mode of origin of its processes. The cell-body has a large vesicular nucleus in which there are one or more nucleoli. It also possesses a centrosome in spinal and sympathetic ganglia. Its reticulated appearance is due to a net-work of fine fibrille which form a close net about the nucleus. These fibrillæ may be seen in all parts of the cell-body and they are continued into the processes given off from it. Masses of deeply staining material, called tigroid bodies or Nissl bodies, produce the granulated aspect of the perikaryon. The tigroid bodies are found in the receptive side of the neurone, viz., in the dendrites and throughout the cell-body, except in the axonehillock (Fig. 50).

The processes of the neurone are from one to eight or a doezn in number and are of two kinds, namely, the dendrites and the axones. Dendrites, like the cell-body, are protoplasmic in composition and of irregular contour, except in sensory nerves, where they are smooth, like axones. In all cases they contain fibrillæ prolonged from the cell-body. They branch richly and end in beaded points. The terminal branches are called telodendria. Dendrites may form synapses with axones of other neurones, may terminate in special end-organs, or end free among the cells of any tissue. They are developed later than the axones and are not always present. An active cell usually has several den- 
drites. If long they become medullated. Their conduction is toward the cell-body, cellulipetal, or afferent. Axones, neuraxones or axis-cylinders (Fig. 50) are smooth and fibrillar in character. One (occasionally two or more) is given off from the axonehillock of the cell-body. It may break up into branches at once, constituting a dendraxone; but usually it runs a considerable distance and sends out side branches, called collaterals. When long, as a rule, it becomes medullated as its function develops, and the medullary sheath presents segmental divisions separated by constrictions, called the nodes of Ranvier, at which points the collaterals are given off. Both the parent axone and all its collaterals end by multiple division in the form of an end-brush, or tassel. This tassel lies in contact with the dendrites or body of another neurone, with a secreting cell, or with a muscle fiber. In the last instance the fibers of the tassel spread out into disc-like platelets, called motorial end-plates (Fig. 50). Axone conduction is from the cell-body, cellulifugal, or efferent.

The myelin sheath of axones and dendrites, which is developed as the neurone begins to functionate, is imbedded in neuroglia within the optic nerve and tracts, and in the brain and spinal cord; but, elsewhere, is surrounded by the neurolemma (Schwann) and the fibrous sheath of Henle. The fibers of the olfactory nerve and most sympathetic fibers are not medullated, but they possess the fibrous sheath. Near the cell-body and near the end-tuft the processes are naked, having neither the fibrous nor the medullary sheath.

Types of Neurones.- - . The first type has a long axone, which preserves its identity, though it may give off many collaterals. Found in brain and spinal cord (Deiters).

2. The second type has a short axone, breaking at once into branches of apparently equal importance, the dendraxone. Found in cerebrum and cerebellum (Grolgi).

3. The third type has two or more axones-diaxone, triaxone, polyaxone - as in the first layer of the cerebral cortex (Cajal).

Orders of Neurones.-I. The first order has distal process in relation with the periphery, as spinal-ganglion and anterior columna neurones, and conducts from the periphery or to it. 


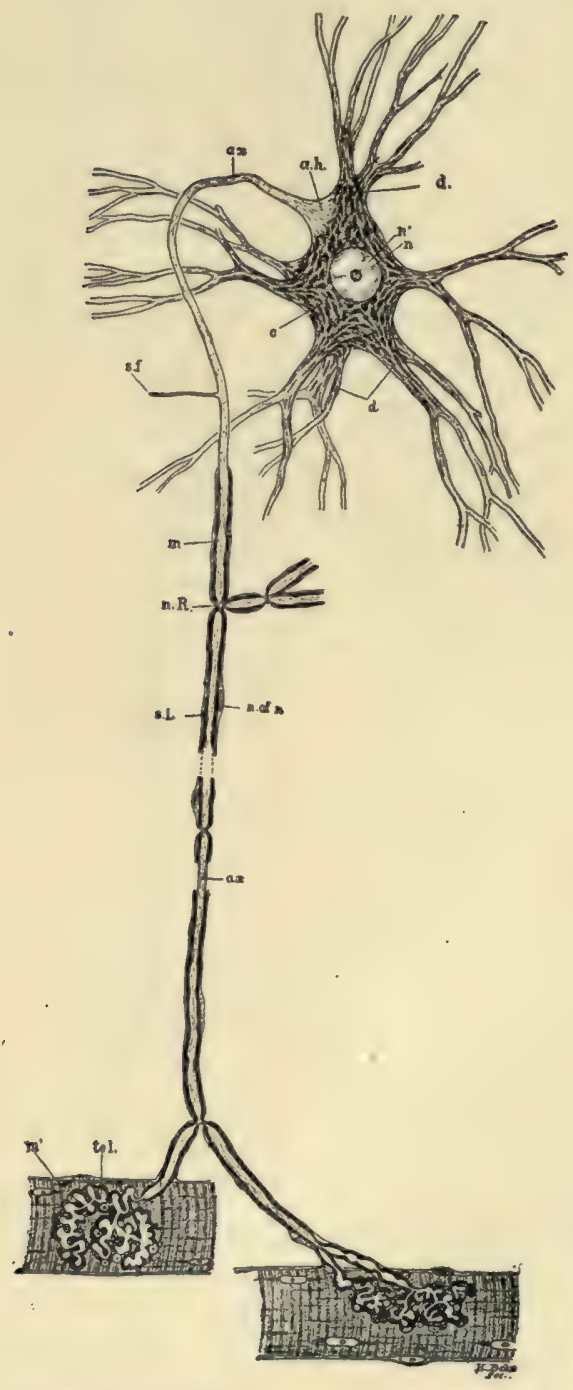

Fig. 50.-Motor neurone. (After Barker.)

a.h. Axone-hillock devoid of Nissl bodies, and showing fibrillation, ax. Axis cylinder or axone; this process near the cell-body, becomes surrounded by myelin. m., and a cellular sheath, the neurilemma, the latter not being an integral part of the neurone. c. Cytoplasm showing Nissl bodies and lighter ground substance. d. Protoplasmic processes (dendrites) containing Nissl bodies. n. Nucleus. n'. Nucleolus. n.R. Node of Ranvier. s.f. Side fibrils, collaterals. n. of $\mathbf{n}$. Nucleus of neurilemma sheath. tel. Motor end plate or telodendrion. m. Striped muscle fiber, s.L. Segmentation of Lantermann. 

2. The second order has cell-body or distal process in relation with neurone of first order. It conducts to a neurone of the first order or conducts centrally from it. In like manner there are neurones of the third, fourth, fifth order, etc.

Functions of Neurones.-I. Afferent. 2. Associative. 3. Efferent.

A neurone is afferent in function when its dendrites are directed toward the periphery; if its axone is peripherally directed, its

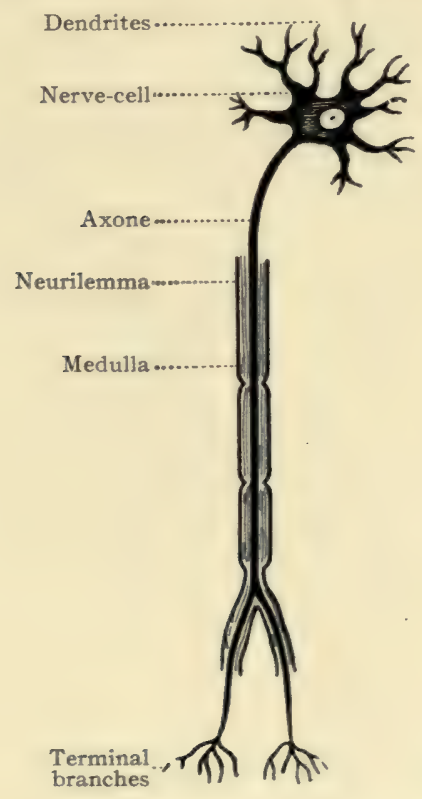

A

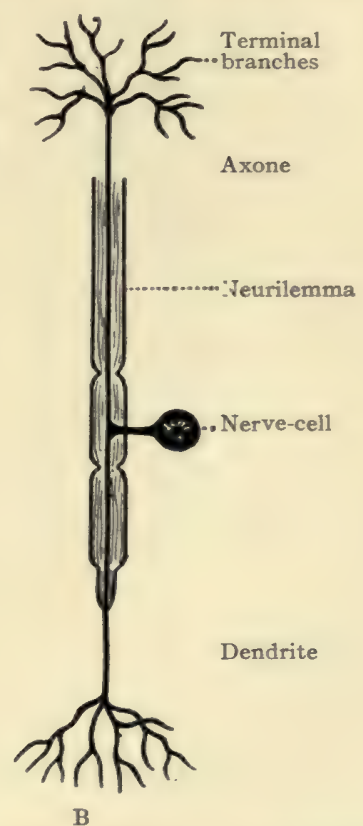

B

Fig. 51.-An efferent neurone and an afferent neurone. (After Brubaker.)
A. Efferent neurone.
B. Afferent neurone.

function is efferent (Fig. 5I). An association neurone connects other neurones together.

Degeneration.--If a neurone is prevented from functionating, it undergoes a slow atrophic change, called degeneration of Nissl. Rapid Wallerian degeneration occurs in an axone or dendrite which is completely cut off from all other parts of the neurone. In all probability neurones differ in chemical constitution and in electric 
status, hence chemicals, electricity and diseases appear to exercise a selective power and affect certain neurones without influencing others.

Development (Figs. 52 and I28).--Every neurone is the derivative of an epiblastic cell, called the germinal cell of His, which is located in the neural tube or crest. This germinal cell, in the neural tube, sends out a process from what was originally its deep end, and becomes a neuroblast. This process forms the axone and, later, dendrites are produced. During development
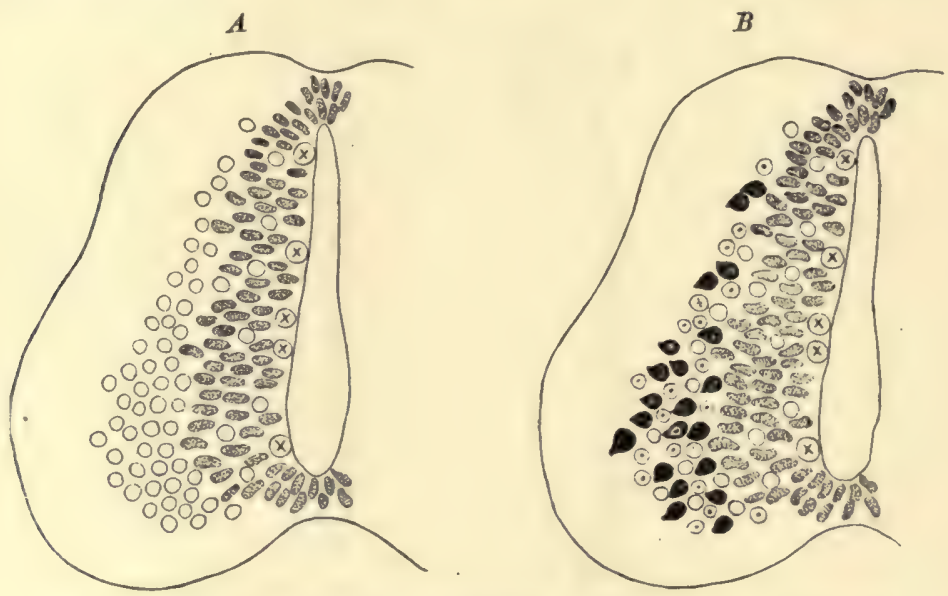

Fig. 52.-Diagram showing development of neurones in the spinal cord. (McMurrich after Schäfer.)

The circles, indifferent cells; circles with dots, neuroglia cells; shaded cells, germinal cells; circles with cross, germinal cells in mitosis; black cells, nerve-cells.

the cell-body wanders more or less from its original position, and thus reaches its adult location. The neurones are in this manner aggregated into nuclei and cortex. The germinal cells of the neural crest form bipolar neuroblasts, which send out a process from each extremity. The axone appears first, the dendrite later, as in the neural tube. The resulting neurones make up the cerebral, spinal and sympathetic ganglia. In the spinal ganglia and in the common sensory cerebral ganglia, the neurones become converted into the unipolar form by the lateral growth of the cellbody and the fusion, for a short distance, of the two processes 
into one common stem (Figs. II 5 and I28). They remain bipolar in the olfactory and acustic ganglia, but form multipolar cells in the sympathetic ganglia.

Sustentacular Tissue (Fig. 53). - In the brain and spinal cord and in the optic nerves two forms of sustentacular tissue are

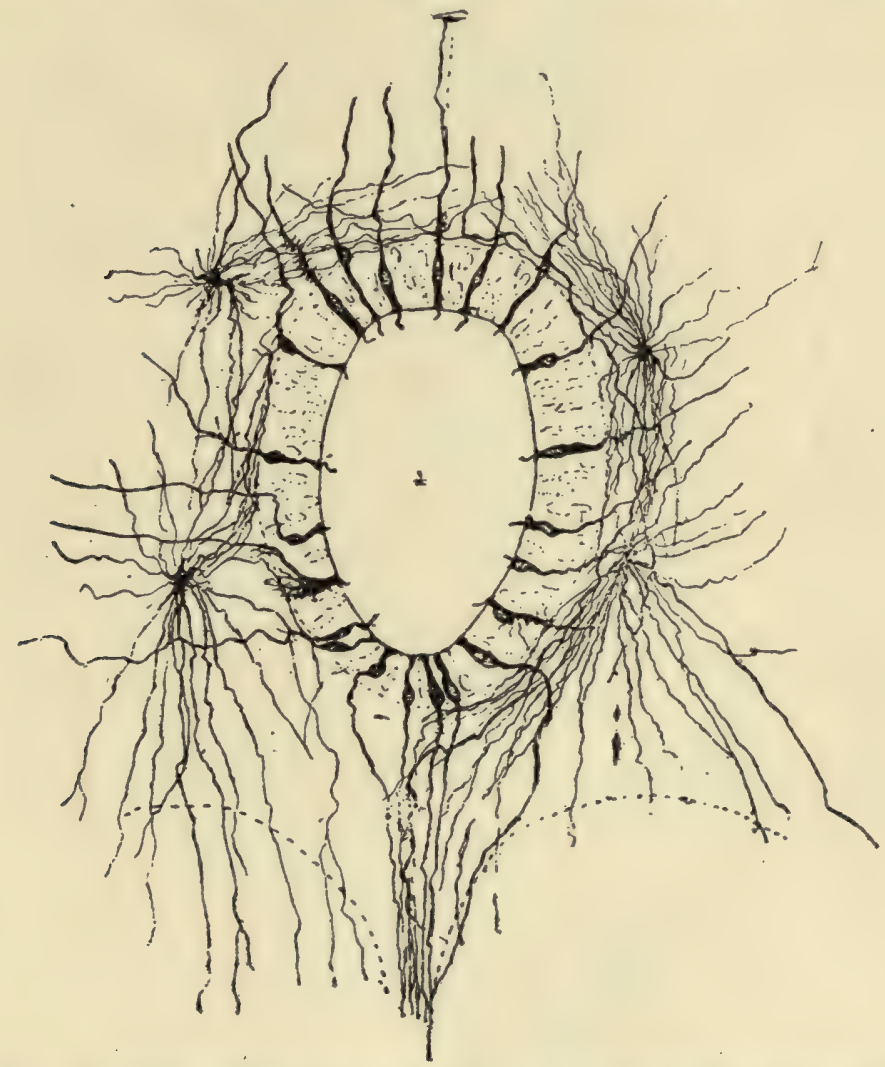

Fig. 53.-A section through the spinal cord of a human fetus, $23 \mathrm{~cm}$. in length. Showing the central canal with its substantia gelatinosa centralis, neuroglia cells and ependyma cells. (After Lenhossek. Gordinier's Nervous System.)

found supporting the neurones, viz.: (I) The epiblastic tissue, comprising the neuroglia and the ependyma: (A) Neuroglia is most abundant in gray matter. It is made up of richly branched nucleated cells whose processes form a fine reticulation in the 
larger meshes of the connective tissue network. There are three varieties of neuroglia cells, viz., (a) the short rayed cells, found in the cortex of cerebellum and cerebrum, the processes of which are strong and richly branched; (b) the long rayed cells, located chiefly in the white substance, whose processes are long and fine and but little branched; and (c) the arborescent cells, which lie near the surface in the cortex. Upon reaching the surface the tree-like branches form a limiting membrane of neuroglia.

(B) The columnar ciliated ependymal cells which line the ventricles are also epiblastic in origin and should be classed with the neuroglia cells. They form the only sustentacular tissue present in the neural tube when it is first formed, at which time they extend from the ventricular to the exterior surface of the tube and, in the peripheral zone, form a rich supporting network. They appear to be of little importance in the adult condition.

(2) Connective Tissue Network.-That is of mesoblastic origin and is formed by branching processes from the inner surface of the pia mater. It transmits the blood-vessels into the nervous substance.

The neurones constitute 53 per cent. of the brain and cord (cell-bodies, 6-per cent.) and the sustentacular tissue 47 per cent. (Donaldson).

The white matter of the cerebro-spinal axis is made up chiefly of bundles of medullated axones imbedded in neuroglia and supported by connective tissue. The fibers possess no neurilemma.

The gray matter of the central nervous system is composed of cell-bodies and dendrites, chiefly, but also contains axones. These nerve elements are supported by connective tissue and blood-vessels and are imbedded in a great abundance of neuroglia. The nerve fibers in the gray matter are to a large extent non-medullated and naked.

The cerebral gray substance (substantia grisea cerebri) is conveniently divided into three groups or classes:

I. Cortical.

II. Ganglionar.

III. Central, or ventricular. 


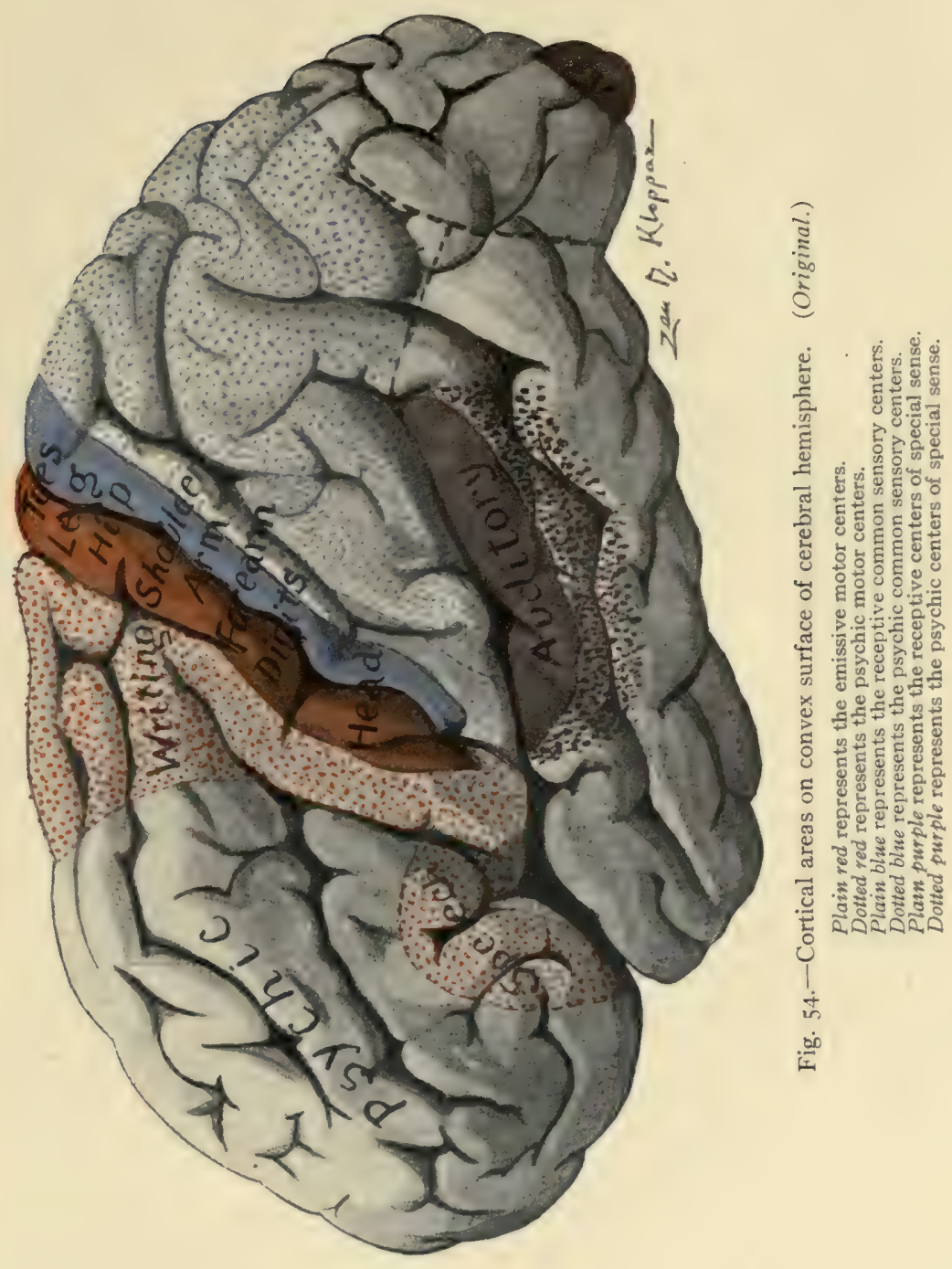





\section{THE CORTICAL GRAY MATTER.}

The substantia corticalis consists of a thin envelope, the cortex (or bark), which forms the surface of the hemispheres and incloses the white medulla, the centrum semiovale. The cortex varies in thickness from a line to a quarter of an inch. Thickest on the surface of the gyrus, it grows thinner to the bottom of the sulci. It is of a reddish, or yellowish-gray color depending on the richness of the blood supply. By repeated observation of the symptoms produced by definite brain lesions and by the anatomical and physiological study of human and lower animal brains, both in the embryonic and adult condition, the cortex has been mapped out into certain definite functional areas (Figs. 54 and 55). Psychic function undoubtedly is dependent upon the associated activity of a number of cortical areas; but motor, common sensory and special sensory regions have been outlined with considerable exactness.

Cortical or Cerebral Localization (Figs. 54 and 55).-In the following study of the cerebral cortex I shall use very extensively the recent work of Dr. Alfred W. Campbell, entitled, "Histological Studies on the Localization of Cerebral Function," Cambridge, England. In this epoch-making work we are shown that certain cortical areas have a characteristic histological structure that distinguishes them from all other areas. This will be referred to later under "cell and fiber lamination of the cortex," but to appreciate this histological evidence of localization one should thoroughly study the above work.

Motor Area (Figs. 54, 55, 56 and 57).- The emissive motor area is situated in the anterior wall of the central sulcus, in the posterior one-half of the gyrus centralis anterior and in that part of the paracentral lobule immediately continuous with it. This is the center for ordinary voluntary motion on the opposite side of the body. Axones from this area descend to the nuclei of all motor nerves. In lateral sclerosis there is degeneration and disappearance of the giant pyramidal cells limited to this motor area (Campbell). It is divided into four segments: the head and neck, the arm, the trunk, and the leg, named from below upward. The first extends to the inferior knee of the sulcus centralis 
(Rolandi), though eye movements appear to be represented in the posterior end of the middle frontal gyrus; the arm area comprises the region between the genu inferius and the genu superius, the thumb, fingers, wrist, fore-arm, arm and shoulder movements being represented in this ascending order; just above the shoulder area, at the genu superius, is the trunk area; and above that, in the anterior central gyrus and in the paracentral lobule, in front of the sulcus centralis, is the center for leg movements, The representation in the leg center is inverted, the ascending order being hip, thigh, leg, ankle, toes and great toe.

The psychic molor areas, or areas for educated movements are located just anterior to the above motor areas, in the anterior central gyrus and in the contiguous ends of the superior, middle and inferior frontal gyri (Figs. 54 and 55). These areas are believed to send their axones to the emissive motor centers in the cortex. The psychic motor center for the lower extremity is probably located just in front of its emissive motor center in the anterior central and superior frontal gyri. In the posterior end of the middle frontal gyrus is the psychic motor center for the arm, the writing center of Gordinier; and in the inferior frontal gyrus the center for the organs of voice and speech, hence the motor speech center. In right handed people these centers are developed only in the left cerebral hemisphere.

Common Sensory Area (Figs. 54 and 55).-According to Dr. Alfred W. Campbell the receptive area of common sensation is limited to the posterior wall of the sulcus centralis, including the anterior one-half of the posterior central gyrus and that part of the paracentral lobule which is continuous with it. This area undergoes exclusive Nissl degeneration in locomotor ataxia (Campbell). It is probably divided into segments similar to those of the motor area (Spiller).

Psychic Sensory Area (Figs. 54, 55, 56 and 57).-A large portion of the remainder of the parietal cortex probably constitutes a number of centers for the interpretation of common sensory impulses, hence the term, psychic sensory area. Impressions of the muscular sense are believed to be interpreted in the supramarginal gyrus and the center of stereognosis is said to be located 


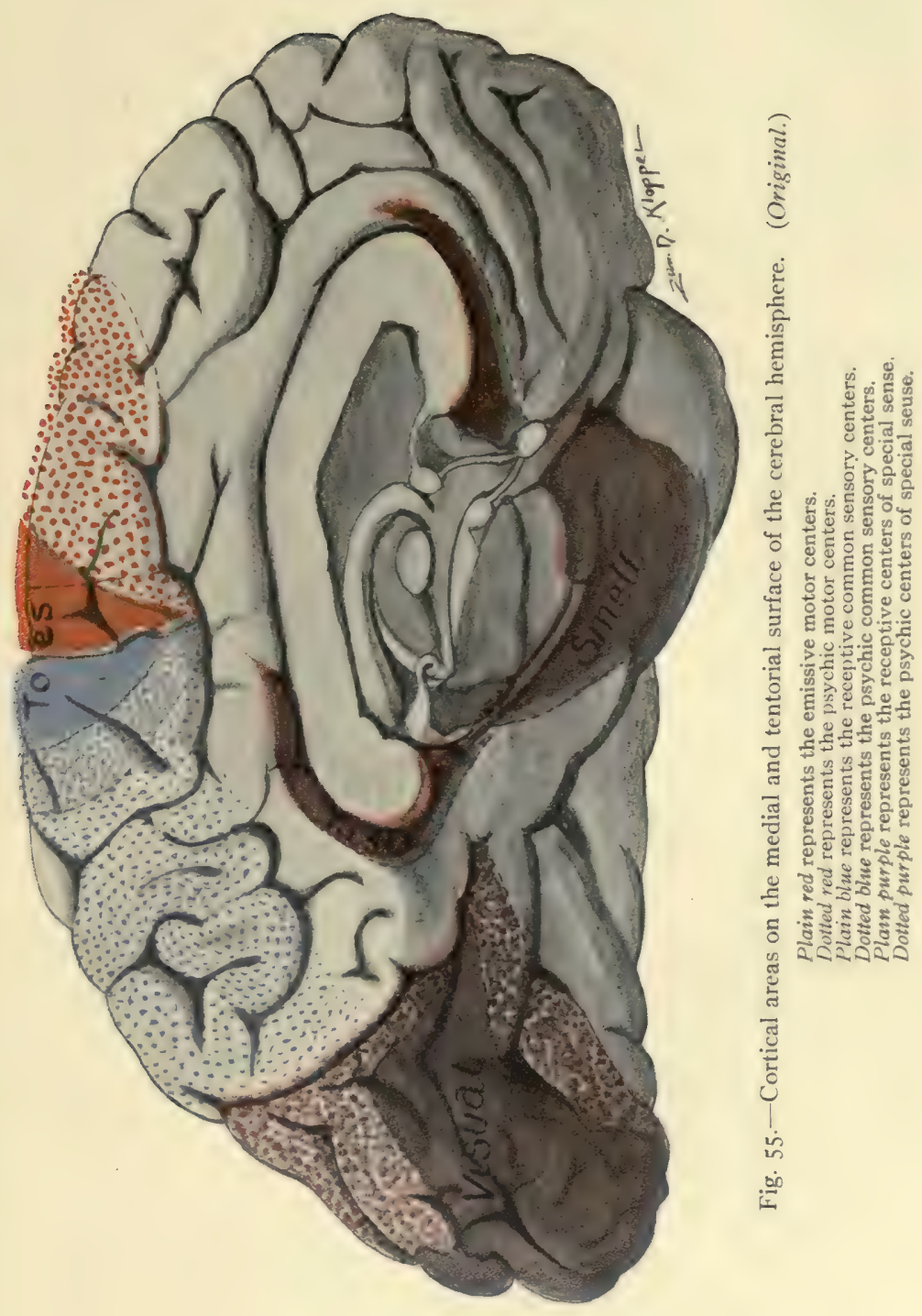



in the superior parietal lobule and præcuneus. Perhaps other parts interpret tactile, pain and temperature impulses. The whole receptive and psychic area of common sensation has been called the somasthetic area (Barker), though the application of this term might better be limited to the receptive area.

Acustic Center (Figs. 54 and 56).-The receptive acusticcenter is located in the transverse temporal gyri and in that part of the superior temporal gyrus which is continuous with them. In the adjacent part of the superior and middle temporal gyri, in the left hemisphere, is the psychic acustic center.

Optic Center (Figs. 54 and 55).--In the cuneus and lingual gyrus is located the receptive optic center for the temporal half of the same retina and the nasal half of the opposite one; perhaps, also, for the macula lutea of both sides. The remainder of the occipital lobe and, according to Mills and others, the angular gyrus, also, form the psychic optic center. This latter center is probably unilateral and developed only in the left hemisphere of right handed people.

Olfactory and Gustatory Centers (Figs. 55 and 57).-The uncus hippocampi forms the chief cortical center of smell, close to which in the fusiform gyrus is probably the gustatory center (Mills). Paul Flechsig in his recent studies of the human brain locates taste in the gyrus cinguli contiguous to the splenium of the corpus callosum (Fig. 55). The olfactory, auditory, visual, common sensory and motor areas are all distinguished by a definite characteristic histological structure. peculiar to each region (Campbell). Medullation of the fibers in these cortical areas occurs at different times; and, according to Flechsig, in the following order: olfactory, tactile and muscular sense, visual, auditory, and gustatory.

In the temporal lobe Mills locates four other centers which include the pole, the inferior temporal gyrus and a part of the middle temporal gyrus (Fig. 56). These are from before backward: the center of intonation at the pole, the naming center, the center of equilibration, and the center of orientation.

All the above motor, somæsthetic and special sense areas are provided with projection fibers which connect them with definite muscle groups and surface regions and with the organs of 
special sense. Large parts of the cerebral cortex possess no projection fibers; they are believed to be associative in function.

Association Centers of Flechsig.-Flechsig describes three association centers, the anterior, middle, and posterior. Anterior Association Center (Fig. 54).-According to Flechsig, that part of the frontal cortex which is anterior to the motor region determines the temperament and individuality of the person; and as Mills declares, is the center of inhibition, self control, attention, concentration, volition. It is the center of "the abstract concept"

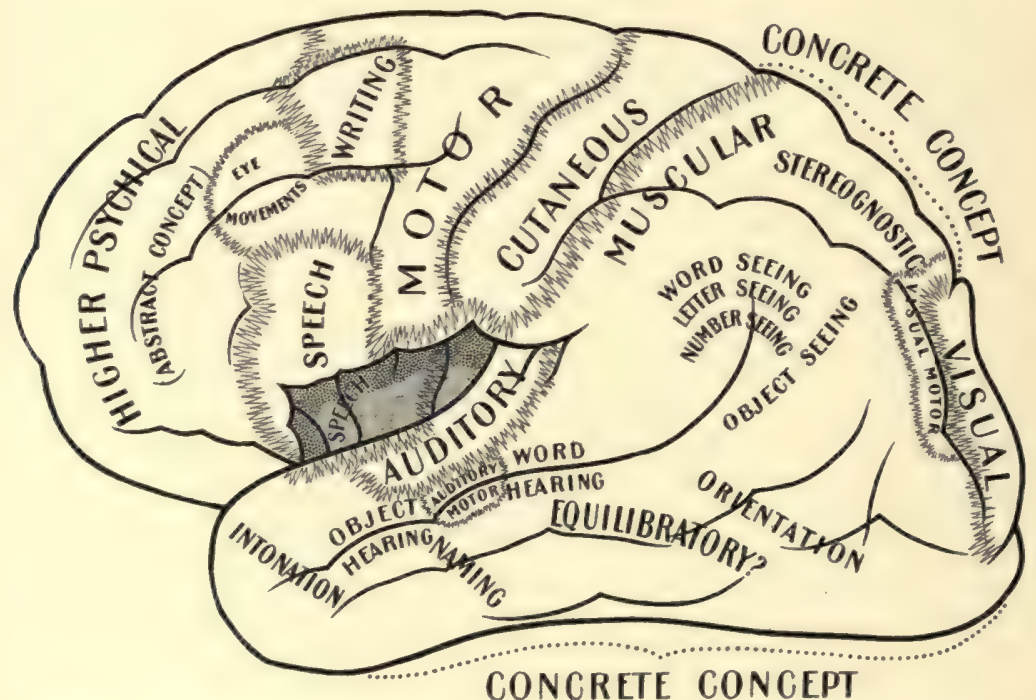

Fig. 56.-Cortical areas after C. K. Mills. Convex surface of cerebral hemisphere. (Brubaker's Physiology.)

(Fig. 56). J. S. Bolton says of this association center that "it is the last part of the cerebrum to be developed, and is the first to undergo dissolution; it is under-developed in amentia of all grades and atrophicd in dementia, according to its degree." "It possesses the highest (mental) function" (Brain, Vol. 29). The posterior association center, composed of those portions of cortex situated between the sensory region of the equatorial zone, in front, and the visual cortex of the occipital lobe, behind, deter- 
mines the intellectuality of the individual (Fig. 54). To acquire knowledge of the external world is thus the function of the posterior association center. Mills calls it the center of "the concrete concept" (Fig. 56). It includes three psychic areas, the common sensory, auditory and visual. Flechsig regards the island (of Reil) as the middle association center (Figs. 25 and 56). Lesions in it are associated with paraphasia.

Destructive lesions of parts of the motor or sensory cortex causè merely loss of certain motions and sensations represented

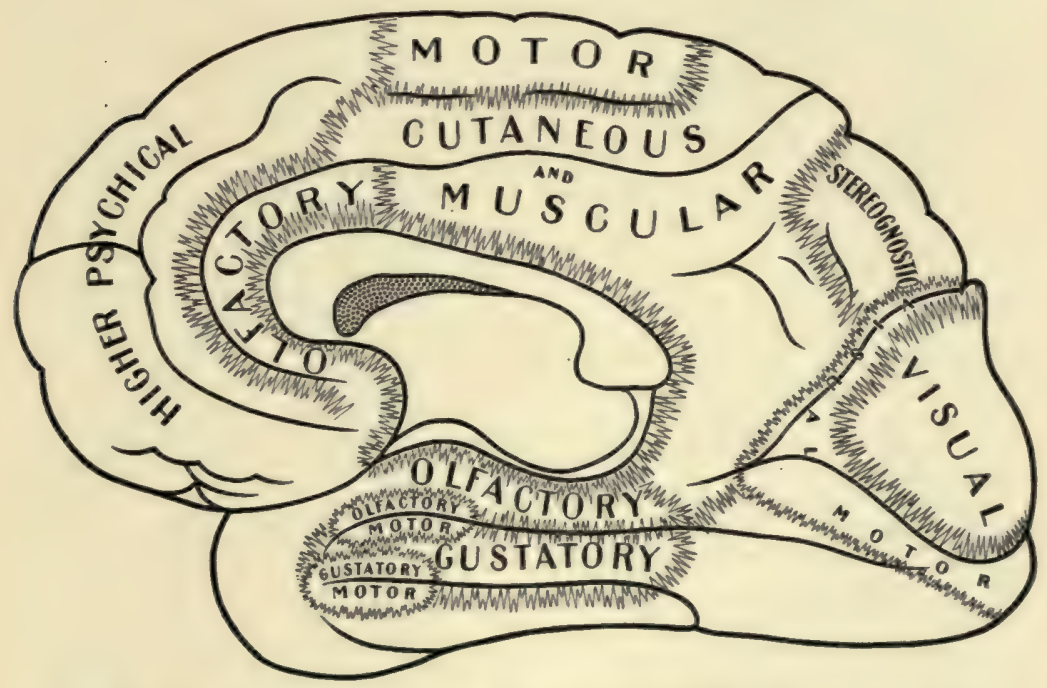

Fig. 57.-Cortical areas after C. K. Mills. Medial and tentorial surface of cerebral hemisphere. (Brubaker's Physiology.)

by those parts, but ablation of association centers disconnects the sensory, the psychic and the motor regions and causes aphasia, agraphia, change of temperament, impairment of the so-called moral and intellectual faculties, etc. Ablation of the visual psychic center or auditory psychic center produces mind-blindness in the former and in the latter mind-deafness.

Cell and Fiber Lamination of the Cerebral Cortex.--There is a type of cerebral cortex which, with small but definite variations, prevails throughout the cerebrum, excepting in the visual 
and olfactory regions (Fig. 58). Though Dr. Campbell's division of the cortex into seven layers of cells is complicated, it is similar to Cajal's description and I think it entirely worthy of general adoption and shall follow it in this work. It is to be regretted that the fiber and the cell layers have not been more satisfactorily correlated, as this would assist in determining function. Dr. Arthur W. Campbell gives the layers as follows:

\section{First, the layers of cells :-}

I. The plexiform or molecular layer.

2. The layer of small pyramids.

3. The layer of medium-sized pyramids.

4. The external layer of large pyramids.

5. The layer of stellate or polymorphous cells.

6. The internal layer of large pyramids.

7. The layer of fusiform cells.

\section{Second, the fiber zones:-}

I. The fiberless layer, or neuroglia zone.

2. The zonal layer, stratum zonale.

3. The supraradiary zone.

4. The baillargic zone, line of baillarger.

5. The radiary zone.

6. The felt-work of Kaes.

I. The plexiform layer has next the surface a fiberless zone of neuroglia, on account of which it is often called the neuroglialayer. Underneath the neuroglia is a more or less dense plexus of nerve fibers, constituting the stratum zonale; and then an area of sparsely scattered fibers that belongs to the supraradiary zone. Scattered here and there in the zonal and supraradiary regions of the plexiform layer are small stellate cell-bodies, four or six microns in diameter, belonging to the types of Golgi and Cajal, whose dendrites and whose double or triple axones ramify in the stratum zonale, some near the cell-body and others at a considerable distance from it. The stratum zonale also contains dendritic processes from subjacent laminæ, the $\mathrm{T}$-branched axones of Martinotti's cells, and, perhaps, the end-tufts of incoming fibers 


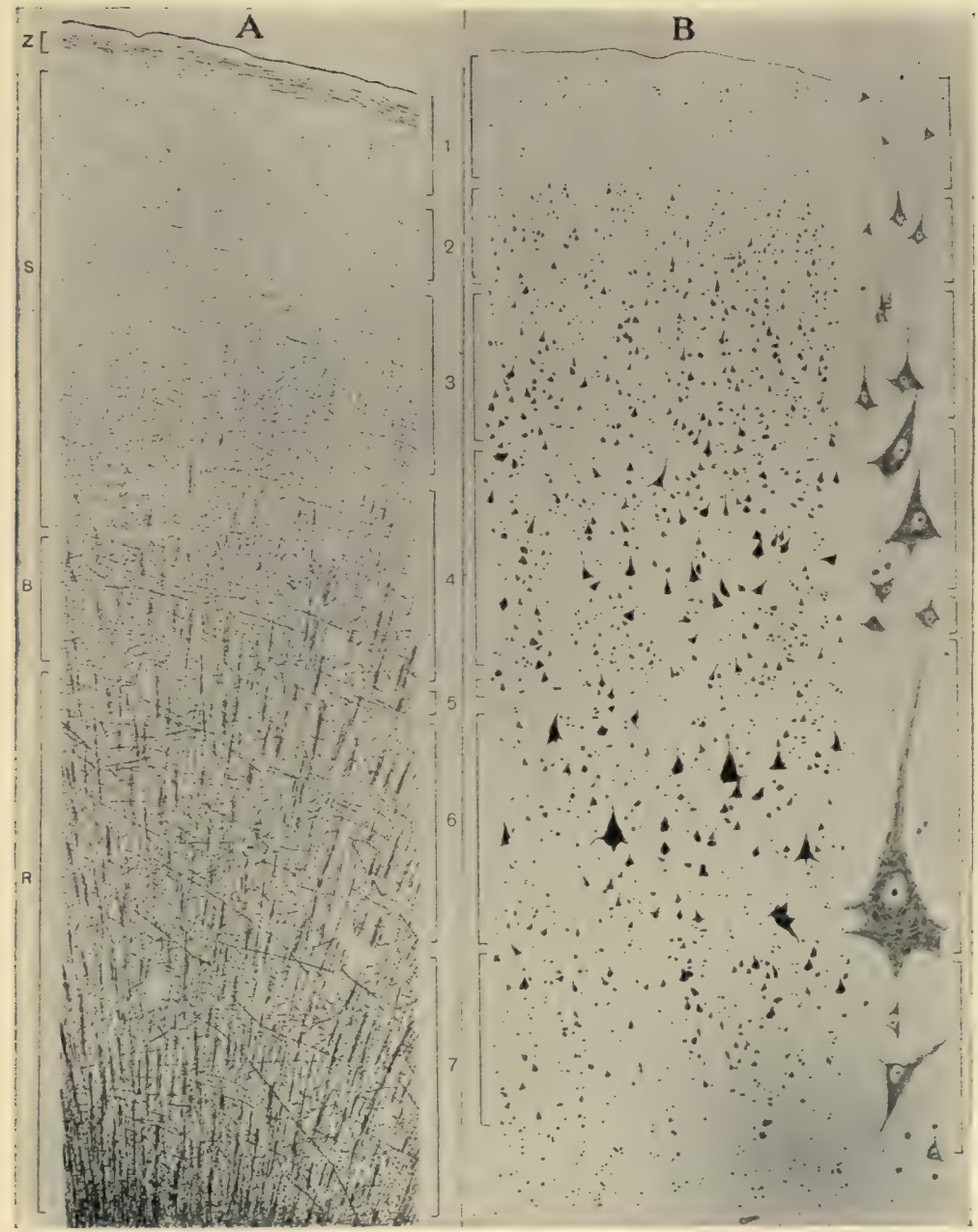

Fig. 58.-Cell and fiber lamination in the posterior half of the anterior central gyrus. The motor area. (After A. W. Campbell's "Histological Studies on the Localization of Cerebral Function." Published by the Syndics of the Cambridge University Press.)

A. Stained to show only fibers. B. Stained to show only cell-bodies. z. Stratum zonale. s. Supraradiary zone. B. Line of Baillarger. R. Radiary zone in the deep part of which is the felt-work of Kaes, I. Plexiform layer. 2. Layer of small pyramids, 3. Layer of medium-sized pyramids. 4. External layer of large pyramids. 5. Stellate or polymorphous cells. 6. Internal layer of large pyramids. 7. Layer of fusiform cells. 

from the commissural, the associative and the projection systems. It is very well marked in the motor area (Fig. 58), not so well in the common sensory area (Fig. 59). In the uncus it is very distinct (Fig. 6r) and is so thick and dense in the gyrus hippocampi (the subiculum) as to be visible to the naked eye (Fig. 62). The zonal layer of fibers is faint in the visuo-sensory and auditosensory cortex. The stratum zonale appears to grow richer with the education of the individual. The function of the plexiform layer is commonly thought to be association.

2. The layer of small pyramids (Figs. 58 and 59), as well as the third layer, is situated in the supraradiary zone. It is composed chiefly of small closely packed cell-bodies, pyramidal in shape. They measure eight or ten microns in diameter. Their apices point toward the surface. From the apices, surfaces and lateral angles, dendrites are given off which ramify in the stratum zonale of the first layer. The axone issues from the base of the pyramid and runs down through the subjacent layers. Among the small pyramids are a few ploymorphous cells.

3. The layer of medium-sized pyramids (Fig. 58) is a nearly pure layer; and, like the overlying layer, is nearly uniform throughout the cerebral cortex. In arrangement of cell-bodies and processes it is like the second layer. The pyramids get farther apart and become larger in size as the layer is descended. They measure ten to fifteen microns in their vertical diameter. In the anterior central gyrus these cells are motor. Layers "two" and "three" might be combined in one as was formerly the custom.

4. External Layer of Large Pyramids. (Figs. 58 and 59).-This layer coincides in position with the line of Baillarger. The pyramids are larger and farther apart than in the above layer, and show a considerable accession of Nissl bodies as compared with the smaller pyramids. They measure $\mathrm{I} 5 \mu$ to $20 \mu$ by $25 \mu$ to $30 \mu$, and form "one of the most important criteria in dividing the brain surface into different histological territories" (Campbell). As these cells, in the anterior central gyrus, degenerate in amyotrophic lateral sclerosis, they are considered motor in function. The apical processes appear to reach the first layer and ramify in the stratum zonale; the lateral and basal dendrites arborize 
within the line of Baillarger; the axone runs inward to the white substance. The external large pyramids are found in nearly all parts of the cortex. They are replaced by a layer of large stellate cells in the receptive visual cortex along the calcarine fissure. The line of Baillarger in the calcarine cortex is so thick and dense that Gennari and, later; Vicq d'Azyr described it from its naked eye appearance (Fig. 60). In the motor and common sensory area the line can scarcely be made out; while in the superior parietal gyrus there are two Baillargic lines, the deep line coinciding in position with the internal large pyramids. There are some peculiar Golgi cells among these pyramids, whose axones run horizontally and give off collaterals that form pericellular baskets around the pyramids (Johnston). They are associative in function.

5. The layer of stellate or polymorphous cells presents great variation in different regions and is a valuable guide for cortical localization. It is best marked in the calcarine area (Fig. 60) where it and the fourth layer are associated with the greatly thickened Baillargic line (Gennari), though elsewhere it is situated in the radiary zone of fibers. It is well marked in the auditory cortex. In the motor area it is hardly distinguishable, but it appears suddenly at the bottom of the central sulcus and forms a distinct feature in the common sensory region of the posterior central gyrus (Fig. 59). The cell-bodies of the stellate layer measure five to eight microns in diameter and are of many shapes, some being pyramidal. They are richly branched.' The dendrites arborize at once near the cell-body. The axones of a considerable number of cells extend horizontally within the layer, helping to form the line of Baillarger; while others run toward the surface and end in the overlying laminæ.

6. Internal Layer of Large Pyramids (Fig. 59).-This is the most important layer of the cortex for localization. It lies in the radiary zone of fibers and is present in almost every part of the cerebral cortex, though distinguished by definite variations in the different regions (Figs. 59, 60 and 6I). Its great pyramids are intermingled with irregular cell-bodies of the Golgi type and with Martinotti cells. The giant pyramids (Betz), or ganglionic 


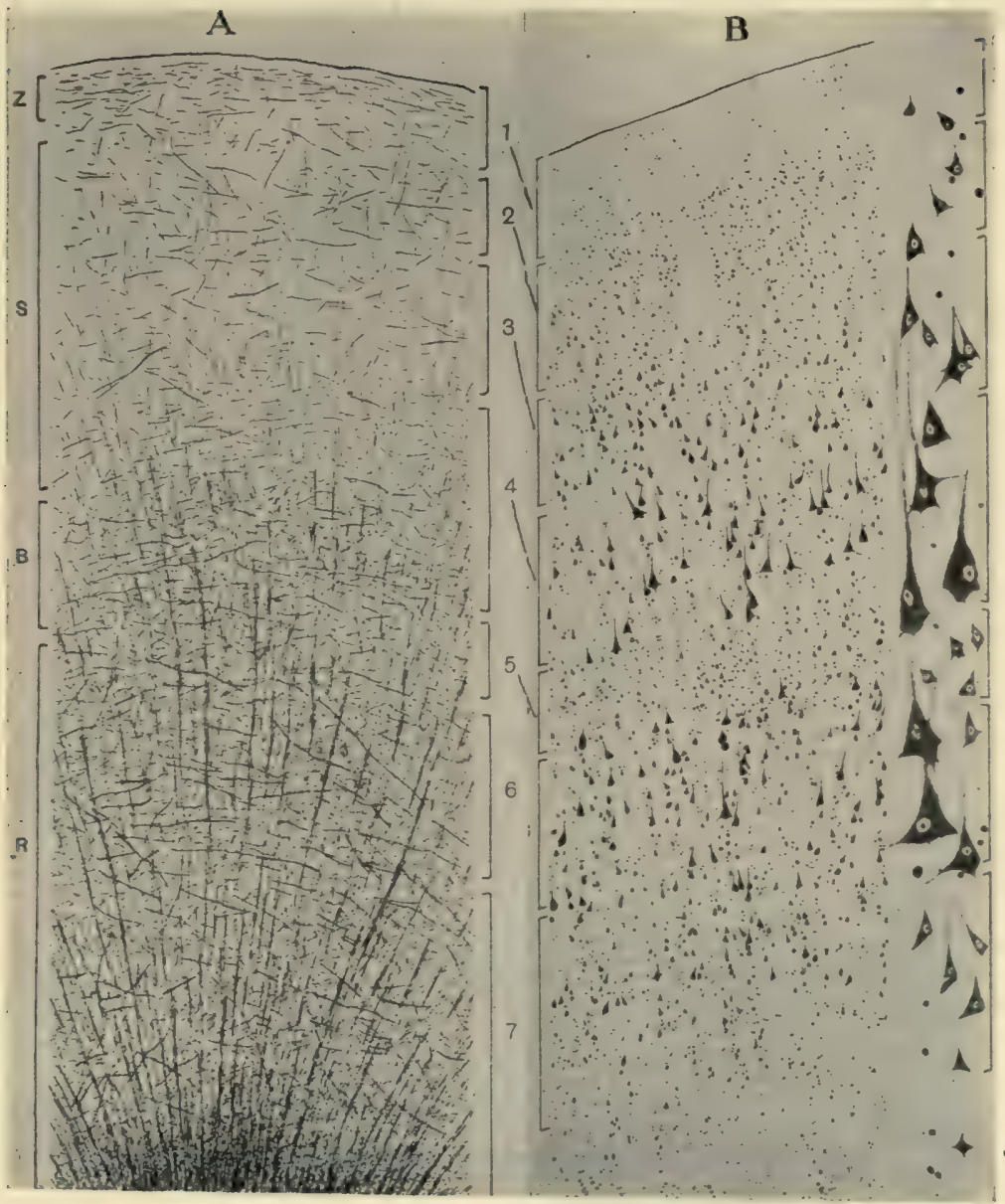

Fig. 59.-Cell and fiber lamination in the anterior half of the posterior central gyrus. The COMMON SENSORY AREA. (After A. W. Campbell's "Histological Studies on the Localization of Cerebral Function." Published by the Syndics of the Cambridge University Press.)

A. Stained to show fibers. B. Showing only cell-bodies. z. Stratum zonale. s. Supraradiary zone. B. Line of Baillarger. R. Radiary zone. I. Plexiform layer. 2. Layer of small pyramids. 3. Layer of medium-sized pyramids. 4. External layer of large pyramids. 5. Layer of stellate cells, 6. Internal layer of large pyramids. 7. Layer of fusiform cells. 


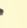


cells (Bevin Lewis), are "pyraform" in shape. They are loaded with $N$ issl bodies and give off one axone and many dendrites. The lateral and basal dendrites ramify in the radiary zone; the dendrite of the summit runs straight out toward the surface and like the same process from other pyramids, arborizes in the stratum zonale of the plexiform layer; the axone enters the medullary substance and becomes a projection, association or commissural fiber. The giant pyramids characterize the motor cortex. No where else are they so large. Neither do they have in any other region the "pyraform" shape or the definite nest-like grouping seen in the anterior central gyrus. They measure twenty-five by sixty microns in the leg area of that gyrus; twenty by forty-five microns in the arm area; and, in the head area, seventeen by thirty-five microns (Bevin Lewis). In cases of amyotrophic lateral sclerosis studied by Campbell, 87.5 per cent. of these cells in the affected area were entirely destroyed and those remaining showed signs of degeneration. Both the internal and external large pyramids were thus affected in the anterior central gyrus, so they are motor in that gyrus; but they were not degenerated in any other part of the cortex.

7. The fusiform layer (Figs. 58, 59, 60 and 6I) is found every where in the cerebral cortex. It presents very little topographical variation. Its spindle-shaped cell-bodies lie in the deep part of the radiary zone and in the felt-work of Kaes. The long axes of the spindles are perpendicular to the surface in the crown of a gyrus but are parallel with it in the fissural walls and floor. From these cell-bodies one axone and several dendrites are given off. The function of the spindle cell is probably association. The felt-work of Kaes is a rich plexus of fibers in which the white and gray substance meet. It is produced by the intermingling of the association, commissural and projection fibers. Scattered here and there through all the layers of the cortex are two atypical neurones, viz., the dendraxones of Golgi, which arborize very richly and are associative in function; and, second, the inverted pyramids of Martinotti. Of the latter the dendrites terminate near the cell-body; but the axone runs out to the first layer and, branching $\mathrm{T}$-like, ramifies in the stratum zonale. 
Martinotti's cells, like those of Golgi, are probably associative in function.

The Radiations of Meynert (Figs. 58, 59, 60 and 6I).-The six fiber zones above enumerated have been as fully indicated in speaking of the cell lamination as the limits of this work will allow, but certain fibers, called the fibers of Meynert, need to be mentioned. The radiations of Meynert are strands of fibers, clearly visible in the radiary zone of the cortex, composed of corticifugal and corticipetal fibers. The corticifugal fibers are very largely axones from the pyramids; the incoming fibers of Meynert's radiations rise in other parts of the cortex or in gray matter situated at a lower level. The radiating fibers belong to the following systems, the projection, the association and the commissural. The radiations are distinct as far out as the external layer of large pyramids and the line of Baillarger, hence the name radiary zone; but in certain regions they are much longer and in some parts of the temporal lobe they extend to the stratum zonale of the first layer. In such regions the line of Baillarger divides the radiary zone into two parts and there is no proper supraradiary zone.

Association Fibers of Meynert.-This name is applied to arcuate fibers of large diameter located in the radiary, the Baillargic and the deep part of the supraradiary zone. Owing to condensation they form a distinct layer in the fissural walls and floor, but are sparsely scattered in the crown of the gyrus (Campbell). They arch over the medullary projection, crossing Meynert's radiations at right angles. The deeper ones appear to be continuous with certain fibers in the radiations and are probably corticipetal in direction (Kaes and Campbell). Meynert's association fibers are said by Vulpius not to be developed before the seventeenth year; and, according to Kaes, are most abundant in the highly developed parts of the brain.

Atypical Cortex. - The decided variations from the typical cortex are found in the visual and olfactory areas.

Visual Receptive Center (Fig. 60).-The cortex in the cuneus and gyrus lingualis presents three marked variations from typical cortex: I. The greatly accentuated line of Baillarger, which 


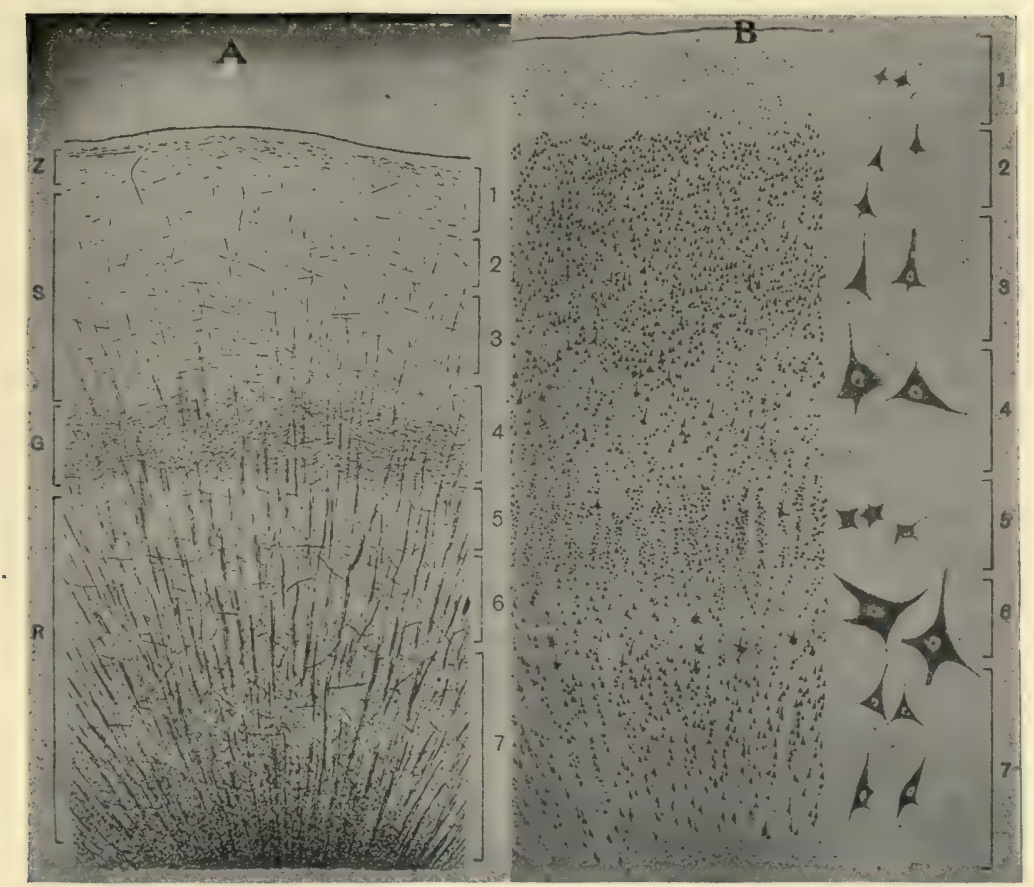

Fig. 60.-Cell and fiber lamination in the calcarine region. RECEPTIVE visual. -AREA. (After A.W. Campbell's "Histological Studies on the Localization of Cerebral Function." Published by the Syndics of the Cambridge University Press.)

A. Shows fibers of occipital cortex. B. Cells of same. z. Stratum zonale. s. Supra-radiary zone. G. Line of Baillarger or Genari. R. Radiary zone. 1. Plexiform layer. 2. Layer of small pyramids. 3. Layer of medium-sized pyramids. 4. External layer of large stellate cells. 5. Small steliate cells. 6 . Layer of giant pyramidal or stellate cells with some small pyramids. 7. Layer of fusiform cells with some medium-sized pyramids. 

may be seen dividing the cortex into two gray layers. This line was first seen and described in the visual area by Gennari and, ten years later, by Vicq d'Azyr. 2. The external layer of large pyramids is replaced by a layer of stellate cells $25 \mu$ in diameter. These stellate cells give off three or four strong processes which appear to arborize in the line of Baillarger. They are found in the receptive and psychic visual areas but not elsewhere in the cerebral cortex. 3. The internal layer of large pyramids is replaced by a conglomerate made up of three varieties of pyramids. From without inward there are: First, small inverted pyramids, cells of Martinotti, which extend their axones out toward the surface. Second, the scattered giant pyramids, $25-30 \mu$ in diameter, arranged in a single row and called the solitary cells of Meynert. The axones of the giant pyramids probably pass into the optic radiation. Third, a layer of medium-sized pyramids.

Olfactory Cortex.-All the divisions of the rhinencephalon are here mentioned, though only a part of them need be described: the olfactory bulb, triangle, parolfactory area, anterior perforated substance, gyrus subcallosus, septum pellucidum, gyrus supracallosus (longitudinal striæ), fasciola cinerea, fascia dentata, hippocampus, subiculum and uncus. The posterior inferior part of the hippocampal gyrus and the gyrus cinguli are ordinarily included in the rhinencephalon; but, according to Elliot Smith, they belong to the neopallium (Figs. 26, 28 and 62).

The cortex of the olfactory bulb (Fig. 63) is divided into five layers as pictured by Barker. These five layers are as follows, named from the surface toward the center: (I) The stratum nervosum, composed of the T-branched fibers from the olfactory nerve and their collaterals. These fibers run nearly parallel with the surface for some distance, then bend centrally and break up into their end-tufts in the second layer. (2) The stratum glomerulosum is made up of round bodies, called glomeruli, which are composed of the end-tufts of olfactory nerve fibers and of brush-like dendrites from the spindle and mitral cells of the third and fourth layers. The glomeruli constitute the synapses between the first and second olfactory neurones. (3) The stratum reticulare. This is a network of mitral dendrites interwoven with 
arborizing processes from the granules in the fifth layer and the branches of a few endogenous spindle cells, called the brush cells. The mitral dendrites are on their way to the glomeruli in the second layer. The spindle cells likewise, both large and small, throw their dendritic processes down into the stratum glomerulosum, where they end in rich tufts or brushes; and their axones penetrate the fourth and fifth layers, enter into the white sheath of the bulb and thence are continued into the olfactory tract. (4) The stratum cellulare, or layer of mitral cell-bodies. The mitral cells have large pyramidal bodies with one axone and rich dendritic processes. The latter arborize through the reticular layer to the glomeruli of the second layer, where they terminate in the form of end-brushes. The axones of the mitral cells run centrally through the granular layer, to which they give off collaterals, and then turn backward in the white sheath and ultimately constitute the olfactory tract. The white sheath incloses a mass of cells derived from the ependymal lining of the ventricle in the embyro. (5) The stratum granulosum is composed of a thick layer of small cell-bodies, "granules," whose processes arborize richly in the granular, cellular and reticular layers. Imbedded in the granular layer are the medullated axones coursing toward the white sheath and the olfactory tract. The function of the granular layer is not understood. The mitral and spindle cells of the olfactory bulb, it should be carefully noted, form the terminal nucleus of the olfactory nerves: the points of contact between them are established in the glomeruli; and the axones of the nucleus constitute the olfactory tract and its strice, which join the bulb to the cerebral hemisphere (Fig. 63). The lateral stria of the olfactory tract runs directly to the uncus, hence we shall study that region next.

The uncus and crown of the hippocampal gyrus (Figs. 55, 6I and 62) probably represent the greater part of the lobus pyraformis of osmatic mammals. It constitutes the chief cortical center of smell. However it is probable that the subiculum, hippocampus, fascia dentata, and anterior end of the gyrus cinguli belong in the cortical area of smell, as all showed arrested development in two cases of congenital absence of the olfactory bulbs 


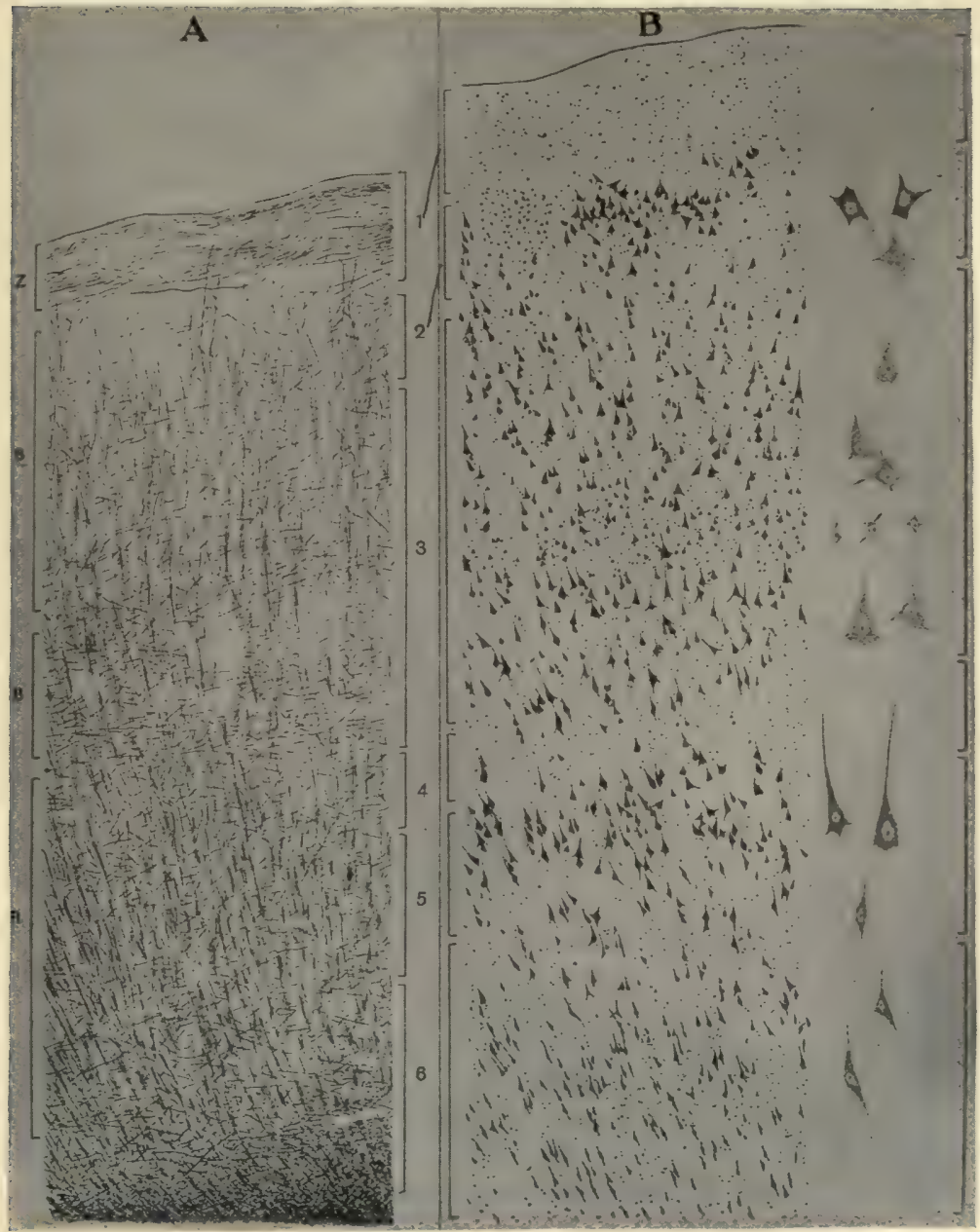

Fig. 6r.-Cell and fiber lamination in the uncus hippocampi (lobus pyraformis). The area of SMell. (After $A$. W. Campbell's "Histological Studies on the Localization of Cerebral Function. Published by the Syndics of the Cambridge University Press.)

A. Showing fibers. B. Showing cell-bodies. z. Stratum zonale, external medullary lamina. S. Supraradiary zone containing radiating filbers. B. Line of Baillarger. R. Radiary zone. x. Plexiform layer. 2. Layer of stellate cells showing cell-nests. 3. Represents third and fourth layers of typical cortex, medium-sized pyramids obliquely placed and stellate cells; with Golgi's silver method shows tassel-cells. 4. Fusiform or triangular cells. 5. Medium-pyramids. 6. Fusiform cells. 

(Zuckerkandl). The fascia dentata is of first importance according to Alexander Hill. He calls attention to the fact that the Narwhal, which has no sense of smell, possesses every part of the hippocampal region excepting the dentate fascia (Campbell). The uncus comprises the whole anterior part of the gyrus hippocampi. In structure the crown of the hippocampal gyrus and the uncus are nearly identical. They have only five layers of cells. (I) As already pointed out the plexiform layer is thick and possesses a dense stratum zonale, only second to that of the subiculum. (2) The place of the small pyramids is usurped by the "olfactory islets" (Calleja) which are curious nests of large stellate cells $(28 \mu)$ interspersed with small nests of very minute pyramidal cells. (3) The tassel-cells of Cajal. Peculiar pyramidal cells, with such rich dendritic arborizations hanging from the bases as to resemble tassels, are seen in the place of the mediumsized pyramids. At the line of Baillarger there are no cells; the fourth layer of typical cortex is entirely wanting. (4) The stellate layer and internal layer of large pyramids are replaced by a layer of intermixed fusiform and triangular cells heavy with Nissl bodies. (5) The fusiform-cell layer is nearly typical.

Nucleus Amygdale (Fig. 32).-In the anterior wall of the inferior horn of the lateral ventricle, near the temporal pole and dorsal to the uncus hippocampi, is the amygdala, a nucleus of doubtful classification. The amygdala is in part continuous with the corpus striatum and, according to Campbell, appears on the surface of the uncus as the gyrus semilunaris.

Cortex of the Lower Wall and Lip of the Hippocampal Fissure (Fig. 62).-This is known as the subiculum. It is especially distinguished for its remarkable stratum zonale, which is visible to the naked eye, and for its long radiations, which reach the zonal layer and give the cortex a striated appearance. (I) The plexiform layer is almost wholly occupied by the stratum zonale, called here the external medullary lamina. (2) The layer of olfactory islets. The islets are closely packed nests of minute triangular cells, $5 \mu$ in diameter, resembling those in the uncus. (3) The stratum radiatum occupies about three-fourths of the depth of this cortex. In its deep part (the stratum lucidum) 
there are several layers of medium-sized pyramids, arranged in columns. The prominent apical processes of these pyramids collect in bundles and proceed outward to the stratum zonale, separating the columns of pyramids and producing the striations above mentioned. As the apical dendrites approach the olfactory islets they branch richly. The axones of the pyramids run straight to the white core of the gyrus or into the alveus. The pyramids continue without interruption through the hippocampus into the nucleus of the dentate fascia. The alveus, which forms the ventricular surface of the hippocampus, is made up largely of the axones of these pyramids; from the alveus they proceed into the crus of the fornix. (4) A few fusiform or stellate cells lie next the alveus. They belong to the type of Golgi, the axone being wonderfully branched. In function they are associative. It is in the region of these associative neurones that the axones of the pyramids bend and adjust themselves so as to enter the alveus nearly parallel with its surface, hence the name stratum oriens applied to it by Edinger.

The fascia dentata (Fig. 62) is a free lip of cortex folded inward anterior to the hippocampal fissure. It presents a type of structure, which is continued, forward, through the band of Giacomin into the reflected part of the uncus; and which extends backward through the fasciola cinerea into the gyrus supracallosus (longitudinal striæ of the corpus callosum). It is similar in structure to the subiculum, the first and the third layers only present a marked variation. The stratum zonale is not so prominent as in the subiculum; and the stratum radiatum is entirely replaced by the nucleus fasciæ dentatæ. The nucleus is composed of pyramids, of polymorphous and fusiform cells and their branches. Their dendrites radiate toward the stratum zonale, their axones proceed into the crus of the fornix. The dentate fascia is absent in anosmatic animals (A. Hill).

Trigonum Olfactorium, Area Parolfactoria (Broca), Gyrus Subcallosus and Septum Pellucidum, and Substantia Perforata Anterior (Figs. 26 and 28).-These are the parts into which run most of the fibers of the medial and intermediate striæ of the olfactory tract. They are more conspicuous in the embryo than 


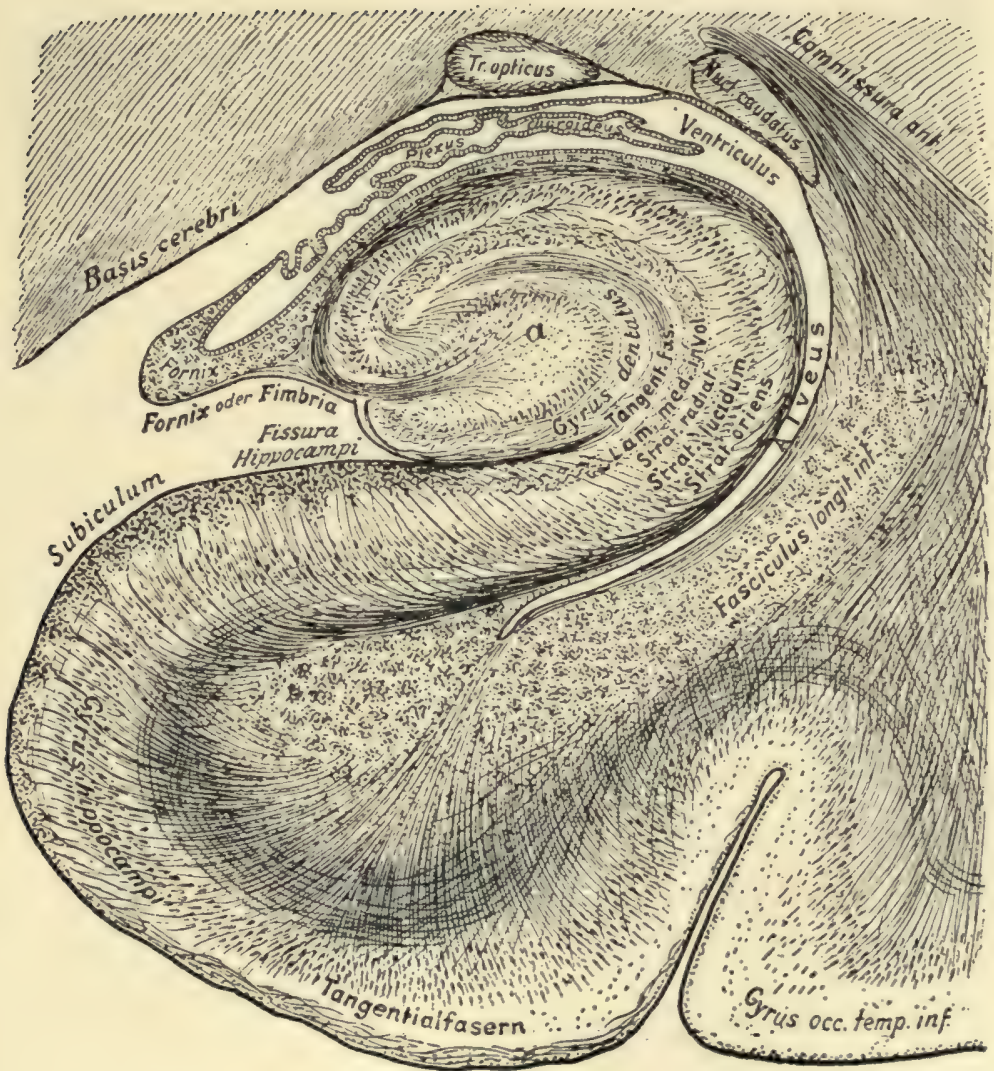

Fig. 62.-Transverse section of the hippocampal region. (After Edinger.)

a. Nucleus of fascia dentata. 

in the adult human brain. The cortex of this whole region is so rudimentary or vestigial as to require but brief description. The plexiform layer may be identified. The whole gray substance beneath that is occupied by scattered pyramids of medium size, separated by strands of fibers belonging to the olfactory tract and, perhaps, to the cingulum.

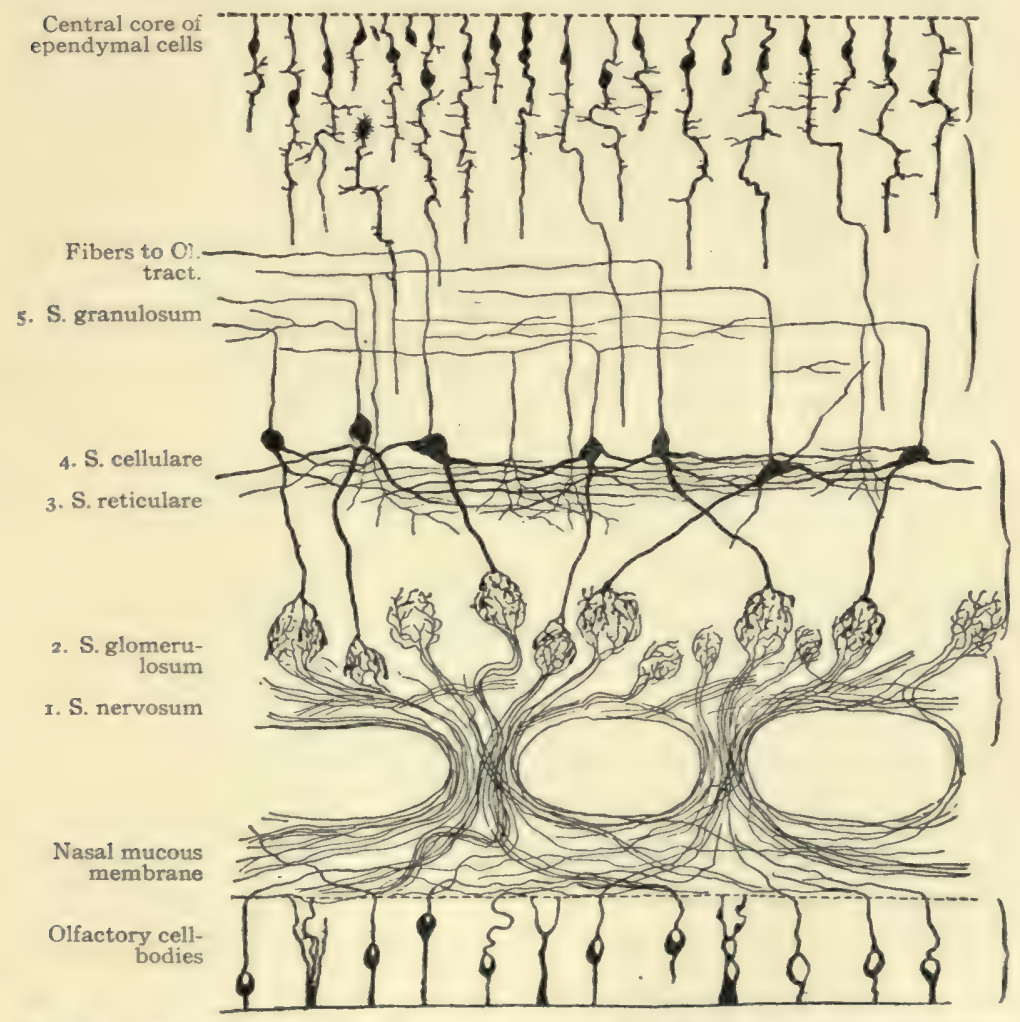

Fig. 63.-Chief elements of the olfactory bulb. (Gordinier after Van Gehuchten.)

The cortex of the gyrus cinguli (Fig. 28) is characterized by an entire absence of large fibers and large cells, by an oblique and irregular direction of the pyramids and by a most remarkable color affinity possessed by the deep cells. There are only four cell layers. (I) The plexiform presents a faint stratum zonale, but nothing characteristic. (2) The layer of small pyramids 
is ill defined. (3) A layer of medium-sized pyramids placed at various angles occupies the place of the third, fourth, fifth and sixth layers of typical cortex. (4) The layer of spindle cells. In the spindle-cell layer are found the remarkable chromophilous cells. They are triangular or pyramidal in shape and have greater affinity for stains than the cells of any other part of the cerebral cortex.

The claustrum (Figs. $3 \mathrm{I}$ and 42 ) is a sheet of peculiar gray substance which, according to Meynert, may be classed as cortical. In structure it resembles the seventh layer of typical cortex, being made up of fusiform cell-bodies. The claustrum is a vertical antero-posterior sheet placed medial to the island, and lateral to the external capsule. The surface in contact with the external capsule is smooth, but the external surface is convoluted to coincide with the gyri insulæ. At its lower border it joins the lentiform nucleus.

\section{GANGLIONAR GRAY MATTER.}

The substantia grisea ganglionaris is found in the great ganglia which, in their situation and relations, have already been considered. They should be re-studied in this connection. They are as follows:

I. In the hemisphere:

The corpus striatum, composed of the caudate and the léntiform nucleus.

2. In the inter-brain:

The thalamus, lateral and medial geniculate bodies, nucleus hypothalamicus (Luysi) and red nucleus.

3. In the mid-brain:

The superior and inferior quadrigeminal colliculi, the substantia nigra, and the nucleus lateralis superior in the tegmentum.

The corpus striatum (Figs. 64, 3I and 66) is an ovoid mass of reddish-gray matter containing pigmented multipolar cellbodies of various sizes, those of large size being more numerous in the nucleus lentiformis than in the nucleus caudatus. The 


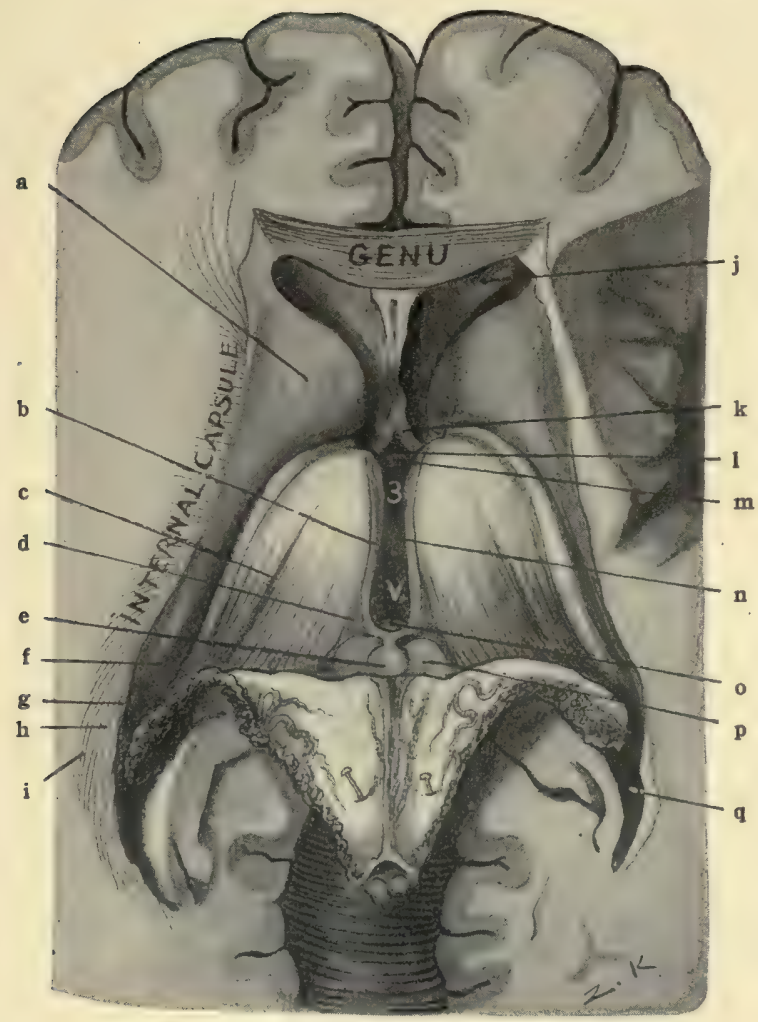

Fig. 64.- Horizontal section of cerebrum through genu and below splenium of corpus callosum. Fornix and chorioid tela turned back, to show inter-brain and third ventricle. (Original.)

a. Head of caudate nucleus. b. Stria medullaris thalami (or pineal stria.) c. Chorioid groove. d. Trigonum habenulae. e. Pineal body. f. Tail of caudate nucleus. g. Tapetum. h. Occipito-thalamic radiation. i. Inferior longitudinal fasciculus. j. Anterior horn of lateral ventricle. k. Columna of fornix. 1. Recessus triangularis. m. Anterior commissure. n. Massa intermedia (or middle commissure). o. Posterior commissure. p. Superioriquadrigeminal colliculus. q. Posterior horn of lateral ventricle. 

axones of those cell-bodies run both toward the pons and toward the cerebral cortex. The corpus striatum, therefore, forms a relay-station in an efferent conduction tract and, perhaps a less important one, in an afferent tract.

Of the centrifugal fibers note the following three groups:

(I) The cortico-striate radiation made up of corticifugal and

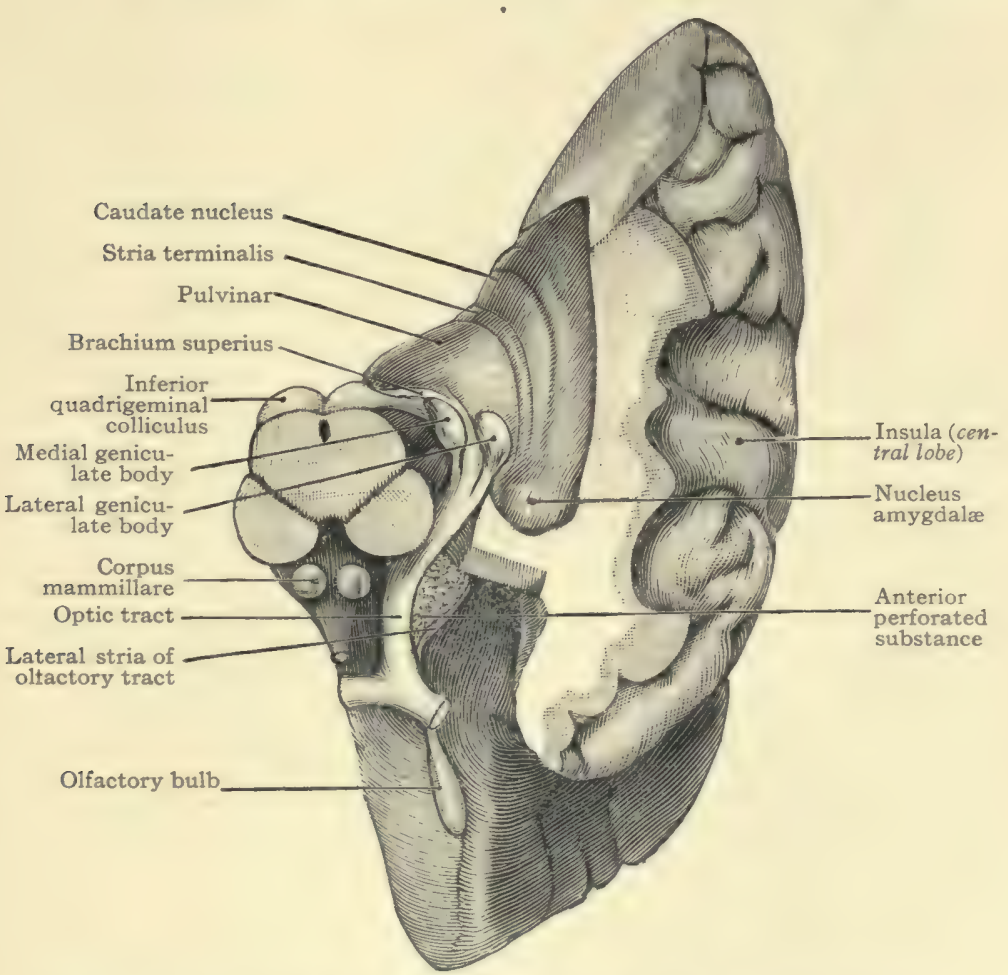

Fig. 65.-Dissection of brain to show geniculate bodies, optic tract, nucleus amygdalæ, etc. (After Morris's Anatomy.)

corticipetal fibers running from and to the equatorial zone of the hemisphere. (2) The striato-thalamic fibers, which are in part crossed fibers, pass through the inferior lamina of the internal capsule and terminate in many nuclei of the inter-brain, mid-brain and pons; some of them pierce the superior lamina of the capsule. They run to the thalamus, hypothalamus and 
hypothalamic nucleus of both sides, the crossing fibers forming the superior commissure (Meynert's) in the tuber cinereum; to the nucleus ruber and quadrigeminal colliculi; and, through the deep part of the basis pedunculi, to the substantia nigra and nucleus pontis (Fig. 70). The last tract is the stratum intermedium, or the intermediate bundle of the basis pedunculi (Fig. 68). The olivary bundle (fasciculus, olivaris) probably rises in the globus pallidus (or thalamus) and descends to the nucleus of the olive in the medulla. In addition to the above, it is probable that the fronto-pontal and temporo-pontal tracts are relayed in the corpus striatum or thalamus.

The centripetal fibers connected with the corpus striatum are also numerous. (I) The thalamo-striate fibers, which belong to the common sensory path, rise partly in the thalamus and partly from nuclei lower down. They run through the frontal stalk and the ventral stalk of the thalamus and, perhaps, through the parietal stalk, and include uninterrupted fibers of the medial fillet and spino-thalamic tract (Fig. 70). (2) Fibers from the globus pallidus to the posterior central cortex in the cortico-striate radiation (the alpha and gamma bundles of Flechsig). These enter the parietal stalk high up in the superior lamina of the capsule.

Lesions of the corpus striatum affect the internal capsule, which impales it; and may cause, if extensive, hemiplegia and hemianesthesia of the opposite side of the body, partial deafness chiefly in the opposite ear and hemianopia due to cortical isolation of the corresponding halves of both retinæ.

The thalamus (Figs. 64, 66 and 31 ) is made up chiefly of gray matter containing multipolar and fusiform cell-bodies. The white matter consists of the stratum zonale on its free surface and of the internal medullary lamina. The latter divides the gray substance into nuclei, of which Nissl has described about twenty. They may be grouped as follows: The medial, lateral, anterior, nucleus of the pulvinar and nucleus habenulæ.

(I) The medial nucleus is joined to the opposite medial nucleus by the massa intermedia and is continuous with the hypothalamic gray matter in the wall and floor of the third ventricle; but the 


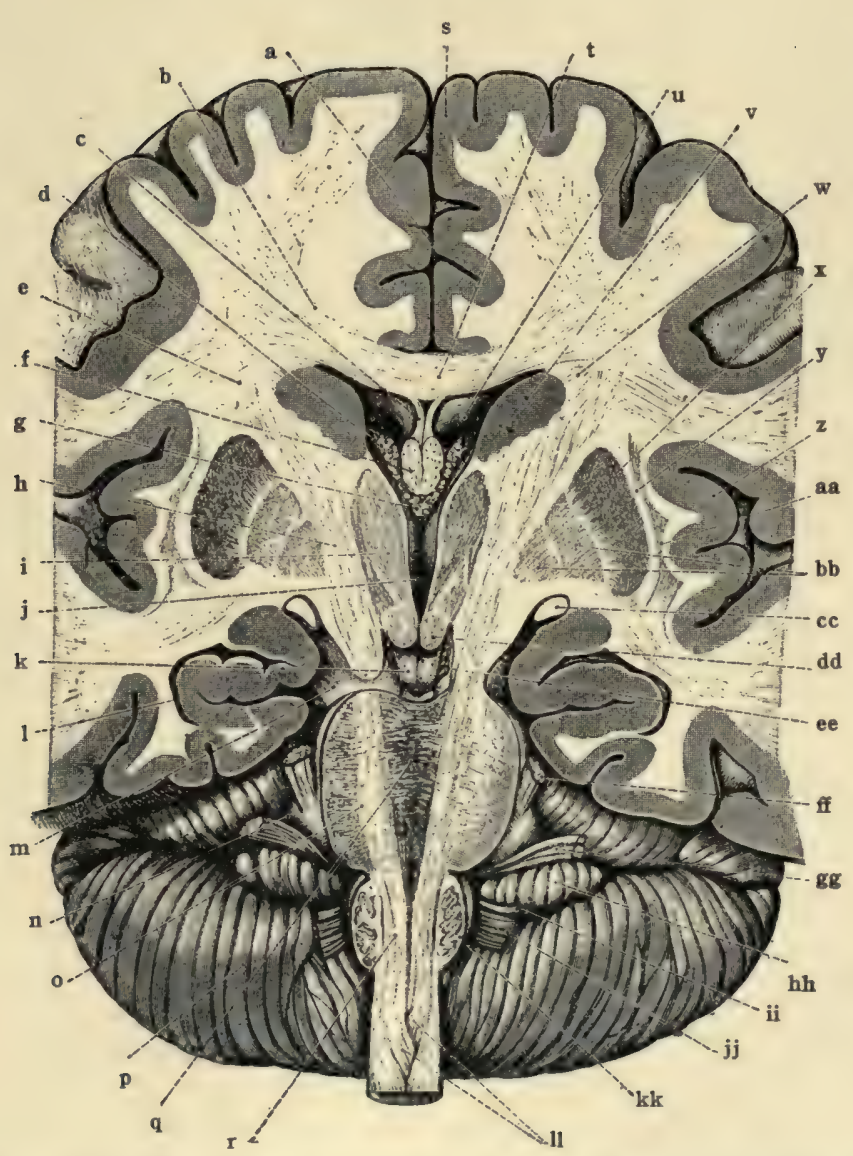

Fig. 66.-Transverse section of the brain in the line of the pyramidal tracts, showing basal ganglia, internal capsules, corpus callosum, lateral and third ventricles, etc. Viewed from front. (Morris's Anatomy after Toldt.)

a. Longitudinal fissure, b. Radiation of corpus callosum. c. Septum pellucidum. d. Chorioid plexus of lateral ventricle. e. Corona radiata. f. Column of fornix. g. Chorioid plexus of third ventricle. h. Internal capsule. i. Thalamus, j. Third ventricle. k. Interpeduncular fossa. 1. Inferior horn of lateral ventricle. m. Cerebral peduncle. n. Brachium pontis. o. Longitudinal pyramidal fasciculi of pons. p. Cerebellum. q. Deep fibers of pons. r. Pyramid. s. Superior frontal gyrus. t. Body of corpus callosum. u. Anterior horn of lateral ventricle. v. Head of caudate nucleus. w. Radiation of corpus striatum. x. Putamen. y. External capsule. z. Insula. aa. Claustrum. bb. Globus pallidus. cc. Optic tract. dd. Corpus mammillare. ee. Oculo-motor nerve. ff. Trigeminal nerve. gg. Facial and acoustic nerves, hh. Flocculus. ii. Glossopharyngeal nerve, jj. Vagus nerve. kk. Inferior olivary nucleus. 11. Decussation of pyramids. 

internal medullary lamina separates it from the other nuclei of the same thalamus. It gives origin to a part of the ventral stalk of the thalamus. According to Bechterew it probably gives rise to some descending fibers that run down the cord. (Jas. S. Collier has traced thalamo-spinal fibers in the cat down the lateral column of the cord.)

(2) The lateral nucleus is the largest. It extends from superior to inferior surface the entire length of the thalamus, and includes the center median (Luysi) and the arcuate nucleus, both of which fuse with it posteriorly. It also fuses with the nucleus of the pulvinar. It forms the terminal nucleus for the larger part of the tegmental fibers, especially of the medial fillet, the spino-thalamic tract, a part of the medial longitudinal bundle and the brachium conjunctivum of the cerebellum; and it constitutes the nucleus of origin for most of the fibers of the cortical fillet. Destruction of this nucleus interrupts the common sensory path, and causes anesthesia and ataxia of the opposite side.

(3) The nucleus of the anterior tubercle (Fig. 44) receives the fasciculus thalamo-mammillaris (Vicq d'Azyri) from the corpus mammillare and is thus connected with the columna of the fornix (Fig. 66).

(4) The nucleus of the pulvinar (Fig. 44) is an important one. It receives about twenty per cent. of the optic fibers and gives rise to a corresponding number of the corticipetal fibers in the optic, or occipito-thalamic radiation; hence, a lesion of the pulvinar impairs vision. It is continuous with the lateral nucleus.

(5) The nucleus of the habenula belongs to the epithalamus (Fig. 64). It lies beneath the trigonum habenulæ. It receives fibers from the rhinencephalon through the medullary stria of the thalamus, and originates a bundle of fibers, the fasciculus retroflexus (Meynerti), which may be traced back through the tegmentum to the interpeduncular ganglion in the substantia nigra. Beyont this, connections are probably established with the motor nuclei of cerebral nerves.

The white matter of the thalamus includes, first, the stratum zonale of the superior surface, which is derived from the occipitothalamic radiation and the lateral root of the optic tract; and, 
second, the interior fibers, a part of which form the internal medullary lamina. Into the thalamus enter the medial fillet, the spinothalamic tract, a small part of the medial longitudinal bundle, the brachium conjunctivum cerebelli and perhaps some other tegmental fibers, all carrying common sensory impulses; they end chiefly in the lateral nucleus, whence the cortical fillet proceeds to the sensory area of the cerebral cortex. The thalamus also receives fibers from the special sense paths, from the optic, auditory, olfactory, and probably the gustatory (?), and gives rise to fibers that continue in those paths to the special sense areas of the cortex or as, in the case of the olfactory, to reflex centers. It is also known that the thalamus is entered by a considerable number of corticifugal fibers, especially through the occipitothalamic and temporo-thalamic radiations. The several bundles of thalamic fibers are as follows:

(I) The columna of the fornix, having pierced the thalamus, descends to the corpus mammillare and terminates in its medial nucleus, whence the bundle of Vicq d'Azyr, the thalamo-mammillary bundle, rises and ascends to the thalamus. It ends in the anterior nucleus.

(2) The stria medullaris thalami (Fig. 64) from the hippocampus and from the region of the olfactory triangle, terminates in the nucleus habenulæ and from this nucleus the fasciculus retroflexus originates and descends to the interpeduncular ganglion. Both "one" and "two" belong to the olfactory paths.

(3) From the lateral surface of the thalamus issue two groups of fibers which rise chiefly in the lateral nucleus. They are common sensory in function: (a) The inferior one is the ventral stalk (ansa peduncularis) (Fig. 70), which rises from the medial and lateral nuclei and is in part relayed in the lentiform nucleus under which it passes toward its destination. It proceeds through the inferior lamina of the internal capsule and contributes fibers to both the medullary laminæ of the lentiform nucleus and to the external capsule. Underneath the lentiform nucleus the ventral stalk is divided into two horizontal laminæ by a thin sheet of gray substance. The upper lamina, the ansa lenticularis, is intermingled with the striato-thalamic fibers and enters into the lenti- 


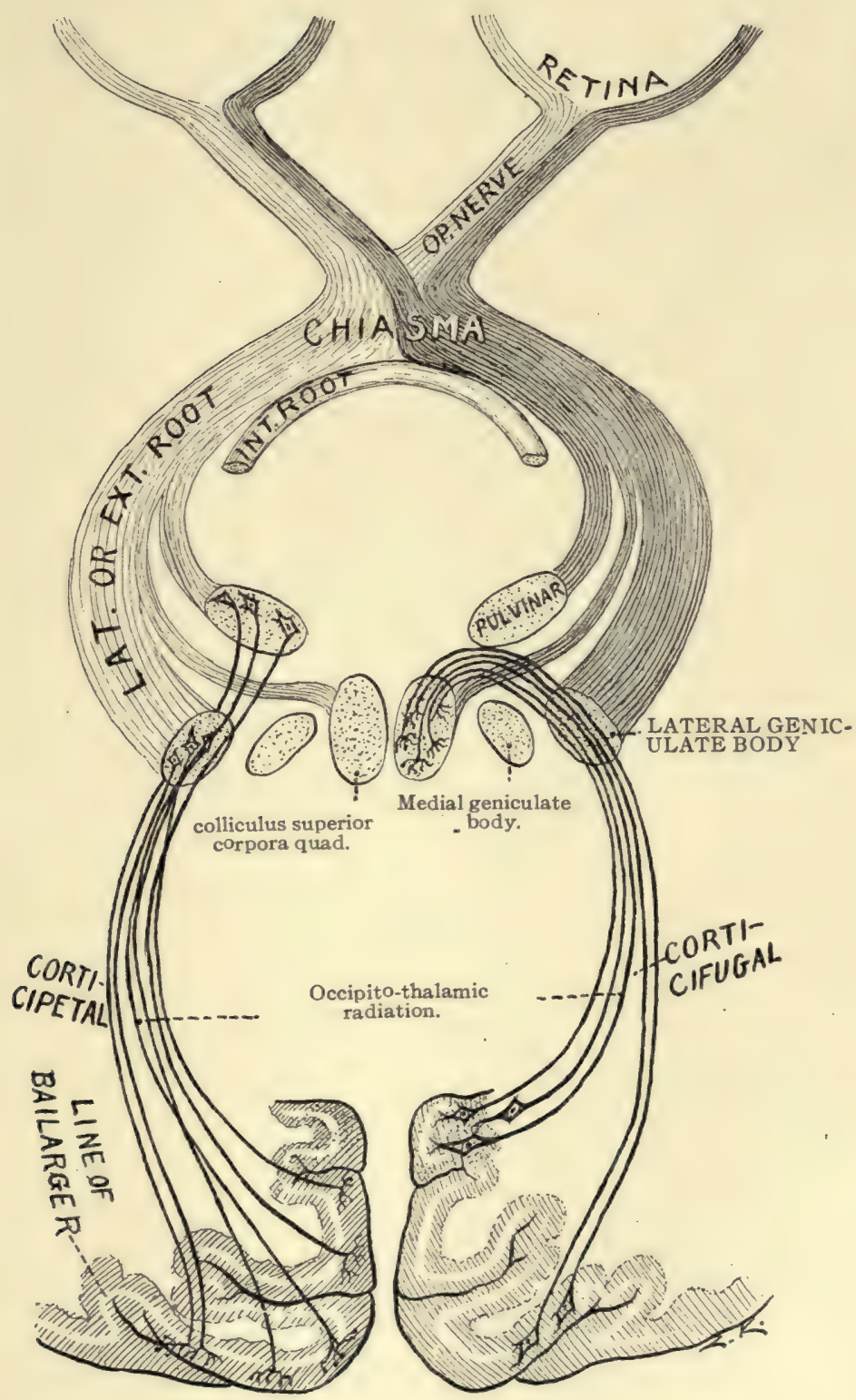

Fig. 6\%--The optic path. (Original.) 

form nucleus, where it probably terminates; but the conduction path to which it belongs is continued to the upper third of the posterior central and the paracentral gyri by the alpha and gamma bundles of Flechsig. The lower lamina of the ventral stalk, the inferior peduncle, runs principally into the external capsule medial to the claustrum, but a part of it dips under the claustrum. It terminates in the temporal lobe and the island. (b) The superior

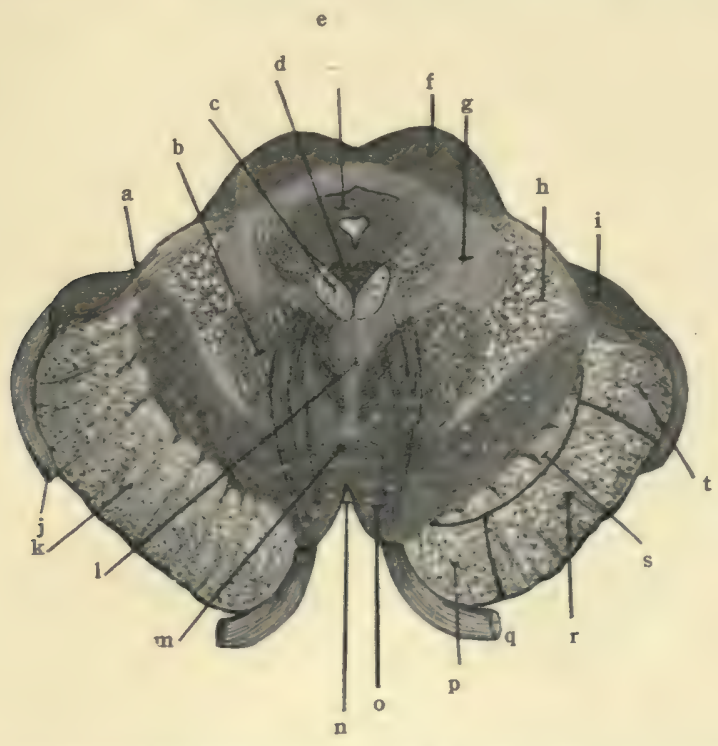

Fig. 68.-Section of the mid-brain through superior colliculi and the apparent origin of the oculomotor nerve. (Original.)

a. Sulcus laternlis of; mid-brain. b. Red nucleus. c. Medial longitudinal bundle. d. Oculomotor nucleus. e. Stratum griseum centrale. f. Colliculus superior of coroora quadrigemina. g. Formatio reticularis. h. Medial fillet. i. Medial geniculate body. j. Optic tract. k. Basis pedunculi. 1. Dorsal tegmental decussation (Meynerti). m. Ventral tegmental decussation (Foreli), n. Fossa interpeduncularis, o. Substantia nigra. p. Fronto-pontal tract. q. 3 d. N. r. Pyramidal tract. s. Intermediate tract. t. Temporo-pontal tract.

group of fibers that issues from the lateral surface of the thalamus is the parietal stalk (Fig. 70). It rises from the lateral nucleus and traverses the superior lamina of the internal capsule. To a slight extent it is probably relayed in the lentiform nucleus. It terminates in the posterior central gyrus and in the paracentral lobule. This parietal stalk, in the upper limits of the internal capsule, contains nearly all of the common sensory fibers going 
to the cortex. Flechsig has found that in development it is built up of six distinct strands of fibers, which receive their medullary sheaths one after the other; and he names the bundles, according to the order of their medullation, after the first six letters of the Greek alphabet (see page 232 ).

(4) The frontal stalk (Fig. 70) streams from the anterior end of the lateral nucleus via the frontal part of the internal capsule. Its fibers end in the corpus striatum and the frontal cortex. In

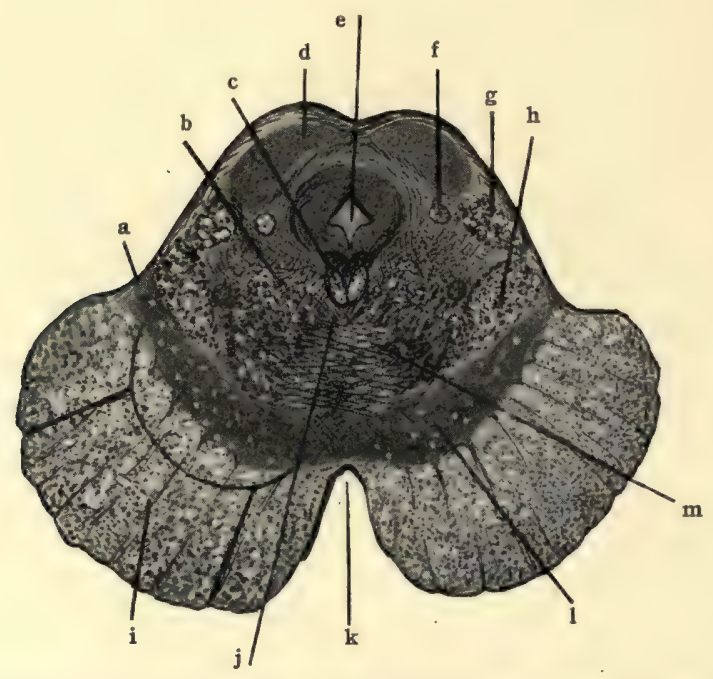

Fig. 69.- Section of the mid-brain cutting the inferior colliculi of the corpora quadrigemina. (Original.)

a. Sulcus lateralis, b. Formatio reticularis. c. Medial longitudinal bundle. d. Nucleus of colliculus inferior. e. Aqueductus cerebri. f. Rubro-spinal tract. g. Lateral fillet. h. Medial filliet. i. Basis pedunculi. j. Location of anterior longitudinal bundle. $\mathbf{k}$. Interpeduncular fossa. 1. Substantia nigra. m. Decussation of brachia conjunctiva.

the globus pallidus they are probably relayed to the somæsthetic cortex.

The ventral, parietal and frontal stalks of the thalamus constitute the cortical fillet; the parietal stalk, high up, contains nearly all the common sensory corticipetal fibers and, alone, is often called cortical fillet. The cortical fillet carries common sensations received from the medial fillet, the spino-thalamic tract, the medial longitudinal bundle and the brachium conjunctivum 
cerebelli, up to the somæsthetic area (Fig. 54). Interruption of the cortical fillet stops all common sensory impulses-tactile, pain and temperature impulses, and impulses of the muscular sense.

(5) Radiatio Occipito-thalamica (Gratioleti).-A large pencil of fibers, the oplic, or occipito-lhalamic radiation (Fig. 70) passes from the lateral geniculate body and the pulvinar through the internal capsule to the visual centers in the occipital lobe. A number of fibers in the occipito-thalamic radiation are corticifugal and end in the superior quadrigeminal colliculus, though many are probably relayed in the thalamus and lateral geniculate body. Destructive lesion of the occipito-thalamic radiation of either side produces hemianopia and atrophy of the same side of both retinæ.

(6) Acustic Radiation or Radiatio Temporo-thalamica (Fig. 70).-Another pencil of fibers radiates from the region of the medial geniculate body through the internal capsule to the auditory area in the temporal lobe. It constitutes the temporothalamic radiation. Its fibers are also in part corticifugal in direction. If the temporo-thalamic radiation be destroyed the result is partial deafness affecting chiefly the opposite ear.

According to von Bechterew certain lesions in the thalamus have been accompanied by loss of facial movements expressing emotion.

The red nucleus (nucleus ruber) of the tegmentum is situated beneath the thalamus (Figs. 42 and 46). It is a relay-station in the indirect sensory tract, receiving the opposite brachium conjunctivum cerebelli and, by its axones, continuing the tract to the thalamus and somæsthetic cortex. It also receives efferent axones from the cerebral cortex (Beevor and Horsley) and gives origin to two bundles: (a) One centrifugal bundle of axones, (the rubro-spinal tract), after crossing over in the ventral decussation of the tegmentum (Forel's) descends, first, with the medial portion of the lateral fillet; second, through the lateral area of the medulla, and, third, through the lateral part of the spinal cord. Gradually diminishing, it disappears at the first lumbar segment. It ends in the lateral columna and center of the gray crescent of the spinal cord. (b) The red nucleus also sends a bundle of axones through the opposite brachium conjunctivum of the cerebellum to the nucleus dentatus. 
The nucleus hypothalamicus (Luysi) (Figs. 30 and 42 ) is a pigmented bi-convex mass of gray matter placed ventro-lateral to the red nucleus, and between it and the basis pedunculi. It is separated from the red nucleus by the zona incerta. It constitutes an important relay for certain corticipetal fibers of the tegmentum and gives origin to others belonging to the cortical fillet. Certain descending fibers from the striate body terminate in this nucleus. The latter run through the tuber cinereum, just above the posterior border of the optic chiasma, and form the commissura superior (Meynerti), Gudden's commissure being called the commissura inferior.

Metathalamus. - The metathalamus is made up of two ganglia at the posterior end of the thalamus, called the geniculate bodies (Fig. 65). The lateral geniculate body (corpus geniculatum laterale) contains pigmented multipolar cells, which receive 80 per cent. of the optic fibers in the corresponding tract and give origin to a like per cent. of the corticipetal fibers in the occipitothalamic radiation; and, also, receive corticifugal fibers from the same radiation and originate a part of the brachium superius. The latter are reflex in function. As the dark cell-bodies are arranged in layers separated by laminæ of fibers, the body has a stratified appearance. The medial geniculate body (corpus geniculatum mediale) is gray in color. Placed at the end of the medial root of the optic tract, it would at first appear to be concerned in vision; but it is not visual in function. Moreover, it is a very important station in the auditory path. The medial optic root is merely Gudden's commissure, the fibers of which are supposed to rise in the medial geniculate body. This body contains chiefly fusiform cell-bodies which, in addition to their connection with the inferior commissure (of Gudden), receive the end-tufts of fibers in the brachium inferius and send their axones through the acustic, or temporo-thalamic radiation to the auditory cortex; the cells also receive corticifugal fibers through the temporo-thalamic radiation and, probably, shoot some of their axones backward through the brachium inferius to the corpora quadrigemina. The latter are reflex in function.

Ablation of the lateral geniculate body interrupts the visual 
path, destruction of the medial geniculate breaks the auditory path.

The superior colliculi of the corpora quadrigemina (Figs. 65 and 68) represent the optic lobes of birds, fishes and reptiles. They contain the center of optic reflexes. In being stratified, they bear some resemblance to the lateral geniculate bodies. They possess three white and two gray layers: (I) The stratum zonale (stratum album superficiale) is a layer of white matter on the surface. This invests the laminated stratum griseum, which forms the deep part of the colliculus and comprises two gray and two white layers: (2) The stratum griseum superficiale is composed of small multipolar cells. (3) The stratum album medium is a layer of fibers separating the small from the large multipolar cells. (4) The large cells make up the stratum griseum profundum, underneath which is another layer of fibers. (5) The stratum album profundum. The fibers of the superficial, middle and deep strata comprise, first, those that enter the colliculus through the optic tract and radiation, through the superior and a part of the lateral fillet, the striato-thalamic tract and the spino-thalamic tract; and, second, those that take origin in the colliculus and leave it through the brachium superius or the anterior longitudinal bundle. Of the fibers originating in the superior colliculus and running through the brachium superius it is supposed that some go as far as the retina; probably others enter the cortical fillet.

The anterior longitudinal bundle is made up of efferent axones of the cell-bodies in the superior colliculus. It crosses at once through the dorsal tegmental decussation (Fig. 68) and descends ventro-lateral to the opposite medial longitudinal bundle, to the anterior columna of gray matter in the spinal cord. Its fibers end largely in the nuclei of the third, fourth and sixth cerebral nerves and in the cervical enlargement of the spinal cord; but perhaps others enter the remaining nuclei of motor cerebral nerves, and a few fibers of the tract have been traced as low as the lumbar region. This bundle is the great optic reflex tract. The fibers to the nuclei of the third, fourth and sixth cerebral nerves bring about the reflex morements of the eyeball, contraction 
of the pupil and accommodation to distance; while those fibers which end in the gray substance of the lower part of the cervical enlargement of the spinal cord, called the cilio-spinal center, through the white rami communicantes and cervical sympathetic, produce dilatation of the pupil. The latter constitute the pupillodilator tract.

Destructive lesions affecting the superior quadrigeminal colliculi produce loss of reflex movement of the eyeballs, loss of pupillary reflex and loss of accommodation.

The inferior colliculi of the corpora quadrigemina form a relay in the auditory path (Figs. 65 and 69). They are made up of a white stratum zonale, whose fibers are continuous chiefly with the lateral fillet and brachium inferius, and of a deep gray mass, the mucleus colliculi iuferioris, which is composed of small multipolar cell-bodies in a network of fibers. The nuclei of the two eminences fuse in the median plane. In the nuclei end a considerable number of fibers belonging to both lateral fillets, but most of them belong to that of the same side; and from them proceed axones of the auditory paths through the brachia inferiora to the medial geniculate bodies. A few fibers of the spino-thalamic tract also end in the inferior colliculus. Again this colliculus receives a small bundle of the striato-thalamic fibers and, probably, corticifugal fibers of the temporo-thalamic radiation.

Though the greater part of the lateral fillet passes by the inferior colliculus without relay, a lesion in this body is apt to involve the entire bundle and cause almost complete deafness in the opposite ear.

Nucleus Lateralis Superior (Fig. 68).--In the reticular formation of the tegmentum at the level of the superior quadrigeminal colliculus is the nucleus lateralis superior. It contains large multipolar cell-bodies. The nucleus forms a relay both for ascending and descending fibers of the formatio reticularis. According to Tschermak, a small fasciculus runs from this nucleus into the medial longitudinal bundle where it divides T-like; and its descending fibers run down through the anterior fasciculus proprius of the cord (Barker). The spino-thalamic tract probably undergoes a partial relay in the superior lateral nucleus. 
Substantia Nigra (Figs. 66, 67, 68 and 69).-The small pigmented multipolar cell-bodies which make up the substantia nigra form, first, a terminal nucleus for certain fibers of the medial fillet and a nucleus of origin for other fibers which continue in that tract (Barker); and, second, a terminal station for the fasciculus retroflexus (Meynerti) and a relay for the intermediate tract from the corpus striatum. Beyond this terminal station the efferent tracts are probably continued, but with the exception of the intermediate tract they have not been traced. The intermediate tract is relayed to the nucleus pontis.

\section{CENTRAL, OR VENTRICULAR GRAY MATTER.}

It is located (I) in the floor and walls of the third ventricle, the hypothalamus; (2) in the middle commissure of that ventricle, the massa intermedia; and (3) around the cerebral aqueduct, the stratum griseum centrale.

(I) The Hypothalamus, Pars Optica.-The lamina cinerea terminalis and the tuber cinereum (Figs. 21 and 27 ) form a sheet of gray substance that connects the inferior and medial surfaces. of the cerebral hemispheres and may be called their inferior gray commissure. The optic chiasma is white matter, and the hypophysis is not composed of nerve tissue at all and, therefore, neither one need be described in this place. From the floor of the third ventricle the gray matter extends laterally beneath the thalamus, and is continuous with the anterior perforated substance. The gray matter of the floor also extends up to the sulcus hypothalamicus on the medial surface of the thalamus. The inferior gray commissure receives efferent fibers from the corpus striatum of both sides. Some of these fibers form a commissure just above that of Gudden; hence, it is called the commissura superior (Meynerti) to distinguish it from the commissura inferior (Guddeni) in the optic chiasma. The fibers of Meynert's commissure cross through the tuber cinereum.

Hypothalamus, Pars Mammillaris (Figs. 26 and 46).-The corpora mammillaria (albicantia), though composed of fornix fibers on the surface, contain in the interior two nuclei, the medial and lateral. The medial nucleus is the larger of the two. It 
receives the end-tufts of the fibers in the columna of the fornix and gives origin to the fasciculus mammillaris princeps. The latter bifurcates, sending one branch, the thalamo-mammillary bundle (of Vicq d'Azyr), up to the anterior nucleus of the thalamus and the other branch, the fasciculus pedunculo-mammillaris, backward into the tegmentum. The fasciculus thalamomammillaris (Vicq d'Azyri) connects the fornix with the thalamus. The tegmental part of the pedunculo-mammillary bundle has been traced, in the mouse by Cajal, downward to the lower part of the pons, running anterior to the medial longitudinal bundle (Barker). The small lateral nucleus of the corpus mammillare gives origin to the basilar part of the pedunculo-mammillary bundle, which according to Flechsig ends in the substantia grisea centrale of the mid-brain and is thence connected with the motor nerve-nuclei and the automatic centers of the medulla (Barker). Through the fornix, the stria medullaris thalami and the fasciculus retroflexus, and through the fornix and the pedunculomammillary bundles the reflex connections of the olfactory nerve are in part established.

(2) The massa intermedia (the middle commissure, Figs. 27 and 64) joins the medial nuclei of the thalami. It is formed, when present, by the approximation and fusion of the thalami in the second month of embryonic life. It is occasionally absent. In the massa intermedia are cell-bodies and transverse fibers. The latter appear to be loops which reach only to the median line; at least many of the fibers do not cross to the opposite side. It is not a commissure in the ordinary sense of that term.

(3) The stratum griseum centrale of the mid-brain (Figs. 46, 68 and 69) surrounds the cerebral aqueduct (Sylvii). This gray matter begins in the lateral wall of the third ventricle. It extends through the mid-brain and is continuous with the gray substance in the floor of the fourth ventricle. Besides the nuclei of the third, fourth and a part of the fifth cerebral nerves, it contains scattered cell-bodies of variable size and shape which give origin to the true commissural fibers of the posterior commissure. According to Flechsig this central gray substance receives the basilar part of the pedunculo-mammillary bundles and probably 
gives origin to fibers that descend to the motor nuclei of the pons and the medulla.

Oculomotor Nucleus (Figs. 45 and 68).- The nucleus of the third cerebral nerve (nucleus nervi oculomotorii) is an elongated mass of gray substance in the ventral part of the stratum griseum centrale, which extends from the lateral wall of the third ventricle down to the level of the transverse groove between the quadrigeminal colliculi. The nuclei are placed somewhat obliquely; at the lower end they fuse in the median plane. According to Perlia, seven distinct cell-nests are found in each nucleus; and nest fifteen occupies the area of fusion and is common to both nerves. The greater number of axones of this nucleus run forward into the nerve of the same side; but those from the median nest go into both nerves, and a small bundle from each nucleus descends with the medial longitudinal bundle to the colliculus facialis, where it joins the facial nerve and through that nerve supplies the muscles of facial expression above the orbit.

Trochlear Nucleus (Fig. 69).--The nucleus nervi trochlearis is a small oval mass of cell-bodies situated anterior to the inferior colliculus of the corpora quadrigemina. It is in the ventral part of the stratum griseum centrale like the oculomotor nucleus. Unlike the third, the axones from the nucleus of the fourth cerebral nerve run backward and issue from the posterior surface of the mid-brain at the isthmus; they are peculiar also, in that the axones decussate before their emergence (Fig. 65).

The nuclei of the oculomotor and trochlear nerves receive fibers from the cerebral cortex through the pyramidal tract and other motor tracts of the internal capsule and thus obtain their voluntary motor and inhibitory impulses. It is probable also that the third nucleus receives fibers, through the medial longitudinal bundle, from the opposite abducent nucleus, and that the part of the nucleus which receives these fibers supplies the internal rectus muscle of the eye. For the purpose of reflex both the oculomotor and trochlear nuclei receive fibers from the anterior and medial longitudinal bundles, from the pedunculo-mammillary bundles (?) and, perhaps, from the cerebellum through the brachia conjunctiva. 
Trigeminal mucleus of the mid-brain is a very small nucleus situated in the extreme lateral part of the central gray matter. It is continuous with the pontine nucleus of the fifth, located under the locus cæruleus, and is merely the superior end of the motor nucleus of the trigeminal. It gives origin to the descending root of the fifth nerve, which descends to the pons and there joins the main motor root. In its course downward the mesencephalic root runs between the central gray matter and the brachium conjunctivum cerebelli. .

Lesions of these cerebral nerve nuclei are apt to involve the tegmentum. If so, the result is paralysis of the nerves on the same side and hemianæsthesia, hemiataxia, loss of taste (?) and deafness on the opposite side.

The white matter of the cerebrum is composed, in the adult condition, of medullated fibers; the medullation begins in the ninth month, in utero, and is continued for a considerable time after birth (Flechsig). Within the cortical substance the myelin sheaths continue to be laid down until late in life (Kaes, McMurrich). The cerebral fibers form three definite systems:

I. Projection, or peduncular fibers.

2. Transverse, or commissural fibers.

3. Association fibers.

\section{PROJECTION FIBERS.}

The projection fibers are connected only with the motor and sensory areas of the cerebral cortex and are, therefore, motor and sensory in function (Figs. 54 and 55). Where they are present they are continuous with Meynert's radiations. They are composed, first, of the medullated axones of the pyramids and the polymorphous neurones; these descend from the cerebral cortex, are motor in function, or corticifugal, and constitute the upper motor segment; and, second, they comprise the medullated axones of neurones whose cell-bodies are situated in gray matter below the cerebral cortex; these axones ascend to the cortex and are sensory in function, or corticipetal. The projection fibers run from cerebral cortex through the corona radiata, the internal 
capsule and the mid-brain, and vice versa (Figs. 30 and 42). They connect the cortex, directly or indirectly, with all parts of the body, throwing or projecting a picture of every part and organ upon the cerebral cortex. Many of the fibers are interrupted in the basal ganglia, especially of the corticipetal fibers. Within the hemisphere all projection fibers run through one great sheet, the internal capsule, with the exception of the olfactory; but in the mid-brain, they are separated into two great groupsthe basis pedunculi and the tegmentum, the substantia nigra intervening.

\section{CORTICIFUGAL, OR MOTOR PROJECTION FIBERS.}

The most important tracts of corticifugal or motor projection fibers are the following, namely, the intermediate tract, the frontopontal tract, the pyramidal tract and the temporo-pontal tract.

The intermediate tract (stratum intermedium pedunculi, Figs. 70,69 and 87) extends from the corpus striatum through the inferior lamina of the capsule and the deep part of the basis pedunculi to the motor cerebral nuclei and to the nucleus pontis, though it is probably relayed in the substantia nigra. From the nucleus pontis axones run by way of the brachium pontis to the cortex of the opposite hemisphere of the cerebellum. The intermediate tract thus forms a segment of an indirect (through the cerebellum) efferent, or motor path.

The fronto-pontal tract (tractus cerebro-cortico-pontalis frontalis. Figs, 70, 7r, 69 and 87) rises from the cortex of the frontal lobe anterior to the precentral sulci. It tranverses the centrum semiovale, corona radiata, frontal part of the internal capsule and medial one-fifth of the basis pedunculi to the ventral area of the pons, where it terminates in the nucleus pontis (chiefly) and in the nuclei of motor cerebral nerves (Flechsig). It is probably relayed in the thalamus (Beevor and Horsley).

According to Dejerine, the temporo-pontal tract (tractus cerebro-cortico-pontalis temporalis, Figs. 70, 71, 69 and 87) extends from the temporal lobe through the inferior lamina (and posterior part of the superior lamina) of the internal capsule and lateral one-fifth of the basis pedunculi to the substantia nigra and the 
nucleus pontis; but according to Spitzka some of its fibers end in the nuclei of motor cerebral nerves. Thus it should be noted that, with the exception of those fibers to motor nuclei of the cerebral nerves, each of the three tracts above mentioned, viz., the intermediate, fronto-pontal and temporo-pontal, constitutes a segment of an indirect efferent path which is interrupted in the nucleus pontis and then continued by the axones of that nucleus through the brachium pontis of the cerebellum. It is probable, though not surely established, that the fronto-pontal and temporo-

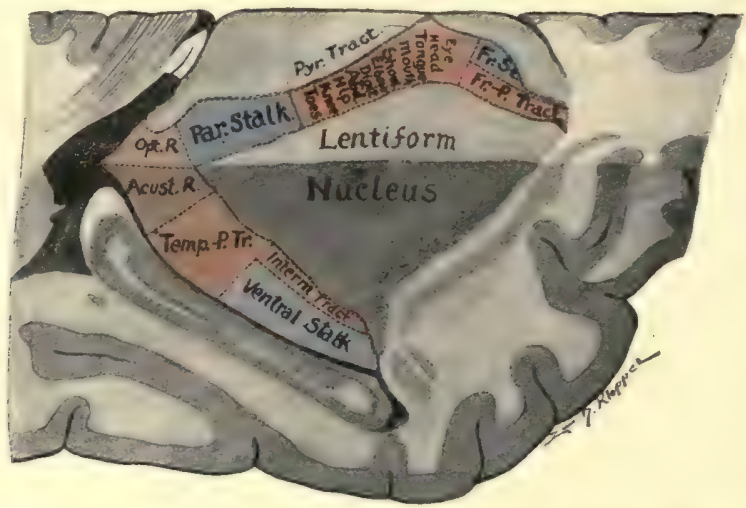

Fig. 70.-Horizontal and sagittal section through internal capsule, much enlarged. (Original.)

Blue, Common sensory tracts: Fr. St., Frontal stalk; Par. Stalk, Parietal stalk; Ventral stalk. RED, Motor tracts: Fr.-P. Tract, Fronto-pontal tract; Pyr. Tract, Pyramidal tract; Temp.-P. Tr., Temporo-pontal tract; Interm. Tract, Intermediate tract. PURPLE, Special sense tracts: Opt. R., Occipito-thalamic radiation; Acust. R., Temporo-thalamic radiation.

pontal tracts are relayed in the corpus striatum or thalamus, as they have been found undegenerated in the base of the peduncle when their cortical origins were destroyed by extensive lesions.

The pyramidal tract (tractus cerebro-spinalis pyramidalis) (Figs. 70 and $7 \mathrm{I}$ ) rises in the anterior central gyrus and the precentral part of the paracentral lobule. It is composed of axones from the giant pyramids and large polymorphous cells of that region. Descending through the corona radiata, genu and anterior two-thirds of the occipital part of the internal capsule, the pyramidal tract comprises the middle three-fifths of the basis pedunculi, enters into the anterior longitudinal fibers of the pons, 
forms the pyramid of the medulla and the anterior and lateral pyramidal tracts of the spinal cord (Figs. 7x, 69, 87, 93 and I02). The fibers of the pyramidal tract, with a few exceptions, cross over to the opposite side; they end in connection with the motor nuclei of cerebral and spinal nerves. Fibers enter the nucleus of the trochlear (or fourth) nerve chiefly on the same side, and a few descend to the motor nuclei of other cerebral nerves and to the gray matter in the spinal cord without decussation; all other pyramidal fibers terminate on the side opposite to their origin. The fibers from the lower one-fourth of the anterior central gyrus, which go to the motor nuclei of thè cerebral nerves, to a large extent

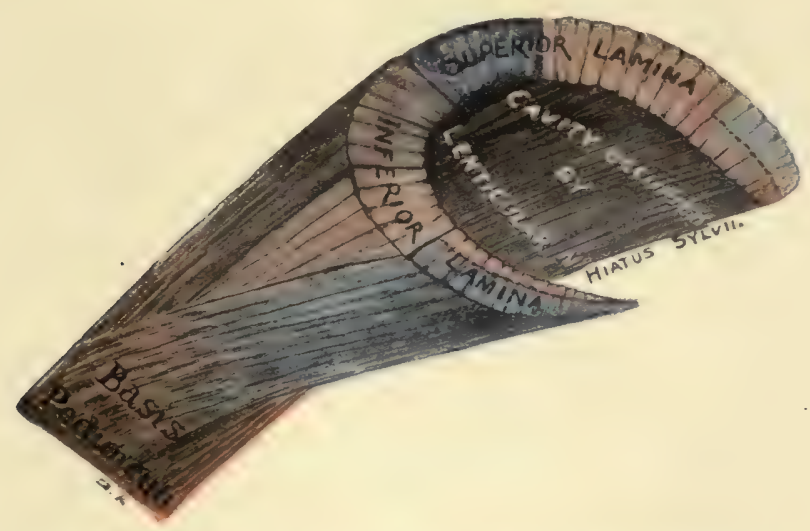

Fig. 71.-Diagram of internal capsule in colors. (Original.)

RED, motor; BLUE, common sensory; PURPLE, special sensory.

leave the pyramidal tract high up in the peduncle and run for some distance through the medial portion of the fillet; they constitute Bechterew's accessory lemniscus. This accessory fillet has been recently traced by Flechsig.

Head and Neck Fibers (Figs. 70, 105 and 106).-Those fibers of the pyramidal tract which end in the nuclei of the cerebral and the upper four cervical nerves rise in the lower segment of the motor area, including that part of the anterior central gyrus below the genu inferius of the central sulcus. They run through the genu of the internal capsule to the peduncle and, then, both through 
the accessory fillet and the inner portion of the middle threefifths of the basis pedunculi. Upper Extremity Fibers (Figs. 70 and 105).- The fibers of the pyramidal tract that end in the cervical part of the spinal cord, and through it innervate the muscles of the upper extremity, take their origin from that part of the anterior central gyrus adjacent to the foot of the middle frontal gyrus: their origin lies between meridians which intersect the central sulcus at the genu inferius and the genu superius, respectively. These fibers run through the pars occipitalis of the internal capsule just behind the genu, and through the basis pedunculi immediately lateral to the head and neck fibers. Those fibers which innervate the muscles of the thumb, fingers and hand, rise lowest down in the arm area of the cortex and occupy the posterior part of the arm bundle in the internal capsule and the lateral part of it in the peduncle. The fibers which control the shoulder muscles rise in the upper part of the cortical area and form the anterior and medial part of the arm bundle in the capsula interna and basis pedunculi, respectively; while the wrist, forearm, elbow and arm are innervated by means of fibers which are intermediate in both origin and course. Trunk Fibers.The trunk fibers of the pyramidal tract rise in that projection of the anterior central gyrus which is situated just above the genu superius of the central sulcus. In the internal capsule, the trunk fibers run just behind those to the fingers and just lateral to them in the basis pedunculi. Lower Extremity Fibers (Figs. 70 and 105). -A large number of the pyramidal fibers terminate in the lumbar enlargement of the spinal cord and carry impulses to the nerves of the lower extremity. They originate in the upper fourth of the anterior central gyrus and in the paracentral lobule. The hip fibers rise farthest downward and the toe fibers farthest upward, immediately in front of the sulcus centralis. The fibers have the same relative position in the internal capsule; in the base of

\section{Description to Fig. 72.}

a, a. Motor cells of cerebral cortex. b, b. End-tufts of sensory fibers in cortex. c. Nucleus of funiculus cuneatus, showing end-tufts of fibers from the cord. d. Nucleus of funiculus gracilis, containing end-tufts of fibers from cord. e. Section of medulla at fillet decussation. $\mathbf{f}$. Section of medulla at pyramidal decussation. g, g. Motorial end-plates. h. Section of cervical cord, showing terminations of fibers of anterior and lateral pyramidal tract. i, i. Spinal ganglia. j, k. Short sensory fibers. 1. Long sensory fibers. m, m, m. Sensory end-organs. n. Section of lumbar cord. 


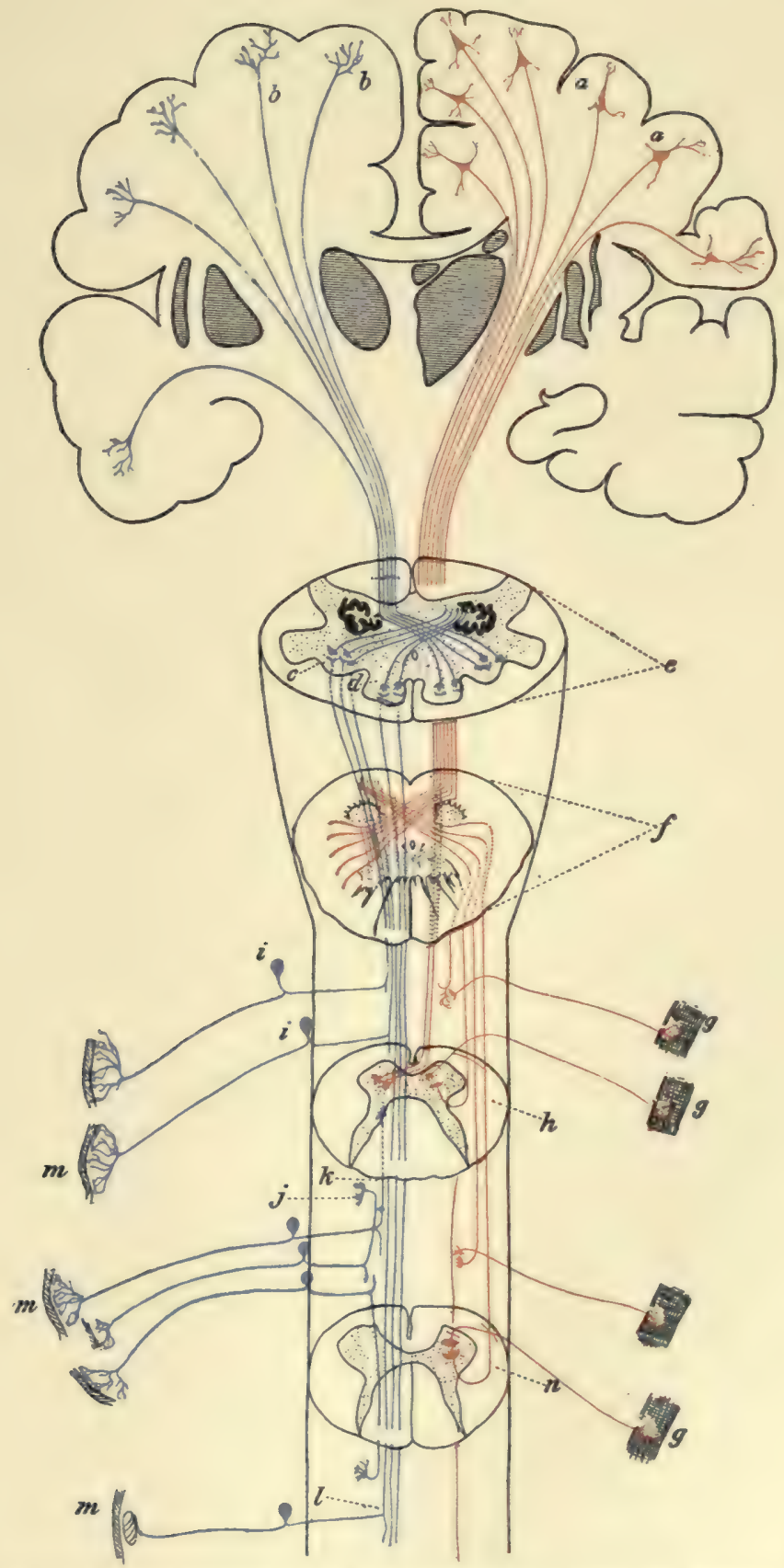

Fig. 72.-A diagram showing motor and sensory paths; motor red, sensory blue. (After Gordinier.) 

the peduncle the hip fibers are medial and the toe fibers lateral. Fibers which innervate the muscles of the thigh, leg and small toes have this same relative position and order between the hip and great toe fibers both in their cortical origin and in their course through the internal capsule and basis pedunculi.

There are other corticifugal fibers in the internal capsule, viz., some within the occipito-thalamic and temporo-thalamic radiations (Figs. 70 and $7 \mathrm{r}$ ) and others running from the special and common sensory areas of the cortex; but these fibers are probably reflex-in function and do not properly belong to the projection group.

Several bundles of descending fibers are found in the tegmentum, namely, the anterior longitudinal bundle, which is reflex in function, the rubro-spinal tract from the red nucleus, a small part of the brachium conjunctivum of the cerebellum, the descending root of the trigeminal nerve, the olivary bundle, and certain other fibers in the formatio reticularis. With these exceptions the tegmentum is ascending in direction and sensory in function.

Destruction by clot or tumor, or otherwise, of any of the above divisions of the pyramidal tract causes upper segment paralysis of the particular muscles innervated through that tract, the muscles being spastic and the reflexes increased.

\section{SENSORY OR CORTICIPETAL PROJECTION FIBERS.}

The sensory or corticipetal projection fibers of the tegmentum comprise the medial, superior and lateral fillets; the.spino-thalamic tract; the brachium conjunctivum of the cerebellum; a part of the medial longitudinal bundle; and certain other ascending fibers of the formatio reticularis. Excepting a small number of fibers, all these bundles terminate in the basal ganglia; but the paths of conduction are continued through the internal capsule. The medial fillet carries impressions of the tactile and the muscular senses; the spino-thalamic tract conducts tactile, pain and temperature impulses; while all varieties of common sensory impulses are carried by the brachium conjunctivum cerebelli. Chiefly through these three tracts, common sensory impressions 
arrive in the lateral nucleus of the thalamus. In the capsula interna the corticipetal projection fibers constitute the cortical fillet and the optic and acustic (and gustatory?) radiations. The former end in the somæsthetic area of the cerebral cortex, the latter in the visual, auditory and gustatory cortex.

The olfactory projection fibers are contained neither in the tegmentum nor in the internal capsule. They proceed from the olfactory bulb, through the olfactory tract and its striæ, directly to the cerebral cortex.

The exact origin of the cortical fillet (Figs. 70 and $7 \mathrm{I}$ ) has not been entirely determined, but it is known to rise, chiefly; in the lateral nucleus of the thalamus. The ventral stalk of the thalamus (Fig. 7o) runs through the internal capsule, in the inferior lamina. It is relayed largely in the globus pallidus. Its fibers enter the medullary laminæ of the nucleus lentiformis and the external capsule; ultimately they terminate in the upper onethird of the posterior central gyrus and in the temporal and insular lobes (see p. 212). From the anterior end of the thalamus streams a great pencil of fibers, called the frontal stalk (Fig. 70). It mingles to a small extent with the fibers of the pyramidal tract, but runs chiefly through the frontal part of the internal capsule. Its termination is largely in the corpus striatum; some of its fibers reach the frontal cortex.

The parietal stalk issues from the lateral surface of the thalamus higher up than the ventral stalk and mingles with the pyramidal fibers in the superior lamina of the internal capsule. Its location is principally in the posterior third of the occipital part of the capsule (Figs. 70 and 7I). It terminates in the paracentral lobule and the posterior central gyrus. According to Flechsig some fibers also end in the upper extremity of the anterior central, the superior frontal gyri and in the underlying part of the gyrus cinguli. The parietal stalk, if we may apply this name to all the common sensory fibers in the occipital part of the capsule, ultimately comprises all the corticipetal fibers to the somæsthetic area. Flechsig gives their origin and termination, in the order of their medullation, as follows:

a. The alpha bundle, a small one, runs from the globus pal- 
lidus to the upper third of the central gyri, chicfly to the anterior central. Origin is in doubt.

$\beta$. A large bundle from the posterior inferior part of the lateral nucleus to the upper third of the central gyri, chiefly to posterior central gyrus and adjacent part of the paracentral lobule.

$\gamma$. The gamma bundle is large. It runs from the globus pallidus to same area as beta bundle. Origin is in doubt.

$\delta$. Runs from the lateral nucleus of thalamus (from the central of Luys and the arcuate nuclei) to the middle third of the central gyri, principally to the posterior central.

$\epsilon$. A bundle from the antero-inferior part of the lateral nucleus to the lower third of the posterior central gyrus.

$\zeta$. The zeta bundle is last to be medullated. It rises in the superior part of the lateral nucleus and terminates in the foot (posterior end) of the superior frontal gyrus and in that part of the gyrus cinguli which underlies the foot of the first frontal.

The cortical fillet conveys common sensory impulses to the somæsthetic area of the cerebral cortex. If the cortical fillet be severed, all common sensory impulses to that hemisphere are interrupted; and complete loss of common sensation on the opposite side of the body and hemiataxia result.

Just where the fibers conducting taste impulses are located in the tegmentum is unknown Auditory impulses run through the lateral fillet and the brachium inferius to the medial geniculate body; while optic impulses run directly to the lateral geniculate body and the pulvinar without passing through the mid-brain at all. Within the internal capsule the gustatory tract cannot at present be located; but the acustic and visual paths are well known.

The acustic, or temporo-thalamic radiation (Figs. 70 and 54) continues the auditory path from the medial geniculate body through the retrolentiform part of the internal capsule, to the transverse temporal gyri and the third and fourth fifths of the superior temporal gyrus (Barker). Interruption of these fibers produces deafness in the opposite ear, which is not complete because the acustic path is not wholly crossed. There are some 
corticifugal fibers in the temporo-thalamic radiation; they are probably reflex in function.

The optic, or occipito-thalamic radiation (Figs. 70, 38, 45 and 55 ) rises in the lateral geniculate body and in the pulvinar of the thalamus. It continues the visual conduction path through the retrolentiform region of the internal capsule to the cortex of the lingual and cuneate gyri. Half-blindness in the same side of both retinæ results from section of the optic radiation. The corticifugal fibers in the occipito-thalamic radiation are believed by Campbell to be axones of the solitary giant cells (Meynert's) in the occipital cortex. They run through the lateral geniculate body and brachium superius to the superior colliculus of the corpora quadrigemina, where they end in contact with the neurones of the anterior longitudinal bundle. Their function is reflex.

\section{COMMISSURAL FIBERS.}

They connect opposite sides of the cerebrum and, like the projection fibers, are continuous with the radiations of Meynert. They are contained chiefly in the corpus callosum, the anterior commissure, and the commissura hippocampi; but are also found in the posterior commissure, commissura hebenularum, inferior (Gudden's) and superior (Meynert's) commissures.

The corpus callosum, as already described, is the great link between the cerebral hemispheres (Figs. 34, 27 and 73). Its fibers connect both similar and dissimilar parts of the cortices; within the hemisphere, they form a prominent radiation, called the radiatio corporis callosi. The corpus callosum is made up almost wholly of cortical axones, a few of them being projection fibers; of a small number of afferent projection fibers, and of collaterals from the association and projection fibers. All callosal fibers, except the few efferent projection fibers, end on the opposite side in arborizations within the cortex. It is the corpus callosum, chiefly, that makes it possible for the two hemispheres of the cerebrum to act together as one organ. Philogenetically, it is of recent development, since it is not found below mammals.

The anterior commissure (Figs. 73, 40 and 30) joins the 
opposite temporal and occipital lobes together (pars occipitotemporalis), the limbic lobes with the contra-lateral olfactory tracts, and the olfactory tracts with each other (pars olfactoria). It is supplementary to the corpus callosum and associates regions not joined by the great commissure, especially the cortex of the tentorial areas of the cerebral hemispheres. In size it varies

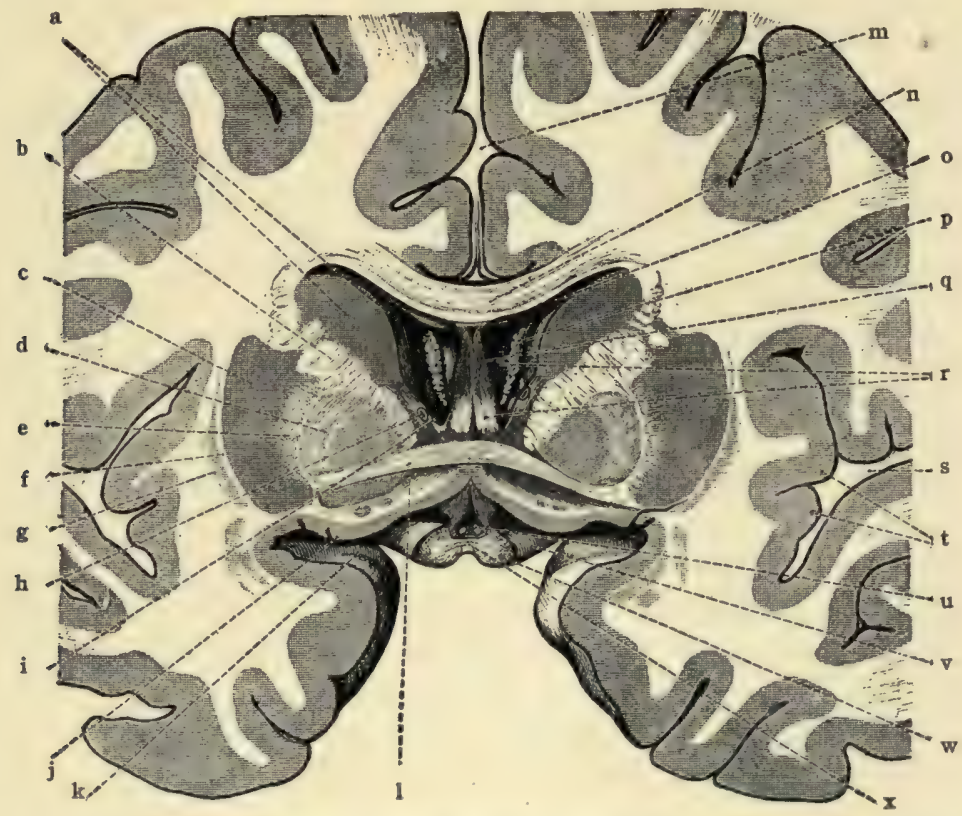

Fig. 73.-Transverse section of cerebrum, cutting corpus callosum, anterior commissure and optic chiasma. Viewed from front. Commissural fibers. (Morris's Anatomy after Toldt.)

a. Caudate nucleus (head). b. Internal capsule (frontal portion). Lentiform nucleus: c. Putamen, d Globus pallidus. e. Medullary lamina. f. External capsule. g. Claustrum. h. Vena terminalis, i. Interventricular foramen (Monroi). j. Anterior perforated substance. k. Uncus. 1. Anterior commissure. m. Longitudinal fissure. n. Corpus callosum. v. Anterior horn of lateral ventricle. p. Chorioid plexus of lateral ventricle. q. Septum pellucidum. r. Columns of fornix. s. Lateral fissure (Sylvii). t. Gyri of insula. u. Optic recess: v. Optic tract. w. Optic chiasma. x. Inferior commissure (Guddeni).

inversely as the corpus callosum. Its importance diminishes with the appearance of the corpus callosum in the lower mammalia and it continues to decrease as the higher forms are approached. Below mammals it is said to be the most important connecting link between the hemispheres and is philogenetically very old. 
The commissura hippocampi, the lyre (Fig. $3^{6}$ ), unites the hippocampal gyrus, dentate fascia, and the hippocampus with their fellows of the opposite side. This is the commissure of the pyraform lobes, the cortical areas of smell.

\section{ASSOCIATION FIBERS.}

These fibers remain on the same side and connect parts of the same hemisphere. They are situated within or beneath the cortex, the various parts of which they serve to unite. Association fibers become medullated and actively functional only as mental effort and education gradually develop them. So far as the brain is concerned education consists, first, in the development of the functional centers of the brain; and, second, in the establishment of lines of rapid communication between them.

The short association fibers are the more numerous and are very important. They unite contiguous parts of the same gyrus and associate together adjacent gyri. They are intralobar. In direction they comprise arcuate and tangential fibers; and they are iniracortical and subcortical, in position. Every zone of the cerebral cortex contains association fibers, from the felt-work of Kaes to the stratum zonale. But they are found chiefly (I) in the radiary zone and adjacent part of the supraradiary zone, along the line of Baillarger (Fig. 60); and (2) in the zonal layer (Figs. $5^{8}$ and 6I). (I) Those sparsely scattered large fibers whose location is indicated by the line of Baillarger are called Meynert's association fibers. The deeper of these fibers are continuous with the radiations of Meynert and probably do not belong to the short association fibers, if associative at all (they are corticipetal fibers); the more superficial, intersect the radiations at right angles and are truly associative in function. The associative fibers of Meynert are compacted together by pressure in the walls and floor of the sulci. In the crown of a gyrus they are scattered. Their exact origins are not yet worked out; but they are probably the horizontal processes of cells in the second to sixth layers. The association fibers of the plexiform layer of the cortex, which constitute the stratum zonale (Fig. 6I), are quite short when compared with those of Meynert; they join together immediately 
contiguous parts within circumscribed areas. The richness of the zonal layer of fibers, as already pointed out in describing the plexiform layer of the cortex, varies greatly in different regions, being best developed in the subiculum. The fibers comprising the zonal layer have four sources of origin: (a) The axones and dendrites of the cells of Cajal in the plexiform layer. (b) The apical dendrites of the subjacent pyramids. (c) The T-branched

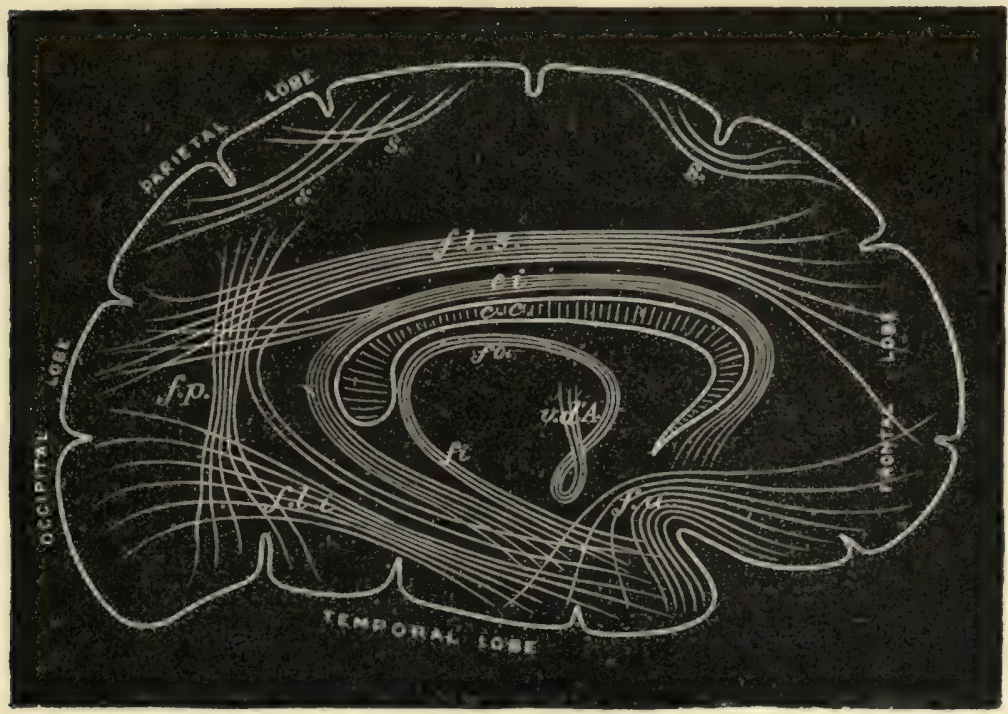

Fig. 74.-Diagram of association fibers in the cerebral hemisphere. (Gordinier and Quain after Meynert.)

s. Short association fibers, connecting adjacent gyri. f.l.s. Fasciculus longitudinalis superior. c.i. Cingulum. f.p. Fasciculus perpendicularis. $f . l . i$. Fasciculus longitudinalis inferior. f.u. Fasciculus uncinatus, fo. Fornix. fi. Crus fornicis, v.d'A. Thalamo-mammillary bundle of Vicq d'Azyr.

axones of Martinotti's cells. (d) The corticipetal axones which terminate in the superficial layer of the cortex.

The short association fibers are almost infinite in their connections. They connect the receptive and psychic sensory areas, and their interruption on the left side causes inability to interpret the sensations, called mind-blindness, mind-deafness, stereagnosis, etc. Again, those short fibers also associate the psychic with the psychic-motor, and the psychic-motor with the emissive- 
motor centers. In this manner the writing center is connected with the motor center for the upper extremity, and the speech center with the motor centers for the lips, tongue, etc.: breaking of the former connection on the left side destroys ability to write, agraphia; and aphasia results, if the latter connection is broken. Besides these and many other connections of associated centers, the short fibers join together the various parts of each cortical area.

The long association fibers (Figs. 74 and 75) are collected into bundles. They rise from the pyramidal, the polymorphous and the fusiform layers of the cerebral cortex (Cajal), and are axones. Proceeding out of the lobe in which they rise, being interlobar, they dip down into the centrum semiovale and arborize about neurones in more or less distant parts of the cortex. Among the best known are the following bundles:

(I) The cingulum of the gyrus fornicatus (Fig. 74) is a bundle of fibers in that gyrus which almost entirely encircles the corpus callosum. It extends from the anterior perforated substance through the gyrus cinguli and hippocampal gyrus, to the uncus and temporal pole. The fibers, which form several systems, have been divided into three groups by Beevor, namely: (a) The anterior, which joins the region of the anterior perforated substance to the fore part of the frontal lobe. (b) The horizontal, which unites the frontal lobe and the gyrus fornicatus. (c) The posterior fasciculus, which associates the lingual and fusiform gyri with the hippocampal gyrus and the pole of the temporal lobe. Like the two following bundles it establishes associations for the sense of smell.

(2) The Fornix (Fig. 74). - In each lateral half of the fornix is a bundle of association fibers as well as of projection and commissural fibers. The projection fibers rise in the olfactory bulb and in the region of the olfactory triangle and, running up through septum pellucidum to the fornix, continue through it to their destination in the hippocampus and uncus. The commissural fibers of the fornix rise in the uncus, the fascia dentata and gyrus hippocampi and run through the alveus of the hippocampus into the crus fornicis, whence they cross through the commissura hip- 
pocampi to the opposite side (Fig. 36). They terminate in the dentate fascia and hippocampal gyrus, including the uncus. The associative group of fibers in the fornix has the same origin as the commissural group. It enters crus fornicis and continues through the body of the fornix (corpus fornicis) into the columna, where

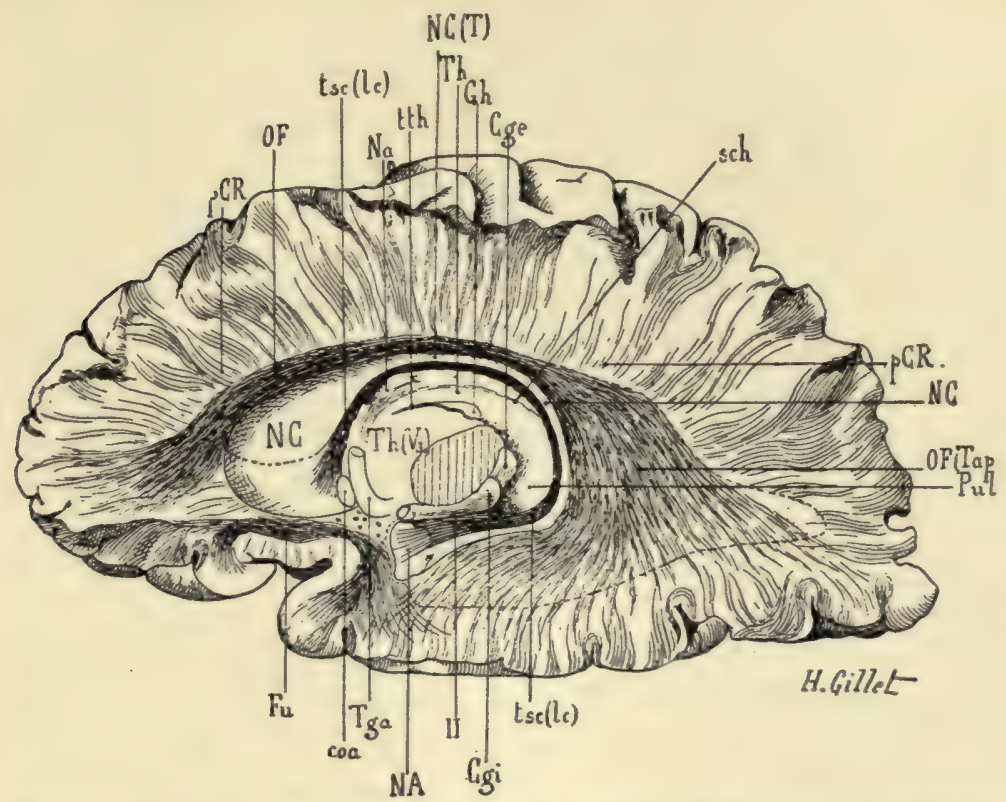

Fig. 75.-Fasciculus occipito-frontalis. Stria terminalis and fasciculus uncinatus. (Gordinier after Dejerine.)

Cge. Lat. geniculate body. Cgi. Med. geniculate body. coa. Anterior commissure. Fu. Fasciculus uncinatus. Gh. Ganglion of the habenula. NA. Amygdaloid nucleus. Na. Anterior nucleus of thalamus. NC. Head of caudate nucleus. NC' Tail of caudate nucleus. NC $(T)$. Body of caudate nucleus. OF. Fasciculus occipito-frontalis. OF (Tap). Part of the fasciculus occipito-frontalis forming the tapetum. pCR. Foot of corona radiata. Pul Pulvinar. sch. Choriodal fissure. Tga. Columna of fornix. Th and $\mathbf{T h}\left(\mathbf{V}_{3}\right)$. Thalamus. tsc(lc). Stria terminalis. th. Stria medullaris thalami. II. Optic tract.

it divides into two unequal bundles: The smaller is the stria medullaris thalami (pineal stria) (Fig. 40), which bends backward and runs along the supero-medial border of the thalamus to the nucleus habenulæ; in part it decussates in the commissura habenularum. By the fasciculus retroflexus of Meynert, it is connected with the inter-peduncular ganglion of the mid-brain. The larger bundle continues as columna fornicis (Fig. 74). As already 
indicated, it terminates in the medial nucleus of the corpus mammillare, partly on the opposite side. Its termination is associated first, with the anterior nucleus of the thalamus by the thalamomammillary bundle (of Vicq d'Azyr) and, second, with the midbrain and pons by the pedunculo-mammillary bundles.

(3) The uncinate fasciculus (fasciculus uncinatus, Fig. 74) is a bundle, with some sharply curved fibers, which arches over the main stem of the fissura cerebri lateralis, and connects the uncus and the anterior temporal region with the orbital gyri and the pole of the frontal lobe. It is situated near the basal surface. Its fibers spread out at both ends in the cortex, and they especially join the medial and posterior orbital and the inferior frontal gyri with the limbic lobe (Barker). Like the cingulum and fornix, it is connected with the rhinencephalon. Lesion in any one of these three bundles causes disturbance of smell.

(4) The superior longitudinal fasciculus (fasciculus longitudinalis superior, Fig. 74) is a sagittal bundle located beneath the convex surface of the hemisphere, just above the posterior ramus of the lateral fissure of the cerebrum. According to Cunningham, it runs just above and behind the putamen of the lentiform nucleus, external to the base of the corona radiata. Its fibers diverge at the posterior end of the lateral fissure and radiate into the parietal, occipital and temporal cortex: some of them, arching around that fissure, run as far forward as the temporal pole. The superior longitudinal bundle joins the frontal cortex with the parietal, occipital and the external temporal. It thus associates the psychic auditory and visual centers with the motor speech center; hence motor aphasia is the result of its interruption.

(5) The inferior longitudinal fasciculus (fasciculus longitudinalis inferior, Fig. 74) is about on a level with the lateral ventricle. It passes near the outer wall of the inferior and posterior horns of that ventricle, being separated from them by the occipitothalamic radiation and the tapetum. It connects the temporal lobe to the occipital. In the temporal lobe its fibers cross at right angles those of the inferior lamina of the internal capsule. This fasciculus unites the auditory and visual psychic centers, and 
thus associates the concepts of things seen with those of things heard. Breaking of this connection produces intercortical sensory aphasia. So, an object seen is recognized, but fails to suggest its name; and the spoken word is heard and understood, but does not recall the visual picture of the object or person it represents. According to Ferrier and Turner the inferior longitudinal bundle is not found in the Macaque monkey, but is present in the chimpanzee and the orang.

(6) The Fasciculus Occipito-frontalis (Foreli).-This is a large bundle of fibers formerly regarded as a part of the corpus callosum (Fig. 75). It is situated below the corpus callosum and, in equitorial sections of the brain, is found in the angle formed between the callosum and the internal capsule, just external to the lateral ventricle. It extends from the cortex of every part of the frontal lobe to the cortex of the convex surface and lateral border of the occipital lobe. Posteriorly, the fibers diverge to form a fan-like sheet in which there is an intermingling of fibers from the corpus callosum (Cunningham); and that sheet enters into the external boundary of the inferior horn of the lateral ventricle and into the floor, lateral wall and roof of the posterior horn, hence the synonym, tapetum. The tapetum is lined by the ventricular ependyma and is separated from the inferior longitudinal bundle by the occipito-thalamic radiation. Its particular function is unknown.

(7) The Perpendicular Fasciculus (Fasciculus Perpendicularis, Fig. 74).-This is a very broad vertical bundle located just in front of the occipital pole. It extends from the inferior parietal and superior occipital gyri, above, down to the middle and inferior temporal, the inferior occipital and the fusiform gyri. It is often classed with the short association fibers. Its function is doubtful. 


\section{CHAPTER IV. \\ THE RHOMBENCEPHALON. SECTION I. THE CEREBELLUM.}

The rhombencephalon is composed of the cerebellum, the pons and the medulla oblongata (Figs. 20, 2I and 27). It is the lozengeshaped brain. The cerebellum is the dorsal and largest portion of it, and is developed in the roof-plate and dorsal zones of the metencephalon. Together with the pons it forms the hind-brain. Its weight is about five ounces, slightly more than one-tenth of the whole brain. It is situated in the posterior fossa of the skull, under the tentorium cerebelli and dorsal to the pons and medulla oblongata. Between it and the last two structures is enclosed the fourth ventricle. The cerebellum is distinguished from the cerebrum by its stratification. Its surface is composed of gray substance, the cortex (substantia corticalis); its interior is white and is called the medullary body (cor pus medullare, Figs. 79 and 8I).

Function.- The cerebellum is an important relay in the indirect motor and indirect sensory paths. In response to impulses received from skin, muscles, tendons, joints and viscera, it is also believed to originate impulses which co-ordinate muscles and maintain equilibrium. Moreover, according to Russel, each cerebellar hemisphere exercises an important inhibitory function, through the brachia conjunctiva, upon the opposite side of the cerebrum.

Divisions. - The cerebellum is made up of two lateral parts, the hemispheres, and a central part, uniting the hemispheres together, called the vermis cerebelli, or worm (Figs. 76, 77, and 80). In the early embryo the cerebellum is a transverse ridge in the roof of the fourth ventricle, partially divided for a time by a median groove on its ventricular surface; and it remains undifferentiated into medial and lateral parts in many lower animals (Edinger).

The cerebellar hemispheres (hemispheria cerebelli) measure 
two inches from before backward and about the same in thickness, antero-medially; but they taper rapidly toward the lateral borders (Figs. 76 and 77). They-are joined together by the worm, or vermis, which forms the central and most elevated part of the cerebellum.

The vermis cerebelli, or worm, is a small elongated lobe,

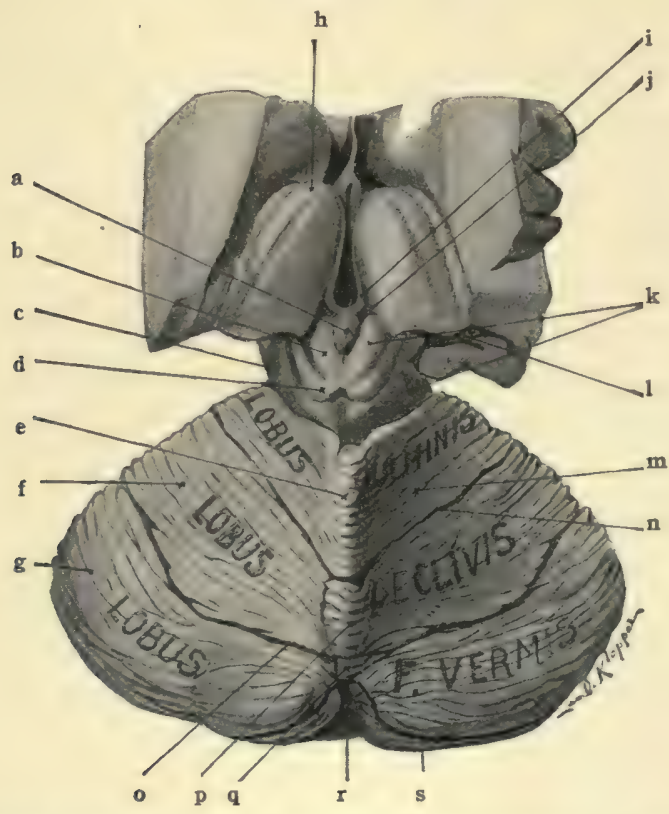

Fig. 76.-Dorsal view of inter-brain, mid-brain and cerebellum. Superior surface of cerebellum. (Original.)

a. Pineal body. b. Colliculus superior of corp. quad. c. Lateral sulcus, d. Colliculus inferior of corp quad. e. Culmen monticuli. f. Pars posterior of quadrangular lobule. g. Superior semilunar lobule. h. Anterior tubercle of thalamus. i. Stria medullaris thalami. j. Trigonum habenulæ. k. Mid-brain. 1. Inferior horn of lateral ventricle, m. Pars anterior of quadrangular lobule. n. Predeclivi] sulcus. o. Postdeclivil sulcus. p. Declive monticuli. q., Folium vermis. r. Posterior cerebellar notch. s. Horizontal sulcus.

shorter and much thinner than the hemisphere (Figs. 76 and 80). In animals lower than mammals, it is not differentiated from the hemispheres and appears to be the only part of the cerebellum present, being very large in birds and swimming reptiles (Edinger). Its transverse ridges give it a worm-like appearance. It unites the upper half of the medial aspect of the two hemispheres, their 
lower halves being separated by an antero-posterior groove, called the valley or vallecula cerebelli. The upper surface of the vermis is called the superior worm, or vermis superior; and the lower surface, the inferior worm, or vermis inferior. The superior and inferior surfaces are separated from one another at the posterior end of the worm by the great horizontal sulcus; anteriorly, the medullary body of the cerebellum separates them. At either end of the worm is a notch bounded by the vermis and the hemispheres, the anterior and posterior cerebellar notches.

The posterior cerebellar notch, incisura cerebelli posterior (Fig. 76), bounded by the posterior end of the worm and the postero-medial border of the hemispheres, is occupied by the falx cerebelli. A prolongation of the medullary body of the cerebellum fills up the incisura cerebelli anterior, or anterior cerebellar notch, which is situated between the antero-medial borders of the hemispheres in front of the vermis cerebelli.

The medullary body (corpus medullare) which is the white center of the cerebellum splits, in its median part, into two lamina: a superior, which forms the superior medullary velum and three pairs of connecting bands (peduncles), and an inferior, which is the inferior medullary velum (Figs. 79 and 78). Separating at an acute angle, the two laminæ form the tent of the fourth ventricle.

The inferior medullary velum (velum medullare inferius, Figs. 79 and 9r) is the inferior lamina of the medullary body. It is a short plate of white matter, not more than a quarter of an inch long and is separated from the superior lamina by the angle, called the fastigium. It ends in a concave border from which a sheet of epithelium continues down over the fourth ventricle; and together they form the inferior half of the roof of that cavity. Laterally, the inferior velum extends to the flocculus of the hemisphere. Of the worm it covers the nodulus, antero-superiorly. It bounds, dorsally, the lateral recesses of the fourth ventricle.

In the BNA the cerebellar vela are called "velum medullare anterius" and "v. m. posterius;" but there is no more reason for those embryological terms in this place than there is elsewhere throughout the central nervous system, and I have used "superius" and "inferius" which properly indicate their positions in adult anatomy. 
The superior lamina of the medullary body joins the cerebellum immediately to the pons. The superior lamina is made up of three pairs of connecting bands (cerebellar peduncles) and the superior medullary velum. It constitutes all the prolongations of the corpus medullare of the cerebellum, except the inferior velum (Fig. 9I).

The brachia conjunctiva (superior peduncles, Figs. 78 and 86) converge as they pass forward and upward to the inferior

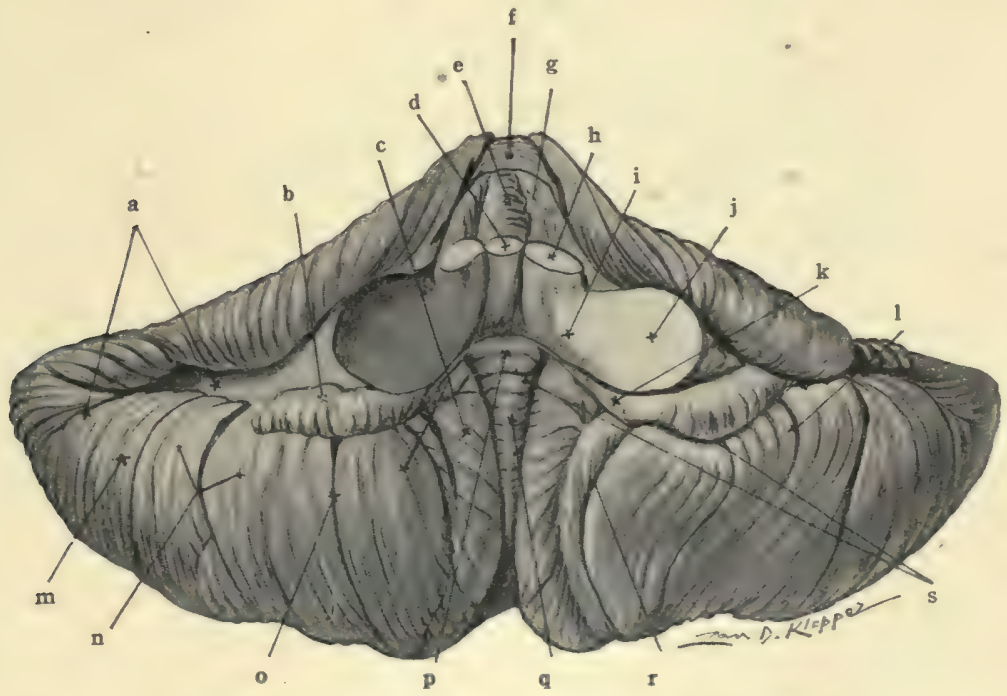

Fig. 77--Anterior aspect of cerebellum. (Original.)

a. Horizontal sulcus. b. Flocculus. c. Tonsil. d. Superior medullary velum. e. Lobulus centralis. f. Culmen monticuli. g. Inferior medullary velum. h. Brachium conjunctivum. i. Restiform body. j. Brachium pontis. k. Peduncle of flocculus. 1. Division in biventral lobule. m. Lobulus gracilis. n. Lobulus biventer. o. Prepyramidal sulcus. p. Nodule. q. Uvula. r. Depression in tonsil. \&. Postnodular sulcus.

quadrigeminal colliculi, where they disappear. They are joined to one another by a thin plate of white matter, the superior medullary velum (velum medullare superius). With the velum, they form the roof and lateral boundaries of the superior half of the fourth ventricle. They gradually bury themselves in the pons as they proceed upward toward the corpora quadrigemina. Beneath the corpora quadrigemina and the cerebral aqueduct, the brachia conjunctiva cerebelli decussate, and pass into the 
hypothalamic region of the opposite side. They end chiefly in the red nuclei, which their fibers surround. Near the corpora quadrigemina each brachium conjunctivum is obliquely crossed by the lateral fillet in its course to the inferior quadrigeminal colliculus.

The superior medullary velum (valve of Vieussens, Figs. 86,87 and 92), is a trapezoidal sheet of white substance, wider where it fuses with the corpus medullare of the cerebellum than at the mesencephalic end. It forms the floor of the groove between the brachia conjunctiva cerebelli and the superior half of the

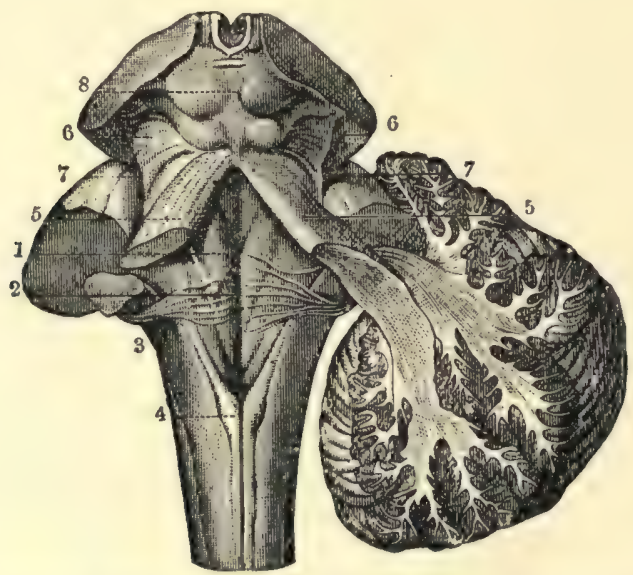

Fig. 78.-Dissection of rhombencephalon to show brachium conjunctivum, brachium pontis and corpus restiforme. (Gordinier, Sappey after Hirschfeld and Leveillc.)

On left side the cerebellar brachia and restiform body have been cut short; the right hemisphere is cut obliquely to show connection with brachium conjunctivum and corpus restiforme. I. Median groove of fourth ventricle, 2. Medullary striæ. 3. Restiform body 4. Clava in funiculus gracilis. 5,5. Brachium conjunctivum. 6. Lateral fillet. 7,7. Lateral sulcus of mid-brain. 8. Corpora quadrigemina.

roof of the fourth ventricle. Its lateral borders fuse with and unite the brachia conjunctiva, hence their name. In the median line its posterior surface presents a slight ridge, the frcmulum veli, from either side of which emerges the trochlear nerve.

The corpora restiformia (inferior peduncles of the cerebellum) issue from the cerebellum between the brachium conjunctivum and the brachium pontis (Figs. 78 and 86). They first run forward to the posterior surface of the pons, near the inferior border; and then, bending downward and backward (a flexion 
of more than 90 degrees), they converge in the posterior areas of the medulla toward the calamus scriptorius. They help to form the floor and to bound laterally the inferior half of the fourth ventricle.

The brachia pontis (middle peduncles) join the cerebellum to the lateral borders of the pons (Figs. 78, 85 and 9x). They are continuous with the transverse fibers in the ventral area of

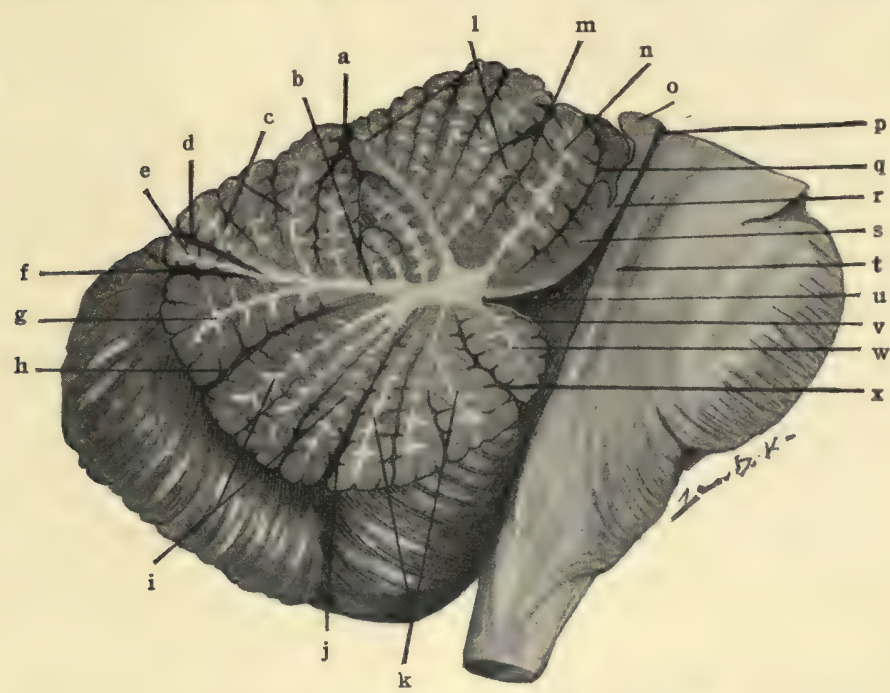

Fig. 79.-Median section of cerebellum, pons and medulla. (Original.)

a. Predeclivil sulcus. b. Arbor vitæ. c. Declive monticuli. d. Postdeclivil sulcus. e. Folium vermis. f. Horizontal sulcus, g. Tuber vermis. h. Postpyramidal sulcus, i. Pyramid. j. Prepyramidal sulcus. k. Uvula. 1. Culmen monticuli. m. Postcentral sulcus. n. Central lobule. o. Inferior colliculus of corp. quad. p. Cerebral aqueduct. q. Precentral sulcus. r. Superior medullary velum. s. Lingula. t. Medial longitudinal bundle. u. Fastigium. v. Inferior medullary velum. w. Nodule. x. Postnodular sulcus.

the pons. The brachia pontis in the anterior cerebellar notch are placed external to the brachia conjunctiva and the restiform bodies, and are opposite the widest part of the fourth ventricle.

Horizontal Sulcus of Cerebellum (Figs. 76, 79 and 80).The cerebellum has one great sulcus which divides it into upper and lower surface. The sulcus horizontalis cerebelli is irregularly circular in shape; anteriorly its lips are separated by the prolongation of the medullary body from which the sulcus runs backward, 
dividing the border of each hemisphere and the posterior end of the worm. Rarely the two halves are not continuous through the posterior extremity of the worm. In the horizontal sulcus the remaining important sulci of the cerebellum terminate. They are nearly parallel with one another; hence, the cerebellum is laminated, not convoluted like the cerebrum. Though the horizontal sulcus is an important landmark in the adult cerebellum, it does not form a primary embryonic division of the cerebellum but appears late in fotal life (Cunningham).

\section{SUPERIOR SURFACE OF THE CEREBELLUM.}

The superior surface of the cerebellum (facies cerebelli superior) is bounded by the horizontal sulcus and the superior lamina of the medullary body (Figs. 76 and 79). The posterior and larger part of this surface is covered by the tentorium cerebelli, the tentorial area; the small anterior part of it bounds the anterior cerebellar notch. The superior surface is divided into five continuous lobes by four crescentic sulci, called interlobular sulci.

Sulci of Upper Surface.-The interlobular sulci (sulci interlobulares) divide the worm and both hemispheres into lobules; and each lobe is composed of a central and two lateral lobules. These sulci are best seen in a median section of the vermis and are named in accordance with their relations to the lobules in the worm, viz.:

(I) The precentral sulcus (s. pracentralis), which is located in the anterior cerebellar notch just above the superior velum (Fig. 79). It is between the lingula and lobulus centralis, in the worm; between the vinculum and ala, in the hemisphere. It terminates in the horizontal sulcus. When the vinculum is wanting the precentral sulcus is present only in the vermis.

(2) The postcentral sulcus (s. postcentalis), in the worm, separates the lobulus centralis from the culmen; and, in the hemisphere, the ala from the anterior part of the quadrangular lobule (Figs. 77 and 79). The sulcus is situated at the upper border of the anterior cerebellar notch and runs just under the anterior border of the tentorial surface of the cerebellar hemis- 
phere. Both central sulci terminate on the dorsum of the superior medullary lamina in the horizontal sulcus.

(3) Predeclivil Sulcus. (S. pradeclivis, s. primarius) (Figs. 76 and 79).-Behind the culmen and anterior part of the quadrangular lobule, a half inch from the anterior border of the tentorial surface, there is the predeclivil sulcus. It bounds the declive and posterior part of the quadrangular lobule in front. It ends at the junction of the anterior and middle thirds of the antero-lateral border of the hemisphere in the horizontal sulcus. Embryologically it is second to appear; it is the deepest sulcus of the cerebellum, hence the name, sulcus primarius, given it by Kuithan. Its development begins near the end of the third month in utero (Cunningham).

(4) The postdeclivil sulcus (s. postdeclivis) (Figs. 76 and 79) is located in the posterior cerebellar notch, from which it curves outward and forward in the superior surface of the hemispheres. It separates the declivil lobe from the folium vermis, in the worm, and from the superior semilunar lobules in the hemispheres. It ends in the horizontal sulcus at the junction of the posterior and middle thirds of the antero-lateral border. Being behind the crescentic gyri of the quadrangular lobule, this sulcus may be called the sulcus postlunatus. It appears a month later than the predeclivil sulcus.

Sulci and lobules of the upper surface of the cerebellum from before backward:

\section{Hemisphere.}

Vinculum

Ala

Lobulus quadrangularis, pars anterior.

Lobulus quadrangularis, pars posterior

Semilunaris superior
Worm.

Lingula

Precentral sulcus

Lobulus Centralis

Postcentral sulcus

Culmen Monticuli

Predeclivil sulcus

Declive Monticuli

Postdeclivil sulcus

Folium Vermis

Horizontal sulcus

\section{Hemisphere.}

Vinculum

$\mathrm{Ala}$

Lobulus quadrangularis, pars anterior.

Lobulus quadrangularis, pars posterior

Semilunaris superior 
Lobes of Superior Surface (Figs. 76, 77 and 79).-The lobes of the superior surface of the cerebellum should be studied first in a median section, where the branches of the medullary body (lamina medullares) will guide the student and where the sulci are most easily identified. These lobes include the divisions of the worm and of the hemispheres, and are five in number.

Lingula and Vincula, Lobus Lingulæ.-The lingula is a very small lobule of the vermis entirely concealed in the anterior cerebellar notch by the overhanging central lobule. It is a tongueshaped group of four or five rudimentary transverse gyri. It rests upon the superior medullary velum, with which its white center is continuous. Laterally, the lingula tapers off and is sometimes represented in the hemisphere by a very thin gyrus called the vinculum lingulæ. The vinculum is bounded by the brachium conjunctivum cerebelli in front, and by the precentral sulcus behind. The precentral sulcus separates the lobe of the lingula from the central lobe.

Central Lobule and Alæ, Lobus Centralis (Figs. 77 and 79).- The lobulus centralis is situated between the precentral and postcentral sulci, in the anterior cerebellar notch. It covers the lingula and in turn is overhung by the culmen. Four or five small transverse gyri make it up. On sagittal section, it is seen to form a single branch of the corpus medullare (arbor vitæ). The gyri of the central lobule, continuing along the anterior cerebellar notch into either hemisphere, form a triangular or winglike lobule, the ala (ala lobuli centralis).

Culmen and Anterior Part of Quadrangular Lobules, Lobus Culminis (Figs. 76 and 79).-In the culmen monticuli the surface of the cerebellum reaches its highest elevation. It is a large lobule and occupies half of the tentorial surface of the worm. It is made up of three or four prominent gyri, which extend laterally into the hemispheres; and, in each, forms the anterior part of the quadrangular lobule. The pars anterior lobuli quadrangularis occupies about one-third of the tentorial surface of the hemisphere. The predeclivil sulcus separates the culmen and the pars anterior of either side (the lobe of the culmen) from the declivil lobe. 
Declive and Posterior Parts of Quadrangular Lobules, Lobus Declivis (Figs. 76 and 79).-The declive monticuli forms the posterior slope, as the culmen forms the summit, of the monticulus cerebelli. The declive has about half the extent of the culmen. Its gyri are continued into either hemisphere, where they form a large crescentic lobule, the pars posterior lobuli quadrangularis. The increased size of the lobe in the hemisphere is due to the expansion of the secondary gyri found in the worm. The anterior and posterior parts of the quadrangular lobule constitute the lobulus quadrangularis, which forms the anterior two-thirds of the tentorial surface of the hemisphere. The declive and its hemispheral extensions are inclosed between the predeclivil and postdeclivil sulci.

The folium vermis and superior semilunar lobules, lobus folii vermis (Figs. 76 and 79), lies behind the postdeclivil and above the horizontal sulcus. The folium vermis is the terminal lobule in the superior worm, and occupies the posterior cerebellar notch. It appears near birth in the bottom of a transverse groove common to the postdeclivil and horizontal sulci (Cunningham). Rarely it is absent. It contains a single medullary lamina beset with rudimentary gyri, which are largely developed in the hemispheres. The superior semilunar lobule is, therefore, very large in comparison with the folium vermis. It expands lateralward to the postero-lateral border of the hemisphere, which it forms. It comprises the posterior third of the hemisphere's tentorial surface, and forms one of the remarkable features of the human cerebellum.

\section{INFERIQR SURFACE OF THE CEREBELLUM.}

The inferior surface of the cerebellum (facies cerebelli inferior) is prominent laterally and depressed centrally (as the organ is viewed inverted), the hemispheres being separated by the anteroposterior groove, called the vallecula cerebelli (Figs. 77 and 80). The vallecula (little valley) is occupied by the inferior worm and is bounded on either side by a small cleft, between the worm and the overhanging hemisphere, called the sulcus vallecule. The 
inferior cerebellar surface is limited by the horizontal sulcus and is separated from the medulla by the transverse fissure of the cerebellum. It is more complex than the superior surface; and its sulci are more sharply curved forward as they pass from the worm into the hemispheres.

Sulci of Lower Surface (Fig. 80).-The interlobular sulci of this surface are very deep. They are three in number, namely:

(I) The postnodular sulcus (s. postnodularis) (Figs. 77, 79 and 80 ) is in the anterior end of the worm between the nodule

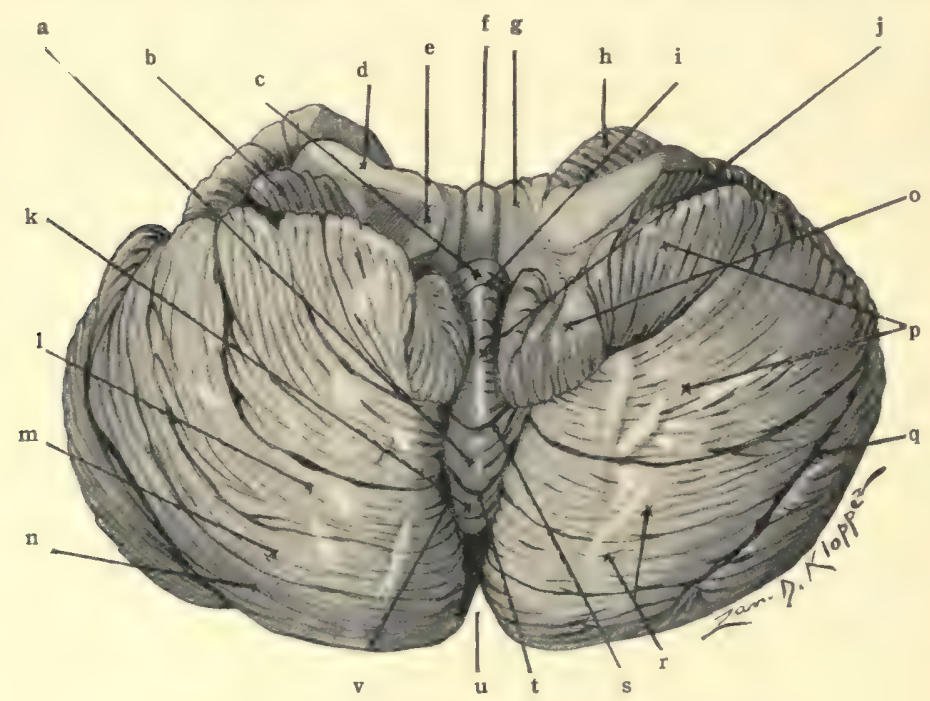

Fig. 80.-Inferior surface of cerebellum. (Original.)

a. Pyramid. b. Flocculus. c. Nodule. d. Brachium pontis. e. Restiform body. f. Superior medullary velum. g. Brachium conjunctivum. h. Quadrangular lobule. i. Postnodular sulcus. j. Uvula. k. Tuber' vermis. 1. Ant. and $\mathbf{m}$. Post. Slender lobules, n. Inferior senuilunar lobule. o. Tonsil. p. Biventral lobule. q. Horizontal sulcus. r. Lobulus gracilis. s. Prepyramidal sulcus. t. Postpyramidal sulcus. u. Post. cerebellar notch. v. Sulcus valleculæ.

and uvula. In the hemisphere it winds forward and outward between the inferior medullary velum and the tonsil, and then continues lateralward between flocculus and biventral lobule to the horizontal sulcus. It is the first cerebellar sulcus to be developed (Cunningham).

(2) The prepyramidal sulcus (s. prepyramidalis) (Figs. 79 and 8o), situated between the uvula and pyramid, is very con- 
cave in the hemispheres. It curves outward and forward around the tonsil, separating it from the biventral lobule. It terminates behind the flocculus in the postnodular sulcus.

(3) The postpyramidal sulcus (s. postpyramidalis) (Figs 79 and 80), between the pyramid and tuber vermis, is near the posterior end of the worm. It forms an oblique groove in either sulcus valleculæ, from which three concentric sulci extend into the hemisphere. The anterior of the three (the pregracile), usually considered the postpyramidal sulcus in the hemisphere, separates the biventral lobule from the slender lobule (1. gracilis); the remaining two (midgracile and postgracile) subdivide the slender lobule into anterior and posterior slender, and separate the lobulus gracilis from the inferior semilunar lobule. The last is bounded behind by the horizontal sulcus.

Sulci and lobules of the lower surface of the cercbellum, from before backward:

Hemisphere.

Flocculus.

Tonsil.

Biventral lobule.

Slender lobule and inferior semilunar lobule.
Worm.

Nodule.

Postnodular sulcus.

Uvula.

Prepyramidal sulcus.

Pyramid.

Postpyramidal sulcus.

Tuber vermis.

Horizontal sulcus.

\section{Hemisphere.}

Flocculus.

Tonsil.

Biventral lobule.

Slender lobule and inferior semilunar lobule.

Lobes of Lower Surface.-They are not continuous from the worm to the hemisphere as on the upper surface (Figs. 76 and 80). Excepting in the posterior lobe, only a small ridge beneath the sulcus valleculæ joins the central and lateral lobules together. The inferior lobes are four in number. Each is composed of a central and two lateral lobules as on the upper surface. The lobule in the worm gives its name to the lobe.

Nodules and Flocculi, Lobus Noduli (Figs. 79 and 80).-The nodule (nodulus vermis) is a small lobule at the anterior end of the inferior worm. It is composed of three or four gyri, which project from the middle of the dorsal surface of the inferior medullary velum. It comprises a single branch of the arbor vitæ. 
Though larger it is the counterpart of the lingula on the superior velum. It is bounded by the sulcus valleculæ on either side. The inferior medullary velum extends laterally from the nodule, and in part blends with the brachium pontis of the cerebellum. In front of the tonsil, a layer of gray matter (pedunculus flocculi) appears on the velum. That gray matter enlarges more externally to a small tufted mass, called the flocculus, in which the velum ends. Embryologically, the flocculus is the oldest lobule of the human cerebellum, as is the floccular sulcus (postnodular sulcus) which bounds it, the first one formed. The flocculus is very small and rudimentary in man. It is divided into an anterior and a posterior part, the latter being called the secondary flocculus. The flocculus is separated from the tonsil and the biventral lobule by the postnodular sulcus. The whole line of structures, namely, the nodule, velum, peduncle and 'flocculus, form the lobe of the nodule.

Uvula and Tonsils, Lobus Uvulæ (Figs. 79 and 80).-The uvula (uvula vermis) comprises a considerable part of the vermis inferior behind the nodule. It broadens backward and is widest next the pyramid. Bounded on either side by the sulcus valleculæ, it projects into the valley like the uvula into the isthmus of the fauces. It comprises one large branch of the arbor vitæ which bifurcates near its origin into two laminæ and presents at the surface six or eight small gyri. A slight ridge, the furrowed band, joins it to the tonsil (tonsilla cerebelli) in the hemisphere. From the furrowed band the tonsil expands downward and backward, forming a lobule of nearly a dozen sagittal gyri. The tonsil overhangs the side of the uvula and conceals the furrowed band, medially; and, behind, it conceals the connecting ridge between the pyramid and biventral lobule. Its large size makes it a prominent feature of the human cerebellum. The fossa containing the tonsil is the bird's nest (nidus avis). Behind the uvular lobe, composed of the above three lobules, is the prepyramidal sulcus.

Pyramid and Biventral Lobules, Lobus Pyramidis (Figs. 79 and 80).-As seen from the surface, three or four distinct gyri make up the pyramid (pyramis vermis); in reality, it covers 
one strong lamina of the arbor vitæ, which divides into two near the surface. It forms the most prominent lobule of the inferior worm. A low connecting ridge joins the pyramid to the biventral lobule in the hemisphere. The biventral lobule (lobulus biventer) is triangular in outline. Its base looks toward the flocculus and is bounded by the postnodular and the horizontal sulcus; its apex is continuous with the connecting ridge joining

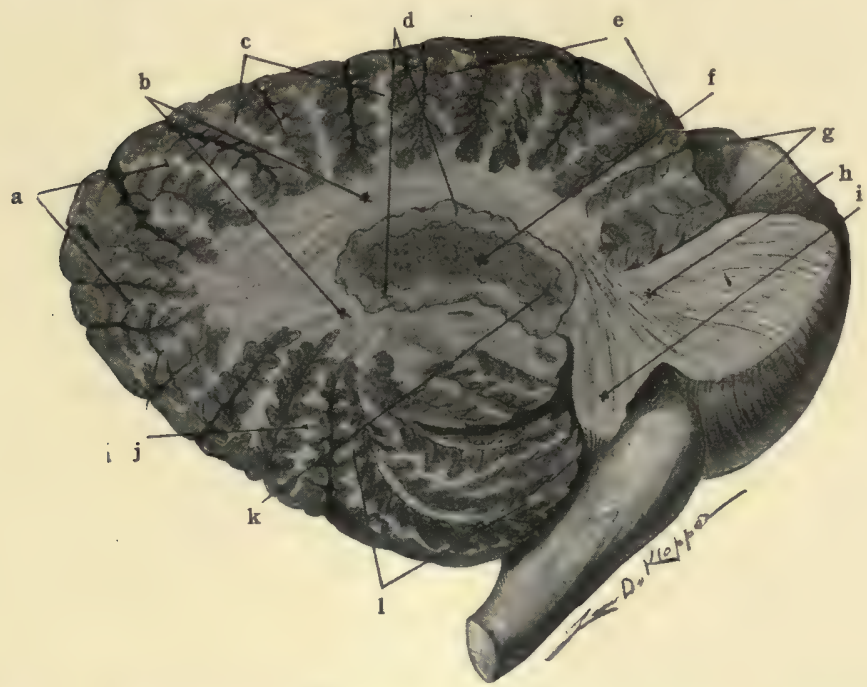

Fig. 81.-Sagittal section of cerebellum, cutting nucleus dentatus. (Original.)

a. Sup. semilunar lobule. b. Corpus medullare. c. Post. part quadrangular lobule. d. Nucleus dentatus. e. Ant. part of quadrangular lobule. f. Interior of dentate nuc. g. Central sulci. h. Brachium pontis. i. Restiform body. j. Inf. semilunar and slender lobules. k. Hilus of nuc. dent. 1. Biventral lobule.

it to the pyramid. The gyri composing it radiate from the apex toward the base, and are divided into two groups by a very deep intralobular sulcus. Its lateral extension is a little beyond the flocculus. The postpyramidal sulcus bounds it postero-externally, and separates it from the slender lobule.

Tuber Vermis, Slender and Inferior Semilunar Lobules, Lobus Tuberis (Figs. 79 and 80).-The tuber vermis forms the posterior end of the inferior worm. It resembles the lobules of the vermis superior, because some of its half dozen tertiary gyri 
are continued into the hemispheres, the sulcus valleculæ not cutting them off. A bifurcated lamina of the arbor vitæ enters into the tuber. The horizontal sulcus separates it from the folium vermis of the superior worm. The slender and inferior semilunar lobules comprise the posterior two-thirds of the inferior surface of each hemisphere, extending from the biventral lobule to the postero-lateral border. Twelve to fifteen gyri compose the lobules. The gyri are divided into three groups by the midgracile and postgracile sulci; the anterior and middle groups are named the anterior slender and posterior slender lobules, they constitute the lobulus gracilis. The posterior is the inferior semilunar lobule. The inferior semilunar lobule, only, is continuous with the gyri of the vermis. The great size of the inferior and superior semilunar lobules is the most characteristic feature of the human cerebellum.

The gray matter of the cerebellum is composed of cortex which covers the cerebellar laminæ and of ganglia imbedded in the medullary body (Figs. 8x and 84).

\section{CORTICAL GRAY MATTER.}

The cortex of the cerebellum (substantia corticalis cerebelli) is made up of two thick layers visible to the naked eye, viz,. (I) a superficial layer, and (2) a deep, granular layer. At the junction of these two layers is a single row of large pitcher-shaped cellbodies, which are characteristic of the cerebellar cortex and are often visible to the unaided eye. They are the bodies of Purkinje's cells, and are considered in the deep part of the first macroscopic layer, where they form the stratum gangliosum. Under the microscope three layers are easily seen, viz., (I) the gray layer (stratum cinereum); (2) the ganglion cell layer (stratum gangliosum); and (3) the granular layer (stratum granulosum).

(I) Superficial Layer (Figs. 82 and 83 ).-Thickest on the laminæ and thinnest beneath the fissures, this layer contains small and large stellate cell-bodies with their processes, which constitute the stratum cinereum proper; and the large Purkinje cell-bodies with their dendrites and recurrent axones, together 
with many corticipetal fibers. The Purkinje cells form the stratum gangliosum.

Cells. - The bodies of Purkinje's cells (Figs. 82 and 83 ) are located near the deep surface of the superficial layer in the stratum gangliosum. They measure from $100 \mu$ to $\mathrm{I} 35 \mu$ in their longest axis. Each has one axone which, after piercing the deep layer, becomes a fiber of the medullary body. It gives off, in the deep layer, several recurrent collaterals, which form contact relations with other cells in both layers. From the outer end of each cell-body antler-like processes, the dendrites, are given off; they ramify toward the surface in a wide plane at right angles to the free border of the gyrus. The edge of the plane only is seen in a longitudinal section of the gyrus and the arborization is very narrow and tall. The stellate cell-bodies, an outer and inner layer, together form the stratum cinereum. They increase in size toward the Purkinje cells. They have rich dendritic processes and one axis-cylinder each. Their processes ramify throughout the stratum cinereum and stratum gangliosum. The inner layer of the stratum cinereum contains the larger cells; they are called the "basket cells." Their axis-cylinder processes run parallel with the surface and at right angles to the border of the gyrus; they give off vertical branches, which descend to Purkinje's corpuscles and inclose them in a basket work of filaments. In the outer layer of the stratum cinereum the stellate cell-bodies are smaller than in the inner layer. They branch freely and terminate in claw-like end-tufts in contact with other stellate cells.

The fibers of the superficial layer (Figs. 82 and 83 ) have three sources: (a) The processes of neurones within the layer, which include the dendrites and axones of the stellate cells and the dendritic planes and recurrent collaterals of Purkinje's cells. (b) The processes of cell-bodies in the deep layer, whose $\mathrm{T}$ branched axones pierce the dendritic planes of Purkinje in the first layer; and, the processes of the large granules whose dendrites ramify toward the surface. (c) The fibers of the medullary projection rise or end largely in the cellular layer. The axones of Purkinje's neurones compose all of the corticifugal fibers. They end in the cerebellar and vestibular nuclei of the cat (Clark 
and Horsley) and probably have these endings in the human brain, with the possible addition of the inferior olivary nucleus. The corticipetal fibers, which rise either in other parts of the brain or in the spinal cord and ganglia, terminate in varicose fibrils chiefly in the superficial layer. These fibrils entwine about the "primary and secondary stems of the Purkinje dendrites" (Cunningham).

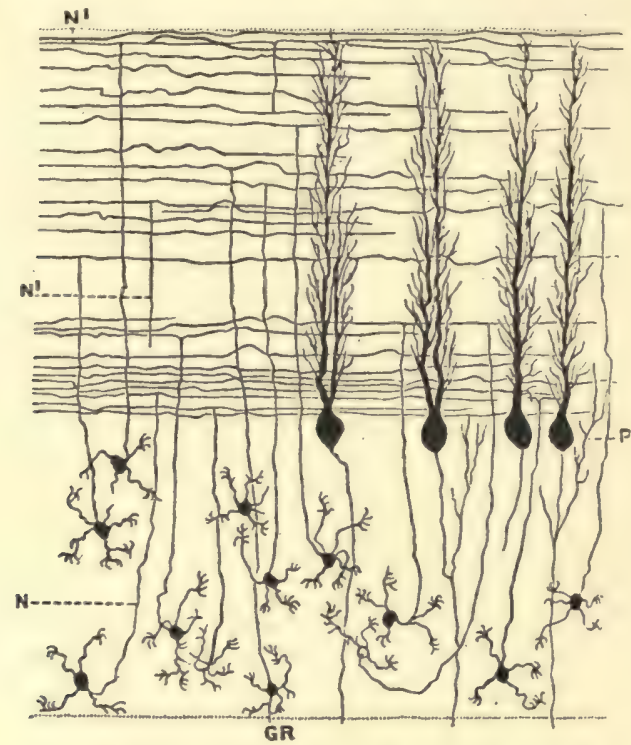

Fig. 82.-Section of cerebellar gyrus made parallel with its free border.

Diagrammatic. (After Kölliker from Cunningham.)

G R. Small granules with claw-shaped dendrites and long axones that run out into the gray layer and divide T-like. N. Axone of small granule. P. Purkinje cells seen in profile, showing border of dendritic planes in gray layer.

(2) The deep, granular layer (stratum granulosum, Figs. 82 and 83 ) is of uniform thickness. It blends centrally with the medullary projection. It contains a few superficial granules which are comparatively large in size and many small granules in which the nucleus occupies nearly the whole cell-body.

Cells of the Granular Layer. - The granules are small, round, or stellate cell-bodies, largest near Purkinje's cells, closely packed externally, but scattered among the projection fibers centrally. 
Each small granule has three to five short dendrites, which soon break up into claw-like tufts in contact with adjacent granules, and one long axone. The axone runs out into the superficial layer, branches T-like, and, piercing the dendritic planes of Pur-

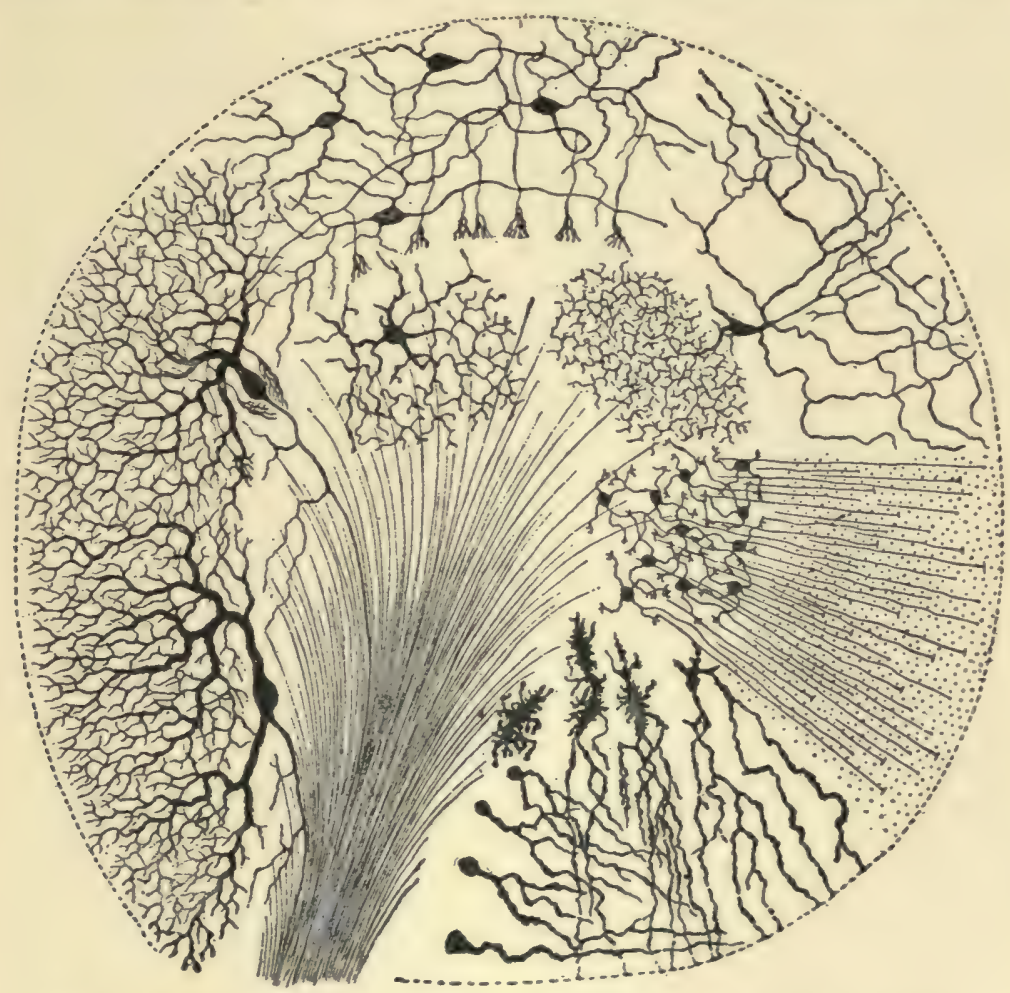

Fig. 83.-Section across a cerebellar gyrus at a right angle to the free border.

Diagrammatic. (Gordinier after Van Gehuchten.)

Showing large stellate cells of first layer with their basket-work endings; the cells of Purkinje, their dendritic planes in the gray layer and their axones running through the granular layer to the medullary lamina of the gyrus; two large granules of Golgi type; the small granules whose T-branches run parallel with the border of the gyrus; moss-jike endings of Cajal, etc.

kinje, gives off collaterals to them until exhausted by multiple division. The large granules are dendraxones, the type of Golgi. The axones form remarkable arborizations toward the medullary projection, touching and associating many granules. The dendrites, branching freely, ramify in the superficial layer. 
Fibers of the Granular Layer (Figs. 82 and 83 ).- The nerve fibers of the granular layer are as follows: (a) The processes of the granules, (b) the axones of Purkinje's cells running down into the medullary projection, together with their recurrent collaterals, and (c) corticipetal fibers, most of which run through the granular layer, without branching, to end in the first layer; the remainder terminate in the deep layer in the moss-like appendages of Cajal.

The functions of the stellate cells, the "basket cells" and the granule cells are probably receptive and associative; they receive impulses through the projection fibers and transfer those impulses to the dendrites or bodies of Purkinje's cells. Purkinje's cells originate impulses for the coordination of muscular action (Gordinier), and for the inhibition of nervous activity in the opposite cerebral hemisphere (Russel). Lesions in the cerebellum produce incoordination, chorea, athetosis and, rarely, convulsions.

The neuroglia of the cerebellum is similar to that in the cerebrum. The short-rayed cells are scattered throughout the gray substance, while the long-rayed are located near, or within, the white substance. In the region of Purkinje's cells, near the surface of the deep layer, are the bodies of the arborescent cells, whose processes form a fine interlacement about the cell-bodies of Purkinje and then extend in parallel lines out to the surface. They form a neuroglia felt-work just beneath the pia mater (lamina basalis).

\section{GANGLIONAR GRAY MATTER.}

The ganglia of the cerebellum are the nucleus dentatus, nucleus emboliformis, nucleus globosus and nucleus fastigii (Figs. 8I and 84). All these nuclei are made up of stellate cell-bodies, which vary in size from six to forty microns. They form relay stations in the paths going out of the cerebellum. In them terminate axones of Purkinje's cells.

The nucleus dentatus (corpus dentatum) is a wavy, sinuous pouch of yellowish-brown gray matter imbedded in the medullary body of each hemisphere. The nucleus dentatus measures a half inch in length and a quarter of an inch in width (Fig. 8I). 
It is filled with white fibers, which issue from its open anterior end, called the hilus, and form the greater part of the brachium conjunctivum cerebelli. It also receives many axones from Purkinje's cells and, thus, forms a relay in the common sensory path.

The small ganglia are just visible to the naked eye under favorable conditions (Fig. 84). One of these, a club-shaped mass,

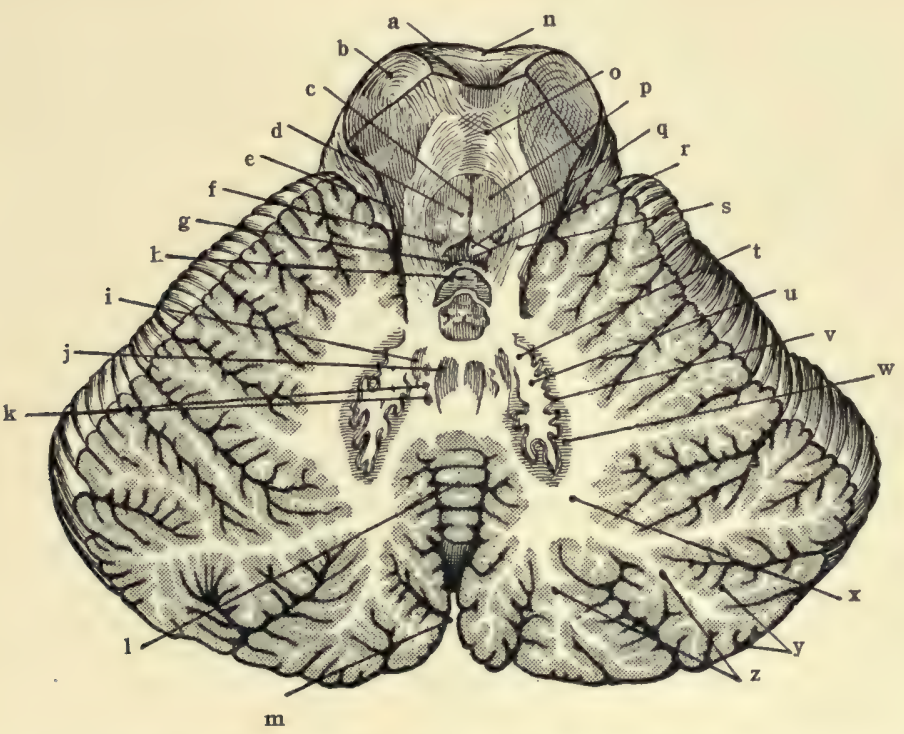

Fig. 84.-Horizontal section of cerebellum cutting nuclei and brachia conjunctiva. (Morris's Anatomy after Toldt.)

a. Interpeduncular fossa, b. Cerebral peduncle. c. Raphe of medulla oblongata. d. Medial longitudinal fasciculus. e. Lateral lemniscus. f. Substantia ferruginea. g. Superior medullary velum. h. Lingula cerebelli. i. Nucleus emboliformis, j. Nueleus fastigii. k. Nucleus globosus. 1. Vermis (superior). m. Posterior cerebellar notch. n. Pons (varolii). o. Decussation of brachium conjunctivum. p. Stratum nucleare. q. Fossa rhomboidea (pars superior). r. Fourth ventricle. s. Brachium conjunctivum. t. Hilus of dentate nucleus. u. Core of the dentate nucleus. v. Dentate nucleus. w. Capsule of dentate nucleus. x. Corpus medullare. y. Cortical substance. z. Medullary lamina.

the cork-like nucleus emboliformis, partly closes the hilus of the dentate nucleus. Medial to that is an elongated antero-posterior ganglion, bulbous behind, called the nucleus globosus. The third nucleus is in the anterior end of the worm, just above the fastigium of the fourth ventricle. It is called the nucleus of the highest 
point of the roof, nucleus fastigii (Stillingi). ' In the last two the stellate cell-bodies are larger than in the nucleus emboliformis or dentatus; but, otherwise, they are alike in structure. The nucleus fastigii and nucleus globosus form a part of the origin of the acustico-cerebellar tract, which descends to the nucleus of Deiters in the medulla; probably axones of Purkinje's cells also enter into the acustico-cerebellar tract in man, as they do in the cat (Clarke and Horsley). Perhaps the nucleus emboliformis and nucleus dentatus have a less important connection with the same tract. The small cerebellar ganglia, and especially the nucleus fastigii and nucleus globosus, constitute a relay in the arc of equilibrium.

The White Substance of the Cerebellum (Figs. 79 and 8I). - The corpus medullare contains all the white matter of the cerebellum. It is a strong body measuring a third of an inch in thickness vertically in the middle of the hemisphere, but in the worm it is a thin sheet and is very slender as seen in median section. Its branches to the cerebellar gyri are called the medullary lamince (lamine medullares). Viewed in a sagittal section of the hemisphere, the medullary laminæ are short and stubby branches of a very thick trunk; but the tree-like appearance of the medullary body and laminæ in the vermis is perfect, hence the name, arbor vite, which is applied to them there. In the anterior cerebellar notch the medullary body divides into a thick superior lamina and a thin inferior lamina which are separated by a transverse furrow, the bottom of which constitutes the peak, or fastigium, of the fourth ventricle. The inferior lamina is the inferior medullary velum, already described; this, with the continuation of its ependymal epithelium, forms the roof of the inferior half of the fourth ventricle. The superior lamina of the corpus medullare forms the three pairs of connecting bands (peduncles) and the superior medullary velum. Medullated axones make up the entire corpus medullare and its divisions. We study these axones in three systems like those of the cerebrum:

I. Projection, or peduncular fibers.

II. Commissural fibers.

III. Association fibers. 


\section{I. - PROJECTION FIBERS.}

All fibers that leave the cerebellum, or enter it, do so through the brachia, the restiform bodies and the superior medullary velum, hence these are composed of projection fibers. At a higher level the projection fibers are contained in the corpus medullare.

Brachium Conjunctivum (Figs. 44, 77 and 78 ).- The brachium conjunctivum (superior peduncle) is the innermost of the three, at its origin in the anterior cerebellar notch; external to it, in the notch, are the restiform body and the brachium pontis; and, in the angle between the brachium conjunctivum and the restiform body, is the vestibular nucleus of Bechterew (the upper part of Deiters's nucleus). The brachium conjunctivum is joined to its fellow of the opposite side by the superior medullary velum (velum medullare superius). Two bundles of fibers run throughout the brachium conjunctivum. They are afferent and efferent. First, the fibers to the cerebrum, which comprise nearly the whole brachium, are axones of cell-bodies situated in the nucleus dentatus. This group of fibers partially buries itself in the dorsal area of the pons, then penetrates the mid-brain and decussates ventral to the inferior quadrigeminal colliculi. It ends largely in the opposite red nucleus, but partly in the thalamus. It constitutes one segment of the indirect sensory path. In the red nucleus this path is relayed to the thalamus and to the cortical fillet, or the relay is directly into the cortical fillet. It is probable that a few fibers of this brachium terminate in the nuclei of the third and fourth, and perhaps the sixth cerebral nerves. The second group of fibers rises in the opposite red nucleus, pursues a reverse course and ends in the nucleus dentatus. A few fibers from Bechterew's nucleus are found in the cerebellar end of the brachium conjunctivum and the fila lateralia pontis traverses the same part of it. Although the greater number of these fibers in the conjoined brachia decussate in the tegmentum, a few run to the red nucleus and thalamus of the same side; while the fila lateralia cross in the pons from the opposite pontine nucleus and the commissural fibers between Bechterew's vestibular nuclei leave the brachium and cross through the superior velum in the isthmus. The superior medullary velum (Fig. 65) arches over 
the fourth ventricle between the brachia conjunctiva. It is composed of longitudinal and transverse fibers. One distinct bundle, derived from the spinal cord, passes through it to the worm. This is the anterior ascending cerebello-spinal tract (Hoche). The decussating root-fibers of the fourth nerve (trochlear) course transversely through it and also the commissural fibers between Bechterew's nuclei.

Brachium Pontis (Middle Peduncle, Figs. 44, 78, 8I and 9I).The brachium pontis comes from the pons, of which it forms the anterior and middle transverse fibers. It enters into the medullary body of the cerebellum lateral to both the brachium conjunctivum and the restiform body. According to Klimoff fibers running to the cerebellum make up the entire brachium pontis. These are axones of the nucleus pontis, chiefly the opposite one. Most of them run to the cortex of the cerebellar hemisphere; a small number runs to the vermis cerebelli. They form a segment in the indirect motor path contained, above the pons, in the medial and lateral fifths and the intermediate bundle of the basis pedunculi. In the cerebellar end of the brachium pontis we may include the acustico-cerebellar tract. The latter is largely a descending tract but may contain some ascending vestibular fibers. It rises in the cerebellar nuclei, and probably in the ganglionar layer of the cortex; it terminates in the nucleus of Deiters. The principle origin of the acustico-cerebellar tract is in the opposite nucleus fastigii and nucleus globosus. 'It forms a part of the descending limb of the arc of equilibrium. By far the greater number of fibers in the brachium pontis, whether efferent or afferent, are crossed fibers.

Possibly there are in the brachium pontis axones of Purkinje's cells which terminate in the nuclei pontis on both sides and in the nuclei of the reticular formation.

The corpus restiforme (inferior peduncle) (Figs. 44, 78, 8I and 9I) can be traced both to and from the upper part of the hemisphere and worm. Inferiorly, it is the restiform body of the medulla oblongata. The bundles of component fibers are very numerous: (I) The posterior cerebello-spinal fasciculus (direct cerebellar tract), whose origin is in the dorsal nucleus of the cord 
and termination in the superior worm, forms its central part. (2) The external arcuate fibers of the medulla (posterior and anterior) form its free surface. They rise in the nucleus funiculi gracilis and nucleus funiculi cuneati and end in the vermis superior, the posterior on the same and the anterior on the opposite side. (3) A bundle from the lateral nucleus of the medulla to the cerebellar cortex on the same side. (4) The internal arcuate fibers to the opposite lower olive, the cerebello-olivary tract. These constitute a large part of the deep area of the restiform body and, with the acustico-cerebellar tract, make up all, or the greater part, of the indirect motor path from the cerebellum to the medulla. According to Cunningham the cerebello-olivary are ascending fibers.

There are probably a few axones of Purkinje's cells that run down through the corpus restiforme and are continued without interruption into the anterior descending cerebello-spinal tract of the spinal cord; but surely the greater part of that tract is relayed in the medulla and pons, chiefly in the nucleus of Deiters and in the inferior olivary nucleus.(?)

It should be noticed that the (posterior) cerebello-spinal fasciculus, the external arcuate fibers and the anterior ascending cerebellospinal tract, with the tract from the lateral nucleus, are the in-coming parts of the indirect sensory path, and that the brachium conjunctivum is the out-going continuation of that path up to the red nucleus and thalamus; and again, that the brachium pontis, the acustico-cerebellar tract and the descending fibers of the restiform body constitute segments of the indirect motor path and have to do with coordination and equilibrium.

\section{COMMISSURAL FIBERS.}

The cerebellar hemispheres are joined by transverse fibers, of which there are two sets, namely: One near the anterior end of the worm beneath the central lobe, and the other at the posterior end of the worm.

\section{ASSOCIATION FIBERS.}

Limited areas of cerebellar cortex are richly associated together as pointed out in the description of the cortical gray substance; 
but there appears to be nothing analogous to the long association fibers found in the cerebrum.

\section{RHOMBENCEPHALON.}

\section{SECTION II. THE PONS (VAROLII).}

The pons and medulla form the ventral part of the rhombencephalon, the cerebellum being its dorsal portion. By a transverse indentation of its roof, the posterior brain-vesicle is partially divided into an upper vesicle, the metencephalon, and a lower vesicle, the myelencephalon; the latter is the embryonic medulla, the former gives rise to the cerebellum and the pons. The pons is developed from the floor of the metencephalon (Fig. 79). It is so named because it forms the connecting link, or bridge, between the mid-brain, above, and the cerebellum and medulla oblongata, below; between the medulla and cerebellum, and between the two cerebellar hemispheres (Fig. 85).

In shape the pons is roughly cylindrical. It has a broad basal or ventral part, the pars basilaris pontis, and a narrower dorsal portion, the pars dorsalis pontis (Fig. 87).

Size.-The pons is about one inch long. It is a little broader than long, and measures an inch, dorso-ventrally.

Position.-It rests in the anterior end of the groove which extends from the foramen magnum to the dorsum sellæ, and lies between and ventral to the hemispheres of the cerebellum. Superiorly, it joins the mid-brain; and, below, it is continuous with the medulla oblongata.

Surfaces of the Pons. - The pons has four surfaces, viz., superior (attached); inferior (attached); anterior (free), and posterior (partially free); and two borders, namely, right and left lateral, continuous with the brachium pontis of the cerebellum.

The superior and inferior surfaces are made by section, and are directly continuous with mid-brain above and the medulla below.

Anterior Surface (Tuber annulare).-The anterior surface of the pons (Fig. 85) looks forward and slightly downward and rests on the sphenoid bone behind the sella turcica. It is divided 


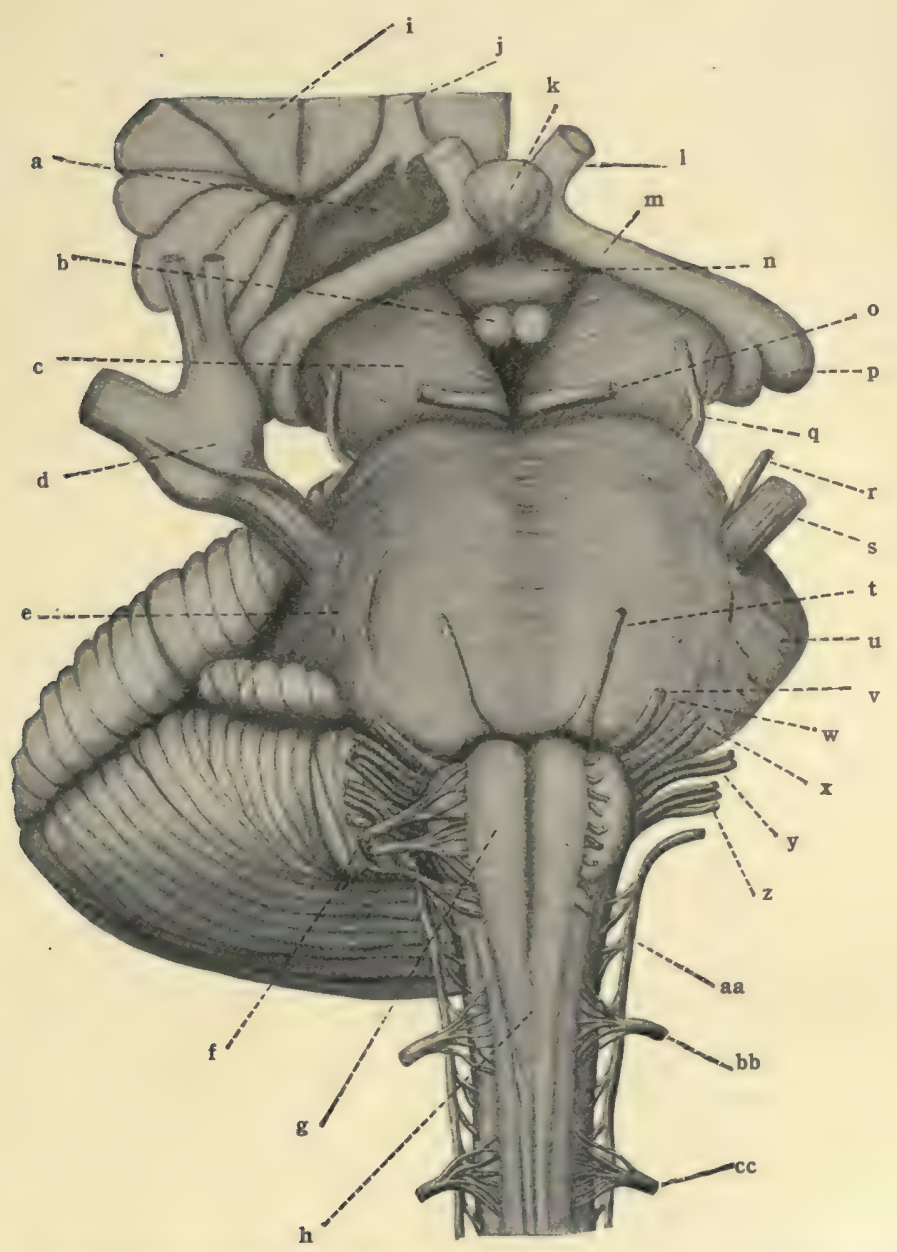

Fig. 85.-Anterior aspect of mid-brain, pons and medulla. (After Morris's Anatomy.)

a. Anterior perforated substance. b. Corpus mammillare. c. Cerebral peduncle. d. Ganglion semilunare (gasseri). e. Oblique fasciculus. f. N. hypoglossus (XII). g. Pyramiu h. Decussation of pyramids. i. Insula. j. Olfactory tract. k. Hypophysis. 1. N. opticu. (II). m. Optic tract. n. Tuber cinereum. o. N. oculomotorius (III). p. Lateral genicu late body. q. $\mathrm{N}$. trochlearis (IV). r. N. trigeminus (M. P.). s. N. trigeminus (V). t. N. abducens (VI), u. Brachium pontis. v. N. facialis (VII), w. N. intermedius. $\mathbf{x} . \mathbf{N}$ acusticus (VIII), y. N. glossopharyngeus (IX). z. N. vagus (X). aa. N. accessorius (XI) (spinal accessory). bb. Cervical I, cc. Cervica :II. 

into lateral halves by the sulcus basilaris, containing the basilar artery; and is bounded laterally by a sagittal plane cutting the root of the trigeminal nerve. Vertically the surface is slightly convex, and is markedly so from side to side. It shows transverse striations, which converge laterally, due to the fibers that form it and enter the brachia pontis of the cerebellum. The fibers of the anterior surface are not exactly transverse in direction. Those at the superior end of the pons bend downward (fasciculus obliquus) and form a rounded margin, which covers the lower part of the bases pedunculi of the mid-brain; at the inferior extremity of the pons, the fibers are convex downward and partially conceal the pyramids of the medulla oblongata. The two roots of the fifth nerve (trigeminal) are attached to the lateral border (Henle) of this surface, a little above the middle.

The posterior surface of the pons is concealed by the cerebellum (Fig. 88). It is free in its middle part, where it forms the floor of the superior half of the fourth ventricle (Fig. 86). The ventricular area of the posterior surface is completely concealed by the superior medullary velum. If examined, it is found to be divided into lateral halves by a median longitudinal groove. Each half presents in its posterior part a rounded eminence, the colliculus facialis, which flanks the median furrow and is in turn bounded, laterally, by a linear valley, the sulcus limitans, lying near the brachium conjunctivum cerebelli and parallel with it. The inferior end of the valley is called the fovea superior; its upper part has a bluish tint, due to underlying pigmented cells, and is called the locus caruleus. Attached Area.-Lateral to this ventricular area, the posterior surface of the pons is attached to the restiform body and the conjoined arms of the cerebellum. The restiform bodies enter the surface near the lower end of the pons and then bend downward into the medulla oblongata; while the brachia conjunctiva, in their course up to the cerebrum, partly imbed themselves in the lateral part of the posterior surface and form the walls of the fourth ventricle. The lateral fillet issues from this surface just external to the brachium conjunctivum. It runs obliquely across the upper end of the brachium to the inferior colliculus of the corpora quadrigemina, and pro- 
duces a flat striated ridge, which may be seen easily in a well hardened specimen.

Structure of the Pons. - The pons is composed of transverse and longitudinal white fibers and of gray matter. The trans-

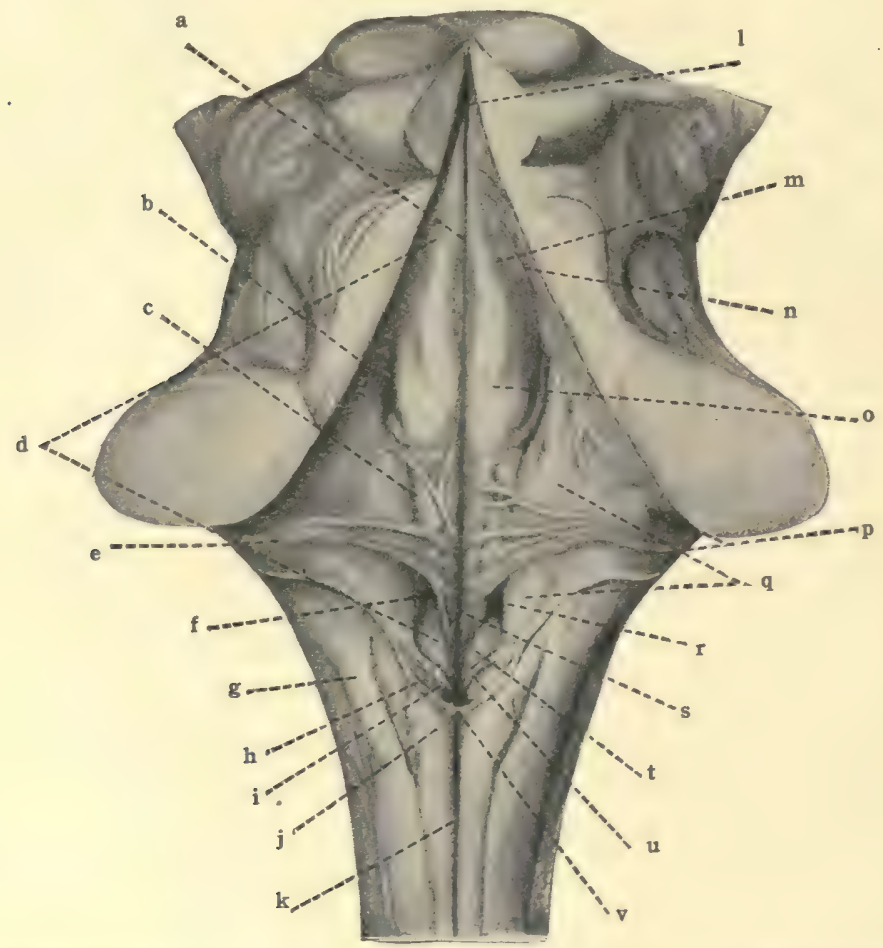

Fig. 86.-Dorsal surface of pons and medulla. (Morris's Anatomy modified from Spalteholz.)

a. Median sulcus. b. Superior fovea. c. Limiting sulcus. d. Medial eminence. e. Stria medullares. f. Inferior fovea. g. Nucleus funiculi cuneati. h. Tænia of fourth ventricle. i. Area postrema. j. Nucleus funiculi gracilis (clava), k. Posterior median fissure. 1. Aquæductus cerebri. m. Nucleus incertus. n. Locus cæruleus. o. Colliculus facialis et nucleus abducentis. p. Nucleus N. cochlearis (tuberculum acusticum). q. Area acustica (nucleus vestibularis). r. Nucleus intercalatus. s. Trigonum N. hypoglossi. t. Ala cinerea. u. Funiculus separans. $\nabla$. Obex.

verse fibers are found chiefly in the basilar portion of the pons; the longitudinal, in both the basilar and the dorsal part. The anterior longitudinal intersect the deep transverse fibers of the pars basilaris. 


\section{TRANSVERSE FIBERS OF PONS.}

The transverse fibers form three consecutive layers in the pons, viz., the anterior, the middle, and the posterior layer. They lie one upon another. The two former are situated in the basilar area of the pons, the latter in the dorsal area (Figs. 87 and 88).

The superficial transverse fibers are anterior in position and form a thin compact layer constituting the anterior surface of the pons (Fig. 87). They are not intersected by longitudinal fibers; but, otherwise, are like the deeper transverse fibers of the pars basilaris pontis.

Deep Transverse Fibers of Pars Basilaris. - These form a thick lamina posterior to the superficial transverse fibers and in contact with the superficial lamina. They are intermingled with longitudinal fibers from the bases pedunculi, viz., the pyramidal, fronto-pontal, temporo-pontal and intermediate tracts (Figs. 87 and 88). In the meshes between the intersecting fibers is a large mass of gray.matter, on either side, called the nucleus pontis. The deep and superficial transverse fibers of the pars basilaris pontis form the brachia pontis cerebelli. Their origin is found in the opposite nucleus pontis (Klimoff). They continue the indirect motor path from the termination of the fronto-pontal, temporo-pontal and intermediate tracts in the nucleus pontis to the cerebellar cortex of the opposite side.

In connection with the superficial and deep transverse fibers in the basilar part of the pons there should be mentioned an independent strand, the fila lateralia pontis, which is situated at the upper border of the pons and buried more or less in the isthmian furrow between the pons and mid-brain. It is called by Henle the tænia pontis. According to Sir Victor Horsley, it rises from the nucleus pontis just ventral to the interpeduncular ganglion, and, winding round the isthmus, enters the cerebellum through the brachium conjunctivum. Its destination is probably the nucleus dentatus and nucleus fastigii (Brain, Vol. 29, No. II3).

The transverse fibers of the pars dorsalis pontis (Fig. 88) compose a thin layer on the dorsum of the middle transverse fibers, separating them from the formatio reticularis. This 
transverse lamina is present only in the inferior part of the pons. It is called the corpus trapezoideum.

The trapezoid body (corpus trapezoideum) lies in the dorsal area of the pons, next the boundary between the pars basilaris and the pars dorsalis pontis. Its fibers rise chiefly from the nuclei of the cochlear nerve and, after decussating in the raphe, are continued up in the lateral fillet to the inferior quadrigeminal colliculus. A few fibers join the tract directly from the cochlear nerve. The nuclei of the trapezoid body and of the superior olive form relays for a number of its fibers. The corpus trapezoideum with the medullary striæ, and the lateral fillet, which is the continuation of both, form the second stage in the auditory conduction path; and the auditory impulses are continued (a) through the brachium quadrigeminum inferius and (b) the temporo-thalamic radiation to the temporal cortex (Fig. 89).

\section{LONGITUDINAL FIBERS OF PONS.}

The longitudinal fibers of the pons are arranged in two distinct groups, viz., the ventral or anterior and the dorsal or posterior (Figs. 87 and 88 ). The groups are separated by the trapezoid body.

The ventral longitudinal fibers are situated in the pars basilaris pontis (Figs. 87 and 88). Four fasciculi make them up. They are the four motor tracts of the basis pedunculi. The frontopontal, temporo-pontal and intermediate tracts terminate in the nucleus pontis. The pyramidal fibers run from the middle threefifths of each basis pedunculi down through the middle transverse layer of the pons to the pyramids of the medulla oblongata. Together with the above cerebro-pontal tracts they form a thick bundle on either side of the median line, which presses down the superficial transverse fibers and produces the sulcus basilaris. The nucleus pontis, one on either side, is situated among the pyramidal fibers. The pyramidal tracts diminish in size during their descent, because of the fibers which leave them to decussate and end in the nuclei of motor cerebral nerves.

The Dorsal Longitudinal Fibers. - These are contained in 
the pars dorsalis pontis in the formatio reticularis (Figs. 87 and 88). They are dorsal to the corpus trapezoideum, and lie in the floor of the fourth ventricle, where they are intermingled with the ventricular gray substance. They do not form a compact layer, but are collected into a number of distinct strands, of which the larger are visible to the naked eye in Weigert-Pal sections. The dorsal longitudinal fibers are mingled with many transverse and oblique fibers; and, thus, there is produced the netlike arrangement suggesting the name, formatio reticularis. The formatio reticularis of the pons is continued down from the tegmentum of the mid-brain and comprises the tegmental region of the pons. The gray matter in the meshes of this network, which is the stratum nucleare continued up from the medulla, contains the nuclei of the fifth, sixth and seventh cerebral nerves and a part of the nucleus of the eighth nerve, and also the nuclei of the formatio reticularis, viz., the nucleus centralis superior, medius and inferior, and the nucleus lateralis medius. In the formatio reticularis are the nine bundles or tracts of fibers that constitute the dorsal longitudinal fibers of the pons. These tracts are as follows: The anterior ascending cerebello-spinal tract, the spinothalamic tract, the medial fillet, the lateral fillet, the medial (posterior) and anterior longitudinal bundles, the rubro-spinal tract, the olivary fasciculus, and the descending root of the fifth cerebral nerve, besides unidentified fibers of the formatio reticularis which are probably both ascending and descending in conduction. The anterior ascending cerebello-spinal tract and the descending root of the trigeminal (or fifth) nerve are the only tracts not already considered in our study of the tegmental region of the mid-brain, page 156 .

(I) The medial fillet (lemniscus medialis, Figs. 87 and 88) is a large bundle of fibers that runs through the pons next the median plane. In the lower part of the pons it lies dorsal to the trapezoid body, though some of the trapezoidal fibers pierce it. Its origin is found on the opposite side in the nucleus funiculi gracilis and nucleus funiculi cuneati and in the terminal nuclei of common sensory cerebral nerves (Fig. 94). It conducts impulses of the tactile and muscular senses. In the mid-brain it 
gives off the superior fillet (lemniscus superior) which terminates in the superior quadrigeminal colliculus. The medial fillet ends in the lateral nucleus of the thalamus (Fig. 47). Interruption of the medial lemniscus causes ataxia on the opposite side.

(2) Lateral Fillet. (Lemniscus lateralis). - The lateral fillet forms a link in the special sense, auditory path (Fig. 89). As stated on page $I 60$ it is but the longitudinal continuation of the corpus trapezoideum and the medullary striæ. It takes form near the middle of the pons, where the fibers of the trapezoid body bend-upward to a longitudinal direction; and it runs just lateral to the medial fillet (Figs. 86 and 87). Very soon it becomes separated from the medial fillet by the brachium conjunctivum of the cerebellum. It runs dorso-medially over the conjoined brachium to the inferior colliculus of the corpora quadrigemina, where a few of its fibers end; but the greater number are continued through the brachium inferius to the medial geniculate body. The chief origin of the lateral fillet is found in the opposite cochlear nuclei, though some of its fibers rise in the nucleus of the corpus trapezoideum, the superior olivary nucleus, and the nucleus of the lateral fillet, which constitute partial relays in the auditory path. It is also true that a few fibers enter the lateral fillet from the cochlear nuclei and nerve of the same side; they are supposed to decussate near or in the quadrigeminal bodies and terminate in the opposite inferior colliculus. Destruction of the lateral fillet causes deafness, almost complete, in the opposite ear.

(3) The spino-thalamic tract occupies the lateral part of the formatio reticularis where it is intermingled with the anterior ascending cerebello-spinal tract (Figs. 87 and 88). As already stated, it rises in the spinal cord from the basal gray substance of the anterior columna and from the terminal nuclei of common sensory cerebral nerves in the medulla and pons. The spinothalamic tract ends in the lateral nucleus of the thalamus. It conducts impulses of the tactile, pain and temperature senses.

(4) The anterior ascending cerebello-spinal tract has the same spinal origin and function as the spino-thalamic tract and the same course up to the isthmus rhombencephali (Figs. 87 and 88). There, it bends backward, medial to the brachium con- 
junctivum, and, through the superior medullary velum, enters the superior worm of the cerebellum. It thus belongs to the indirect sensory path (through the cerebellum). From the cerebellar cortex the path is continued by the axones of Purkinje's cells to the nucleus dentatus, whence the brachium conjunctivum completes it up to the opposite red nucleus and thalamus. The anterior ascending cerebello-spinal and the spino-thalamic tracts

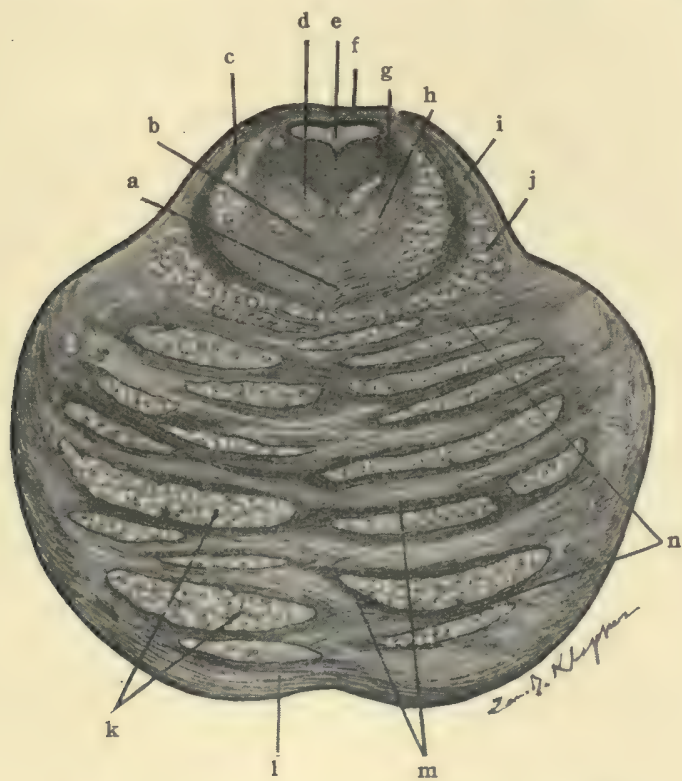

Fig. 87.-Superior section of the pons. (Original.)

a. Beginning of decussation of brachium conjunctivum. b. Formatio reticularis. c. Brachium conjunctivum. d. Medial longitudinal bundle. e. Fourth ventricle. f. Superior medullary velum. g. Descencding root of $5^{\text {th }} \mathbf{n}$. h. Spino-thalamic tract. i. Lateral fillet. j. Medial fillet. k. Long. fibers from basis pedunculi. 1. Superficial transverse fibers. m. Nucleus pontis. n. Deep transverse fibers of pars basilaris pontis.

are the chief bundles of a spino-encephalic system of fibers which terminates very largely in the cerebellum and thalamus, but also sends fibers to the nucleus lateralis inferior and other reticular nuclei, to the substantia nigra, to the inferior and superior colliculi of the corpora quadrigemina, to the nucleus ruber, the nucleus hypothalamicus, and the corpus striatum. In this spinoencephalic system there are two important paths-the direct 
path (No. 3) and the indirect path (No. 4)-for tactile, pain and temperature impulses. It is called Gowers's tract.

(5) The medial (posterior) longitudinal bundle (fasciculus longitudinalis medialis) (Figs. 87 and 88 ) runs next the median plane and just under the ventricular gray matter in a position similar to the one it occupies in the mid-brain (Figs. 47 and 48), see page ${ }^{5} 6$. It is in the pontine portion of this bundle that the fibers from the oculomotor nucleus pass to the genu of the facial nerve, ultimately to innervate the frontalis, corrugator and orbicularis oculi; it is in the pons, that fibers from the abducent nucleus join this bundle, and run upward through it to the oculomotor nucleus of the opposite side and make possible the conjugate movements of the eyeballs; it is also here, that fibers, which rise in the hypoglossal nucleus, leave the longitudinal bundle and enter the facial nerve at the genu to be distributed by way of the facial to the orbicularis oris. As in the mid-brain, the longitudinal bundle includes the two functional tracts, the descending strand and the ascending strand.

(6) Anterior Longitudinal Bundle.-This ocular-reflex bundle is continued from the mid-brain down through the pons in nearly the same relative position. Diverging a little from the medial longitudinal bundle as it descends through the pons, it is located in the formatio reticularis a short distance ventro-lateral from it. The anterior longitudinal bundle can be recognized in normal adult tissue only in the dorsal tegmental decussation (Meynerti) of the mid-brain: lower down it can be distinguished from the surrounding tissues by degeneration and medullation but in no other way (Fig. 87). Having given fibers to the oculomotor and trochlear nuclei above, it sends fibers to the nucleus of the abducent nerve at this level, and perhaps to other pontine nuclei (see Mid-brain, page $15^{8}$ ).

(7) Rubro-spinal Tract.-In the mid-brain, we have traced this tract from the red nucleus, through the ventral tegmental decussation (Foreli), to the opposite side, where it mingles with the lateral fillet down near the isthmus (Figs. 47 and 48). It occupies the same position in the upper half of the pons; it is close to the posterior surface of the corpus trapezoideum in the lower 
part of the pons (Figs. 87 and 88). In the center of the gray crescent of the spinal cord the rubro-spinal tract ends. Its function is unknown, perhaps it has to do with locomotion (Horshy).

(8) The olivary bundle (fasciculus olivaris) is regarded by many as an ascending tract, but the weight of evidence at present is in favor of a descending course. Rising in the lentiform nucleus and descending through the mid-brain, it enters the center of the

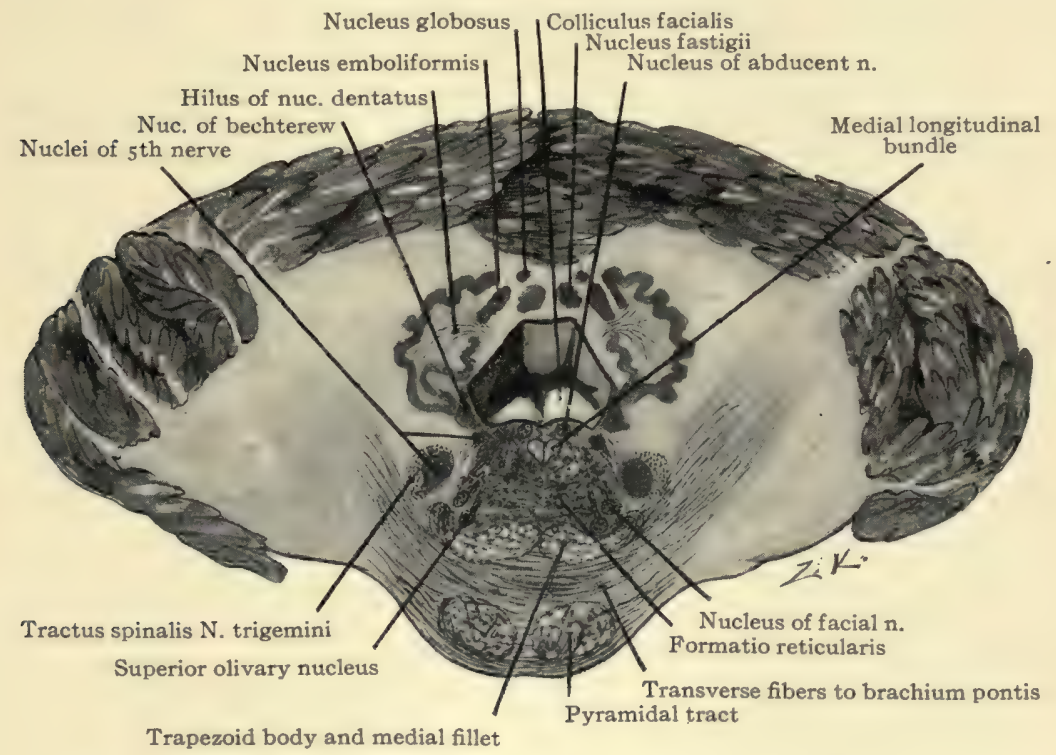

Fig. 88.- Inferior section of the pons together with the cerebellum. (Original.)

reticular formation of the pons, hence the name, central tegmental tract (Fig. 88). It runs dorsal to the medial fillet and the corpus trapezoideum in the lower part of the pons, just medial to the superior olivary nucleus. It terminates in the inferior olive.

(9) The descending root of the trigeminal nerve (Fig. 87) is crescentic in section and runs between the ventricular gray substance and the brachium conjunctivum of the cerebellum down to the middle of the pons, where it joins the principle motor root. It passes lateral to the motor nucleus situated under the locus 
cæruleus, and runs between it and the superior end of the terminal or sensory nucleus.

In the lowest part of the pons there is seen the spinal tract of the fifth nerve (racus spinalis nervi trigemini). This is a part of its sensory root and is composed of the descending limbs of T-branched axones from the semilunar ganglion (Gasseri). The spinal tract of the trigeminal nerve runs lateral to the gray substance, between the substantia gelatinosa (Rolandi), on the inner side, and the vestibular root of the acustic nerve and the restiform body on the outer side.

\section{GRAY MATTER OF THE PONS.}

In the pons gray matter is found in two situations: (I) In the interstices between the deep transverse fibers of the pars basilaris pontis and the ventral longitudinal fibers, the nuclei pontis; and (2) in the formatio reticularis, just under the fourth ventricle where it forms the stratum nucleare.

The nucleus pontis is a mass of gray matter, on either side the raphe, containing the bodies of large multipolar nerve cells whose axones run through the brachium pontis of the cerebellum to the cortex on the opposite side. It extends vertically throughout the pons and is continuous with the arcuate nucleus of the medulla. The nucleus pontis receives the terminals of the descending tracts which form the inner and outer fifths of the basis pedunculi and the intermediate bundle of the same, and thus connects these tracts with the cerebellum. It forms a relay in the indirect motor path.

The gray matter of the stratum nucleare includes (I) the olivary group of nuclei, viz., the superior olivary nucleus, the nucleus of the corpus trapezoideum, the preolivary nucleus and the semilunar nucleus; (2) the nuclei of the formatio reticularis, viz., the nucleus centralis superior, medius and inferior, and the nucleus lateralis medius; and (3) the nuclei of cerebral nervesthe fifth, sixth and seventh, and a part of the vestibular nucleus of the eighth nerve.

(I) Olivary Group.-The superior olivary nucleus (n. olivaris 
superior) is situated in the lateral part of the formatio reticularis in the dorsal portion of the corpus trapezoideum (Fig. 88). It lies just ventral to the nucleus of the facial nerve and ventrolateral to the olivary bundle of fibers. The nucleus contains small bodied nerve cells; and, in this respect, resembles the olive of the medulla. Its outline is crescentic, convex toward the median line. In size it is microscopic. According to Bruce and Cunningham it is continuous with the nucleus of the lateral fillet. The superior olive constitutes a subordinate relay in the auditory path, receiving fibers from the cochlear nuclei of both sides and contributing fibers to both lateral fillets (Fig. 89).

The superior olivary nucleus gives off a small strand of fibers, called the olivary pedicle, which runs dorso-medially, between the recurrent and emergent parts of the root of the facial nerve, to the nucleus of the abducent nerve; there some of its fibers end, the remainder join the medial longitudinal bundle and run to the trochlear and oculomotor nuclei. The pedicle forms part of an auditory-ocular reflex arc.

A small accessory nucleus, called the nucleus proolivaris, is situated just a little ventral to the superior olivary nucleus; and a second one embraces the convexity of the nucleus olivaris superior, lying on the medial side of it. The latter is the nucleus semilunaris.

Nucleus of the Trapezoid Body (N. Corporis Trapezoidei).This nucleus is deeply imbedded in the trapezoid body ventromedial to the superior olivary nucleus (Fig. 88). Its cell-bodies are scattered and, like the other nuclei of the olivary group, it forms a partial relay for the auditory path. This nucleus is peculiar; the fibers it receives terminate in the form of cup-shaped.discs, acustic cups, which are in direct contact with its cell-bodies (Held).

(2) The nuclei of the reticular formation contained in the pons are the $n$. centralis superior, $n$. centralis medius, $n$. centralis inferior and n.lateralis medius. All are microscopic. They are made up of large scattered cell-bodies whose axones, dividing $\mathrm{T}$ like, are both ascending and descending in direction (Tschermak). We may divide these axones into two groups, a crossed and an uncrossed. The crossed fibers pierce the median plane and become 
longitudinal in the formatio reticularis near the ventricular gray matter and medial to the root of the abducent nerve. At that point they bifurcate and one branch runs upward and the other downward. The descending branches of the crossed fibers (lateral ponto-spinal tract) pass through the substantia reticularis grisea of the medulla and the lateral area of the spinal cord throughout its length; they end in the center of the gray crescent in successive segments until exhausted near the end of the cord (Tschermak,

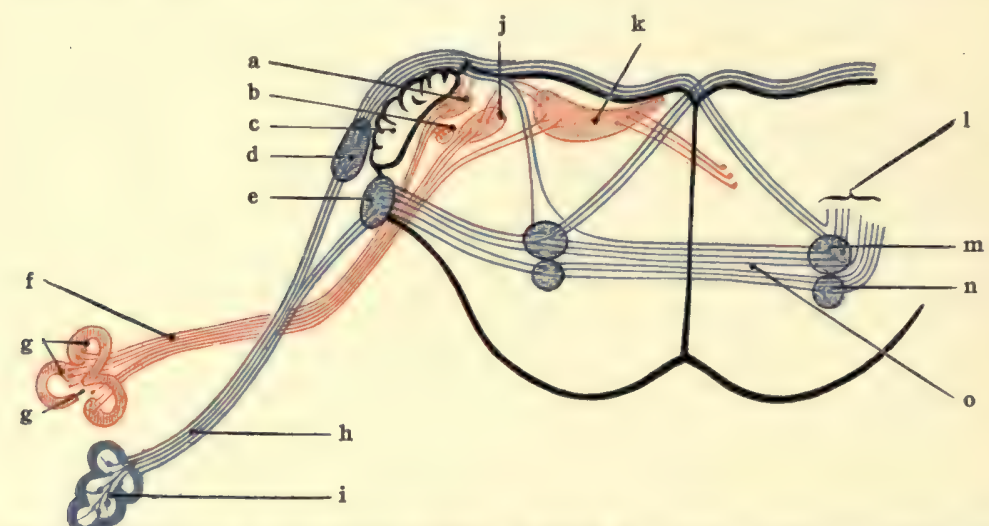

Fig. 89.-Diagram of a transverse section through the junction of the medulla and pons showing the roots and nuclei of the eighth cranial nerve and the auditory paths in the pons. (After Morris's Anatomy.)

a. Bechterew's nucleus. b. Nuc. of descending root. c. Restiform body. d. Lat. cochlear nucleus. e. Ventral nucleus. f. Vestibular nerve. g. Semicircular canals. h. Cochlear nerve. i. Cochlea. j. Dorso-lateral nucleus (Deitersi). k. Dorso-medial nucleus. 1. Lateral fillet. m. Superior olivary nucleus. n. Nucleus of trapezoid body. o. Trapezoid body.

Barker). The uncrossed fibers from the reticular nuclei enter the medial longitudinal bundle of the same side and there branch T-like. The descending branches (anterior ponto-spinal tract) run with this bundle into the anterior column of the spinal cord, through which some of them continue to the end. They occupy the outer side of the anterior funiculus and end in succession in the anterior columna of gray matter (Tschermak and Barker). Just what is the destination of the ascending branches of either group of fibers has not been determined. J. S. Collier suggests that these tracts from the reticular nuclei should be called the crossed and uncrossed ponto-spinal tracts (Brain, Vol. 24, I90I). 
(3) Nerve Nuclei.-The nuclei of the trigeminal nerve (nuclei nervi trigemini) are two in number. The genetic or motor nucleus of the fifth nerve ( $n$. motorius) in the pons is a continuation of the mesencephalic nucleus. It is rather close to the fourth ventricle in the extreme lateral part of its floor, underneath the locus cæruleus (Fig. 86). It extends as far down as the middle of the pons, where the whole group of axones passes forward into the motor root of the nerve. Cortical Connection.-The nucleus receives motor fibers from the opposite pyramidal tract and perhaps from the cerebro-pontal tracts of the same side; and sensory fibers terminate in it from the sensory root of the fifth nerve, and from the terminal nuclei of other common sensory nerves, through the medial longitudinal bundle and establish its reflex connections.

The terminal or sensory nucleus ( $n$. terminalis or sensibilis) of the trigeminal nerve begins at the middle of the pons and extends to the second segment of the spinal cord. At its superior end it is ventro-lateral to the motor nucleus and under cover of the brachium conjunctivum of the cerebellum (Figs. 86 and 87). Near the medulla it lies ventro-medial to the restiform body and the vestibular root of the acustic nerve. This part of it is almost in contact with the nucleus of the facial nerve, and its distance from the ventricle is greater than it is higher up. The nucleus is gelatinous in character and is continuous with the same substance in the posterior columna of the spinal cord. It receives the whole sensory root of the trigeminal nerve. Just lateral to it runs the spinal tract of the fifth nerve, the fibers of which gradually bend into the nucleus and terminate in rich arborizations. Axones from the nucleus pursue several different courses: (a) Reflex fibers go directly to the motor nucleus of the fifth and through the medial longitudinal bundle to other motor nuclei. (b) Tactile, pain and temperature fibers are supposed to enter the opposite spino-thalamic tract through which they reach the thalamus; perhaps, some run through the anterior cerebello-spinal tract to the cerebellum. (c) Tactile and muscular sense fibers proceed to the thalamus, probably through the medial fillet on the opposite side.

Nucleus of the Abducent Nerve (N. Nervi Abducentis).-This 
motor nucleus is close to the median plane and is separated from the ependyma of the ventricular floor only by the fibers of the seventh or facial nerve. It is situated in the colliculus facialis (Figs. 86 and 88). The root-fibers of the facial nerve run lateral to the sixth nucleus, describe a loop on its dorsal surface and then return lateral to it. Cortical Connection.-The abducent nucleus receives the end-tufts of motor fibers from the opposite pyramidal tract and from the cerebro-pontal tracts. It receives reflex impulses through the anterior and medial longitudinal bundles and the pedicle of the superior olivary nucleus; and perhaps, also, through the brachium conjunctivum from the cerebellum. The axones of the cell-bodies in the adbucent nucleus run in two directions: The greater number run ventro-lateralward and emerge at the lower part of the pons, as abducent nerve; a small bundle of axones runs to the oculomotor nucleus on the opposite side by way of the medial longitudinal bundle. The former innervates the external rectus muscle of the eye on the same side as the nucleus; the latter, through the third nerve, innervates the internal rectus of the opposite eye, though that muscle receives independent fibers from the third, also.

The nucleus of the facial or seventh nerve ( $n$. nervi facialis) is genetic or motor (Fig. 88). It is situated deep in the pons, in the lateral part of the formatio reticularis, beneath the superior fovea. Immediately ventral to it is the superior olivary nucleus, and the substantia gelatinosa (Rolandi) lies dorso-lateral to it. The nucleus is placed midway between the spinal tract of the fifth nerve and the olivary fasciculus. Cortical Connections.It receives voluntary motor impulses from the cerebral cortex of the opposite hemisphere via the pyramidal tract; and, probably, fibers of the cerebro-pontal tracts terminate in it. These establish its motor connections. The reflex. connections of the facial nucleus are established by fibers from the spinal tract of the trigeminal nerve, from the trapezoid body (Cunningham), and from the medial longitudinal bundle. The axones of the cellbodies in the nucleus facialis all enter the root of the facial nerve. By its direction this root is divided into three parts, viz., two distinct parallel parts, joined by a very short ascending portion. 
(I) The recurrent part, the pars prima, runs dorso-medianward to the colliculus facialis, passing lateral and then dorsal to the lower end of the abducent nucleus; (2) it then ascends about onefifth of an inch (Cunningham) between the ventricular ependyma, dorsally, and the abducent nucleus and medial longitudinal bundle, ventrally, and this part is called the genu internum; and (3) the pars secunda, bending sharply outward over the nucleus of the sixth nerve, then plunges ventrally through the pons; this emergent part of the root runs between the nuclei of the facial and trigeminal nerves. The root of the facial nerve is joined at the genu internum by fibers from the medial longitudinal bundle which rise in the oculumotor and hypoglossal nuclei and supply the facial muscles above the orbit and the orbicularis oris respectively.

Just dorsal to the facial nucleus is a small group of cell-bodies which is said to constitute a salivary nucleus (Cunningham). Its axones run to their destination through the nervus intermedius and its chorda tympani branch, and form the efferent root of the nervus interdemedius. They are secretory and vasodilator fibers to the submaxillary and sublingual glands.

Vestibular Nucleus of the Auditory Nerve (N. Nervi Vestibularis) (Fig. 88).--This nucleus is made up of three parts: (I) The chief nucleus (Schwalbe); (2) the nucleus of the descending root, and (3) the mucleus of Deiters, which is lateral in position. The superior parts of Schwalbe's and of Deiters's nuclei extend into the pons just medial to the restiform body, and the nucleus of Deiters is prolonged dorsally along that body toward the cerebellum. This dorsal extension of Deiters's nucleus is called Flechsig's or Bechterew's nucleus. We shall recur to the vestibular nucleus in the medulla where the greater part of it is located.

Lesions in the pons are usually attended by crossed paralysis. The paralysis and anæsthesia of parts supplied by spinal and by bulbar cerebral nerves are on the opposite side, but the fifth, sixth and seventh cerebral nerves of the same side as the lesion are apt to be involved. If the spino-thalamic and anterior ascending cerebello-spinal tracts are involved and not the medial fillet, the pain and temperature sense is lost, but there is no ataxia; if 
the medial fillet be destroyed and not the spino-thalamic and anterior ascending cerebello-spinal tracts, then the pain and temperature sense is intact, but the muscular sense is lost on the opposite side of the body. The tactile sense is impaired in both cases. A lesion of the trapezoid body produces almost total deafness; of the lateral fillet, slightly impaired hearing on the same side and nearly complete deafness in the opposite ear. Conjugate deviation occurs when the nucleus of the sixth nerve is affected; and strabismus when the root fibers, but not the nucleus, are involved. The strabismus is external if the lesion be irritative and internal if the root fibers are destroyed. Destructive lesion in the nucleus of the seventh nerve causes inferior paralysis of the face, the frontalis, corrugator, orbicularis oculi and orbicularis oris not being affected. Complete facial paralysis occurs if the root-fibers of the facial nerve be destroyed in the pars secunda or in the genu internum.

\section{RHOMBENCEPHALON.}

\section{SECTION III. THE MEDULLA OBLONGATA. (MYELENCEPHALON.)}

Situation. - The medulla oblongata is the distal, or caudal part of the brain (Figs. 2I and 27). It may be regarded as the expanded intra-cranial portion of the spinal cord, hence the synonym, spinal bulb. It occupies the basilar groove of the occipital bone, posterior to the pons; and is continuous with the spinal cord below the foramen magnum. Dorsally, it is in part concealed in the valley of the cerebellum. The vertebral arteries wind forward around it, and form the basilar at its junction with the pons.

Size.-The medulla is about an inch long, and dorso-ventrally, is a half-inch thick. Its width at the lower end is a half-inch, also. At the upper extremity it measures from three-quarters of an inch to one inch in width (Figs. 85 and 86).

Its shape resembles an inverted frustum of a cone flattened dorso-ventrally at the base. The truncated apex of the frustum, which is nearly circular in outline, is continuous with the spinal 
cord; and the flattened base joins the pons. On the anterior surface, a transverse groove marks the boundary between the medulla and pons. The medulla is a bilateral organ composed of symmetrical halves (Figs. 85 and 86). In the interior, the two halves are united by both gray and white matter in the raphe, but on the surface they are partially separated by the anterior and the posterior median fissures (fissura mediana anterior and $\mathrm{f}$. m. posterior). These fissures are continued through the spinal cord, but neither extends the whole length of the medulla. The anterior median fissure is interrupted in the lower part of the medulla by the crossing of two large tracts of fibers, forming the

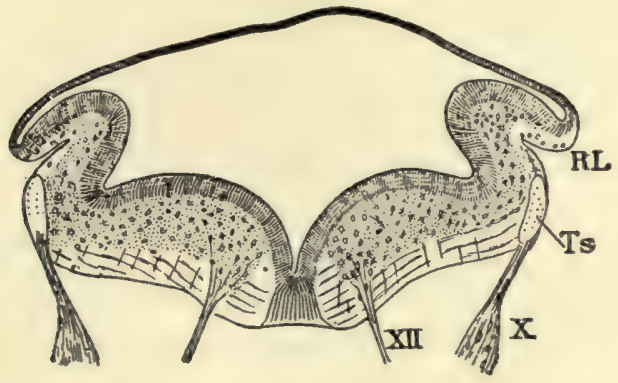

Fig. 90.-Section of embryonic medulla. Length of back, 9.I mm.

(Gordinier and Minot after His.)

RL. Rhomboid lip. Ts. Tractus solitarius. X. Vagus nerve, XII. Hypoglossal nerve.

decussation of the pyramids; while only through the lower half of the medulla does the posterior median fissure extend.

Origin.-The medulla oblongata is developed from the myelencephalon of the embryo (Figs. 90 and II8). The myelencephalic floor and walls thicken and form the greater part of the medulla. Inferiorly, the roof undergoes some thickening; but it stretches out into a single layer of epithelium, superiorly, which is continuous at its upper end with the inferior medullary velum of the cerebellum.

Ventricle.-The common cavity of the posterior brain vesicle persists in the mature brain as the fourth ventricle (Figs. II8, 90 and 86). The fourth is, therefore, the ventricle of the adult rhombencephalon (see page 3 I3). 


\section{SURFACES.}

The medulla oblongata presents four surfaces: The anterior, posterior and two lateral, separated by the anterior lateral and posterior lateral grooves. In the upper medulla, the surfaces are clearly defined; but they become less distinct as they descend to the inferior and nearly circular extremity (Figs. 8I, 85 and 86).

The anterior lateral sulcus (s. lateralis anterior) separates the anterior from the lateral surface, and is in line with the exits of the anterior roots of the spinal nerves. No corresponding groove exists in the cord. From the anterior lateral groove issue the roots of the hypoglossal nerve and the anterior root of the first cervical nerve. The abducent (or sixth) nerve rises nearly in line with it from the transverse groove between the pons and the medulla (Fig. 86).

Posterior Lateral Sulcus. (S. lateralis posterior).-The posterior lateral sulcus of the medulla separates the lateral from the posterior surface (Figs. 86 and 87). It descends between the olive and the restiform body and is continued through the spinal cord. Through this sulcus into the cord run the posterior roots of the spinal nerves, and, likewise, the sensory roots of the vagus and glossopharyngeal nerves run through it into the medulla; while the motor roots of the ninth and tenth and the cerebral root of the eleventh nerve emerge from the medulla through the posterior lateral sulcus. The roots of the seventh, eighth and intermediate nerves are found at the superior end of the sulcus in the transverse groove between the medulla and pons. The posterior lateral sulcus is not parallel with the axis of the medulla, but bends outward and forward as it ascends. Inferiorly, it is obliterated for a short distance by the crossing of the fasciculus cerebellospinalis (direct cerebellar tract) from the lateral to the posterior surface.

The anterior surface (facies anterior) of the medulla, bounded on either side by the anterior lateral sulcus, extends from the transverse sulcus below the pons down to the spinal cord (Fig. 85). It is made up of symmetrical halves united, below, by the decussation of the lateral (crossed) pyramidal tracts; but separated, above, by the anterior median fissure, which terminates at the 
inferior end of the pons in a blind foramen (foramen cæcum of Vicq d'Azyr). On either side of the median fissure, the anterior surface presents a fusiform eminence, most prominent near the pons, called the pyramid. The pyramidal tract, which we have already traced through the internal capsule, basis pedunculi and pons, forms the pyramid of the medulla. In the lower part of the medulla the pyramid divides into two tracts, viz., the lateral (or crossed) pyramidal tract and the anterior (or direct) pyramidal tract, the former comprising about the medial four-fifths and the anterior pyramidal tract the lateral one-fifth of the pyramid. Transverse fibers, called the anterior external arcuate, are also seen crossing the pyramid from within outward. They form a more or less continuous sheet of fibers, which emerges from the anterior median fissure and winds around the medulla to the posterior surface, where its fibers enter the restiform body. The anterior surface is identical with the surface of the two anterior areas of the medulla.

Lateral Surface. (Facies lateralis, Figs. 8I and 85).-There are two lateral surfaces, a right and a left. Each is bounded by the anterior lateral and the posterior lateral sulcus; and is inclosed between the roots of the hypoglossal nerve, ventrally, and those of the ninth, tenth and the cerebral portion of the eleventh, dorsally. Lateral surface is synonymous with the surface of the lateral area. The lateral surface is formed, above, by the olive, below, by the lateral column and, winding backward over both, are the anterior external arcuate fibers.

The olive (oliva) is an elongated eminence, a half inch in length, situated just below the pons (Fig. 85). It is produced by the inferior olivary nucleus in the lateral area of the medulla; and, superficially, is composed of fibers continuous with the fasciculus proprius of the lateral column in the spinal cord (Fig. 93).

Lateral Column. (Funiculus lateralis, Fig. 85). - It is made up of three great bundles of fibers (Figs. 93, 94 and 95): The lateral fasciculus proprius, which, splitting into a superficial and a deep lamina, incloses the inferior olivary nucleus; the anterior descending cerebello-spinal tract, running down the anterior lateral sulcus; and the anterior ascending cerebello-spinal and spino- 
thalamic tract, which runs up the posterior lateral groove. At the junction of the medulla with the spinal cord the cerebellospinal fasciculus (direct cerebellar tract) passes from the lateral to the posterior surface. The anterior external arcuate fibers, running from the anterior surface backward to the restiform body, may be so numerous as to conceal the lateral column and lower part of the olive.

The posterior surface (facies posterior) of the medulla comprises all the surface inclosed between the diverging posterior lateral sulci (Fig. 86). It embraces the surfaces of the two posterior areas of the medulla.

Inferiorly, it is divided into lateral halves by the posterior median fissure, and presents four bundles of fibers in each half (Figs. 9I, 94 and 96). From the fissure outward they are as follows: The funiculus gracilis, funiculus cuneatus, tractus spinalis $n$. trigemini, and the cerebello-spinal fasciculus. The funiculus gracilis (Fig. 96) is a continuation of the medial tract of the posterior column of the spinal cord, and the funiculus cuneatus is in direct continuity with the lateral tract in the same column of the cord. These two bundles leave the surface and end in the nuclei of these columns in the medulla. The spinal tract of the trigeminal nerve is here situated on the surface; its fibers end in the under-lying gelatinous substance. The fasciculus cerebellospinalis (direct cerebellar tract) is continued up from the lateral column of the spinal cord. Remaining on the surface it runs up to the cerebellum through the restiform body.

Superiorly, the posterior surface, on either side, is formed by a large rounded band of fibers, the restiform body (Figs. 91 and 96). The restiform body (corpus restiforme) is continued upward to the pons and then bends backward into the corpus medullare of the cerebellum in connection with which it has already been studied. Of the superficial fibers in the medulla, the cerebellospinal fasciculus, and the anterior and posterior external arcuate fibers are continued into the restiform body; and, as we have already learned, the restiform body contains, in addition to the above three tracts, the tract from the lateral nucleus and the cerebello-olivary tract. A single layer of flattened epithelial cells 
stretches between the two restiform bodics, and roofs over the inferior part of the fourth ventricle. That is the roof epithelium. It is continuous with the surface layer of the ependyma lining the fourth ventricle. By it the posterior surface is completed.

The roof epithelium (Figs. 9o and 9I), seen in the mid-dorsal surface of the medulla, is of triangular shape; its base is attached to the inferior medullary velum of the cerebellum; its apex, which is directed downward, terminates at the obex and covers the

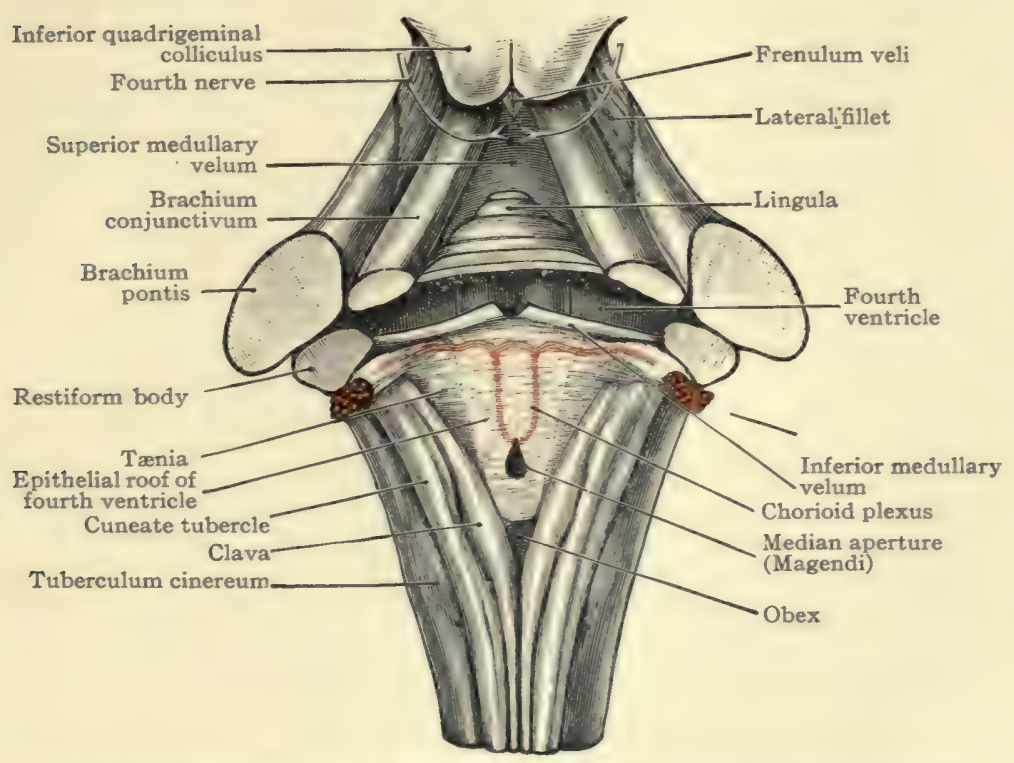

Fig. 9r.-Roof and lateral walls of fourth ventricle, and its chorioid plexuses. (After Morris's Anatomy.)

inferior angle of the fourth ventricle; and, laterally, it is attached to the clava, the cuneate funiculus and the restiform body. The line of attachment to the restiform body runs, first, obliquely upward and outward; and then, transversely outward, inferior to the lateral recess. The borders of the epithelial lamina become thickened by the addition of neuroglia, and are in continuity with the ependyma of the ventricle. The thickened apex of the epithelial lamina is called the obex. With the pia mater investing 
it, termed the chorioid tela of the fourth ventricle, this roof epithelium is perforated in the median line near the obex by a foramen, the median aperture (apertura mediana ventriculi quarti, Magendi) and over each lateral recess by the lateral aperture (apertura lateralis ventriculi quarti, Key and Retzii). These foramina establish communication between the subarachnoid space and the ventricle. On either side of the median line there is a longitudinal invagination of the epithelial lamina into the ventricle and a similar transverse one just below the inferior medullary velum, both of which are occupied by a vascular fold of pia mater. This fold constitutes the chorioid plexus of the fourth ventricle (plexus chorioideus ventriculi quarti). If the roof epithelium be torn away, as it usually is with the pia, a rough line of separation is seen winding over the restiform body. That line is the tania of the fourth ventricle. Two layers of ependyma form it.

When the roof epithelium is thus removed, the lower triangle of the floor of the fourth ventricle is brought into view (Figs. 86 and 96). Notice the median longitudinal furrow bounded by the eminentice mediales, which form the calamus scriptorius; then the little fossa in the sulcus limitans, called the fovea inferior, situated lateral to the middle of the pen, and the ala cinerea (trigonum vagi), whose superior angle is formed by the fovea inferior; and, last, the large lateral area, located above the ala cinerea external to the eminentia medialis, and crossed by the nearly transverse medullary stria. This region is called the area acustica.

\section{WHITE MATTER OF MEDULLA.}

The medulla is made up of white and gray matter, which together bound ventrally and laterally the inferior part of the fourth ventricle, and surround the upper extremity of the sixth ventricle (Figs. 92 and 95).

For the most part the white matter of the medulla is continuous with the longitudinal fibers of the pons and restiform bodies, above, and with the spinal cord, below; the bulbar roots of the eighth to the twelfth cerebral nerves, and many decussating or 
commissural fibers of the reticular substance are also included in the white substance.

Substantia Reticularis.-Superficially, the white matter is collected into great bundles of fibers, such as the pyramids, lateral column and restiform body; but, in the deep parts of the medulla, the white matter enters into a great network called the substantia reticularis, which has gray matter in its meshes (Figs. 92 and 94). It is continuous, above, with the reticular formation of the pons, and, below, with the fasciculi proprii of the spinal cord. The substantia reticularis contains many scattered fibers, processes of its intrinsic neurones, which form a frequently interrupted and, for the most part, a crossed ascending and descending tract. Transverse and oblique fibers are, likewise, numerous in the reticular substance. They are chiefly the arcuate fibers. The distinct tracts of longitudinal fibers contained in it will be noticed later (page 293). The gray substance of the substantia reticularis is composed of the mucleus lateralis inferior, the eighth to the twelfth cerebral nerve nuclei and the olivary nuclei.

Raphe (Figs. 92 and 94).- The raphe is, primarily, a sagittal lamina of neuroglia derived from the floor-plate of the myelencephalon. It lies in the median plane and joins the lateral halves of the medulla together. It is very distinct in the superior part of the medulla. Below the level of the olive, it is entirely obliterated by the fillet and pyramidal decussations. The raphe is pierced transversely by decussating and commissural fibers and is traversed dorso-ventralward by the anterior external arcuate fibers.

The white matter of the medulla is divided by the direction of its fibers into three classes or systems: (I) Transverse fibers; (2) dorso-ventral fibers; and (3) longitudinal fibers.

(I) The transverse fibers of the medulla are really more or less oblique in direction and most of them are arched. They include the fibers of the following: (a) The pyramidal decussation (decussatio pyramidum), (b) the medial fillet in the decussatio lemniscorum, (c) the anterior external arcuate fibers on the surface and, also, in the decussatio lemniscorum, and (d) the cerebello-olivary fibers. The pyramidal decussation (Fig. 95) is lo- 
cated in the lower half of the medulla. About four-fifths of the pyramid crosses over through it, and becomes the lateral pryamidal tract of the spinal cord. The lower level of the pyramidal decussation marks the boundary between medulla and cord. The medial fillet, the anterior and the posterior external arcuate fibers all rise in the nucleus funiculi gracilis and nucleus funiculi cuneati. The two former run forward and cross over in the middle of the medulla, forming the fillet decussation (decussatio lemniscorum), after which they separate (Fig. 94). The medial fillet bends upward and ascends between the inferior olives, hence its name in the medulla, stratum interolivare lemnisci. The anterior external arcuate fibers, continuing their ventral direction, issue from the anterior median fissure and anterior surface of the medulla (Fig. 93), then arch backward around the medulla to the restiform body, through which they enter the cerebellum. The posterior external arcuate fibers run through the restiform body to the cerebellum without decussating. The external arcuate fibers, like the medial fillet, conduct impulses of the tactile and muscular senses. The cerebello-olivary tract is a large one (Fig. 92). It comprises most of the internal arcuate fibers of the medulla. Its origin is in the cerebellar cortex, and perhaps in the cerebellar ganglia; it terminates in the opposite inferior olivary nucleus. This tract probably belongs to the indirect motor path, though Cunningham believes it to be ascending in direction.

(2) The dorso-ventral fibers of the medulla are found in five situations: (a) In the median raphe, (b) in either half of the medulla between the anterior and lateral areas, running in a linear series of ten or twelve fascicles toward the anterior lateral sulcus, and (c) in several bundles not exactly in linear series, which run inward or outward through the posterior lateral sulcus between the lateral and posterior areas of each side.

(a) The dorso-ventral fibers of the raphe are the anterior external arcuate fibers (Fig. 92). These can be traced to the cortex of the cerebellum through the restiform body. Their origin is in the nuclei funiculi gracilis and funiculi cuneati (Fig. 94). Some of them seem to be interrupted in the arcuate nucleus on the anterior surface of the pyramid. 
The root-bundles of the eighth to the twelfth cerebral nerves constitute the remaining groups of dorso-ventral fibers. By them the medulla is divided into areas.

(b) The root-bundles of the hypoglossal nerve (Fig. 93) run from the ventricular gray matter, near the median line, ventro-lateralward to the anterior lateral sulcus, where they emerge. Inclosing between them and the raphe, the anterior area, they also separate it from the lateral area. The anterior and lateral areas are bounded dorsally by the thick sheet of gray matter in the floor of the fourth ventricle.

(c) The vestibular root of the auditory nerve, the roots of the glosso-pharyngeal and vagus and the cerebral root of the accessory nerve form the third group of dorso-ventral fibers (Fig. 93).

The motor fibers of the ninth, tenth and cerebral part of the eleventh nerves take their origin in nuclei of the medulla and emerge from the posterior lateral sulcus; while the sensory fibers of the vagus, glossopharyngeal and vestibular nerves enter that sulcus from without and run through the medulla to their terminal nuclei in the ventricular gray matter. These nuclei, both genetic and terminal, are located lateral to the hypoglossal nucleus. The nerve roots rising or terminating in them separate the lateral from the posterior area. The posterior area comprises everything dorsal to the above roots of the eighth to eleventh cerebral nerves. It thus includes the gray matter in the floor of the fourth ventricle, called the siratum nucleare.

(3) Longitudinal Fibers.-In the medulla, the longitudinal fibers, are chiefly continuations of the same in the pons and the restiform bodies; they are also continuous with the tracts of the spinal cord. They can be best located by reference to the three areas bounded by the above dorso-ventral fibers, namely, anterior, lateril and posterior areas, which are distinctly outlined in the upper half of the medulla (Figs. 92 and 93).

Longitudinal Fibers of the Anterior Area.-The anterior area of the medulla lies betwen the raphe and the roots of the hypoglossal nerve, and between the anterior surface and the gray matter in the floor of the fourth ventricle (Figs. 92 and 93). It contains the pyramid, the medial fillet, the medial and anterior 
longitudinal bundles, the substantia reticularis alba and two nuclei, the arcuate nucleus and the medial accessory olivary nucleus. Excepting that part forming the lateral pyramidal tract and the medial fillet, the anterior area is continued in the anterior column of the spinal cord. It is naturally divided into a ventral and a dorsal part, or the region of the pyramid and the region of the substantia reticularis alba.

The pyramid (pyramis) with the arcuate nucleus imbedded in it and the arcuate fibers winding over it, occupies the ventral portion of the anterior area (Figs. 92 and 94). It is the continuation of the pyramidal tract and is composed of the axones of cortical cells in the anterior central gyrus of the cerebrum. The pyramidal tract diminishes in size as it descends through the pons and medulla, because some of its fibers terminate in ramifications about the cells of cerebral nerve nuclei. In the lower part of the medulla, the pyramid breaks up into the anterior (direct) pyramidal tract (Io per cent. of the pyramid), which descends along the anterior median fissure in the same side of the spinal cord; and the lateral (crossed) pyramidal tract ( 80 per cent. of the pyramid), which, after decussating with its fellow through the anterior median fissure, runs down in the lateral column of the opposite side of the cord (Fig. 95). The fibers of the anterior tract cross in succession to the opposite side of the cord, through the white anterior commissure; and there, with the fibers of the lateral pyramidal tract, terminate in fibrillar end-tufts about the cell-bodies in the gray matter. Thus the pyramid forms a crossed cerebral tract for motor cerebral and spinal nerves. A small number of pyramidal fibers (Io per cent.) diverge lateralward from the pyramid in the medulla and descend in the lateral funiculus of the cord without decussation. They account for the weakness on the well side, and for slight motion on the paralyzed side, which are commonly observed in hemiplegia. If, as E. A. Schäfer claims, the fibers of the pyramidal tracts end in the posterior columna of gray matter, then at least one neurone intervenes between them and the motor neurones of the spinal nerves; but without doubt, they enter into either direct or indirect relations with those neurones. 
The dorsal part of the anterior area is occupied by the substantia reticularis alba (Figs. 92 and 93). It contains the bodies of very few nerve cells and is of a light color. The medial accessory olivary nucleus is imbedded in it near the pyramid and among the fibers of the fillet; and three definite bundles of longitudinal

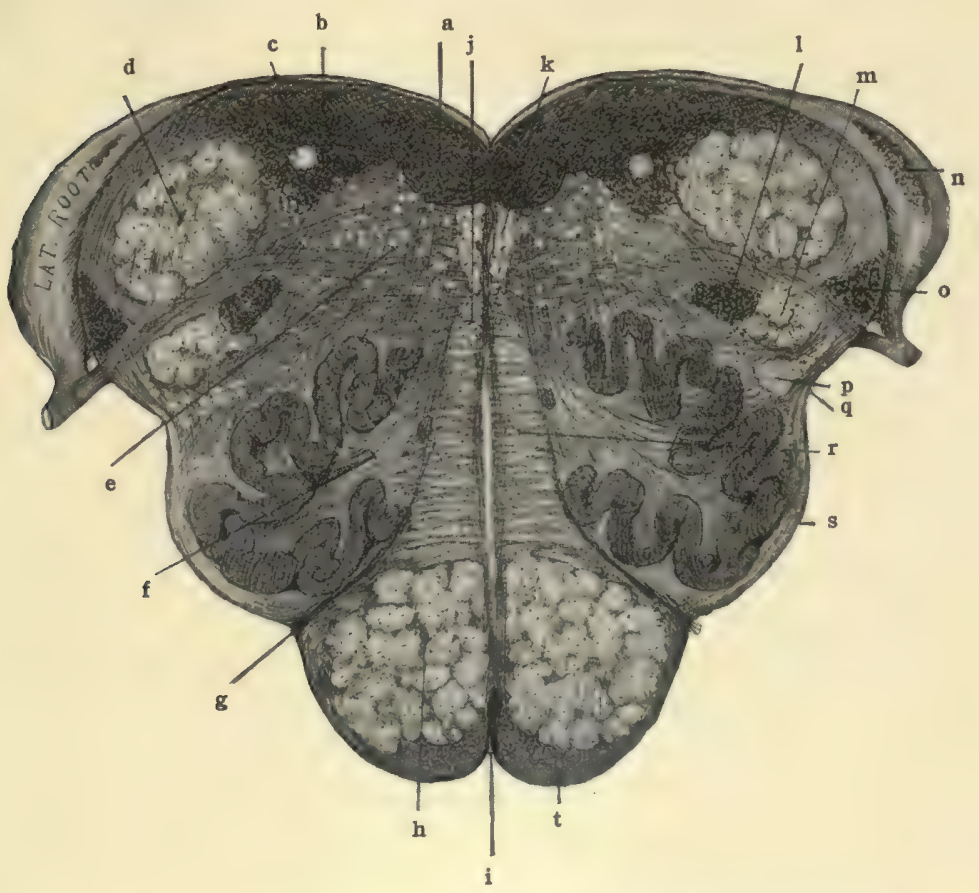

Fig. 92.-Section of medulla oblongata near the pons. (Original.)

a. Hypoglossal nucleus. b. Vestibular nucleus. c. Tractus solitarius. d. Restiform body. e. Substantia reticularis, f. Hilus of olivary nucleus containing cerebello-olivary fibers. g. Anterior lateral sulcus. h. Pyramid. i. Anterior median fissure, j. Anterior longitudinal bundle. k. Medial longitudinal bundle. 1. Nuc. tractus spinalis n. trigemini. m. Tractus spinalis n. trigemini. n. Lateral cochlear nucleus. o. Ventral cochlear nucleus. p. Ascending anterior cerebello-spinal, spino-thalamic, and rubro-spinal tracts. q. Posterior lateral sulcus. r. Medial fillet, interolivary stratum. s. Anterior external arcuate fibers. t. Arcuate nucleus.

fibers have been demonstrated in it, namely, the medial fillet and the medial and anterior longitudinal bundles.

Medial Fillet (Lemniscus Medialis).- -Just dorsal to the pyramid in the anterior area of the medulla at the level of the olives, is a large bundle of fibers called the interolivary stratum of the fillet 
(Figs. 92 and 94). Situated between the inferior olivary nuclei, it is on that account so named. Superiorly, it is continued as the medial fillet. The medial fillet rises from the nucleus funiculi gracilis and nucleus funiculi cuneati of the medulla and crosses through the median raphe in the fillet decussation. As the fillet runs brain-ward, it receives fibers from the terminal nuclei of common sensory cerebral nerves and from the vestibular nuclei. Along its lateral border, it is accompanied for a short distance in the pons by the lateral fillet. The medial fillet is composed of ascending axones which constitute a "cerebral" tract for the sensory fibers of spinal and cerebral nerves. It carries ordinary sensations (tactile and muscular) to the superior quadrigeminal colliculus by the superior fillet, and to the thalamus by the greater part of the medial fillet.

The medial longitudinal bundle (fasciculus longitudinalis medialis) (Figs. 92 and 95) which we have studied in the midbrain and pons, constitutes a very distinct strand in the superior half of the medulla; but below the level of the olive it can be identified in the anterior fasciculus proprius only by a study of its medullation or of its degeneration. It is continuous with the anterior fasciculus proprius of the spinal cord. Its location is next the median raphe and the ventricular gray substance, immediately anterior to the hypoglossal nucleus, in the upper medulla. The same position is occupied by it in the mid-brain and pons. It is here in the medulla that the hypoglossal fibers are supposed to enter it and run up to the colliculus facialis, where they join the facial nerve at the internal genu. At the middle of the medulla the decussation of the fillet pushes this bundle forward and somewhat away from the median plane, so that it runs between the fillet decussation and the medial accessory olivary nucleus. Below the level of the fillet decussation it runs between the decussatio pyramidum and the isolated head of the anterior columna of gray substance. Rising primarily in the gray matter of the cord, the ascending part of the medial longitudinal bundle is augmented in the medulla and pons by fibers from the terminal nuclei of sensory cerebral nerves. Most of its ascending fibers cross the median line and terminate in the motor cerebral nuclei on the 
opposite side; these form the middle links in many reflex arcs; a few decussate in the posterior commissure and end in the thalamus. The latter are sensory conduction fibers. The descending

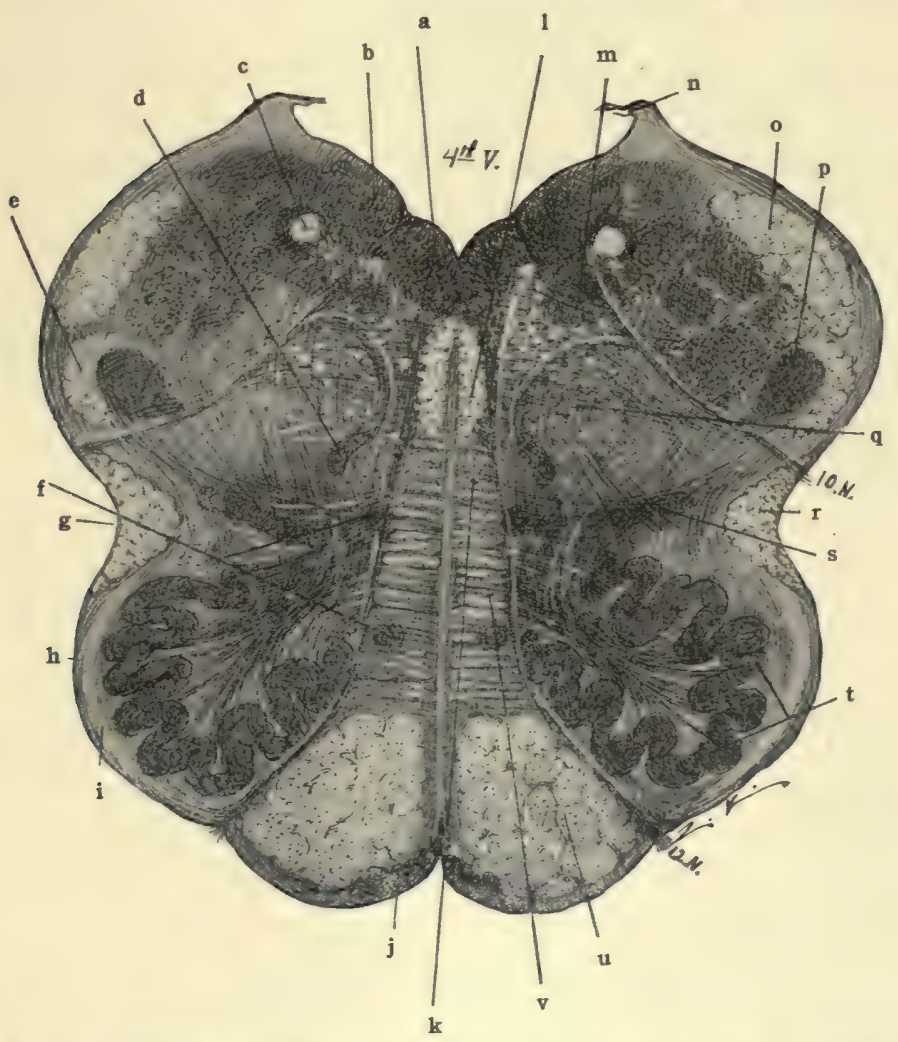

Fig. 93.-Section of the medulla oblongata at the middle of olive. (Original.)

a. Nucleus of Iath $\mathrm{n}$, b. Vestibular nucleus, c. Tractus solitarius, d. Nucleus ambiguus. e. Tractus spinalis $\mathrm{n}$. trigemini. f. Medial accessory olivary nuclei. g. Posterior lateral sulcus, h. Ant. external arcuate fibers, i. Fasciculus proprius, j. Arcuate nucleus. k. Anterior longitudinal bundle in substantia reticularis alba. 1. Medial longitudinal bundle m. Nucleus alæ cinerea, n. Tænia of 4 th ventricle. o. Restiform body. p. Gelatinous substance. q. Substantia reticularis grisea nucleus lateralis inferior. $\mathbf{r}$. Ascending ant. cerebello-spinal, spino-thalamic, and rubro-spinal tracts. $\mathbf{s}$. Dorsal accessory olivary nucleus. t. Inferior olivary nucleus. $u$. Pyramid. v. Medial fillet, interolivary stratum.

part of the medial longitudinal bundle is the medial ponto-spinal tract of Collier (see pages 280,360 and 373 ).

The anterior longitudinal bundle (Figs. 92 and 95) descends as a distinct strand, from the opposite superior colliculus of the 
corpora quadrigemina, to the lower part of the medulla; there it approaches the medial longitudinal bundle and is continued along the fissural surface of the anterior column in the cord. Its termination is in the central gray substance, chiefly the cilio-spinal centers. It forms the middle link in the visual reflex arc. Its bulbar and spinal portions constitute, chiefly, the pupillo-dilator tract (see pages 158,276 and 355 ).

Longitudinal Fibers of the Lateral Area.-The contents of the lateral area (Figs. 92 and 93) are as follows: Superficially, the lateral fasciculus proprius, the anterior descending, and the anterior ascending cerebello-spinal tracts and the spino-thalamic tract. Deeply, lies the substantia reticularis grisea. Imbedded in the substantia reticularis are the nucleus ambiguus, the nucleus lateralis inferior and the accessory olivary nuclei, and in the fasciculus proprius is the main inferior olivary nucleus. The gray matter of the substantia reticularis grisea is a part of the disintegrated anterior columna of the cord and, unlike that of the anterior area, it contains the bodies of many large nerve cells.

\section{Lateral Fasciculus Proprius (Fasciculus Lateralis Pro-} prius, Figs. 93 and 94).- The whole lateral column of the spinal cord, except the lateral pyramidal and cerebello-spinal tracts, is continued into the lateral area of the medulla. Composed of ascending and descending axones which are commissural and associative for different segments of the spinal cord, the lateral fasciculus proprius ascends into the lateral area of the medulla, and runs in part beneath and in part superficial to the inferior olivary nucleus; beyond the olive, it is continued in the substantia reticularis grisea of the medulla and reticular formation of pons and mid-brain. Among the fibers of the lateral fasciculus proprius, ventral to the olive, is the triangular tract of Helwig and, dorsal to the olive, the olivary bundle. The former is believed to rise in the olive of the medulla and the latter to end in it. They are made up of descending axones and, according to Bechterew, form a functionally continuous tract.

The anterior descending cerebello-spinal tract (fasciculus descendens cerebello-spinalis anterior) rises in the cerebellar ganglia 
and cortex. Between the cerebellum and the medulla it comprises the acustico-cerebellar tract and, possibly, the cerebello-olivary tract; and, therefore, is almost completely relayed in the vestibular nuclei, the nuclei of the reticular formation, and, possibly, in the inferior olivary nucleus (Figs. 94 and 95). It forms part of the brachium pontis and restiform body through which it reaches the medulla. In the lateral area, it descends along the anterior lateral sulcus, to the corresponding area of the cord. It ends (?) in the anterior columna of gray matter. It was once regarded as a part of the anterior pyramidal tract. The anterior descending cerebello-spinal tract forms a segment of the indirect motor path, and the motor arm of the reflex arc of coordination and equilibrium.

\section{Anterior Ascending Cerebello-spinal and Spino-thalamic} Tract. (Fasciculus ascendens cerebello-spinalis anterior) (Figs. 92 and 95).-These two tracts are combined into one throughout the cord, medulla and pons. Near the isthmus the former turns backward through the superior medullary velum and ends in the cortex of the superior vermis cerebelli; while the spino-thalamic tract continues in the original direction, to the lateral nucleus of the thalamus. The common spino-encephalic tract made up of these two bundles takes its origin from the base of the anterior columna and center of the crescent of gray matter, chiefly on the opposite side of the spinal cord; and, crossing through the white anterior commissure, it ascends, mingled somewhat with the fibers of the above descending cerebello-spinal tract, along the lateral surface of the cord (Barker). It runs beneath the posterior lateral groove of the medulla and through the formatio reticularis of the pons, to the point of division near the isthmus rhombencephali, whence the two divisions proceed to their cerebellar and thalamic terminations, as above stated. The tract is probably reinforced in the medulla and pons by the addition of axones from the opposite terminal nuclei of common sensory cerebral nerves; and, rising primarily in relation with the posterior roots of spinal nerves, it thus forms a crossed path for common sensations, spinal and cerebral. It conducts tactile, pain and temperature impulses (see pages I6I and 356). 
The rubro-spinal tract is the crossed descending tract of the red nucleus (Figs. 92 and 95). Running dorsal to the inferior olive in the medulla it mingles with the fibers of the anterior ascending cerebello-spinal tract. It is continued down the cord, in the lateral column, to the lateral columna and center of the gray crescent as far as the first lumbar segment (see pages I62, 276 and $\left.35^{8}\right)$.

Longitudinal Fibers of the Posterior Area. - The longitudinal fibers of the posterior area form many bundles; and the bundles are different in upper and lower medulla. The substantia reticularis is small. The ventricular gray substance belongs to this area.

The lower medulla contains: The funiculus gracilis, funiculus cuneatus, tractus spinalis nervi trigemini and cerebellospinal tract, named from the posterior median fissure outward (Figs. 94 and 96). In the upper medulla are: The restiform body and the spinal tract of the fifth cerebral nerve at the surface; and the tractus solitarius in the interior (Figs. 92, 93 and 96).

The funiculus gracilis is the superior end of the ascending postero-medial column (Goll's column) of the spinal cord. Near its extremity it expands and forms the clava, and then tapers off and disappears along the side of the fourth ventricle. The clava is due to the nucleus funiculi gracilis, in which the fibers of the column end. The funiculus gracilis is composed of ascending branches of the posterior roots of the spinal nerves, which enter the cord below the seventh or eighth thoracic segment.

Funiculus Cuneatus (Figs. 94 and 96).- - It is separated from the posterior median fissure by the gracile bundle; and is the continuation of the ascending postero-lateral column (Burdach's column) of the spinal cord. It ends about the cells of the nucleus funiculi cuneati and accessory nucleus funiculi cuneati, which form the cuneate tubercle seen on the surface. The fibers of the funiculus cuneatus are ascending branches of the posterior roots of the spinal nerves. The nerves contributing to this column are the cervical and the six or eight upper thoracic. The funiculi gracilis and cuneatus carry to the nuclei of these columns common sensations belonging to the tactile and muscular senses. Interference with these tracts produces ataxia. 
Spinal Tract of the Trigeminal Nerve. (Tractus spinalis nervi trigemini, Figs. 92 and 96).-It forms a narrow strip of the posterior surface of the medulla, which is broadest near the restiform body and tapers downward toward the spinal cord. It is composed of the descending fibers from the sensory root of the trigeminal nerve: the sensory fibers of this nerve on entering the pons divide T-like, into an ascending and a descending branch, and the descending branches form the spinal tract of the nerve, which for a short distance is visible on the surface of the medulla. This tract is continued through two segments in the spinal cord. The nucleus of the spinal tract of the trigeminal nerve, over which it runs and in which it terminates, is but the continuation of the gelatinous substance of the posterior columna of gray matter in the cord; in the upper medulla.it is situated ventro-medial to the restiform body. The nucleus produces a slight eminence below the level of the clava, called the tuberculum cinereum.

The cerebello-spinal tract (jasciculus cerebello-spinalis, Figs. 94 and 96) in the lower medulla crosses the posterior lateral groove and the spinal tract of the fifth nerve, going from the lateral column of the cord to the posterior area of the medulla; it then ascends to form a considerable part of the restiform body. It takes its origin from the nucleus dorsalis (Clarki) in the spinal . cord. It ends, very largely on the opposite side, in the cortex of the superior cerebellar worm. The cerebello-spinal tract (direct cerebellar tract) probably conducts common sensory impulses received from viscera. They should be classed largely under the muscular sense, as they seem to concern equilibrium and orientation. They are sympathetic sensory.

Restiform Body. (Cor pus restiforme). - In the upper medulla, forming the lateral part of each posterior area, is a large rounded bundle of fibers, called the restiform body (Figs. 92, 93 and 96). It is the largest bundle in the medulla, and joins it to the cerebellum. It is made up of the following: Ascending tracts-(I) the cerebello-spinal tract; (2) the external arcuate fibers, anterior and posterior; and (3) the tract from the lateral nucleus of the medulla to the cortex of the cerebellum. Descending tracts(4) the cerebello-olivary fibers; and (5) a few axones of Purkin- 
je's cells which enter into the descending cerebellar tract (?) (see cerebellum, page 264).

The restiform body is inclosed between the vestibular and cochlear roots of the auditory nerve (eighth) (Fig. 89). Ventral to it and between the roots, is the ventral part of the cochlear nucleus; on its lateral surface and among the fibers of the lateral root, is the dorsal, or lateral part of the same nucleus. The vestibular nuclei-the chief dorso-medial nucleus (Schwalbe), the dorsolateral (Deiters), the superior (Flechsig and Bechterew) and the nucleus of the descending root are situated dorsal and medial to the restiform body.

Tractus Solitarius (Figs. 92 and 94).- The solitary tract is a small, round bundle imbedded in the middle of the ventricular gray matter of the posterior area. In Weigert-Pal sections of the upper medulla it is clearly visible to the naked eye. It is formed by the sensory roots of the nervus intermedius and the ninth and tenth cerebral nerves. It descends through the $m u$ cleus tractus solitarii lateral to the nucleus of the ala cinerea, and along the medial aspect of the posterior columna of gray matter in the cord. It ends on both sides of the median line in gelatinous gray matter, called the nucleus of the solitary tract, which surrounds it. The nuclei of these tracts converge downward; according to Cajal, they are united near their inferior extremities by a central mass, called the nucleus commissuralis (Barker). The solitary tract may be traced from the upper part of the medulla down to the spinal cord (Kölliker). It is believed to associate the nuclei of the nerves controlling respiration, hence the synonym, respiratory bundle.

\section{GRAY MATTER OF MEDULLA.}

The gray matter of the medulla is composed (I) of that continuous with the nucleus pontis, called the arcuate nucleus; (2) of the ventricular gray substance, the stratum nucleare, and that of the substantia reticularis, also continued downward from the pons; and (3) of the special nuclei of the medulla, which are not represented in the pons or in the spinal cord.

(I) Nucleus of the External Arcuate Fibers (Figs. 92 and 
93).-This is the mucles arcuatus. It forms a large crescentic mass on the ventral and medial surface of the pyramid. Running over and through it there are the anterior external arcuate fibers, for certain of which it constitutes a relay. The arcuate nucleus is continuous above with the nucleus pontis. It is brought to the surface at the lower border of the pons by the disappearance of most of the transverse fibers.

(2) The gray substance in the substantia reticularis and in the immediate floor of the fourth ventricle (Figs. 92 and 93) is continuous with the same in the pons and mid-brain, above; and is represented in the spinal cord by the $\mathrm{H}$-shaped column of gray matter. By the posterior and lateral expansion of the neural canal, in the upper half of the medulla and in the pons, which forms the fourth ventricle, the posterior columnæ of the $\mathrm{H}$-shaped column of gray matter are pushed outward to a transverse direction; and the entire bases of the anterior columnæ are brought into the floor of the ventricle. The expansion of the canal, together with the decussation of the lateral pyramidal tracts through the anterior columnæ and of the medial fillets through the posterior and anterior columnæ, disposes the $\mathrm{H}$-shaped column as follows:

Anterior Columna.-From the base of the anterior columna is derived a column of cells, the hypoglossal nucleus (Figs. 92 and 93), which is two-thirds of an inch in length and extends along the median raphe in the upper medulla, beneath the eminentia medialis. It is continued into the lower medulla as far as the pyramidal decussation. By commissural fibers it is joined to the nucleus of the opposite side according to Kölliker. The nucleus gives origin to the hypoglossal nerve proper; and probably to a small fasciculus which by way of the medial longitudinal bundle joins the facial nerve and supplies the orbicularis oris. The hypoglossal axones run in linear series forward through the medulla to the anterior lateral sulcus, whence they emerge between the pyramid and the olive (Fig. 85). They separate the anterior from the lateral area. The main body of the anterior columna is broken up into the nucleus lateralis inferior, the nucleus ambiguus and the motor part of the mucleus of the ala cinerea. 
The nucleus lateralis inferior (Figs. 92 and 94) is situated in the reticular substance of the lateral area. Though it is made up of large cell-bodies, they are so scattered among the fibers of this region that the nucleus is invisible to the naked eye. It probably gives origin to descending fibers which are continued both in the anterior and lateral column of the spinal cord (Tschermak). It is known to receive fibers from the anterior ascending cerebellospinal tract and from the nuclei funiculi gracilis and funiculi cuneati, and it gives rise to the ascending tract already traced to the cerebellum, which probably transmits tactile, muscular, pain and temperature impressions to the cerebellar cortex.

The nucleus ambiguus (Fig. 93) forms an irregular sheet of gray substance, pear-shaped in section, which extends longitudinally through two-thirds of the medulla. It is prolonged from near the ventricle ventro-lateralward into the substantia reticularis. It is not visible to the naked eye. Its axones form the cerebral root of the eleventh nerve and probably a part of the motor roots of the tenth and ninth nerves. The root fibers of the accessory nerve run out through the posterior lateral sulcus below the level of the olive.

The nucleus of the ala cinerea (Fig. 93) is in part derived from the base of the anterior columna; this part is motor in function and its neurones resemble those of the anterior columna in the cord. It belongs to the ninth and tenth nerves. It is situated, above, close to the floor-ependyma of the fourth ventricle under the ala cinerea; and it extends, inferiorly, into the closed medulla nearly as far as the hypoglossal nucleus. It is immediately lateral to the nucleus of the hypoglossal. Its axones run in a curve, convex toward the median plane, between the restiform body and the olive. They are joined medially by those axones of the nucleus ambiguus which enter the roots of the ninth and tenth nerves. By this nucleus and the nucleus ambiguus, many distinct fascicles are formed, belonging to the roots of the ninth, tenth and the cerebral part of the eleventh nerves. They run in slightly different planes, but all of them emerge in the region of the posterior lateral sulcus of the medulla (Fig. 85). Intermingled with the motor cells of the nucleus alæ cinereæ, there are the small spindle 
cells of the terminal nucleus of the vagus nerve, which represent neurones of the posterior columna of gray matter. They receive the end-tufts of the sensory root fibers of the vagus nerve and possibly of a small number from the glossopharyngeal nerve.

The hypoglossal nucleus, the motor part of the nucleus of the ala cinerea and the nucleus ambiguus receive many fibers from the opposite pyramidal tract, and probably from the cerebro-pontal tracts, which bring to them voluntary motor and inhibitory im-

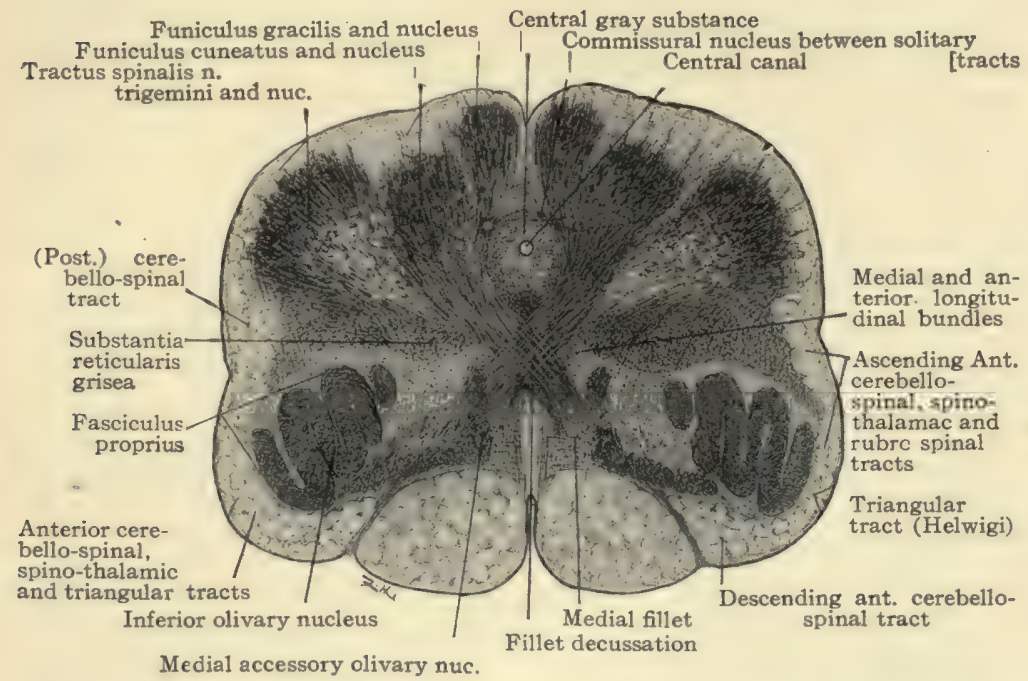

Fig. 94.-Section of the medulla oblongata at the fillet decussation. (Original.)

pulses from the cerebral cortex; and their reflex connection is established by fibers of the medial longitudinal bundle which rise in sensory nuclei.

The posterior columna is decapitated by the fillet. It is represented in the medulla (I) by the following terminal nuclei, viz., the sensory part of the nucleus alæ cinereæ of the vagus and glossopharyngeal nerves, the vestibular and cochlear nuclei of the auditory nerve, the nucleus tractus solitarii and the nucleus of the spinal tract of the trigeminal nerve; and (2) by the gray matter of the reticular substance of the posterior area. 
The mucleus ala cinerea of the vagus and glossopharyngeal nerves (Fig. 93) contains in its lateral part a group of small fusiform cell-bodies like those in the posterior columna. These fusiform cells constitute the terminal nucleus of the sensory fibers of the vagus, and it is probable that a few glossopharyngeal fibers also arborize and end in the nucleus. Cortical Connection.Axones of this nucleus probably enter into the medial fillet, the spino-thalamic tract and the medial longitudinal bundle. The two former conduct tactile, muscular, pain and temperature impulses to the thalamus, whence the cortical fillet carries them to the cortex; the latter establishes its reflex connection with motor nerves.

Nucleus Tractus Solitarii (Figs. 92 and 94).-The nucleus of the solitary tract surrounds the tractus solitarius with which it coincides in extent. It is gelatinous in appearance and is situated just lateral to the nucleus of the ala cinerea. In its descent it trends dorsally and toward the median line. It is joined to the opposite nucleus, at its spinal end, by the mucleus commissuralis (Cajal). The nucleus of the solitary tract is the terminal nucleus of the afferent fibers of the intermediate and glossopharyngeal nerves and, probably, receives a few fibers from the vagus. It is thus the nucleus of the nerves of taste and forms the first relay station in the gustatory path. The axones of the cell-bodies in the nucleus tractus solitarii establish reflex connections with efferent nuclei and continue the taste path toward the thalamus, but their exact course has not been worked out.

Nucleus Tractus Spinalis Nervi Trigemini (Figs. 92 and 95).The nucleus of the spinal tract of the trigeminal nerve is gelatinous in character. It is continuous with the sensory pontine nucleus of the trigeminal nerve, above; and, below, is continued in the gelatinous substance of the posterior columna of the spinal cord. As low down as the second cervical segment it receives fibers from the trigeminal nerve, so the terminal nucleus of this nerve extends from the middle of the pons to the second cervical nerve. The nucleus of the trigeminal is embraced between the emergent part of the facial nerve, medially, and the vestibular nerve, laterally, in the lower portion of the pons; in the upper medulla, the 
nucleus lies along the ventro-medial surface of the restiform body; it enlarges in bulk and approaches the surface near the middle of the medulla, where it produces the tuberculum cinereum; and it is then continued down into the cord as a cap of the posterior columna of gray substance. In the lower part of the medulla the nucleus underlies the visible part of the tractus spinalis nervi trigemini.

The sensory root of the trigeminal nerve (fifth) enters the pons on its ventral surface, in line with the roots of the seventh, eighth, ninth, tenth and accessory nerves (Fig. 85). The root fibers divide T-like; the short ascending branches end in the pontine nucleus of the fifth nerve and the long descending branches, forming the spinal tract, terminate in the nucleus of that tract. A certain few of these root-fibers go directly to the motor nucleus of the trigeminal nerve and perhaps to other motor nuclei; these are reflex in function.

From the trigeminal nucleus axones establishing reflex and cortical relations run: (a) To motor nuclei by way of the medial longitudinal bundle and directly without entering that bundle, forming the middle link of reflex arcs, and (b) by way of two paths they run toward the cerebral cortex as far as the thalamus. The latter cross the median raphe and probably enter the medial fillet and the spino-thalamic tract. The axones bearing impulses of the muscular sense enter the medial fillet and are continued through it to the lateral nucleus of the thalamus; those fibers which conduct pain and temperature impressions run through the spino-thalamic tract to the same nucleus. Both sets of fibers conduct tactile impulses. From the thalamus the cortical fillet completes the path to the somæsthetic cortex of the cerebrum.

Vestibular Nuclei (Nn. Nervi Vestibularis, Figs. 92 and 93).These are located partly in the pons as already pointed out, and extend as low as the mid-medulla. Their function is equilibrium. The principal mucleus (Schwalbe's) is dorso-medial in position and lies beneath the acustic area of the ventricular floor, crossed by the medullary striæ. It extends transversely from near the eminentia medialis almost to the restiform body. It appears to receive nearly all the fibers of the vestibular nerve, which arbor- 
ize and terminate about its cells. Lateral to the principal nucleus are the mucleus of Deiters and the nucleus of the descending root. Deiters's nucleus is spread along the medial surface of the restiform body, chiefly in the pons. It becomes a distinct nucleus as the lower border of the pons is approached and grows larger for some distance above that point. In the pons it is bent backward with the restiform body toward the cerebellum. Its upper end is thus placed in the lateral wall of the fourth ventricle between the restiform body and the brachium conjunctivum. This portion is called the superior mucleus (of Bechterew or Flechsig). Deiters's nucleus is made up of cell-bodies which are large in comparison with those of the principal nucleus. It receives the descending fibers of the acustico-cerebellar tract from cerebellar ganglia and cortex and originates axones that proceed down the cord in the anterior descending cerebello-spinal tract, thus forming a relay in the cerebello-spinal path; and this is its chief function. It is only a subordinate terminal nucleus of the vestibular nerve. The nucleus of the descending root is, in all probability, a relay station in the conduction path from the vestibular nerve, but not a terminal nucleus of that nerve. It is composed of cell-bodies scattered through a strand of fibers, called the descending root, which extends from the level of the principal nucleus down to the nucleus funiculi cuneati (Bruce). It is placed somewhat under cover of the medial border of the restiform body and, with the enveloping descending root, separates this body from the principal nucleus. Certain fibers of the descending root terminate in the nucleus of the same name.

Cortical Connections.-Axones of the terminal nuclei of the vestibular nerve enter the opposite medial fillet and, by way of the descending root, also the nucleus funiculi cuneati. The medial fillet continues the direct path to the thalamus and the cortical fillet completes it up to the temporal cortex. The descending root and the arcuate fibers from the nucleus funiculi cuneati constitute the connecting link between the vestibular nuclei and the cortex of the cerebellum. The reflex connections of the vestibular nerve are established, first, with cerebral nerves, by fibers which join the medial longitudinal bundle and terminate 
in the nuclei of motor nerves; second, with spinal nerves by the axones of Purkinje's cells to the nucleus fastigii and nucleus globosus; from the cerebellar ganglia by the acustico-cerebellar tract to the nucleus of Deiters, and then by way of the anterior descending cerebello-spinal tract to the motor nuclei of the spinal nerves in the anterior columna.

Cochlear Nuclei (Nn. Nervi Cochlearis, Fig. 92).-There are two cochlear nuclei, the ventral and the lateral. They concern hearing proper. The ventral cochlear mucleus appears in section as a triangular mass of cell-bodies imbedded in the medulla at the upper end of the posterior lateral sulcus. It lies between the restiform body and the olive; the vestibular root of the auditory nerve separates it from the olive. It receives the greater number of fibers in the cochlear nerve and gives rise to those of the trapezoid body and, through that, to a large part of the lateral fillet of the opposite side; a few of its fibers enter the fillet of the same side. In the corpus trapezoideum, the cochlear tract is largely relayed by the neurones forming the nuclei of the superior olivary group. The lateral cochlear nucleus embraces the outer surface of the restiform body. It is situated both lateral and dorsal to the ventral nucleus and, stretching around the posterior surface of the restiform body, it produces the ventricular eminence in the lateral part of the acustic area, called the tuberculum acusticum. The lateral nucleus receives that part of the cochlear root which does not end in the ventral nucleus, and the fibers arborize about its cells. The axones of the lateral nucleus form the medullary striæ; a few of them enter the trapezoid body (Figs. 86, 89 and 92). The medullary striæ run somewhat obliquely across the ventricular floor to the median groove, plunge forward to the superior olivary nuclei of the opposite side where they are partially relayed and then, bending upward, are continued in the lateral fillet. At the superior olivary nuclei of the opposite side the fibers from the lateral and ventral nuclei become intermingled, hence the trapezoid body and medullary striæ combine in the formation of the lateral fillet. The lateral fillet suffers a partial relay in its own nucleus, after which it separates into two parts; the principal part runs to the internal geniculate body, by way of the 
brachium inferius; the smaller part ends in the quadrigeminal colliculi, chiefly in the inferior colliculus on the same side. From the medial geniculate body to the transverse and superior temporal gyri, the acustic path is formed by the temporo-thalamic (acustic) radiation. This completes the cortical connection of the cochlear nuclei. Their reflex connections are established, first, by the olivary pedicle and medial longitudinal bundle and, second, by that part of the lateral fillet which ends in the colliculi of the corpora quadrigemina (see pages I60 and I6I).

(3) There are Certain Special Nuclei of the Medulla.These are not represented either in the pons, above, or the spinal cord, below. They are the nucleus funiculi gracilis, the nucleus funiculi cuneati and the nucleus olivaris inferior.

Nucleus Funiculi Gracilis and Nucleus Funiculi Cuneati (Figs. 94 and 95).-The nucleus funiculi gracilis and nucleus funiculi cuneati are large nuclei, extending from the level of the olive to the lower end of the medulla. They are situated near the pos. terior surface beneath the gracile and cuneate funiculi, whose fibers terminate in them; they give origin to the medial fillet, and the anterior and posterior external arcuate fibers, and they produce, respectively, the clava and cuneate tubercle on the posterior surface of the medulla. In successive sections from below upward, the nucleus funiculi gracilis is first seen as an isolated mass of gray substance imbedded in the funiculus gracilis at the level of the pyramidal decussation. It enlarges dorso-ventrally and transversely toward its upper end, as is shown in consecutive sections, and reaches its greatest size at the clava, where it receives the terminal end-tufts of the funiculus gracilis. At the level of the clava the ventral border of the nucleus funiculi gracilis fuses with the gray matter about the central canal. The axones of this nucleus form about one-half of the medial fillet and the external arcuate fibers. The nucleus funiculi cuneati (Fig. 95) appears at the same inferior level as the nucleus funiculi gracilis. It is from the first and throughout its length continuous with the central gray substance on which it appears as a bud-like outgrowth in the lower medulla. It gradually broadens and elongates dorsalward when traced upward (Fig. 94). Beneath the cuneate tubercle 
it reaches its full stature and gathers into itself the fibers of the funiculus cuneatus and the descending root of the vestibular nerve; thence it sends its own axones upward in the medial fillet and the external arcuate fibers. Near the lower end of the medulla there is a small lateral bud of gray matter connected with the nucleus funiculi cuneati, to which it is accessory and, like it, is imbedded in the funiculus cuneatus. It occupies the position of the dorsal nucleus (Clarki) in the spinal cord, and is called the accessory mucleus funiculi cuneati.

The nuclei funiculi gracilis et cuneati form the first relay station in the spino-cerebral path for impressions of the muscular and

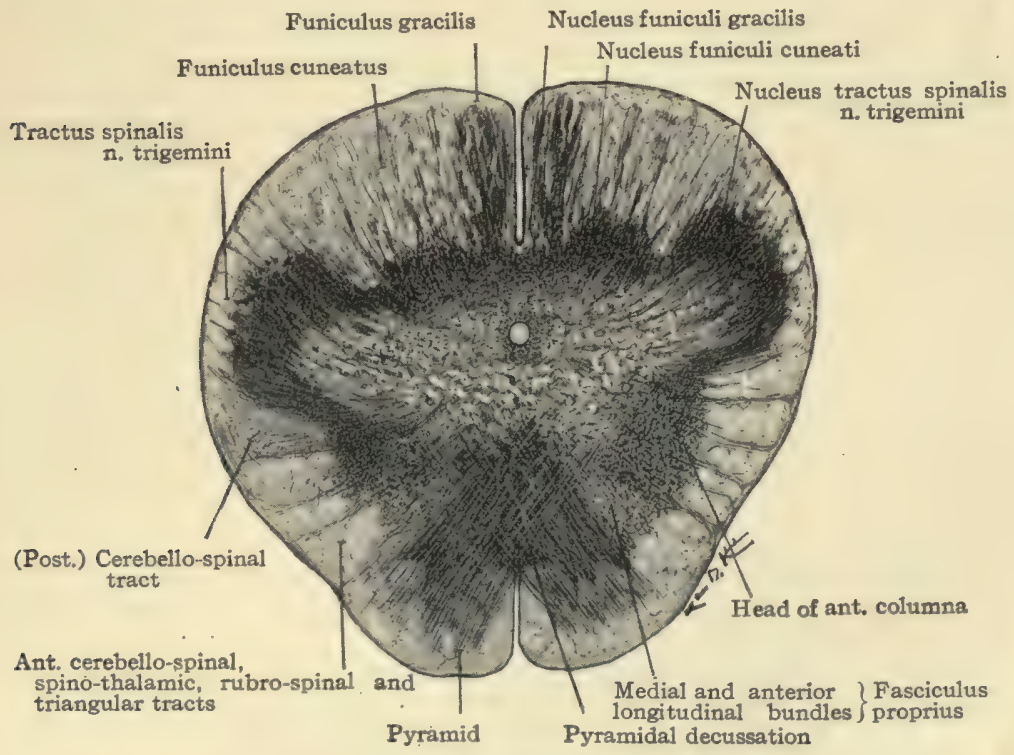

Fig. 95.-Section of the medulla oblongata at the pyramidal decussation. (Original.)

tactile senses, and lesions in them cause ataxia. They also lie at the dividing of the ways; the direct path continuing through the fillet decussation and the medial fillet to the thalamus, and the indirect path running through the arcuate fibers to the cerebellar cortex. From the cerebellar cortex the impulses proceed cerebralward through Purkinje's neurones to the dentate nucleus 
and, thence, through the dentate neurones by way of the brachium conjunctivum cercbelli to the opposite red nucleus and thalamus. The cortical fillet conducts all common sensory impulses from the thalamus to the cerebral cortex.

The nucleus olivaris inferior, the olivary nucleus of the medulla (Figs. 92 and 94), is a sinuous, pouch-like collection of gray matter resembling the nucleus dentatus of the cerebellum. It is situated near the lateral surface of the medulla and is invested superficially and deeply by fibers from the lateral fasciculus proprius. Its open hilus looks medially and is filled with fibers, the cerebello-olivary fibers, which join it to the opposite hemisphere of the cerebellum. On either side of the olivary nucleus is an accessory nucleus-the medial accessory, in the anterior area among the fibers of the interolivary part of the medial fillet, and the dorsal accessory in the lateral area. The olivary nucleus, covered by fibers of the lateral fasciculus proprius, forms the olive (oliva). The olive shows the longitudinal extent of the nucleus and on section it is seen to measure a quarter of an inch in depth. The olivary nucleus is said to be a modern structure; it is found well developed only in the higher mammals and does not appear in the human brain until the sixth month. It probably contains both ascending and descending neurones. Axones enter it from the nucleus funiculi gracilis and nucleus funiculi cuneati and probably some of the cerebello-olivary fibers are ascending in direction. It receives two groups of descending fibers, viz.: (a) The descending cerebello-olivary fibers pass down from the cortex of the cerebellum through the restiform body to the medulla, decussate and, entering the hilus of the opposite nucleus, arborize and end about itscellbodies; and (b) the olivary fasciculus (central tract of the tegmentum), having descended from the lentiform nucleus through the mid-brain and pons, fades away just dorsal to the olive and probably ends in it. Axones of the olivary nuclei of the medulla have been traced by Kölliker and others down the lateral column of the spinal cord. The triangular tract of Helwig is probably composed of such fibers. It descends along the surface of the cord lateral to the anterior roots of the spinal nerves and ends in the gray matter of the cord. 
Lesions in the medulla are very fatal and death usually occurs before any sensory or motor phenomena can be observed; but rarely the pyramidal tracts alone have been involved or the pyramidal tracts together with one or more of the roots of the ninth to the twelfth cerebral nerves. In the last case, crossed paralysis is produced, as in the pons, affecting the cerebral nerves on the same side and the opposite spinal nerves. In progressive bulbar paralysis the motor nuclei of the medulla are involved as a preliminary to the degeneration of the anterior gray columna in the spinal cord.

\section{RHOMBENCEPHALON.}

\section{SECTION IV. THE FOURTH VENTRICLE.}

The common cavity of the rhombencephalon is the fourth ventricle (ventriculus quartus) (Fig. II 8). The fourth ventricle is contained chiefly in the pons and medulla, and is ventral to the cerebellum (Fig. 79). It is broadest at the junction of the pons and medulla (Figs. 86 and 9I). Above and below that junction, it gradually contracts to the size of the cerebral aqueduct and central canal of the spinal cord, with which it is continuous. Inferiorly it communicates through its roof with the subarachnoid space via three apertures, a median and two lateral. The fourth ventricle is a gable-roofed chamber with a diamond-shaped floor. The gables (Fig. 79) are directed lateralward and are prolonged in tunnel-like extensions around the restiform body. The long axis of the ventricular floor (Figs. 86 and 96) is parallel with the spinal cord, and extends from the superior extremity of the pons to the middle of the medulla. The transverse axis coincides with the junction of the pons and medulla. Thus the superior triangle of the floor is formed by the pons; the inferior, by the medulla oblongata. The fourth ventricle is lined with ependyma, which is complete throughout, except in the roof of the inferior part, where below the inferior medullary velum only the epithelial layer is present.

Boundaries. - The floor is formed by the pons and medulla. 
The lateral wall (superior triangle) is formed by the brachium conjunctivum of the cerebellum; and (inferior triangle) by the tænia of the fourth ventricle winding across the restiform body; funiculus cuneatus and funiculus gracilis to the obex. The roof is formed by the superior medullary velum (valve of Vieussens) superiorly; and by the inferior medullary velum and roof epithelium, inferiorly (Fig. 9r). The superior and inferior halves of the roof meet at an acute angle, the fastigium, and form the tent of the fourth ventricle (Fig. 79). On either side, the gable is pushed out over the restiform body and thus is formed the lateral recess. The lateral recess is a tunnel-like extension of the ventricular cavity, reaching almost to the posterior lateral sulcus. The recess is bounded, superiorly and ventrally, by the restiform body; dorsally, by the inferior medullary velum; and inferiorly, by the roof epithelium. The chorioid plexuses of the fourth ventricle invaginate the roof epithelium and hang from the roof into the lateral recesses and the inferior part of the cavity (Fig. 9I).

Floor of the Fourth Ventricle. (Fossa rhomboidea).-Because it contains the nuclei of one or more roots of the posterior eight (fifth to twelfth) cerebral nerves, the floor of the fourth ventricle is a very important area (Figs. 86 and 96). A median groove bounded by the eminentiæ mediales forms the long axis of the diamond-shaped floor and divides it into two lateral halves; the lateral halves are bisected transversely by a number of lines, the medullary striæ (striæ medullares). The striæ are produced by bundles of fibers which rise from the cochlear nucleus of the auditory nerve. Diverging somewhat and plunging into the medulla at the median groove, the fibers of the strix enter the opposite trapezoid body and lateral fillet. The medullary strix divide each lateral half of the floor into a superior and an inferior triangle.

\section{Description to Fig. 96.}

a. Nucleus of olfactory nerves. b. Nucleus of oculomotor nerve. c. Nucleus of trochlear nerve. d. Nucleus of descending root of trigeminus. e. Chief motor nucleus of trigeminus. f. Nucleus of facial. g. Nucleus of abducens. h. Nucleus ambigutus (vagus and glossopharyngeus). i. Nucleus of thypoglossus. j. Nucleus of accessory nerve. Nuclei of optic nerve: k. Pulvinar of thalamus, 1. Lateral geniculate body, $\mathbf{m}$. Nucleus of superior colliculus. n. Sensory nucleus of trigeminus. o. Nucleus of vestibular nerve, p. Ventral nucleus of cochlear nerve. q. Lateral nucleus of cochlear nerve. r. Nucleus alæcinereæ (vagus and glossopharyngeus). 8. Solitary tract (vagus and glossopharyngeus). t. Nucleus of spinal tract of trigeminus. 


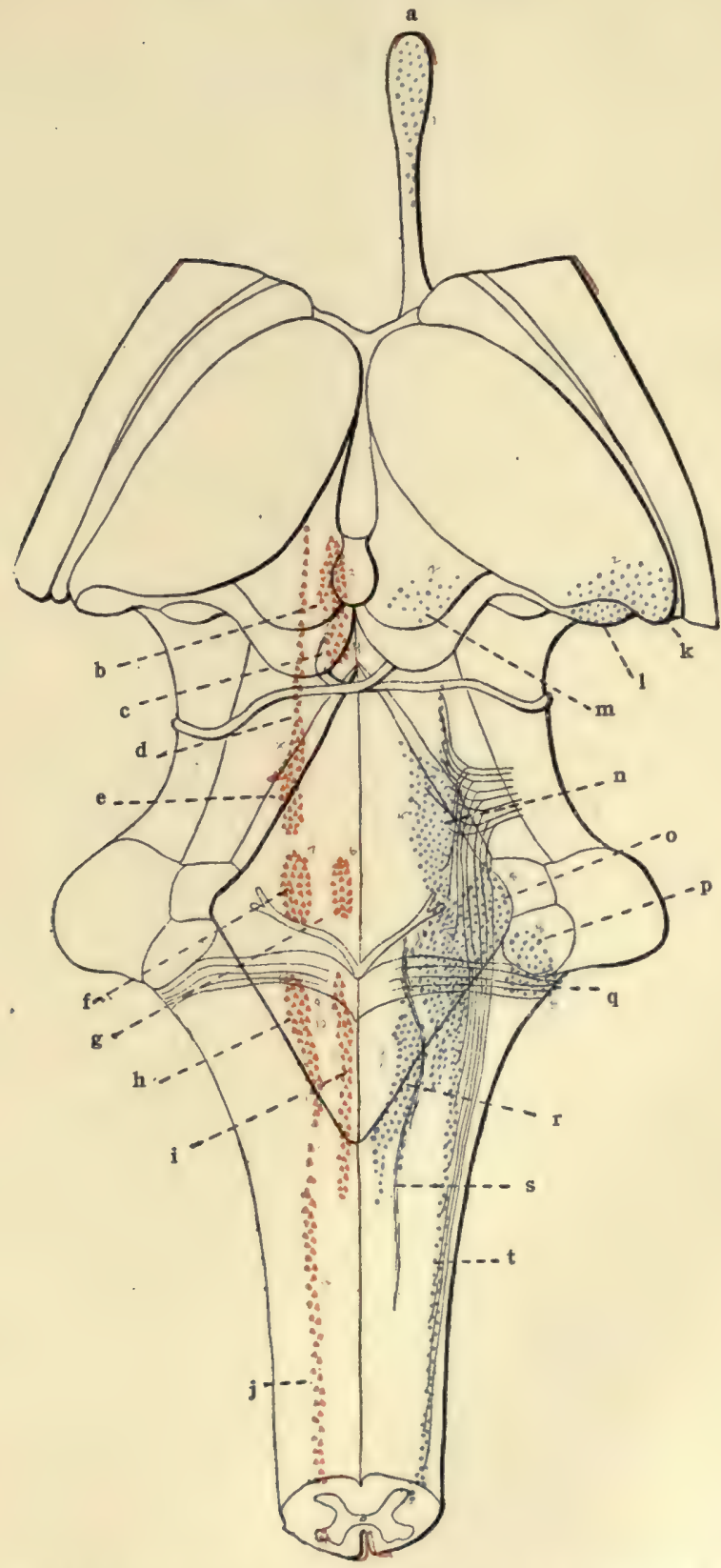

1'ig. 96.-Nuclei of the cerebral nerves in the medulla, pons, mid-brain, inter-brain and olfactory bulb. Motor (or genetic) nuclei, red; terminal (or sensory) nuclei, blue. (After Morris's Anatomy.) 

The superior triangle of the floor presents the colliculus facialis, superior fovea, locus cæruleus and a part of the acustic area.

The colliculus facialis (Fig. 86), the superior extremity of the eminentia medialis, is located next the median groove. It is produced largely by the genu of the facial nerve. Beneath it is the nucleus of the abducent (sixth) nerve (Fig. 96). External to it and in front of the striæ medullares is a small fossa, the fovea superior.

Fovea Superior (Fig. 86).- The fovea superior is near the lateral wall of the ventricle and marks the location of the facial nucleus (seventh) and the salivary nucleus of the intermediate nerve, which are deeply seated in the pons. Running upward along the wall of the ventricle from the superior fovea, is the sulcus limitans. It is a blue-floored groove in the pons, called locus cæruleus.

The locus ccruleus (Fig. 86) continues to the superior angle of the ventricle. The blue color is due to the substantia ferruginea, a pigmented layer of cell-bodies underlying the ependyma. The principle motor nucleus of the trigeminal nerve (fifth) lies beneath the superior part of the locus cæruleus (Fig. 96).

Inferior Triangle of the Ventricular Floor.-It presents: The trigonum hypoglossi, fovea inferior, ala cinerea and eminentia cinerea, and most of the area acustica (Fig. 86).

The hypoglossal triangle (Fig. 86) is produced by the inferior half of the eminentia medialis. Its apex is in the inferior angle of the ventricle, and forms one nib of the calamus scriptorius; its base looks upward and is situated under the medullary striæ. The twelfth nerve rises from the column of cells whose upper onehalf is covered by it (Fig. 96). External to the trigonum hypoglossi and inferior to the striæ medullares is the inferior fovea, which forms the apex of the ala cinerea.

Ala Cinerea (Trigonum Vagi, Fig. 86).-The vagus triangle is of a darker color than the ventricular floor around it, hence the name, ala cinerea. The inferior fovea forms the depressed and superiorly directed apex of the ala; its floor rises inferiorly to the base, eminentia cinerea, which is directed toward the clava. The 
nucleus alæ cinereæ, the nucleus tractus solitarii and the nucleus ambiguus, three nearly parallel columns of cell-bodies a halfinch in length, are in part covered by the ala cinerea (Fig. 96).

The superior and inferior foveæ and the sulcus limitans fossæ rhomboideæ in which they are located represent the lateral sulcus, which in the embryo separates the ventral from the dorsal zone of the rhombencephalon.

Area Postrema.-Below the ala cinerea and between it and the tænia ventriculi quarti, there is a small fusiform strip of the ventricular floor which Retzius has called the area postrema. An oblique stria separates it from the base of the ala cinerea.

The area acustica occupies the lateral angle of the ventricular floor (Fig. 86). It is partly in the superior triangle, but chiefly in the inferior. Inclosed between the ala cinerea and the tænia, its apex points downward, and its base looks upward and is crossed by the medullary striæ. A slight eminence, the tuberculum acusticum, makes the lateral angle of the acustic area most prominent. Beneath the acustic area are the vestibular nuclei of the auditory nerve; also the lateral part of the cochlear nucleus, which is found in the acustic tubercle (Fig. 96).

\section{ORIGIN OF CEREBRAL NERVES.}

According to Sömmering, there are twelve pairs of cerebral nerves (nervi cerebrales), but to this must be added the nervus intermedius (pars intermedia) which, though associated with the facial nerve in the facial canal, is of itself a true mixed nerve. The first, second and eighth cerebral nerves are purely sensory; six of them, the third, fourth, sixth, seventh, eleventh and twelfth, are purely motor; while the fifth, the intermediate, the ninth and tenth are mixed nerves and contain both efferent and afferent fibers.

Cerebral Nerves, Nervi Cerebrales (Figs. 86 and 96).-

I. Olfactory (nn. olfactorii) - special sense of smell.

2. Optic (n. opticus)-special sense of sight.

3. Oculomotor (n. oculomotorius)-motor.

4. Trochlear (n. trochlearis)-motor. 
5. Trigeminal (n. trigeminus)-motor and common sensory.

6. Abducent (n. abducens) - motor.

7. Facial (n. facialis)-motor.

Intermediate (n. intermedius)-special sense of taste, secretory and trophic.

8. Acustic (n. acusticus) - special senses of hearing and equilibrium.

9. Glossopharyngeal (n. glossopharyngeus) - Special sense of taste, common sensory, secretory, trophic and motor.

Io. Vagus (n. vagus) - motor, vaso-motor, viscero-motor, inhibitory, secretory, trophic and common sensory.

II. Accessory (n. accessorius)-motor.

I2. Hypoglossal (n. hypoglossus)-motor.

All cerebral nerves are connected with the brain and, when their functions were not understood, these points of connection were indiscriminately called origins; but with our present knowledge of the functions and development of the pure sensory and the mixed nerves such use of the term "origin" is not admissible. Pure sensory nerves and the sensory roots of mixed nerves take their origins from ganglia situated wholly outside the brain. From those ganglia the dendrites grow outward to the peripheral distribution of the respective nerves; the axones grow centrally into the brain, where they arborize and end in groups of cell-bodies forming nuclei. Such nerves conduct impulses from the periphery to these nuclei, hence the name applied to them is terminal nuclei (muclei terminales). See the blue nuclei, Fig. 96. The motor nerves and the motor roots of mixed nerves take their origins inside the brain from groups of cell-bodies also called nuclei. The axones grow outward from these latter nuclei toward the periphery; they conduct impulses from the nuclei to the muscles or to the secreting cells in their respective areas of distribution, hence the nuclei of motor nerves and motor roots are genetic nuclei (nuclei origines). See the red nuclei, Fig. 96. Thus it is seen that the brain connection of a motor nerve is its true origin, while this connection is the real termination of a sensory nerve. 


\title{
TABLE II.
}

\section{SENSORY NERVES AND SENSORY ROOTS.}

\section{Ganglion of Origin. Entrance Into Brain. Terminal Nucleus.}

\author{
I. Oljactory (Smell).
}

Olfactory cells in nasal Under surface of olfac- Mitral and brush cells mucous membrane. tory bulb. of bulb (a part of cere. bral hemisphere).

\section{Optic (Sight).}

Ganglionar layer of the Surface of lateral genic- Nuclei of same in interretina. ulate body, pulvinar brain and mid-brain. of thalamus and su. perior quadrigeminal colliculus.

5. Trigeminal (Sensory Root).

Semilunar ganglion.

Anterior surface of the Nucleus tractus spinalis pons.

n. trigemini reaching from mid-pons to second cervical nerve.

Intermediate Nerve (Sensory Root) (Taste).

Geniculate ganglion. Groove between pons Nucleus tractus solitarii and medulla, between beneath inferior fovea seventh and eighth in medulla. nerves.

8. Acustic (Hearing and Equilibrium).

Cochlear Root.-Spiral Groove between pons Cochlear Nuclei.-Venganglion (of Corti). and medulla. tral and lateral, placed ventral and latcral to restiform body in medulla.

Vestibular Root.-Vestib-

Vestibular Nuclei-Prinular ganglion. cipal, Deiters's and nucleus of descending root, in floor of fourth ventricle in medulla.

\section{Glossopharyngeal (Sensory Root) (Taste, etc.).}

Superior and petrosal Posterior lateral sulcus Nucleus tractus solitarii ganglia in jugular of medulla. foramen.

and nucleus alæ cinerex in medulla. 
ro. Vagus (Sensory Root).

Jugular and nodular Posterior lateral sulcus Nucleus alæcinereæ, and ganglia in jugular of medulla. foramen and below it. perhaps nucleus tractus solitarii, in medulla.

\section{TABLE III.}

\section{MOTOR NERVES AND MOTOR ROOTS.}

\section{Genetic Nucleus.}

Apparent Origin (Exit from Brain).

3. Oculomotor (Motor Nerve).

Nucleus in floor of cerebral aqueduct Interpeduncular fossa of mid-brain. in mid-brain under superior colliculus.

\section{Trochlear (Motor Nerve).}

Nucleus in floor of cerebral aqueduct in mid-brain under inferior colliculus.
Superior medullary velum in isthmus rhombencephali.

\section{Trigeminal (Motor Root).}

Nucleus in floor of cerebral aqueduct Anterior surface of pons. in mid-brain and under locus cæruleus of pons.

\section{Abducent (Motor Nerve).}

Nucleus under colliculus facialis in Groove between pons and medulla. pons.

\section{Facial (Motor Nerve).}

Nucleus under fovea superior in pons. Groove between pons and medulla.

\section{Intermediate (Efferent Part, Secretory).}

Dorsal part of facial nucleus in pons. Groove between pons and medulla.

9. Glossopharyngeal (Motor Root).

Nucleus alæ cinereæ and nucleus am- Posterior lateral sulcus of medulla, biguus in the medulla. upper end.

I0. Vagus (Motor Root).

Same as ninth, but chiefly nucleus Posterior lateral sulcus below ninth, ambiguus. and between olive and restiform body. 


\section{Accessory (Motor Nerve).}

Cerebral Root.-Nucleus ambiguus in closed medulla.

Spinal Root.-Nucleus in lateral part of base of anterior columna-upper five segments of cord.
Posterior lateral sulcus of medulla below level of olive.

Lateral surface of cord between ligamentum denticulatum and posterior roots of spinal nerves.

12. Hypoglossal (Motor Nerve).

Nucleus under trigonum hypoglossi, floor of fourth ventricle, and in Anterior lateral sulcus of medulla closed medulla.

Terminal Nuclei.-The terminal nuclei of the first and second cerebral nerves are peculiar and cannot as yet be classified with the nuclei of other sensory nerves and sensory roots (Figs. 26, 43 and 63). The terminal nuclei of the fifth, the intermediate, the eighth, ninth and tenth nerves may be called the posterior columna series; because they are formed by masses of cell-bodies representing the upward prolongation of the posterior columna of gray substance in the spinal cord. Terminal nuclei are common sensory and special sensory.

Common Sensory Nuclei (Fig. 96). - Of the posterior columna series of nuclei, the terminal nucleus of the fifth and of the tenth nerves and a part of the terminal nucleus of the ninth nerve receive common sensory impulses, and transmit them to the opposite thalamus by two routes, viz., through the medial fillet and through the spino-thalamic tract. Impulses of the muscular sense travel over the former route; those of the pain and temperature senses, over the latter; and tactile impressions are believed to traverse both routes alike. From the thalamus these impulses are carried to the cortex of the posterior central gyrus. Thus is the cortical connection of these nuclei established; and each is brought into reflex connection with motor nuclei by axones of the terminal nuclei which run chiefly through the medial longitudinal bundle and terminate in the motor nuclei.

Special Sense Nuclei.-The cortical connection of the nucleus tractus solitarii, which receives taste impulses from the glossopharyngeal and intermediate nerves, has not been definitely traced; but it is probably relayed in the thalamus and is established by 
fibers of the formatio reticularis, in the pons and mid-brain, and, in the hemisphere, by certain fibers of the internal capsule which end in the fusiform gyrus (?). The cochlear muclei (ventral and lateral) receive true impulses of hearing and conduct them on toward the cerebral cortex by way of their axones, which form the trapezoid body and medullary striæ and then unite in forming the lateral fillet (Fig. 89). The remaining links of the cortical connection are formed by the brachium inferius and the acustic radiation (radiatio temporothalamica). The reflex connection of these nuclei is somewhat indirect. It is established in part by certain fibers of the lateral fillet which end in the quadrigeminal colliculi, together with the anterior longitudinal bundle; but is chiefly brought about by the olivary pedicle and the medial longitudinal bundle. The vestibular nuclei-the principal (Schwalbe's), Deiters's and the nucleus of the descending rootconcern equilibrium. They receive impulses from the vestibule and semicircular canals of the internal ear. They have a'cerebral and a cerebellar cortical connection. The former is established as far as the thalamus, by the opposite medial fillet, and completed by the cortical fillet; and the latter is formed by the descending root, which ends in the nucleus funiculi cuneati, and the external arcuate fibers. With the cerebral motor nerves, these nuclei are brought into reflex connection, first, by axones entering the medial longitudinal bundle and terminating in the motor nuclei; and, second, probably by fibers of the brachium conjunctivum which become connected with the nuclei of the third, fourth and sixth cerebral nerves. The reflex connection with spinal nerves is formed by the anterior descending cerebello-spinal tract, viz., by axones of Purkinje's cells, the acustico-cerebellar tract, the cerebello-olivary fibers and descending fibers from Deiters's and the inferior olivary nuclei.

The terminal nuclei of the optic nerve are situated in the lateral geniculate body, the pulvinar of the thalamus and the superior colliculus of the corpora quadrigemina (Fig. 43). Like the terminal nucleus of the olfactory nerve, these cannot at present be included in the posterior columna series, because the ventral and dorsal zones of the embryonic fore-brain have not been sufficiently 
elucidated. If the sulcus hypothalamicus really separate ventral from dorsal zone in the inter-brain, as is claimed by many, it would seem that both the optic and olfactory terminal nuclei might be included in the posterior series; but there is need of further investigation, as this places the whole cerebral hemisphere in the dorsal zone.

The cortical connection of the terminal nuclei of the optic nerve (Fig. 67) is established by fibers of the optic radiation (radiatio occipito-thalamica) which rise in the lateral geniculate body and in the pulvinar of the thalamus and terminate in the cortex of the calcarine region of the occipital lobe. From this cortical center, corticifugal fibers run through the occipito-thalamic radiation and brachium superius to the superior quadrigeminal colliculus. This colliculus also receives a few fibers directly from the outer root of the optic tract; it thus becomes the center of optic reflexes; and axones of the superior colliculus form the anterior longitudinal bundle, which completes the connection with opposite motor nuclei.

The terminal nucleus of the olfactory nerve is situated in the olfactory bulb (Figs. 26 and 63). The axones of this nucleus establish direct cortical connection, first, with the uncinate region and anterior perforated substance by way of the lateral olfactory stria and, probably, by way of the intermediate stria; and, second, with the parolfactory area, the olfactory triangle, the gyrus subcallosus and the gyrus cinguli through the fibers of the medial stria of the olfactory tract. The reflex connection of the olfactory nerve is but little understood. The following probabilities may be stated: First, the fornix, stria medullaris thalami, fasciculus retroflexus and axones of interpeduncular ganglion; second, the fornix, thalamo-mammillary bundle and descending axones of the thalamus; and, third, the fornix and the pedunculo-mammillary bundles running into mid-brain, pons and medulla.

Genetic Nuclei (Nn. Origines) (Fig. 96).-The nuclei of the oculomotor, trochlear, abducent, facial, accessory and hypoglossal nerves and the nuclei of the motor roots of the trigeminal, glossopharyngeal and vagus nerves represent the anterior columna of gray matter in the cord and constitute the anterior columna series. These nuclei are connected with the cerebral cortex on both 
sides, but chiefly with that of the opposite hemisphere. The connection is established first and principally by the pyramidal tracts, some of the fibers running directly from the tract to the nucleus, and others, leaving the tract high up, run through the accessory fillet (Bechterewi) to a point near the respective nuclei which they are about to enter; and, second, the fronto-pontal, temporopontal and intermediate tracts are believed to send some fibers to the genetic nuclei of the same side. The reflexconnection of these genetic nuclei is established for all of them by the medial longitudinal bundle; by the anterior longitudinal bundle and by the olivary pedicle (for the third, fourth, and sixth), by the trapezoid body (for the seventh) and by the spinal tract of the trigeminal nerve (for the fifth, seventh, and twelfth). 


\section{CHAPTER V.}

\section{MEMBRANES OF THE SPINAL CORD. (MENINGES SPINALIS.)}

Dura Mater.- Through the foramen magnum the membranes of the brain are continuous with those of the cord with which they are very similar in structure. The dura mater spinalis is attached to the margin of the great foramen and to the bodies of the first two or three cervical vertebræ; elsewhere, though joined to the vertebræ by fibrous bands, its surface is free from immediate bony attachment and it does not possess the periosteal layer. Thus suspended, it hangs as an open sack, or sheath (Fig. IoO) and reaches down to the third sacral vertebra, where it is constricted to a fibrous cord which blends with the periosteum on the posterior surface of the coccyx. The arachnoid and pia, and the spinal cord and cauda equina are contained in the dural sack (Figs. 97, 98 and 100). Externally, the surface of the dura is separated from the wall of the spinal canal by the internal vertebral plexus of veins, areolar tissue and fat. The outer surface is composed of flat polygonal cells, like the inner surface. Its internal, serous surface. is bathed with a small amount of cerebro-spinal fluid which separates it from the arachnoid. For every segment of the spinal cord, the dura presents, on either side, a pair of foramina, through which run the anterior and posterior roots of the spinal nerves (Fig. 97). Those nerve roots are invested by a sheath of dura prolonged from the margins of the foramina. The dura mater of the cord does not separate into two layers, and forms neither sinuses nor processes. It performs no periosteal function and possesses no arachnoid granulations (pacchionian bodies). Its two surfaces are formed by endothelium.

Arachnoid.-The arachnoid of the spinal cord (arachnoidea spinalis) forms a sack of the same length as the dural sheath, with which it is externally in contact (Figs. 97, 98 and 100). It 

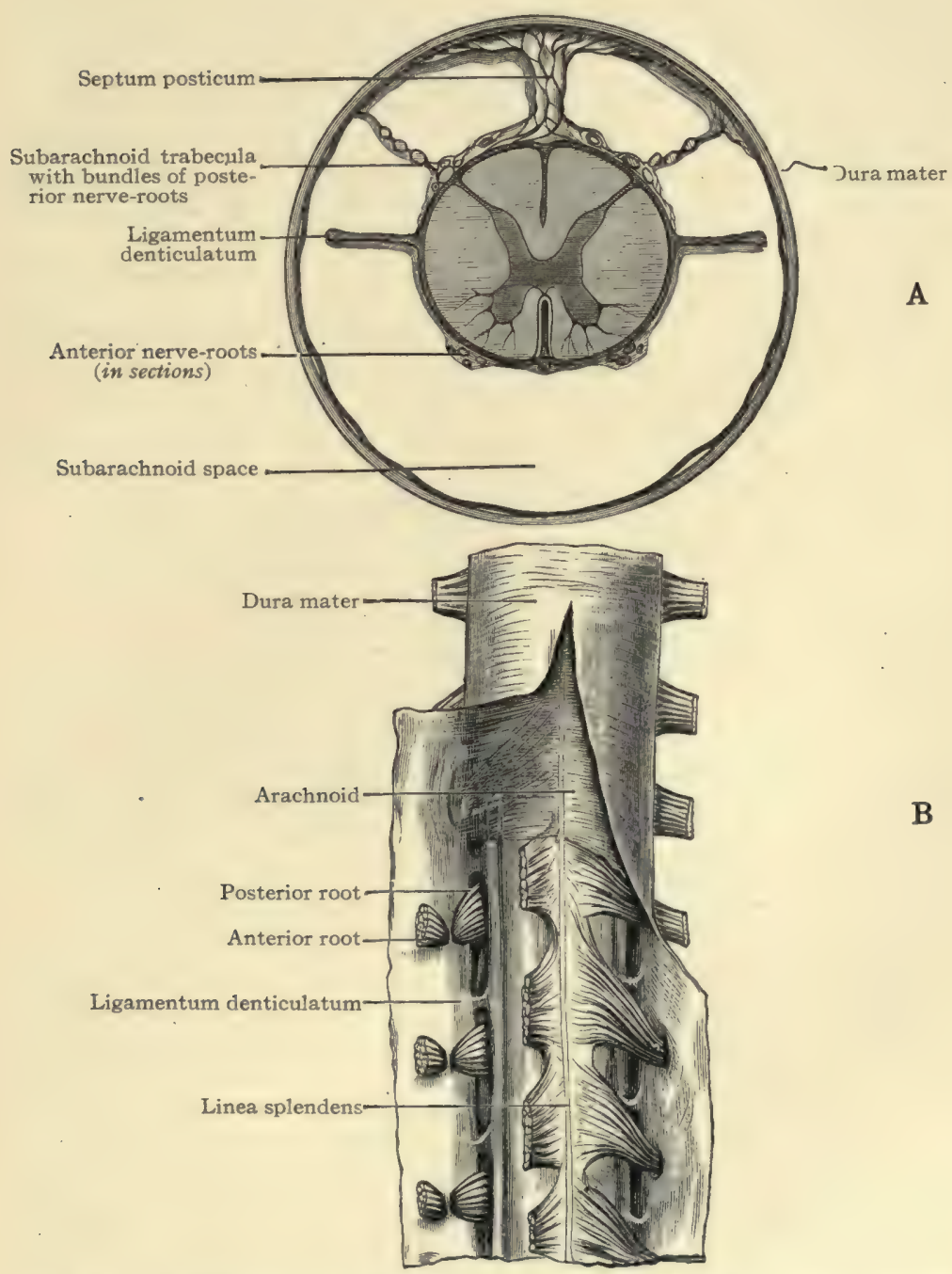

Fig. 97--Meninges of the spinal cord. A. Transverse section. (After Key and Retzius.) BB. Anterior view. (After Ellis. Morris's Anatomy.) 

presents two serous surfaces. Internally, bands of fibro-elastic tissue attach it to the pia mater along the posterior median line of the cord and form the subarachnoid sepium (Fig. 97). The external spinal veins and a considerable space separate the arachnoid from the pia mater. That subarachnoid space is filled with fluid. By the ligamenta denticulata it is divided into the anterior and posterior subarachnoid spaces, which, through the foramen magnum, are continuous with the same spaces in the cranial cavity (Figs. 97 and 6).

Pia Mater.-The pia of the cord (pia mater spinalis) is much stronger than that of the brain (Figs. 97 and 98). It has two

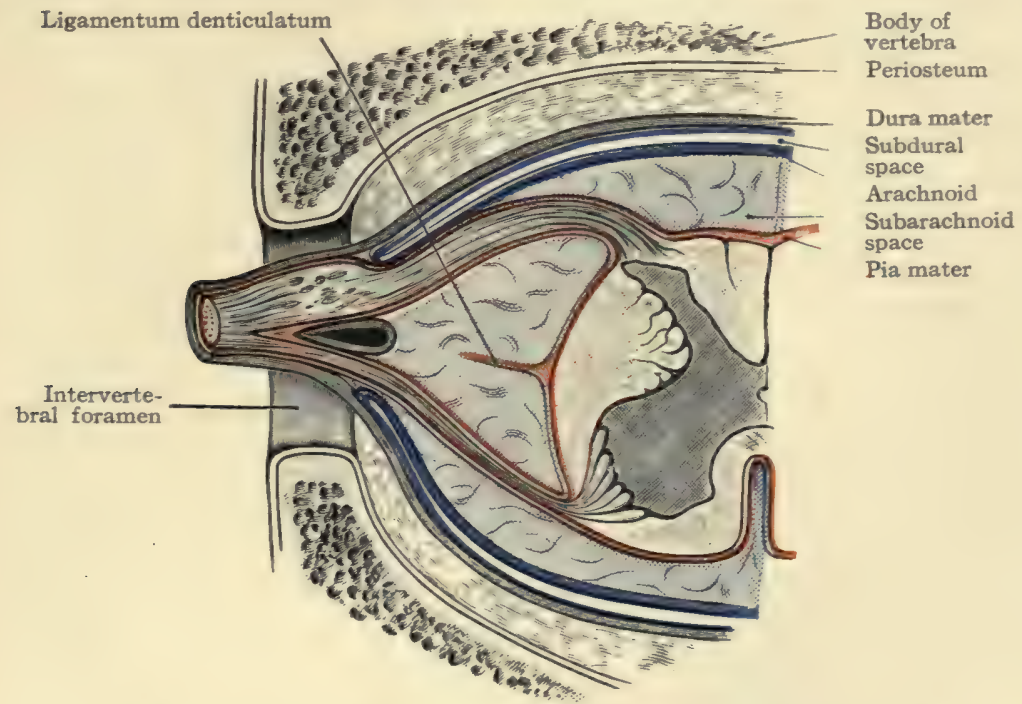

Fig. 98.-Diagrammatic section of the spinal meninges and spinal cord.

(After Morris's Anatomy.)

distinct layers, the inner of which is continuous with the brain pia and forms an epineurium for the cord and roots of the spinal nerves. The outer is the more vascular layer. Both layers dip into the anterior median fissure; they form the anterior septum which contains the anterior spinal artery. The inner layer is attached to the septum in the posterior median fissure. The outer layer forms the linea splendens along the front of the cord, and the 
ligamentum denticulatum on either side. The denticulate ligament is a longitudinal band whose smooth medial border is continuous with the pia along the middle of the lateral surface of the cord; its lateral border is notched and its twenty teeth, invested with arachnoid, are attached to the dura opposite the first twenty vertebræ. The two ligaments subdivide the space between the pia and arachnoid into anterior and posterior subarachnoid spaces. A filamentous extension of the pia below the cord proper helps to form the filum terminale internum. It descends in the arachnodural sheath with the roots of the lumbar and sacral nerves, and all together constitute the cauda equina (Fig. Ioo). For some distance, about half its length, the filum terminale internum contains gray matter and rudimentary fibers continuous with the spinal cord. The filum unites with the arachnoid and dura at the third sacral vertebra in forming the filum terminale externum which forms a sort of ligament for the spinal cord. That ligament is inserted into the coccyx. The pia mater of the cord contains the trunks and large branches of the anterior and the two posterior spinal arteries, and the tributaries of the external spinal veins.

Nerve Supply.-The membranes of the spinal cord are supplied by recurrent branches of the spinal nerves and by the sympathetic. The recurrent branches are sensory in function.

\section{BLOOD SUPPLY OF THE SPINAL CORD.}

The vessels supplying the cord are the anterior spinal artery and the two posterior spinal arteries, which rise at the foramen magnum from the vertebral arteries, and are reinforced by cervical, intercostal and lumbar arteries. The anterior spinal artery (a. spinalis anterior) descends along the entrance to the anterior median fissure (Fig. 99); it is formed by the union of two vessels, one from each vertebral. The posterior spinal artery (a. spinalis posterior) of either side, is in reality a pair of vessels which freely communicate, and are so placed as to embrace the posterior nerve roots. The larger vessel of the pair is anterior to the nerve roots, while the smaller is between them and the posterior median fissure 
(Fig. 100). The spinal arteries give origin to two sets of branches, namely, the fissural or centrifugal, and, the centripetal arteries. Both sets are end-arteries and form rich longitudinal plexuses, which overlap each other but do not anastomose.

The fissural or centrifugal arteries rise, first and chiefly, from the anterior spinal artery (Fig. 100). These enter the anterior median fissure and, running lateralward, supply the

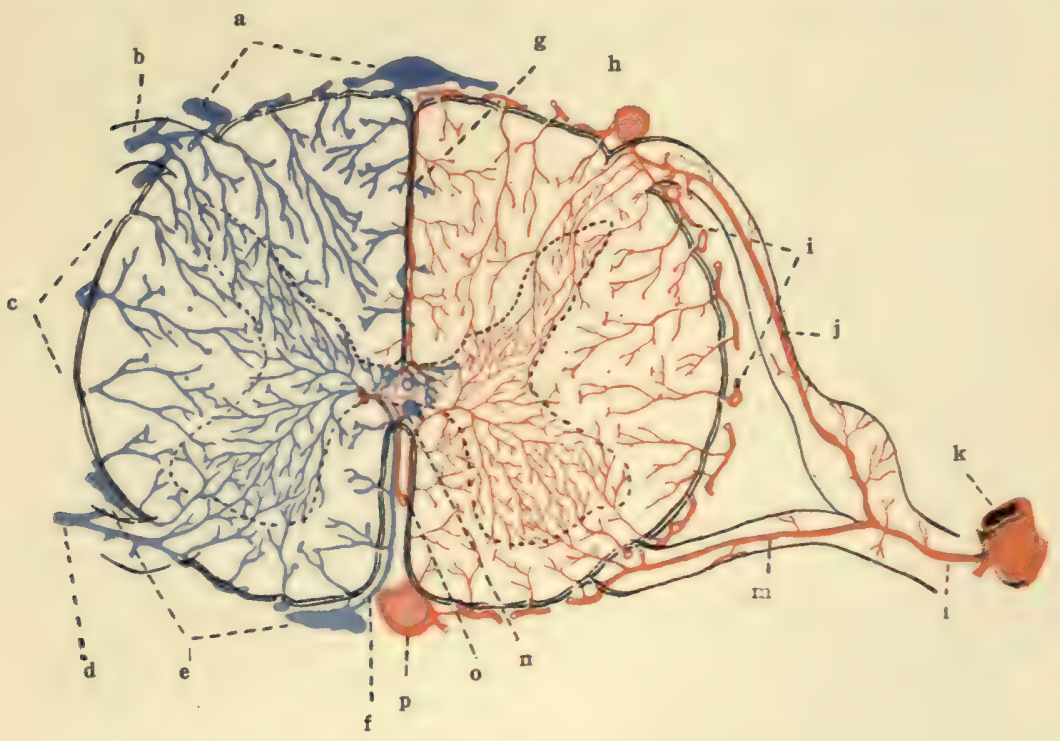

Fig. 99.-The arteries and veins in the spinal cord. Diagrammatic. (After Morris's Anatomy.)

a. Dorsal external spinal veins. b. Posterior radicular vein. c. Peripheral venous plexus. d. Anterior radicular vein. e. Ventral external spinal veins. f. Anterior central vein. g. Posterior central artery and vein. h. Posterior spinal artery. i. Peripheral arterial plexus. j. Posterior radicular artery. ik. Intercostal artery. 1. Spinal ramus. m. Anterior radicular artery. n. Interior spinal vein, o. Anterior central artery. p. Anterior spinal artery.

greater part of the gray matter. Second, a few centrifugal arteries rise from the posterior spinal arteries. Running into the posterior fissure, they are distributed to the posterior white columns, the posterior commissure and to the nucleus dorsalis (Clarki).

The centripetal arteries rise from both the anterior and posterior spinal arteries (Fig. I00). They enter the cord at right angles to the surface, and supply the white matter and the periph- 
eral parts of the gray substance, including the tips of the columnæ. Those branches to the columnæ accompany the root-fibers.

Veins.- The veins that carry the blood from the interior of the cord, the venæ spinales internæ, are the fissural veins, which issue from the fissures, the root-veins, which accompany the anterior and posterior root-fibers to the surface of the cord, and a small number of veins that issue from other parts of the surface of the spinal cord. All unite in forming the external spinal plexus (venæ spinalis externæ) spread over the entire surface of the cord beneath the arachnoid membrane. According to Cunningham, the plexus includes six longitudinal veins-anterior and posterior median and, on either side, an antero-lateral and a postero-lateral vein placed just behind the respective nerve roots. In the upper cervical region, the plexus forms two or three small veins which empty into the vertebral or inferior cerebellar veins; elsewhere, by a branch along each spinal nerve, the plexus communicates with the internal vertebral plexus (plexus venosi vertebrales interni) outside the dura mater, and is drained into the vertebral, intercostal, lumbar and sacral veins. No valves are found in the spinal veins.

Lymphatics.-Perivascular and perineural spaces carry the lymph from the spinal cord. There are no lymphatic vessels in the cord. 


\section{CHAPTER VI. \\ THE SPINAL CORD.}

The spinal cord (medulla spinalis) is developed from the posterior part of the neural tube, and forms the corresponding portion of the central axis of the nervous system.

Extent. - It is continuous with the medulla oblongata, above; and, in the adult, reaches to the lower border of the first lumbar vertebra (Fig. I00). Its length is seventeen to eighteen inches. In a very slender process, the filum terminale internum, the cord is continued beyond the first lumbar vertebra. That process and the lower spinal nerves form the cauda equina, which is inclosed in a sheath composed of the arachnoid and dura mater. The filum terminale internum for about three inches contains a prolongation of the central gray matter and ventricle of the cord; and, also, a few fibers, which suggest the coccygeal nerves of lower animals.

In the foetus before the third month, the cord and spinal canal are of equal length. At birth the cord reaches the third lumbar vertebra, and it continues to recede with the rapid growth of the vertebræ to adult life.

Diameters (Fig. Ior).-The spinal cord is shaped like a cylinder, slightly flattened from before backward (dorso-ventrally). Its longest diameter is transverse and measures less than half an inch, except in the cervical and lumbar enlargements of the cord. In the latter, it equals a half-inch; and, in the former, it slightly exceeds it. The thoracic portion of the cord is small and nearly cylindrical in shape. Divested of its meninges and nerves the spinal cord weighs about one ounce and a half, avoirdupois.

The cervical enlargement (intumescentia cervicalis) extends from the medulla oblongata to the second thoracic vertebra (Figs. IOO and IOI). Its greatest diameter is on a level with the fifth intervertebral disk. It gives origin to the motor fibers and 
receives the sensory fibers of the nerves which form the cervical and brachial plexuses.

The lumbar enlargement (intumescentia lumbalis) begins at the tenth thoracic vertebra and increases to the twelfth (Figs. IOO and IOI). Opposite the first lumbar vertebra, it tapers off
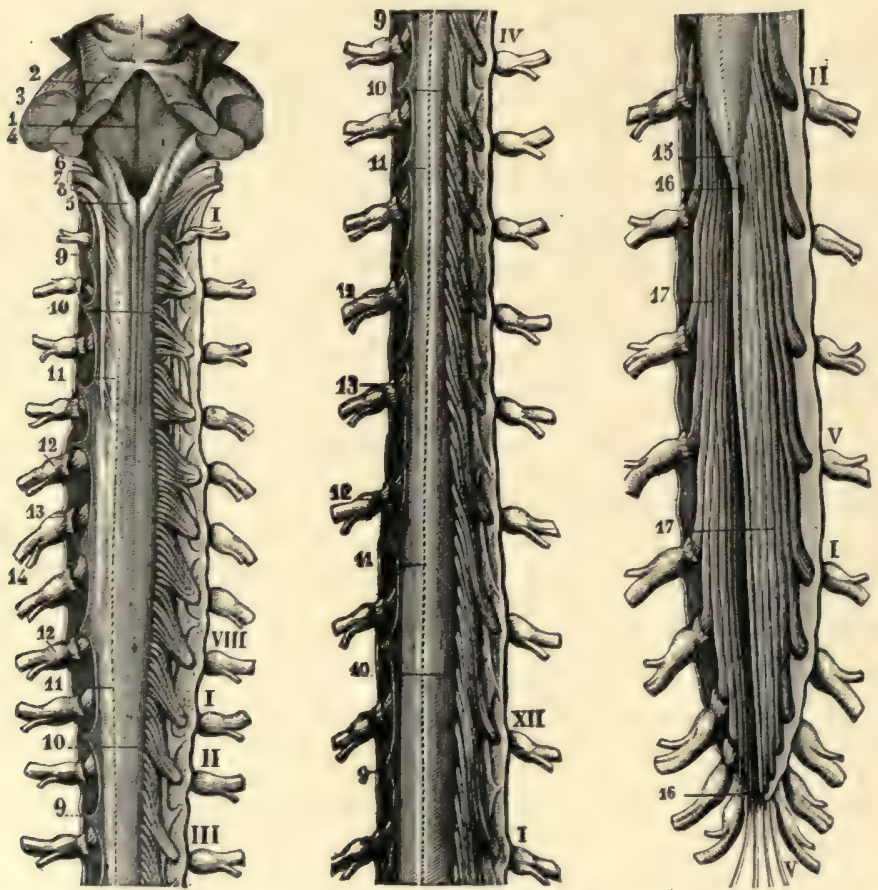

Superior or Cervical Segment Middle or Dorsal Portion Inferior Portion of Cord and of Spinal Cord. of Cord. Cauda Equina.

Fig. I00.-Posterior view of the spinal cord, the dura mater and the arachnoid being laid open and turned aside. (Brubaker after Sappey.)

I. Floor of fourth ventricle. 2. Brachium conjunctivum. 3. Brachium pontís. 4. Restiform body. 5. Clava. 6. Glossopharyngeal nerve. 7. Vagus. 8. Accessory nerve. 9, 9, 9, 9. Ligamentum denticulatum. 10, 10, 10, 10. Posterior roots of spinal nerves. II, II, II, II. Posterior lateral sulcus, 12, 12, 12, 12. Spinal ganglia. 13, 13. Anterior roots of spinal nerves. 14. Anterior and posterior divisions of spinal nerve. 15. Conus medullaris. I6, I6. Filum terminales internum. I7, I7. Cauda equina. I-VIII. Cervical nerves. I-XII. Thoracic nerves. I-V. Lumbar nerves. I-V. Sacral nerves.

almost to a point, the conus medullaris; but a very small process continues in the filum terminale internum. From the lumbar enlargement rise the motor fibers of the nerves contained in the lumbar and sacral plexuses, and into it enter the sensory fibers of the same plexuses. 


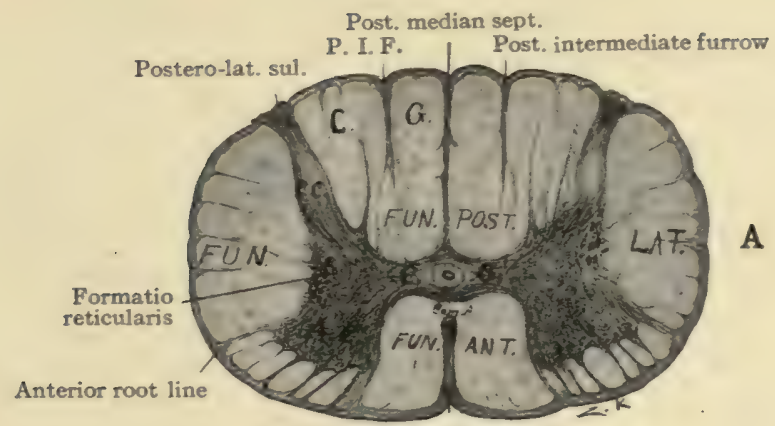

Ant. median fis.

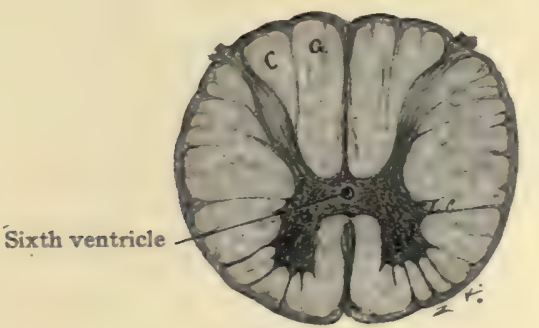

B

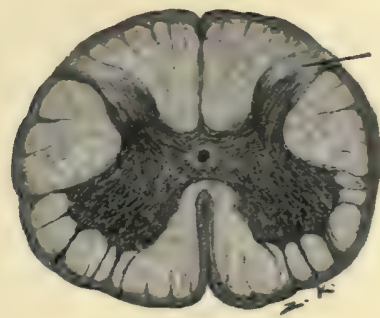

Gelatinous substance

$\mathrm{C}$

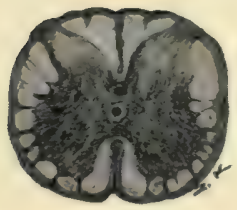

D

Fig. ror.-Sections of the spinal cord; A. The cervical, B. The thoracic, C. The lumbar, and $\mathrm{D}$. The sacral. (Original.)

A. Section of cervical cord. A. C. Anterior columna, P. C. Posterior columna, C. G. Gray commissure, anterior gray and posterior. Com. A. White anterior commissure. Fun. Ant. Funiculus anterior, Fun. Lat. Funiculus lateralis. Fun. Post. Funiculus posterior. G. Fasciculus gracilis, C. Fasciculus cuneatus. B. Section of thoracic cord. L. C. Lateral columna. C. Section lumbar cord. D. Section of lower sacral cord. 

Sixth Ventricle (Fig. Ior, B).-The central canal of the spinal cord (canalis centralis spinalis) is the representative of the cavity of the neural tube. It is just visible to the naked eye; but it extends throughout the cord and expands above into the fourth ventricle. In the filum terminale internum it is also dilated, forming the ventriculus terminalis (Krausei). It is lined with columnar ciliated cells which stand on a thick lamina of substantia gelatinosa.

Fissures of the Spinal Cord (Fig. Ior).-The spinal cord is incompletely divided into symmetrical lateral halves by the anterior and the posterior median fissure.

The anterior median fissure (fissura mediana anterior) is the broader and shallower of the two (Fig. IOI). It extends in length from the inferior end of the ventral surface of the pons (foramen cæcum of Vicq d'Azyr) down the anterior median line of the medulla and cord. As to depth, it equals one-third of the cord's axis. Its floor is formed by the white anterior commissure. Both layers of pia mater dip down into it and inclose the anterior spinal artery and its branches. The anterior median fissure is interrupted at the junction of the cord and medulla by the decussation of the pyramids. In the lumbar enlargement it gradually disappears.

The posterior median fissure (fissura mediana posterior) is narrow and deep (Fig. Ior). It extends, longitudinally, down the posterior median line of the cord from the middle of the posterior surface of the medulla. It divides the cord, dorsoventrally, beyond its middle. The floor of the fissure is formed by the posterior commissure, which, with the gray and white anterior commissures, separates the posterior from the anterior median fissure. The posterior median fissure is not an open fissure; it is occupied by a lamina of connective tissue, the posterior septum, which is attached to the deep layer of the pia mater. In the posterior septum ramify branches of the two posterior spinal arteries and tributaries of the external spinal veins.

PosteriorLateral Sulcus. (S. lateralis posterior).-Each lateral half of the spinal cord is partially divided, near the junction of the posterior fourth with the anterior three-fourths of its semi- 
circumference, by the posterior lateral sulcus (Fig. IOI). The sulcus is situated opposite the posterior columna of gray matter, to which it transmits the posterior roots of the spinal nerves. It is continuous above with the posterior lateral sulcus of the medulla. It separates the posterior surface and the antero-lateral surface from each other.

Anterior Root-line. (S. lateralis anterior).-As a landmark, it is convenient to call the longitudinal line through which issue the most lateral fibers of the anterior roots of the spinal nerves, the anterior root-line of the spinal cord (Fig. IOI). There is no groove on the surface of the cord along this line and it is misleading to call it a sulcus, as has been the custom. It is situated opposite the anterior columna of gray matter and in line with the anteriorlateral groove of the medulla oblongata. Through it and through the surface, just medial to it, emerge the anterior roots of the spinal nerves. It subdivides the antero-lateral surface into anterior and lateral surfaces.

The posterior intermediate sulcus (s. intermedius posterior) is a slight longitudinal groove in the cord which subdivides the upper three-fourths of the posterior surface into postero-medial surface and postero-lateral surface (Fig. Ior). From it a connective tissue septum extends into the cord and separates the fasciculus gracilis and fasciculus cuneatus from each other. The posterior intermediate furrow is found only in the cervical and in the upper eight thoracic segments of the cord.

\section{GRAY MATTER OF THE CORD.}

The spinal cord is composed of (I) gray matter (substantia grisea spinalis), in the central part; and (2) white matter (substantia alba spinalis) in the peripheral area. It is like the medulla and pons in having the white matter on the surface (Fig. IOI).

A column of gray matter (Fig. IOI), crescentic in section, extends through the center of each lateral half of the spinal cord. The crescent is convex medially; and is joined to its fellow, a little in front of the middle, by a vertical transverse lamina of gray matter, called the gray commissure (commissura grisea). 
It is joined to the white matter of the opposite side by the white anterior commissure. The points of the crescent are directed forward and backward, respectively, and form the anterior and posterior columnæ. A lateral projection from the center of the crescent, visible only in the thoracic region, is called the lateral columna; it fuses with the anterior columna in the cervical and and lumbar enlargements. Together, the two crescents and the gray commissure form an $\mathrm{H}$-shaped column of gray matter. The H-shaped column is well marked in the cervical and thoracic regions; but, toward the lower end of the cord, the crescents become short and thick, and the gray column is almost cylindrical.

The H-shaped column is composed of two kinds of gray substance, viz.:(I) The substantia gelatinosa (Rolandi), which forms (a) a cap for the head of the posterior columna and (b) an envelope for the central canal, or ventricle, of the cord. (2) The substantia spongiosa. The latter forms all the $\mathrm{H}$-shaped column except the tips of the posterior columnæ and the thick sheath of the central canal. Imbedded in the neuroglia, there is a network of medullated nerve fibers; and these, with the common stains, give rise to a spongy appearance under the microscope.

Gray Crescent (Fig. IOI).- - It is made up of (I) the anterior columna; (2) the center, which is joined to its fellow of the opposite side by the gray commissure and which forms the lateral projection, called the lateral columna; and, (3) the posterior columna. The lateral border of the crescent is not everywhere clear cut and definite, especially in the cervical region, but is intermingled for a short distance with the white matter, forming the formatio reticularis. The formatio reticularis is found in the cervical region, elsewhere it is very feebly developed.

(I) The anterior columna (columna anterior) (Fig. IOI) as seen in sections is short and thick compared with the posterior columna. It is thickest in the cervical and lumbar enlargements, where it swells out sharply toward the lateral surface of the cord; in the mid-thoracic region it is more slender. It does not reach the surface of the cord as does the posterior columna. It ends in a bulbous, serrated head, which points toward the anterior root-line, and is joined to the center of the crescent by the cervix 
or base. From it the anterior roots of the spinal nerves rise; and, together with the anterior root-fibers, it separates from each other the anterior and lateral white columns of the cord.

Cells of the Anterior Columna (Figs. 102 and I03).-The gray matter of the spinal cord contains multipolar neurones of the Golgi and Deiters types. The Golgi cells ramify richly in the gray matter about the cell-bodies, and, both their axones and dendrites terminate in relation with other neurones in the adjacent gray substance. The long axones of the Deiters cells either enter into the anterior roots (radices anterior), and the neurones are called radicular cells, or they enter into a longitudinal tract or strand of fibers, and the neurones are named strand-cells (Cunningham). The dendrites of the Deiters cells arborize in both the gray and white substance. The cell-bodies in the anterior columna are large and vesicular in character. They are motor or efferent in function, and their axones form, in great part, the anterior roots of the spinal nerves. Together with the neurones of the genetic nuclei of cerebral nerves, these of the anterior columna constitute the lower segment motor neurones.

Two chief columns of cell-bodies are located in the anterior columna, the medial column and the lateral column (Figs. IO2 and IO3). The former is continuous throughout the cord with the exception of the fifth lumbar and the first sacral segments (Bruce-Cunningham); while the lateral column is found only in the cervical and lumbar enlargements. The medial column of cells shows a double group in sections of the lower three cervical, all the thoracic and the first lumbar segments of the cord. These subgroups are called the ventro-medial and the dorsomedial cells. Only the ventro-medial group is present above the sixth cervical segment and below the first lumbar segment. The dendrites of the cell-bodies in the medial column arborize in the gray substance of the same columna, in the adjacent white matter of the anterior column of the cord and, to some extent, in the opposite anterior columna, having passed through the white anterior commissure; the axones of these medial cell-bodies enter very largely into the anterior roots of the spinal nerves on the same side; but a certain number probably run through the white 

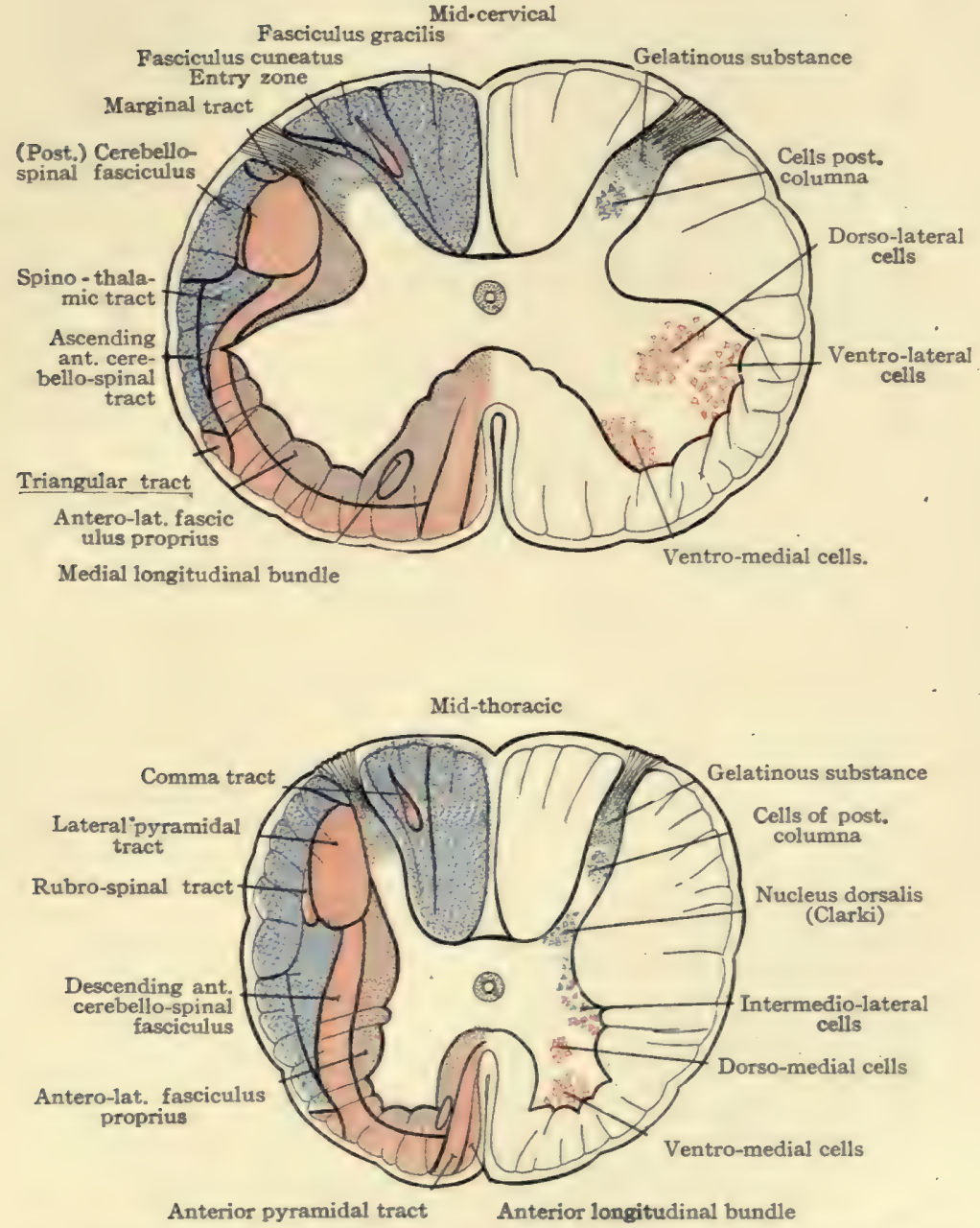

Fig. ro2.- Tracts of fibers 'and columns? of cells, in the cervical and thoracic regions of the cord. Diagrammatic. (In part after Bruce and Cunningham.) 

anterior commissure into the anterior nerve-roots of the opposite side, and others enter into the fasciculi proprii of the cord.

The lateral column of cells in the anterior columna is a large one (Figs. I02 and 103). It is found only in the regions which innervate the extremities, that is, in the cervical and lumbar enlargements. It is everywhere divided into a ventro-lateral and a dorso-lateral cell-group, and in most of the segments of the lumbar enlargement there are two other cell-groups, according to Alexander Bruce. One of them is located behind the dorso-lateral cells and is called the post-dorso-lateral group; and the other, which occupies the angle between the ventro-lateral and the dorso-lateral cells, lying medial to both, is called the central group (Cunningham). The dendrites of the cell-bodies in the lateral column arborize and end both in the gray matter of the anterior columna and in the white matter adjacent to its lateral surface; the axones proceed largely into the anterior roots of the spinal nerves but partly into the longitudinal white columns of the cord. Probably the medial column innervates the trunk muscles; the lateral column, the muscles of the extremities. Cortical Connection.-These cell-columns are brought into relation with the anterior (direct) pyramidal fibers and the lateral (crossed) pyramidal fibers by means of intermediate neurones. In this manner motor and inhibitory impulses descend to them from the cerebral cortex, coming from the opposite hemisphere, chiefly, but also from the same side. They also are in relation with the end-tufts of posterior root-fibers and with axones whose cell-bodies are located in the center and posterior columna of the gray crescent. The latter neurones form contact relations with fibers of the posterior roots of the spinal nerves on both sides. Thus both by immediate contact between anterior and posterior root-neurones and by the intervention of an intrinsic spinal neurone the simple reflex mechan$i s m$ of the spinal cord is formed. It has been the belief that the end-tufts of the fibers in the anterior and lateral pyramidal tracts are in direct contact with the dendrites or cell-bodies of the neurones in the anterior columna; but the investigations of Schäfer, Collier and others, indicate that this connection between the neurones of the anterior columna and the lateral pyramidal fibers, 
at least, is established by intervening neurones whose cell-bodies are located near the base of the posterior columna in the region of the nucleus dorsalis (Clark's column). The evidence of such termination of the anterior pyramidal tract is not conclusive.

Lesions.-Paralysis due to lesions of the anterior columna and of the genetic nuclei of cerebral nerves is often called lower segment paralysis. The cells in the anterior columna are the seat of hemorrhagic inflammation and rapidly degenerate in acute anterior poliomyelitis. In progressive muscular atrophy and in amyotrophic lateral sclerosis they degenerate slowly. As a result of the first, sudden flaccid paralysis occurs. The muscles waste away in the second and third because the nerves controlling the muscles and their blood supply are gradually destroyed. In the last, the muscles are also spastic, because the involvement of the pyramidal tracts cuts off cerebral inhibition.

(2) Center of Crescent and Columna Lateralis (Fig. Ior).In the center of the crescent there are many small, closely packed cell-bodies, which are probably sympathetic in function. This part of the crescent appears to contain the automatic spinal centers, such as, the cilio-spinal, cardiac-accelerator, vaso-motor, secretory, trophic, inhibito-secretory, viscero-motor, viscero-inhibitory, etc. That the cell-bodies in the center of the crescent are of sympathetic function is suggested by two facts; first, the cell-bodies are small, which indicates that the axones run but a short distance from the neurone center, as is the case with spinal sympathetic neurones; and, second, the distribution of these central neurones is limited to those regions of the spinal cord whence the efferent sympathetic fibers rise.

The intermedio-lateral column of cell-bodies (Fig. IO2, B) is the only one found in the center of the crescent. In the thoracic segments of the cord, where the lateral columna is visible, this column is contained in the lateral columna and in the white matter immediately adjacent to it; so far as it is found in the cervical and lumbar enlargements it is situated in the base of the anterior columna near its lateral surface. The intermedio-lateral column is found in the last cervical, all the thoracic and the first and second lumbar segments, in a nearly continuous column; it is also found 


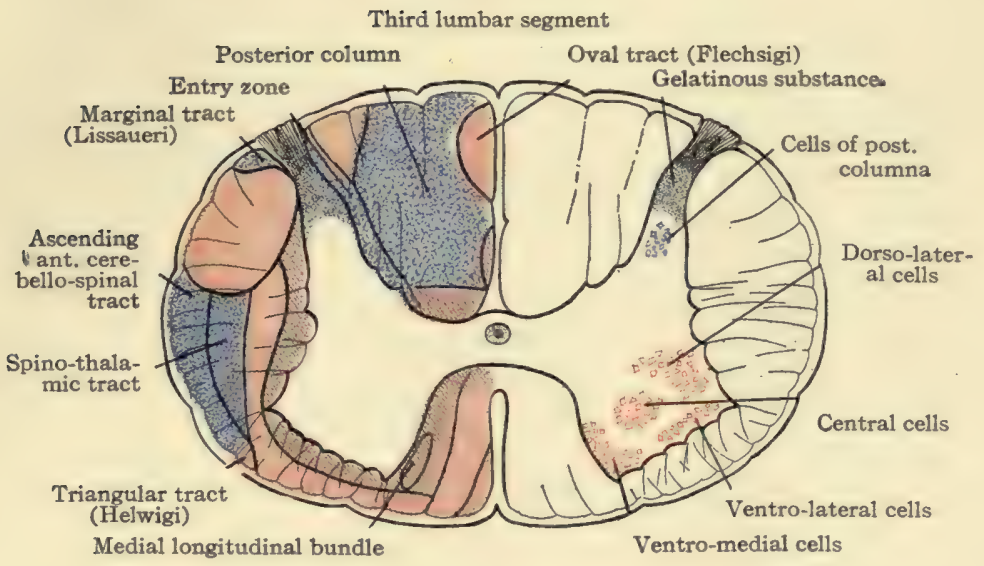

Third sacral segment

Septomarginal tract

Postero-lateral descending tr. Cornu-commissural tr

Lateral pyramidal tr.

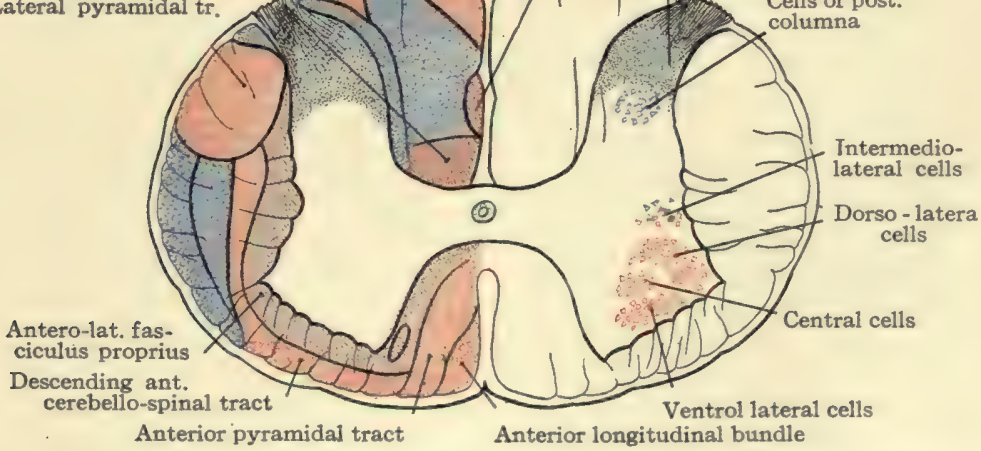

Fig. I03.-Tracts of fibers and columns of cells in the lumbar and sacral regions of the cord. Diagrammatic. (In part after Bruce and Cunningham.) 

in the third and fourth sacral segments of the cord and in the first three cervical (Cunningham). The first region (from last cervical to second lumbar segment) corresponds in position and extent to the origins of the white rami communicantes; the second region is at the level of origin of the pelvic splanchnics; and the cell-groups representing this column in the upper cervical segments probably contribute sympathetic fibers to the accessory and phrenic nerves.

The cells of the intermedio-lateral column are largely of the radicular variety, their slender axones enter into the anterior roots of the spinal nerves. Whether any belong to the strandvariety is not known. They are in contact relation with posterior root-fibers and are also connected with the posterior roots by intervening neurones. Their cerebral connection has not been traced.

(3) The posterior columna (columna posterior) except in the lower cord, is slender (Fig. Ior). It is longer than the anterior columna and reaches the surface in the posterior lateral sulcus, where it receives the posterior roots of the spinal nerves. The posterior columna presents a slight enlargement near its extremity, called the caput columnce, which tapers off to the apex columna. The head is joined to the base by a constricted part, the cervix. The head of the posterior columna is capped by a V-shaped mass of substantia gelatinosa. Spongy substance makes up the remainder of it. The posterior columna separates the posterior from the lateral column of the cord (Figs. IO2 and I03).

The cells of the posterior columna are numerous. In the head of the posterior columna they have smaller bodies than the cells of the columna anterior. They are less definitely grouped and are fusiform in shape throughout the caput columnæ; but in the base of the horn, near its medial surface, they have large vesicular bodies and form one of the most definite cell-columns in the spinal cord. The posterior columna neurones are afferent or sensory in function.

The neurones of the head of the posterior columna (Figs. I02 and I03) belong largely to the type of Golgi, as the processes of the greater number of them neither enter into the anterior roots 
of the spinal nerves, nor help to form the longitudinal fiber-tracts of the cord. Their axones arborize and end in the gray substance of both crescents. Those axones of the Golgi cells which cross over through the posterior commissure to the opposite crescent help to constitute the "immediate decussation" of the pain and temperature path. There are also Deiters cells in the head of the posterior columna; their axones enter into the posterior fasciculus proprius of the cord, hence they belong to the strand-variety. They appear to form the ventral field of the posterior column (Hoche) called the cornu-commissural tract, and at least a part of the comma, oval and septo-marginal tract. The neurones in the caput of the posterior columna receive posterior root-fibers which carry excito-reflex impulses and impulses of the tactile, pain and temperature senses. The former are transmitted forward largely in the same crescent to the center and anterior columna; the latter, to a considerable extent, are carried through the posterior commissure to the anterior columna of the opposite crescent.

Nucleus Dorsalis (Stillingi and Clarki).-This column, which was discovered by Stilling, is composed of cell-bodies measuring from $40 \mu$ to $90 \mu$ in diameter (Figs. IO2 and I04). It forms a most striking feature of the gray crescent throughout the thoracic region. It is situated near the medial surface of the base of the posterior columna, bounded laterally by a curved strand of posterior root-fibers; and forms a continuous column from the seventh cervical segment to the second lumbar segment. The column is largest in the lower two thoracic segments, where it bulges out the medial surface of the posterior columna. It is represented by separated groups of cell-bodies in the third and fourth sacral and first three or four cervical segments of the cord and in the accessory nucleus of the cuneate column in the medulla oblongata. The limitation of the dorsal nucleus, as an unbroken column, to the region of the white rami communicantes has suggested its connection with the sympathetic system; and it is the terminal nucleus of afferent sym pathetic fibers, but it gives rise to no efferent fibers of that system. All the axones of the dorsal nucleus appear to run toward the lateral surface of the cord and enter into the cere- 
bello-spinal tract of the same side. The several dendrites of each neurone ramify richly in the vicinity of the cell-body. Together with the cell-bodies, the dendrites are in contact relation with fibers of the posterior roots of the spinal nerves. The nucleus dorsalis probably has to do with muscular tonicity and equilibrium, chiefly. It is supposed to receive afferent sympathetic impulses from the internal organs.

Concerning the relation of the gray crescent to the spinal nerves (Fig. I04), it may be remarked, here, that in the anterior columna

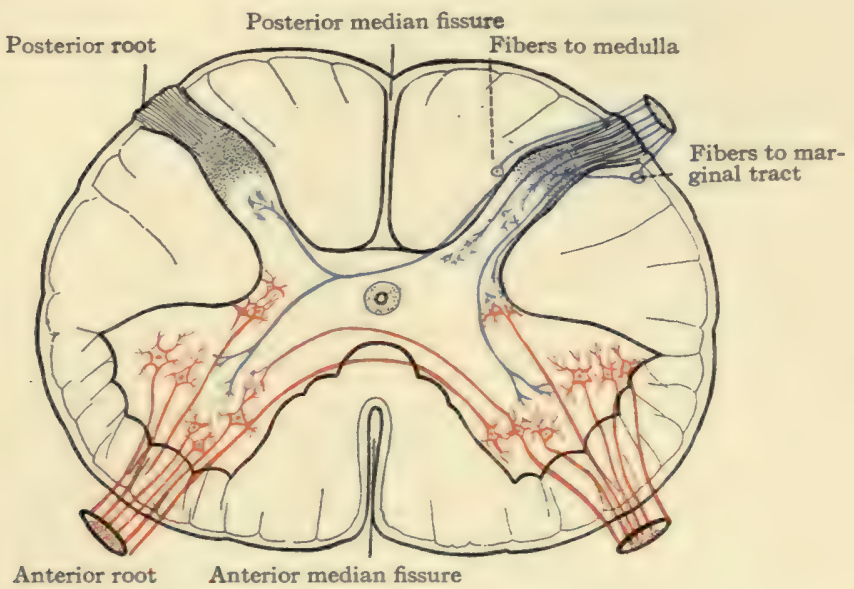

Fig. I04.-The roots of the spinal nerves. Diagrammatic. (Original.)

Anterior root rises from medial, lateral and intermedio-lateral cells of same crescent and from medial cells of opposite crescent: it is also connected with the opposite side by certain dendrites.

Posterior root terminates in gelatinous substance, in the center and base of the posterior columna, in the intermedio-lateral column, and in the anterior columna of the same crescent: in the anterior and posterior columna of the opposite side (?); and in the nucleus funiculi gracilis or nucleus funiculi cuneati of the medulla oblongata.

and center of the crescent are located the genetic nuclei of the motor or efferent fibers (anterior roots) of the spinal nerves; and that the terminal nuclei of the sensory fibers (posterior roots) of the spinal nerves are located chiefly in the posterior columna, but also in the center and anterior columna of the crescent in the cord, and in the nucleus funiculi gracilis and nucleus funiculi cuneati in the medulla oblongata. 
The gray commissure of the spinal cord (the gray anterior, and the posterior commissure) is the vertical, transverse sheet of gray substance connecting the two crescents together (Fig. IOI). This commissure (commissura grisea) completes the gray matter of the cord. It unites the gray crescents together a little in front of their center, except in the lumbar region where it joins their centers. It forms the floor of the posterior median fissure; and, in front, is in relation with the white anterior commissure. It is pierced longitudinally by the central canal of the spinal cord, which is surrounded by a thick envelope of substantia gelatinosa. This canal, the sixth ventricle, divides the commissure into two parts. That part of the commissure in front of the canal is the gray anterior commissure (commissura anterior grisea) and that behind it is the posterior commissure (commissura posterior, Fig. IOI). The gray commissure, comprising both these divisions, is composed of spongy and gelatinous substance in which there are imbedded the bodies of many nerve cells and a large number of medullated fibers. The medullated fibers are derived from the posterior roots of the spinal nerves, and from intrinsic neurones of the cord, whose centers are situated chiefly in the commissure and in the posterior columna. The posterior commissure is said to contain a long sensory tract, between the ventricle and dorsal surface (Ciaglinski). This long sensory tract is found in the thoracic portion of the cord and the discoverer believes it to be made up of ascending root-fibers which conduct pain and temperature impulses. It is in need of further investigation (Barker).

Lesions of the gray substance, as in syringomyelia, completely abolish the pain and temperature senses at the level of the lesion, while the muscular and tactile senses are preserved. The dissociation of sensations is most complete and bilateral when the lesion destroys the posterior commissure; if the lesion be limited to one crescent, the pain and temperature senses are affected on the side opposite to the lesion. These facts show that the pain and temperature paths to the cerebral cortex run through the gray substance of the cord and that they decussate. through it immediately upon entering the cord. It also shows that muscular and 
tactile impressions may reach the cerebrum without passing through the gray substance in the spinal cord; but it does not exclude the possibility of a part of the impulses of the muscular and tactile senses being transferred from a lower to a higher neurone in the spinal gray substance.

\section{WHITE MATTER OF THE CORD.}

The white matter (Fig. Ior) of the spinal cord (substantia alba spinalis) is disposed in its peripheral area and in the white anterior commissure. It is composed of medullated nerve fibers (axones and collaterals) imbedded in a small amount of neuroglia, and supported by a connective tissue network derived from the pia mater. Like the gray matter, it is richly supplied with bloodvessels. The fibers of the spinal cord run transversely, dorsoventrally and longitudinally.

The transverse fibers, which are usually somewhat oblique in direction, comprise (I) those running from the longitudinal tracts into the gray matter or out of the gray matter into such tracts; (2) the axones of intrinsic neurones which connect the two crescents at nearly the same level; and (3) posterior root-fibers running through the posterior commissure to the opposite crescent.

The white anterior commissure of the spinal cord (commissura anterior $a l b a$ ) is the only definite lamina of transverse fibers in the cord (Fig. IOI). It connects the anterior and lateral white columns of the cord with the opposite gray crescent and the two crescents with each other. It is located in front of the gray anterior commissure, forming the floor of the anterior median fissure. It is composed of medullated fibers belonging to (a) the anterior pyramidal tract, (b) the anterior fasciculus proprius, (c) the ascending anterior cerebello-spinal and spino-thalamic tracts; (d) it comprises the crossed fibers to the anterior roots of the spinal nerves, and (e) the decussating dendrites between the anterior columnæ.

The dorso-ventral fibers of the spinal cord (Fig. I04) are (a) those of the anterior roots of the spinal nerves, in their course from the gray matter to the surface of the cord; (b) those of the 
posterior roots, running from the posterior-lateral sulcus to their destination in the gray matter, and (c) axones of intrinsic neurones connecting posterior with anterior parts of the crescent.

The longitudinal fibers comprise most of the white matter in the cord, forming the funiculus anterior, funiculus lateralis and funiculus posterior (Fig. Ior). These three great columns occupy the anterior, lateral and posterior areas of the cord. They are disposed around the gray crescent in bundles or tracts. The tracts which make up the funiculi are not visible to the naked eye, nor under the microscope in a healthy adult cord; they have been located by embryological, experimental and pathological investigations. The longitudinal fibers rise in the brain, in the spinal cord and in the spinal ganglia; some run upward and others downward, constituting the tracts of the cord. Thus the tracts are characterized as ascending, descending and mixed tracts.

Ascending Tracts: (I) Ascending anterior cerebello-spinal and spino-thalamic tract. (2) Cerebello-spinal tract (direct cerebellar) with the spino-vestibular tract. (3) Marginal tract (of Lissauer). (4) Fasciculus cuneatus. (5) Fasciculus gracilis. Ciaglinski's long sensory tract, in the posterior commissure.

Descending Tracts: (I) Anterior (direct) pyramidal tract, including the anterior longitudinal bundle. (2) Lateral (crossed) pyramidal tract. (3) Descending anterior cerebello-spinal tract, including Helwig's tract. (4) Rubro-spinal tract (Pawlow). Descending postero-medial tract, called at different levels the comma, oval, and septo-marginal tract. (6) Descending posterolateral tract.

Mixed, Ascending and Descending Tracts: (I) The anterior fasciculus proprius, includiug the medial longitudinal bundle. (2) Lateral fasciculus proprius. (3) The posterior fasciculus proprius, composed of the cornu-commissural tract, the septal bandalette of Hoche, and possibly a part of the comma, oval, septo-marginal and the descending postero-lateral tract (Cunningham).

The methods of locating tracts of fibers may be summarized briefly, as follows:

The embryological method was first employed successfully 
by Flechsig. He found that nerve fibers when first laid down are naked axones without any insulating white substance of Schwann ensheathing them. That the medullary sheaths are developed at different times and that the medullation is nearly coincident with the beginning of function. Thus the fibers of motor and sensory nerves are first to become medullated, since life cannot be sustained without the automatic mechanism. Second, the fasciculi proprii of the cord are medullated and, third, the cerebellar tracts. At this stage the simple automatic and coordinating mechanisms are complete. Fourth, the voluntary motor mechanism is established by the medullation of the tracts connecting the lower neurones with the cerebral cortex, the fibers of the pyramidal tracts being the last to receive their medullary sheaths. This last occurs just before birth. Fibers of the cerebrum concerned with the higher psychic functions of the brain become medullated gradually, year after year, keeping pace with the mental development; and the process of medullation there is not completed until late in life (Kaes).

The pathological and experimental methods depend upon the fact that a nerve fiber when severed from the cell-body undergoes degeneration in accordance with the law of Waller. If the severed fiber be above the cell-body, the degeneration occurs above the lesion and is called ascending degeneration; but, if the degeneration extends from the lesion down the nerve fiber, the cell-body being above, then the condition is called descending degeneration, though all parts of the severed fibers really degenerate simultaneously. Thus by studying the paths of degeneration, above and below a destructive lesion in the human cerebro-spinal axis, the various tracts of fibers have been discovered and many of them charted and traced from origin to termination. These investigations have been greatly aided by the study of degenerations in the brain and cord of lower animals. These degenerations are the results of definite experimental lesions, as cutting of certain posterior nerve roots, partial section, hemisection or complete section of the spinal cord, etc. The pathological and experimental methods are commonly called the physiological method. 


\section{TRACTS OF THE SPINAL CORD.}

The antero-lateral fasciculus proprius (fasciculus anterolateralis proprius) occupies the deep part of the anterior and lateral columns (Figs. I02 and 103). It embraces the anterior columna of gray matter and covers the outer surface of the center of the crescent and the base of the posterior columna. By the most lateral anterior root-fibers it is subdivided into anterior and lateral fasciculi. It approaches, but does not quite reach, the surface of the cord. Notice that it is separated from the anterior median fissure by the anterior pyramidal tract, and that the anterior cerebello-spinal and spino-thalamic tracts run between it and the surface of the cord. Behind, it is in relation with the lateral pyramidal tract. The antero-lateral fasciculus proprius is composed of ascending and descending fibers which are the $\mathrm{T}$-branches of axones from the gray crescent. It is largely a short fiber tract, associative and commissural in function. That part situated in the anterior column, the anterior fasciculus proprius, is largely commissural, between the anterior columnæ; while the lateral fasciculus proprius is chiefly associative, and connects different segments of the cord on the same side. The antero-lateral fasciculus proprius is continued in the substantia reticularis of the medulla, and the reticular formation of pons and mid-brain, constituting a short fiber tract which extends from the lower part of the cord to the basal ganglia of the cerebrum. In the anterior fasciculus proprius there is a strand of long fibers called the medial longitudinal bundle (fasciculus longitudinalis medialis).

The medial (posterior) longitudinal bundle is composed of an ascending and a descending strand of fibers (Figs. IO2 and I03). The ascending strand rises from the anterior columna in each segment of the spinal cord and runs upward to the motor nuclei of cerebral nerves and terminates in them. Perhaps a few fibers reach the thalamus. It ascends just ventro-medial to the anterior columna in the cord; in the medulla, it runs between the head of the anterior columna and the pyramidal decussation, then just lateral to the fillet decussation, after which, it takes its dorso-medial position along the raphe. Its function is reflex. The descending 
strand is the medial ponto-spinal tract (Collieri). It rises from all the nuclei of the reticular formation but chiefly from the nuclei centrales and the nucleus lateralis medius in the pons. Forming a part of the medial longitudinal bundle of the same side, its fibers end in the center of the crescent as it descends the cord. Within the lateral fasciculus proprius, descends the lateral pontospinal tract (Collieri). This tract has the same origin and termination as the medial ponto-spinal tract except that it decussates. It crosses near its origin right through the medial longitudinal bundles. Both ponto-spinal tracts extend to the lower part of the cord.

The anterior pyramidal tract (fasciculus cerebro-spinalis anterior), occupies a thin area next the anterior median fissure (Figs. I02 and 103). It is the direct continuation of about ro per cent. of the pyramidal tract in the medulla. Its fibers are axones of cortical cells whose bodies are situated in the anterior central gyrus of the cerebrum. As the tract descends in the cord, the fibers decussate through the white anterior commissure, and terminate in relation with the cells of the opposite gray crescent, probably, in the posterior columna. It reaches to the fifth sacral segment (Collier). Imbedded in the anterior pyramidal tract is a small strand first described by Held, the anterior longitudinal bundle.

Anterior Longitudinal Bundle (Figs. I02 and I03).--Held called it the fasciculus longitudinalis ventralis. It occupies a very narrow strip in the anterior column just beside the entrance of the anterior median fissure. The anterior longitudinal bundle has already been traced from its origin in the superior quadrigeminal colliculus, through the dorsal tegmented decussation (Meynerti) to a position in the mid-brain ventro-lateral from the medial longitudinal bundle. It descends in that relative position through the pons and half the medulla; near the pyramidal decussation the anterior and medial longitudinal bundles are brought together and lie between that decussation and the isolated head of the anterior columna; they diverge upon entering the cord and remain separate to the end. The anterior longitudinal bundle ends in both anterior columnæ (Collier). It forms the middle link in the ocular and pupillary reflex arcs. 
The descending anterior cerebello-spinal tract (fasciculus descendens cerebello-spinalis anterior-Marchi's, Tschermak's and Loewenthal's column) together with the ascending anterior cerebello-spinal tract, occupies a thin peripheral area, broadest posteriorly, which extends from the anterior pyramidal tract outward and backward, over the antero-lateral fasciculus proprius, to the middle of the lateral surface of the cord (Figs. I02 and 103). Its posterior border is in relation with the (posterior) cerebello-spinal tract and the lateral pyramidal tract. The fibers of the two anterior cerebello-spinal tracts are mingled together; but the descending fibers are found, chiefly, in the anterior and posteromedial part of the common area and the ascending in the posterolateral part. The tract is almost completely relayed in the medulla. The descending fibers, in the first stage, are axones from the cortical cells of Purkinje in the cerebellum and from the nucleus fastigii and perhaps the other cerebellar ganglia. They descend to the nucleus of Deiters through the brachium pontis cerebelli. There the greater number end and new fibers rise which continue the tract down the cord. In all probability the cerebello-olivary fibers in the restiform body, and the olivo-spinal fibers, forming the tract of Helwig in the cord, should be included in the descending cerebellar tract. It terminates in the gray substance of the spinal cord and directly or by the intervention of intrinsic spinal neurones is connected with the motor cells of the anterior columna. The descending cerebello-spinal tract forms one segment of an indirect motor path; and, together with the intrinsic and anterior columna neurones, it forms the efferent limb of a reflex arc of muscular tonicity, coordination and equilibrium. Scattered among the vestibolu-spinal fibers in the posterior part of the anterior descending cerebello-spinal tract are descending fibers from the thalamus and quadrigeminal colliculi which end in the gray crescent.

\section{Ascending Anterior Cerebello-spinal and Spino-thalamic} Tract. (Fasciculus ascendens cerebello-spinalis anterior, Gowersi).These form a single tract in the cord which is found chiefly in the postero-lateral part of the area common to it and the descending anterior cerebello-spinal tract (Figs. I02 and 103). It is composed 
of axonic processes of cells whose bodies are situated in the center of the crescent and base of the anterior columna, chiefly on the opposite side of the cord (v. Lenhossek). Most of the fibers of the ascending anterior cerebello-spinal and spino-thalamic tract cross near their origin through the white anterior commissure of the cord. In the medulla, the tract ascends through the dorsal part of the lateral area, sending collaterals to the inferior lateral nucleus; it then continues, through the formatio reticularis of the pons, to a point near the inferior quadrigeminal colliculus, where the cerebellar part is bent backward under the brachium conjunctivum, and enters the vermis cerebelli superior through the superior medullary velum (Hoche). The spino-thalamic portion continues to the thalamus, sending some fibers to the quadrigeminal colliculi (Mott) and others to the substantia nigra and lentiform nucleus (Rossolimo). The anterior ascending cerebello-spinal and spino-thalamic tract carries tactile, pain, and temperature impulses. The triangular tract of Helwig (fasciculus olivaris) is found on the surface near the middle of the area common to the anterior cerebello-spinal tracts (Figs. I02 and I03). It descends just lateral to the anterior nerve roots and may be traced to the lumbar region, where its longest fibers end. It probably rises in the olive of the medulla oblongata and is efferent in conduction.

The (posterior) cerebello-spinal tract (direct cerebellar) (fasciculus cerebello-spinalis [posterior]) runs posterior to the other cerebello-spinal tracts (Fig. IO2). It is superficially located, and in section extends from the middle of the lateral surface of the cord back to the posterior lateral sulcus as far down as the lumbar cord. Below the second lumbar segment its absence allows the lateral pyramidal tract to come to the surface. The (posterior) cerebello-spinal tract runs from the dorsal nucleus (Clarki) of the cord to the superior worm of the cerebellum. Its fibers are axones of vesicular cells in that nucleus. In the medulla, it forms a part of the restiform body. It conveys impulses of the muscular sense, received, especially, from the viscera (?). In the dorso-lateral part of the cerebello-spinal tract is a small strand of fibers discovered by Horsley and Thiele in I901, called the spino-vestibular tract. It rises in the lumbo-sacral region 
of the cord and, ascending along the surface of the cerebello-spinal tract to the medulla, it winds inward dorsal to the restiform body and terminates in the nucleus of the descending root of the vestibular nerve.

The lateral pyramidal tract (fasciculus cerebro-spinalis lateralis) forms a considerable part of the lateral column of the spinal cord (Figs. I02 and I03). It is covered, superficially, by the cerebello-spinal tracts in the cervical and thoracic cord; but in the lumbar and sacral cord it forms part of the surface. Its deep surface is in relation with the posterior columna of gray matter, the lateral fasciculus proprius and the marginal bundle. The fibers composing it are axones of cell-bodies in the anterior central gyrus of the cerebral cortex. They rise with those of the anterior pyramidal tract, and the two run as one tract down through the genu and anterior two-thirds of the occipital part of the internal capsule, the middle three-fifths of the basis pedunculi, the anterior longitudinal fibers of the pons and the pyramid of the medulla. In the medulla the two tracts separate. The lateral tract, comprising four-fifths of the pyramid, decussates with its fellow through the anterior median fissure, pierces the anterior gray columna and descends with some uncrossed fibers in the lateral column of the cord. It terminates in relation with the cell-bodies within the posterior columna, according to Schäfer, Collier and others. The anterior tract follows the anterior median fissure as already described. Both end chiefly in the gray crescent opposite to their cortical origin. According to Marchi, ten or twenty per cent. of the fibers remain uncrossed. The pyramidal tracts are the cerebral motor tracts. By them motor and inhibitory impulses are carried to the cord. In the outer part of the lateral pyramidal area is found the crossed descending tract of the red nucleus, the rubro-spinal tract (Pawlow).

The Rubro-spinal Tract (Fig. IO2).--It extends as far as the first lumbar segment and ends in the center of the gray crescent. Its origin in the nucleus ruber, its crossing through the ventral tegmental decussation (Foreli) and its course down the brain stem have been described. The rubro-spinal tract is descending in direction. 
Lesions.-The pyramidal tracts (especially the lateral) are involved in lateral sclerosis and in amyotrophic lateral sclerosis; and, as a consequence of it, both voluntary and inhibitory impulses from the brain are interfered with, hence the spastic paralysis and exaggerated reflexes. The pyramidal tract may be more or less involved in insular sclerosis and in bulbar paralysis, and the symptoms vary with the amount of sclerosis. Gliosis of the pyramidal, cerebello-spinal and posterior tracts (Dejerine and Letulle) has been demonstrated in Friedreich's hereditary ataxia, and the involvement of the pyramidal tracts explains the spastic paralysis which affects both arms and legs. In ataxic paraplegia (Gowersi) there is diffuse sclerosis of the lateral and posterior columns of the cord. It is the degeneration in the pyramidal tracts that causes the spastic gait, incoordinated arm movements and early increase of the reflexes, observed in that affection.

The marginal tract (fasciculus marginalis, Lissaueri) is a small tract composed of the ascending branches from the outer set of fibers in the posterior roots of the spinal nerves (Figs. IO2 and 103). It is situated in the lateral column on the outer surface and apex of the posterior columna. Its fibers are of small caliber and, after ascending a short distance, end about the cell-bodies of the substantia gelatinosa.

Tracts of the Posterior Column of the Cord (Fig. IOr).In the posterior column of the spinal cord, there are, first, two ascending and two descending tracts, derived from posterior nerve roots; second, the posterior fasciculus proprius, which is much scattered and is little understood; and, third, the entry zone occupied by the incoming fibers of the posterior roots.

Entry Zone (Figs. I02 and I03).-Over the apex and along the medial surface of the posterior columna of gray substance the posterior roots of the spinal nerves enter the cord and divide T-like into ascending and descending branches. The name entry zone is well applied to this region. The presence of horizontal fibers distinguishes the entry zone from the longitudinal tracts. The root-fibers of small caliber and many collaterals very soon enter the gray substance. The large fibers, in part, enter the dorsal nucleus, but the greater number form the longit- 
udinal tracts of the posterior column. The ascending fibers begin their upward course in the entry zone. As they ascend they are crowded toward the median septum by the entrance of new root-fibers in the succeeding upper segments. So the fibers near the septum are those which enter low down in the cord and those close to the posterior columna are of recent entrance into the cord. The descending T-branches and collaterals of the posterior roots begin their descent also in the entry zone. Some of them run a long course, even from the sixth cervical to the sacral segments (Collier). The greater number are much shorter. From above downward they are crowded in a dorso-medial direction, like the ascending fibers; and, after a considerable downward course, they plunge forward through the white column to end in the posterior columna. These ascending and descending fibers, whose origin is in the spinal ganglia on the posterior roots of the spinal nerves, together with the fibers of the posterior fasciculus proprius which rise in the posterior columna, constitute the longitudinal tracts of the posterior column of the spinal cord. That posterior column is undivided by any sulcus below the eighth thoracic segment, where the posterior intermediate furrow and septum fade away; but, above that level, it is subdivided into two distinct ascending tracts, a postero-medial, the fasciculus gracilis, and a postero-lateral, the fasciculus cuneatus (Fig. I02). These two tracts are alike in constitution. They have the same function, carrying impressions of the muscular and tactile senses. They differ only in length; the fibers of the fasciculus gracilis come from the spinal nerves below the eighth thoracic, while those of the fasciculus cuneatus come entirely from thoracic and cervical nerves.

Fasciculus Gracilis (Ascending Postero-medial Tract, Goll's Column).- This tract may be said to begin at the entrance of the posterior root of the coccygeal nerve (Figs. IO2 and I03). It ascends along the posterior median septum to the nucleus funiculi gracilis of the medulla. Up to the lower thoracic nerves it gradually enlarges, due to the acquisition of successive posterior rootfibers; and in this region, excepting only the entry zone and the descending branches of the posterior roots, it comprises the whole 
extrinsic part of the posterior column. Its size is uniform in the upper three-fourths of the cord, where the posterior intermediate furrow separates it from the fasciculus cuneatus. In depth it reaches to the posterior commissure. Its fibers are the ascending branches of the spinal ganglia cells. Arriving at the clava, all the fibers of the fasciculus gracilis arborize and end in the nucleus funiculi gracilis.

Fasciculus Cuneatus (Ascending Postero-lateral Tract, Burdach's Column).-Beginning in the lower thoracic segments (Fig. I02) the fasciculus cuneatus ascends between the entry zone and the fasciculus gracilis to the nucleus funiculi cuneati of the medulla. It acquires new fibers from every spinal nerve above its origin and grows stronger up to the first cervical nerve. In section it is wedge-shaped, being broadest at the surface; the edge of the wedge touches the junction of the posterior columna and posterior commissure. Its fibers are ascending, and are branches of the axones of spinal ganglia cells, like the fasciculus gracilis. In the nucleus funiculi cuneati all its fibers arborize and terminate (Collier).

Descending Tracts Derived from Posterior Roots.-The descending fibers from the posterior roots of the spinal nerves arrange themselves somewhat roughly into two tracts, a posterolateral and a postero-medial (Figs. I02 and I03). These descending radicular tracts greatly expand and multiply the terminal relations of the posterior root-fibers. Mingled with them, there are many fibers of the posterior fasciculus proprius.

The descending postero-medial tract (Figs. I02 and I03) has received various names at different levels. In the cervical and upper nine thoracic segments, it is the comma tract (of Schultze), situated in the fasciculus cuneatus. Its ventral part disappears in the posterior columna above the tenth segment, but the remainder continues down the cord. Shifting its position in a dorso-medial direction, it takes its place beside the median septum and gradually approaches the cord's posterior surface. It continues in that situation to the end of the cord, and is called, in succession, the oval tract (of Flechsig), the septo-marginal tract (of Bruce and Muir), and the median triangular tract 
(of Gambault and Phillipe). Fibers from the spinal nerves enter this postero-medial descending tract in the cervical, thoracic, and, at least, the upper lumbar segments. After a variable course within the tract, the fibers plunge forward into the posterior columna of gray substance where they terminate.

The descending postero-lateral tract (Fig. I03), situated at the posterior surface of the cord medial to the entry zone, in the lumbo-sacral region, appears to have been first described by Thiele and Horsley. It is very largely endogenous, but contains a number of root-fibers (Collier). In section it is triangular. Its fibers terminate in the posterior columna of gray substance. Both the descending radicular tracts are intermingled with endogenous fibers that belong to the fasciculus proprius.

Posterior Fasciculus Proprius. (Fasciculus posterior proprius).- This is made up of association fibers that connect different segments of the cord. It is not a compact strand. Its fibers are scattered throughout the posterior column, but they are especially numerous in the descending radicular tracts, in the region along the ventral one-third of the septum (the bandelette of Hoche) and in the cornu commissural field of Marie (Figs. IO2 and I03).

The cornu commissural tract (Fig. I03) is placed between the posterior columna (cornu), the posterior commissure and the posterior septum. It extends up to the eleventh thoracic segment and downward to the end of the cord. It contains both ascending and descending fibers as do other parts of the fasciculus proprius.

Lesions in the posterior columns cause disturbances of the muscular and tactile senses, and ataxia and incoordination result. If the entry zone is involved there is disturbance of all kinds of common sensation, at the level of the lesion. These columns are usually involved, by extension from the posterior roots, in locomotor ataxia (posterior sclerosis), hence the paræsthesia, crises, loss of reflexes, disturbed equilibrium and ataxic gait.

\section{ROOTS OF THE SPINAL NERVES.}

Thirty-one pairs of spinal nerves connect the cord with the periphery. Each nerve is joined to the cord by two roots: an ante- 
rior, efferent or motor root and a posterior, afferent or sensory root (Figs. I00 and 104). These roots descend more or less from their cord attachment to the inter-vertebral foramen in which they unite to form the spinal nerve. The roots of the first cervical nerve are horizontal; those of the first thoracic nerve descend the width of two vertebræ, and those of the twelfth thoracic, the width of four vertebræ; while the roots of the coccygeal nerve extend from the first lumbar vertebra to the second piece of the coccyx, through ten vertebræ.

Anterior Root.-In all spinal nerves, except the first, the anterior root (radix anterior) is smaller than the posterior. It is composed of from four to six fasciculi, which soon combine into two bundles. After piercing the dura mater, the anterior root unites with the posterior, beyond the latter's ganglion, and forms a spinal nerve. The anterior root is efferent, or motor, in function.

Apparent Origin (Fig. I04).-The anterior root is composed of medullated axones which issue from the narrow longitudinal area at the junction of the anterior one-fourth with the posterior three-fourths of the cord's surface. This area is bounded laterally by the anterior root-line, commonly called the anterior lateral sulcus.

Real Origin (Fig. 104).- These medullated axones rise from the medial, lateral, and intermedio-lateral columns of cell-bodies on the same side of the cord and from the medial column of the opposite side. These cell-bodies of the anterior columnæ and the intermedio-lateral column constitute the genetic nuclei (nuclei origines) of the spinal nerves. The fibers of large caliber in the anterior roots rise from the cell-bodies in the anterior columnæ. They are voluntary motor fibers. In the intermedio-lateral column, the small fibers of the anterior roots take their origin. They are probably sympathetic in function, that is, involuntary motor, vaso-motor, viscero-motor, inhibitory, secretory, trophic, inhibitosecretory, and inhibito-trophic.

Lesions.-The lower motor neurones (spinal and cerebral) are probably in a state of toxic irritation in laryngismus stridulus, tetanus, acute ascending paralysis (Landry), strychnine poisoning, 
etc., hence the twitchings, spasms and convulsions. Their destruction causes flaccid paralysis (lower segment paralysis). In spinal meningitis both the anterior and posterior roots are affected.

The posterior root (radix posterior) is the sensory, or afferent root (Figs. Ioo and I04). It is larger than the anterior root, except in the case of the first cervical nerve; and is composed of from six to eight fasciculi, which also combine at once into two bundles. The posterior root pierces the dura mater separately from the anterior root. It unites with the anterior root in the intervertebral foramen. Near the outer end, it presents a swelling which contains large vesicular unipolar cell-bodies and is called a spinal ganglion (ganglion spinale). The ganglion (Figs. 100 and I28) and posterior root are occasionally absent on the first nerve. The posterior root, external to the ganglion, is made up of the dendritic processes (Cajal) of the ganglion cells. These dendrites, which in appearance are axones, extend to the most distant parts of the body; they are the sensory fibers of the spinal nerves. On the proximal side of the ganglion, the posterior root is composed of axones, which rise from the ganglion cells. Both the axonic and dendritic processes are medullated.

A pparent Central Termination (Fig. I04).-The posterior roots of the spinal nerves enter the posterior-lateral sulcus; and, at once, divide into an outer set of small fibers and an inner set of large fibers with some small ones interspersed. The fibers of each set bifurcate into a large ascending and a smaller descending branch. Collaterals rise from the parent axone and from both branches.

Real Central Termination, Terminal Nuclei (Fig. 104).-The ascending divisions of the outer set of fibers run a short distance along the external surface of the posterior columna, and end in ramifications about the cell-bodies of the substantia gelatinosa. They form the marginal tract (of Lissauer). Probably their descending branches have the same ending. The T-branches and collaterals of the inner set of fibers from the posterior root run: (I) To the gray matter of the cord, viz.: To all parts of the posterior columna, to the center of the crescent, and to the anterior columna on the same side, and, through the posterior commis- 
sure, to the center and columnæ of the opposite crescent. These fibers end in relation with the dendrites or cell-bodies situated in those several regions of the crescent at various levels: (a) At the same level as the nerve, (b) at a lower level than the nerve, through the descending radicular tracts, and (c) at a higher level, through the collaterals given off by the ascending tracts. (2) The large ascending $\mathrm{T}$-branches of the inner set of fibers run to the medulla oblongata. They form the fasciculus gracilis and fasciculus cuneatus and ascend to the nuclei of those columns in the medulla oblongata. They terminate in the nuclei funiculi gracilis and cuneati. Hence the terminal nuclei (nn. terminales) of any spinal nerve are composed of the gray crescents of the cord, chiefly at the same level, and of the mucleus funiculi gracilis or the mucleus funiculi cuneati in the medulla.

Physiologically, we may divide the fibers of the posterior roots of the spinal nerves into four groups, viz.: I. Excito-reflex fibers which terminate in all parts of both crescents. 2. Fibers bearing impulses of the muscular and tactile senses from muscles, tendons, joints and skin, through the posterior column, to the nucleus funiculi gracilis and nucleus funiculi cuneati. 3. Fibers carrying impulses of the muscular sense from the viscera to the dorsal nucleus. 4. Fibers transmitting pain, temperature and tactile impulses to that part of the same and opposite crescent whence the spino-thalamic and ascending anterior cerebello-spinal tracts take their origin.

Lesions.- The posterior roots of the spinal nerves and the spinal ganglia are affected in locomotor ataxia, and the lesion extends to the marginal tract (of Lissauer) and the posterior column of the cord. Excepting the fasciculus proprius, the whole posterior column becomes involved. 


\section{CHAPTER VII. \\ TRACING OF IMPULSES.}

Having studied the grouping and chaining together of neurones, let us now make the knowledge practical by tracing impulses through the better known paths formed by these various neurone groups. The paths thus formed are of three kinds, namely: I. Efferent, or motor. II. Afferent, or sensory - general and special sense. III. Reflex.

\section{EFFERENT, OR MOTOR PATHS.}

The CEREBRO-SPINAL OR PYRAMIDAL PATHS (Fig. I05) are direct, as they do not pass through the cerebellum. Their impulses ultimately run either through the spinal or the cerebral nerves, and are both motor and inhibitory. Hence the increased reflexes and spastic contractions of lateral sclerosis in which these tracts are diseased.

I. Through the Spinal Nerves (Fig. 105).-Starting in the upper three-fourths of the gyrus centralis anterior of the cerebral cortex, motor and inhibitory impulses run down through the corona radiata, the anterior two-thirds of the occipital part of the internal capsule, the middle three-fifths of the basis pedunculi, the anterior longitudinal fibers of the pons, and the pyramid of the medulla oblongata, whence they proceed by the lateral and anterior pyramidal tracts to the gray crescent, partly in the same side but chiefly in the opposite side of the spinal cord. By the former route, the impulses cross over in the medulla, through the decussation of the pyramids, and descend in the lateral column of the spinal cord to the gray substance in the vicinity of the nucleus dorsalis (Clarki), where the path is relayed, and intrinsic neurones carry the impulses forward into the anterior columna; but by the anterior route, they descend in the anterior column of the cord and decussate, in succession, through the white anterior com- 


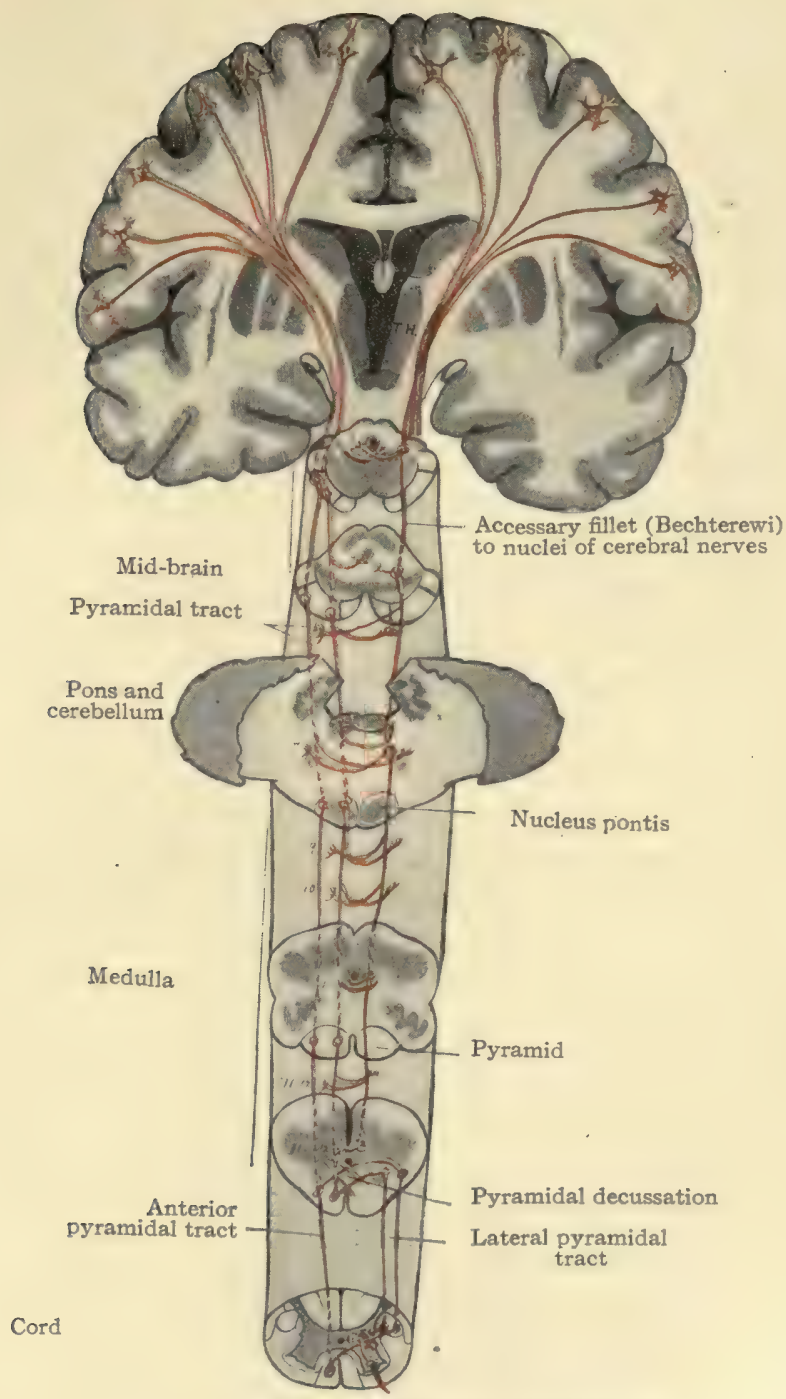

Anterior root of spinal nerve

Fig. 105.-Direct motor paths from cerebral cortex, to cerebral and spinal nerves. Diagrammatic. (Original.)

Motor paths extending from the cortex of the anterior central gyrus to the nuclei of the motor cerebral nerves and of the anterior roots of the spinal nerves; 0 . represents point where the section is pierced by a longitudinal fiber; $3,4,5,6,7,9,10$, II, and I2, nuclei of cerebral nerves. 

missure. Impulses by either route finally reach the anterior gray columna of the spinal cord and, with the exception of a small per cent. of them, they reach the columna opposite to their cortical origin. The few undecussated fibers in the lateral pyramidal tract conduct uncrossed impulses to the anterior columna of the same side. Thus are explained two symptoms of hemiplegia due to cerebral lesion, viz., weakness on the well side and slight motion on the paralyzed side. From the anterior gray columna of the spinal cord the nerve commotions are conducted by the efferent, or motor fibers of the spinal nerves to the muscles.

2. Through the Cerebral Nerves (Fig. I05).-Impulses destined to the cerebral nerves run chiefly from the lower two-fourths of the anterior central gyrus through corona radiata, genu of internal capsule and on, by the same path as the impulses to spinal nerves, down to the point where they leave the pyramidal tract to enter the nuclei of the cerebral nerves, which some of them do in the vicinity of the several nuclei. According to Bechterew they run, at least in part, through pyramidal fibers which constitute the accessory lemniscus. These fibers leave the pyramidal tract near the internal capsule, and descend through the medial portion of the fillet to points near the respective nuclei in which they end by multiple division. From either hemisphere impulses proceed to the nuclei of both sides. But the greater number enter the nucleus of the fourth nerve on the same side, and the nuclei of the third, fifth, sixth, seventh, ninth, tenth, eleventh and twelfth cerebral nerves of the opposite side. By the above nine nerves innervation is conducted to the muscles of the orbit; the muscles of mastication, and expression; the muscles of the tongue, palate and ear; the digastric and styloid muscles; the muscles of the larynx, trachea and bronchi, and of the pharynx, esophagus, stomach, and the intestines down to the descending colon. And inhibitory impulses are carried to the heart; also vasodilator, secretory, trophic and inhibito-secretory impulses to alimentary glands, etc.

THE CEREBRO-PONTAL PATHS, FRONTAL, TEMPORAL AND INTERMEDIATE.-These paths are indirect, for spinal nerves, since they run through the cerebellum. 
Fronto-pontal (Fig. I06).-The impulses originate in the frontal region (?), and descend through corona radiata, the frontal part of the internal capsule, and the medial fifth of the basis pedunculi to the nucleus pontis. Temporo-pontal.-They rise in the temporal cortex and run through corona radiata, the occipital part and inferior lamina of the internal capsule, and the lateral fifth of the basis peduncli to the nucleus pontis. Both the fronto-pontal and the temporo-pontal paths are probably relayed in the corpus striatum or the thalamus. Intermediate Bundle.-Cortical impulses of unknown origin àre received by the corpus striatum and conveyed, by a bundle of centrifugal axones which form the deep portion of the basis pedunculi, to the substantia nigra and then to the nucleus pontis, chiefly of the same side. The impulses thus traverse the internal capsule and a broad but thin area in the basis pedunculi just ventral to the substantia nigra and dorsal to the pyramidal tract. In all three of these paths the impulses run to the nucleus pontis of the same side and to motor nuclei of the cerebral nerves. Whence they proceed from nucleus pontis: (I) To Spinal Nerves. They run through the brachium pontis to the cerebellar cortex and, thence, continue down the anterior descending cerebello-spinal tract to the anterior gray columna of the spinal cord. Their course from the cerebellar cortex is through the acustico-cerebellar tract and the restiform body, the lateral area of the medulla and the anterolateral column of the cord. From the gray matter of the spinal cord the impulses are conveyed by the motor fibers of the spinal nerves to the muscles which they supply. (2) To Cerebral Nerves. The impulses run from the synapses formed in the cerebral nuclei, by the fibers of the cerebro-pontal tracts, through the motor fibers of these nerves to their distribution.

PATHS THROUGH THE RED NUCLEUS (Fig. IO6).There are two, a direct and an indirect. Impulses run from some part of the cerebral cortex to the thalamus and red nucleus or to the corpus striatum and red nucleus. From the red nucleus they pursue either a direct or indirect route.

(I) Direct Route, The Rubro-spinal Path.-By the direct route, impulses run through the crossed descending tract of the 


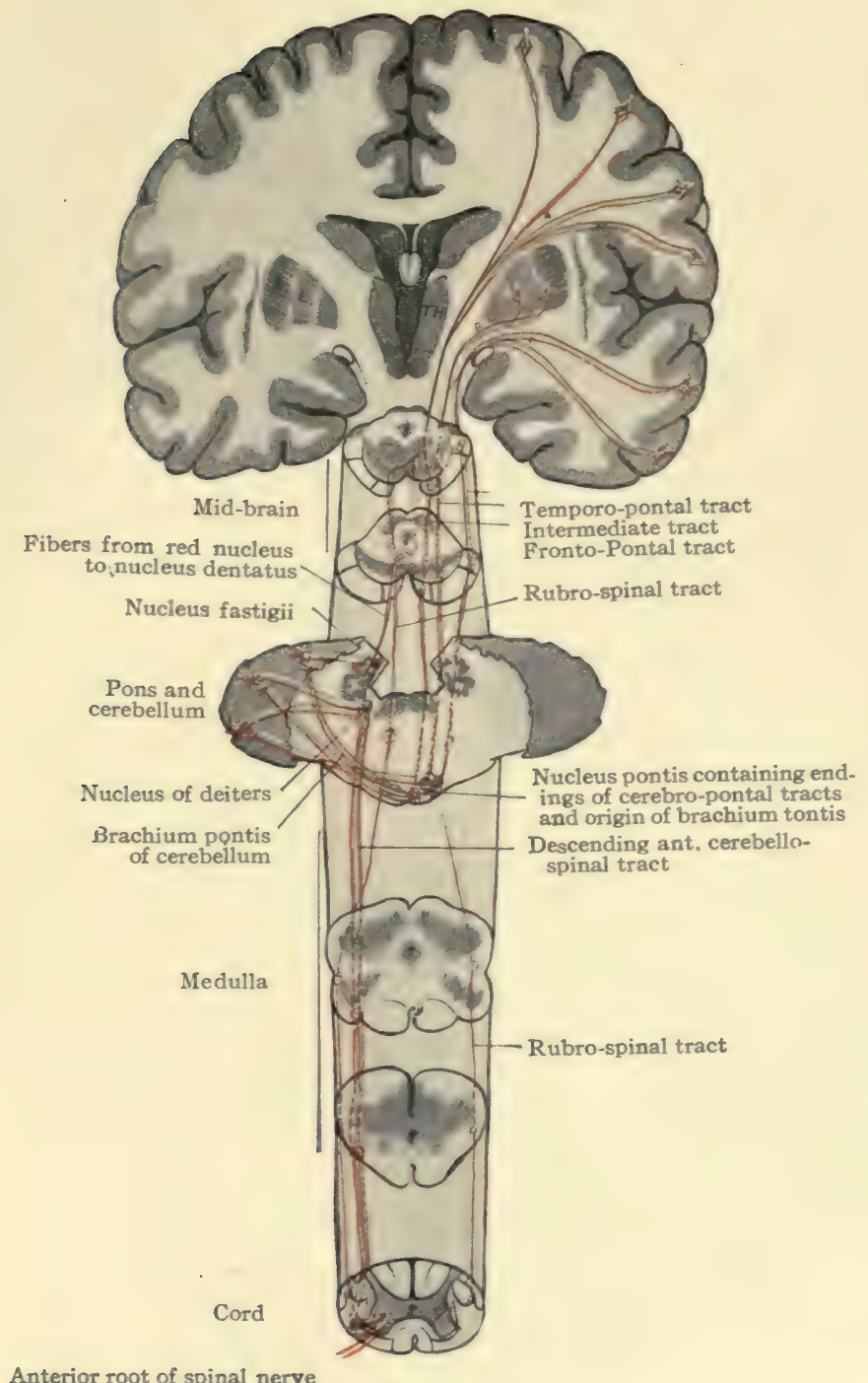

Anterior root of spinal nerve

Fig. 106.-Indirect motor paths to the spinal nerves. Diagrammatic. (Original.)

Motor paths from cerebral cortex through the cerebellum to spinal nerves. A. Frontopontal tract rising in frontal lobe. B. Intermediate tract rising in lentiform nucleus. C. Temporo-pontal tract rising in middle and inferior temporal gyri. Also rubro-spinal tract. - C represent points of perforation in the sections. 

red nucleus to the center of the gray crescent in the opposite side of the spinal cord. Crossing the median raphe at once, in the hypothalamic region through the ventral tegmental decussation, (Foreli), the impulses descend by way of the rubro-spinal tract, through the ventral part of the formatio reticularis of the midbrain and pons, in the medial part of the lateral fillet, then through the lateral area of the medulla, among the fibers of the anterior ascending cerebello-spinal tract, and finally down the spinal cord, through the ventral portion of the lateral pyramidal area, to their destination in the gray matter. From the center of the gray crescent they proceed with or without transferring to the roots of the spinal nerves, and are conducted to the muscles.

(2) Indirect Route.-Impulses from the red nucleus may also run through the brachium conjunctivum, chiefly the opposite one, to the nucleus dentatus; and thence, by axones from that nucleus, down the anterior descending cerebello-spinal tract, which continues their path to the columna anterior of the cord.

PONTO-SPINAL PATHS. - The ponto-spinal tract is the name suggested by Collier for the tracts originating in the nuclei of the reticular formation, chiefly in the pons, and descending to the gray matter of the spinal cord. There are two of them on either side. The medial ponto-spinal tract accompanies the medial longitudinal bundle down the anterior ground bundle of the cord without decussating, unless the crossing occurs near the termination. The lateral ponto-spinal tract, which is crossed, decussates in the brain stem near its origins and descends in the lateral column of the spinal cord. Just how the impulses reach the nuclei centrales and nuclei laterales of the formatio reticularis, in which the ponto-spinal tracts take their origins, cannot be definitely stated; but, having arrived in them, they descend to both crescents of the spinal cord and apparently enter into all its segments. The anterior nerve roots complete the paths.

Short Fiber Paths. - Those are paths in the formatio reticularis chiefly. (I) Impulses having reached the great ganglia of the cerebrum and mid-brain may run through many relays down the formatio reticularis of mid-brain, pons and medulla and the antero-lateral fasciculus proprius of the spinal cord, to the gray 
crescent of the same, and continue through the anterior root-fibers to their destination. On the other hand, the impulses, leaving formatio reticularis in mid-brain, pons or medulla, may enter the nuclei of motor cerebral nerves and be conducted by them to the muscles and glands supplied by cerebral nerves. (2) The impulses may leave the formatio reticularis in the pons, and run to the cerebellar cortex through the brachium pontis. From the cerebellum they may follow the ordinary course through the anterior descending cerebello-spinal tract, by way of the nucleus of Deiters, to the anterior gray columna of the cord. (3) Impulses having arrived at the cerebellar cortex by way of the brachia conjunctiva and brachia pontis, may descend through the cerebello-olivary tract, in the restiform body, to the opposite inferior olive, and be carried on through descending axones of the olive, in the lateral column, to the gray matter of the cord (Kölliker). (4) Impulses descend from the nucleus of the third cerebral nerve by way of certain fibers in the medial longitudinal bundle to the seventh nerve, where it enters into the colliculus facialis, and through the facial nerve innervate the following muscles-the frontalis, procerus, corrugator and orbicularis oculi. Hence these muscles are not paralyzed in nuclear facial paralysis. But if the lesion be in the colliculus fascialis or distal to it, then facial paralysis is complete. (5) At the same eminence, impulses pass into the facial nerve from the hypoglossal nucleus via a small strand of axones which rises in the hypoglossal nucleus and runs through the medial longitudinal bundle into the facial at the genu (?). These impulses innervate the orbicularis oris. (6) Through certain short fibers in the medial longitudinal bundle which rise in the nucleus of the sixth cerebral nerve and cross to the opposite nucleus of the motor oculi, impulses run from the nucleus of the abducent through the opposite third nerve to the internal rectus oculi. They explain the conjugate action of the eyes in health, and also the conjugate deviation observed in lesions affecting the nucleus of the sixth nerve. In nuclear lesions of the abducent nerve the external rectus of the same eye and the internal rectus of the other eye are paralyzed if the nucleus is destroyed and stimulated if the nucleus is only irritated. 


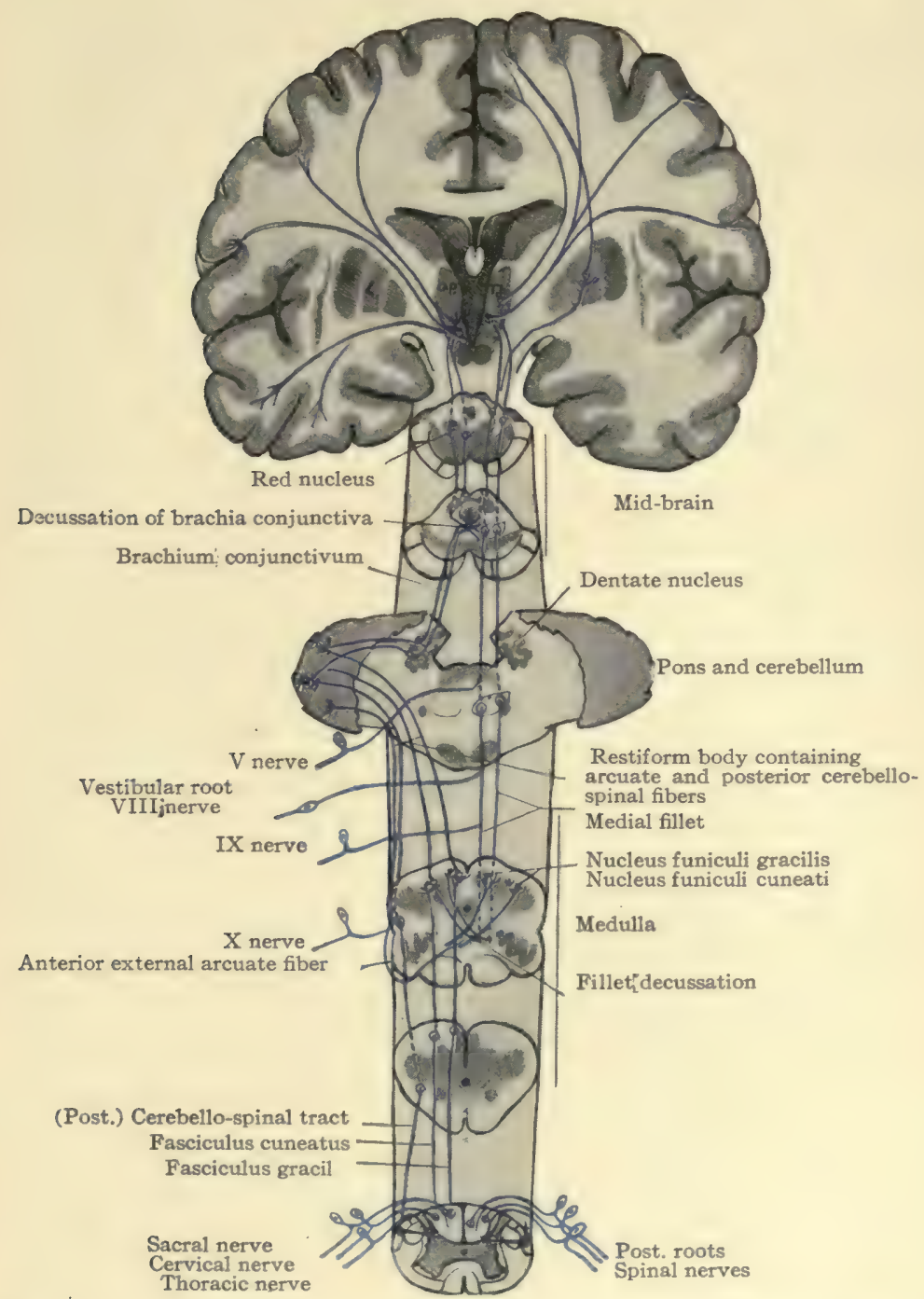

Fig. I07.-Common sensory paths, muscular and tactile, by way of the posterior column and (posterior) cerebello-spinal tract. Diagrammatic. "(Original.)

These paths terminate in the posterior central gyrus and in the middle and inferior temporal gyri. 



\section{AFFERENT, OR SENSORY PATHS.}

The sensory paths conduct two varieties of impulses, viz., general and special. The impulses originate in the end-organs of the cerebral and spinal nerves, and by those nerves are conveyed to the cerebro-spinal axis, through which they reach the proper cortical area in the cerebrum.

\section{General Sensations.}

General sensation is the function of the sense of touch. This sense has four important subdivisions - the tactile sense, muscular sense, pain sense, and temperature sense. Stereognosis is only an associated interpretation of all the impulses of the sense of touch and not a subdivision of it. Tactile sensations appear to be most elemental and, according to Spiller and Mills, may be conducted by all common sensory nerve fibers. Other common sensations seem to require some specialization, as yet not understood, in their conducting media; and pain and temperature impulses pursue a path entirely distinct from that followed by impressions of the muscular sense. In giving the common sensory tracings, the following classification will be adhered to, though conclusive evidence of certain points in it is still lacking.

I. Paths conducting impulses of the muscular and tactile senses, chiefly, from muscles, tendons, joint surfaces, and the skin. Spinal and cerebral (Fig. I07).

II. Paths carrying impulses of the muscular and tactile senses, chiefly from viscera (?) (Fig. I07).

III. Paths conveying pain, temperature, and tactile impulses. Spinal and cerebral (Fig. I08).

\section{PATHS TRANSMITTING IMPULSES OF THE MUS-} CULAR AND TACTILE SENSES, chiefly, from muscles, tendons, ligaments, joint surfaces and the skin.

Through Posterior Column and Fasciculi Gracilis et $\mathrm{Cu}-$ neatus (Fig. I07).- Impulses originating in the end-organs of the spinal nerves traverse the dendrites of the spinal ganglion neurones (Cajal), the cell-bodies in the ganglia, and then the axones of the same. They enter the cord through the posterior roots of the 
spinal nerves, and ascend through the posterior column as far as the eighth thoracic segment and then through the fasciculus gracilis, or, entering above the eighth thoracic segment, they ascend through the fasciculus cuneatus. In either case they arrive in one of the nuclei of the posterior column, namely, the nucleus funiculi gracilis or the nucleus funiculi cuneati. Thence the impulses may proceed either by a direct or by an indirect route.

(I) The direct route carries the impulses by way of the medial fillet through the sensory decussation of the medulla, the formatio reticularis of pons and mid-brain, to the lateral nucleus of the thalamus, from which they are conducted by the cortical fillet to the somæsthetic area of the cerebral cortex. In their last stage the impulses run from the thalamus through the internal capsule and corona radiata to the posterior central gyrus in the equatorial zone of the hemisphere.

(2) Indirect Route.-By that route impulses from the nucleus funiculi gracilis and nucleus funiculi cuneati run to the cortex of the vermis cerebelli superior through the external arcuate fibers; then on, through the brachium conjunctivum, to the red nucleus and thalamus. They traverse the restiform body of the same side, by way of the posterior external arcuate fibers; or, by way of the anterior external arcuate fibers, they traverse the fillet decussation of the medulla and the opposite restiform body to reach the vermis cerebelli superior. From the cerebellar cortex, the impulses continue through cortical axones to the nucleus dentatus, whose axones conduct them to the red nucleus and thalamus of the opposite side. The greater number, therefore, cross over in the tegmentum of the mid-brain. Their course from the red nucleus and thalamus is through the cortical fillet to the cortex.

These impulses from the spinal nerves go to the upper twothirds of the posterior central gyrus, those from the lower extremity to the upper third and those from the arms to the middle third (Spiller).

Through Cerebral Nerves and Medial Fillet (Fig. I07).As crossed fibers from the terminal nuclei of the trigeminal, the vestibular, the glossopharyngeal and the vagus nerves join the 


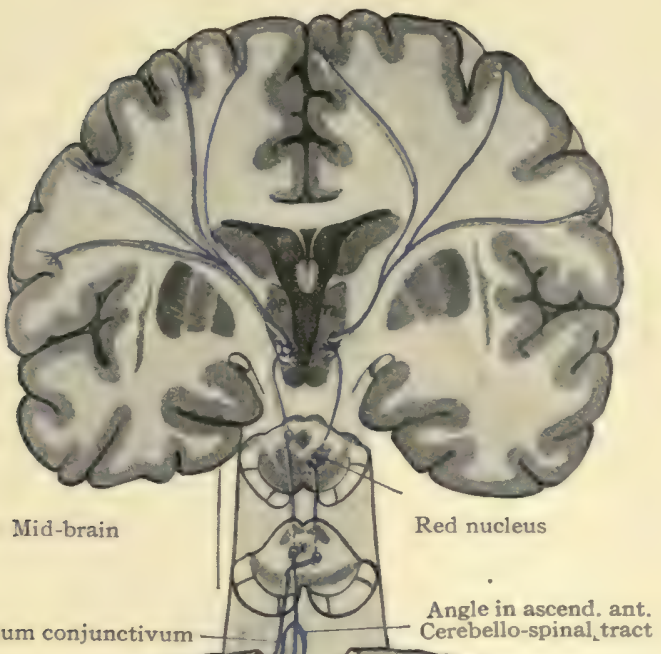

Pons and cerebellum

Tract from inferior lateral nucleus to cerebellum via the restiform body

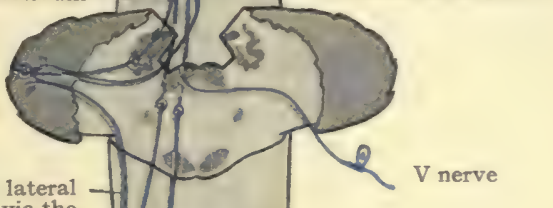

Gowers's $\left\{\begin{array}{l}\text { Spino-thalamic tract } \\ \text { Ascending ant. cere- }\end{array}\right.$

Tract $\} \begin{gathered}\text { Ascending ant. cere- } \\ \text { bello-spinal tract }\end{gathered}$

Medulla

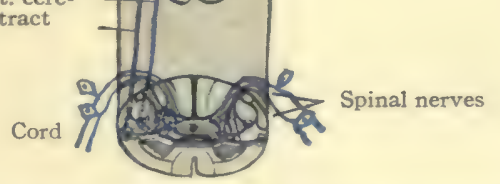

Fig. I08.-Common sensory paths, pain, temperature and touch, by way of ascending anterior cerebello-spinal and spino-thalamic tracts. Diagrammatic. (Original.)

Posterior root-fibers connected with this path end in the center of the crescent and in the base of the anterior columna of both sides; and the ascending fibers rise partly on the same and partly on the opposite side; the crossed fibers run through the white anterior commissure. 

medial fillet and run to the thalamus, so muscular and tactile sensations transmitted by those cerebral nerves to their nuclei in the medulla and pons, are carried by the medial fillet to the lateral nucleus of the thalamus on the opposite side. The cortical fillet conducts them to the lower portion of the posterior central gyrus in the somæsthetic area.

II. PATHS CARRYING IMPULSES OF THE MUSCULAR AND TACTILE SENSES, CHIEFLY FROM VISCERA (?).

Through (Posterior) Cerebello-spinal Tract (Direct Cerebellar) (Fig. I07).-The column of cell-bodies, forming the nucleus dorsalis (Clarki), receives impulses concerning equilibrium (?) from the posterior roots of the spinal nerves and transmits them at once to the (posterior) cerebello-spinal tract, through which they ascend along the dorso-lateral surface of the cord, along the posterior surface and through the restiform body of the medulla to the cortex of the superior worm of the cerebellum. To a small extent they cross in the worm to the opposite side. These impulses probably excite, within the cerebellar cortex, impulses of coordination and equilibrium which traverse the descending anterior cerebello-spinal tract to the gray crescent of the cord, and are transmitted to the motor neurones of the anterior columna. The motor neurones conduct the impulses to the muscles. From the cerebellar cortex the journey to the cerebrum is completed in four stages, as already described, namely: Nucleus dentatus, opposite red nucleus, thalamus and some part of the cerebral cortex, probably the middle and inferior temporal gyri.

Probably the vagus nerve conducts impulses of similar nature from the viscera to its terminal nucleus; but, if so, their path from the terminal nucleus is not certainly known.

\section{PATHS CONVEYING PAIN, TEMPERATURE AND TAC- TILE IMPRESSIONS. SPINAL AND CEREBRAL.}

Through Spino-thalamic and Ascending Anterior Cerebello-spinal Tract (Fig. I08).-In the spinal cord, medulla and pons these constitute one tract, commonly called Gowers's tract. They separate just. below the isthmus, whence the spino-thalamic tract continues to the thalamus and the other turns back to the cerebellum. They appear to form the only paths for pain and 
temperature impulses. These impulses enter the gray crescent of the cord, on both sides, through the posterior nerve roots. A large number decussate via the posterior nerve roots in the gray commissure; the rest decussate in the first stage of the ascending tracts, crossing in the white anterior commissure, and run upward through the spino-thalamic and ascending anterior cerebellospinal tracts of the opposite side; they run to the thalamus and to the cortex of the superior worm of the cerebellum. In the cord they ascend along the lateral surface. They run dorsal to the olive in the lateral area of the medulla oblongata, and through the lateral part of the formatio reticularis of the pons to the angle in Gowers's tract situated near the isthmus. From the angle, just below the quadrigeminal bodies, the cerebellar impulses run backward with the tract through the superior medullary velum to the cortex of the vermis cerebelli superior; the remainder run upward to the thalamus, and from that to the posterior central cortex. The common course of sensory impulses from the cerebellar to the cerebral cortex is, as already described, through nucleus dentatus and brachium conjunctivum to opposite red nucleus and thalamus. Having arrived in the thalamus, they proceed thence by the cortical fillet to the somæsthetic cortex.

Certain fibers of the ascending anterior cerebello-spinal tract diverge from the others, in the medulla oblongata, and terminate in the inferior lateral nucleus. Impulses of pain and temperature, following the same course, enter the lateral nucleus, and are carried on through the restiform body to the cerebellum by the tract from the lateral nucleus to the cerebellar cortex, thence to the somæesthetic area as previously given.

\section{Through Cerebral Nerves and the Spino-thalamic Tract} (Fig. 108).--Pain and temperature impulses are transmitted by certain fibers of the vagus, glossopharyngeal and trigeminal nerves to their terminal nuclei. From those nuclei they are conducted by axones which probably enter into the spino-thalamic tract, and, perhaps, into the ascending anterior cerebello-spinal tract, to the thalamus and to the cerebellar cortex. The path from either point to the posterior central gyrus is now familiar.

The Short Fiber Paths.-What special varieties of common 
sensation are conducted through these paths is unknown. Under certain conditions, perhaps, they may carry all varieties. (I) The antero-lateral fasciculus proprius and formatio reticularis contain ascending axones which may convey sensory impulses from the gray matter of the cord, received from the posterior roots of the spinal nerves, and from terminal nuclei in medulla and pons which receive the common sensory fibers of cerebral nerves, upward to the thalamus of the opposite side. The course from the thalamus is by way of the cortical fillet. (2) Again, impulses may leave the formatio reticularis in the pons and run through the brachium pontis cerebelli, by way of axones from the nuclei pontis, to the cortex of the cerebellum; and then continue by the ordinary course through the brachium conjunctivum to the red nucleus and thalamus.

Destruction of any of the above sensory paths causes diminution or loss of the especial variety of impulse which travels that path. Destruction of the posterior white columns produces loss of muscular sensations and gives rise to ataxia. Interruption of Gowers's tract (spino-thalamic and ascending anterior cerebello-spinal tracts) abolishes pain and temperature sensations while touch is not much affected.

\section{Special Sensations.}

Impulses producing the sensations of smell, sight, hearing and taste are carried from the respective organs of sense to the brain by the following nerves: The olfactory; the optic; the auditory; and the glossopharyngeal and intermediate nerves.

Olfactory Path (Figs. IO9 and 2I). - Impulses of smell originate in the upper third of the nasal mucous membrane. They run through the olfactory nerves to the second layer in the bulb, where they are transferred to the dendrites of the mitral and brush cells. By the axones of these cells they are carried backward through the olfactory tract and strixe to the cerebral hemisphere. The medial stria conducts them to the parolfactory area (Brocae), the triangle, the gyrus subcallosus and anterior end of the gyrus cinguli, whence, through the cingulum, the fornix and uncinate fasciculus they may reach the cortical area of smell in the uncus 
hippocampi. By the intermediate olfactory stria the impulses enter the triangle and anterior perforated substance, and complete their journey either in the same manner as given above or via the anterior commissure. The lateral stria of the olfactory tract conducts the impulses directly to the uncus of the hippocam-

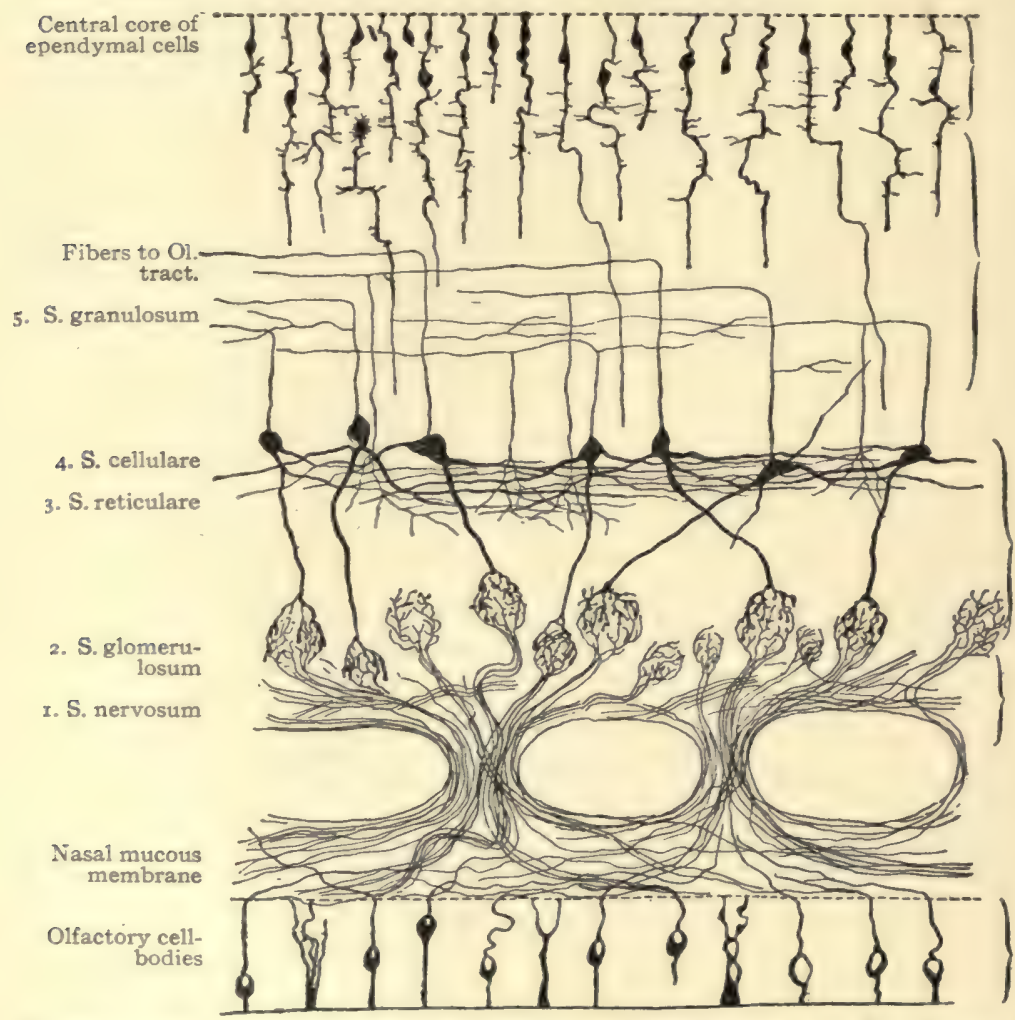

Fig. I09.-Chief elements of the olfactory bulb. (Gordinier after Van'Gehuchten.)

pal gyrus. Note that olfactory impulses are chiefly, if not entirely, uncrossed.

Optic Path (Figs. I Io and III).--Impulses of sight originate in the rods and cones of the retinæ and traverse three or more series of neurones to the terminal nuclei of the optic tracts; namely, the rod and cone, the bipolar, and the ganglionar neurones. The axones of the last form the optic nerves and the visual part 
of the optic tracts. From the right halves of both retinæ and from the left halves of both, impulses run through the corresponding tract to the lateral geniculate body and the pulvinar of the thalamus; also to the superior quadrigeminal colliculus. The latter produces ocular and pupillary reflexes. From the lateral geniculate body and pulvinar the occipito-thalamic radiation carries the impulses through the pars occipitalis of the internal capsule to the half-visual center in the cuneus, gyrus lingualis and the pole of the occipital lobe. Impulses from the nasal halves of the retinæ decussate in the optic commissure; those from the

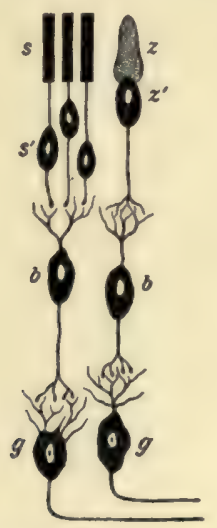

Fig. Iro.-The chief retinal elements. (After Brubaker.)

Cells. $s^{\prime} z^{\prime}$. Visual cells with their peripheral terminations. $s$. Rods. $z$. Cones. $b$. Bipolar cells. g. Ganglion cells from which arise the axones of the optic nerve.

temporal halves, for the most part at least, remain on the same side, but a few may cross through the quadrigeminal colliculi and brachia superiora. Impulses from the nasal half and from the temporal half of the macula lutea are conducted equally by both optic tracts. Hence destruction of one tract causes hemianopsia, preserving the vision in the corresponding half of each visual field, and also diminishes the acuteness of macular vision in both eyes.

Auditory Paths.-There are two auditory paths, cochlear and the vestibular. The former is concerned with hearing and the latter with equilibrium. 
I. The Cochlear Path (Figs. 89, 70 and 54).--Impulses of hearing originate in the organ of Corti. They are transmitted by the rods and hair cells of Corti to the dendrites of the spiral ganglion. Traversing the dendrites and cell-bodies of that ganglion, they enter the axones, which form the cochlear nerve, and run backward to the terminal nucleus of that nerve in the medulla. Both the ventral and the lateral portions of the cochlear nucleus receive the impulses of hearing. From the cochlear nucleus they run either lateral and dorsal to the restiform body and cross to the opposite side through the medullary striæ and trapezoid body, or they run medial to the restiform body and enter at once into the trapezoid body. By either course they reach the lateral fillet, and chiefly the opposite one. The lateral fillets conduct the impulses to the inferior quadrigeminal colliculi; the brachia inferiora to the medial geniculate bodies, and the temporo-thalamic radiations to the third and fourth fifths of the superior temporal and to the transverse temporal gyri of the cerebrum. Through the lateral fillet, impulses producing reflex reach the quadrigeminal colliculi and, thence, by the anterior longitudinal bundle, pass to motor nuclei; and, also, through the olivary pedicle and medial longitudinal bundle, they reach the nuclei of the sixth, fourth and third cerebral nerves.

2. Vestibular. Path.-The extent of the vestibular conduction path is from the acustic areas of the utricle, saccule and semicircular canals to the vestibular nuclei in the floor of the fourth ventricle; and, thence, to the cerebellum, and to the cortical area of equilibrium, according to Mills, in the temporal cortex. It is the path of space sense. Through the vestibular nerve the impulses reach the dorso-medial, the dorso-lateral and superior nucleus, and the nucleus of the descending root in the floor of the fourth ventricle.

The impulses may pursue, from the terminal nuclei in the ventricular floor, either a direct or an indirect course to the cerebral cortex.

(I) By the direct course they run through the opposite medial fillet and certain fibers in the cortical fillet, perhaps the ventral stalk of the thalamus, to the middle and inferior temporal gyri. 


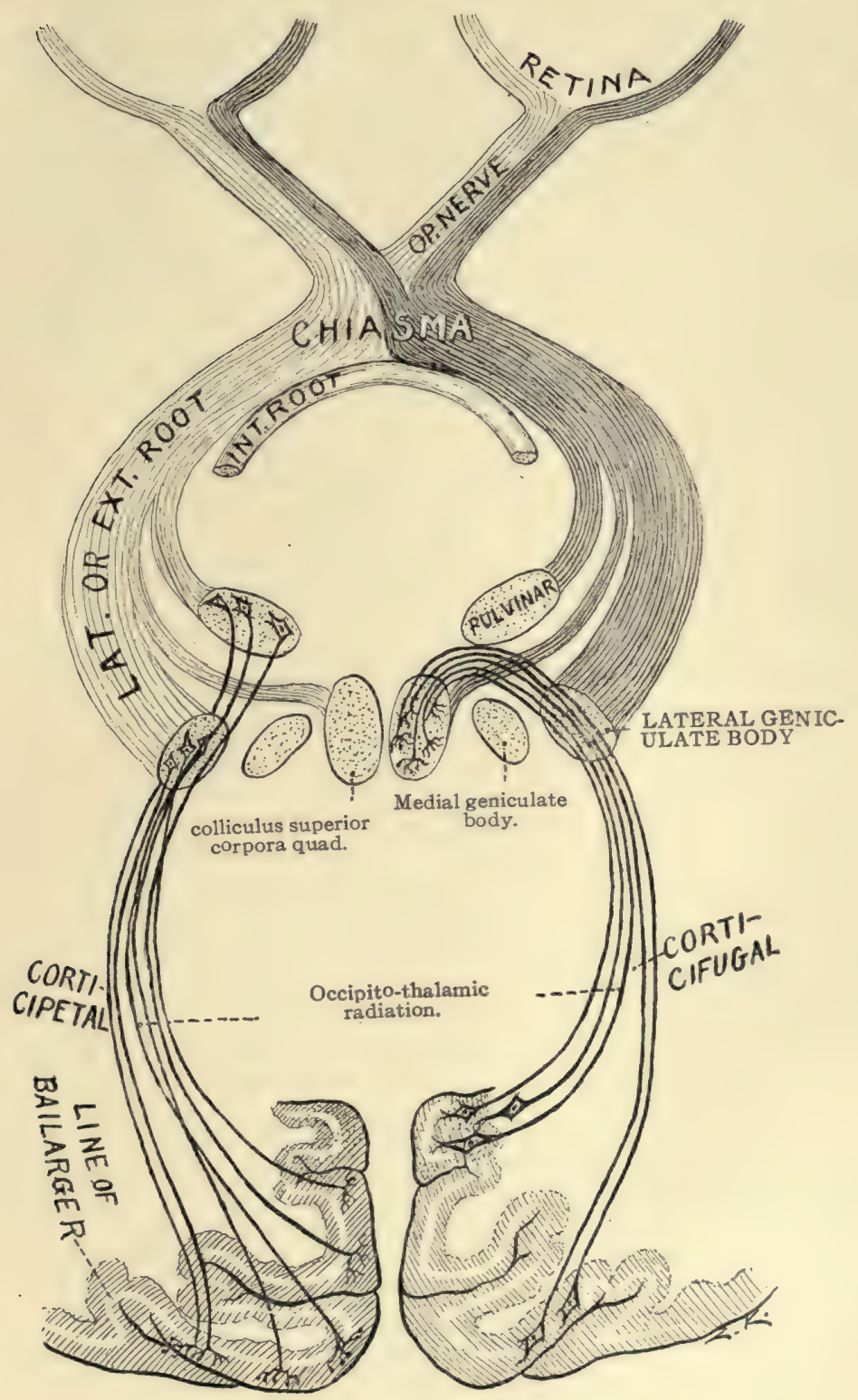

Fig. trx.-The optic path. (Original.) 

(2) The impulses run to the cerebellum, by the indirect course, through the descending root, and the external arcuate fibers in the restiform body. They thus reach the cerebellar cortex. They may excite in the cerebellum impulses of equilibrium and then continue upward. From the cerebellum the course of the impulses is, presumably, through the brachium conjunctivum to the red nucleus and thalamus of both sides and thence to the cortex.

Impulses, believed to be concerned with reflexes, run from the vestibular nuclei in the floor of the fourth ventricle, (a) to the opposite nuclei of motor cerebral nerves via the medial longitudinal bundle; (b) to the quadrigeminal colliculi through the superior fillet; (c) to the cerebellum by way of the descending root and arcuate fibers, whence they reach the spinal nerves through the descending anterior cerebello-spinal tract; (d) to the spinal cord and nerves through the axones of Deiters's nucleus; and, perhaps, less directly through the vestibulo-olivary and olivo-spinal fibers of Kölliker.

The Gustatory Paths.-They extend from the tongue to the nucleus tractus solitarii in the medulla and thence, probably, through the opposite formatio reticularis and internal capsule to the taste area in the gyrus fusiformis (Mills) or gyrus cinguli (Flechsig). There are two paths from the tongue to the nucleus of the solitary tract. Those impulses from the base of the tongue and the palate run through the ninth nerve and those from the anterior two-thirds of the tongue through the chorda tympani and intermediate nerve to the medulla (A. F. Dixon, Keen and Spiller, H. Cushing, etc.). Possibly, gustatory impulses originating in the palate may traverse the descending branches of Meckel's ganglion and the great superficial petrosal nerve to reach the geniculate ganglion on the facial; and, then, continue through the intermediate nerve to the solitary tract. All impulses arriving at this nucleus of the solitary tract probably complete their journey in two stages: First, through the formatio reticularis to the opposite thalamus; and, second, through internal capsule to the cortex.

Destruction of the olfactory conduction path on one side causes anosmia on the same side; of the optic tract or radiation, atrophy and destruction in the corresponding halves of both retinæ; 
of the auditory path above the pons, deafness chiefly on the opposite side; and interruption of the gustatory path above the medulla oblongata abolishes taste on the opposite (?) side.

\section{REFLEX PATHS.}

There is no visible limit to the number of reflex paths. Hence no attempt will be made to give them completely, but a few examples of various kinds will be given which may assist the student to trace others and be suggestive of their great multiplicity and importance. Under certain conditions, unquestionably, the sensory and motor paths that have been traced are but the afferent and efferent limbs of reflex arcs.

Reflex arcs are formed (I) by the sensory and motor fibers of spinal nerves, associated in the gray matter of the cord; (2) by the sensory and motor fibers of cerebral nerves, which are connected in the brain; (3) by afferent spinal fibers connected by the ascending fibers of the medial longitudinal bundle, with efferent cerebral fibers; and (4) by afferent cerebral and efferent spinal nerve fibers, the two being associated by the anterior longitudinal bundle, the ponto-spinal tracts, the fasciculi proprii, the spinal tract of the fifth nerve, the vestibulo-spinal tract, the solitary tract, etc.

(I) Spinal Reflexes (Figs. II2 and II3). - In the simplest spinal reflexes, the afferent fibers of the arc arborize about the cell-bodies whose axones constitute the efferent fibers; the afferent and efferent fibers are connected by one set of intervening neurones in the next grade of reflex arc. Among these are the skin and muscle reflexes, such as the plantar, the patellar, the gluteal and the cremaster reflexes, the involuntary withdrawing of a part from a source of irritation, etc.

Coordinating and equilibrating reflex impulses traverse much longer arcs. They are composed of the spinal ganglion neurones, the external arcuate fibers (and probably the [posterior] cerebellospinal tract) in the afferent limb, and the descending anterior cerebello-spinal tract and the anterior root neurones in the efferent limb of the reflex arc. 
More complicated spinal reflexes are those of defecation, micturition, parturition, vasomotor reflexes, cardio-accelerator reflexes, etc. The impulses traverse at least three neurones in these reflexes; because all efferent white rami communicantes terminate in some ganglion proximal to the organ supplied. As an example, trace a defecation reflex.

Defecation Reflex.-The rectum is supplied by the third and fourth sacral nerves and by branches of the inferior mesenteric and hypogastric plexuses. Irritation of the sensory endings in the mucous membrane is caused, normally, by the presence of feces. The impulses caused thereby run to the special defecation center in the lumbar enlargement of the spinal cord, either by

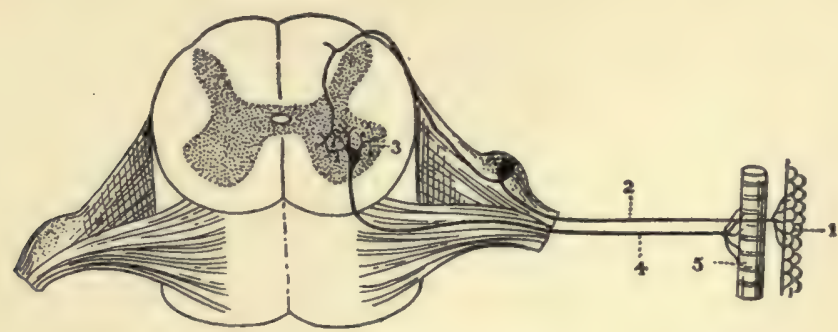

Fig. Ir2.-Diagram of a simple reflex arc. (After Brubaker.)

I. Sentient surface. 2. Afferent nerve. 3. Emissive or motor cell. 4. Efferent nerve. 5. Muscle.

way of the sacral nerves or through the sympathetic plexuses, the ganglionated cord, and the rami communicantes to the lumbar nerves, through the posterior roots of which they reach the center in the cord. From the defecation center the impulses pursue two courses: (a) They descend through the third and fourth sacral nerves and cause inhibition in the circular fibers of the rectum and contraction of the longitudinal muscle. (b) This action is immediately followed by impulses which pursue the sympathetic course, through the anterior roots of the lumbar nerves, the rami communicantes, the ganglionated cord, and the inferior mesenteric and hypogastric plexuses, to the rectum. They cause, in succession from above downward, contraction of the circular muscle of the rectum. The two series of impulses 
thus open a way for the passage of fecal matter; and, then, force it through the opening unless prevented by the voluntary contraction of the external sphincter.

(2) Cerebral Reflexes.-The simplest of these reflexes are such as spasm of the muscles of mastication caused by a bad tooth, in which both limbs of the arc are formed by the trigeminal nerve. Again, the facial expression of pain due to the same cause. In this the impulses traverse the trigeminal nerve and

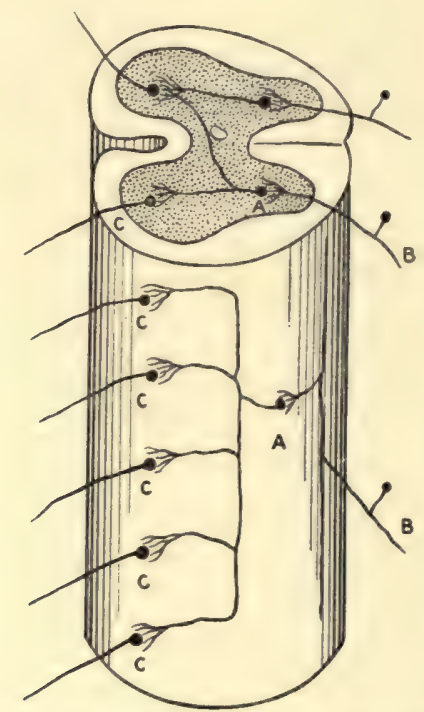

Fig. Ir 3.-A more complicated spinal reflex arc, involving the fasciculi proprii.

(Brubaker after Kölliker.)

Diagramishowing the relation of the third neurone $\mathbf{a}$, to the afferent neurone $\mathbf{b}$, and to the efferent neurones $\mathrm{c}$, $\mathrm{c}$, c.

by the collaterals of its root-fibers reach the nucleus of the facial. Through the facial they cause contraction of certain muscles of expression. Facial spasm in tic douloureux is due to the same reflex. The involuntary expansion of the nostrils upon the detection of a faint odor is due to an olfactory-facial reflex. The connection of the terminal nucleus and cortical center of the olfactory nerve with the genetic nucleus of the facial nerve is very much 
in doubt; it may be established in part (I) by the fornix, the thalamo-mammillary bundle and thalamo-spinal fibers, (2) by the fornix, medullary stria, fasciculus retroflexus and descending fibers of the interpeduncular ganglion, and, (3) by the fornix and the pedunculo-mamillary bundles. Squinting, due to bright light, is produced by an arc composed of the visual path, the corticifugal part of the occipito-thalamic radiation, the anterior longitudinal bundle and the facial nerve. Substitute the oculomotor nerve for the facial and we have the arc for pupillary contraction under the same conditions.

Salivary reflexes, in which the sight of a fine dinner or the smell of it causes the flow of saliva; coughing, sneezing, vomiting reflexes and deglutition reflexes are complicated, but, knowing the nerve supply of the parts involved, the student should try to trace the impulses.

(3) Spino-cerebral Reflexes.-Impulses received by the spinal cord through the afferent fibers of its nerves are transmitted by the medial longitudinal bundle, the fasciculi proprii and formatio reticularis to the nuclei of motor cerebral nerves. Thus is brought about the movement of the head and eyes toward the source of impulse, a change of facial expression to agree with the painful or pleasing character of the impulses, etc.

(4) Cerebro-spinal Reflexes.-Of these there are many. Let us notice three.

Respiratory Reflex.-Any obstruction or irritation in the larynx or trachea sends an impulse through the vagus nerve to its sensory nucleus and, through its descending branches, in the solitary tract, to the nucleus ambiguus and nucleus of the phrenic nerve in the cervical cord, causing increased respiratory efforts, coughing, spasm of muscles closing glottis, etc.

Equilibrium Reflex (Vestibulo-spinal Reflex).-The simplest arc of equilibrium between the eighth cerebral nerve and the spinal nerves is formed by the neurones of the vestibular ganglia (Scarpa's), the vestibulo-spinal part of the descending anterior cerebello-spinal tract and the motor neurones of the anterior columna of the spinal cord. A more complicated arc includes the descending root of the vestibular nerve, the external arcuate 
fibers from the nucleus funiculi cuneati and the descending anterior cerebello-spinal tract.

Pupillary Reflexes. - Pupillary dilatation belongs to the cerebrospinal group of reflexes. The cilio-spinal center is in the cervical enlargement of the spinal cord. It receives optic impulses through the anterior longitudinal bundle from the corpora quadrigemina. The superior quadrigeminal colliculi receive those impulses by two routes: First, directly, through the fibers of the lateral root of the optic tract, and, second, indirectly, through corticifugal fibers in the occipito- thalamic radiation, and the brachium superius. By the latter route, the optic impulses which have reached the visual area of the occipital lobe, by way of the intrinsic retinal ncurones and the optic nerves, tracts and radiation, are returned to the lateral geniculate and superior quadrigeminal bodies. Thence, reaching the cilio-spinal center through the anterior longitudinal bundle, the impulses take the following course: They leave the spinal cord through the anterior roots of the upper thoracic nerves and run, in succession, through the rami communicantes, the cervical cord of the sympathetic, the cavernous plexus, the ciliary ganglion and the short ciliary nerves to the radiating fibers of the iris, causing dilatation of the pupil.

For pupillary constriction, the impulses run directly from the superior quadrigeminal colliculus to the oculomotor nucleus, traversing the anterior longitudinal bundle only through the dorsal tegmental decussation (Meynerti). Then, through the third and short ciliary nerves, they reach the sphincter pupillæ muscle. 


\section{CHAPTER VIII.}

\section{EMBRYOLOGY OF THE BRAIN AND SPINAL CORD.}

The brain and spinal cord are developed from the neural tube; the spinal and sympathetic ganglia and the ganglia of sensory cerebral nerves are derivatives of the neural crest (Figs. I6 and II4).

Very soon after conception there appears along the median line, in the back of the embryo, a plate of epiblast, called the medullary plate. That plate soon presents two longitudinal elevations, the medullary ridges, separated by a median furrow, the neural groove (Fig. I6). At first the medullary plate is formed of a single layer of columnar cells; but those cells undergo rapid mitosis, as the medullary ridges are developed, and arrange themselves in several layers which thus bound the neural groove (Fig. II4). The infolding of the medullary ridges, together with their rapid growth, roofs over and closes in the neural groove and produces the neural tube (Fig. 16). The tube is first formed in the cervical region, whence the fusion of the medullary ridges extends in both'directions. By the approximation of the medullary ridges a slight paramedian crest is produced on either side, called the neural crest. The neural tube and the neural crest are nearly complete by the fifteenth day after conception, the groove is open only at the position of the future median aperture of Magendie. Both ends of the tube are open for a short time, the anterior closing first. The mesoblast which later grows around the neural tube is developed into the meninges and the surrounding bones, which inclose the cerebro-spinal axis.

The Neural Tube (Figs. I6 and II4).-It is well formed at the fifteenth day. The cephalic end of the tube is much larger than the caudal end, and presents at this time two constrictions that separate the primary brain vesicles from one anotherthe anterior, the middle, and the posterior. Behind the posterior 
primary vesicle, the neural tube remains small and of nearly uniform size; that part forms the spinal cord, in which the general plan of development may be observed in its simplest form.
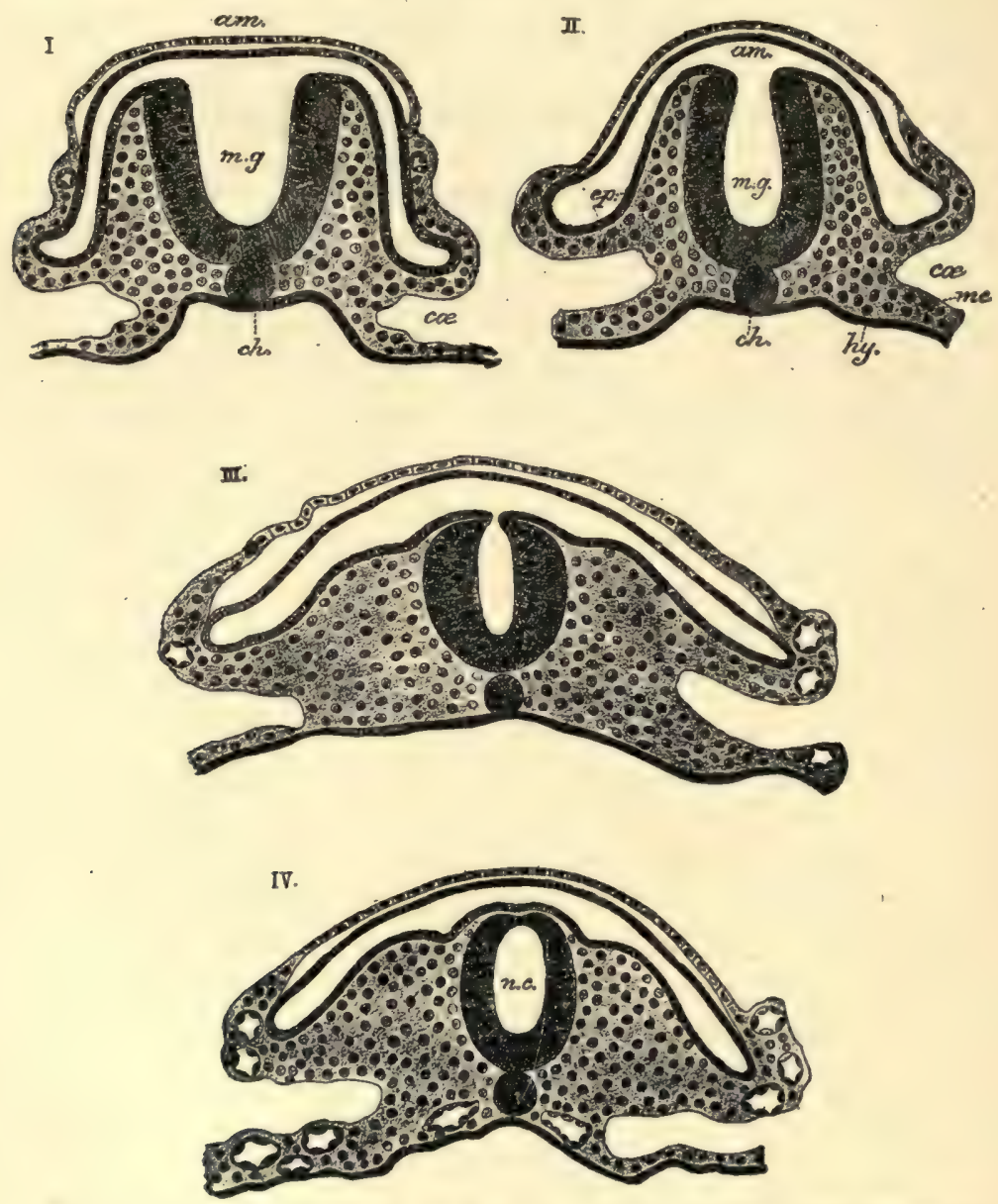

Fig. II 4.- Sections showing stages in the conversion of the medullary groove into the neural canal. From the tail end of an embryo of the cat. (Gordinier after E. A. Schäfer.)

ep, me, hy. Epiblast, mesoblast, and hypoblast. m.g. Medullary groove. n.c. (in IV), Neural canal. $c h$. Notochord. $c a$. Celom. am. Tail-fold of the amnion.

In the very early stages of development two varieties of cells make up the neural tube (Fig. Ir5). Certain very long cells, during the karyokinetic growth, arrange themselves at right angles 
to the axis of the tube. They are the ependymal cells, which form a supporting framework for the more important elements. They reach from the lumen of the tube to its peripheral surface and possess nuclei which are situated largely in the central half of the cell. The peripheral parts of the ependymal cells, by irregular branching and vacuolation, form a protoplasmic network destitute of nuclei, called the marginal velum. This marginal velum forms a peripheral zone which comprises the outer one-

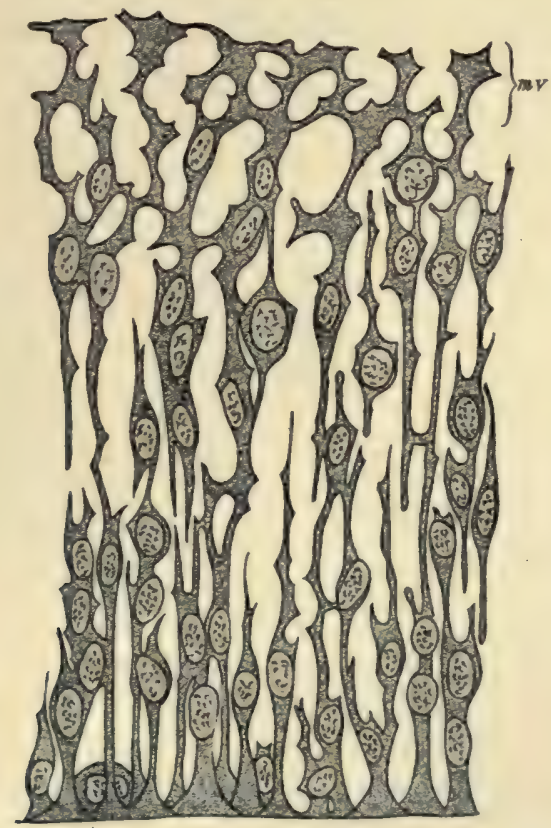

Fig. II 5.-Two histologic layers in the embryonic spinal cord, embryo $4.25 \mathrm{~mm}$. long. (McMurrich after $H$ is.)

$m v$. Marginal velum formed by peripheral ends of ependymal cells, indifferent cells among their bases.

sixth of the neural tube. The inner three-fourths or five-sixths of the tube constitutes a thick central zone in which are the bodies of the ependymal cells and, among their bases, a variety of cells possessing large nuclei and small protoplasmic bodies (Fig. II 5). The destination of the latter cells cannot yet be determined, so they are named the indifferent cells (Fig. I16). In the nuclei 
of the indifferent cells all forms of karyokinetic figures may be seen. This fact shows that they are rapidly multiplying.

In a somewhat later stage of development the indifferent cells wander outward toward the marginal velum and arrange themselves into a definite layer, known as the mantle layer (Figs. Ir6 and 127 ). Three concentric zones are clearly visible at this time in a cross section of the neural tube; the marginal velum, the mantle layer, and the ependymal layer formed by the bases of the ependymal cells. As the indifferent cells multiply in the mantle layer they become specialized into two types, the neuroblasts and the
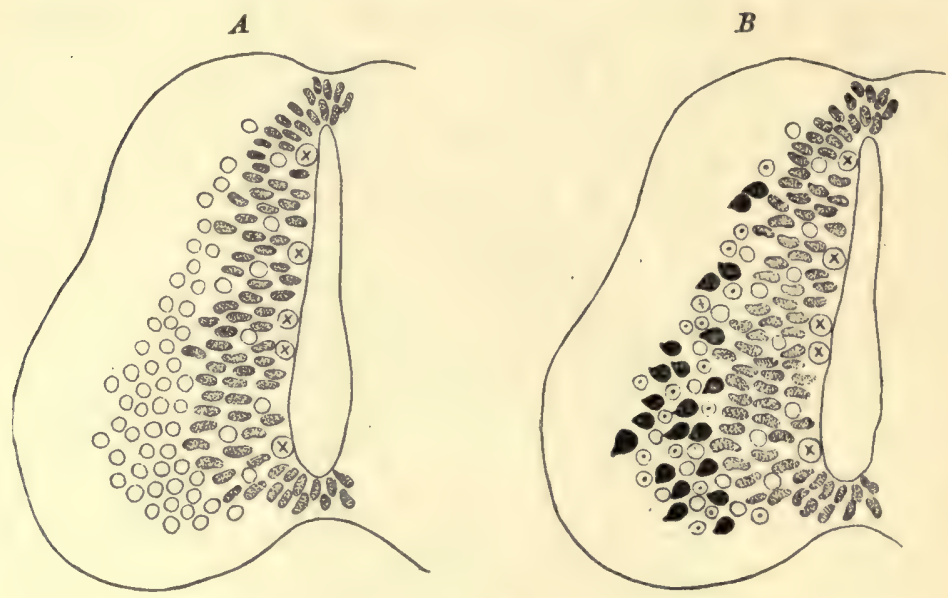

Fig. I 16.-Diagram showing development of neurones in the spinal cord.

(McMurrich after Schäfer.)

The circles, indifferent cells; circles with dots, neuroglia cells; shaded cells, germinal cells; circles with cross, germinal cells in mitosis; black cells, nerve-cells.

spongioblasts. The neuroblasts are the larger. They are the embryonic neurones. Each neuroblast throws out a protoplasmic process which develops into an axone and grows outward into the marginal velum. Within the marginal velum the axone either runs some distance and returns to the mantle layer, or it passes outward through the marginal velum and becomes an efferent or motor root-fiber. Collaterals are given off from the axones, the end-tufts are formed and the medullary sheath is laid down as development proceeds and function begins. While these events 
are occurring in the efferent part of the neuroblast, other processes, called dendrites, are given off by the cell-body. They belong to the afferent side of the cell. There are usually several dendrites. They branch richly close to the cell-body and terminate, as a rule, within the mantle layer. The spongioblasts are the smaller of the indifferent cells. They are primitive neuroglia cells (Fig. 53). The spongioblasts develop many very slender and richly branched processes of ray-like or arborescent form, which by their interlacement constitute a delicate supporting reticulum. The neurones are held in the meshes of this reticulum, and both the neurones and neuroglia cells are still further supported by a coarse mesoblastic framework produced by the ingrowth of blood vessels.

Zones (Figs. II 7 and I27).-While the neurones and neuroglia cells are forming, there occurs in each lateral wall of the neural

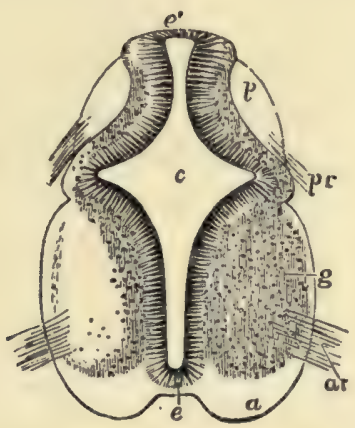

Fig. II 7.- Transverse section of the cervical part of the spinal cord of a human embryo of six weeks. (Gordinier after Kölliker, from Quain.)

c. Central canal. e. Its epithelial lining. e. (Superiorly) The original place of closure of the canal. $a$. The white substance of the anterior columns. g. Gray substance of anterior columna. p. Posterior column. ar. Anterior roots. pr. Posterior roots.

canal a longitudinal evagination. This evagination produces a longitudinal groove on the ventricular surface of the neural tube, which divides it into a ventral and a dorsal zone. The ventral zone is largely efferent in function, hence from it issue the outgoing tracts (Figs. 127 and 128 ). It is composed of a median floor plate and, on either side, of a basal, or ventral lamina. The dorsal zone is made of up two alar, or dorsal laminæ joined by 
the median roof-plate. The dorsal zone is afferent in function and in connection with it the sensory nerves terminate. The roof-plate and the floor-plate take little or no part in forming neurones; they lack the mantle layer, and are composed of neuroglia and ependymal cells.

The Neural Crest (Figs. I6 and II4).-(I) The cephalic portion of the neural crest becomes broken into five pairs of ganglia, which, during development, shift their positions to the ventral side of the brain. Those ganglia are called the vagus, glossopharyngeal, geniculate, auditory and semilunar (Gasseri). Later, the auditory ganglia lie between the geniculate and glossopharyngeal ganglia. These five ganglia give origin to the sensory parts of the vagus, glossopharyngeal, intermediate and trigeminal nerves; and to all of the acustic nerve. In all the ganglia except the auditory, the cells develop into unipolar neurones; they remain bipolar in the auditory ganglia. The unipolar condition is produced by the growth of the cell-body toward the surface of the ganglion and the shifting of both processes to the same side of the cell-body, together with the elongation of the common point of attachment. The single processes of the unipolar neurones immediately divide, $\mathrm{T}$-like, into peripheral and central fibers, which in appearance are axones. The peripheral fibers form the sensory part of the respective nerves and conduct impulses toward the cell-body, hence they may be considered dendrites (Cajal); the central fibers, the axones proper, form that part of the nerve which extends from the ganglion into the dorsal zone of the embryonic brain. All the central axones of the several ganglia divide, $\mathrm{T}$-like, upon entering the brain, and collaterals rise from the undivided fibers and from both branches of them. These axones and collaterals arborize, chiefly, in the terminal nuclei of the respective nerves; but certain of them, the excito-reflex fibers, terminate in nuclei of motor nerves.

The development of peripheral common sensory neurones, both cerebral and spinal, has been observed in certain lower vertebrates. In the amblystoma punctatum the following phases have been observed: $r$. The elongation of the cells in the neural crest to a spindle-form. 2. The projection of a slender central process, the axone, which grows into the neural tube. 3. A thick 
irregular process, the dendrite, is thrown out from the peripheral end of the cell; later it becomes smooth and fibrillar in character. This bipolar condition persists in very low forms, such as the amphyoxus and cyclostomes. 4. In bony fishes some of the neurones become unipolar. The greater number become unipolar in higher vertebrates (J. B. Johnston's Nervous System of Vertebrates).

(2) The spinal portion of the neural crest forms the thirty-one pairs of spinal ganglia situated on the posterior nerve roots; and, also, the vertebral, prevertebral and terminal ganglia of the sympathetic system. The sympathetic ganglia wander widely. In them the epiblastic cells develop into multipolar neurones, the nonmedullated processes of which constitute the larger number of gray fibers in the sympathetic system. The cells of the spinal ganglia form unipolar neurones, like those of the vagus, glossopharyngeal, geniculate and semilunar ganglia. Like them, also, the single processes divide, T-like, the peripheral arms of the T-branches forming the sensory part of each spinal nerve and the central arms (the axones) the posterior roots of those nerves. The latter enter the cord at the posterior lateral sulcus and, before and after dividing $\mathrm{T}$-like into an ascending and a descending branch, give off collaterals. The descending fibers, long and short, arborize and end in the gray matter of the posterior columna, the center of the crescent and the anterior columna, the long fibers forming the postero-medial and postero-lateral descending radicular tracts; the ascending axones and collaterals terminate in the gray substance of the spinal cord and in the nuclei of its posterior columns, namely, the nucleus funiculi gracilis and nucleus funiculi cuneati of the medulla. The long ascending fibers form the postero-medial tract (Goll's) and, the posterolateral tract (Burdach's); and, the shorter ones, those reaching but a few segments, make up the marginal tract of Lissauer. The greater number of posterior root-fibers terminate in the gray substance at or near their level of entrance into the cord.

\section{THE BRAIN.}

The primary brain vesicles grow rapidly. By the end of the fourth week a constriction is visible in the anterior primary vesicle 
and another in the posterior primary vesicle, dividing each into two and making in all five secondary brain vesicles, which freely communicate with one another and are numbered from before backward. They are:

I. Telencephalon.

2. Diencephalon.

3. Mesencephalon.

4. Metencephalon.

5. Myelencephalon.

These vesicles form the brain, their cavities becoming the ventricles (Figs. II8, I20 and I7). The neuroblasts of the mantle layer produce the neurones, whose cell-bodies and dendrites are found in the cortex and ganglia, and whose medullated axones form the white substance.

Flexures (Fig. II8).--The cephalic portion of the neural tube is the seat of three flexures, two ventral and one dorsal. (I) The mesencephalic flexure (ventral) begins very early and amounts to nearly 180 degrees by the twenty-eighth day. It bends ventrally the diencephalon until it almost touches the metencephalon. Thus the inter-brain and pons are approximated and the mid-brain almost concealed. (2) The cervical flexure is also a ventral one. It is located at the junction of the fifth vesicle with the spinal cord, and corresponds to the bending of the head upon the body of the embryo. This flexion begins about the twenty-first day. By the end of the fourth week, it is completed and amounts to 90 degrees. (3) The dorsal flexure is beginning to form at the same time (fourth week). It occurs between the fourth and fifth brain vesicles, and is often called the metencephalic flexure. It reaches 180 degrees by the eighth week, when the dorsal part of the metencephalon (the cerebellum) rests upon the medulla oblongata. The convexity is formed by the pons, hence the synonym, pontine flexure. The cervical and metencephalic flexures almost entirely disappear, but the mesencephalic flexure is permanent. 


\section{TABLE IV.}

\section{BRAIN VESICLES, THEIR DERIVATIVES AND VENTRICLES}

(Figs. I7, II8 and I20).

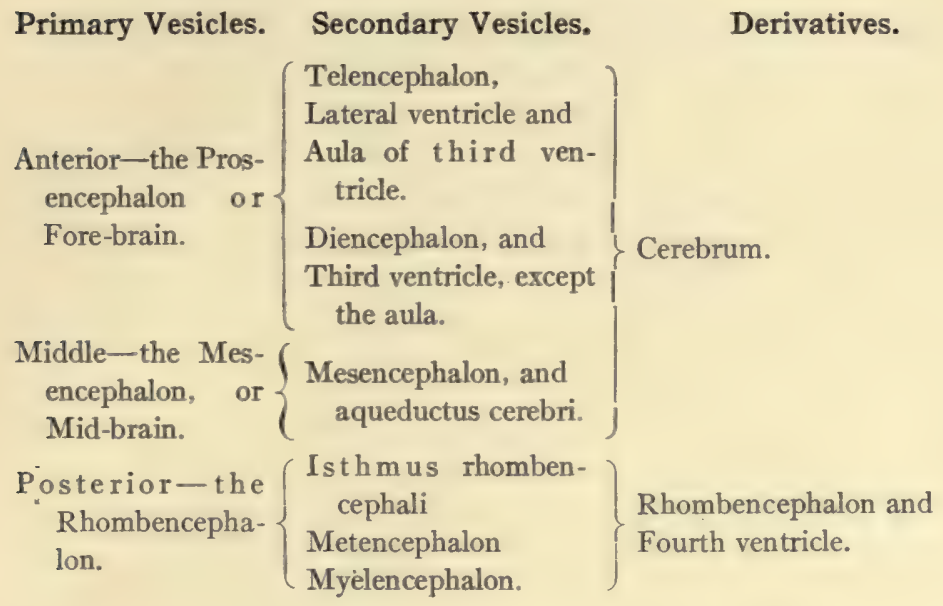

\section{TABLE V.}

\section{SECONDARY BRAIN VESICLES AND THEIR DERIVATIVES.}

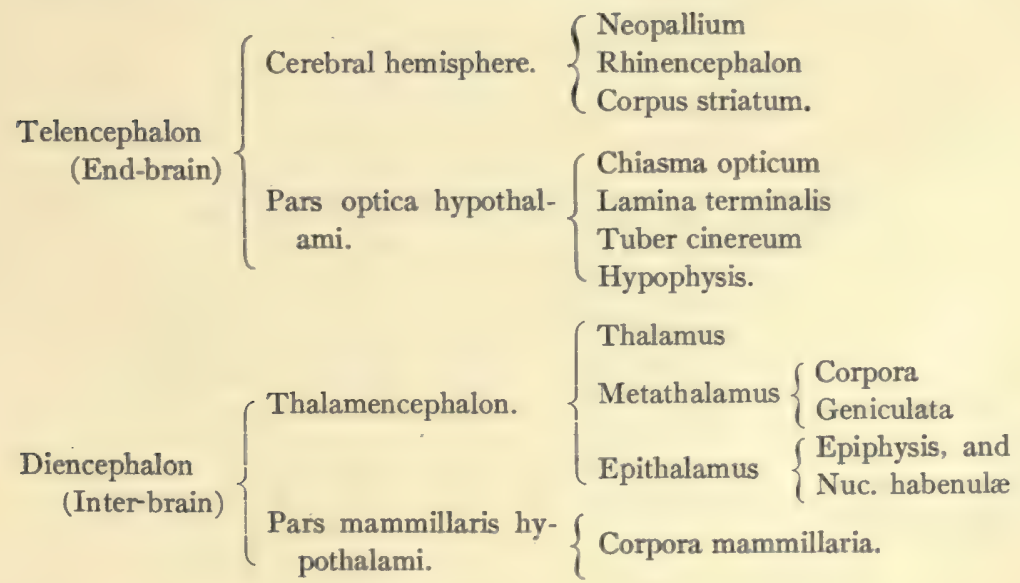


Mesencephalon

(Mid-brain)

Lamina quadrigemina.

$\int$ Bases pedunculi

$\{$ Substantia nigra

( Tegmenta.

Corpora quadrigeminaColliculi superiores Colliculi inferiores

Brachia-

Brachia superiora

Brachia inferiora.

$\left\{\begin{array}{c}\text { Isthmus rhomben- } \\ \text { cephali } \\ \text { are) }\end{array}\right.$ (in which $\left\{\begin{array}{l}\text { Pedunculi cerebri } \\ \text { Brachia conjunctiva } \\ \text { Velum medullare superius. }\end{array}\right.$

Metencephalon

Cerebellum:

Pons Varolii.

Cortex

Corpus medullare

Ganglia

(Pedunculi cerebelli)

Vela medullaria.

Myelencephalon $\{$ Medulla oblongata.

In the following resume of the formation of the central nervous system, I have drawn so largely from Prof. McMurrich's "Development of the Human Body" that I wish at this point, to make grateful acknowledgment of my indebtedness to him.

\section{TELENCEPHALON.}

\section{TABLE VI.}

\section{DERIVATIVES OF TELENCEPHALON.}

(Modified from McMurrich's Embryology.)

In Median Structures.

Roof-plate $\left\{\begin{array}{l}\text { Roof epithelium of the aula } \\ \text { Lamina terminalis (in part). }\end{array}\{\right.$ Floor of chorioidal fissure

DorsalLamina $\left\{\begin{array}{l}\text { Lamina terminalis (in part) } \\ \text { Optic vesicle. }\end{array}\left\{\begin{array}{l}\text { Neopallium } \\ \text { Rhinencephalon } \\ \text { Corpus striatum. }\end{array}\right.\right.$ 
Ventral Zone $\left\{\begin{array}{l}\text { Anterior part of hypothal- } \\ \text { amic region } \\ \text { Tuber cinereum } \\ \text { Infundibulum. }\end{array}\right.$

Surrounding
Mesoblast $\left\{\begin{array}{l}\text { Falx cerebri } \\ \text { Part of chorioid tela of third } \\ \text { ventricle. }\end{array}\{\right.$ Meninges.

The telencephalon is at first a single vesicle forming the fore part of the anterior primary vesicle, and for a time is open in front at the neuropore (Fig. II8, VI). The median portion of its anterior wall, lamina terminalis, remains almost stationary; laterally, it is the seat of rapid growth.

Optic Vesicle.-Almost before the telencephalon is differentiated from diencephalon, a club-shaped diverticulum, called the optic vesicle, is thrown out from the ventral part of its dorsal zone. It grows outward and forward, separates from the telencephalon and, becoming indented, forms the optic cup, from which the retina is developed. The point of origin of the optic vesicle is indicated in the adult by a slight pit, termed the optic recess. The optic recess is situated between the lamina terminalis and the columna fornicis at the anterior end of the sulcus hypothalamicus (Monroi). This sulcus, in the anterior primary vesicle, separates the ventral from the dorsal zone.

Hemisphere of Cerebrum (Figs. I7, II8 and 120).- Soon after the appearance of the optic vesicle, a large bulging occurs on either side in the dorsal zone of the telencephalon. That bulging produces a hollow diverticulum, whose cavity is the primitive lateral ventricle and whose walls form the substance of the hemisphere. The outgrowth is called the hemisphere vesicle. Its constricted stalk contains the primitive interventricular foramen (Monroi, Fig. I7). The vesicles grow forward and outward at first, separated from one another by mesoblast which forms the falx. Later, growth occurs in succession, upward, backward and downward, until by the seventh month the hemisphere overhangs every other part of the brain. The hemisphere vesicle is formed almost wholly by the dorsal lamina of the telencephalon which grows and develops wonderfully and will be considered further 
on. It also contains a narrow prolongation of the roof-plate. This prolongation of the roof-plate undergoes very little development. At first it is placed in the roof of the hemisphere vesicle; but the upward growth of the hemisphere shifts it to the medial surface and, later, the backward and downward extension of the hemisphere carries the roof-plate through a horse-shoe curve down toward the pole of the temporal lobe. No thickening occurs in this prolongation. Becoming indented longitudinally it forms the floor of the chorioidal fissure in which is developed, from

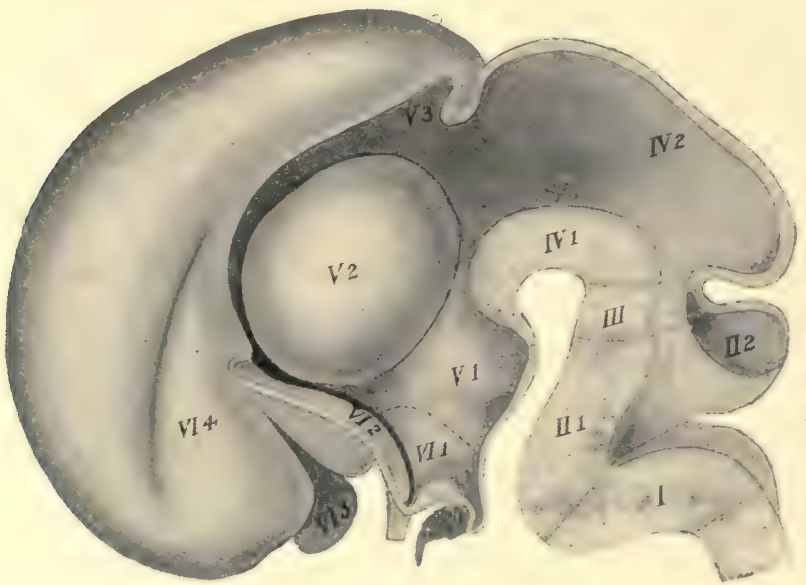

Fig. I 18.-Median section of embryonic brain of the third month. (McMurrich after His.)

I. Myelencephalon. II. Metencephalon: I, Pons, 2, Cerebellum. III. Isthmus rhombencephali. IV. Mesencephalon: I, Pedunculi, 2, Corpora quadrigemina. V. Diencephalon: I, Pars mammillaris hypothalami, 2, Thalamus, 3, Epithalamus. VI. Telencephalon: 1, Pars optica hypothalami, 2, Corpus striatum, 3, Rhinencephalon, 4, Neopallium.

ingrowing mesoblast, the chorioid plexus of the lateral ventricle (Fig. II9).

Rhinencephalon (Figs. I7, Ir8 and I20). - In the fifth week a hollow diverticulum grows out from the antero-inferior wall of the hemisphere vesicle and forms a prominent lobe. It preserves a lobular form in the horse, and in some other animals; but in man it soon becomes constricted by the sulcus parolfactorius posterior into an anterior and a posterior part, and loses its ventricular cavity. The anterior part develops the olfactory bulb, 
B

A
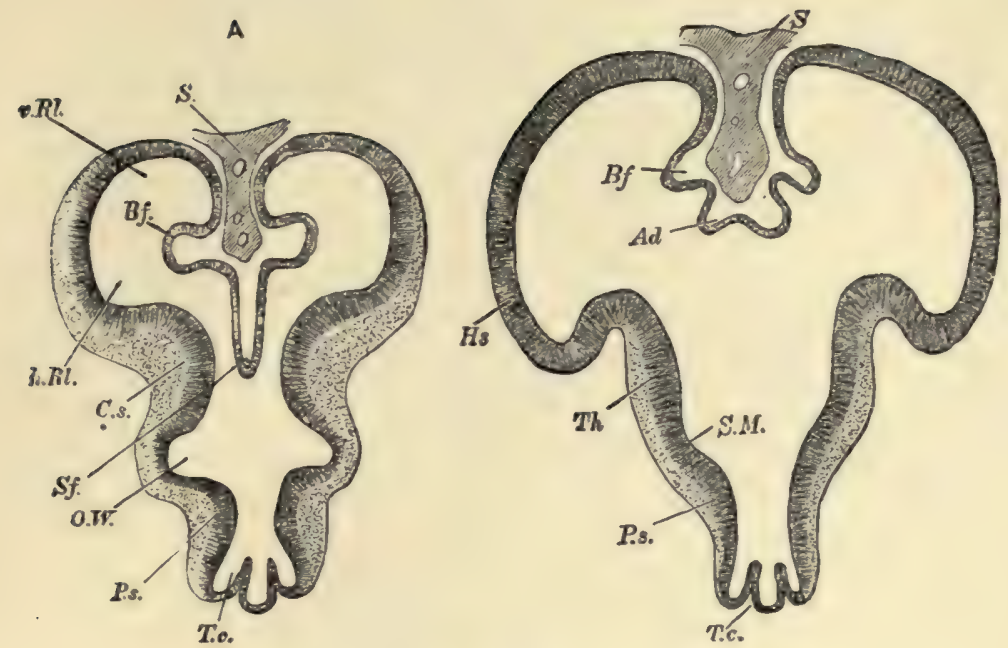

C

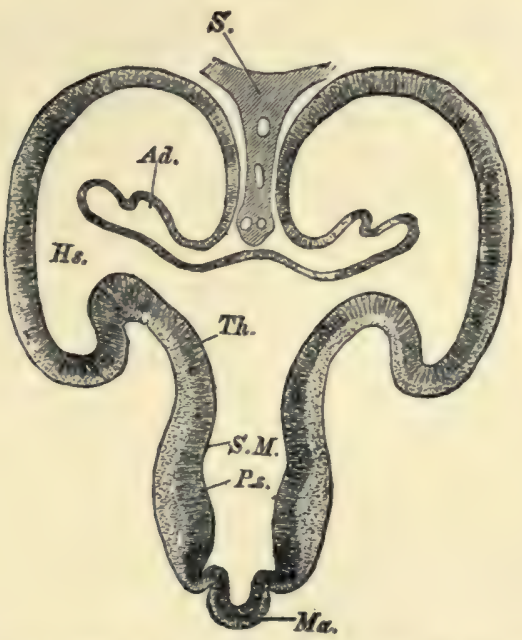

Fig. Ir9.-Transverse sections through the fore-brain of a four and one-half weeks embryo. (Gordinier and Quain after $H$ is.)

A. Through the lower anterior part of the fore-brain. S. Falx. Sf. Fold of roof passing below falx toward the thjrd ventricle. Bf. Fold forming the fissura hippocampi, v. Rl., h. Rl. Anterior and posterior parts of olfactory lobe. Cs. Corpus striatum. O.W. Groove continuous with optic stalk. P.s. Hypothalamus. T.c. Tuber cinereum,

B. Section a little farther back. Sf is replaced by a less prominent but broader fold of the roof, $A d$, which subsequently receives the chorioid vessels, and is, therefore, the chorioid fold. Hs. Hemisphere vesicle. Th. Thalamus. S.M. Sulcus hypothalamicus (Monroi), below and behind the thalamus.

C. Still farther back. Ad. Chorioid fold here projecting into lateral ventricles, but still free from mesoblast and blood-vessels. Ma. Mammillary tubercle. The other lettering as before. 

tract, triangle, and the parolfactory area (Brocæ), the posterior portion forms the anterior perforated substance, etc.

Primary Fissures (Figs. I2I and I22).--The vesicle walls are of uniform thinness up to the second month. During their rapid growth in the second and third months they become thrown into folds which encroach upon the cavity and present on the surface of the vesicle (or hemisphere) the primary fissures. The cause of the infoldings is, perhaps, the resistance of the slower growing cranium. The primary fissures are best developed in the third month. They consist of the chorioidal fissure, already mentioned, the hippocampal fissure which, surrounding it, is concentric with

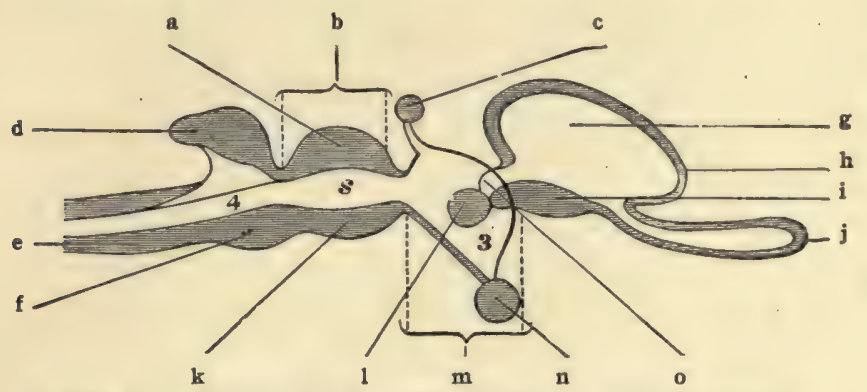

Fig. 120.-Diagrammatic sagittal section of vertebrate brain. (Morris's Anatomy after Huxley.)

a. Corpora quadrigemina. b. Mid-brain. c. Pineal body. d. Cerebellum (hind-brain). e. Medulla oblongata (after-brain). f. Pons Varolii (hind-brain). g. Lateral ventricle. h. Cerebral hemisphere. i. Corpus striatum. j. Olfactory diverticulum. k. Pedunculi cerebri. 1. Thalamus, m. Inter-brain. $\mathbf{n}$. Hypophysis. o. Interventicular foramen. 4. Fourth ventricle, s. Aqueduct of cerebrum. 3. Third ventricle.

it, and the fossa lateralis cerebri (Sylvii). The hippocampal fissure (Fig. I2I) begins near the frontal pole on the medial surface of the hemisphere vesicle and extends backward, downward and, lastly, forward toward the temporal pole. It divides the medial surface into a broad marginal gyrus and the dentate gyrus, the latter is between it and the chorioidal fissure. In its whole course it is parallel with the chorioidal fissure (Figs. I I9 and I2I). The ventricular eminence caused by the hippocampal fissure is called the hippocampus. The hippocampal fissure gives off two branches at its most posterior part, which represent the occipitoparielal and the calcarine fissures (Fig. I2I). Both branches produce ventricular eminences, but only that of the calcarine per- 
sists, in the calcar avis. The primary occipito-parietal fissure entirely disappears. The whole superior part of the hippocampal fissure is represented in the adult, as to position, by the callosal fissure. The lateral cerebral fossa (Fig. I22) is a deep bay in the ventral border of the hemisphere vesicle. It is due to the relatively limited growth of the corpus striatum in comparison with the more rapid and greater growth of the surrounding parts. The thickened floor of the fossa cerebri lateralis develops, internally, the corpus striatum and, externally, forms the insula (Reili). The lateral fossa and the four fissures mentioned above are well formed by the third month, but the lateral cerebral fossa is not converted into a fissure until the end of the fifth month. Even then only the posterior ramus of the fissura cerebri lateralis (Sylvii) is formed, and this is brought about by the meeting of the temporal and the fronto-parietal parts of the operculum. It is during the first year after birth that the development of the orbital and frontal parts of the operculum produces the anterior horizontal ramus and the anterior vertical ramus of this fissure.

Secondary Sulci and Secondary Fissure (Fig. I23).-The secondary sulci are linear indentations of the surface only; they cause no ventricular eminences. About the middle of the fifth month the first secondary sulcus makes its appearance. It is the sulcus cinguli of the medial surface, which separates the superior frontal gyrus and paracentral lobule from the gyrus cinguli of the adult brain (Fig. 27). Its development in two or three pieces explains its irregularity and occasional want of continuity. In the sixth month the subparietal and occipito-parietal sulci are present. There is no sign of the latter sulcus in the early part of the fifth month and the permanent sulcus does not produce a ventricular eminence, hence the occipito-parietal indentation is a sulcus and not a fissure (see Cunningham Memoirs). Early in the sixth month the most important of the remaining sulci are formed, such as, the central sulcus (Rolandi), the precentral, the postcentral, the superior temporal sulcus and the remaining larger sulci of the frontal, parietal, occipital and temporal lobes (Figs. 23 and 123). The only secondary fissure, the collateral fissure appears in the same month. It is developed on the tentorial sur- 
face of the hemisphere, at first, in three separate parts, an anterior and a posterior temporal and an occipital part. Its middle part is a total fissure, so is its anterior part sometimes. When both are total they produce a long eminence in the inferior horn of the lateral ventricle, external to the hippocampus and parallel with it, hence its name, the eminentia collateralis.

There are many tertiary sulci which do not appear until near birth, some of them one or two years after birth.

Transverse Fissure of the Cerebrum.-Only the anterior part

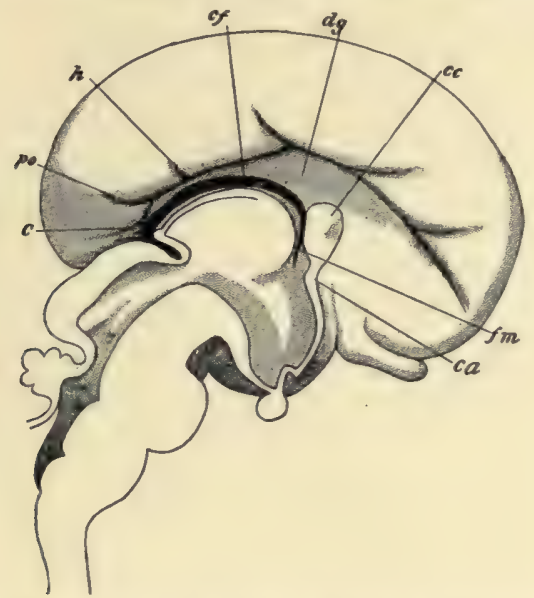

Fig. 1 2x.- Medial sagittal section through the brain of an embryo of three months, showing the primitive fissures on the medial surface of the cerebral hemisphere. (McM urrich after Mihalkovicz.)

c. Calcarine fissure, ca. Anterior commissure. $c c$. Corpus callosum. $c f$. Chorioidal fis sure. dg. Dentate gyrus. $f m$. Foramen interventriculare (Monroi). $h$. Hippocampal fissure. po. Occipito-parietal fissure.

of this fissure, which is between the fornix and the inter-brain, belongs wholly to the cerebrum; the posterior part separates cerebrum from cerebellum. The anterior part of the transverse fissure is produced by the backward growth of the united cerebral hemispheres over the free dorsal surface of the diencephalon, the inter-brain. It is continuous laterally with the chorioidal fissure in each hemisphere and contains the chorioid tela of the third ventricle.

Cerebral Cortex and Medulla.-As the gyri of the cerebrum 
are acquiring their adult forms and producing the various fissures and sulci, the walls of the hemisphere vesicle thicken rapidly and give rise to the corpus striatum, the cortex and the medullary substance; but the order of development is not yet understood. The development of the gray and the white substance contracts the cavity of the hemisphere vesicle to the size of the lateral ventricle; and the cormua of the ventricle are produced by the forward, backward, and downward growth of the vesicle in the successive formative stages. Little is known of the time at which the cortical neurones are formed; but it would seem that they continue to undergo medullation and to become functional up to a late period of life, and the investigations of Kaes support this inference.

Fornix.-A ridge appears, about the fourth month, on the medial surface of the hemisphere vesicle, along the convexity of the chorioidal fissure. That ridge reaches from the lower end of the hippocampus to the roof of the interventricular foramen. It becomes converted into a bundle of fibers which is continued into the lamina terminalis and then through the lateral wall and floor of the diencephalon to the corpus mammillare. It forms a lateral half of the fornix. The union of the two halves in the lamina terminalis forms the primitive body, or corpus fornicis. The body is extended by the upward and backward growth of the lamina terminalis and by the crossing over of certain fibers from one crus fornicis to the other, which results in the formation of the commissura hippocampi.

Another ridge, a slight one, appears about the same time on the opposite lip of the chorioidal fissure. It represents the stria terminalis. This latter ridge and the fornix ridge bound the roofplate as represented in the hemisphere. This roof-plate extension undergoes no development. It forms the chorioid epithelial lamina of the lateral ventricle, which loosely joins the fornix and the stria terminalis, being folded over the chorioid plexus of that ventricle (Fig. II9).

Internal Capsule. - It is formed largely along the line of fusion between the medial surface of the hemisphere vesicle and the lateral surface of the diencephalon. Motor fibers grow downward through the corpus striatum from the cerebral cortex, and 
sensory fibers ascend through the striated body to the cortex from the thalamus and other ganglia. All these motor and sensory fibers together constitute the internal capsule. Its bell-shape in the hemisphere is due to the rotary growth of the hemisphere, upward, backward, downward and forward, around the corpus striatum (Figs. 32 and 33).

Anterior Commissure (Fig. I2I).-The lamina terminalis at the fourth week is a thin median plate bounding the aula anteriorly and joining the hemisphere vesicles together just in front of the foramina interventricularia (Monroi) (Fig. I7). Its ventral portion remains thin; but its dorsal part thickens greatly and,

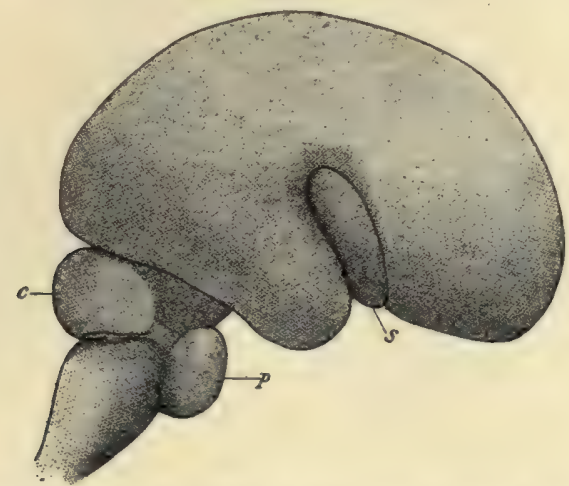

Fig. 122. - The fossa lateralis cerebri, in embryonic brain of the fourth month. (After McMurrich.)

c. Cerebellum. p. Pons, s. Fossa lateralis cerebri.

with the upward and backward growth of the hemisphere, it is extended in a crescent, dorsal to the interventricular foramen, as far backward as the splenium of the corpus callosum. Within this thickened crescentic part of the lamina terminalis are formed the anterior commissure, the body of the fornix, the corpus callosum and the septum pellucidum. The anterior commissure is first formed. Through the ventral angle of the thickened portion grow, transversely, the commissural and the decussating fibers of the rhinencephalon and the commissural fibers connecting the occipito-temporal cortices. These fibers make up the anterior commissure. The fornix fibers grow for the most part 
longitudinally through the zone next the ventral surface of the crescentic lamina and, on reaching the anterior commissure, bend backward into the lateral wall of the aula and third ventricle; a certain number of fornix fibers cross over in the posterior border of the lamina terminalis and produce the commissura hippocampi. The corpus callosum is formed in the dorsal zone of the crescentic lamina terminalis.

The corpus callosum (Fig. I2I) is produced by fibers that grow from one cerebral hemisphere to the other through the fronto-

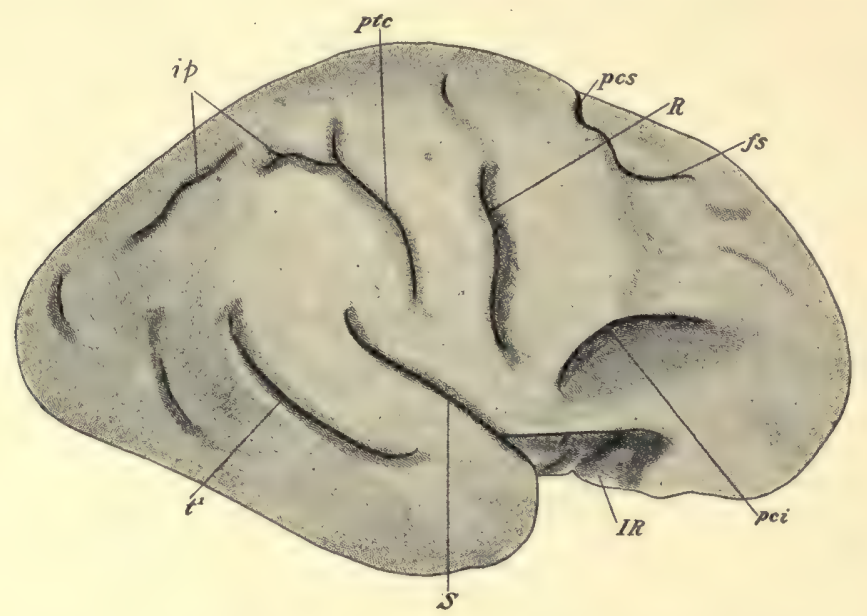

Fig. 123.-Permanent fissures and sulci on the convex surface of the cerebrum as seen in a seven months embryo. (McMurrich after Cunningham.)

fs. Superior frontal fissure. ip. Interparietal. IR. Island (Reili), pci. Inferior pre-central. pcs. Superior pre-central. ptc. Post-central. R. Central (Rolandi). S. Lateral (Sylvii). $t^{1}$. First temporal.

dorsal zone of the lamina terminalis. The different parts of the corpus callosum are formed in regular order-rostrum, genu, truncus, splenium-and this order coincides with the lines of growth in successive stages of cerebral development. The fibers of the corpus callosum pierce the dentate gyrus of the hemisphere vesicle. In so doing, they completely obliterate the superior part of the hippocampus and leave but rudiments of the dentate gyrus, namely, the gyrus supracallosus and gyrus subcallosus (longitudinal striæ and peduncle of the corpus callosum), the fasciola 
cinerea and the small dentate fascia of the adult are the rudimentary remains.

Septum Pellucidum. - That part of the lamina terminalis which is included between the corpus callosum and the body of the fornix persists as the septum pellucidum. It develops a lymph space in the median plane, called the fifth ventricle (cavum septi pellucidi) and becomes so thin as to be translucent.

The above description, giving the origin of the septum pellucidum, corpus callosum and anterior commissure from lamina terminalis, is the one commonly accepted; but it is not their origin in the rabbit (Marshall) or in the rat (Zuckerkandl), and there is still room for doubt concerning their origin in the human brain. It is possible that all or a part of them are developed in a crescentic area of fusion between the cerebral hemispheres, as in lower animals.

The pars optica hypothalami (Fig. II8) is developed in the ventral zones of the telencephalon. The tuber cinereum and the infundibulum are true derivatives of these telencephalic zones, but the optic chiasma is not. It is produced by the ingrowth of fibers from the retinæ and the medial geniculate bodies.

\section{DIENCEPHALON.}

This is the posterior division of the anterior primary vesicle. It forms the inter-brain and contains the greater part of the third ventricle (Figs. I7, II8, V, and II9). Its lateral walls present on each side a distinct ventricular sulcus, the sulcus hypothalamicus (Monroi) (Fig. II9, B and C) which separates the rentral zone from the dorsal zone (?).

\section{TABLE VII.}

\section{DERIVATIVES OF THE DIENCEPHALON.}

Roof-plate
Dorsal Lamina
Ventral Zone $\left\{\begin{array}{l}\text { Derivatives. } \\ \text { Roof epithelium of third ventricle. } \\ \text { Corpus pineale (epiphysis). } \\ \text { Thalamus } \\ \text { Corpora geniculata. } \\ \text { Pars mammillaris hypothalami } \\ \text { Corpus mammillare } \\ \text { A small part of tuber cinereum. } \\ \text { Chorioid tela and plexus of third ven- } \\ \text { tricle. }\end{array}\right.$


The roof-plate of the diencephalon stretches out and becomes very thin, except at its posterior extremity (Figs. II8, V3, and I20). Its major portion forms the epithelium covering the third ventricle. Posteriorly, fibers grow through it from opposite sides and form a transverse white band, the posterior commissure. Immediately in front of this commissure, a diverticulum of the roofplate appears which is the primitive pineal body. The pineal body soon becomes solid and is joined to the diencephalon by a

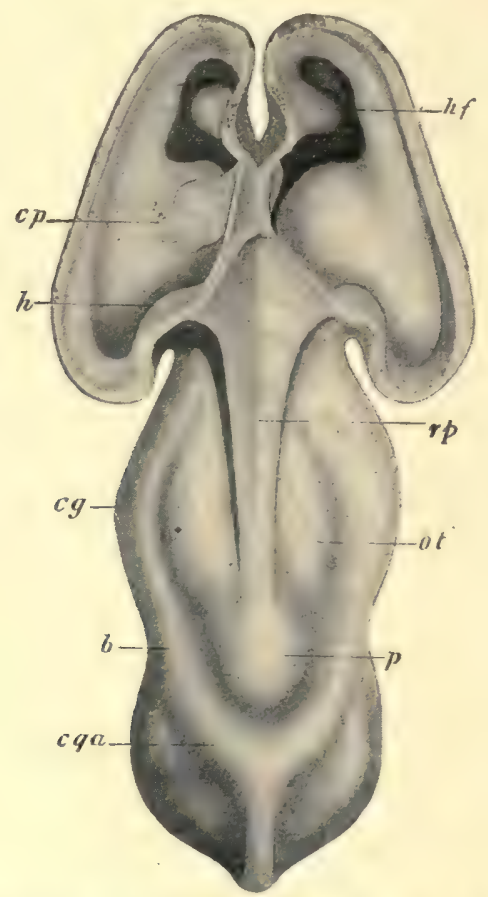

Fig. I24.-Dorsal view of an embryonic brain, the roof of the lateral ventricles having been cut away. Embryo of $12.6 \mathrm{~mm}$. (McMurrich after His.)

b. Brachium superius. cg. Corpus geniculatum laterale. $C p$. Chorioid'plexus of llateral ventricle. $C q a$. Colliculus superior of corpora quadrigemina. $h$. Hippocampus. $h f$. Hippocampus and fissure. Ot. Thalamus. $p$. Pineal body. $r p$. Roof-plate.

constricted stalk in which there is a conical prolongation of the ventricular cavity, called the pineal recess (Fig. 120). Anterior to the pineal body, two longitudinal folds of the roof-plate dip down into the ventricular cavity. These are followed by two 
like downward projecting folds from the inferior lamina of the chorioid tela, which constitute the chorioid plexuses of the third ventricle.

The dorsal lamina of the diencephalon thickens greatly and so encroaches upon the ventricle as to convert it into a narrow median slit. Externally, the dorsal lamina fuses with the cerebral hemisphere, as a result of the formation of the internal capsule; and the fusion at one point of the medial surfaces of the two dorsal laminæ gives rise to the massa intermedia (middle commissure). The development of the thickened dorsal lamina of the diencephalon produces the thalamus with its pulvinar and the geniculate bodies (Fig. I24).

The ventral lamina and floor-plate of the diencephalon constitute the pars mammillaris hypothalami, which embraces some gray and white matter beneath the thalamus, and the corpora mammillaria (Fig. II8, VI). A single oval eminence situated in the median line, represents the mammillary bodies up to the third month; but, during the third month, that eminence is divided into the two white bodies (corpora albicantia) of the adult. The gray substance immediately in front of the corpora mammillaria also belongs to this region, hence a part of the tuber cinereum is included among the derivatives of the diencephalon.

\section{MESENCEPHALON.}

This is the mid-brain (Figs. I7, II8, IV, and I20). It is the third of the secondary vesicles. The elbow of the mesencephalic flexure of 180 degrees is formed by it; and that flexure almost

- brings the diencephalon and metencephalon in contact with one another beneath it. The mesencephalon remains small, but its walls thicken greatly. As a result of the thickening, its cavity is reduced to a slender canal, the cerebral aqueduct (Fig. I20).

\section{TABLE VIII.}

\section{DERIVATIVES OF MESENCEPHALON.}

Dorsal Zones $\quad\left\{\begin{array}{l}\text { Lamina quadrigemina } \\ \text { Colliculi } \\ \text { Brachia (in part) } \\ \text { Red nuclei. }\end{array}\right.$


Ventral Zones

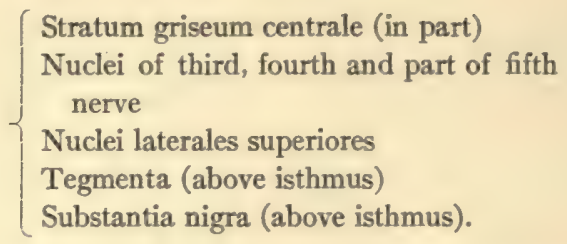

Little is known about the development of the mid-brain. The origin of the derivatives tabulated above is largely inferred from their position and function. The formation of the corpora quadrigemina has been observed (Figs. I20 and I24). In the dorsal part of the mesencephalon, an elongated eminence on either side of median line is present at the beginning of the third month. Those two eminences resemble the corpora bigemina of birds, fishes and reptiles. Two months later a transverse groove divides each eminence into the superior colliculus and the inferior colliculus. By the growth of fibers through the marginal velum between the colliculi and the corpora geniculata the brachium superius and the brachium inferius are produced (Fig. 43). The bases pedunculi are produced, likewise, by the down-growth of fibers from the fore-brain, which traverse the marginal velum in the ventral region and, when medullated, form the prominent strands of the adult.

\section{METENCEPHALON.}

The metencephalon is the fourth of the secondary brain vesicles. From it are derived the isthmus, the cerebellum and the pons (Figs. I7, I I 8, III, and IV, and I20). Its dorsal wall, which forms the cerebellum, presents a transverse indentation, the metencephalic angle; and, as a result of that angle the opposite wall is bulged forward. The ventral wall of the metencephalon forms the pons. The cavity of this vesicle forms the upper half of the fourth ventricle. Superiorly, this cavity contracts to the size of the cerebral aqueduct; it expands inferiorly and is broadest at the junction of the pons with the medulla oblongata. The ventricular surface presents on each lateral wall a longitudinal furrow which divides the metencephalon into a ventral and a dorsal zone. In the adult the location of that furrow is indicated by the sulcus 
limitans of the rhomboid fossa, containing the fovea superior and the locus coruleus, hence, the pons comprises the greater portion of the metencephalon, including the entire ventral zone and a part of the dorsal zone.

\section{TABLE IX.}

\section{DERIVATIVES OF METENCEPHALON (McMurrich, modified).}

\begin{tabular}{|c|c|c|}
\hline & Metencephalon Proper & Isthmus \\
\hline Roof-plate & $\left\{\begin{array}{l}\text { Inferior velum } \\
\text { Cerebellar vermis }\end{array}\right.$ & $\begin{array}{l}\text { Superior velum, or valve of } \\
\text { Vieussens - contains de- } \\
\text { cussation of trochlear } \\
\text { nerves. }\end{array}$ \\
\hline Dorsal Zones & $\begin{array}{l}\text { Lobes of cerebellum } \\
\text { Terminal nuclei, of sensory } \\
\text { nerves (part of fifth and } \\
\text { eighth) } \\
\text { Ganglia of cerebellum (?) } \\
\text { Nucleus pontis (?) }\end{array}$ & $\begin{array}{l}\text { Brachium conjunctivum of } \\
\text { cerebellum grows through } \\
\text { it. }\end{array}$ \\
\hline Ventral Zones & $\begin{array}{l}\text { Genetic nuclei of motor } \\
\text { nerves (fifth, sixth, sev- } \\
\text { enth) } \\
\text { Nuclei centrales and nucleus } \\
\text { lateralis medius of the re- } \\
\text { ticular formation }\end{array}$ & $\begin{array}{l}\text { Inferior part of tegmen- } \\
\text { tum and of substantia } \\
\text { nigra. } \\
\text { The basis pedunculi grows } \\
\text { down through it. }\end{array}$ \\
\hline Floor-plate & Median raphe & Median raphe. \\
\hline
\end{tabular}

Cerebellum.-It forms the roof of the upper part of the fourth ventricle, that is as far down as the transverse fold of invaginated roof-plate, called the plica chorioidea, produced by the metencephalic flexure (Fig. II8, between II and I). Above the plica chorioidea the dorsal laminæ of the metencephalon thicken rapidly and form a prominent transverse ridge on either side of the median line. The two thickenings are partially separated from each other in the median line by a deep ventricular sulcus but joined dorsally by a thin bridge of tissue, the metencephalic roof-plate. At this early stage there is no representative of the vermis cerebelli, the ridges represent the hemispheres. But very soon, cells from the dorsal laminæ invade and thicken the 
roof-plate to the extent of obliterating the median ventricular sulcus and completely uniting the two dorsal laminæ. The cerebellum is now represented by one continuous transverse ridge arching over the fourth ventricle. It develops slowly in comparison with the cerebrum. The lateral parts develop more rapidly than the median portion after the third month. Thus the hemispheres are differentiated from the vermis. The flocculus is the first lobule to be formed and it reaches a high development in the third month; other lobules of the hemisphere which are more characteristic of the human cerebellum, the tonsil, the quadrangular lobe, the superior semilunar and the inferior semilunar lobules, are not fully formed until near birth. The folium vermis of the worm is developed after birth (Figs. 76 and 80).

Sulci (Fig. 79).-The chief sulci of the worm appear in the third month; with two exceptions, those of the hemisphere develop later. The lateral part of the postnodular sulcus is first manifest. In the second month it cuts off a strip of the cerebellar ridge, just above the chorioidal plica, which is the primitive flocculus. A little later the sulcus extends across the median line and forms the posterior boundary of the nodule. The next sulcus to develop is the predeclivil sulcus (Fig. 79). It cuts very deeply into the vermis between the culmen and the declive. From the vermis it extends into the hemispheres, where it separates the anterior and the posterior parts of the quadrangular lobe. The prepyramidal and the postpyramidal sulci are formed near the end of the third month (Fig. 79). In the fourth month the hemispheral part of the postdeclivil sulcus is first visible behind the quadrangular lobule (Fig. 76). Soon it becomes continuous through the vermis with that of the opposite hemisphere. It is the beginning of the fifth month before the prepyramidal sulcus is extended into the hemisphere between the tonsil and biventral lobule (Fig. 80). At about the same time the lateral extension of the postpyramidal sulcus establishes the posterior boundary of the biventral lobule. According to O. C. Bradley's study of the rabbit's cerebellum, the postcentral sulcus and the precentral sulcus appear at about the same time as the postdeclivil. The last important sulcus to appear is the horizontal sulcus (Figs, 76 
and 80). It is not well formed until near birth and is produced almost wholly by the enormous growth of the superior and the inferior semilunar lobules which are so characteristic of the human cerebellum (Cunningham).

The cerebellar lobules are subdivided into gyri by intralobular sulci which develop in the later months of pregnancy and the early months of extrauterine life. There are two, called the midgracile and postgracile sulci, which Bradley says are found only in man and the anthropoid apes.

Cortex and Ganglia.-Little is known of the particular order and manner of development in either the cortex or ganglia of the cerebellum. As to the cortex, it consists in general, (I) of the multiplication and development of the cells in the mantle layer, some of whose processes descend to the cerebellar ganglia and Deiters's nucleus, and, (2) of the formation of contacts with ingrowing fibers from pons, medulla and spinal cord.

Corpus Restiforme of Cerebellum.-Fibers from the cerebellar cortex descending to the medulla, and ascending fibers from the cord and medulla in their course to the cerebellar cortex, give rise to the corpus restiforme in the third month. Fibers from the nucleus pontis and nucleus fastigii form the brachium pontis a month later and in the fifth month the brachium conjunctivum is produced by fibers from the nucleus dentatus.

Pons (Figs. II8, II, I, I2I and I22).-The pons develops simultaneously with the cerebellum. The ventral zone of the metencephalon thickens greatly. The neuroblasts formed therein constitute the nuclei of the pons (?) and of the reticular formation, and the motor muclei for the fifth, sixth and seventh pairs of cerebral nerves; the dorsal lamina in the lateral wall of the metencephalon, produces the neuroblasts which form the superior olivary nucleus and the superior part of the terminal nucleus of the trigeminal and of the acustic nerve. From the nucleus pontis axones ascend through the lateral walls of the metencephalon to the cerebellum. They form most of the brachia pontis. At the same time, about the fourth month, the motor tracts composing the basis pedunculi grow downward into the ventral portion of the pons and the fronto-pontal, temporo-pontal and intermediate tracts 
end in the nuclei pontis. The pyramidal fibers to motor nuclei of spinal nerves grow down through the pons, intersecting its transverse fibers.

\section{MYELENCEPHALON.}

The myelencephalon forms the medulla oblongata (Figs. II 8 , I, and I22). It is constricted off from the metencephalon at the twenty-eighth day; but, later, that constriction largely disappears and the common cavity of the two vesicles, broad in the middle

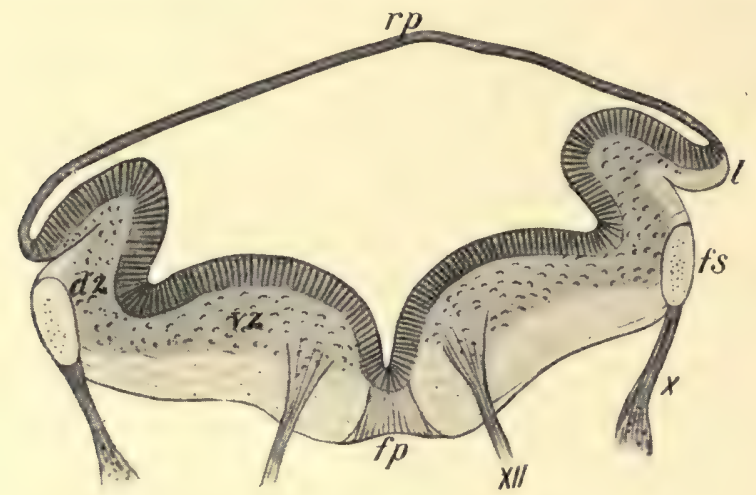

Fig. I25.-Transverse section of medulla from an embryo of 91. mm. (McMurrich after $H$ is.)

dz. Dorsal zone. fp. Floor-plate, fs. Tractus solitarius. l. Lip. rp. Roof-plate. vz. Ventral zone. $X$ and $X I I$. Tenth and twelf th nerves.

and contracted to a slender canal at each end, persists as the fourth ventricle of the mature brain. Like the metencephalon, the fifth brain vesicle is divided at the third week into a ventral and a dorsal zone by a deep furrow on the ventricular surface of each lateral wall (Figs. I25 and I26). That lateral furrow actually persists in the sulcus limitans and inferior fovea of the fourth ventricle. The roof-plate of the superior half of the myelencephalon stretches out widely and remains a single layer of epithelial cells. It forms no nerve tissue. Other portions of the myelencephalon develop quite uniformly. But by the expansion of the roof, just mentioned, the dorsal extremities of the lateral walls are pushed outward and 
forward almost to the plane of the floor, and a transverse section of the vesicle in that region presents the form of a very broad capital $\mathrm{V}$ with the roof epithelium stretching between the two arms and converting the letter into a triangle. Transverse section through the lower half of the myelencephalon at the third or fourth week shows an elongated ellipse with a dorso-ventral major axis.

Internal Surface (Fig. 125).-The lateral wall, in both upper and lower regions, presents the longitudinal groove which separates the ventral and dorsal zones. The median ventral groove persists throughout and the lateral grooves are represented by the

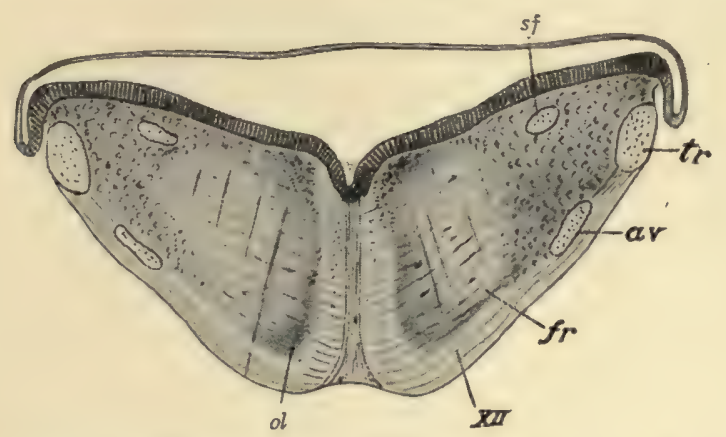

Fig. I26.-Transverse section of the medulla from an embryo of eight weeks. (McMurrich after $H$ is.)

av. Spinal tract of the trigeminal nerve. fr. Substantia reticularis. Ol. Olivary nucleus of medulla. sf. Tractus solitarius. tr. Restiform body. XII. Hypoglossal nerve.

sulcus limitans and inferior fovea of the fourth ventricle (Fig. 96). The low eminence situated between the median and each lateral groove becomes the eminentia medialis.

External Surface (Figs. 125 and 126).-On the exterior surface of the myelencephalon, along the ventral border of the dorsal zone, there appears very early an oval bundle of descending fibers, called the solitary tract. It is composed of axones from the geniculate, glossopharyngeal and vagus ganglia, and constitutes the root-fibers of the intermediate, the ninth and tenth pairs of nerves. At about the same time axones from the semilunar ganglion form a bundle of descending 
fibers on the lateral surface ventral to the solitary tract. They constitute the spinal tract of the trigeminal nerve. Soon after the appearance of the solitary tract and the spinal tract of the fifth nerve, the posterior margin of the dorsal lamina is folded outward and forward until it rests upon the external surface. That fold, which is called the rhombic lip, covers both the above bundles of fibers and places them in the position they occupy in the mature brain. Between the rhombic lips the roof epithelium stretches across the ventricle and pushes a transverse fold into it. The mesoblast which dips into that fold develops the chorioid plexus of the fourth ventricle (Fig. 9I).

\section{TABLE X.}

\section{DERIVATIVES OF MYELENCEPHALON.}

(Modified from McMurrich.)

\begin{tabular}{|c|c|}
\hline Roof-plate & Roof epithelium \\
\hline Dorsal Zone & $\begin{array}{l}\text { Nuclei funiculi-gracilis, and cuneati, } \\
\text { and nucleus tractus spinalis n. tri- } \\
\text { gemini. } \\
\text { Olivary nuclei (inferior) } \\
\text { Arcuate nuclei } \\
\text { Terminal nuclei of sensory roots of cere- } \\
\text { bral nerves (intermediate, eighth, ninth } \\
\text { and tenth) } \\
\text { Sensory tracts grow up through it. }\end{array}$ \\
\hline Ventral Zone & $\begin{array}{l}\text { Nucleus lateralis inferior } \\
\text { Genetic nuclei of motor roots and nerves, } \\
\text { ninth, tenth and eleventh (its bulbar } \\
\text { root) and twelfth } \\
\text { Motor tract (py ramid) grows down } \\
\text { through it. }\end{array}$ \\
\hline Surrounding Mesoblast & $\begin{array}{l}\text { Meninges- } \\
\text { Chorioid plexus of fourth ventricle. }\end{array}$ \\
\hline
\end{tabular}

The substance of the myelencephalon, like other divisions of the neural tube, presents under the microscope three distinct layers at the fourth week of embryonic life (Fig. 125). The 
outer layer, the marginal velum, is composed of neuroglia; the middle, or mantle layer, of neuroblasts; and the inner, or ependymal layer is made up of columnar epithelial cells.

The cells of the inner layer become ciliated and form the lining of the ventricle.

The mantle, or middle layer undergoes most development. Its neuroblasts form the substantia reticularis and the cerebral nerve nuclei and other nuclei of the medulla oblongata. In the dorsal zone the neuroblasts form the terminal nuclei for the eighth, ninth and tenth cerebral nerves and the nucleus funiculi gracilis, nucleus funiculi cuneati and nucleus of the spinal tract of the trigeminal nerve. As early as the fourth week axones may be traced from the nucleus funiculi gracilis and nucleus funiculi cuneati, ventro-medially, toward the point where they very soon form the fillet decussation. Neuroblasts which have wandered from the dorsal zone form the arcuate, the olivary and accessory olivary nuclei. The olivary nuclei are developed quite late in the intrauterine life (sixth month). The nucleus of the cuneate funiculus, the nucleus of the spinal tract of the $\mathrm{n}$. trigeminus, the olivary and arcuate nuclei are all products of the rhombic lip (Cunningham). From the neuroblasts of the ventral zone are developed the gray matter and fibers of the substantia reticularis alba et grisea, and the motor nuclei of the twelfth, eleventh (cerebral part), tenth and ninth pairs of cerebral nerves.

The neuroglia layer, or marginal velum, forms the supporting matrix for the tracts of fibers in the medulla. By the third month the funiculus gracilis and funiculus cuneatus, extensions of the same fasciculi in the cord, have grown up to their terminal nuclei in the medulla. The restiform body is at that time well developed, and the tracts of the lateral area of the medulla are visible. The medial longitudinal bundles appear near the median raphe in the ventral zone at about the same time; and, ventral to them, fibers from the fillet decussation insinuate themselves and form the interolivary stratum of the medial fillets. The great motor tracts from the anterior central gyrus of the cortex reach the medulla at the fourth month. Growing downward in the neuroglia layer, on either side of the median line, they conceal the 
medial fillets and form the pyramids of the medulla oblongata. The form of the medulla is completed two months later (the sixth month) by the appearance of the olivary bodies (Fig. 93).

\section{SPINAL CORD.}

That portion of the neural tube which is situated behind the metencephalon is the embryonic spinal cord (Figs. I6 and II8). It is of nearly uniform size from cephalic to caudal end. The lumen of the neural tube in this region is at first large and elliptical in shape. Later, at the sixth week, it has a diamond shape, the acute angles of the diamond being formed by the roof-plate and floor-plate of the canal; it is lined with columnar ciliated cells (Fig. II7). As the walls thicken the canal is contracted more and more until it reaches the capillary size of the adult cord. The canal is continuous with the fourth ventricle above and dilates to form the ventriculus terminalis in the filum terminale internum (Fig. IIO). The spinal part of the neural tube forms the whole substance of the spinal cord, with the exception of the great motor tracts that grow into it from the brain, and the sensory tracts and fibers that enter it from the spinal ganglia. At the sixth week of embryonic life the anterior and posterior roots of the spinal nerves are clearly seen; they are horizontal in direction. The cord extends to the fourth mesoblastic somite of the coccyx, when the somites are first laid down; but, as no neuroblasts are developed by the three lower segments of the human cord, they form a connective tissue strand, the primitive filum terminale (McMurrich). The cord occupies the entire length of the spinal canal until the third month, when the caudal end begins to recede and the filum terminale to lengthen. It reaches only to the third lumbar vertebra at birth and, in the adult, but to the lower border of the first lumbar vertebra. With the rapid growth of the spinal column, the roots of the lumbar, sacral and coccygeal nerves and the filum terminale become greatly elongated and form the cauda equina.

Meninges.-The investing mesoblast of the neural tube develops the meninges of the spinal cord.

Zones (Figs. II 7 and I $27 \mathrm{~A}$ ).-By the sixth week of intrauterine 
life the neural tube, in the spinal region, is divided into a ventral and a dorsal zone by a lateral groove on either side, continuous with that formed in the rhombencephalon two weeks earlier. The tube presents externally, opposite to each lateral groove, a furrow in the mantle layer representing the concavity of the gray crescent and called the central fissure; it is occupied later by the lateral pyramidal and other tracts. The whole of the spinal cord ventral to the bases of the posterior columnæ of gray matter is represented by the ventral zones. From them grow out the
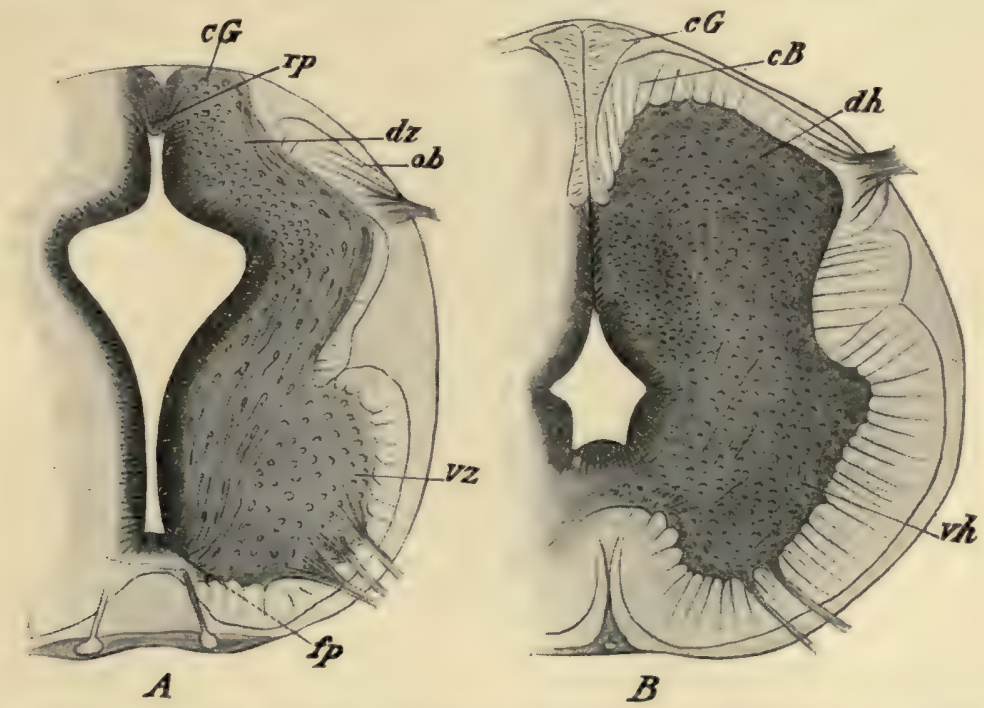

Fig. 127.- Transverse sections through the spinal cords of embryos of (A) about four and a half weeks and (B) about three months. (McMurrich after His.)

$c B$. Fasciculus cuneatus (Burdachi). $c G$. Fasciculus gracilis (Golli). dh. Posterior columna. dz. Dorsal zone. fp. Floor-plate. ob. Oval bundle (Hisi). rp. Roof-plate. vh. Anterior columna. v\&. Ventral zone.

anterior roots of the spinal nerves. In the dorsal zones the posterior columnæ and the posterior columns of fibers are developed. They receive the posterior roots of the spinal nerves (Figs. II 7 , 127 and 128).

Three Histologic Layers (Figs. II6 and 127A).-At a time somewhat earlier than the division into ventral and dorsal zones, even at the fourth or fifth week, the spinal part of the neural tube 
presents three microscopic layers, like those seen earlier in the brain vesicles.

The outer layer, or marginal velum, composed of neuroglia, is very thin except in the central fissure and in the floor and roof of the tube on either side of the median line. In those situations are formed the lateral, the anterior and the posterior funiculi of the cord.

The mantle layer is gray matter. It contains the neuroblasts. At the fourth or sixth week it is very thick, comprising nearly all of the neural tube. The $H$-shaped column of gray sub-

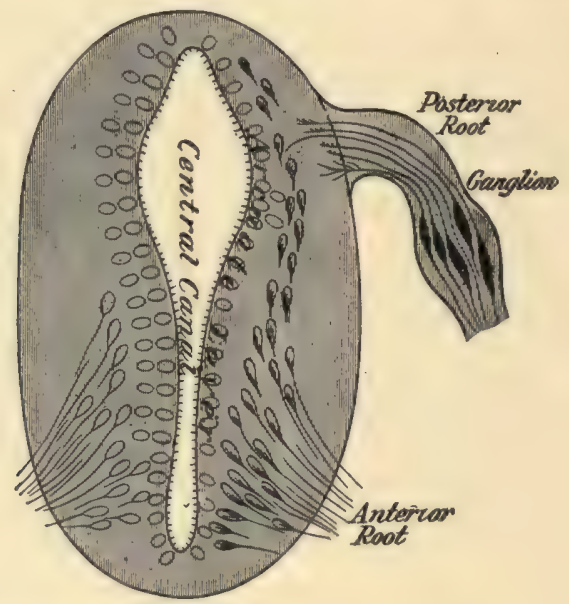

Fig. 128.-Mode of origin of anterior and posterior roots of spinal nerves. Diagrammatic. (Brubaker and Edinger after His.)

stance is derived from this layer. In the ventral zone the neuroblasts develop earliest. They collect near the floor of the tube and form a large column on either side of the median line, the anterior columna. The axones of the more ventrally located neuroblasts grow centrifugally out of the antero-lateral surface of the neural tube. They form the anterior roots of the spinal nerves. The more dorsally located neuroblasts develop axones also; but on account of meeting resistance, according to His, they become longitudinal and form the fasciculi proprii of the cord and, perhaps, the ascending anterior cerebello-spinal and the spino-thala- 
mic tracts. The gray matter in the dorsal zone develops later. It becomes transformed into the posterior columna; its neuroblasts form largely intrinsic neurones, their processes remaining in the spinal cord, others give rise to the posterior cerebello-spinal tract which ascends through the marginal velum to the vermis superior of the cerebellum. Axones from the spinal ganglia, forming the posterior roots of the spinal nerves, grow into the dorsal zone; and each axone divides T-like into a descending and an ascending branch, and also gives off.many collaterals both before and after division. The ascending fibers for a time form the oval bundle of $H$ is on the surface of the tube; later, they constitute the marginal tract (Lissaueri) and the fasciculus cuneatus (Burdachi) and fasciculus gracilis (Golli).

The third, or ependymal layer, lines the ventricle. It is composed of neuroglia covered internally by columnar ciliated cells, which appear about the fifth week.

The Longitudinal Tracts (Figs. IOI, IO2 and I03).- The white. columns of the spinal cord are formed in the superficial, or neuroglia layer. At first they are composed of nonmedullated fibers, and it is a remarkable fact that the various tracts receive their myelin sheaths at definite periods between the fifth and ninth months of intrauterine life (Flechsig). The anterior nerveroots and the anterior and lateral fasciculi proprii, and the posterior nerve-roots and the oval bundles of His are first to make their appearance. The axones of these nerves-roots and tracts may be seen at the sixth week. The ascending cerebello-spinal tracts follow, and the descending cerebello-spinal and the pyramidal tracts are last to appear; they are not developed before the fourth or fifth month. According to Kahler, as quoted by Cunningham, the tracts of the spinal cord are medullated as follows:

I. Fibers of the fasciculus cuneatus with the posterior nerveroots, preceded by the medullation of the anterior nerve-roots (fifth month).

2. Fibers of anterior and lateral fasciculi proprii (fifth and sixth months).

3. Fibers of fasciculus gracilis (sixth month).

4. Posterior cerebello-spinal tract (seventh month). 
5. Ascending anterior cerebello-spinal and spino-thalamic tracts

(?) (eighth month).

6. Pyramidal tracts (ninth month).

Fissures (Fig. IOI).-The so-called posterior median fissure is in reality a septum of neuroglia. At no time is it a true fissure. Its mode of formation is still in doubt. It appears to be produced by thickening in the roof-plate due to the elongation of ependymal cells. From the sixth week this neuroglia septum extends ventrally until it reaches the center of the cord.

The posterior lateral sulcus is the groove between the lateral border of the posterior column and the dorsal border of the lateral column. It is the development of the posterior and lateral columns that produces the fissure. The embryonic central fissure is obliterated and the postero-lateral surface of the cord rendered prominent by the formation of the lateral fasciculus proprius, the cerebello-spinal and the pyramidal tracts.

Anterior Median Fissure.-That is a true fissure. It begins to be formed at the sixth week, when the anterior columnæ and the earliest fibers of the anterior fasciculus proprius are developing. It deepens with the growth of the gray columnæ and of the anterior fasciculus proprius and its walls are further heightened, in the fourth or fifth month, by the descent of the anterior pyramidal tracts. In this manner there is produced a bulging of the anterior surface on either side of the median line, which increases with the medullation of the longitudinal tracts up to the ninth month. The ridges thus produced, failing to fuse completely, become the walls of the anterior median fissure. The partial fusion which does occur between the two ridges is due to the formation of the white anterior commissure of the cord. 


\section{ADDENDA.}

According to Head, Rivers and Sherren (Brain, 1905) three distinct mechanisms operate in the production of common sensation, one concerned with deep sensibility and two with superficial sensibility. The mechanism of decp sensibility includes the nerves supplying muscles, tendons, joints and ligaments. Though it may be affected by environment, it is chiefly acted upon by stimuli originating within the organism, such as pressure (resulting from weight and inertia) and tension. It may give rise to painful sensations, but its principal concern is with muscular tonicity and equilibrium. The nerves of the deep mechanism constitute the "proprio-ceptors" of Sherrington. They are so named because their stimuli are furnished chiefly by the organism itself (Brain, I906). The mechanisms of superficial (cutaneous) sensibility are called by Head, Rivers and Sherren the Protopathic and the Epicritic. The protopathic mechanism responds to painful cutaneous stimuli and to extremes of heat and cold. It may set up reflexes without arousing consciousness. Cutaneous localization is no part of its function. The mechanism of epicritic sensibility is adapted to mild stimuli, to light touch, to warmth, to coolness. By it the localization of cutaneous stimuli is accomplished and its activity usually involves consciousness. It may also produce reflexes through the efferent nerves. Sherrington speaks of these two mechanisms as the "extero-ceptors" and gives the name "intero-ceptors" to the nerves of the interior surfaces, those of the alimentary tract, etc. The "intero-ceptors" are especially adapted to chemical stimuli and give rise to digestive reflexes; but they may also produce pain and temperature sensations, and may respond to stimuli occasioned by tension and pressure. See pages 365 and 377 . 



\section{INDEX.}

Abducent nerve, 45, 153, $32 \mathrm{I}$

Accessory lemniscus, 227 nerve, 5, 49, I53, 293, 322 nucleus funiculi cuneati, 3 II

Acustic center, I8I nerve, 46, I53, 209, 320 radiation, 100, 104, 217, 226,

$$
232,233,310
$$

Acustico-cerebellar tract, 264

Afferent neurone, I7 I

paths, 377

root, 363

sympathetic fibers, 348

After-brain, 33

Ala cinerea, I49, 270, 290, 317

Alveus. 200, 201

Amygdala, I23, I24

Angular gyrus, 43, 61, 63, 67

Ansa lenticularis, 100,145 peduncularis, I45, 2 I2

Anterior area of medulla, 293 association center, 182

brain vesicle, $3 \circ$

calcarine fissure, 89

central gyrus, 39, 59, 60, 6 I

central artery, $33 \mathrm{I}$

cerebral artery, 4, 6, I5, I6, 25 vein, 23

chorioidal artery, I5, I8, 19

communicating artery, I4, I 5, I6 commissure of cerebrum, 30,95 , I09, I3I, I36, I 37, 234, 235, $4 \mathrm{II}, 4 \mathrm{I} 3$

columna, 335, 339, 399, 427, 428

neurones, 165,340

external arcuate fibers, 292, 295, 297

fasciculus proprius, 354

horn of lateral ventricle, 120,137 , 209, 235

inferior cerebellar artery, 15,27 , 29

internal frontal arteries, I6 28
Anterior lateral sulcus of cord, 335, $33^{8}$

lateral sulcus of medulla, $4 \mathrm{I}, \mathbf{1 5 3}$, 295

longitudinal bundle, $158,16 \mathrm{I}$, $163,219,276,295,297,305$,

34I $, 345,355$

median fissure of cord, 335,337 . 349,430

median fissure of medulla, $4 \mathrm{I}$, $285,286,295,337$

median vein of medulla, 24

orbital gyrus, 74

parolfactory sulcus, 86

perforated substance, 38,47 , 79 , $207,235,267$

pyramidal tract, 289, 294, 34 I, 345,353

radicular artery, $33 \mathrm{I}$

root-line, $335,33^{8}$

root of spinal nerve, 327,334 ,

$349,363,399,427,428$

slender lobule, 252, 253, 256

spinal artery, $\mathbf{5}, \mathbf{1 5}, \mathbf{2 4}, \mathbf{2 7}, 330$, 33 I

subarachnoid space, $8,327,329$

surface of medulla, 267, 286

of mid-brain, 267

of pons, 266, 267

temporal artery, I9

tubercle of thalamus, I40, I49, 243

Antero-lateral ganglionic arteries, 15, I8, 19,22

fasciculus proprius, 354

median ganglionic arteries, 18 , 19

Apertura mediana ventriculi quarti (Magendi), 9, 290

lateralis ventriculi quarti (Key and Retzii), 9, 290

Aqueduct of cerebrum, 37

Aqueductus cerebri, 37, 75, I52, I6 I, 270 
Arachnoid granulations, 5, 6, 65 of brain, 8, 9 of cord, 326, 327, 329

Arachnoid of brain and cord compared, 9

Arachnoidea encephali, 8 spinalis, 326

Arbor vitæ, 247, 262

Archipallium, 98

Arcuate fibers, 29r

nucleus of medulla, 295, 297, 302

nucleus of thalamus, 2 II

Arcus occipito-parietalis, 64,69

Area acustica, 149, 270, 290, 318

parolfactoria (Brocæ), 47, 75 ,

$$
\text { 9 I, 94, } 200
$$

postrema, 270, 318

for educater movements of cerebrum, 178

Arteria basilaris, 14

carotis interna, I4, 25

cerebelli inferior anterior, 27, 29 inferior posterior, 27,29

superior, 27

cerebri anterior, 16, 25

media, I6

posterior, 17,25

chorioidea anterior, I8

communicans anterior, 14,25

posterior, 14, 25

meningea media, 6,7

spinalis anterior, $24, \mathbf{2 7}, 330$

posterior, 24, 27, $33^{\circ}$

vertebralis, 14,27

Arteriæ chorioideae posteriores, I7 pontales, 24

Arterial circle, I4

Arteries of dura, 6

of pia mater, ro

of spinal cord, $330,33 \mathrm{I}$

Artery of cerebral hemorrhage (Charcot), 18

Ascending anterior cerebello-spinal tract, $287,295,297,298$, 305,3 I I , 34 I , 345, 356

anterior ramus of lateral fissure of cerebrum, 57

frontal arteries, $16,19,22$

parietal arteries, $17,19,22$

part of medial longitudinal bundle, 157

postero-medial tract, 360
Ascending postero-lateral tract, $36 \mathrm{r}$ tracts of spinal cord, $35^{2}$

Association fibers of cerebellum, 262, 265

fibers of cerebrum, 224, 236

Meynert, I92

Associative centers of Flechsig, 182

Atypical cortex, I92 neurones, I9I

Auditory artery, 5 center, $70,7 \mathrm{r}$ conduction path, I60 paths, 280, 385

Auditory and facial nerve, $\mathbf{5}$

Aula of third ventricle, 30, 137 , I 4 I

Axones, I68, I69, 398, 400

Axone hillock, 167, 169

Axis cylinders, I68, I69

Baillargic line (Gennari), I88 zone of cerebral cortex, 184

Bandalette of Hoche, 345, 362

Base of brain, 36 of cerebral hemisphere, 73,75 of fore-brain, $52,73,75$

Basilar artery, 5, I4, I5 plexus, 4,5 vein, 23

Basis pedunculi, 4, 30, 38, 47, 75, II $5, I_{45}, I_{46}, I_{47}, I_{53}$, I 57, I 59, I6I, 418

Basket cells of cerebellar cortex, 257 , 259

Bechterew's accessory lemniscus, 227

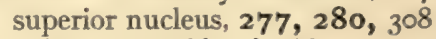

Betz, giant pyramids of, 188 .

Bipolar neurones, 400, 401

Biventral lobules, 252, 254, 255

Blood supply of brain, 14 of cerebellum, 27 of cerebrum, I4 of medulla oblongata, 24 of pons varolii, 24 of spinal cord, $33^{\circ}$

Body of fomix, I0I, I09

Boundaries of fourth ventricle, $3 \mathrm{I} 3$,

Brachia of corpora quadrigemina, $3^{\circ}$, I 46,164

Brachium conjunctivum cerebelli (or brachia conjunctiva), I3, I46, I 49, I62, 23I, 245, 246, $252,26 \mathrm{I}, 263,275,289$ 
Brachium inferius of orpora quadrigemina,

I $46, \mathrm{I} 47, \mathrm{I} 49, \mathrm{I}_{3}, \mathrm{I} 64,4 \mathrm{I} 8$ pontis, I $3,4 \mathrm{r}, 149,209,245$, $246,247,252,255,264$, 267,289

superius of corpora quadrigemina, $147,157,163,164$, $207,4 \mathrm{r} 6,418$

Brain, 3I

general considerations, 34

vesicles, $30,34,395,402,403$

Bruce and Muir's septo-marginal tract, $36 \mathrm{I}$

Bulb of posterior horn of lateral ventricle, 123, $\mathbf{I}_{3} \mathbf{I}$

Bulbus olfactorius, 77

Burdach's column, 300, 35r

Buried gyrus, 64

Cajal's moss-like appendages, 260 , 259

tassel cells, I99

Calamus scriptorius, 149, 317

Calcar avis, I23, I25, I3 I, I33, 410

Calcarine artery, 17

fissure, $43,75,86,89,91,95$, I 2 I, 409, 4 I I

Calleja's olfactory islets, 199

Callosal sulcus, 80, 86, 9I, 95, 10 I, 410

Calloso-marginal sulcus, 86

Canalis centralis spinalis, 337

Capsula interna, 99

Cauda equina, 333,334

Caudate nucleus, IO I, I05, I09, IJ 5 , II6, I $23,125, I_{37}, 1_{45}$, $204,207,209,235$

Cava subarachnoidealia, 8

Cavernous sinus, 3,5

Cavum septi pellucidi, III, 4I5

Cell-body of neurone, 167,169

Cell-columns of cord, 340, 34I, 344, 345,347

Cells of anterior columna, 340 of granular layer of cerebellum, 258

of posterior columna, 347 of Purkinje, 257, 258, 259

Cell and fiber lamination of cerebral cortex, I8 $, 185,189,193$, 197
Cellulifugal conduction, 168

Cellulipetal conduction, I68

Center median (Luysi)of thalamus, 211 of abstract concept, 182,182 of concrete concept, 182,183 of crescent, 344 of equilibrium, $7 \mathrm{I}, \mathrm{r} 8 \mathrm{I}$ of intonation, $7 \mathrm{I}, 18 \mathrm{I}$ of macular vision, 67 of muscular sense, 178 of optic reflexes, 324 of orientation, $7 \mathrm{I}, \mathrm{I} 8 \mathrm{I}$ of smell, 82, 94, I8I of stereognosis, 178 of taste,. 94,98, I 8 I of visual memories, 67,69

Central canal of cord, 399,428

of lower medulla, 305

cells of anterior columna, 343, 345

gray substance of medulla, 305 lobule, 247,250

part of lateral ventricle, II 2 sulcus (Rolandi), 56, 57, 410, 4I 4 sulcus of island, 72 tegmental tract, 277 or veutricular gray matter, $22 \mathrm{I}$

Centrifugal arteries, 33I fibers of corpus striatum, 207

Centripetal arteries, 33I fibers of corpus striatum, 208

Centrosome, r67

Centrum semiovale, $\mathbf{I}_{77}$

Cerebellar hemispheres, 242 notches, 244 vermis or worm, 243

Cerebello-olivary fibers, 265, 292, 295, 312

Cerebello-spinal fasciculus (posterior), 288,3 ㅇ

Cerebellum, 3I, 33, 35, 36, 37, 38 , $43,53,209,242,418,419$ blood supply of, $27,29,30$ veins of 29,30

Cerebral aqueduct (Sylvii), 4, 30, 34

$87, \mathrm{I}_{32}, \mathrm{I} 47, \mathrm{I}_{52}, 247,4 \mathrm{I} 7$ cortex and medulla, I77, $4 \mathrm{II}$ gray substance, 174 hemispheres, $30,35,37,5^{1}, 9^{8}$ localization, I77 nerves, 47,318 peduncle, 209,267 reflexes, $39^{2}$ 
Cerebro-pontal paths, frontal, temporal and intermediate, 369

Cerebro-spinal paths, 366 reflexes. 393

Cerebrum, 30, 31, 33, $5^{\circ}$

Cervical enlargement of spinal cord, $333,334,335$

flexures, 402

Charcot's artery of cerebral hemorrhage, $x 8$

Chiasma opticum, $3^{8}, 82,83$

Chief nucleus of vestibular nerve (Schwalbe), 283

Chorioid epithelial lamina of lateral ventricle, $124,4 \mathrm{I} 2$

plexus of fourth ventricle, I3, 290,3 I 4,424

of lateral ventricle, I0, 80,90 , I20, I24, I25, I27, I36, I $45,209,235,289,406$, $4 \mathrm{I} 2,4 \mathrm{I} 6$

of third ventricie, IO, I 29, I 32 , $209,4 \mathrm{I} 7$

tela of fourth ventricle, Io

of third ventricle, Io, I I, 90, 95, I3 $1,132,135$

Chorioidal fissure, $80,86,90,95$, I o I, I24, I 27, I $33,406,407$, 409,4 I I

groove of thalamus, I37, I40, 147

vein, II, 2I, I 29

Chromophilous cells, 204

Ciaglinski's sensory tract, $35^{\circ}$

Cilio-spinal centers, 298

Cingulum, 237, $23^{8}$

Circular sinus, 4,5 sulcus, 70

Circulation of cerebrum, I4 of rhombencephalon, I4, 24

Circulus arteriosis (Willisi), I4

Cisterna cerebello-medullaris, 9 interpeduncularis, 9 pontis, 9

Claustrum, I01, I45, 204, 209, 235

Clava, I3, 246, 289, 300, 310

Cochlear nerve, 280 nucleus, 280, 302, 305, 309, 323 path, 386

Cohnheim's end arteries, I8

Collaterals, $168, \mathbf{1 6 9}, 398$

Collateral fissure, $75,80,8 \mathrm{I}, 86,90$, 9I, 95, IOI, 4 IO
Colliculi of corpora quadrigemina, $\mathrm{r} 47, \mathrm{r} 49,163,243,246$

Colliculus facialis, 149, 269, 270, 277,317

inferior of corpora quadrigemina, I $49, \mathrm{I}_{3}, 220$

superior of corpora quadrigemina, $149,159,16_{3}, 219$, 243,416

Columna anterior, 339

fornicis, I05, ITO, I3I, 239

lateralis, $335,34 \mathrm{I}, 344$

posterior, 347

Columnæ of fornix, IIO, I 3 I , I32, I 37, I $39,209,212,235$

Comma tract of Schultze, 34I, 36I

Commissura anterior alba, 35I anterior cerebri, 31, I36, $20 \mathrm{I}$ anteria grisea, $35^{\circ}$ grisea of spinal cord, $338,35^{\circ}$ habenularum, III, I35 hippocampi (Fornix), 5I, IIO, I25, I3I, 236, 4I 2, 4I 4 inferior (Guddeni), 218, 221 posterior cerebri, I $3^{2}$ posterior of cord, $35^{\circ}$ superior (Meynerti), 218, 221

Commissural fibers of cerebellum, 262,265

of cerebrum, 224, 234

nucleus, 305, 306

Common sensory area of cerebrum, 178 nuclei of cerebral nerves, 322 paths $375,37 \%, 379$

Confluens sinuum, 2

Conus medullaris, 3 r, 334

Co-ordinating and equilabrating reflex, 390

Convex surface of fore-brain, 52

Corona radiata, 209

Cornu anterius ventriculi lateralis, 120 commissural tract, 345,362 inferius ventriculi lateralis, 123 , 133

posterius ventriculi lateralis, 123 , I33

Corpora albicantia (see corpus maminilla), $85,22 \mathrm{I}$

geniculata, 30

quadrigemina, 28, 30, 37, 1०9, I $46, \mathrm{I}_{3}, 219,4 \mathrm{I} 8$

mamillaria (see corpus mam. millare) 
Corpus callosum, 6, 25, 30, 5I, 87, 9 I, I I I, IO4, IO9, I I 7, II 2, I45, 209, 234, 235, 4I I, $4 \mathrm{I} 3,4 \mathrm{~J} 4$

dentatum, 260

geniculatum mediale, I43, 147 , $149,218,221$

geniculatum laterale, I43, I47, 218,416

Luysi, I43, I45

mammilare (or corpora mammillaria), $30,38,47,75,82$, $85,87,95,1_{31}, I_{45}, 1_{53}$, $207,209,221,267,417$

medullare cerebelli, $242,244,247$, $255,261,262$

pineale, $30,75, \mathrm{~J} 32, \mathrm{x} 37,243$

restiforme, I 49, 246, 264, 288, $30 \mathrm{I}, 42 \mathrm{I}$

striatum, $28,36,37,98,115$, 204, 410

trapezoideum, 272

Cortex of cerebellum, $242,256,258$, 259

and ganglia of cerebellum, $42 \mathrm{I}$

Cortical areas of convex surface of cerebrum, 175,182

of medial surface of cerebrum, 179,183

of smell, $82,94, \mathrm{I} 8 \mathrm{r}$

gray matter of cerebrum, 177

connection of abducent nucleus, 282

of anterior columna, 343

of cochlear nuclei, 3 Io

of facial nucleus, $\mathbf{2 8 2}$

of genetic nuclei of cerebral nerves, 324

of motor nuclei in medulla, 305 of nucleus alæe cinerex, 306

of oculomotor and trochlear nuclei, 223

of olfactory nucleus, 324

of terminal nuclei of optic nerve, 324

of trigeminal nucleus, 28I, 307

of vestibular nuclei, 308, 323

fillet, 104, 160, 216, 232, 233

localization, 177

system of arteries, I5

Corticifugal fibers of cerebrum, 225

Corticipetal projection fibers, 23I

Cortico-striate radiation, 207
Crista galli, 4

Crossed descending tract of red nucleus (see rubro-spinal tract)

ponto-spinal tract, 280

pyramidal tract, 287

Crown of hippocampal gyrus, I96

Crus fornicis, 95, I01, I05, I IO, I24, I 3 I, I 33, 237

Culmen monticuli, $87,243,245$, 247,250

Cuneate gyrus (see cuneus) tubercle, I 3, 289, 310

Cuneus, 25, 97

Declive monticuli, $87,243,247,25$ I

Decussatio lemniscorum, 291, 292

pyramidum, 4I, 29I

Decussation of brachia conjunctiva, I56, I6r, 245, 26r, 263 , 275

of pyramids, 209, 267, 29ז, 294

Deep transverse fibers of pars basilaris pontis, 271, 275

veins of pons, 27

vena cerebri media, 23

Defecation reflex, 39I

Degeneration of neurones, I7I

of Nissl, I7 I

of Waller, I 7 I

Deiter's cells, 340,348

dorso-lateral vestibular nucleus, 280, 308

Dendraxone, I68

Dendraxones of Golgi, rg

Dendrites, $165,167,169,399,40 \mathrm{r}$

Dentate fascia, 82, Ior, I24, I27, 4I5

gyrus, $4 \mathrm{II}, 4 \mathrm{I} 4$

nucleus, 255, 260, 277

Derivatives and ventricles of brain vesicles, 403

of dicncephalon, 4I5

of mesencephalon, $4 \mathrm{I} 7$.

of metencephalon, $4 \mathrm{r} 9$

of myelencephalon, 424

of teiencephalon, 404

Descending anterior cerebello-spinal tract, $287,298,305,3 \mathrm{II}$, $34 \mathrm{I}, 345,352,356$

part of medial longitudinal bundle, 158

postero-medial tract, $36 \mathrm{r}$ postero-lateral tract, $3^{62}$ 
Descending root of trigeminal nerve, I $6.3,275,277$

tracts of spinal cord, $35^{2}$

Destruction of special sense paths, $3^{89} 9$

Destructive lesions of cerebral cortex, 183

Development of brain, $40 \mathrm{r}$

of neurones, 172

of peripheral common sensory neurones, 400

Diameters of spinal cord, 333, 334, 335

Diagram of brain ventricles, I4 I

Diaphragma sellæ, 5,84

Diencephalon, 28, 30, $127,402,4 \mathrm{r} 5$,

Digitations of hippocampus, I24

Direct cerebellar tract (see posterior cerebello-spinal tract), 264, 3 or, 357

motor paths, 367

pyramidal tract (see anterior pyramidal tract)

Dorsal accessory olivary nucleus, 297, 3I 2

longitudinal fibers of pons, 272

tegmental decussation (Meynerti), I56, $157,{ }_{55}$

view of brain ventricles, I4 I

zone, 422, 427

Dorso-medial cells of cord, $340,34 \mathrm{I}$ vestibular nucleus (of Schwalbe), 280, 307

Dorso-lateral cell-group, 34I, 343, 345

vestibularnucleus(Deitersi), 280, 308

Dorso-ventral fibers of medulla, 292 of spinal cord, $35 \mathrm{I}$

Dura mater encephali, I

of brain, I, 6

of cord, 326, 327, 329

spinalis, 326

of brain and cord compared, 7

Ectorhinal sulcus, 80, 86, 90, 95

Efferent neurone, 169,171

paths, 366

root, $3^{6} 3$

svmpathetic filsers, 344

Elements of olfactory bulb, 196, 203

Embryologic divisions of the braintable, 30
Embryological method of locating tracts, $35^{2}$

Embryology of brain and spinal cord, 395

Embryonic neurones of spinal cord, I 72

Eminentia cinerea, 317

collateralis, 93, I24, I 31, I33, 4 II

medialis, 290,423

Emissive motor area of cerebrum, 63,177

Fncephalon, 3 I

general considerations, 34

End arteries of Cohnheim, I8

End-brain, 30, 33, 50, 5I, 73

End-brush of axones, $x 68$

Entry zone, 34I, 345, 359

Ependymal rells, I73, 174, 397

layer of embryo, 398, 425, 429

Epiblast, 395, 396

Epiblastic sustentacular tissue, $\mathbf{I 7 3}$

Epiphysis, 135

Epithalamus, 28

Equilibrium reflex, 393

Experimental methods of locating tracts, 353

Exterior surface of fore-brain, 5I

External arcuate fibers, 265,292

capsule, I0I, I05, II3, I45, 209, 235

layer of large pyramids of cerebrum, 184,187

perpendicular sulcus, 67,69

spinal veins, 331,332

surface of temporal lobe, 70

veins of cerebrum, 22

Facial nerve, 45, I53, 209, $32 \mathrm{I}$

Facies anterior of medulla, 286

cerebelli inferior, $25^{\mathrm{I}}$

superior, 248

convexa cerebri, 52

lateralis of medulla, 287

posterior of medulla, 288

Falx cerebelli, 2, 3

cerebri, $1,2,3,4,6,407$

Fascia dentata, 90,07 , I 3I, 200, 20 I

Fasciculus antero-lateralis proprius. 297, 305, 3I I, 34 I, 345, 354

ascendens cerebelln-spinalis anterior, 298,356 
Fasciculus cerebello-spinalis posterior, 301,357

cerebro-spinalis anterior, 355

lateralis, $35^{8}$

cuneatus, 335, 34 I, 36r, 427

descendens cerebello-spinalis anterior, 298, 356

gracilis, $335,341,360,427$

lateralis proprius, 298

longitudinalis inferior, I 31,201 , 237,240

medialis, $156,276,296,354$

pyramidalis, 148

superior, 237,240

ventralis (see anterior longitudinal bundle)

mammillaris princeps, IIO, 222

marginalis (Lissaueri), 34 I , 345, 359

occipito-frontalis (Foreli), 239, $24 \mathrm{I}$

olivaris, 208,277

pedunculo-mammillaris, 85 , IIo, 222

perpendicularis, $237,24 \mathrm{r}$

posterior proprius, 362

thalamo-mammillaris (Vicq d' Azyri), 85, IIo, 237

uncinatus, $237,239,240$

Fasciola cinerea, 82, 97, 414

Fastiguum, 244, 247

Felt-work of Kaes, I84, I9I

Fiber zones, 184

Fibers of granular layer of cerebellar cortex, 260

of Meynert, 192

of superficial layer of cerebral cortex, 257

Fibrillie of neurones, 167

Fibrous sheath of Henle, r68

Fifth nerve, 3 ventricle, III, I2I, 4I5

Fila lateralia pontis, $27 \mathrm{I}$

Fillet, 159 decussation, 292, 305, 425

Filum terminale externum, $33 \circ$ terminale internum, $33^{\circ}, 333,334$, 334

Fimbria hippocampi, I24

First cerebral vesicle, 35

Fissura calcarina, 25, 86, 89

cerebri lateralis, $53,55,57,6 \mathrm{r}$, $63,9 \mathrm{x}$
Fissura chorioidea, $80,86,90,95$, 4 II

collateralis, $75,80,8 \mathrm{I}, 86,90$, I 3 I hippocampi, 80, 36, 90, 95, I 3 I, 201

longitudinalis cerebri 35,39 , $43,52,53$

mediana anterior, 337

posterior, 337

occipito-parietalis, 409

rhinalis, 93

transversa cerebelli, 36

cerebri, 35,55

Fissural arteries, $33 \mathrm{I}$

Fissure, 52

Fissures of convex surface of cerebrum, 52

of medial and tentorial surface, 85

of spinal cord, $335,337,430$

Flechsig's oval tract, $36 \mathrm{I}$

sensory bundles of internal cap sule, 232

superior nucleus, 308

Flexures, 402

Flocculus, i. of cerebellum, 209, 245, $\mathbf{2 5 2}, 25.3,420$

Floor of fourth ventricle, 246, 270, 290, 314, 334

Floor-plate of neural tube, 400,422 , 427

Folium vermis, $87,243,247,251$

Foramen crecum (Vicq d'Azyri), I53 interventriculare (foramina interventricularia) (Monroi), 30 , 87, II 2, I 32, I 4 I , 4I I

Forceps major of corpus callosum, I08, 123

minor of corpus callosum, ro8, I 20

Foreli's ventral tegmental decussation, ${ }_{5} 6$, I $57, I_{59}$

Fore-brain, 30, 3I, 35, 50, 51, 407

Formatio reticularis of cord, 335 of mid-brain, 155, 157, 159, I6r, 273, 339 of pons, $273,275,277$

Fornix, II, 30, 87, 9I, 95, 97, ror, I09. I 2 I, I 29, I3I, I 45, $237,238,4 \mathrm{I} 2,4^{\mathrm{I}} 3$

Fossa cerebri lateralis, 4I 3 interpeduncularis, $x_{4} 6, \mathbf{I}_{53}, I_{59}$ rhomboidea, $3^{1} 4$ 
Fourth nerve, 3, 5, 1 3, 289 ventricle, $9,13,33,37, \mathbf{1} 47$, $242,275,285,289,3$ I 3 , 422

Fovea inferior, I49, 290, 317 superior, I49, 269, 3I 7

Foveolæ granulares, 6

Frænuluin veli, I3, I47, 246

Frenulum of Giacomin, 94

Frontal lobe of cerebrum, 37, 53, 59, $73,75,87,91,98$

part of internal capsule, Io3 pole of cerebrum, 50,53 stalk of thalamus, $10_{3}, 216,226$, 232

Fronto-marginal sulcus, 60

Fronto-pontal tract, IO3, I5I, I59, 225,226

Function of cerebellum, 242 of neurones, I $7 \mathrm{I}$ of posterior root, 364,365

Functional areas of cerebrum, I75, I 77, I 79, I 82, I 83

Funiculus anterior, 335,352 cuneatus, 288, 300, 305, 3 I I gracilis, 288, 300, 305, 3 I I lateralis, 335,352 of medulla, I 53, 287

posterior, $335,35^{2}$ separans, 270

Fusiform gyrus, 79, 8I, 95 layer, IgI

Galen's great cerebral vein, $\mathbf{1}_{3} 6$ internal cerebral veins, 129

Gambault and Philippe's median triangular tract, $36 \mathrm{I}$

Ganglia, 400, $40 \mathrm{x}$ of cerebral nerves, 400

Ganglion interpedunculare, I5 I jugulare, 49

nodosum, 49

semilunare (Gasseri), 45, 267 spinale, 364

Ganglionar gray matter of cerebellum, 260

of cerebrum, 204

Ganglionic cells (Bevin Lewis), IgI

system of arteries, $\mathrm{I} 8$

Gelatinous substance (Rolandi), 339, 34 I

Genetic nucleus, i., 42, 319, $3^{6} 3$
Genetic nuclei of anterior roots of spinal nerves, 349,363 of cerebral nerves, 3 I9, 324 nucleus oculomotor nerve, 45, I 52,223

trochlear nerve, $45,152,22.3$

Geniculate bodies (metathalamus), 5 r, $204,207,4 \times 7$

Gennari's or Baillargic line, I8 8

Genu capsulæ internæ, Io3, I I 3 inferius of central sulcus, 56 internum of facial nerve, 283 superius of central sulcus, 56 of corpus callosum, I I, 95, I08, IOQ, I20, I 2 I

Germinal area, 35 cells of neural crest. I72 of His in neural tube, I 72

Giant pyramids (Betz), I88

Globus pallidus of lentiform nucleus, II6, I 45, 209, 235

Glomus chorioideum, II, I2O, I25, I 29

Glossopharyngeal nerve, 5, 46, I53, $209,293,320,32 \mathrm{I}$

Golgi cells, I 65 of spinal cord, $165,340,347$ dendraxones, I9I

Goll's column, 300,360

Gordinier's writing center, I75, I78, 182

Gowers's tract, 276,356

Granulationes arachnoideales, 5

Granular layer of cerebellar cortex 258

Granule cells of cerebellum, 258, 259

Gray anterior commissure, $335,35^{\circ}$ commissure of cord, $335,33^{8}$, $35^{\circ}$

crescent of spinal cord, 339

matter of cerebellum, 256

of cerebrum, I64

of medulla, 302

of pons, 278

of spinal cord, $33^{8}$

of stratum nucleare, 278,302

Gratiolet's radiatio occipito-thalamica, 217

Great anastomotic vein (of Trolard), 23

cerebral vein (Galeni), 3, 10, 2r, I36

middle meningeal artery, 6, 7 
Gudden's inferior commissure, 218 , 221, 235

Gustatory center, $\mathbf{1 7 9}, \mathbf{1} 8$ r, 183 paths, 389 radiations, 232

Gyri breves insulæe, 65,72 insulee, $65,72,235$ occipitales laterales, 68 superiores. 68 temporales transversi, 70 transitivi, 64

of convex surface of cerebrum, 59

of medial and tentorial surface, 93

Gyrus angularis, $61,63,67$ centralis anterior, $59,60,6 \mathrm{I}$ posterior, $6 \mathrm{I}, 63,64$ cinguli, 93, 95, 97, 203 cortex, of 203 circumambiens, 82, 94 cunei, 89

cuneo-lingualis, 90 fornicatus, 93, 95, 97 frontalis inferior, 59, 6I medius, 59. $6 \mathrm{I}$ superior, 59, 6I, 95, 97

fusiformis, 75, 8I, 97

hippocampi, 79, 81, 93, 94, 95, 97

lingualis. $8 \mathrm{r}, 95,97$ longus (furcalis) insulæ, 65, 72 marginalis, 97

orbitalis anterior, 74,75 lateralis, 74 medialis 74,75 posterior, 74,75 profundus transitivus, 64 rectus, $74,75,95,97$ semilunaris, 82,94 subcallosus, $75,87,9$, 97 , I07, $200,4 \mathrm{I} 4$

supracallosus, Io7, 4I 4

supramarginalis, $6 \mathrm{r}, 63,67$

temporalis inferior, $6 \mathrm{I}, 70,7 \mathrm{I}$, 75 medius, 6r, 70,71 superior, $6 \mathrm{I}, 70,7 \mathrm{I}$

transversus insul:e (of Eberstaller), 72

H-shaped column, 339

Helwig's triangular tract, 298, 305 , $3^{12}, 357$
Hemisphere vesicle, 405, 409

of cerebellum, 4I, 242, 245, 252, 4 I9

of cerebrum, $35,39,43,47$, 53, 405

Hemispheria cerebelli, 242, 245, 252

Hiatus (Sylvii), 99, I I 5

Hind-brain, 3 I, 33

Hippocampal fissure, $80,86,90,9 I$, 95, IOI, 20I, 409, 4 II, 416

gyrus, $8 \mathrm{I}, 94,95$

region, $20 \mathrm{I}$

Hippocampus, 80, 90, Ior, II 3 , I24, I 3 I, I 33, I 45, 409. $4 I 4,4$ I 6

minor (see calcar avis), I 25, I 33

His (germinal cell in neural tube), I 72

Histologic layers of neural tube, 427 of spinal cord, 397

Hoche's bandalette, 362,345

Horizontal anterior ramus of lateral fissure $\mathbf{5 7}$,

part of interparietal sulcus, 57

sulcus of cerebellum, 243,245 , 247,420

Hypoblast, 396

Hypoglossal nerve, 5, 49, I 53, 29.3 , 322

nucleus, 295, 303

triangle, $3\ulcorner 7$

Hypophyseal region, 37

Hypophysis cerebri, $37,38,47,82$. 84,267

Hypothalamic nucleus, I.43, I 45

Hypothalamus, 82, I43, 22 I

Impressio petrosa, 53

Incissura pratoccipitalis, $6 \mathrm{r}$, temporalis, 80 tentorii, 2

Indifferent cells, 397

Indirect motor paths, $37 \mathrm{x}$

Inferior cerebral veins, I3, 23 . colliculi of corpora quadrigem. ina, I47, I49, I63, 220 , 418

commissure (Guddeni), 2I8, 22I, 235

efferent veins of pons, 27

external cerebellar veins, 29 frontal artery, I9, 22

fovea, $270,290,317$ 
Inferior frontal gyrus, $39,59,60 ; 6$ I sulcus, $39,57,59$

gray commissure of fore-brain, 84

horn of lateral ventricle, Ior, I05, I23, I $33, I_{4}$ I, I 45

internal frontal artery, 19

lamina of internal capsule, 99, I I 3, I I 5, 123

lateral occipital gyrus, 6I

longitudinal fasciculus, 137,237 , 240

medullary velum, 13, 244, 245, 247, 262

olivary nucleus, 209, 297, 305, 3T2, 425

parieial lobule, $61,63,64$

peduncles of cerebellum, 246,264

of thalâmus, I00, I 45

petrosal sinus, $3,3,4,5$

postcentral sulcus, $\mathbf{5 7}, \mathbf{6}_{3}$

precentral sulcus, 39, 57, 59, $4 \mathrm{I} 4$

quadrigeminal colliculus, I 3 , I47, I49, 163, 204, 207, 220,418

sagittal sinus, 2, 3

semilunar lobule, 252, 255, 255, 420

surface of cerebellum, 25I, 252 of island, 74

striate veins, 23

temporal gyrus, 6I, 70, 7I, 79

sulcus, 70, 80, 8I, 86, 93

Infero-lateral border, 52

Infundibulum, $4,9,47,75,82,84$, I09, I3I

Insula (Reili), 7I, 74, 75, I 05, I 25 , $207,209,267,410$

Inter-brain, $30,33,37,50,5 \mathrm{x}, 73$, I27, 4I 5

Interior surface of fore-brain, 98

Interlobar boundaries, 55

Intermediate nerve, 45, I 53, 320

olfactory stria, 77,78

tract Ico, I48, I 59, 225, 226

Intermedio-lateral column of cellbodies, 34I, 344

Internal capsule. 99, ror, r 05, I09, I I 3 , I I 5, I 45, 209, 226 , 412,417

carotid artery, 3, 4, 5, I4, I 5, I9 cerebellar veins, 29
Internal layer large pyramids of cerebral cortex, I84, I88

medullary lamina of thalamus, 208

orbital artery, I6

spinal veins, 33 r, 332

veins of cerebrum, 21

Interparietal sulcus, $39,43,57,63$, $4 I 4$

Interpeduncular fossa, I 45, I46, I 53, I 59, I6I, 209

ganglion, I5I

space, $3^{8}$

Interventricular foramen (of Monro), $36,37,95$, II $2,128,235$, 405

Intumescentia cervicalis, 333, 334, 335

lumbalis, 334,335

Inverted pyramids of Martinotti, I9I Island (of Reil), 71, 74, 75, 98, I05, 414

Isthmus gyri fornicati, 94

rhombencephali, 28, 33, 4I8, 4I9

Jugular ganglion, 49

Kaes, medullation late in life, 4 I 2

Key and Retzii, apertura lateralis ventriculi quarti, 9, 290

Lamina affixa, II9

chorioidea epithelialis, II5, II9, 124

cinerea terminalis, $30,36,38,82$, $83,87,9 \mathrm{I}, 95$, I09, I3I, I39, 22I, 4I3

quadrigemina, $30,16_{3}$

rostralis of corpus callosum, ro8

Laminx medullares, 262

Lateral apertures (Key and Retzii), 9, 10, 290

area of medulla, 293, 298

cerebral fossa, 4IO, 4 I 3

cochlear nucleus, 309

column of cell-bodies, 340,34 I, 345

column of medulla, 287

columna of cord, 335,344

lasciculus proprius, 287,298 , 354

fillet, I3, I59, I60, I6 I, 246, $274,275,309$ 
Lateral fissure of cerebrum (Sylvii), $53,55,57,6 \mathrm{I}, 63,9 \mathrm{I}, 4 \mathrm{I} 4$ geniculate bndy, I40, I43, I57, 204, 207, 21 8

longitudinal stria (see longitudinal stria)

nucleus of thalamus, 2 I I

occipital gyri, $43,68,69$

sulci, $\mathbf{5 7}, 68$

olfactory stria, 75, 78, 207

orbital arteries, 16

gyrus, 74

ponto-spinal tract (Collieri), 355, 373

pyramidal tract, $287,294,34 \mathrm{I}$, $345,35^{8}$

recess of fourth ventricle, 289

sulcus of mid-brain, I59, 243

surface of medulla, 287

ventricles, $30,34,36,37$, III, II 2 , I 45, 405, 4I 2

view of brain ventricles, $\mathbf{r} \mathbf{4} \mathrm{I}$

Layers of cells of cerebral cortex, 184

Layer of fusiform cells of ccrebral cortex, 184

of large pyramids, external, 184 , I 87

of large pyramids, internal, I84, I 88

of medium sized pyramids of ceretral cortex, 184,187

of small pyramids of cerebral cortex, 184,187

of stellate and polymorphous cells of cerebral, cortex, I84, I88

Law of Waller, 353

Lemniscus, 159

lateralis, 160,274

medialis, $160,273,295$

superior, 274

I.enticulo-striate arterp, 18

Lentiform nucleus, I0I, I05, II 3 , I $6,145,204,226,235$

Lesions of anterior columna, 344

of corpus striatum, 208

of gray substance in cord, $35^{\circ}$

of special sense paths. 389

in medulla, $3 \mathbf{I} 3$

in posterior columns, 362

Ligamentum denticulatum, 3, 327, 329, $33 \circ$

Limbic lobe, 73, 8I, 87, 91, 93, 97, 98

Limen insulæe, 72, 77, 79
Line of Baillarger, I84, I88, 213

Linea splendens, 327,329

Lingual gyrus, 95, 97

I.ingula cerebelli, I3 $_{3} 87,247,250$

Lissauer's fasciculus marginalis, $34 \mathrm{I}$, 345,359

Lobes of convex surface of cerebrum, 59

of lower surface of cerebellum, 253

of medial and tentorial surface of cerebrum, 93

of superior surface of cerebellum, 250

Lobules of cerebellar vermis, $\mathbf{2 4 7}$, 249,253

Lobulus biventer, 245, 254

centralis, $87,245,250$

gracilis, 245,256

paracentralis, 64, 97

parietalis inferior, $6_{3}, 6_{4}$

superior, $6_{3}, 6_{4}$

I,obus centralis cerebelli, $25^{\circ}$

culminis cerebelli, $\mathbf{2 4 3}, 25^{\circ}$

declivis cerebelli, $\mathbf{2 4 3}, 250$

folii vermis, $243,25 \mathrm{I}$

frontalis, 59

limbus, $8 \mathbf{I}$

lingulie cerebelli, $25^{\circ}$

noduli, 253

occipitalis, 67

parietalis, 63

pyraformis, 97

pyramidis, 254

temporalis. 69

tuberis, 255

uvulæe, 254

Localization of cerebral function, $x 77$

Locus cæruleus, $269,270,317$

Long association fibers of cerebrum, 238

I ongitudinal fibers of medulla, 293

of lateral area of medulla, 298

of pons, 272, 275

of posterior area of medulla. 300

of spinal cord, $35^{2}$

fisssure of cerebrum, 35, 39, 43, $52,53,209,235$

strice, medial and lateral, $97, \mathrm{IO}_{7}$, I 7

Lower segment paralysis, 344

surface of cerebellum, 253 
Lumbar enlargement of spinal cord, 334,335

Luys, center median of thalamus, 211 nucleus hypothalamicus of, I43, I5 1,218

Lymph spaces of cerebellum, 30

of cerebrum, 23

of medulla, 24

of spinal cord, 332

Magendi, apertura mediana ventriculi quarti, 290

Mammillary bodies, $38,5 \mathrm{I}, \mathbf{7 5}, 85$

Mantle layer, $398,425,428$

Marginal gyrus, 95, 97

sinus, 5

tract, Lissauer's, 34I, 345, 359

velum, $397,397,398,425,428$

Margo-infero-lateralis, 52, 75

Margo occipitalis lateralis, 52, 75

medialis, 52,75

orbitalis medialis, 52,75

superciliaris, $39,52,75$

supero-medialis, 39,52

Martinotti's inverted pyramids, I9I

Massa intermedia, 95, I 37, I39, I 45, 22I, 222, 4I 7

Medial accessory olivary nucleus, $295,297,305,312$

cerebral veins, 22

column of cell-bodies, 340

fillet, $157,159,160,161,23 \mathrm{I}$, $273,275,277,292,295$, $295,297,305$

geniculate body, I40, I43, I46, I 47, I 49, I 57, I 59, 204 207, 218

longitudinal bundle, 156,157 , I 59, I6I, 275, 276, 277, 295, 296, 297, 305, 3 I I, $34 \mathrm{I}, 345,354$

stria (see longitudinal stria), nucleus of thalamus, 208

occipital border, 52,75

olfactory stria, $75,77,78$

orbital border, 52,75

gyrus, 74,75

ponto-spinal tract, Collieri, ${ }_{5} 8$, $297,355,373$

surface of fore-brain, 92, 95

and lateral olfactory strix, 47 , $75,77,78$
Median aperture (Magendi), 9, 9, 10, I $3,289,290$

triangular tract (of Gambault and Phillipe), $36 \mathrm{r}$

Medulla oblongata, 24, 3 I, 33, 35, 36, $37,38,87,242,284,422$

spinalis, $31,333,334,335$

Medullary body of cerebellum, 242, 244

groove, 396

laminæ, 262

plate, 395

ridges, 395

striæ, I 49, 270, 290, 309

Medullation, $168,169,429$

Membranes, of brain, I of spinal cord, $326,327,329$

Meninges encephali, I spinalis, $326,327,329$ of brain, $I$ of spinal cord, 426,327

Mesencephalic flexure, 402

Mesencephalon, 28, 30, 3I, I44, $402,4 \mathrm{I} 7$

Mesoblast, 396

Mesoblastic sustentacular tissue, 174

Metathalamus, $140,143,218$

Metencephalic flexure, 402

Metencephalon, 28, 31, 33, 36, $266,402,418$

Methods of locating tracts of fibers, $35^{2}$

Meynert's association fibers, 192,236 commissura superior, 208, 218 , 221

dorsal tegmental decussation, ${ }_{5} 6$ I 57, I 59

solitary cells, 195

Mid-brain, 30, 3I, 33, 36, 37, 50,'87,

I $44,243,417$

Middle association center, 183

brain vesicle, $3 \circ$

cerebral artery, 4, I5, I6

commissure, 137,222

frontal gyrus, 39, 59, 6r sulcus, 57,60

internal frontal arteries, $\mathbf{1 6}$

meningeal artery, 3, 5, 6, 7

or lateral occipital gyrus, $6 \mathbf{r}$

peduncles of cerebellum, 247 . 264

temporal gyrus, 6I, 70, 7x sulcus, 63,70 
Midgracile sulcus, 42I

Mixed ascending and descending tracts, $35^{2}$

Monro, interventricular foramen of, 95, II2, I28, I4I, 405 sulcus hypothalamicus of, $9 \mathrm{I}, 95$, 415

Moss-like appendages of Cajal, 259, 260

Motor area of cerebrum, 177 center for foot, 98,178 cerebral nerves, $32 \mathrm{I}$ fibers of cerebrum 225, of internal capsule; 100, I03 memory center 67 , neurone, I69 paths, $229,366,367,37 \mathrm{I}$ roots of cerebral nerves, $32 \mathrm{I}$ root of spinal nerve, 363 speech center, 178

Motorial end-plates, 168, 169

Myelencephalon, 28, 31, 33, 266, $284,402,422$

Myelin sheath of axones and lendrites, $\mathbf{1 6 8}$

Myelinization, 4I2, 429

Naming center, 7 I, I8I

Neopallium, 28, 98

Nerves of arachnoid, 9 of dura mater, 7 of pia mater, 13

Nervi cerebrales, 3 I 8,42 I, 425 olfactorii, 42,320

Nervus abducens, 45, 267, 321 accessorius, 49, 267, 322 acusticus, 46, 267, 320 facialis, $45,267,32 \mathrm{I}$ glossopharyngeus, $46,267,320$, 321.

hypoglossus, $49,267,322$ intermedius, $45,267,320$ oculomotorius, $42,267,32 \mathrm{I}$ opticus, $25,42,83,267,320$ trigeminus, $45,267,320,321$ trochlearis, $45,267,32 \mathrm{I}$ vagus, $46,267,321$

Neural canal, 396 crest, 35, I72, 395, 400 groove, 35, 395 tube, 35, I 72, 395

Neuraxones, I68

Neuroblasts, 398
Neuroglia, I72, 399

cells, $\mathbf{I} 73$

zone, 184

of cerebellum, 260

Neurolemma (Schwann), I68, I69

Neurone I65, I67, I69, I7 1,398 center, ${ }^{5} 57$

Neurones of head of posterior columna, 347

Neuropore, 405

Nuclei of cerebellum, 260, $26 \mathrm{I}$ of cerebral nerve, $278,28 \mathrm{I}, 303$ of reticular formation, 156,278 , 279, 304

of thalamus, 208

Nucleolus of neurone, 169

Nucleus alæ cinereie, 297, 303, 304, $305,306,3$ I 5

ambiguus, 297, 303, 304, 3 I 5

amygdalie, II 3, II9, I99, 207, 239

arcuatus, 303

caudatus, in 6

centralis inferior, 278,279 medius, 278,279

superior, 278,279

commissuralis, 305,306

corporis trapezoidei, 270

dentatus, 255, 260, 261, 277

dorsalis (Stillingi and Clarki), 348

dorso-lateralis (of Dieters), $28_{3}$, 30.3

emboliformis, 260, 26r, 277

fastigii, $261,277,260,262$

funiculi cuncati, $270,305,3 \mathrm{I}$ I, 310

gracilis, 270, 305, 3 I I, 3IO

globosus, 260, 26r, 277

habenula, I 10, 2 II

hypothalamicus Luysi, I43, I5I, 204,218

incertus, 270

intercalatus, 270

lateralis inferior, 297, 303, 304 medius, 278,270

superior, 156,220

lentiformis, I0I, 105, xI6, 209

n. abducentis, 28r

cochlearis, 270, 315, 309

facialis, 282

oculomotorii, 45, I55, 315

trochlearis, 45, 155, 315 
Nucleus vestibularis, 270, 283, 305, 307,3 I 5

olivaris inferior, $3^{12}$

originis, $42,319,324,363$

pontis, $272,278,421$

ruber, $2 \mathrm{I} 7$

terminalis, 42, 319, 365

tractus solitarii, 302, 305, 306

spinalis n. trigemini, 295, 305 , 3I I, 3 14, 306

of anterior tubercle, 2II

of abducent nerve, $277,28 \mathrm{r}$, 315

of accessory nerve, $304,3^{1} 5$

of Bechterew, 277, 308

of colliculus inferior, $161, \mathrm{r}_{4}$, 220

of corpus mammillare, $85, \mathbf{I} 57$

of descending root of trigeminal nerve, 155

of descending ront of vestibular nerve, $280,283,308$

of external arcuate fibers, 302

of facial nerve, $277,282,3$ I 5

of fascia dentata, 201

of fourth nerve, 155,3 I 5

of hypoglossal nerve, 303,315

of neurone, 167,169

of oculomotor nerve, $\mathrm{J}_{52}, 3$ I 5

of optic nerve. 315

of pulvinar, 208

of third nerve, 152,315

of trapezoid body, 272, 278, 279, 280

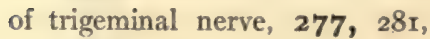
3 5

of trochlear nerve, $152,223,315$

Ninth, tenth and eleventh nerves, 3

Nissl bodies, 167,169 degeneration, $\mathbf{I} 7 \mathbf{I}$

Nodes of Ranvier, 168 , I69

Nodular ganglion, 49

Nodule of cerebellum, 245, 247,

$$
252,253
$$

Nodulus cerebelli, 87, 253

Notochord, 396

Obex, 13, 270

Oblique fasciculus of pons, 267, 269

Occipital lobe, $\mathbf{5 3}, 67, \mathbf{7 5}, \mathbf{8 7}, 9 \mathrm{I}, 98$ part of internal capsule, Io3

pole, 5o, 53, 68

sinus, 2,5
Occipito-parietal fissure, 409, $4 \mathrm{I} \mathrm{I}$ sulcus, $17,43,53,56,57,86$, $89,91,95$

Occipito-thalamic radiation, $\mathbf{1 3 7}$, $2 x_{3}, 217,226,234$

Ocular reflex arcs, 355

Oculomotor nerve, 42, I45, I53, 209, 32I

sulcus, I 46,159

Olfactory bulb, $37,47,75,77,195$, 203,207

center, 97

cortex, I95

diverticulum, 36, 37

islets (Calleja), 199

lobe, $73,77,98$

nerves, 42,320

path, $3^{8} 3$

projection fibers, 232

sulcus, 74

strix, 75,78

tract, $47,75,77,78,267$

triangle, $75,77,79$

Oliva, 287

Olivary fasciculus, $162,208,277,298$ nucleus, inferior, 295, 297, 312, 423,425

pedicle, 279

Olive, 3I, 4r, I 53, 287

Operculum. 64, 70, 410

Ophthalmic division of fifth nerve, $\mathbf{5}$

Optic center, $18 \mathrm{x}$ chiasma, $30,38,47,75,83,95$, $147,153,2$ I $3,235,415$

commissure (see optic chiasma), I 3 I

cup, 405

nerve, $4,38,42,83,153,213$, 320

path, 21 3,384

radiation, IO4, 21 3, 217, 226, 232, 234

recess, 9I, I39, 235, 405

reflex center, 163,324

tract, 219, 355

tract, $4,38,47,75,83,145$, $147,1_{53}, 1_{57}, 1_{59}, 209$, 235,267

vesicle, 35,405

Orbital lobe, 73

Orders of neurones, I68

Origin of anterior root of spinal nerve. $3^{6} 3$ 
Origin of posterior root of spinal nerve, 364

Oval bundle of His, 427,429 tract (Flechsigi), 345, 36r

Pacchionian bodies, 5

Paracentral lobule, 64

Paramedial sulcus, $\mathbf{3 9}, \mathbf{5 7}, 59,60$

Parietal lobe, 53, 63, 87, 91, 98 stalk of thalamus, I03, I04, 226

Parieto-temporal artery, I 7, 22

Parolfactory area (of Broca), 79, 86

Pars anterior rhinencephali, 77 basilaris pontis, 266

dorsalis pontis, 266,273

frontalis capsulæ internæ, I03, I 3

mammillaris hypothalami, $\mathbf{2 8}, 82$, 22I, 4I 7

occipitalis capsulæ internæ, то3, I 3

optica hypothalami, 28, 82, 22I, 415

posterior rhinencephali, 77

Pathological method of locating tracts, 353

Path carrying visceral, muscular and tactile impulses, $38 \mathrm{r}$

for tactile and muscular sense, 375,377

for tactile, pain and temperature impulses, $276,379,3^{8 r}$

through red nucleus, 370,37 I

Peduncle of corpus callosum (see gyrus subcallosus)

of flocculus, 245,254

Peduncular fibers of cerebellum, 262 of cerebrum, 224

Pedunculi cerebri, 28, 30, 37, 146, I 47

Pedunculus flocculi, 254

Perikaryon, 167

Permanent fissures and sulci of cerebrum, 409, 4I4

Perpendicular fasciculus, 237, 241

Petrosal glossopharyngeal ganglion, 46

Petro-squamosal sinus. $\mathbf{5}$

Physiological method of locating tracts, 353

Pia mater encephali, 9

spinalis, 329

of brain, 9,9
Pia mater of spinal cord, $\mathbf{3 2 9}, 329$

of brain and cord compared, 13

Pineal body, 36, 37, 5I, 9I, 95, ro9, ${ } 3^{1}, I_{32}, I_{37}, I_{47}, I_{57}$, 243,4 I 6,4 I 6

recess. 132,416

stria, I35, I37, 239 (see stria medullaris thalami)

Pituitary body, 84 (see hypophysis)

Plexiform layer of cerebral cortex, I84

Plexus basilaris, 4

chorioideus ventriculi lateralis, I2O, 201

chorioideus ventriculi quarti, 290 venosi vertebrales interni, 332

Plica chorioidea of fourth ventricle, 4 I9

Pole of island, $7 \mathrm{I}$

Polus insulæ, $7 x$

Pons (varolii), 24, 28, 31, 33, 37, 38,87 , I O I, I 45, I 53, 242 , $266,418,42 \mathrm{I}$

Pontal arteries, 24

Pontine flexure, 402

Ponto-spinal tracts, 280, 373

Postcentral lobule, 72

sulcus of cerebellum, $\mathbf{2 4 7}, 248$, 420

of cerebrum, 63, 4IO

Postdeclivil sulcus, 243, 247, 249, 420

Post-dorso-lateral cells, 343 (see inintermedio-lateral)

Postgracile sulcus, $42 \mathrm{I}$

Postnodular sulcus, 247, 252, 252 . 420

Postparietal gyrus, 63,67

Postpyramidal sulcus, 245, 247, 252, 253,420

Posterior anastomotic vein, 23

area of medulla, 293, 300

association center, I 82

brain vesicle, 33,35

calcarine fissure, 89

central gyrus, 39, 6I, 63, 64

cerebellar notch, $36,243,244$ 252

cerebello-spinal fasciculus, 264 , 305,3 I I, 288, 34I, 357

cerebral artery, 15, 17, 25

chorioidal artery, I5, I7, I9

columna, 335, 339, 347, 427, 429 
Posterior commissure of cerebrum, 95, I09, I3I, I32, I 37, 416 of cord, $335,35^{\circ}$

communicating artery, 4, I4, I 5 fasciculus proprius. 362

horn of lateral ventricle, 123 , I 3 I $, I_{33}, x_{37}, I_{4}$ I

inferior cerebellar artery, 15,24 , 27,29

intermediate sulcus of cord, 335 , 338

internal frontal arteries, 16,25

lateral sulcus of cord, 335,337 430

lateral sulcus of medulla, 4I, I49, I $53,286,295,297$

longitudinal bundle (see medial longitudinal bundle)

median fissure of cord, 335,337 , $349,43^{\circ}$

median fissure of medulla, 285

median vein, 24

orbital gyrus, 74,75

parolfactory sulcus, 86

perforated substance, 38,47 , I09, I32, I46, I5I

pillar of fornix, 133 (see crus fornicis)

radicular artery, $33^{I}$

vein, $33 \mathrm{I}$

ramus of lateral fissure of cerebrum, 57

root of spinal nerve, 327,334 , $349,363,364,399,427$, 428,429

slender lobule, 252, 255

spinal artery, 24, 27, 33 I

subarachnoid space, 8,329

surface of medulla, 270,288

of pons, 269,270

white column of cord, 345,399

Postero-lateral column (Burdachi), 300

descending tract, 345,362

ganglionic arteries, 19, 21

Postero-medial column (Golli), 300

Postero-median ganglionic arleries, $15,18,19$

Precentral lobule of island, 72

sulcus of cerebellum, 247, 248, 420

of cerebrum, 59, 4IO, 4I4

Precuneate gyrus (see precuneus)
Precuneus, 95, 97

Predeclivil sulcus, 243, 247, 249, 420

Preolivary nucleus, 278,279

Prepyramidal sulcus, 245, 247, 252, 252, 420

Prevertebral ganglia, 40r

Primary brain vesicles, $30,35,395$ fissures of cerebrum, 409, 4II, $4 \mathrm{I} 3$

Primitive streak, 35

Principle or chief vestibular nucleus (Schwalbe's), 307

Processes of dura mater, $\dot{I}$ of neurone, 167,169

Processus duræe matris, I

Projection fibers of cerebellum, 262, 263

of cerebrum, 224

Prosencephalon, 30, 3I, 5 I

Protoplasmic processes of neurones, 169

Psychic acustic center, 7I, I8I center of abstract concept, 60, 182,183

center of concrete concept, $\mathbf{1 8 2}$, 183

motor area, 178

optic center, $18 \mathrm{r}$

sensory area, 178,67

Pulvinar of thalamus, $140,149,207$, 2II, 3 I 5

Pupillary constriction, 394 dilation, 394

reflexes, 394

Pupillo-dilator tract, 220, 298, 355

Purkinje's cells, 257, 258, 259, 265

Putamen of lentiform nucleus, II6 $145,209,235$

Pyramid of cerebellum, 247, 252, 254

of medulla, 4I, $153,209,267$, $295,297,3$ I I, 293, 294

Pyramidal cells of cerebral cortex, I 65

decussation, 291, 3 I I

motor paths, 366

tract, 103, 148, 159, 226, 272, $277,294,355,35^{8}$

Pyramis cerebelli, 87,254 medullæ oblongatæ, 294 vermis, 254 
Quadrangular lobule $243,25025 \mathrm{I}$. 255,420

Quadrigeminal bodies, 4, 146, 147, 163

colliculus, i., $75,87,163$

lamina, $x 46,147,163$

Radiatio occipito-thalamica (optic), I04, I3I, 2 I 7

corporis callusi, 234

temporo-thalamica (acustic), I04, 217

Radiations of corpus callosum, 209, 234

of corpus striatum, 207, 209

of Meynert, 192

Radiary zone of cerebral cortex, 184

Radicular cells, 340

veins of medulla 24

Radix anterior of spinal nerve, 349, $3^{6} 3$

posterior of spinal nerve, 349, 364

Ranvier's nodes, 168,169

Receptive auditory center, 70, 7 1 , 18 I

olfactory center, $82,94,97,179$, 183

visual center, $8 \mathrm{I}, 98, \mathrm{I} 8 \mathrm{I}$

Recessus triangularis, $128, I_{3} 6,1_{37}$

Red nucleus, I I I, I 57, I 59, 204, 2 I 7

Refex arcs, 390, 391,392

connections of abducent nucleus, 282

of cochlear nucleus, 310, 323

of facial nucleus, 282

of genetic nuclei, 325

of motor nuclei in medulla, 305

of nucleus alæ cinerea, 306

of olfactory nucleus, 324

of trigeminal nuclei, 28I, 307

of vestibular nuclei, 308,323 , 389

mechanism of cord, 343

paths, 390

Regio tegmentalis hypothalami, I43

Relations of arachnoid, 8, 326, 327, 329

of dura mater, $1,326,329$

of pia mater, 9, 327. 329

Respiratory reflex, 393

Restiform body, I 3, 4I, 246, 252, $255,280,288,295,297$, 301,423
Rhinencephalon, 28, 77, 97, 98, 406

Rhombencephalon, 3I, 33, 38, 242, 266,284

Rhomboid lip, 285, 422, 423

Rolandic angle, 56

Rolando, central sulcus of, 56,57 , 61,410

gelatinous substance of, 339, 34 I 345

Roof epithelium of fourth ventricle, I3, 289, 3r4

of third ventricle, $\mathrm{r}_{32}$

of fourth ventricle, 289,289 , $3 \mathrm{I} 4$

Ronf-plate, 400, 416, 422, 422, 427

Roots of optic tract, $83,147,153$

of spinal nerves, 349,362

of twelve cerebral nerves, 42

Rostrum of corpus callosum, 95, 108, 120

Rubro-spinal path, $370,37 \mathrm{I}$

tract, I61, I62, 276, 295, 297, $300,305,3 \mathrm{II}, 34 \mathrm{I}, 35^{8}$, 370,371

Salivary nucleus, 283

Schultze, comma tract of, 34 I, 36 I

Schwalbe's dorso-medial vestibular nucleus, 280,307

Schwann's sheath, I68

Second cerebral vesicle, 35 cervical nerve, 3

Secondary brain vesicles, 402,403 sulci and fissure, 4IO, 4I I , 4I 4

Sections of fore-brain, 407 of spinal cord, 335

Semilunar ganglion (Gasseri), 45 nucleus, 278

Sense of Louch, 377

Sensory aphasia, $24 \mathrm{I}$

cerebral nerves, 320

conduction paths, 377

paths, 229, 377

projection fibers, 23I

roots of cerebral nerves, 320

of spinal nerve, $349,363,364$

root of trigeminal nerve, 307

tract Ciaglinski, $35^{\circ}$

Septo-marginal tract, $345,36 \mathrm{I}$

Septum pellucidum, $30,87,95,97$, III, II5, I2I, I23, 209, $235,4 \mathrm{I} 3,4 \mathrm{I} 5$

Seventh and eighth nerves, 3 
Short association fibers of cerebrum, 236,237

fiber motor paths, 373

sensory paths, 382

Simple reflex arc, 39 I

Sinus alæ parvæ, 3

cavernosus, 3

circularis, 4

duræ matris, 2

intercavernosus anterior, 3

posterior, 3

occipitalis, 2

petrosus inferior, 3

superior, 3

rectus, 2

sagittalis inferior, 2

superior, 2

transversi, 2

Sixth nerve, 3

ventricle, 335,337

Slender lobules, 255, 255

Smelling brain, 77

Solitary cells of Meynert, I95 tract, 295, 297, 302, 305, 423

Somæsthetic area, 64,98, I 81

Special nuclei of medulla, 302, 310 sensations, 383

sense fibers of internal capsule, IO4, II 5, 226

nuclei, 322

Speech center, 6o, I75, I78, I82

Spheno-parietal sinus, 3, 4

Spinal bulb, 284

cord, 3I, 333. 334, 335, 34 I, 426

ganglion. $334,364,40 \mathrm{r}, 428$

neurones, I65, 400, $40 \mathrm{I}$

reflexes, 390

tract of trigeminal nerve, 278 , $301,423,424$

Spino-cerebral reflexes, 393

Spino-thalamic tract, I6I, 23I, 275, $274,287,295,297,299$, $305,3 \mathrm{II}, 34 \mathrm{I}, 345,356$

Spino-vestibular tract, 357

Splenium of corpus callosum, $\mathbf{7 5}, \mathbf{9 5}$, 105, I08, I I 3, 109, I 2 I, I 3 I

Spongioblasts, 398,399

Stalk of pineal hody, 87, 149

Stellate cells of cerebellum, 257,259

Stem of fissura cerebri lateralis, $\mathbf{5 7}$

Stereognosis, 377
Stereognostic center, $64,98, \mathbf{1 8 2}, \mathbf{1} 83$

Stilling's nuclei, 262

Stilling and Clark (nucleus of), 348

Straight gyrus, 74, 75 sinus, $2,3,4$

Strand cells, 340

Stratum cinereum, 256 gangliosum, 256, 257 granulosum, 256, $25^{8}$ griseum centrale. $\mathbf{5} 5 \mathbf{2}, \mathbf{I}$ 57, 159 $22 \mathrm{I}, 222$

of superior colliculus, I6 3,219 intermedium, 208, 225

interolivare lemnisci, 292

nucleare, $278,293,302,303$

zonale of cerebral cortex, 184 of corpora quadrigemina, $\mathrm{I}_{3}$, 219 of thalamus, 140,208

Stria intermedia, $77,78,267$

lateralis, 78,267

medialis, $77,78,267$

medullaris thalami, $87,9 \mathrm{r}, 95$, I 35, I 37, 2 I 2, 239, 239

terminalis, I0I, I09, 115, II9, I 24, I 25, 207, 239, 4I 2

Strix medullares, 149, 270, 290, 309 olfactoriæ, 78,267

Striato-thalamic fibers, 207

Structure of arachnoid, 8,

of cerebrum, $\mathrm{I}_{4}$

and relations of dura mater, I and relations of pia mater, 9

Subarachnoid rivulets, 8

septum, 327, 329

space, $6,8,9,329$

Subcallosal gyrus, 97, I07, 4I4

Subdural space, 329

Subiculum, 199, 201

Subparietal point, 55 sulcus, 86, 9 I, 95

Substantia alba spinalis, 35I corticalis cerebelli, $242,25^{6}$ cerebri, I77

gelatinosa centralis, I73, 339

(Rolandi), 339, 345, 347

nigra, 30, ror, 145 , I46, J5I, I57, I 59, I6 I, 22I

perforata anterior, $38,75,79,200$

posterior, 38,75, I $5 \mathrm{I}$

reticularis, 291, 295, 297, 302, $303,305,423$

spongiosa, 339 
Sulci, 52

occipitales laterales, $6 \mathrm{r}, 68$

of cerebellum, 247, 248, 252, 420

of convex surface of cerebrum, 52 of lower surface of cerebellum, $25^{2}, 253$

of lower surface of cerebrum, 73 of medial surface of cerebrum, 85

of upper surface of cerebellum, 248,249

Sulcus basilaris, 153,272

centralis (Rolandi), 39, 53, 56, $57,61,410$

insulæ, 65,72

cinguli, $25,86,9 x, 95,4$ Iо

circularis insulæe, $70,7 \mathrm{I}, 75, \mathrm{I} 25$

corporis callosi, $86,9 \mathrm{I}$

ectorhinalis, $80,86,90$

frontalis inferior, $59,6 \mathrm{I}$

medius, 59, 60, $6 \mathrm{I}$

superior, 59,6 I

horizontalis cerebelli, 247

hypothalamicus, 9I, I39, 405, 4 I 5

intermedius posterior, $\mathbf{3 3 5}, 338$

interparietalis, 6r, 63

lateralis anterior, $4 \mathrm{I}, 286,338$

mesencephali, I46, I59, I6 I

posterior, 4I, 286, 337

limitans, 269, 270, 290, 4I8

nervi oculomotorii, I 46

occipitalis transversus, 6 I, 68

occipito-parietalis, 58, 6I, 86

orbitalis, 74,75

paramedialis, $59,6 \mathrm{r}$

parolfactorius anterior, 47,75 , 79

posterior, $75,77,79,95$

precentralis cerebelli, 248

inferior, $59,6 \mathrm{r}$

superior, 59,61

prædeclivis cerebelli, 249

præpyramidalis cerebelli, $25^{2}$

postcentralis cerebelli, 248

inferior, $6 \mathbf{r}, 6_{3}$

superior, $6 \mathrm{I}, 63$

postdeclivis cerebelli, 249

postnodularis cerebelli, $25^{2}$

postpyramidalis cerebelli, 25.3

rhinalis, 80

subparietalis, 86,9 I
Sulcus temporalis inferior, $70,80,86$ medius, $6 \mathrm{r}, 6_{3}, 70$ superior, $61,63,70$

valleculer, 251,252

Superciliary border, 39,52

Superficial annectant gyrus, 64

layer of cerebellar cortex, 256

middle cerebral vein, 23

transverse fibers of pons, $27 \mathrm{I}$, 275

Superior cerebellar artery, 15,27

peduncle (see brachium conjunctivum)

cerebral veins, 22

colliculus of corpora quadrigemina, 157, 219

commissure (Meynerti), 208, 218, 221

efferent veins of pons, 27

external cerebellar veins, 29

fillet, $159,160,274,296$

fovea, 270

frontal gyrus, 39, 59, 6o, 6r, 209

sulcus, $39,57,59,414$

glossopharyngeal ganglion, 46

lamina of internal capsule, 100 , I I 3, I I 5, 226

longitudinal fasciculus, 240

medullary velum, $\mathbf{1}_{\mathbf{3}}, \mathbf{8 7}, \mathbf{1 4 6}$, I47, I 49, 245, 246, 247, $252,262,263,275$

nucleus of Bechterew and Flechsig, 308

uccipital gyrus, 43, 6I, 68, 69

olivary nucleus, $277,278,279$ 280

parietal lobule, $43,61,63,64$

peduncle of cereluellum, 245

petrosal sinus, $3,4,5$

postcentral sulcus, 57,63

precentral sulcus, $39,57,59$, 4IO, 4 I 4

quadrigeminal colliculus, I37, 204, 2I9, 4I 8

sagittal sinus, $2,3,5,6$

semilunar lobule, 243, 251, 255, 420

surface of cerebellum, $\mathbf{2 4 3}, 248$

of temporal lobe, 70

temporal gyrus, $6 \mathrm{r}, 70,7 \mathrm{I}$

sulcus. $57,63,70,410$

Supero-medial border, $39,5^{2}$ 
Supracallosal gyrus, 97, 107, 4I 4

Supramarginal gyrus. 43, 61, 63, 67 Supraradiary zone of cerebral cortex, I 84

Surface of cerebellum, 248 of cerebrum, 34, 5 I

Surfaces of medulla oblongata, 286 of mid-brain, I44

of pons, 256

of spinal cord, 337

of thalamus, 140

Sustentacular tissue, 167, I 73

Sylvian point, 55

Tænia semicircularis, II5, II9, I25 (see stria terminalis)

terminalis, II9

ventriculi quarti, 1499, 270, 297

Tapetum, I23, I24, I 3 I, I 37, 239

Tassel neurones, I99

Tegmental decussations, ${ }_{5} 6$

hypothalamic region, I43

Tegmentum, a, of mid-brain, 30,75 , I09, I32, I46, I52

Tela chorioidea ventriculi quarti, to tertii, ro

Telencephalon, 28, 30, 402, 403, 404

Telodendria, 167,169

Temporal artery, $\mathbf{I} 7$

lobe of cerebrum, $37,53,69,75$, 9r, 98

pole, 53,70

Temporo-occipital gyrus, 8 I, 97 sulcus, $8 \mathrm{I}$

Temporo-parietal artery, is

Temporo-pontal tract, I00, I48, I 59, 225,226

Temporo-thalamic radiation, roo, $226,233,310$

Tentorial area of cerebellum, 248 of cerebrum, 79

notch, 2,4

Tentorium cerebelli, 2, 3, 4

Terminal ganglia, 4 어 nuclei of optic nerve, 3 I 5, 323

nucleus, 42, 319, 322, 349, 364, 365 of olfactory nerves, 42,196

Tertiary sulci, 4 II I

Thalamus, i, 28, 30, 37, 51, I o r, I05, II9, I25, I39, I45, I47, I57, 208, 209, 4I 6, 417

Thalamo-mammillary fasciculus (of Vicq d'Azyr), 145, 222, 237
Thalamo-striate fibers, 208

Third cerebral vesicle, 35

nerve, $3,4,5,42$ ventricle, $9,30,34,37$, I O I, III $127,137,145,209$

Threshold of island, 72. 77

Tigroid bodies, I67

Tonsil of cerebellum, 245, 252, 254, 420

Torcular herophili, 2

Tracing of impulses, 366

Tracts of fibers in tegmentum, 156 of spinal cord, $34 \mathrm{I}, 345,354$, 429

Tractus cerebro-cortico-pontalis frontalis, $103,15 x, 225$

temporalis, $100,148,225$

cerebro-spinalis pyramidalis, 226 intermedius, I00, T48, 225

olfactorius, 77,78

optici, 83

solitarius, $285,295,297,302$, 422,423

spinalis $\mathrm{n}$. trigemini, 277,278 , $288,295,297,301,305$, 3 I I

Transverse fibers of medulla, 29I

of pons, $27 \mathrm{~T}$

of spinal cord, $35^{\mathrm{I}}$

fissure of cerebellum, 36

of cerebrum, $35,43,53,55$, IO9, 4II

occipital sulcus, $43,57,68,69$

sinuses, $2,3,4,5$

temporal gyri, 70, I05, I2I, 125

sulci, 70

Trapezoid body, 271, 272, 277, 280

Triangle of habenula, $\mathbf{I}_{4} \mathrm{O}$

of lateral fillet, I52, I64

Triangular tract of Helwig, 298, 305, 3I I, 3I 2, 34 I , 345, 357

Trigeminal nerve, 4r, 45, I53, 209, $320,32 \mathrm{I}$

nucleus, 224

Trigonum vagi, 290,317 , (see ala cinerea)

collaterale, I23, I24

habenulæ, 140, 137, 243

n. hypoglossi, I49, 270

olfactorium, $47,75,77,79,200$

Trochlear nerve, 45, 146, 147, 32 I

nucleus, 223 
'Trolard's great anastomotic vein, 23

Truncus of corpus callosum, 95, ro8

Tuber annulare, 266 cinereum, $30,38,47,75,82$, $84,87,22 \mathrm{I}, 267,4 \mathrm{I} 5$ vermis, $87,247,252,255$

Tuberculum anterior thalami, I40 acusticum, 149, 309 cinereum, I $3,289,30$ I

Twelfth nerve, $3,49,322$

Types of neurones. 168

Uncinate fasciculus, $237,239,240$

Uncrossed ponto-spinal tract, 280,373

Uncus hippocampi, 82, 94, I96, 235

Unipolar neurones, 400, 401

Uvula vermis cerebelli, $87,245,247$, 252,254

Vagus nerve, 5, 46, I53, 209, 293 , $32 \mathrm{I}$

Vallecula cerebelli, 36, 244, 25 $\mathrm{I}$

Valve of Vieussens, 246

Veins of cerebellum, 29

of cerebrum, $2 \mathrm{I}$

of medulla, 24

of pia mater, 13

of spinal cord, $331,33^{2}$

Velum interpositium, $\mathbf{I} 35$ (see chorioid tela of third ventricle)

medullare inferius, 244 superius, 245,263

Vena basilaris, 23

cerebri interna, 21

magna, 21

media, 23

terminalis, 235

Venæ cerebelli inferiores, 29 superiores, 29

cerebri, 2 I

externæ, 22

inferiores, 23

mediales, 22

superiores, 22

spinales extrenx, 332

internæ, 332

Ventral cochlear nucleus, 309

longitudinal fibers of pons, 272

stalk of thalamus, $100,212,226$, $23^{2}$
Ventral tegmental decussation, 156 , I 57, I 59

zone, $399,422,427$

Ventro-lateral cells of cord, $34 \mathrm{I}$, 345,343

Ventro-medial cells of cord, 34 I , 345, 340

Ventricle of corpus callosum, 89

Ventricular gray matter of cerebrum. $22 \mathrm{I}$

Ventriculus lateralis, II 2

quartus, 3 I 3

terminalis, 337,426

tertius, I 27

Vermis cerebelli, 242, 243, 4I9

inferior, 244

superior, 244

Vertebral artery, 3, 5, 14, 15, 27 ganglia, $40 \mathrm{I}$

Vessels of arachnoid, 9

Vestibular nerve, 280, 293, 320, 386 mucleus, 295, 297, 283, 302, 307

path, 386

Vestibulo-spinal reflex, 393

Vicq d'Azyr (foramen of), I 53

Vicq d'Azyr's fasciculus thalamomammillaris, 85 , IIO, 222, 237

Vieussens's medullary velum, 146 valve, 246

Vinculum, a, cerebelli, 250

Vision, center for macular, 67

Visual center (receptive), 8I, 98, 192 memory center, 67,69

Wallerian degeneration, I $7 \mathbf{I}$

White anterior commissure, 335, 35I matter of cerebellum, 262

of cerebrum, 224

of medulla, 290

of pons, 270

of spinal cord, $35^{\mathrm{I}}$

of thalamus, 208

Worm of cerebellum, 243

Writing center of Gordinier, $\mathbf{1 7 5}, 178$, 182

Zonal layer of cerebral cortex, 184

Zones of embryo, ventral and dorsal, 399 






